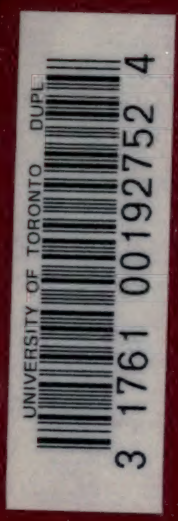



Digitized by the Internet Archive in 2007 with funding from Microsoft Corporation 


28 
(90) 


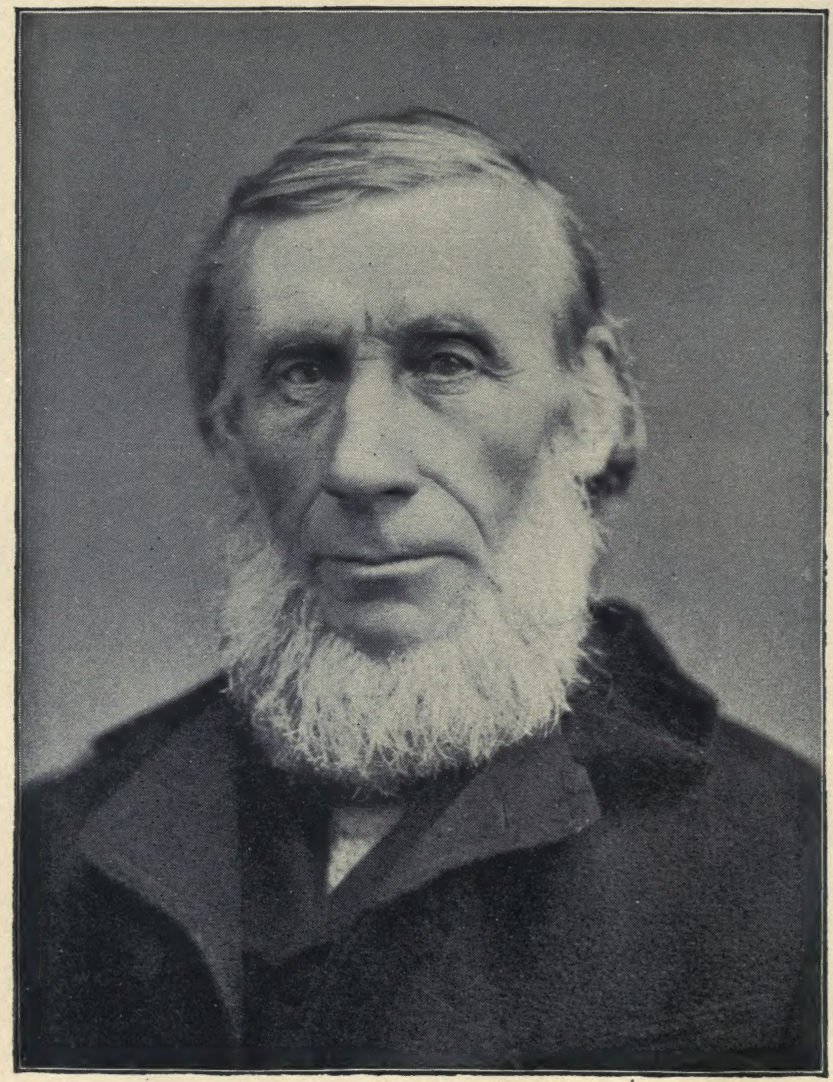

JOHN TYNDALL. 


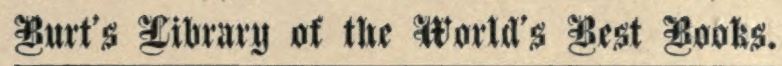

\title{
FRAGMENTS OF SCIENCE
}

\author{
A SERIES OF DETACHED
}

ESSAYS, ADDRESSES AND REVIEWS.

\section{By JOHN TYNDALL, F.R.S.}

FROM THE SIXTH LONDON EDITION.

COMPLETE IN ONE VOLUME.

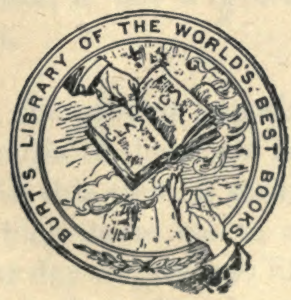

NEW YORK:

A. L. BUR'T, PUBLISHER. 


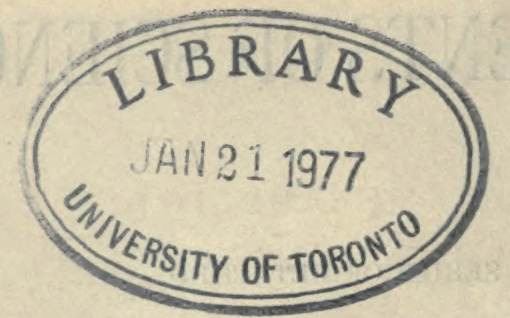

$$
\begin{aligned}
& 1942 \\
& Q \\
& 141 \\
& 18 \\
& 1910 \\
& \text { Cop.2 }
\end{aligned}
$$




\section{PREFACE.}

TO

\section{THE SIXTH EDITION。}

To A VOID unwieldiness of bulk this edition of the "Fragments" is published in one volume, instead, of as heretofore, in two.

The first part deals almost exclusively with the laws and phenomena of matter. The second trenches upon questions in which the phenomena of matter interlace more or less with those of mind.

New Essays have been added, while old ones have been revised, and in part recast. To be clear, without being superficial, has been my aim throughout.

In neither part have $\mathbf{I}$ aspired to sit in the seat of the scornful, but rather to treat the questions touched upon with a tolerance, if not a reverence, befitting their . difficulty and weight.

Holding, as I do, the nebular hypothesis, I am logically bound to deduce the life of the world from forces inherent in the nebula. With this view, it seemed but fair to associate the reasons which cause me to conclude that every attempt made in our day to generate life independently of antecedent life has utterly broken down. 



\section{CONTENTS.}

CHAPTER I.

Page.

The Coustitution of Nature..................... 1

CHAPTER II.

Radiation

CHAPTER III.

On Radiant Heat in Relation to the Color and Chemical Constitution of Bodies............................ 54

CHAPTER IV.

New Chemical Reactions Produced by Light............ 71 CHAPTER V.

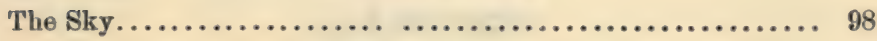

CHAPTER VI.

Voyage to Algeria to Observe the Eclipse................ 107

CHAPTER VII.

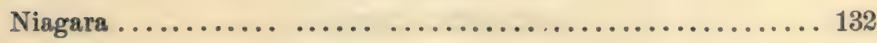

CHAPTER VIII.

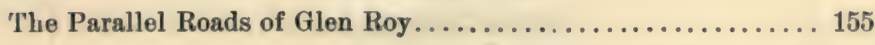

CHAPTER IX.

Alpine Sculpture.............................. 175

CHAPTER $\mathrm{X}$.

Recent Experiments on Fog-Signals................. 193

CHAPTER XI.

On the Study of Physics............................. 215 
CHAP'TER XII.

On C'rystalline and Slaty Cleavage.................. 232

CHAPTER XIII.

On Paramagnetic and Diamagnetic Forces................ 244

CHAPTER XIV.

Pliysical Basis of Solar Chemistry .................. 250

CHAPTER XV.

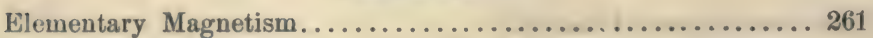

CHAPTER XVI.

On Force..................................... 281

CHAPTER XVII.

Contributions to Molecular Physics..................... 294

CHAPTER XVIII.

Life and Letters of Faraday... . . . . . . . . . . . . . . . 303

CHAPTER XIX.

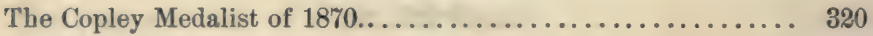

CHAPTER XX.

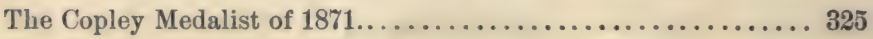

CHAPTER XXI.

Desth by Lightning. ........................... 332

CHAPTER XXII.

Science and the Spirits. ........................... 336

CHAPTER XXIII.

Reflections on Prayer and Natural Law................ 342

CHAPTER XXIV.

Miracles and Special Providences.................... 348

CHAPTER XXV.

On Prayer as a Form of Physical Energy ................ 371

CHAPTER XXVI.

Vitality. ................................. 376 
CHAPTER XXVII.

Matter and Force

CHAPTER XXVIII.

Scientific Materialism. 398

CHAPTER XXIX.

An Address to Students. 410

CHAPTER XXX.

Scientific Use of the Imagination.

CHAPTER XXXI.

The Belfast Address.

CHAPTER XXXII.

Apology for the Belfast Address.

CHAPTER XXXIII.

The Rev. James Martineau and the Belfast Address

CHAPTER XXXIV.

Fermentation, and Its Bearings on Surgery and Medicine..... 532 CHAPTER XXXV.

Spontaneous Generation. . .................... 562

CHAPTER XXXVI.

Science and Man. 596

CHAPTER XXXVII.

Professor Virchow and Evolution....................625

CHAPTER XXXVIII.

The Electric Light............................660 


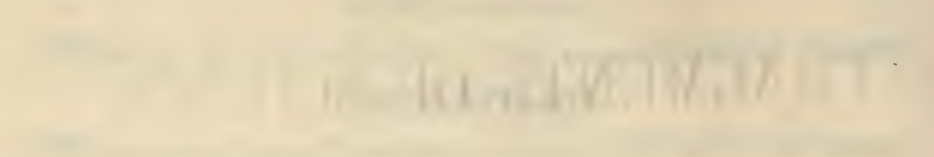




\section{FRAGMENTS OF SCIENCE.}

\section{CHAP'TER I.}

\section{THE CONSTItUTION OF NATURE.*}

WE CANNOT think of space as finite, for wherever in imagination we erect a boundary, we are compelled to think of space as existing beyond it. Thus by the incessant dissolution of limits we arrive at a more or less adequate idea of the infinity of space. But though compelled to think of space as unbounder, there is no mental necessity compelling us to think of it either as filled or empty; whether it is so or not must be decided by experiment and observation. That it is not entirely void the starry heavens declare; but the question still remains, are the stars themselves hung in vacuo? Are the vast regions which surround them, and across which their light. is propagated, absolutely empty? A century ago the inswer to this question, founded on the Newtouian theory, would have been, "No, for particles of light are incessantly shot through space." The reply of modern science is also negative, but on different grounds. It has the best possible reasons for rejecting the idea of luminiferous particles; but in support of the conclusion that the celestial spaces are occupied by matter, it is able to offer proofs almost as cogent as those which can be adduced of the existence of an atmosphere round the earth. Men's minds, indeed, rose to a conception of the celestial and universal atmosphere through the study of the terrestrial and local one. From the phenomena of sound, as displayed in the air, they ascended to the phenomena of light, as displayed in the ether; which is the name given to the interstellar medium.

The notion of this medium must not be considered as a

* “Fortnightly Review,” 1865, vol. iii. p. 129. 
vague or fanciful conception on the part of scientific men. Of its reality most of them are as convinced as they are of the existence of the sun and moon. The luminiferous ether has definite mechanical properties. It is almost infinitely more attenuated than any known gas, but its properties are those of a solid rather than of a gas. It resembles jelly rather than air. This was not the first conception of the ether, but it is that forced upon us by a more complete knowledge of its phenomena. A body thus constituted may have its boundaries; but although the ether may not be co-extensive with space, it must at all events extend as far as the most distant visible stars. In fact it is the vehicle of their light, and without it they could not be seen. 'This all-prevading substance takes up their molecular tremors, and conveys them with inconceivable rapidity to our organs of vision. It is the transported shiver of bodies cuuntless millions of miles distant, which translates itself in human consciousness into the splendor of the firmament at night.

If the ether have a boundary, masses of ponderable matter might be conceived to exist beyond it, but they could emit no light. Beyoud the ether dark suns might burn; there, under proper conditions, combustion might be carried on; fuel might consume unseen, and metals be fused in invisible fires. A body, moreover, once heated there, would continue forever heated; a sun or planet once molten, would continue forever molten. For, the loss of heat being simply the abstraction of molecular motion by the ether, where this medium is absent no cooling could occur. A sentient being, on approaching a heated body in this region, would be conscious of no augmentation of temperature. The gradations of warmth dependent on the laws of radiation would not exist, and actual contact would first reveal the heat of an extra ethereal sun.

Imagine a paddle-wheel placed in water and caused to rotate. From it, as a center, waves would issue in all directions, and a wader as he approached the place of disturbance would be met by stronger and stronger waves. This gradual augmentation of the impression made upon the wader is exactly analogous to the auginentation of light when we approach a luminous source. In the one case, however, the coarse common nerves of the body sultice; for the other we must have the finer optic nerve. 
But suppose the water withdrawn; the action at a distance would then cease, and, as far as the sense of touch is concerned, the wader would be first rendered conscious of the motion of the wheel by the blow of the paddles. The transference of motion from the paddles to the water is mechanically similar to the transference of molecular motion from the heated body to the ether; and the propagation of waves through the liquid is mechanically similar to the propagation of light and radiant heat.

As far as our knowledge of space extends, we are to conceive it as the holder of the luminiferous ether, throngh which are interspersed, at enormous distances apart, the ponderous nuclei of the stars. Associated with the star that most concerns us we have a group of dark planetary masses revolving at various distances round it, each again rotating on its own axis; and, finally, associated with some of these planets we have dark bodies of minor notethe moons. Whether the other fixed stars have similar planetary companions or not is to us a matter of pure conjecture, which may or may not enter into our conception of the universe. But probably every thoughtful person believes, with regard to those distant suns, that there is, in space, something besides our system on which they shine.

From this general view of the present condition of space, and of the bodies contained in it, we pass to the inquiry whether things were so created at the beginning. Was space furnished at once, by the fiat of Omnipotence, with these burning orbs? In presence of the revelations of science this view is fading more and more. Behind the orbs we now discern the nebulæ from which they have been condensed. And without going so far back as the nebulæ, the man of science can prove that out of common non-luminous matter this whole pomp of stars might have been evolved.

The law of gravitation enunciated by Newton is, that every particle of matter in the universe attracts every other particle with a force which diminishes as the square of the distance increases. Thus the sun and the earth mutually pull each other; thus the earth and the moon are kept in company; the force which holds every respective pair of masses together being the integrated force of their component parts. Under the operation of this force 
a stone falls to the ground and is warmed by the shock; under its operation meteors plunge into our atmosphere and rise to incandescence. Showers of such meteors doubtless fall incessantly upon the sun. Acted on by this force, the earth, were it stopped in its orbit to-morrow, would rush toward, and finally combine with the sun. Heat would also be developed by this collision. Mayer first, and Helmholtz and 'Thomson afterward, have calculated its amount. It would equal that produced by the combustion of more than 5,000 worlds of solid coal, all this heat being generated at the instant of collision. In the attraction of gravity, therefore, acting upon non-luminous matter, we have a source of heat more powerful than could be derived from any terrestial combustion. And were the matter of the universe thrown in cold detached fragments into space, and there abandoned to the mutual gravitation of its own parts, the collision of the fragments would in the end produce the fires of the stars.

The action of gravity upon matter originally cold may, in fact, be the origin of all light and heat, and also the proximate source of such other powers as are generated by light and heat. But we have now to inquire what is the light and what is the heat thus produced? This question has already been answered in a general way. Both light and heat are modes of motion. Two planets clash and come to rest; their motion, considered as that of masses, is destroyed, but it is in great part continued as a motion of their ultimate particles. It is this latter motion, taken up by the ether, and propagated through it with a velocity of 186,000 miles a second, that comes to us as the light and heat of suns and stars. The atoms of a hot body swing with inconceivable rapidity-billions of times in a second-but this power of vibration necessarily implies the operation of forces between the atoms themselves. It reveals to us that while they are held together by one force, they are kept asunder by another, their position at any moment depending on the equilibrium of attraction and repulsion. The atoms behave as if connected by elastic springs, which oppose at the same time their approach and their retreat, but which tolerate the vibration. called heat. The molecular vibration once set up is instantly shared with the ether, and diffused by it throughout space. 
We on the earth's surface live night and day in the midst of ethereal commotion. The medium is never still. The cloud canopy above us may be thick enough to shut out the light of the stars; but this canopy is itself a warm body, which radiates its thermal motion through the ether. The earth also is warm, and sends its heat-pulses incessantly forth. It is the waste of its molecular motion in space that chills the earth upon a clear night; it is the return of thermal motion from the clouds which prevents the earth's temperature, on a cloudy night, from falling so low. To the conception of space being filled, we must therefore add the conception of its being in a state of incessant tremor.

The sources of this vibration are the ponderable masses of the universe. Let us take a sample of these and examine it in detail. When we look to our planet, we find it to be an aggregate of solids, liquids, and gases. Subjected to a sufficiently low temperature, the two last would also assume the solid form. When we look at any one of these, we generally find it composed of still more elementary parts. We learn, for example, that the water of our rivers is formed by the union, in definite proportions, of two gases, oxygen and hydrogen. We know how to bring these constituents together, so as to form water: we also know how to analyze the water, and recover from it its two constituents. "So, likewise, as regards the solid portions of the earth. Our chalk hills, for example, are formed by a combination of carbon, oxygen, and calcinm. These are the so-called elements the union of which, in definite proportions, has resulted in the formation of chalk. The flints within the chalk we know to be a compound of oxygen and silicium, called silica; and our ordinary clay is, for the most part, formed by the union of silicium, oxygen, and the well-known light metal, aluminium. By far the greater portion of the earth's crust is compounded of the elementary substances mentioned in these few lines.

The principle of gravitation has been already described as an attraction which every particle of matter, however small, exerts on every other particle. With gravity there is no selection; no particular atoms choose, by preference, other particular atoms as objects of attraction; the attraction of gravitation is proportional simply to the quantity 
of the attracting matter, regardless of its quality. But in the molecular world which we have now entered matters are otherwise arranged. Here we have atoms between which a strong attraction is exercised, and also atoms between which a weak attraction is exercised. One atom can jostle another out of its place, in virtue of a superior force of attraction. But, though the amount of force exerted varies thus from atom to atom, it is still an attraction of the same mechanical quality, if I may nse the term, as that of gravity itself. Its intensity might be measured in the same way, namely by the amount of motion which it can generate in a certain time. Thus the attraction of gravity at the earth's surface is expressed by the number 32 ; because, when acting freely on a body for a second of time, gravity imparts to the body a velocity of thirtytwo feet a second. In like manner the mutual attraction of oxygen and hydrogen might be measured by the velocity imparted to the atoms in their rushing together. Of course such a unit of time as a second is not here to be thought of, the whole interval required by the atoms to cross the minute spaces which separate them amounting only to an inconceivably small fraction of a second.

It has been stated that when a body falls to the earth it is warmed by the shock. Here, to use the terminology of Mayer, we have a mechanical combination of the earth and the body. Let us suffer the falling body and the earth to dwindle in imagination to the size of atoms, and for the attraction of gravity let us substitute that of chemical affinity; we have then what is called a chemical combination. The effect of the union in this case also is the development of heat, and from the amount of heat generated we can infer the intensity of the atomic pull. Measured by ordinary mechanical standards, this is enormous. Mix eight pounds of oxygen with one of hydrogen, and pass a spark through the mixture; the gases instantly combine, their atoms rushing over the little distances which separate them. Take a weight of $4 \%, 000$ pounds to an elevation of 1,000 feet above the earth's surface, and let it fall; the energy with which it will strike the earth will not exceed that of the eight pounds of oxygen atoms, as they dash against one pound of -hydrogen atoms to form water. 
It is sometimes stated that gravity is distinguished from all other forces by the fact of its resisting conversion into other forms of force. Chemical affinity, it is said, can be converted into heat and light, and these again into magnetism and electricity: but gravity refuses to be so converted; being a force maintaining itself under all circumstances, and not capable of disappearing to give place to another. The statement arises from vagueness of thought. If by it be meant that a particle of matter can never be deprived of its weight, the assertion is correct; but the law which affirms the convertibility of natural forces was never intended, in the minds of those who understood it, to affirm that such a conversion as that here implied occurs in any case whatever. As regards convertibility into heat, gravity and chemical affinity stand on precisely the same footing. The attraction in the one case is as indestructible as in the other. Nobody affirms that when a stone rests upon the surface of the earth, the mutual attraction of the earth and stone is abolished; nobody means to affirm that the mutual attraction of oxygen for hydrogen ceases, after the atoms have combined to form water. What is meant, in the case of chemical affinity, is, that the pull of that affinity, acting through a certain space, imparts a motion of translation of the one atom toward the other. This motion is not heat, nor is the force that produces it heat. But when the atoms strike and recoil, the motion of translation is converted into a motion of vibration, which is heat. The vibration, however, so far from causing the extinction of the original attriction, is in part carried on by that attraction. The atoms recoil, in virtue of the elastic force which opposes actual contact, and in the recoil they are driven too far back. The original attraction then triumphs over the force of recoil, and urges the atoms once more together. Thus, like a pendulum, they oscillate, until their motion is imparted to the surrounding ether; or, in other words, until their heat becomes radiant heat.

In this sense, and in this sense only, is chemical affinity converted into heat. There is, first of all, the attraction between the atoms; there is, secondly, space between them. Across this space the attraction urges them. They collide, they recoil, they oscillate. 'There is here a change in the 
form of the motion, but there is no real loss. It is so with the attraction of gravity. T'o produce motion by gravity space must also intervene between the attracting bodies. When they strike together motion is apparently destroyed, but in reality there is no destruction. Their atoms are suddenly urged together by the shock; by their own perfect elasticity these atoms recoil; and thus is set up the molecular oscillation which, when communicated to the proper nerves, announces itself as heat.

It was formerly universally supposed that by the collision of unelastic bodies force was destroyed. Men saw, for example, that when two spheres of clay, painter's putty, or lead, for example, were urged together, the motion possessed by the masses, prior to impact, was more or less annihilated. 'They believed in an absolute destruction of the force of impact. Until recent times, indeed, no difficulty was experienced in believing this, whereas, at present, the ideas of force and its destruction refuse to be united in most philosophic minds. In the collision of elastic bodies, on the contrary, it was observed that the motion with which they clashed together was in great part restored by the resiliency of the masses, the more perfect the elasticity the more complete being the restitution. 'This led to the idea of perfectly elastic bodies-bodies competent to restore by their recoil the whole of the motion which they possessed before impactand this again to the idea of the conservation of force, as opposed to that destruction of force which was supposed to occur when unelastic bodies met in collision.

We now know that the principle of conservation holds equally good with elastic and unelastic bodies. Perfectly elastic bodies would develop no heat on collision. They would retain their motion afterward, though its direction might be changed; and it is only when sensible motion is wholly or partly destroyed, that heat is generated. This always occurs in unelastic collision, the heat developed being the exact equivalent of the sensible motion extinguished. This heat virtually declares that the property of elasticity, denied to the masses, exists among their atoms; by the recoil and oscillation of which the principle of conservation is vindicated.

But ambiguity in the use of the term "force" makes itself more and more felt as we proceed. We have called 
the attraction of gravity a force, without any reference to motion. A body resting ou a shelf is as much pulled by gravity as when, after having been pushed off the shelf, it falls toward the earth. We applied the term force also to that molecular attraction which we called chemical affinity. When, however, we spoke of the conservation of force, in the case of elastic collision, we meant neither a pull nor a push, which, as just indicated, might be exerted upon inert matter, but we meant force invested in motion-the vis viva, as it is called, of the colliding masses.

Furce in this form has a definite mechanical measure, in the amount of work that it can perform. The simplest form of work is the raising of a weight. A man walking uphill, or upstairs, with a pound weight in his hand, to an elevation say of sixteen feet, performs a certain amount of work, over and above the lifting of his own body. If he carries the pound to a height of thirty-two feet, he does twice the work; if to a height of furty-eight feet, he does three times the work; if to sixty-four feet, he does four times the work, and so on. If, moreover, he carries up two pounds instead of one, other things being equal, he does twice the work; if three, four, or five pounds, he does three, four, or five times the work. In fact, it is plain that the work performed depends on two factors, the weight raised and the height to which it is raised. It is expressed by the product of these two factors.

But a body may be caused to reach a certain elevation in opposition to the force of gravity, without being actually carried up. If a hodman, for example, wished to land a brick at an eleration of sixteen feet above the place where he stood, he would probably pitch it up to the bricklayer. Ile would thus impart, by a sudden effort, a velocity to the brick sufficient to raise it to the required height; the work accomplished by that effort being precisely the same as if he had slowly carried up the brick. The initial velocity to be imparted, in this case, is well known. 'I'o reach a height of sixteen feet, the brick must quit the man's band with a velocity of thirtytwo feet a second. It is needless to say, that a body starting with any velocity, would, if wholly unopposed or unaided, continue to move forever with the same velocity. But when, as in the case before us, the body is thrown 
upward, it moves in opposition to gravity, which incessintly returds its motion, and finally brings it to rest at an elevation of sixteen feet. If not here caught by the bricklayer, it would return to the hodman with an accelerated motion, and reach his hand with the precise velocity it possessed on quitting it.

An important relation between velocity and work is here to be pointed out. Supposing the hodmau competent to impart to the brick, at starting, a velocity of sixty-four feet a second, or twice its former velocity, would the amount of work performed be twice what it was in the first instance? No; it would be four times that quantity; for a body starting with twice the velocity of another, will rise to four times the height. In like manner, a threefold velocity will give a ninefold elevation, a fourfold velocity will give a sixteenfold elevation, and so on. The height attained, then, is not proportional to the initial velocity, but to the square of the velocity. As before, the work is also proportional to the weight elevated. Ilence the work which any moving mass whatever is competent to perform, in virtue of the motion which it at any moment possesses, is jointly proportional to its weight and the square of its velocity. Here, then, we have a second measure of work, in which we simply translate the idea of leight into its equivalent idea of motion.

In mechanies, the product of the mass of a moving body into the square of its velocity, expresses what is called the vis viva, or living force. It is also sometimes called the "mechanical effect." If, for example, a camnon pointed to the zenith urge a ball upward with twice the velocity imparted to a second ball, the former will rise to four times the height attained by the latter. If directed against a target, it will also do four times the execution. Hence the importance of imparting a high velocity to projectiles in war. Having thus cleared our way to a perfectly definite conception of the vis viva of moving masses, we are prepared for the announcement that the heat generated by the shock of a falling body against the earth is proportional to the vis viva annihilated. 'The heat is proportional to the square of the velocity. In the ase, therefore, of two cannon-balls of equal weight, if one strike a target with twice the velocity of the other, it will generate four times the heat, if with three times 
the velocity, it will generate nine times the heat, and so on.

Mr. Joule has shown that a pound weight falling from a height of 772 feet, or 772 pounds falling through one foot, will generate by its collision with the earth an amount of heat sufficient to raise a pound of water one degree Fahrenheit in temperature. 7972 "foot-pounds" constitute the mechanical equivalent of heat. Now, a body falling from a height of 772 feet, has, npon striking the earth, a velocity of 223 feet a second; and if this velocity were imparted to the body, by any other means, the quantity of heat generated by the stoppage of its motion would be that stated above. Six times that velocity, or 1,338 feet, would not be an inordinate one for a cannon-ball as it quits the gun. Hence, a cannon-ball moving with a verocity of 1,338 feet a second, would, by collision, generate an amount of heat competent to raise its own weight of water 36 degrees Fahrenheit in temperature. If composed of iron, and if all the heat generated were concentrated in the ball itself, its temperature would be raised about 360 degrees Fahrenheit; because one degree in the case of water is equivalent to about ten degrees in the case of iron. In artillery practice, the heat generated is usually concentrated upon the front of the bolt, and on the portion of the target first struck. By this concentration the heat developed becomes sufficiently intense to raise the dust of the metal to incandescence, a flash of light often accompanying collision with the target.

Let us now fix our attention for a moment on the gunpowder which urges the cannon-ball. This is composed of combustible matter, which if burned in the open air would yield a certain amount of heat. It will not yield this amount if it perform the work of urging a ball. The heat then generated by the gunpowder will fall short of that produced in the open air, by an amount equivalent to the vis viva of the ball; and this exact amount is restored by the ball on its collision with the target. In this perfect way are heat and mechanical motion connected.

Broadly enunciated, the principle of the conservation of force asserts, that the quantity of force in the universe is as unalterable as the quantity of matter; that it is alike 
impossible to create force and to annihilate it. But in what sense are we to understand this assertion? It would be manifestly inapplicable to the force of gravity as defined by Newton; for this is a force varying inversely as the square of the distance; and to affirm the constancy of a varying force would be self-contradictory. Yet, when the question is properly understood, gravity forms no exception to the law of conservation. Following the method pursued by Helmholtz, I will here attempt an elementary exposition of this law. Though destined in its applications to produce momentous changes in human thought, it is not difficult of comprehension.

For the sake of simplicity we will consider a particle of matter, which we may call $\mathrm{F}$, to be perfectly fixed, and a second movable particle, $\mathrm{D}$, placed at a distance from $\mathrm{F}$. We will assume that these two particles attract each other according to the Newtonian law. At a certain distance, the attruction is of a certain definite amount, which might be determined by means of a spring balance. At half this distance the attraction would be augmented four times; at a third of the distance, nine times; at onefourth of the distance, sixteen times, and so on. In every case, the attraction might be measured by determining, with the spring balance, the amount of tension just sufficient to prevent $\mathrm{D}$ from moving toward $\mathrm{F}$. 'Thus far we have nothing whatever to do with motion; we deal with statics, not with dynamics. We simply take into account the distance of D from F, and the pull exerted by gravity at that distance.

It is customary in mechanics to represent the magnitude of a force by a line of a certain length, a force of double magnitude being represented by a line of double length, and so on. Placing then the particle $D$ at a distance from $\mathrm{F}$, we can, in imagination, draw a straight line from $\mathrm{D}$ to $\mathrm{F}$, and at $\mathrm{D}$ erect a perpendicular to this line, which shall represent the amount of the attraction exerted on $D$. If $\mathrm{D}$ be at a very great distance from $\mathrm{F}$, the attraction will be very small, and the perpendicular consequently very short. If the distance be practically infinite, the attraction is practically nil. Let us now suppose at every point in the line joining $\mathrm{F}$ and $\mathrm{D}$, a perpendicular to be erected, proportional in length to the attraction exerted at that point; we thus obtain an infinite number of perpendicu- 
lars, of gradually increasing length, as D approaches $\mathrm{F}$. Uniting the ends of all these perpendiculars, we obtain a curve, and between this curve and the straight line joining $\mathrm{F}$ and $\mathrm{D}$ we have an area containing all the perpendiculars placed side by side. Each one of this infinite series of perpendiculars representing an attraction, or tension, as it is sometimes called, the area just referred to represents the sum of the tensions exerted upon the particle $\mathrm{D}$ during its passage from its first position to $\mathrm{F}$.

Up to the present point we have been dealing with tensions, not with motion. Thus far vis viva has been entirely foreign to our contemplation of $\mathrm{D}$ and $\mathrm{F}$. Let us now suppose $\mathrm{D}$ placed at a practically infinite distance from F; here, as stated, the pull of gravity would be infinitely small, and the perpendicular representing it would dwindle almost, to a point. In this position the sum of the tensions capable of being exerted on $\mathrm{D}$ would be a maximum. Let $\mathrm{D}$ now begin to move in obedience to the infinitesimal attraction exerted upon it. Motion being once set up, the idea of vis viva arises. In moving toward F the particle D consumes, as it were, the tensions. Let us fix our attention on $\mathrm{D}$, at any point of the path over which it is moving. Between that point and $\mathrm{F}$ there is a quantity of unused tensions; beyond that point the tensions have been all consumed, but we have in their place an equivalent quantity of vis viva. After $\mathrm{D}$ has passed any point, the tension previously in store at that point disappears, but not without having added, during the infinitely small duration of its action, a due amount of motion to that previously possessed by $\mathrm{D}$. The nearer $\mathrm{D}$ approaches to $\mathrm{F}$, the smaller is the sum of the tensions remaining, but the greater is the vis viva; the farther $\mathrm{D}$ is from $F$, the greater is the sum of the unconsumed tensions, and the less is the living force. Now the principle of conservation affirms not the constancy of the value of the tensions of gravity, nor yet the constancy of the vis viva, taken separately, but the absolute constancy of the value of both taken together. At the beginning the vis viva was zero, and the tension area was a maximum; close to $\mathrm{F}$ the vis viva is a maximum, while the tension area is zero. At every other point the work-producing power of the particle $\mathrm{D}$ consists in part of vis viva, and in part of tensions. 
If gravity, instead of being attraction, were repulsion, then, with the particles in contact, the sum of the tensions between $\mathrm{D}$ and $\mathrm{F}$ would be a maximum, and the vis viva zero. If, in obedience to the repulsion, D moved away from F, vis viva would be generated; and the farther D retreated from $F$ the greater would be its vis viva, and the less the amount of tension still available for producing motion. Taking repulsion as well as attraction into account, the principle of the conservation of force affirms that the mechanical value of the tensions and vires vivce of the material universe, so far as we know it, is a constant quantity. The universe, in short, possesses two kinds of property which are mutually convertible. The diminution of either carries with it the enhancement of the other, the total value of the property remaining unchanged.

The considerations here applied to gravity apply equally to chemical affinity. In a mixture of oxygen and hydrogen the atoms exist apart, but by the application of proper means they may be caused to rush together across that space that separates them. While this space exists, and as long as the atoms have not begun to move toward each other, we have tensions and nothing else. During their motion toward each other the tensions, as in the case of gravity, are converted into vis viva. After they clash we have still vis viva, but in another form. It was translation, it is vibration. It was molecular transfer, it is heat.

It is possible to reverse these processes, to unlock the combined atoms and replace them in their first positions. But, to accomplish this, as much heat would be required as was generated by their union. Such reversals occur daily and hourly in nature. By the solar waves, the oxygen of water is divorced from its hydrogen in the leaves of plants. As molecular vis viva the waves disappear, but in so doing they re-endow the atoms of oxygen and hydrogen with tension. 'The atoms are thus enabled to recombine, and when they do so they restore the precise amount of heat consumed in their separation. The same remarks apply to the compound of carbon and oxygen, called carbonic acid, which is exhaled from our lungs, produced by our fires, and found sparingly diffused everywhere throughout the air. In the leaves of plants the sun- 
beams also wrench the atoms of carbonic acid asunder, and sacrifice themselves in the act; but when the plants are burned, the amount of heat consumed in their production is restored.

'This, then, is the rhythmic play of Nature as regards her forces. Throughout all her regions she oscillates from tension to vis viva, from vis viva to tension. We have the same play in the planetary system. The earth's orbit is an ellipse, one of the foci of which is occupied by the sun. Imagine the earth at the most distarit part of the orbit. Her motion, and consequently her vis viva, is then a minimum. The planet rounds the curve, and begins its approach to the sun. In front it has a store of tensions, which are gradually consumed, an equivalent amount of vis viva being generater. When nearest to the sun the motion, and consequently the vis viva, reach a maximum. But here the available tensions have been used up. The earth rounds this portion of the curve and retreats from the sun. 'Tensions are now stored up, but vis viva is lost, to be again restored at the expense of the complenentary force on the opposite side of the curve. 'Thus beats the heart of the universe, but without increase or diminution of its total stock of force.

I have thus far tried to steer clear amid confusion, by fixing the mind of the reader upon things rather than upon names. But good names are essential; and here, as yet, we are not provided with such. We have had the force of gravity and living force-two utterly distinct things. We have had pulls and tensions; and we might have had the force of heat, the force of light, the force of magnetism, or the force of electricity - all of which terms have been employed more or less loosely by writers on physics. This confusion is happily avoided by the introduction of the term "energy," which embraces both tension and vis viva. Energy is possessed by bodies already in motion; it is then actual, and we agree to call it actual or dynamic energy. It is our old vis viva. On the other hand, energy is possible to bodies not in motion, but which, in virtue of attraction or repulsion, possess a power of motion which would realize itself if all hindrances were removed. Looking, for example, at gravity; a body on the earth's surface in a nosition from which it cammot fall to a lower one possesses no energy. It has neither motion nor power 
of motion. But the same body suspended at a height above the earth has a power of motion, though it may not have exercised it. Energy is possible to such a body, and we agree to call this potential energy. It consists of our old tensions. We, moreover, speak of the conservation of energy, insteal of the conservation of force; and say that the sum of the potential and dynamic energies of the material universe is a constant quantity.

A body cast upward consumes the actual energy of projection, and lays up potential energy. When it reaches its utmost height all its actual energy is consumed, its potential energy being then a maximum. When it returns, there is a reconversion of the potential into the actual. A pendulum at the limit of its swing possesses potential energy; at the lowest point of its arc its encrgy is all actual. A patch of snow resting on a mountain slope has potential energy; loosened, and shooting down as an avalanche, it possesses dynamic energy. The pine-trees growing on the Alps have potential energy; but rushing down the Holzrinne of the woodcutters they possess actual energy. 'The same is true of the mountains themselves. As long as the rocks which compose them can fall to a lower level, they possess potential energy, which is converted into actual when the frost ruptures their cohesion and hands them over to the action of gravity. The stone avalanches of the Matterhorn and Weisshorn are illustrations in point. The hammer of the great bell of Westminster, when raised before striking, possesses potential energy; when it falls, the energy becomes dynanic; and after the stroke, we have the rhythmic play of potential and dynamic in the vibrations of the bell. The same holds good for the molecular oscillations of a heated body. An atom is driven against its neighbor, anu recoils. The ultimate amplitude of the recoil being attained, the motion of the atom in that direction is checked, and for an instant its energy is all potential. It is then drawn toward its neighbor with accelerated speed; thus, by attraction, converting its potential into dynamic energy. Its motion in this direction is also finally checked, and again, for an instant, its energy is all potential. It once more retreats, converting, by repulsion, it.s potential into dynamic energy, till the latter attains a maximum, after which it is again changed into potential energy. 'Thus, what is true of the 
earth, as she swings to and fro in her yearly journey round the sun, is also true of her minutest atom. We have wheels within wheels, and rhythm within rhythm.

When a body is heated, a change of molecular arrangement always occurs, and to produce this change heat is consumed. Hence, a portion only of the heat communicated to the body remains as dynamic energy. Looking back on some of the statements made at the beginning of this article, now that our knowledge is more extensive, we see the necessity of qualifying them. When, for example, two bodies clash, heat is generated; but the heat, or molecular dynamic energy, developed at the moment of collision, is not the exact equivalent of the sensible dynamic energy destroyed. The true equivalent is this heat, plus the potential energy conferred upon the molecules by the placing of greater distances between them. This molecular potential energy is afterward, on the cooling of the body, converted into heat.

Wherever two atoms capable of uniting together by their mutual attractions exist separately, they form a store of potential energy. 'Thus our woods, forests, and coal-fields on the one hand, and our atmospheric oxygen on the other, constitute a vast store of energy of this kind - vast, but far from infinite. We have, besides our coalfields, metallic bodies more or less sparsely distributed through the earth's crust. 'These bodies can be oxydized; and hence they are, so far as they go, stores of energy. But the attractions of the great mass of the earth's crust are already satisfied, and from them no further energy can possibly be obtained. Ages ago the elementary constituents of our rocks clashed together and produced the motion of heat, which was taken up by the ether and carried away through stellar space. It is lost forever as far as we are concerned. In those ages the hot conflict of carbon, oxygen, and calcium produced the chalk and limestone hills which are now cold; and from this carbon, oxygen, and calcium no further energy can be derived. So it is with almost all the other constituents of the earth's crust. 'I'hey took their present form in obedience to molecular force; they turned their potential energy into dynamic, and yielded it as radiant heat to the universe, ages before man appeared upon this planet. For him a residue of potential energy remains, vast, truly, in relation to the life 
and wants of an individual, but exceedingly minute in comparison with the earth's primitive store.

'To sum up. The whole stock of energy or workingpower in the world consists of attractions, repulsions, and motions. If the attractions and repulsions be so circumstanced as to be able to produce motion, they are sources of working-power, but not otherwise. As stated a moment ago, the attraction exerted between the earth and a body at a distance from the earth's surface, is a source of working-power; because the body can be moved by the attraction, and in falling can perform work. When it rests at its lowest level it is not a source of power or energy, because it can fall no farther. But though it has ceased to be a source of energy, the attraction of gravity still acts as a force, which holds the earth and weight together.

'The same remarks apply to attracting atoms and molecules. As long as distance separates them, they can move across it in obedience to the attraction; and the motion thus produced may, by proper appliances, be caused to perform mechanical work. When, for example, two atoms of hydrogen unite with one of oxygen, to form water, the atoms are first drawn toward each other-they move, they clash, and then by virtue of their resiliency, they recoil and quiver. To this quivering motion we give the name of heat. This atomic vibration is merely the redistribution of the motion produced by the chemical affinity; and this is the only sense in which chemical affinity can be said to be converted into heat. We must not imagine the chemical attraction destroyed, or converted into anything else. For the atoms, when mutually clasped to form a molecule of water, are held together by the very attraction which first drew them toward each other. That which has really been expended is the pull exerted through the space by which the distance between the atoms has been diminished.

If this be understood, it will be at once seen that gravity, as before insisted on, may, in this sense, be said to be convertible into heat; that it is in reality no more an outstanding and inconvertible agent, as it is sometimes stated to be, than is chemical affinity. By the exertion of a certain pull through a certain space, a body is cansed to clash with a certain definite velocity against the earth. Ifeat is thereby developed, and this is the only sense in which gravity 
can be said to be converted into heat. In no case is the force which produces the motion annihilated or changed into anything else. The mutual attraction of the earth and weight exists when they are in contact, as when they were separate; but the ability of that attraction to employ itself in the production of motion does not exist.

The transformation, in this case, is easily followed by the mind's eye. First, the weight as a whole is set in motion by the attraction of gravity. This motion of the mass is arrested by collision with the earth, being broken up into molecular tremors, to which we give the name of heat.

And when we reverse the process, and enploy those tremors of heat to raise a weight-which is done through the intermediation of an elastic fluid in the steam-engine - a certain definite portion of the molecular motion is consumed. In this sense, and in this sense only, can the heat be said to be converted into gravity; or, more correctly, into potential energy of gravity. Here the destruction of the heat has created no new attraction; but the old attraction has conferred upon it a power of exerting a certain definite pull, between the starting-point of the falling weight and the earth.

When, therefore, writers on the conservation of energy speak of tensions being "consumed" and "generated," they do not mean thereby that old attractions have been annihilated, and new ones brought into existence, but that, in the one case, the power of the attraction to produce motion has been diminished by the shortening of the distance between the attracting bodies, while, in the other case, the power of producing motion has been augnented by the increase of the distauce. These remarks apply to all bodies, whether they be sensible masses or molecules.

Of the inner quality that enables matter to attract matter we know nothing; and the law of conservation makes no statement regarding that quality. It takes the facts of attraction as they stand, and affirms only the constancy of working-power. That power may exist in the form of MOTION; or it may exist in the form of FORCE, with distance to act through. The former is dynamic energy, the latter is potential energy, the constancy of the sum of both being affirmed by the law of conservation. The convertibility of natural forces consists solely in transformations of dynamic into potential, and of potential into dynamic 
energy. In no other sense has the convertibility of force any scientific meaning.

Grave errors have been entertained as to what is really intended to be conserved by the doctrine of conservation. This exposition I hope will tend to remove them.

\section{CHAPTER II. \\ RADIATION.* \\ 1. Visible and Invisible Radiation.}

BETWEEN the mind of man and the outer world are interposed the nerves of the human body, which translate, or enable the mind to translate, the impressions of that world into facts of consciousness and thought.

Different nerves are suited to the perception of different impressions. We do not see with the ear, nor hear with the eye, nor are we rendered sensible of sound by the nerves of the tongue. Out of the general assemblage of physical actions, each nerve, or group of nerves, selects and responds to those for the perception of which it is specially organized.

The optic nerve passes from the brain to the back of the eyeball and there spreads out, to form the retina, a web of nerve filaments, on which the images of external objects are projected by the optical portion of the eye. This nerve is limited to the apprehension of the phenomena of radiation, and, notwithstanding its marvelous sensibility to certain impressions of this class, it is singularly obtuse to other impressions.

Nor does the optic nerve embrace the entire range even of radiation. Some rays, when they reach it, are incompetent to evoke its power, while others never reach it at all, being absorbed by the humors of the eye. To all rays which, whether they reach the retina or not, fail to excite vision, we give the name of invisible or obscure rays. All non-luminons bodies emit such rays. There is no body in nature absolutely cold, and every body not absolutely cold emits rays of heat. But to render radiant heat fit to affect

* The Rede Lecture delivered in the Senate House before the University of Cambridge, May 16, 1865. 
the optic nerve a certain temperature is necessary. A cool poker thrust into a fire remains dark for a time, but when its temperature has become equal to that of the surrounding coals, it glows like them. In like manner, if a current of electricity, of gradually increasing strength, be sent through a wire of the refractory metal platinum, the wire first becomes sensibly warm to the touch; for a time its heat augments, still however remaining obscure; at length we can no longer touch the metal with impunity; and at a certain definite temperature it emits a feeble red light. As the current augments in power the light augments in brilliancy, until finally the wire appears of a dazzling white. The light which it now emits is similar to that of the sun.

By means of a prism Sir Isaac Newton unraveled the texture of solar light, and by the same simple instrument we can investigate the luminous changes of our platinum wire. In passing through the prism all its rays (and they are infinite in variety) are bent or refracted from their straight course; and, as different rays are differently refracted by the prism, we are by it enabled to separate one class of rays from another. By such prismatic analysis Dr. Draper has shown, that when the platinum wire first begins to glow, the light emitted is sensibly red. As the glow augments the red becomes more brilliant, but at the same time orange rays are added to the emission. Augmenting the temperature still further, yellow rays appear beside the orange; after the yellow, green rays are emitted; and after the green come, in succession, blue, indigo, and violet rays. To display all these colors at the same time the platinum wire must be white-hot: the impression of whiteness being in fact produced by the simultaneous action of all these colors on the optic nerve.

In the experiment just described we began with a platinum wire atan ordinary temperature, and gradually raised it to a white heat. At the beginning, and even before the electric current had acted at all upon the wire, it emitted invisible rays. For some time after the action of the current had commenced, and even for a time after the wire had become intolerable to the touch, its radiation was still invisible. The question now arises, What becomes of these invisible rays when the visible ones make their appearance? It will be proved in the sequel that they main- 
tain themselves in the radiation; that a ray once emitted continues to be emitted when the temperature is increased, and hence the emission from our platinum wire, even when it has attained its maximum brilliancy, consists of a mixture of visible and invisible rays. If, instead of the platinum wire, the earth itself were raised to incandescence, the obscure radiation which it now emits would continue to be emitted. To reach incandescence the planet would have to pass through all the stages of non-luminous radiation, and the final emission would embrace the rays of all these stages. There can hardly be a doubt that from the sun itself rays proceed similar in kind to those which the dark earth pours nightly into space. In fact, the various kind of obscure rays emitted by all the planets of - our system are included in the present radiation of the sun.

The great pioneer in this domain of science was Sir William Herschel. Causing a beam of solar light to pass through a prism, he resolved it into its colored constituents; he formed what is technically called the solar spectrum. Exposing thermometers to the successive colors he determined their heating power, and found it to augment from the violet or most refracted end, to the red or least refracted end of the spectrum. But he did not stop here. Pushing his thermometers into the dark space beyond the red he found that, though the light had disappeared, the radiant heat falling on the instruments was more intense than that at any visible part of the spectrum. In fact, Sir William Herschel showed, and his results have been verified by various philosophers since his time, that, besides its luminous rays, the sun pours forth a multitude of other rays, more powerfully calorific than the luminous ones, but entirely unsuited to the purposes of vision.

At the less refrangible end of the solar spectrum, then. the range of the sun's radiation is not limited by that of the eye. The same statement applies to the more refrangible end. Ritter discovered the extension of the spectrum into the invisible region beyond the violet; and, in recent times, this ultra-violet emission has had peculiar interest conferred upon it by the admirable researches of Professor Stokes. The complete spectrum of the sun consists, therefore, of three distinct parts: first, of ultra-red rays of high heating power, but unsuited to the purposes of vision; secondly, of luminous rays which display the succession of 
colors, red, orange, yellow, green, blue, indigo, violet; thirdly, of ultra-violet rays which, like the ultra-red ones, are incompetent to excite vision, but which, unlike the ultra-red rays, possess a very feeble heating power. In consequence, however, of their chemical energy these ultraviolet rays are of the utmost importance to the organic world.

\section{Origin and Character of Radiation. The Ether.}

When we see a platinum wire raised gradually to a white heat, and emitting in succession all the colors of the spectrum, we are simply conscious of a series of changes in the condition of our own eyes. We do not see the actions in which these successive colors originate, but the mind irresistibly infers that the appearance of the colors corresponds to certain contemporaneous changes in the wire. What is the nature of these changes?. In virtue of what condition does the wire radiate at all? "We must now look from the wire, as a whole, to its constituent atoms. Could we see those atoms, even before the electric current has begun to act upon them, we should find them in a state of vibration. In this vibration, indeed, consists such warmth as the wire then possesses. Locke enunciated this idea with great precision, and it has been placed beyond the pale of doubt by the excellent quantitative researches of Mr. Joule. "Heat," says Locke, "is a very brisk agitation of the insensible parts of the object, which produce in us that sensation from which we denominate the object hot; so what in our sensations is heat in the object is nothing but motion." When the electric current, still feeble, begins to pass through the wire, its first act is to intensify the vibrations already existing, by causing the atoms to swing through wider ranges. Technically speaking the amplitudes of the oscillations are increased. The current does this, however, withont altering the periods of the old vibrations, or the times in which they were execnted. But besides intensifying the old vibrations the current generates new and more rapid ones, and when a certain definite rapidity has been attained, the wire begins to glow. The color first exhibited is red, which corresponds to the lowest rate of vibration of which the eye is able to take cognizance. By angmenting the strength of the electric current more rapid vibrations are introduced, and orange rays appear. $\Lambda$ 
quicker rate of vibration produces yellow, a still quicker, green; and by further augmenting the rapidity, we pass through blue, indigo, and violet, to the extreme ultraviolet rays.

Such are the changes recognized by the mind in the wire itself, as concurrent with the visual changes taking place in the eye. But what connects the wire with this organ? By what means does it send such intelligence of its varying condition to the optic nerve? Heat being as defined by Locke, "a very brisk agitation of the insensible parts of an object," it is readily conceivable that on touching a heated body the agitation may communicate itself to the adjacent nerves, and announce itself to them as light or heat. But the optic nerve does not touch the hot platinum, and hence the pertinence of the question, By what agency are the vibrations of the wire transmitted to the eye?

The answer to this question involves one of the most important physical conceptions that the mind of man has yet achieved: the conception of a medium filling space and fitted mechanically for the transmission of the vibrations of light and heat, as air is fitted for the transmission of sound. This medium is called the luminiferous ether. Every vibration of every atom of our platinum wire raises in this ether a wave, which speeds through it at the rate of 186,000 miles a second. The ether suffers no rupture of continuity at the surface of the eye, the inter-molecular spaces of the various humors are filled with it; hence the waves generated by the glowing platinum can cross these humors and impinge on the optic nerve at the back of the eye.* 'Thus the sensation of light reduces itself to the acceptance of motion. Up to this point we deal with pure mechanics; but the subsequent translation of the shock of the ethereal waves into consciousness eludes mechanical science. As an oar dipping into the Cam generates systems of waves, which, speeding from the center of disturbance, finally stir the sedges on the river's bank, so do the vibrating atoms generate in the surrounding ether undulations, which finally stir the filaments of the retina. The motion thus imparted is transmitted with measurable, and not very great velocity to the brain, where, by a process

* The action here described is analogous to the passage of soundwaves through thick felt whose interstices are occupied by air. 
which the science of mechanics does not even tend to unravel, the tremor of the nervous matter is converted into the conscious impression of light.

Darkness might then be defined as ether at rest; light as ether in motion. But in reality the ether is never at rest, for in the absence of light-waves we have heat-waves always speeding through it. In the spaces of the universe both classes of undulations incessantly commingle. Here the waves issuing from uncounted centers cross, coincide, oppose, and pass through each other, without confusion or ultimate extinction. Every star is seen across the entanglement of wave-motions produced by all other stars. It is the ceaseless thrill caused by those distant orbs collectively in the ether, that constitutes what we call the "temperature of space." As the air of a room accommodates itself to the requirements of an orchestra, transmitting each vibration of every pipe and string, so does the inter-stellar ether accommodate itself to the requirements of light and heat. Its waves mingle in space without disorder, each being endowed with an individuality as indestructible as if it alone had disturbed the universal repose.

All vagueness with regard to the use of the terms "radiation" and "absorption" will now disappear. Radiation is the communication of vibratory motion to the ether; and when a body is said to be chilled by radiation, as for example the grass of a meadow on a starlight night. the meaning is, that the molecules of the grass have lost a portion of their motion, by imparting it to the medium in which they vibrate. On the other hand, the waves of ether may so strike against the molecules of a body exposed to their action as to yield up their motion to the latter; and in this transfer of the motion from the ether to the molecules consists the absorption of radiant heat. All the phenomena of heat are in this way reducible to interchanges of motion; and it is purely as the recipients or the donors of this motion, that we ourselves become conscions of the action of heat and cold.

\section{The Atomic Theory in reference to the Ether.}

The word "atoms" has been more than once employed in this discourse. Chemists have taught us that all matter is reducible to certain elementary forms to which they give this name. These atoms are endowed with powers of 
mutual attraction, and under suitable circumstances they coalesce to form compounds. Thus oxygen and hydrogen are elements when separate, or merely mixed, but they may be made to combine so as to form molecules, each consisting of two atoms of hydrogen and one of oxygen. In this condition they constitute water. So also chlorine and solium are elements, the former a pungent gas, the latter a soft metal; and they unite together to form chloride of sodium or common salt. In the same way the element nitrogen combines with hydrogen, in the proportion of one atom of the former to three of the latter, to form ammonia. Picturing in imagination the atoms of elementary bodies as little spheres, the molecules of compound bodies must be pictured as gromps of such spheres. This is the atomic theory as Dalton conceived it. Now if this theory have any foundation in fact, and if the theory of an ether pervading space, and constituting the vehicle of atomic motion, be founded in fact, it is surely of interest to examine whether the vibrations of elementary bodies are modified by the act of combination-whether as regards radiation and absorption, or, in other words, whether as regards the communication of motion to the ether, and the acceptance of motion from it, the deportment of the uncombined atoms will be different from that of the combined.

\section{Absorption of Radiant Heat by Gases.}

We have now to submit these considerations to the only test by which they can be tried, namely, that of experiment. An experiment is well defined as a question put to Nature; but; to avoid the risk of asking amiss, we ought to purify the question from all adjuncts which do not necessarily belong to it. Matter has been shown to be composed of elementary constituents, by the compounding of which all its varieties are produced. But, besides the chemical unions which they form, both elementary and compound bodies can unite in another and less intimate way. Gases and vapors aggregate to liquids and solids, without any change of their chemical nature. We do not yet know how the transmission of radiant heat may be affected by the entanglement due to cohesion; and, as our object now is to examine the influence of chemical union alone, we shall render our experiments more pure by liber- 
ating the atoms and molecules entirely from the bonds of cohesion, and employing them in the gaseous or vaporous form.

Let us endeavor to obtain a perfectly clear mental image of the problem now before us. Limiting in the first place our inquiries to the phenomena of absorption, we have to picture a succession of waves issuing from a radiant source and passing through a gas; some of them striking against the gaseous molecules and yielding up their motion to the latter; others gliding round the molecules, or passing through the intermolecular spaces without apparent hindrance. The problem before us is to determine whether such free molecules have any power whatever to stop the waves of heat; and if so, whether different molecules possess this power in different degrees.

In examining the problem let us fall back upon an actual piece of work, choosing as the source of our heat waves a plate of copper, against the back of which a steady sheet of flame is permitted to play. On emerging from the copper, the waves, in the first instance, pass through a space devoid of air, and then enter a hollow glass cylinder, three feet long and three inches wide. The two ends of this cylinder are stopped by two plates of rock-salt, a solid substance which offers a scarcely sensible obstacle to the passage of the calorific waves. After passing through the tube, the radiant heat falls upon the anterior face of a thermoelectric pile, ${ }^{*}$ which instantly converts the heat into an electric current. This current conducted round a magnetic needle deflects it, and the magnitude of the deflection is a measure of the heat falling upon the pile. This famous instrument, and not an ordinary thermometer, is what we shall use in these inquiries, but we shall use it in a somewhat novel way. As long as the two opposite faces of the thermo-electric pile are kept at the same temperature, no matter how high that may be, there is no current generated. The current is a consequence of a difference of temperature between the two opposite faces of the pile. Hence, if after the anterior face has received the heat from our radiating source, a second source, which we may call the compensating source, be permitted to radiate against the posterior face, this latter radiation will tend to neu-

*In the Appendix to the first chapter of "Heat as a Mode of Motion," the construction of the thermo-electric pile is fully explained. 
tralize the former. When the neutralization is perfect, the maynetic needle connected with the pile is no longer deflected, but points to the zero of the graduated circle over which it hangs.

And now let us suppose the glass tube, tlırough which the waves from the heated plate of copper are passing, to be exhausted by an air pump, the two sources of heat acting at the same time on the two opposite faces of the pile. When by means of an adjusting screen, perfectly equal quantities of heat are imparted to the two faces, the needle points to zero. Let any gas be now permitted to enter the exhausted tube; if its molecules possess any power of intercepting the calorific waves, the equilibrium previously existing will be destroyed, the compensating source will triumph, and a deflection of the magnetic needle will be the immediate consequence. From the deflections thus produced by different gases, we can readily deduce the relative amounts of wave-motion which their molecules intercept.

In this way the substances mentioned in the following table were examined, a small portion only of each being admitted into the glass tube. The quantity admitted in each case was just sufficient to depress a column of mercury associated with the tube one inch; in other words, the gases were examined at a pressure of one-thirtieth of an atmosphere. The numbers in the table express the relative amounts of wave-motion absorbed by the respective gases, the quantity intercepted by atmospheric air being taken as unity.

RADIATION THROUGH GASES.

Name of gas.

Relative

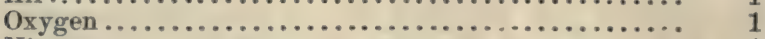

Nitrogen. . . . . . . . . . . . . . . . . . . . . . 1

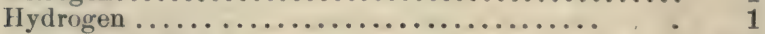

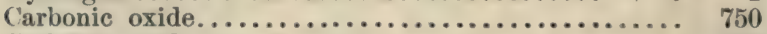

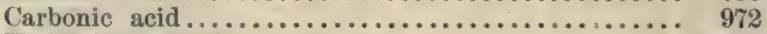

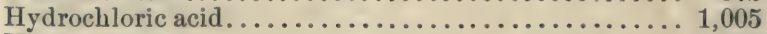

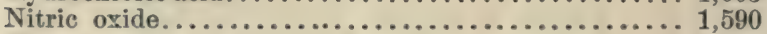

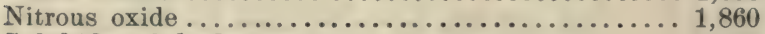

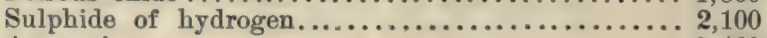

Ammonia. ............................ 5,460

Olefiant gas . ................................ 6,030

Sulphurous acid......................... 6,480 
Every gas in this table is perfectly transparent to light, that is to say, all waves within the limits of the visible spectrum pass through it without obstruction; but for the waves of slower period, emanating from our heated plate of copper, enormous differences of absorptive power are manifested. These differences illustrate in the most unexpected manner the influence of chemical combination. Thus the elementary gases, oxygen, hydrogen, and nitrogen, and the mixture atmospheric air, prove to be practical vacua to the rays of heat; for every ray, or, more strictly speaking, for every unit of waye-motion, which any one of them intercepts, perfectly transparent ammonia intercepts 5,460 units, olefiant gas 6,030 units, while sulphurous acid gas absorbs 6,480 units. What becomes of the wave-motion thus intercepted? It is applied to the heating of the absorbing gas. Through air, oxygen, hydrogen, and nitrogen, the waves of ether pass without absorption, and these gases are not sensibly changed in temperature by the most powerful calorific rays. The position of nitrous oxide in the foregoing table is worthy of particular notice. In this gas we have the same atoms in a state of chemical union, that exist uncombined in t? $\mathrm{e}$ atmosphere; but the absorption of the compound is 1,800 times that of air.

\section{Formation of Invisible Foci.}

'This extraordinary deportment of the elementary gases naturally directed attention to elementary bodies in other states of aggregation. Some of Melloni's results now attained a new significance. This celebrated experimenter had found crystals of sulphur to be highly pervious to radiant heat; he had also proved that lampblack, and black glass (which owes its blackness to the element carbon) were to a considerable extent transparent to calorific rays of low refrangibility. These facts, harmonizing so strikingly with the deportment of the simple gases, suggested further inquiry. Sulphur dissolved in bisulphide of carbon was found almost perfectly diathermic. The dense and deeply-colored element bromine was examined, and found competent to cut off the light of our most brilliant flames, while it transmitted the invisible calorific rays with extreme freedom. Iodine, the companion element of bromine, was next thought of, but it was found impracticable 
to examine the substance in its usual solid condition. It however dissolves freely in bisulphide of carbon. 'There is no chemical union between the liquid and the ioaine; it is simply a case of solution, in which the uncombined atoms of the element can act upon the radiant heat. When permitted to do so, it was found that a layer of dissolved iodine, sufficiently opaque to cut off the light of the midday sun, was almost absolutely transparent to the invisible calorific rays.*

By prismatic analysis Sir William Herschel separated the luminous from the non-luminous rays of the sun, and he also sought tender the obscure rays visible by concentration. Intercepting the luminous portion of his spectrum he brought, by a converging lens, the ultra-red rays to a focus, but by this condensation he obtained no light. The solution of iodine offers a means of filtering the solar beam, or failing it, the beam of the electric lamp, which renders attainable far more powerful foci of invisible rays than could possibly be obtained by the method of Sir William Herschel. For to form his spectrum he was obliged to operate upon sular light which had passed through a narrow slit or through a small aperture, the amount of the obscure heat being limited by this circumstance. But with our opaque solution we may employ the entire surface of the largest lens, and having thus converged the rays, luminous and non-luminous, we can intercept the former by the iodine, and do what we please with the latter. Experiments of this character, not only with the iodine solution, but also with black glass aud layers of lampblack, were publicly performed at the Royal Institution in the early part of 1862 , and the effects at the foci of invisible rays, then obtained, were such as had never been witnessed previously.

In the experiments here referred to, glass lenses were employed to concentrate the rays. But glass, though highly transparent to the luminous, is in a high degree opaque to the invisible heat-rays of the electric lamp, and hence a large portion of those rays was intercepted by the glass. The obvious remedy here is to employ rock-salt lenses instead of glass ones, or to abandon the use of lenses

* Professor Dewar has recently succeeded in producing a medium highly opaque to light, aud highly transparent to obscure heat, by fusing together sulphur and iodine. 
wholly, and to concentrate the rays by a metallic mirror. Both of these improvements have been introduced, and, as anticipated, the invisible foci have been thereby rendered more intense. The mode of operating remains however the same, in principle, as that made known in 1862. It was then found that an instant's exposure of the face of the thermo-electric pile to the focus of invisible rays dashed the needles of a coarse galvanometer violently aside. It is now found that on substituting for the face of the thermo-electric pile a combustible body, the invisible rays are competent to set that body on fire.

\section{Visible and Invisible Rays of the Electric Light.}

We have next to examine what proportion the nonluminous rays of the electric light bear to the luminous ones. This the opaque solution of iodine enables us to do with an extremely close approximation to the truth. The pure bisulphide of carbon, which is the solvent of the iodine, is perfectly transparent to the luminous, and almost perfectly transparent to the dark rays of the electric lamp. Supposing the total radiation of the lamp to pass through the transparent bisulphide, while through the solution of iodine only the dark rays are transmitted. If we determine, by means of a thermo-electric pile, the total radiation, and deduct from it the purely obscure, we obtain the value of the purely luminous emission. Experiments performed in this way prove that if all the visible rays of the electric light were converged to a focus of dazzling brilliancy, its heat would only be one-eighth of that produced at the unseen focus of the invisible rays.

Exposing his thermometers to the successive colors of the solur spectrum, Sir William Herschel determined the heating power of each, and also that of the region beyond the extreme red. Then drawing a straight line to represent the length of the spectrum, he erected, at various points, perpendiculars to represent the calorific intensity existing at those points. Uniting the ends of all his perpendiculars, he obtained a curve which showed at a glance the manner in which the heat was distributed in the solar spectrum. Professor Müller of Freiburg, with improved instruments, afterward male similar experiments, and constructed a more accurate diagram of the same kind. We have now to examine the distribution of heat in the spec- 
trum of the electric light; and for this purpose we shall employ a particular form of the thermo-electric pile, devised by Melloni. Its face is a rectangle, which by means of movable side-pieces can be rendered as narrow as desired. We can, for example, have the face of the pile the tenth, the hundredth, or even the thousandth of an inch in breadth. By means of an endless screw, this linear thermo-electric pile may be moved through the entire spectrum, from the violet to the red, the amount of heat falling upon the pile at every point of its march being declared by a magnetic needle associated with the pile.

When this instrument is brought up to the violet end of the spectrum of the electric light, the heat is found to be insensible. As the pile is gradually moved from the violet end toward the red, heat soon manifests itself, augmenting as we approach the red. Of all the colors of the visible spectrum the red possesses the highest heating power. On pushing the pile into the dark region beyond the red, the heat, instead of vanishing, rises suddenly and enormously in intensity, until at some distance beyond the red it attains a maximum. Moving the pile still forward, the thermal power falls, somewhat more rapidly than it rose. It then gradually shades away, but, for a distance beyond the red greater than the length of the whole visible spectrum, signs of heat may be detected.

Drawing a datum line, and erecting along it perpendiculars, proportional in length to the thermal intensity at the respective points, we obtain the extraordinary curve, shown on the oppossite page, which exhibits the distribution of heat in the spectrum of the electric light. In the region of dark rays, beyond the red, the curve shoots up to B, in a steep and massive peak-a kind of Matterhorn of heat, which dwarfs the portion of the diagram C D E, representing the luminous radiation. Indeed the idea forced upon the mind by this diagram is that the light rays are a mere insignificant appendage to the heat-rays represented by the area A B C D, thrown in, as it were, by nature for the purpose of vision.

The diagram drawn by Professor Müller to represent the distribution of heat in the solar spectrum is not by any means so striking as that just described, and the reason, doubtless, is that prior to reaching the earth the solar rays have to traverse our atmosphere. By the aqueous vapor 


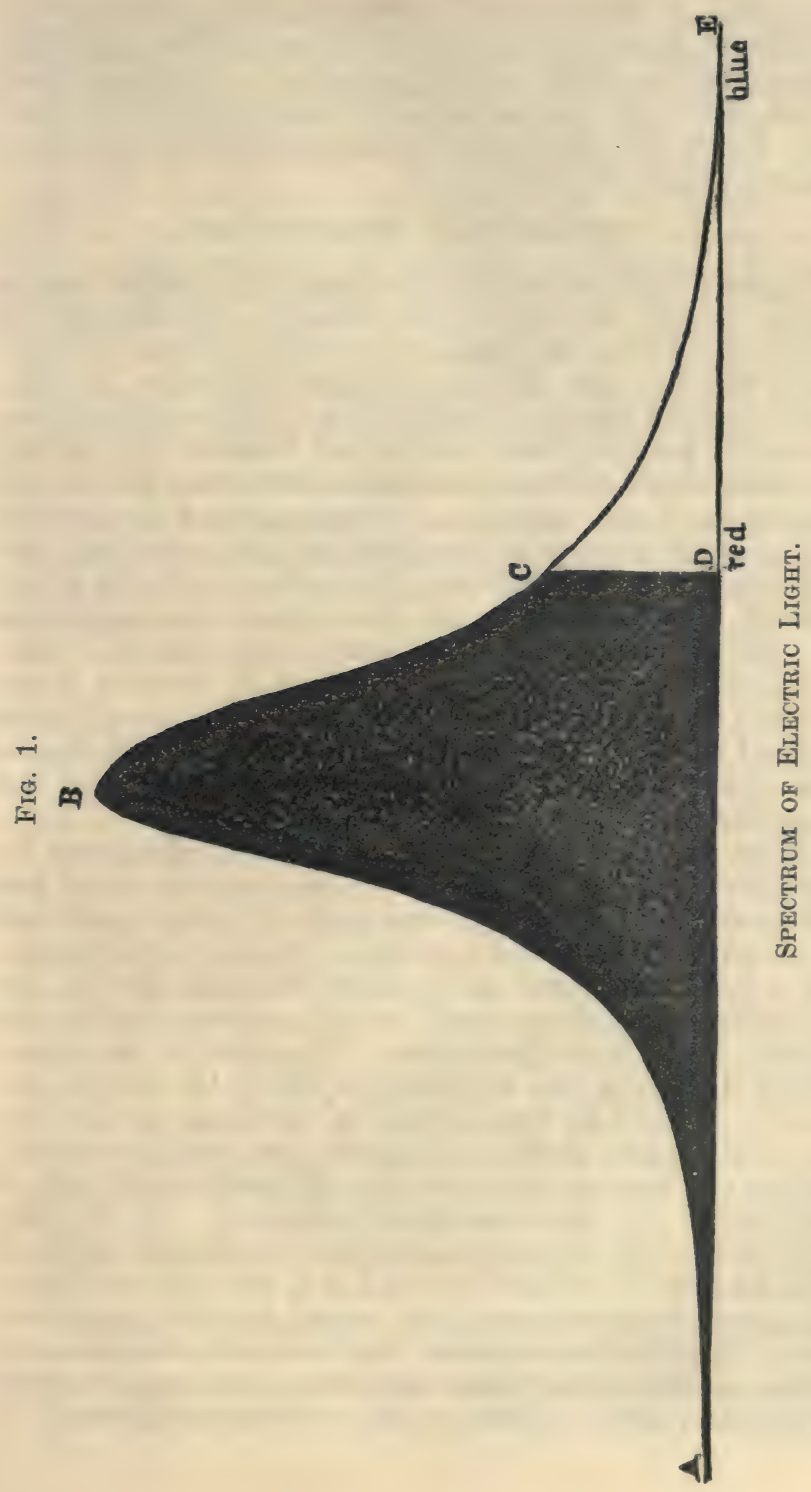


there diffused, the summit of the peak representing the sun's invisible radiation is cut off. A similar lowering of the mountain of invisible heat is observed when the rays from the electric light are permitted to pass through a film of water, which acts upon them as the atmospheric vapor acts upon the rays of the sun.

\section{\%. Combustion by Invisible Rays.}

The sun's invisible rays far transcend the visible ones in heating power, so that if the alleged performances of Archimedes during the siege of Syracuse had any foundation in fact the dark solar rays would have been the philosopher's chief agents of combustion. On a small scale we can readily produce, with the purely invisible rays of the electric light, all that Archimedes is said to have performed with the sun's total radiation. Placing behind the electric light a small concave mirror, the rays are converged, the cone of reflected rays and their point of convergence being rendered clearly visible by the dust always floating in the air. Placing between the luminous focus and the source of rays our solution of iodine, the light of the cone is entirely cut away; but the intolerable heat experienced when the hand is placed, even for a moment, at the dark focus, shows that the calorific rays pass unimpeded through the opaque solution.

Almost anything that ordina:y fire can effect may be accomplished at the focus of invisible rays; the air at the focus remaining at the same time perfectly cold, on account of its transparency to the heat-rays. An air thermometer, with a hollow rock-salt bulb, would be unaffected by the heat of the focus: there would be no expansion, and in the open air there is no convection. The ether at the focus, and not the air, is the substance in which the heat is embodied. A block of wood, placed at the focus, absorbs the heat, and dense volumes of smoke rise swiftly upward, showing the manner in which the air itself would rise, if the invisible rays were competent to heat it. At the perfectly dark focus dry paper is instantly inflamed; chips of wood are speedily burned up; lead, tin, and zinc are fused; and disks of charred paper are raised to vivid incandescenee. It might be supposed that the obscure rays would show no preference for black over white; but they do show a preference, and to obtain rapid combustion, the body, if 
not already black, ought to be blackened. When metals are to be burned, it is necessary to blacken or otherwise tarnish them, so as to diminish their reflective power. Blackened zinc foil, when brought in to the focus of invisible rays, is instantly cansed to blaze, and burns with its peculiar purple light. Magnesium wire flattened, or tarnished magnesium ribbon, also bursts into flame. Pieces of charcoal suspended in a receiver full of oxygen are also set on fire when the invisible focus falls upon them; the dark rays after having passed through the receiver, still possessing sufficient power to ignite the charcoal, and thus initiate the attack of the oxygen. If, instead of being plunged in oxygen, the charcoal be suspended in vacuo, it immediately glows at the place where the focus falls.

\section{Transmutation of Rays: * Calorescence.}

Eminent experimenters were long occupied in demonstrating the substantial identity of light and radiant heat, and we have now the means of offering a new and striking proof of this identity. A concave mirror produces, beyond the object which it reflects, an inverted and magnified image of the object. Withdrawing, for example, our iodine solution, an intensely luminous inverted image of the carbon points of the electric light is formed at the focus of the mirror employed in the foregoing experiments. When the solution is interposed, and the light is cut awav, what becomes of this image? It disappears from sight; but an invisible thermograph remains, and it is only the peculiar constitution of our eyes that disqualifies us from seeing the picture formed by the calorific rays. Falling on white paper, the image chars itself out: falling on black paper, two holes are pierced in it, corresponding to the images of the two coke points: but falling on a thin plate of carbon in vacuo, or upon a thin sheet of platinized platinum, either in vacuo or in air, radiant heat is converted into light, and the image stamps itself in vivid incandescence upon both the carbon and the metal. Results similar to those obtained with the electric light have also been obtained with the invisible rays of the lime-light and of the sun.

Before a Cambridge audience it is hardly necessary to

* I borrow this term from Professor Challis, "Philosophical Magazine," vol. xii., p. 521. 
refer to the excellent researches of Professor Stokes at the opposite end of the spectrum. The abore results constitute a kind of complement to his discoveries. Professor Stokes named the phenomena which he has discovered and investigated Fluorescence; for the new phenomena here described I have proposed the term Calorescence. He, by the interposition of a proper medium, so lowered the refrangibility of the ultra-violet ravs of the spectrum as to render them visible. Here, by the interposition of the platinum foil, the refrangibility of the ultra-red rays is so exalted as to render them visible. Looking through a prism at the incandescent image of the carbon points, the light of the image is decomposed, and a complete spectrum is obtained. The invisible rays of the electric light, remolded by the atoms of the platinum, shine thus visibly forth; ultra-red rays being converted into red, orange, yellow, green, blue, indigo, violet, and ultra-violet ones. Could we, moreover, raise the original source of rays to a sufficiently high temperature, we might not only obtain from the dark rays of such a source a single incandescent image, but from the dark rays of this image we might obtain a second one, from the dark rays of the second a third, and so on-a series of complete images and spectra being thus extracted from the invisible emission of the primitive source.*

* On investigating the calorescence produced by rays transmitted through glasses of various colors, it was found that in the case of certain specimens of blue glass, the platinum foil glowed with a pink or purplish light. The effect was not subjective, and considerations of obvious interest are suggested by it. Different kinds of black glass differ notably as to their power of transmitting radiant heat. When thin, some descriptions tint the sun with a greenish hue: others make it appear a glowing red without any trace of green. The latter are far more diathermic than the former. In fact, carbon when perfectly dissolved and incorporated with a good white glass, is highly transparent to the calorific rays, and by employing it as an absorbent the phenomena of "calorescence" may be obtained, though in a less striking form than with the iodine. The black glass chosen for thermometers, and intended to absorb completely the solar heat, may entirely fail in this object, if the glass in which the carbon is incorporated be colorless. To render the bulb of a thermometer a perfect absorbent, the glass ought in the first instance to be green. Soon after the discovery of fluorescence the late Dr. William Allen Miller pointed to the lime-light as an illustration of exalted refrangibility. Direct experiments have since entirely confirmed the view expressed at page 210 of his work on "Chemistry," published in 1855 . 


\section{Deadness of the Optic Nerve to the Calorific Rays.}

The layer of iodine used in the foregoing experiments intercepted the rays of the noonday sun. No trace of light from the electric lamp was visible in the darkest room, even when a white screen was placed at the focus of the mirror employed to concentrate the light. It was thought, however, that if the retina itself were brought into the focus the sensation of light might be experienced. The danger of this experiment was twofold. If the dark rays were absorbed in a high degree by the humors of the eye the albumen of the humors might coagulate along the line of the rays. If, on the contrary, no such high absorption took place, the rays might reach the retina with a force sufficient to destroy it. To test the likelihood of these results, experiments were made on water and on a solution of alum, and they showed it to be very improbable that in the brief time requisite for an experiment any serious damage could be done. The eye was therefore cansed to approach the dark focus, no defense, in the first instance, being provided; but the heat, acting upon the parts surrounding the pupil, could not be borne. An aperture was therefore pierced in a plate of metal, and the eye, placed behind the aperture, was caused to approach the point of convergence of invisible rays. The focus was attained, first by the pupil and afterward by the retina. Remoring the eye, but permitting the plate of metal to - remain, a sheet of platinum foil was placed in the position occupied by the retina a moment before. The platinum became red-hot. No sensible damage was done to the eye by this experiment; no impression of light was produced; the optic nerve was not even conscious of heat.

But the humors of the eye are known to be highly impervious to the invisible calorific rays, and the question therefore arises, "Did the radiation in the foregoing experiment reach the retina at all?" The answer is, that the rays were in part transmitted to the retina, and in part absorbed by the humors. Experiments on the eye of an ox showed that the proportion of obscure rays which reached the retina amounter to 18 per cent. of the total radiation; while the luminous emission from the electric light amounts to no more than 10 per cent. of the same total. Were the purely luminous rays of the electric lamp con- 
verged by our mirror to a focus, there can be no doubt as to the fate of a retina placed there. Its ruin would be inevitable; and yet this would be accomplished by an amount of wave-motion but little more than half of that which the retina, without exciting consciousness, bears at the focus of invisible rays.

'This subject will repay a moment's further attention. At a common distance of a foot the visible radiation of the electric light employed in these experiments is 800 times the light of a candle. At the same distance, the portion of the radiation of the electric light which reaches the retina, but fails to excite vision, is about 1,500 times the luminous radiation of the candle.* But a candle on a clear night can readily be seen at a distance of a mile, its light at this distance being less than $\frac{\overline{2} 0,000,000}{1}$ of its light at the distant of a foot. Hence, to make the candle-light a mile off equal in power to the non-luminous radiation received from the electric light at a foot distance, its intensity would have to be multiplied by $1,500 \times 20,000,000$, or by thirty thousand millions. Thus the thirty thousand millionth part of the invisible radiation from the electric light, received by the retina at the distance of a foot, would, if slightly changed in character, be amply sufficient to provoke vision. Nothing could more forcibly illustrate that special relationship supposed by Melloni and others to subsist between the optic nerve and the oscillating periods of luminous bodies. 'The optic nerve responds, as it were, to the waves with which it is in consonance, while it refuses to be excited by others of almost infinitely greater energy, whose periods of recurrence are not in unison with its own.

\section{Persistence of Rays.}

At an early part of this lecture it was affirmed, that when a platinum wire was gradually raised to a state of high incandescence, new rays were constantly added, while the intensity of the old ones was increased. 'Thus, in Dr. Draper's experiments, the rise of temperature that generated the orange, yellow. green, and blue angmented the intensity of the red. What is true of the red is true of

\footnotetext{
* It will be borne in mind that the heat which any ray, luminous or non-luminous, is competent to generate is the true measure of the energy of the ray.
} 
every other ray of the spectrum, visible and invisible. We cannot indeed see the augmentation of intensity in the region beyond the red, but we can measure it and express it numerically. With this view the following experiment was performed: A spiral of platinum wire was surrounded by a small glass globe to protect it from currents of air; through an orifice in the globe the rays cuuld pass from the spiral and fall afterward upon a thermo-electric pile. Placing in front of the orifice an opaque solution of iodine, the platinum was gradually raised from a low dark heat to the fullest incandescence, with the following results:

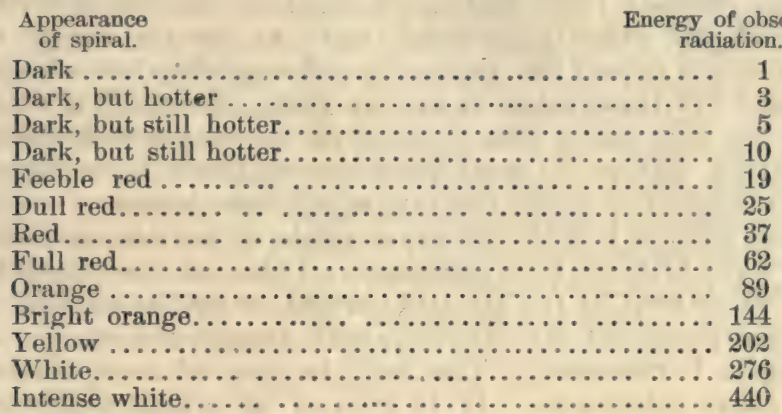

Thus the augmentation of the electric current, which raises the wire from its primitive dark condition to an intense white heat, exalts at the same time the energy of the obscure radiation, until at the end it is fully 440 times what it was at the beginning.

What has been here proved true of the totality of the ultra-red rays is true for each of them singly. Placing our linear thermo-electric pile in any part of the ultra-red spectrum, it may be proved that a ray once emitted continues to be emitted with increased energy as the temperature is augmented. The platinum spiral, so often referred to, being raised to whiteness by an electric current, a brilliant spectrum was formed from its light. A linear thermoelectric pile was placed in the region of obscure rays beyond the red, and by diminishing the current the spiral was reduced to a low temperature. It was then caused to pass through various degrees of darkness and incandescence, with the following results: 
Appearance

Energy of of spiral.

obscure rays.

Dark ................................... 1

Dark ................................ 6

Faint red .............................. 10

Dull red................................. 13

Red .................................. 18

Full ręd................................. 27

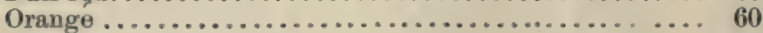

Yellow ............................... 93

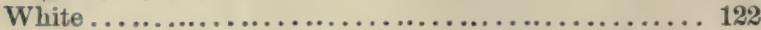

Here, as in the former case, the dark and bright radiations reached their maximum together; as the one augmented, the other augmented, until at last the energy of the obscure rays of the particular refrangibility here chosen became 122 times what it was at first. To reach a white heat the wire has to pass through all the stages of invisible radiation, but in its most brilliant condition it embraces, in an intensified form, the rays of all those stages.

And thus it is with all other kinds of matter, as far as they have hitherto been examined. Coke, whether brought to a white heat by the electric current, or by the oxyhydrogen jet, pours out invisible rays with augmented energy, as its light is increased. The same is true of lime, bricks, and other substances. It is true of all metals which are capable of being heated to incandescence. It also holds good for phosphorus burning in oxygen. Every gush of dazzling light has associated with it a gush of invisible radiant heat, which far transcends the light in energy. This condition of things applies to all bodies capable of being raised to a white heat, either in the solid or the molten condition. It would doubtless also apply to the luminous fogs formed by the condensation of incandescent vapors. In such cases when the curve representing the radiant energy of the body is constructed, the obscure radiation towers upward like a mountain, the luminous radiation resembling a mere "spur" at its base. From the very brightness of the light of some of the fixed stars we may infer the intensity of that dark radiation, which is the precursor and inseparable associate of their luminous rays.

We thus find the luminous radiation appearing when the radiant body has attained a certain temperature; or, in other words, when the vibrating atoms of the body have 
attained a certain width of swing. In solid and molten bodies a certain amplitude cannot be surpassed without the introduction of periods of vibration, which provoke the sense of vision. How are we to figure this? If permitted to speculate, we might ask, are not these more rapid vibrations the progeny of the slower? Is it not really the mutual action of the atoms, when they swing through very wide spaces, and thus encroach upon each other, that causes them to tremble in quicker periods? If so, whatever be the agency by which the large swinging space is obtained, we shall have light-giving vibrations associated with it. It matters not whether the large amplitudes be produced by the strokes of a hammer, or by the blows of the molecules of a non-luminous gas, like air at some height above a gasflame; or by the shock of the ether particles when transmitting radiant heat. The result in all cases will be incandescence. Thus, the invisible waves of our filtered electric beam may be regarded as generating synchronous vibrations among the atoms of the platinum on which they impinge; but, once these vibrations have attained a certain amplitude, the mutual jostling of the atoms produces quicker tremors, and the light-giving waves follow as the necessary product of the heat-giving ones.

\section{Absorption of Radiant Heat by Vapors and Odors.}

We commenced the demonstrations brought forward in this lecture by experiments on permanent gases, and we have now to turn our attention to the vapors of volatile liquids. Here, as in the case of the gases, vast differences have been proved to exist between various kinds of molecules, as regards their power of intercepting the calorific waves. While some vapors allow the waves a comparatively free passuge, the faintest mixture of other vapor's causes a deflection of the magnetic needle. Assuming the absorption effected by air, at a pressure of one atmosphere, to be unity, the following are the absorptions effected by a series of vapors at a pressure of one-sixtieth of an atmosphere:

Name of vapor.

Bisulphide of carbon.................... 47

Iodide of methyl ............................ 115

Benzol .................................. 136

Amylene. ................................. 321

Sulphuric ether.......................... 440

Formic ether ................................. 548

Acetic ether.............................6 612 
Bisulphide of carbon is the most transparent vapor in this list; and acetic ether the most opaque; one-sixtieth of an atmosphere of the former, however, produces 47 times the effect of a whole atmosphere of air, while one-sixtieth of an atmosphere of the latter produces 612 times the effect of a whole atmosphere of air. Reducing dry air to the pressure of the acetic ether here employed, and comparing them then together, the quantity of wave-motion intercepted by the ether would be many thoisand times that intercepteil by the air.

Any one of these vapors discharged into the free atmosphere, in front of a body emitting obscure rays, intercepts more or less of the radiation. A similar effect is produced by perfumes diffused in the air, though their attenuation is known to be almost infinite. Carrying, for example, a current of dry air over bibulous paper, moistened by patchouli, the scent taken up by the current absorbs 30 times the quantity of heat intercepted by the air which carries it; and yet patchouli acts more feebly on radiant heat than any other perfume yet examined. Here follow the results obtained with various essential oils, the odor, in each case, being carried by a current of dry air into the tube alrealy employed for gases and vapors:

Name of perfume.

Absorption.

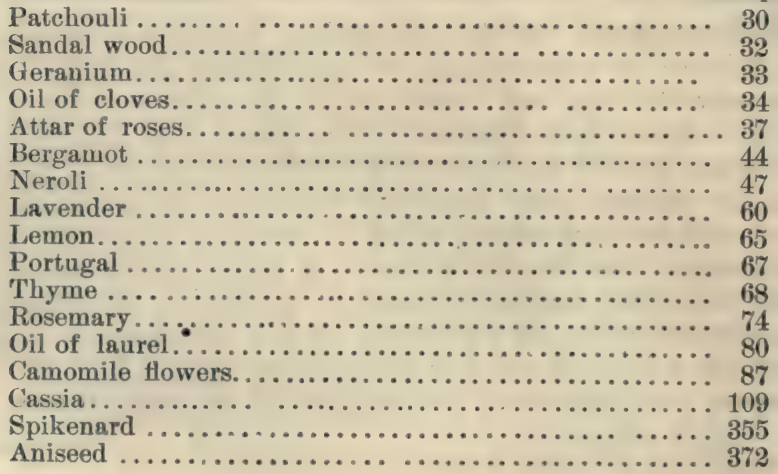

Thus the absorption by a tube full of dry air being 1 , that of the odor of patchouli diffused in it is 30 , that of lavender 60, that of rosemary 74, while that of aniseed 
amounts to 372 . It would be idle to speculate on the quantiiies of matter concerned in these actions.

\section{Aqueous Vapor in Relation to the Terrestrial Tem- peratures.}

We are now fully prepared for a result which, without such preparation, might appear incredible. Water is, to some extent, a volatile body, and our atmosphere, resting as it does upon the surface of the ocean, receives from it a continual supply of aqueous vapor. It would be an error to confound clouds or fog or any visible mist with the vapor of water, which is a perfectly impalpable gas, diffused, even on the clearest days, throughout the atmosphere. Compared with the great body of the air, the aqueous vapor it contains is of almost infinitesimal amount, $99 \frac{1}{2}$ out of every 100 parts of the atmosphere being composed of oxygen and nitrogen. In the absence of experiment, we should never think of ascribing to this scant and varying constituent any important influence on terrestrial radiation; and yet its influence is far more potent than that of the great body of the air. 'To say that on a day of average humidity in England, the atmospheric vapor exerts 100 times the action of the air itself, would certainly be an understatement of the fact. Comparing a single molecule of aqueous vapor with an atom of either of the main constituents of our atmosphere, I am not prepared to say how many thousand times the action of the former exceeds that of the latter.

But it must be borne in mind that these large numbers depend, in part, on the extreme feebleness of the air; the power of aqueous vapor seems vast, because that of the air with which it is compared is infinitesimal. Absolutely considered, however, this substance, notwithstanding its small specific gravity, exercises a very potent action. Probably from 10 to 15 per cent. of the heat radiated from the earth is absorbed within 10 or 20 feet of the earth's surface. This must evidently be of the utmost consequence to the life of the world. Imagine the superficial molecules of the earth agitated with the motion of heat, and imparting it to the surrounding ether; this motion would be carried rapidly away, and lost forever to our planet, if the wares of ether had nothing but the air to contend with in their outward course. But the aqueous vapor takes up the motion, 
and becomes thereby heated, thus wrapping the earth like a warm garment, and protecting its surface from the deadly chill which it would otherwise sustain. Various philosophers have speculated on the influence of an atmospheric envelope. De Saussure, Fourier, M. Pouillet, and Mr. Hopkins, have, one and all, enriched scientific literature with contributions on this subject, but the considerations which these eminent men have applied to atmospheric air, have, if my experiments be correct, to be transferred to the aqueous vapor.

The observations of meteorologists furnish important, though hitherto unconscious evidence of the influence of this agent. Wherever the air is dry we are liable to daily extremes of temperature. By day, in such places, the sun's heat reaches the earth unimpeded, and renders the maximum high; by night, on the other hand, the earth's heat escapes unhindered into space, and renders the minimum low. Hence the difference between the maximum and minimum is greatest where the air is driest. In the plains of India, on the heights of the Himalaya, in central Asia, in Australia-wherever drought reigns, we have the heat of day forcibly contrasted with the chill of night. In the Sahara itself, when the sun's rays cease to impinge on the burning soil, the temperature runs rapidly down to freezing, because there is no vapor overhead to check the calorific drain. And here another instance might be added to the numbers already known, in which nature teuds, as it were, to check her own excess. By nocturnal refrigeration, the aqueous vapor of the air is condensed to water on the surface of the earth; and, as only the superficial portions radiate, the act of condensation makes water the radiating body. Now experiment proves that to the rays emitted by water, aqueous vapor is especially opaque. Hence the very act of condensation, consequent on terrestrial cooling, becomes a safeguard to the earth, imparting to its radiation that particular character which renders it most liable to be prevented from escaping into space.

It might, however, be urged that, inasmuch as we derive all our heat from the sun, the selfsame covering which protects the earth from chill must also shut out the solar radiation. 'This is partially true, but only partially; the sun's rays are different in quality from the earth's rays, and it does not at all follow that the substance which 
absorbs the one must necessarily absorb the other. Through a layer of water, for example, one-tenth of an inch in thickness, the sun's rays are transmitted with comparative freedom; but through a layer half this thickness, as Melloni has proved, no single ray from the warmed earth could pass. In like manner, the sun's rays pass with comparative freedom through the aqueous vapor of the air; the absorbing power of this substance being mainly exerted upon the invisible heat that endeavors to escape from the earth. In consequence of this differential action upon solar and terrestrial heat, the mean temperature of our planet is higher than is due to its distance from the sun.

\section{Liquids and their Vapors in relation to Radiant Heat.}

The deportment here assigned to atmospheric vapor has been established by direct experiments on air taken from the streets and parks of London, from the downs of Epsom, from the hills and sea-beach of the Isle of Wight, and also by experiments on air in the first instance dried, and afterward rendered artificially humid by pure distilled water. It has also been established in the following way: 'Ten volatile liquids were taken at random and the power of these liquids, at a common thickness, to intercept the waves of heat, was carefully determined. The vapors of the liquids were next taken, in quantities proportional to the quantities of liquid, and the power of the vapors to intercept the waves of heat was also determined. Commencing with the substance which exerted the least absorptive power, and proceeding onward to the most energetic, the following order of absorption was observed:

Liquids.

Bisulphide of carbon.

Chloroform.

Iodide of methyl.

Iodide of ethyl.

Benzol.

Amylene.

Sulphuric ether.

Acetic ether.

Formic ether.

Alcohol.

Water.
Vapors.

Bisulphide of carbon.

Chloroform.

Iodide of methyl.

Iodide of ethyl.

Benzol.

Amylene.

Sulphuric ether.

Acetic ether.

Formic ether.

Alcohol.

We here find the order of absorption in both cases to be 
the same. We have liberated the molecules from the bonils which trammel them more or less in a liquid condition; but this change in their state of aggregation does not change their relative powers of absorption. Nothing could more clearly prove that the act of absorption depends upon the individual molecule, which equally asserts its power in the liquid and the gaseous state. We may safely conclude from the above table that the position of a vapor is determined by that of its liquid. Now at the very foot of the list of liquids stands water, signalizing itself above all others by its enormous power of absorption. And from this fact, even if no direct experiment on the vapor of water had ever been maje, we should be entitled to rank that vapor as our most powerful absorber of radiant heat. Its attenuation, however, diminishes its action. I have proved that a shell of air two inches in thickness surrounding our planet, and saturated with the vapor of sulphuric ether, would intercept 35 per cent. of the earth's radiation. And though the quantity of aqueous vapor necessary to saturate air is much less than the amount of sulphuric ether vapor which it can sustain, it is still extremely probable that the estimate already made of the action of atmospheric vapor within 10 feet of the earth's surface, is under the mark; and that we are indebted to this wonderful substance, to an extent not accurately determined, but certainly far beyond what has hitherto been imagined, for the temperature now existing at the surface of the globe.

\section{Reciprocity of Radiation and Absorption.}

Thronghout the reflections which have hitherto occupied us, the image before the mind has been that of a radiant source sending forth calorific waves, which on passing among the molecules of a gas or vapor were intercepted by those molecules in various degrees. In all cases it was the transference of motion from the ether to the comparatively quiescent molecules of the gas or vapor that occupied our thoughts. We have now to change the form of our conception, and to figure these molecules not as absorbers but as radiators, not as the recipients but as the originators of wave-motioli. 'That is to say, we must figure them vibrating, and generating in the surrounding ether undulations which speed through it with the velocity of light. Our 
object now is to inquire whether the act of chemical combination, which proves so potent as regards the phenomena of absorption, does not also manifest its power in the phenomena of radiation. For the examination of this question it is necessary, in the first place, to heat our gases and vapors to the same temperature, and then examine their power of discharging the motion thus imparted to them upon the ether in which they swing.

A heated copper ball was placed above a ring gas-burner possessing a great number of small apertures, the burner being counected by a tube with vessels containing the various gases to be examined. By. gentle pressure the gases were forced through the orifices of the burner against the copper ball, where each of them, being heater, rose in an ascending column. A thermo-electric pile, entirely screened from the hot ball, was exposed to the radiation of the warm gas, while the deflection of a magnetic needle connected with the pile declared the energy of the radiation.

By this mode of experiment it was proved that the selfsame molecular arrangement which renders a gas a powerful absorber, render's it a powerful radiator-that the atom or molecule which is competent to intercept the calorific wares is, in the same degree, competent to send them forth. Thus, while the atoms of elementary gases proved themselves unable to emit any sensible amount of radiant heat, the molecules of compound gases were shown to be capable of powerfully disturbing the surrounding ether. By special modes of experiment the same was proved to hold good for the vapor's of volatile liquids, the radiative power of every vapor being found proportional to its absorptive power.

'The method of experiment here pursued, though not of the simplest character, is still easy to grasp. When air is permitted to rush into an exhausted tube, the temperature of the air is raised to a degree equivalent to the vis viva extinguished.* Such air is said to be dynamically heated, and, if pure, it shows itself incompetent to radiate, even when a rock-salt window is provided for the passage of its rays. But if, instead of being empty, the tube contain a

t See page 10 for a definition of vis viva. 
small quantity of vapor, the warmed air communicates its heat by contact to the vapor, the molecules of which convert into the radiant form the neat imparted to them by the atoms of the air. By this process also, which I have called Dynamic Radiation, the reciprocity of radiation and absorption has been conclusively proved.*

In the excellent researches of Leslie, De la Provostaye and Desains, and Balfour Stewart, the same reciprocity, as regards solid bodies, has been variously illustrated; while the labors, theoretical and experimental, of Kirchhoff have given this subject a wonderful expansion, and enriched it by applications of the highest kind. To their results are now to be added the foregoing, whereby gases and vapors, which have been hitherto thought inaccessible to experiments with the thermo-electric pile, are proved by it to exhibit the indissoluble duality of radiation and absorption, the influence of chemical combination on both being exhibited in the most decisive and extraordinary way.

\section{Influence of Vibrating Period and Molecular Form. Physical Analysis of the Human Breath.}

In the foregoing experiments with gases and vapors we have employed throughout invisible rays, and found some of these bodies so impervious to radiant heat, that in lengths of a few feet they intercept every ray as effectually as a layer of pitch. 'The substances, however, which show themselves thus opaque to radiant heat are perfectly transparent to light. Now the rays of light differ from those of invisible heat merely in point of period, the former failing to affect the retina because their periods of recurrence are too slow. Hence, in some way or other, the transparency of our gases and vapors depends upon the periods of the waves which impinge upon them. What is the nature of this dependence? The admirable researches of Kirchhoff help us to an answer. The atoms and molecules of every gas have certain definite rates of oscillation, and those waves of ether are most copiously absorbed whose

\footnotetext{
* When heated air imparts its motion to another gas or vapor, the transference of heat is accompanied by a change of vibrating period. The Dynamic Radiation of vapors is rendered possible by this trans. mutation of vibrations.
} 
periods of recurrence synchronize with those of the atomic groups among which they pass. Thus, when we find the invisible rays absorbed and the visible ones transmitted by a layer of gas, we conclude that the oscillating periods of the atoms constituting the gaseous molecules coincide with those of the invisible, and not with those of the visible spectrum.

It requires some discipline of the imagination to form a clear picture of this process. Such a picture is, however, possible, and ought to be obtained. When the waves of ether impinge upon molecules whose periods of vibration coincide with the recurrence of the undulations, the timed strokes of the wares augment the vibration of the molecules, as a heavy pendulum is set in motion by well-timed puffs of breath. Millions of millions of shocks are received every second from the calorific waves; and it is not difficult to see that as every wave arrives just in time to repeat the action of its predecessor, the molecules must finally be caused to swing through wider spaces than if the arrivals were not so timed. In fact, it is not difficult to see that an assemblage of molecules, operated upon by contending waves, might remain practically quiescent. This is actually the case when the waves of the visible spectrum pass through a transparent gas or vapor. There is here no sensible transference of motion from the ether to the molecules; in other words, there is no sensible absorption of heat.

One striking example of the influence of period may be here recorded. Carbonic acid gas is one of the feeblest absorbers of the radiant heat emitted by solid bodies. It is, for example, to a great extent transparent to the rays emitted by the heated copper plate already referred to. There are, however, certain rays, comparatively few in number, emitted by the copper, to which the carbonic acid is impervious; and could we obtain a source of heat emitting such rays only, we should find carbonic acid more opaque to the radiation from that source than any other gas. Such a source is actually found in the flame of carbonic oxide, where hot carbonic acid constitutes the main radiating body. Of the rays emitted by our heated plate of copper, olefiant gas absorbs ten times the quantity absorbed by carbonic acid. Of the rays emitted by a carbonic oxide flame, carbonic acid absorbs twice as much as 
olefiant gas. This wonderful change in the power of the former, as an absorber, is simply due to the fact that the periods of the hot and cold carbonic acid are identical, and that the waves from the flame freely transfer their motion to the molecules which synchronize with them. 'Thus it is that the tenth of an atmosphere of carbonic acid, enclosed in a tube four feet long, absorbs 60 per cent. of the radiation from a carbonic oxide flame, while one-thirtieth of an atmosphere absorbs 48 per cent. of the heat from the same source.

In fact, the presence of the minutest quantity of carbonic acid may be detected by its action on the rays from the carbonic oxide flame. Carrying, for example, the dried human breath into a tube four feet long, the absorption there effected by the carbonic acid of the breath amounts to 50 per cent. of the entire radiation. Radiant heat may indeed be employed as a means of determining practically the amount of carbonic acid expired from the lungs. My late assistant, Mr. Barrett, while under my direction, made this determination. The absorption produced by the breath freed from its moisture, but retaining its carbonic acid, was first determined. Carbonic acid, artificially prepared, was then mixed with dry air in such proportions that the action of the mixture upon the rays of heat was the same as that of the dried breath. The percentage of the former being known, immediately gave that of the latter. The same breath, analyzed chemically by Dr. Frankland, and physically by Mr. Barrett, gave the following results:

Percentage of Carbonic Acid in the Human Breath.

Chemical analysis

Physical analysis

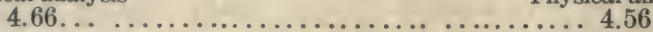

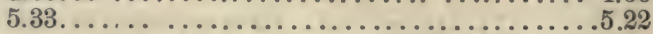

It is thus proved that in the quantity of ethereal motion which it is competent to take up, we have a practical measure of the carbonic acid of the breath, and hence of the combustion going on in the human lungs.

Still this question of period, though of the utmost importance, is not competent to account for the whole of the observed facts. 'The ether, as far as we know, accepts vibrations of all periods with the same readiness. 'To it the oscillations of an atom of free oxygen are just as 
acceptable as those of the atoms in a molecule of olefiant gas; that the vibrating oxygen then stands so far below the olefiant gas in radiant power must be referred not to period, but to some other peculiarity. The atomic group which constitutes the molecule of olefiant gas, produces many thousand times the disturbance caused by the oxygen, it may be, because the group is able to lay a vastly more powerful hold upon the ether than the single atoms can. Another and probably very potent cause of the difference may be that the vibrations, being those of the constituent atoms of the molecule, ${ }^{*}$ are generated in highly condensed ether, which acts like condensed air upon sound. But whatever may be the fate of these attempts to visualize the physics of the process, it will still remain true, that to account for the phenomena of radiation and absorption we must take into consideration the shape, size, and condition of the ether within the molecules, by which the exterual ether is disturbed.

\section{Summary and Conclusion.}

Let us now cast a momentary glance over the ground that we have left behind. The general nature of light and heat was first briefly described: the compounding of matter flom elementary atoms, and the influence of the act of combination on radiation and absorption, were considered and experimentally illustrated. Through the transparent elementary gases radiant heat was found to pass as through a vacuum, while many of the compound gases presented almost impassable obstacles to the caloritic waves. This deportment of the simple gases directed our attention to other elementary bodies, the examination of which led to the discovery that the element iodine, dissolved in bisulphide of carbon, possesses the power of detaching, with extraordinary sharpness, the light of the spectrum from its heat, intercepting all luminous rays up to the extreme red, and permitting the calorific rays beyond the red to pass freely through it. This substance was then employed to filter the beams of the electric light, and to form foci of invisible rays so intense as to produce almost all the effects obtainable in an ordinary fire. Combustible bodies were

* See "Physical Considerations," Art. iv., p. 102. 
burned, and refractory ones were raised to a white heat, by the concentrated invisible rays. Thus, by exalting their refrangibility, the invisible rays of the electric light were rendered visible, and all the colors of the solar spectrum were extracted from utter darkness. The extreme richness of the electric light in invisible rays of low refrangibility was demonstrated, one-eighth only of its radiation consisting of luminous rays. The deadness of the optic nerve to those invisible rays was proved, and experiments were then added to show that the bright and the dark rays of a solid body, raised gradually to incandescence, are strengthened together; intense dark heat being an invarible accompaniment of intense white heat. A sun could not be formed, or a meteorite rendered luminous, on any other condition. 'The light-giving rays constituting only a small fraction of the total radiation, their unspeakable importance to us is due to the fact that their periods are attuned to the special requirements of the eye.

Among the vapors of volatile liquids vast differences were also found to exist, as regards their powers of absorption. We followed various molecules firom a state of liquid to a state of gas, and found, in both states of aggregation, the power of the individual molecules equally asserted. The position of a vapor as an absorber of radiant heat was shown to be determined by that of the liquid from which it is derived. Reversing our conceptions, and regarding the molecules of gases and vapors not as the recipients but as the originators of wave-motion; not as absorbers but as radiators; it was proved that the powers of absorption and radiation went hand in hand, the selfsame chemical act which rendered a body competent to intercept the waves of ether rendering it competent, in the same degree, to generate them. Perfumes were next subjected to examination, and, notwithstanding their extraordinary tenuity, they were found vastly superior, in point of absorptive power, to the body of the air in which they were diffused. We were led thus slowly up to the examination of the most widely diffused and most important of all vapors - the aqueous vapor of our atmosphere, and we found in it a potent absorber of the purely calorific rays. The power of this substance to influence climate, and its general influence on the temperature of the earth, were 
then briefly dwelt upon. A cobweb spread above a blossom is sufficient to protect it from nightly chill; and thus the aqueous vapor of our air, attenuated as it is, checks the drain of terrestrial heat, and saves the surface of our planet from the refrigeration which would assuredly accrue, were no such substance interposed between it and the voids of space. We considered the influence of vibrating period, and molecular form, on absorption and radiation, and finally deduced, from its action upon radiant heat, the exact amount of carbonic acid expired by the human lungs.

Thus, in brief outline, were placed before you some of the results of recent inquiries in the domain of radiation, and my aim throughout has been to raise in your minds distinct physical images of the various processes involved in our researches. It is thought by some that natural science has a deadening influence on the imagination, and a doubt might fairly be raised as to the value of any study which would necessarily have this effect. But the experience of the last hour must, I think, have convinced you, that the study of natural science goes hand in hand with the culture of the imagination. Throughout the greater part of this discourse we have been sustained by this faculty. We have been picturing atoms, and molecules, and vibrations, and waves, which eye has never seen nor ear heard, and which can only be discerned by the exercise of imagination. This, in fact, is the faculty which enables us to transcend the boundaries of sense, and connect the phenomena of our visible world with those of an invisible one. Without imagination we never could have risen to the conceptions which have occupied us here to-day; and in proportion to your power of exercising this faculty aright, and of associating definite mental images with the terms employed, will be the pleasure and the profit which you will derive from this lecture. The outward facts of nature are insufficient to satisfy the mind. We camnot be content with knowing that the light and heat of the sun illuminate and warm the world. We are led irresistibly to inquire, "What is light, and what is heat?" and this question leads us at once out of the region of sense into that of imagination.*

* This line of thought was pursued further five years subsequently. See "Scientific Use of the Imagination". 
'Thus pondering, and questioning, and striving to supplement that which is felt and seen, but which is incomplete, by something unfelt and unseen.which is necessary to its completeness, men of genius have in part discerned, not only the nature of light and heat, but also, through them, the general relationship of natural phenomena. 'The working power of Nature consists of actual or potential motion, of which all its phenomena are but special forms. This motion manifests itself in tangible and in intangible matter, being incessantly transferred from the one to the other, and incessantly transformed by the change. It is as real in the waves of the ether as in the waves of the sea; the latter-derived as they are from winds, which in their turn are derived from the sun-are, indeed, nothing more than the heaped-up motion of the ether waves. It is the calorific waves emitted by the sun which heat our air, produce our winds, and hence agitate our ocean. And whether they break in foam upon the shore, or rub silently against the ocean's bed, or subside by the mutual friction of their own parts, the sea waves, which cannot subside without producing heat, finally resolve themselves into waves of ether, thus regenerating the motion from which their temporary existence was derived. This connection is typical. Nature is not an aggregate of independent parts, but an organic whole. If you open a piano and sing into it, a certain string will respond. Change the pitch of your voice; the first string ceases to vibrate, but another replies. Change again the pitch; the first two strings are silent, while another resounds. Thus is sentient man acted on by Nature, the optic, the auditory and other nerves of the human body being so many strings differently tuned, and responsive to different forms of the universal power.

\section{CHAPTER III.}

ON RADIANT HEAT IN RELATION TO THE COLOR AND CHEMICAL CONSTITUTION OF BODIES.*

ONE OF the most important functions of physical science, considered as a discipline of the mind, is to enable us by means of the sensible processes of Nature to apprehend the

* A discourse delivered in the Royal Institution of Great Britain, Jan. 19, 1866. 
insensible. The sensible processes give direction to the line of thought; but this once given, the length of the line is not limited by the boundaries of the senses. Indeed, the domain of the senses, in Nature, is almost infinitely small in comparison with the vast region accessible to thought which lies beyond them. From a few observations of a comet, when it comes within the range of his telescope, an astronomer can calculate its path in regions which no telescope can reach: and in like manner, by means of data furnished in the narrow world of the senses, we make ourselves at home in other and wider worlds, which are traversed by the intellect alone.

From the earliest ages the questions, "What is light?" and "What is heat?" have occurred to the minds of men; but these questions never would have been answered had they not been preceded by the question, "What is sound?" Amid the grosser phenomena of acoustics the mind was first disciplined, conceptions being thus obtained from direct observation, which were afterward applied to phenomena of a character far too subtle to be observed directly. Sound we know to be due to vibratory motion. A vibrating tuning-fork, for example, molds the air around it into undulations or waves, which speed away on all sides with a certain measured velocity, impinge upon the drum of the ear, shake the anditory nerve, and awake in the brain the sensation of sound. When sufficiently near a sounding body we can feel the vibrations of the air. A deaf man, for example, plunging his hand into a bell when it is sounded, feels through the common nerves of his body those tremors which, when imparted to the nerves of healthy ears, are translated into sound. There are various ways of rendering those sonorous vibrations notoniy tangible but visible; and it was not until numberless experiments of this kind had been executed that the scientific investigator abandoned himself wholly, and without a shadow of misgiving, to the conviction that what is sound within us is, ontside of us, a motion of the air.

But once having established this fact-once having proved beyond all doubt that the sensation of sound is produced by an agitation of the auditory nerve-the thought soon suggested itself that light might be due to an agitation of the optic nerve. 'This was a great step in advance of that ancient notion which regarled light as something 
emitted by the eye, and not as anything imparted to it. But if light be produced by an agitation of the retina, what is it that produces the agitation? Newton, you know, supposed minute particles to be shot through the humors of the eye against the retina, which he supposed to hang like a target at the back of the eye. The impact of these particles against the target, Newton believed to be the cause of light. But Newton's notion has not held its ground, being entirely driven from the field by the more wonderful and far more philosophical notion that light, like sound, is a product of wave-motion.

The domain in which this motion of light is carried on lies entirely beyond the reach of our senses. The waves of light require a medium for their formation and propagation; but we cannot see, or feel, or taste, or smell this medium. How, then, has its existence been established? By showing, that by the assumption of this wonderful intangible ether, all the phenomena of optics are accounted for, with fullness, and clearness, and conclusiveness, which leave no desire of the intellect unsatisfied. When the law of gravitation first suggested itself to the mind of Newton, what did he do? He set himself to examine whether it accounted for all the facts. He determined the courses of the planets; he calculated the rapidity of the moon's fall toward the earth; he considered the precession of the equinoxes, the ebb and flow of the tides, and found all explained by the law of gravitation. He therefore regarded this law as established, and the verdict of science subsequently confirmed his conclusion. On similar, and, if possible, on stronger grounds, we found our belief in the existence of the universal ether. It explains facts far more various and complicated than those on which Newton based his law. If a single phenomenon could be pointed out which the ether is proved incompetent to explain, we should have to give it up; but no such phenomenon has ever been pointed out. It is, therefore, at least as certain that space is filled with a medium, by means of which suns and stars diffuse their radiant power, as that it is traversed by that force which holds in its grasp, not ouly our planetary system, but the immeasurable heavens themselves.

'There is no more wonderful instance than this of the production of a line of thought, from the world of the senses into the region of pure imagination. I mean by 
imagination here, not that play of fancy which can give to airy nothings a local habitation and a name, but that power which enables the mind to conceive realities which lie beyond the range of the senses-to present to itself distiuct images of processes which, though mighty in the aggregate beyond all conception, are so minute individually as to elude all observation. It is the waves of air excited by a tuning-fork which render its vibrations audible. It is the waves of ether sent forth from those lamps overhead which render them luminous to us; but so minute are these waves, that it would take from 30,000 to 60,000 of them placed end to end to cover a single inch. Their number, however, compensates for their minuteness. 'Trillions of them have entererl your eyes, and hit the retina at the backs of your eyes, in the time consumed in the utterance of the shortest sentence of this discourse. 'This is the steadfast result of modern research; but we never could have reached it without previous discipline. We never could have measured the waves of light, nor even imagined them to exist, had we not previously exercised ourselves among the waves of sound. Sound and light are now mutually helpful, the conceptions of each being expanded, strengthened, and defined by the conceptions of the other.

The ether which conveys the pulses of light and heat not only fills celestial space, swathing suns, and planets, and inoons, but it also encircles the atoms of which these bodies are composed. It is the motion of these atoms, and not that of any sensible parts of bodies, that the ether conveys. 'This motion is the objective cause of what, in our sensations, are light and heat. An atom, then, sending its pulses through the ether, resembles a tuning-fork sending its pulses through the air. Let us look for a moment at this thrilling medium, and briefly consider its relation to the bodies whose vibrations it conveys. Different bodies, when heated to the same temperature, possess very different powers of agitating the ether: some are good radiators, others are bad radiators: which means that some are so constituted as to communicate their atomic motion freely to the ether, producing therein powerful undulations; while the atoms of others are unable thus to communicate their motions, but glide through the medium without materially disturbing its repose. 
Recent experiments have proved that elementary bodies, except under certain anomalous conditions, belong to the class of bad radiators. An atom, ribrating in the ether, resembles a naked tuning-fork vibrating in the air. The amount of motion communicated to the air by the thin prongs is too small to evoke at any distance the sensation of sound. But if we permit the atoms to combine chemically and form molecules, the result, in many cases, is an enormous change in the power of radiation. "The amount of ethereal disturbance, produced by the combined atoms of a body, may be many thousand times that produced by the same atoms when uncombined.

The pitch of a musicul note depends upon the rapidity of its vibrations, or, in other words, on the length of its waves. Now, the pitch of a note answers to the color of light. Taking a slice of white light from the sun, or from an electric lamp, and causing the light to pass through an arrangement of prisms, it is decomposed. We have the effect obtained by Newton, who first unrolled the solar beam into the splendors of the solar spectrum. At one end of this spectrum we have red light, at the other, violet; and between those extremes lie the other prismatic colors. As we advance along the spectrum from the red to the violet, the pitch of the light-if I may use the expression-heightens, the sensation of violet being produced by a more rapid succession of impulses than that which produces the impression of red. 'The vibrations of the violet are about twice as rapid as those of the red; in other words, the range of the visible spectrum is about an octave.

There is no solution of continuity in this spectrum; one color changes into another by insensible gradations. It is as if an infinite number of tuning-forks, of gradually augmenting pitch, were vibrating at the same time. But turning to another spectrum-that, namely, obtained from the incandescent vapor of silver-you observe that it consists of two narrow and intensely luminous green bands. Here it is as if two forks only, of slightly different pitch, were vibrating. The length of the waves which produce this first band is such that $4 \%, 460$ of them, placed end to end, would fill an inch. The waves which produce the secoml band are a little shorter; it would take of these 47,920 to fill an inch. In the case of the first band, the 
number of impulses imparted, in one second, to every eye which sees it, is 577 millions of millions; while the number of impulses imparted, in the same time, by the second band is 500 millions of millions. We may project upon a white screen the beautiful stream of green light from whicir these bands were derived. 'This luminous stream is the incandescent vapor of silver. 'The rates of vibration of the atoms of that vapor are as rigidly fixed as those of two tuning-forks; and to whatever height the temperature of the vapor may be raised, the rapidity of its vibrations, and consequently its color, which wholly depends upon that rapidity, remain unchanged.

'The vapor' of water, as well as the vapor of silver, has its definite periods of vibration, and these are such as to disqualify the vapor, when acting freely as such, from being raiseu to a white heat. The oxyhydrogen flame, for example, cousists of hot aqueous vapor. It is scarcely visible in the air of this room, and it would be still less visible if we could burn the gas in a clean atmosphere. But the atmosphere, even at the summit of Mont Blanc, is dirty; in London it is more than dirty; and the burning dirt gives to this flame the greater portion of its present light. But the heat of the flame is enormous. Cast iron fuses at a temperature of $2,000^{\circ}$ Fahr.; while the temperature of the oxyhydrogen flame is $6,000^{\circ} \mathrm{Fahr}$. A piece of platinum is heated to vivid redness, at a distance of two inches beyond the visible termination of the flame. The vapor which produces incandescence is here absolutely dark. In the flame itself the platinum is raised to dazzling whiteness, and is even pierced by the flame. When this flame impinges on a piece of lime, we have the dazzling Drummond light. But the light is here due to the fact that when it impinges upon the solid body, the vibrations excited in that body by the flame are of periods different from its own.

'I'hus far we have fixed our attention on atoms and molecules in a state of vibration, and surrounded by a medium which accepts their vibrations, and transmits them through space. But suppose the waves generated by one system of molecules to impinge upon another system, how will the waves be affected? Will they be stopped, or will they be permitted to pass? Will they transfer their motion to the molecules on which they impinge, or will 
they glide round the molecules, through the intermolecular spaces, and thus escape?

The answer to this question depends upon a condition which may be beautifully exemplified by an experiment on sound. These two tuning-forks are tuned absolutely alike. They vibrate with the same rapidity, and, mounted thus upon their resonant cases, you hear them loudly sounding the same musical note. Stopping one of the forks, I throw the other into strong vibration, and bring that other near the silent fork, but not into contact with it. Allowing them to continue in this position for four or five seconds, and then stopping the vibrating fork, the sound does not cease. The second fork has taken up the vibrations of its neighbor, and is now sounding in its turn. Dismounting one of the forks, and permitting the other to remain upon its stand, I throw the dismounted fork into strong vibration. You cannot hear it sound. Detached from its case, the amount of motion which it can communicate to the air is too small to be sensible at any distance. When the dismounted fork is brought close to the mounted one, but not into actual contact with it, out of the silence rises a mellow sound. Whence comes it? From the vibrations which have been transferred from the dismounted fork to the mounted one.

That the motion should thus transfer itself through the air it is necessary that the two forks should be in perfect unison. If a morsel of wax not larger than a pea be placed on one of the forks, it is rendered thereby powerless to affect, or to be affected by the other. It is easy to understand this experiment. The pulses of the one fork can affect the other, because they are perfectly timed. A single pulse causes the prong of the silent fork to vibrate through an infinitesimal space. But just as it has completed this small vibration, another pulse is ready to strike it. Thus, the impulses add themselves together. In the five seconds during which the forks were held near each other, the vibrating fork sent 1,280 waves against its neighbor and those 1,280 shocks, all delivered at the proper moment, all, as I have said, perfectly timed, have given such strength to the vibrations of the mounted fork as to render them andible to all.

Another curious illustration of the influence of synchronism on musical vibrations, is this: Three small gas- 
flames are inserted into three glitss tubes of different lengths. Each of these flames can be caused to emit a musical note, the pitch of which is determined by the length of the tube surrounding the flame. The shorter the tube the higher is the pitch. 'T'he flames are now silent within their respective tubes, but each of them can be caused to respond to a proper note sounded anywhere in this room. With an instrument called a svren, a powerful musical note, of gradually increasing pitch, can be produced. Beginning with a low note, and ascending gralually to a higher one, we finally attain the pitch of the flame in the longest tube. The moment it is re:ched the flame bursts into song. The other flames are still silent within their tubes. But by urging the instrument on to higher notes, the second flame is started, and the third alone remains. A still higher note starts it also. Thus, as the sound of the syren rises gradually in pitch, it awakens every flame in passing, by striking it with a series of waves whose periods of recurrence are similar to its own.

Now the wave-motion from the syren is in part taken up by the flame which synchronizes with the waves; and were these waves to impinge upon a multitude of flames, instead of upon one flame only, the transference might be so great as to absorb the whole of the orignal wave motion. Let us apply these facts to radiant heat. 'This blue flame is the flame of carbonic oxide: this transparent gas is carbonic acid gas. In the blue flame we have carbonic acid intensely heater, or, in other words, in a state of intense vibration. It thus resembles the sounding fork, while this cold carbonic acid resembles the silent one. What is the consequence? 'Through the synchronism of the hot and cold gas, the waves emitted by the former are intercepted by the latter, the transmission of the radiant heat being thus prevented. The cold gas is intensely opaque to the radiation from this particular flame, though highly transparent to heat of every other kind. We are here manifestly dealing with that great principle which lies at the basis of spectrum analysis, and which has enabled scientific men to determine the substances of which the sun, the stars, and even the nebulæ are composed; the principle, namely, that a body which is competent to emit any ray, whether of heat or light, is competent in the same degree to absorb 
that ray. The absorption depeuds on the synchronism existing between the vibrations of the atoms from which the rays, or more correctly the waves, issue, and those of the atoms on which they impinge.

'To its almost total incompetence to emit white light, aqueous vapor adds a similar incompetence to absorb white light. It cannot, for example, absorb the luminous rays of the sun, though it can absorb the non-luminous rays of the earth. This incompetence of the vapor to absorb luminous rays is shared by water and ice-in fact, by all really transparent substances. Their transparency is due to their inability to absorb luminous rays. 'The molecules of such substances are in dissonance with the luminous waves; and hence such waves pass through transparent bodies without disturbing the molecular rest. A purely luminous beam, however intense may be its heat, is sensibly incompetent to melt ice. We can, for example, converge a powerful luminous beam upon a surface covered with hoar frost, without melting a single spicula of the crystals. How then, it may be asked, are the snows of the Alps swept away by the sunshine of summer? I answer, they are not swept away by sunshine at all, but by rays which have no sunshine whatever in them. The luminous rays of the sun fall upon the snow-fields and are flashed in echoes from crystal to crystal, but they find next to no lodgment within the crystals. They are hardly at all absorbed, and hence they cannot produce fusion. But a body of powerful dark rays is emitted by the sun; and it is these that cause the glaciers to shrink and the snows to disappear; it is they that fill the banks of the Arve and Arveyron, and liberate from their frozen captivity the Rhone and the Rhine.

Placing a concave silvered mirror behind the electric light its rays are converged to a focus of dazzling brilliancy. Placing in the path of the rays, between the light and the focus, a vessel of water, and introducing at the focus a piece of ice, the ice is not melted by the concentrated beam. Matches, at the same place, are ignited, and wood is set on fire. 'The powerful heat, then, of this luminous beam is incompetent to melt the ice. On withdrawing the cell of water, the ice immediately liquefies, and the water trickles from it in drops. Reintrolucing the cell of water, the fusion is arrested, and the drops cease to fall. The 
transparent water of the cell exerts no sensible absorption on the luminous rays, still it withdraws something from the beam, which, when permitted to act, is competent to melt the ice. 'This something is the dark radiation of the electric light. Again, I place a slab of pure ice in front of the electric lamp; send a luminous beam first through our cell of water and then through the ice. By means of a lens an image of the slab is cast upon a white screen. 'The beam, sifted by the water, has little power upon the ice. But observe what occurs when the water is removed; we have here a star and there a star, each star resembling a flower of six petals, and growing visibly larger before our eyes. As the leaves enlarge, their edges become serrated, but there is no deviation from the six-rayed type. We have here, in fact, the crystallization of the ice reversed by the invisible rays of the electric beam. They take the molecules down in this wonderful way, and reveal to us the exquisite atomic structure of the substance with which Nature every winter roofs our ponds and lakes.

Numberless effects, apparently anomalous, might be adduced in illustration of the action of these lightless rays. 'These two powders, for example, are both white, and undistinguishable from each other by the eye. The luminous rays of the sun are unabsorbed by both-from such rays these powders acquire no heat; still one of them, sugar, is heated so highly by the concentrated beam of the electric lamp, that it first smokes and then violently inflames, while the other substance, salt, is barely warmed at the focus. Placing two perfectly transparent liquids in test-tubes at the focus, one of them boils in a comple of seconds, while the other, in a similar position, is hardly warmed. The boiling-point of the first liquid is 78 degrees C., which is speedily reached; that of the second liquid is only 48 degrees $\mathrm{C}$. , which is never reached at all. These anomalies are entirely due to the unseen element which mingles with the luminous rays of the electric beam, and indeed constitutes 90 per cent. of its calorific power.

A substance, as many of you know, has been discovered, by which these dark rays may be detached from the total emission of the electric lamp. This ray-filter is a liquid, black as pitch to the luminous, but bright as a diamond to the non-luminous, raliation. It mercilessly cuts off the former, but allows the latter free transmission. When 
these invisible rays are brought to a focus, at a distance of several feet from the electric lamp, the dark rays form an invisible image of their source. By proper means, this image may be transformed into a visible one of dazzling brightness. It might, moreover, be shown, if time permitted, how, out of those perfectly dark rays, could be extracted, by a process of transmutation, all the colors of the solar spectrum. It might also be proved that those rays, powerful as they are, and sufficient to fuse many metals, can be permitted to enter the eye, and to break upon the retina, without producing the least luminous impression.

The dark rays being thus collected, you see nothing at their place of convergence. With a proper thermometer it could be proved that even the air at the focus is just as cold as the surrounding air. And mark the conclusion to which this leads. It proves the ether at the focus to be practically detached from the air-that the most violent ethereal motion may there exist, without the least aërial motion. But, though you see it not, there is sufficient heat at that focus to set London on fire. The heat there is competent to raise iron to a temperature at which it throws off brilliant scintillations. It can heat platinum to whiteness, and almost fuse that refractory metal. It actually can fuse gold, silver, copper, and aluminium. The moment, moreover, that wood is placed at the focus it bursts into a blaze.

It has been already affirmed that, whether as regards radiation or absorption, the elementary atoms possess but little power. This might be illustrated by a long array of facts; and one of the most singular of these is furnished by the deportment of that extremely combustible substance, phosphorus, when placed at the dark focus. It is impossible to ignite there a fragment of amorphous phosphorus. But ordinary phosphorus is a far quicker combustible, and its deportment toward radiant heat is still more impressive. It may be exposed to the intense radiation of an ordinary fire without bursting into flame. It may also be exposed for twenty or thirty seconds at an obscure focus, of sufficient power to raise platinum to a rer heat, without ignition. Notwithstanding the energy of the ethereal waves here concentrated, notwithstanding the extremely inflammable character of the elementary body exposed to their action, the atoms of that body refuse to 
partake of the motion of the powerful waves of low refrangibility, and consequently cannot be affected by their heat.

The knowledge we now possess will enable us to analyze with profit a practical question. White dresses are worn in summer, because they are found to be cooler than dark ones. The celebrated Benjamin Franklin placed bits of cloth of various colors upon snow, exposed them to direct sunshine, and found that they sauk to different depths in the snow. 'The black cloth sank deepest, the white did not sink at all. Franklin inferred from this experiment that black bodies are the best absorbers, and white ones the worst absorbers, of radiant heat. Let us test the generality of this conclusion. One of these two cards is coated with a very dark powder, and the other with a perfectly white one. I place the powdered surfaces before a fire, and leave them there until they have acquired as high a temperature as they can attain in this position. Which of the cards is then most highly heated?. It requires no thermometer to answer this question. Simply pressing the back of the card, on which the white powler is strewn, against the cheek or forehead, it is found intolerably hot. Placing the dark card in the same position, it is found cool. 'The white powder has absorbed far more heat than the dark one. This simple result abolishes a hundred conclusions which have been hastily drawn from the experiment of Franklin. Again, here are suspended two delicate mercurial thermometers at the same distance from a gasflame. The bulb of one of them is covered by a dark substance, the bulb of the other by a white one. Both bulbs have received the radiation from the flame, but the white bulb has absorbed most, and its mercury stands much higher than that of the other thermometer. 'This experiment might be varied in a hundred ways: it proves that from the darkness of a body you can draw no certain conclusion regarding its power of absorption.

'The reason of this simply is, that color gives us intelligence of only one portion, and that the smallest one, of the rays impinging on the colored body. Were the rays all luminous, we might with certainty infer from the color of a body its power of absorption; but the great miss of the radiation from our fire, our gas-flame, and even from the sun itself, consists of invisible calorific rays, regarding which color teaches us nothing. A body may be highly 
transparent to the one class of rays, and highly opaque to the other. 'Thus the white powler, which has shown itself so powerful an absorber, has been specially selected on account of its extreme perviotisness to the visible rays, and its extreme imperviousness to the invisible ones; while the dark powder was chosen on account of its extreme transparency to the invisible, and its extreme opacity to the visible rays. In the case of the radiation from our fire, about 98 per cent. of the whole emission consists of invisible rays; the body, therefore, which was most opaque to these triumphed as an absorber, though that body was a white one.

And here it is worth while to consider the manner in which we obtain from natural facts what may be called their intellectual value. Throughout the processes of Nature we have interdependence and harmony; and the main value of physics, considered as a mental discipline, consists in the tracing out of this interdependence, and the demoustration of this harmony. The outward and visible phenomena are the counters of the intellect; and our science would not be worthy of its name and fame if it halted at facts, however practically useful, and neglected the laws which accompany and rule the phenomena. Let us endeavor, then, to extract from the experiment of Franklin ail that it can yield, calling to our aid the knowledge which our predecessors have already stored. Let us imagine two pieces of cloth of the same texture, the one black and the other white, placed upon sunned snow. Fixing our attention on the white piece, let us inquire whether there is any reason to expect that it will sink in the snow at all. 'There is knowledge at hand which enables us to reply at once in the negative. There is, on the contrary, reason to expect that, after a sufficient exposure, the bit of cloth will be found on an eminence instead of in a hollow; that insteal of a depression, we shall have a relative elevation of the bit of cloth. For, as regards the luminous rays of the sun, the cloth and the snow are alike powerless; the one cannot be warmed, nor the other melted, by such rays. The cloth is white and the snow is white, because their confusedly mingled fibers and particles are incompetent to absorb the luminous rays. Whether, then, the cloth will sink or not depends entirely upon the dark rays of the sun. Now the substance which absorbs these dark rays with the 
greatest avidity is ice-or snow, which is merely ice in powder. Hence, a less amount of heat will be lodged in the cloth than in the surrounding snow. The cloth must therefore act as a shield to the snow on which it rests; and, in consequence of the more rapid fusion of the exposed snow, its shield must, in due time, be left behind, perched upon an eminence like a glacier-table.

But though the snow transcends the cloth, both as a radiator and absorber, it does not much transcend it. Cloth is very powerful in both these respects. Let us now turn our attention to the piece of black cloth, the texture and fabric of which I assume to be the same as that of the white. For, our object being to compare the effects of color, we must, in order to study this effect in its purity, preserve all the other conditions constant. Let us then suppose the black cloth to be obtained from the dyeing of the white. 'The cloth itself, without reference to the dye, is nearly as good an absorber of heat as the snow around it. But to the absorption of the dark solar rays by the undyed cloth, is now added the absorption of the whole of the luminous rays, and this great additional influx of heat is far more than sufficient to turn the balance in favor of the black eloth. 'The sum of its actions on the dark and luminous rays exceeds the action of the snow on the dark rays alone. Hence the cloth will sink in the snow, and this is the complete analysis of Franklin's experiment.

'Throughout this discourse the main stress has been laid on chemical constitution, as influencing most powerfully the phenomena of radiation and absorption. With regard to gases and rapors, and to the liquids from which these vapors are derived, it has been proved by the most varied and conclusive experiments that the acts of radiation and absorption are molecular-that they depend upon chemical, and not upon mechanical condition. In attempting to extend this principle to solids I was met by a multitude of facts, obtained by celebrated experimenters, which seemed flatly to forbid such an extension. Melloni, for example, had found the same radiant and absorbent power for chalk and lanıblack. MM. Masson and Courtépée had performed a most elaborate series of experiments on chemical precipitates of various kinds, and found that they one and all manifested the same power of radiation. 'They concluced from their researches, that when bodies are reduced 
to an extremely fine state of division, the influence of this state is so powerful as entirely to mask and override whatever influence may bedue to chemical constitution.

But it appears to me that through the whole of these researches an oversight has run, the mere mention of which will show what caution is essential in the operations of experimental philosophy; while an experiment or two will make clear wherein the oversight cousists. Filling a brightly polished metal cube with boiling water, I determine the quantity of heat emitted by two of the bright surfaces. As a radiator of heat one of them far transcends the other. Both surfaces appear to be metallic; what, then, is the catuse of the observed difference in their radiative power? Simply this: one of the surfaces is coated with transparent gum, through which, of course, is seen the metallic luster behind; and this varnish, though so perfectly transparent to luminous rays, is as opaque as pitch, or lampblack, to non-luminous ones. It is a powerful emitter of dark rays; it is also a powerful absorber. While, therefore, at the present moment, it is copiously pouring forth radiant heat itself, it does not allow a single ray from the metal behind to pass through it. The varnish then, and not the metal, is the real radiator.

Now Melloni, and Masson, and Courtépée experimented thus: they mixed their powders and precipitates with gumwater, and laill them, by means of a brush, upon the surfaces of a cube like this. 'True, they saw their red powders red, their white ones white, and their black ones black, but they saw these colors through the coat of varnish which surrounded every particle. When, therefore, it was concluded that color had no influence on radiation, no chance had been given to it of asserting its influence; when it was found that all chemical precipitates radiated alike, it was the radiation from a varnish, common to them all, which showed the observed constancy. Hundreds, perhaps thousands, of experiments on radiant heat have been performed in this way, by various inquirers, but the work will, I fear, have to be done over again. I am not, indeed, acquainted with an instance in which an oversight of so trivial a character has been committed by so many able men in succession, vitiating so large an amount of otherwise excellent work. 
Basing our reasonings thus on demonstrated facts, we arive at the extremely probable conclusion that the envelope of the particles, and not the particles themselves, was the real radiator in the experiments just referred to. 'T'o reason thus and deduce their more or less probable consequencesfrom experimental facts, is an incessant exercise of the student of physical science. But having thus followed, for a time, the light of reason alone through a series of phenomena, and emerged from them with a purely intellectual conclusion, our duty is to bring that conclusion to an experimental test. In this way we fortify our science.

For the purpose of testing our conclusion regarding the influence of the gum, I take two powders presenting the same physical appearance; one of them is a compound of mercury, and the other a compound of lead. On two surfaces of a cube are spread these bright red powders, without varnish of any kind. Filling the cube with boiling water, and determining the radiation from the two surfaces, one of them is found to emit thirty-nine units of heat, while the other emits seventy-four. 'This, surely, is a great difference. Here, however, is a second cube, having two of its surfaces coated with the same powders, the only difference being that the powder's are laid on by means of a transparent gum. Both surfaces are now absolutely alike in radiative power. Both of them emit somewhat more than was emitted by either of the unvarnished powders, simply becuuse the gum employed is a better radiator than either of them. Excluding all varuish, and comparing white with white, vast differences are found; comparing black with black, they are also different; and when black and white are compared, in some cases the black radiates far more than the white, while in other cases the white radiates far more than the black. Determining, noreover, the absorptive power of those powders, it is found to go hand-in-hand with their radiative power. The good radiator is a good absorber, and the bad radiator is a bad absorber. From all this it is evident that as regards the radiation and absorption of non-luminous heat, color teaches us nothing; and that even as regards the radiation of the sun, consisting as it does mainly of non-luminous rays, conclusions as to the influence of color may be altogether delusive. This is the strict scientific upshot of our 
researches. But it is not the less true that in the case of wearing apparel-and this for reasons which I have given in analyzing the experiment of Franklin-black dresses are more potent than white ones as absorbers of solar heat.

'Thus, in brief outline, have been brought before you a few of the results of recent inquiry. If you ask me what is the use of them, I can hardly answer you, unless you define the term use. If you meant to ask whether those dark rays which clear away the Alpine snows, will ever be applied to the roasting of turkeys, or the driving of steamengines-while affirming their power to do both, I would frankly confess that they are not at present capable of competing profitably with coal in these particulars. Still they may have great uses unknown to me; and when our coal-fields are exhausted, it is possible that a more ethereal race than we are may cook their victuals, and perform their work, in this transcendental way. But is it necessary that the student of science should have his labor's tested by their possible practical applications? What is the practical value of Homer's Iliad? You smile, and possibly think that Homer's Iliad is good as a means of culture. 'There's the rub. 'The people who demand of science practical uses, forget, or do not know, that it also is great as a means of culture-that the knowledge of this wonderful universe is a thing profitable in itself, and requiring no practical application to justify its pursuit.

But while the student of nature distinctly refuses to have his labors judged by their practical issues unless the term practical be made to include mental as well as material good, he knows full well that the greatest practical triumphs have been episodes in the search after pure natural truth. 'The electric telegraph is the standing wonder of this age, and the men whose scientific knowledge, and mechanical skill, have made the telegraph what it is, are deserving of all honor. In fact, they have had their reward, both in reputation and in those more substantial benefits which the direct service of the public always carries in its train. But who, I would ask, put the soul into this telegraphic body? Who snatched from heaven the fire that flashes along the line? 'This, I am bound to say, was done by two men, the one a dweller in Italy, ${ }^{*}$ the 
other a dweller in England, * who never in their inquiries conscionsly set a practical object before them-whose only stimulus was the fascination which draws the climber to a never-trodden peak, and would have made Cæastr quit his victories for the sources of the Nile. 'That the knowledge brought to us by those prophets, priests, and kings of science is what the world calls " useful knowlerlge," the triumphant application of their discoveries proves. But science has another function to fulfill, in the storing and the training of the human mind; and I would base my appeal to you on the specimen which has this evening been bronght before you, whether any system of education at the present day can be deemed even approximately complete, in which the knowledge of Nature is neglected or ignored.

\section{CHAPTER IV.}

NEW CHEMICAL REACTIONS PRODUCED BY LIGHT.

1868-69.

Measured by their power, not to excite vision, but to produce heat--in other words, measured by their absolute energy - the ultra-red waves of the sun and of the electric light, as shown in the preceding articles, far transcend the visible. In the domain of chemistry, however, there are numerous cases in which the more powerful waves are ineffectual; while the more minute waves, through what may be called their timeliness of application, are able to produce great effects. A series of these, of a novel and beautiful character, discovered in 1868, and further illustrated in subsequent years, may be exhibited by subjecting the vapors of volatile liquids to the action of concentrated sunlight, or to the concentrated beam of the electric light. Their investigation led up to the discourse on "Dust and Disease" which follows in this volume; and for this reason some account of them is introduced here. 
A glass tube 3 feet long and 3 inches wide, which had been frequently employed in my researches on radiant heat, was supported horizontally on two stands. At one end of the tube was placed an electric lamp, the height

Fia. 2.

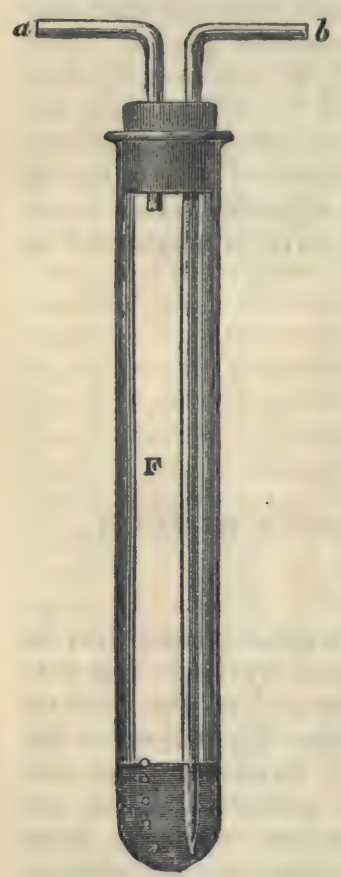

and position of both being so arranged that the axis of the tube, and that of the beam issuing from the lamp, were coincident. In the first experiments the two ends of the tube were closed by plates of rock-salt and subsequently by plates of glass. For the sake of distinction, I call this tube the experimental tube. It was connected with an air-punp, and also with a series of drying and other tubes used for the purification of the air.

A number of test-tubes, like $\mathbf{F}$, fig. 2 (I have used at least fifty of them), were converted into Woulf's flasks. Each of them was stopped by a cork, through which passed two glass tubes: one of these tubes $(a)$ ended immediately below the cork, while the other $(b)$ descended to the bottom of the flask, being drawn out at its lower end to an orifice about 0.03 of an inch in diameter. It was found necessary to coat the cork carefully with cement. In the later experiments corks of vulcanized india-rubber were invariably employed.

The little flask, thus formed, being partially filled with the liquid whose vapor was to be examined, was introduced into the path of the purified current of air. The experimental tube being exhausted, and the cock which cut off the supply of purified air being cautiously turned on, the air entered the flask throngh the tube $b$, and escaped by the small orifice at the lower end of $b$ into the liquid. Through this it bubbled, loading itself with vapor, after which the mixed air and vapor, passing from the flask by the tube $a$, entered the experimental tube, where they were subjected to the action of light. 


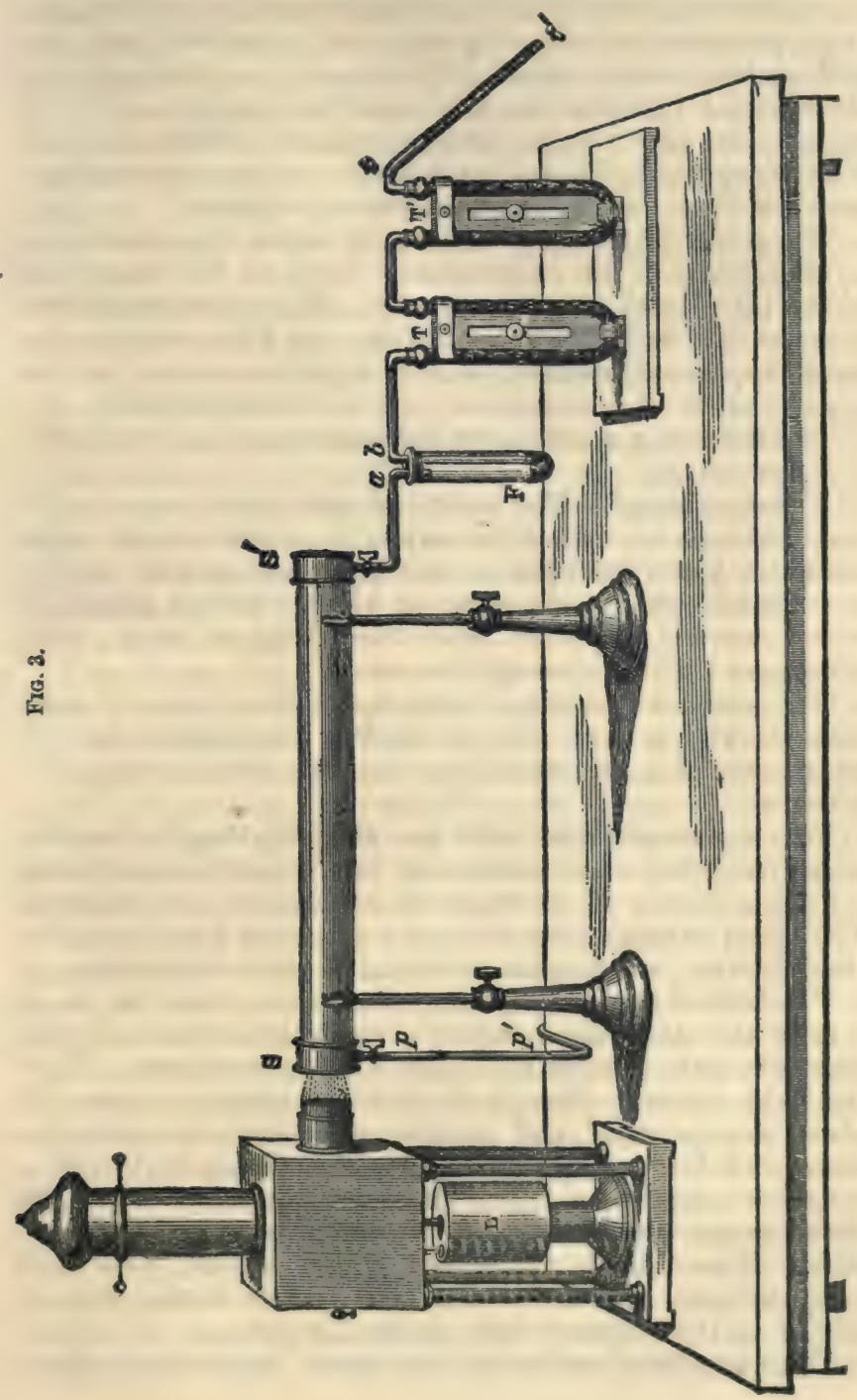


The whole arrangement is shown in fig. 3, where $\mathrm{L}$ represents the electric lamp, s s' the experimental tube, $p p^{\prime}$ the pipe leading to the air-pump, and $\mathrm{F}$ the test-tube containing the volatile liquid. The tube $t t^{\prime}$ is plugged with cotton-wool intended to intercept the floating matter of the air; the bent tube $\mathrm{T}^{\prime}$ contains caustic potash, the tube T sulphuric acid, the one intended to remove the carbonic acid and the other the aqueous vapor of the air.

'The power of the electric beam to reveal the existence of anything within the experimental tube, or the impurities of the tube itself, is extraordinary. When the experiment is made in a darkenel room, a tube which in ordinary daylight appears absolutely clean, is often shown by the present mode of examination to be exceedingly filthy.

The following are some of the results obtained with this arrangement:

Nitrite of amyl. - The vapor of this liquid was in the first instance permitted to enter the experimental tube, while the beam from the electric lamp was passing through it. Curious clouds, the cause of which was then unknown, were observed to form near the place of entry, being afterward whirled through the tube.

The tube being again exhausted, the mixed air and vapor were allowed to enter it in the dark. The slightly convergent beam of the electric light was then sent through the mixture.

For a moment the tube was optically empty, nothing whatever being seen within it; but before a second had elapsed a shower of particles was precipitated on the beam. The cloud thus generated became denser as the light continued to act, showing at some places vivid iridescence.

The lens of the electric lamp was now placed so as to form within the tube a strongly convergent cone of rays. The tube was cleansed and again filled in darkness. When the light was sent through it, the precipitation upon the bean was so rapid and intense that the cone, which a moment before was invisible, flashed suddenly forth like a solid luminous spear. 'The effect was the same when the air and vapor were allowed to enter the tube in diffuse daylight. The cloud, however, which shone with such extraordinary radiance under the electric beam, was invisible in the ordinary light of the laboratory.

The quantity of mixed air and vapor within the experi- 
mental tube could of course be regulated at pleasure. The rapidity of the action diminished with the attenuation of the vapor. When, for example, the mercurial column associated with the experimental tube was depressed only five inches, the action was not nearly so rapid as when the tube was full. In such cases, however, it was exceedingly interesting to observe, after some seconds of waiting, a thin streamer of delicate bluish-white cloud slowly forming along the axis of the tube, and finally swelling so as to fill it.

When dry oxygen was employed to carry in the vapor, the effect was the same as that obtained with air.

When dry hydrogen was used as a vehicle, the effect was also the same.

The effect, therefore, is not due to any interaction between the vapor of the nitrite and its vehicle.

This was further demonstrated by the deportment of the vapor itself. When it was permitted to enter the experimental tube unmixed with air or any other gas, the effect was substantially the same. Hence the seat of the observed action is the vapor.

This action is not to be ascribed to heat. As regards the glass of the experimental tube, and the air within the tube, the beam employed in these experiments was perfectly cold. It had been sifted by passing it through a solution of alum, and through the thick double-convex lens of the lamp. When the unsifted beam of the lamp was employed, the effect wats still the same; the obscure calorific rays did not appear to interfere with the result.

My object here being simply to point out to chemists a method of experiment which reveals a new and beautiful series of reactions, I left to them the examination of the products of lecomposition. The group of atoms forming the molecule of nitrite of amyl is obviously shaken asunder by certain specific waves of the electric beam, nitric oxide and other products, of which the nitrate of amyl is probably one, being the result of the decomposition. 'The brown fumes of nitrous acid were seen mingling with the cloud within the experimental tube. The nitrate of amyl, being less volatile than the nitrite, and not being able to maintain itself in the condition of vapor, would be precipitated as a visible cloud along the track of the beam.

In the anterior portions of the tube a powerful sifting of 
the beam by the vapor occurs, which diminishes the chemical action in the posterior portions. In some experiments the precipitated cloud only extended halfway down the tube. When, under these circumstances, the lamp was shifted so as to send the beam through the other end of the tube, copious precipitation occurred there also.

Solar light also effects the decomposition of the nitriteof-amyl vapor. On October 10, 1868, I partially darkened a small room in the Royal Institution, into which the sun shone, permitting the light to enter through an open portion of the window-shutter. In the track of the beam was placed a large plano-convex lens, which formed a fine convergent cone in the dust of the room behind it. The experimental tube was filled in the laboratory, covered with a black cloth, and carried into the partially darkened room. On thrusting one end of the tube into the cone of rays behind the lens, precipitation within the cone was copious and immediate. The vapor at the distant end of the tube was in part shielded by that in front, and was also more feebly acted on through the divergence of the rays. On reversing the tube, a second and similar cone was precipitated.

\section{Physical Considerations.}

I sought to determine the particular portion of the light which produced the foregoing effects. When, previous to entering the experimental tube, the beam was caused to pass through a red glass, the effect was greatly weakened, but not extinguished. 'This was also the case with various samples of yellow glass. A blue glass being introduced before the removal of the yellow or the red, on taking the latter away prompt precipitation occurred along the track of the blue beam. Hence, in this case, the more refrangible rays are the most chemically active. The color of the liquid nitrite of amyl indicates that this must be the case; it is a feeble but distinct yellow: in other words, the yellow portion of the beam is most freely transmitted. It is not, however, the transmitted portion of any beam which produces chemical action, but the absorbed portion. Blue, as the complementary color to yellow, is hereabsorbed, and hence the more energetic action of the blue rays.

This reasoning, however, assumes that the same rays are absorbed by the liquid and its vapor. The assumption is 
worth testing. A solution of the yellow chromate of potash, the color of which may be made almost, if not altogether, identical with that of the liquid nitrite of amyl, was founil far more effective in stopping the chemical rays than either the red or the yellow glass. But of all substances the iiquid nitrite itself is most potent in arresting the rays which act upon its vapor. A layer one-eighth of an inch in thickness, which scarcely perceptibly affected the luminous intensity, absorbed the entire chemical energy of the concentrated beam of the electric light.

The close relation subsisting between a liquid and its vapor, as regards their action upon radiant heat, has been already amply demonstrated.* As regards the nitrite of amyl, this relation is more specific than in the cases hitherto adduced; for here the special constituent of the beam, which provokes the decomposition of the vapor, is shown to be arrested by the liquid.

A question of extreme importance in molecular physics here arises: What is the real mechanism of this absorption, and where is its seat? $\dagger$ I figure, as others do, a molecule as a group of atoms, held together by their mutual forces, but still capable of motion among themselves. The vapor of the nitrite of amyl is to be regarded as an assemblage of such molecules. The question now before us is this: In the act of absorption, is it the molecules that are effective, or is it their constituent atoms? Is the vis viva of the intercepted light-waves transferred to the molecule as a whole, or to its constituent parts?

The molecule, as a whole, can only vibrate in virtue of the forces exerted between it and its neighbor molecules. The intensity of these forces, and consequently the rate of vibration, would, in this case, be a function of the distance between the molecules. Now the identical absorption of the liquid and of the vaporous nitrite of amyl indicates an identical vibrating period on the part of liquid and rapor, and this, to my mind, amounts to an experimental proof that the absorption occurs in the main within the molecule. For it can hardly be supposed, if the absorption were the

* “Phil. Trans." 1864; “Heat, a Mode of Motion," chap. xii.; and p. 45 of this volume.

$+\mathrm{My}$ attention was very forcibly directed to this subject some vears ago by a conversation with wy excellent friend Professor Clausius. 
act of the molecule as a whole, that it could continue to affect waves of the same period after the substance had pissed from the vaporous to the liquid state.

In point of fact, the decomposition of the nitrite of amyl is itself to some extent an illustration of this internal molecular absorption; for were the absorption the act of the molecule as a whole, the relative motions of its constituent atoms would remain unchanged, and there would be no mechanical cause for their separation. It is probably the synchronism of the vibrations of one portion of the molecule with the incident waves, that enables the amplitule of those vibrations to augment, until the chain which binds the parts of the molecule together is snapped asunder.

I anticipate wide, if not entire, generality for the fact that a liquid and its vapor absorb the same rays. A cell of liquid chlorine would, I imagine, deprive light more effectually of its power of causing chlorine and hydrogen to combine than any other filter of the luminous rays. 'The rays which give chlorine its color have nothing to do with this combination, those that are absorbed by the chlorine heing the really effective rays. A highly sensitive bulb, containing chlorine and hydrogen, in the exact proportions necessary for the formation of hydrochloric acid, was placed at one end of an experimental tube, the beam of the electric lamp being sent through it from the other. 'The bulb did not explode when the tube was filled with chlorine, while the explosion was violent and immediate when the tube was filled with air. I anticipate for the liquid chlorine an action similar to, but still more energetic than that exhilited by the gas. If this should prove to be the case, it will faror the view that chlorine itself is molecular, and not monatomic.

\section{Production of Sky-blue by the Decomposition of Nitrite of Amyl.}

When the quantity of nitrite vapor is considerable, and the light intense, the chemical action is exceedingly rapid, the particles precipitated being so large as to whiten the luminous beam. Not so, however, when a well-mixed and highly attenuated rapor fills the experimental tube. The effect now to be described was first obtained when the 
vapor of the nitrite was derived from a portion of its liquid which had been accilentally introduced into the passage through which the dry air flowed into the experimental tube.

In this case, the electric beam traversed the tube for several seconds before any action was visible. Decomposition then visibly commeuced, and advanced slowly. When the light was very strong, the cloud appeared of a milky blue. When, on the contrary, the intensity was moderate, the blue was pure and deep. In Brücke's important experiments on the blue of the sky and the morning and evening red, pure mastic is dissolved in alcohol, and then dropped into water well stirred. When the proportion of mastic to alcohol is correct, the resin is precipitated so finely as to elude the highest microscopic power. By reflected light, such a medium appears bluish, by transmitted light yellowish, which latter color, by augmenting the quantity of the precipitate, can be caused to pass into orange or red.

But the development of color in the attenuated nitriteof-amyl rapor is doubtless more similar to what takes place in our atmosphere. The blue, moreover, is far purer and more sky-like than that obtained from Brücke's turbid medium. Never, even in the skies of the Alps, have I seen a richer or a purer blue than that attainable by a suitable disposition of the light falling upon the precipitated vapor.

Iodide of Allyl.-Among the liquids hitherto subjected to the concentrated electric light, iodide of allyl, in point of rapidity and intensity of action, comes next to the nitrite of amyl. With the iodide I have employed both oxygen and hydrogen, as well as air, as a vehicle, and found the effect in all cases substantially the same. 'The cloudcolumn here was exquisitely beantiful. It revolved round the axis of the decomposing beam; it was nipped at certain places like an hour-glass, and round the two bells of the glass delicate cloud-filaments twisted themselves in spirals. It also folded itself into convolutions resembling those of shells. In certain conditions of the atmosphere in the Alps I have often observed clouds of a special pearly luster, when hydrogen was made the vehicle of the iodide-of-allyl vapor a similar luster was most exquisitely shown. With a suitable disposition of the light, the purple hue of iodinevapor came out very strongly in the tube. 
The remark already made, as to the bearing of the decomposition of nitrite of amyl by light on the question of molecular absorption, applies here also; for were the absorption the work of the molecule as a whole, the iodine would not be dislodged from the allyl with which it is combined. The non-synchronism of iodine with the waves of obscure heat is illustrated by its marvelous transparency to such heat. May not its synchronism with the waves of light in the present instance be the cause of its divorce from the allyl?

Iodide of Isopropyl. - The action of light upon the vapor of this liquid is, at first, more languid than upon iodide of allyl; indeed many beautiful reactions may be overlooked, in consequence of this languor at the commencement. After some minutes' exposure, however, clouds begin to form, which grow in density and in beauty as the light continues to act. In every experiment hitherto made with this substance the column of cloud filling the experimental tube was divided into two distinct parts near the middle of the tube. In one experiment a globe of clond formed at the center, from which, right and left, issued an axis uniting the globe with two adjacent cylinders. Both globe and cylinders were animated by a common motion of rotation. As the action continued, paroxysms of motion were manifested; the various parts of the cloud would rush through each other with sudden violence. During these motions beautiful and grotesque cloud-forms were developed. At some places the nebulous mass would become ribbed so as to resemble the graining of wood; a longitudinal motion would at times generate in it a series of curved transverse bands, the retarding influence of the sides of the tube causing an appearance resembling, on a small scale, the dirt-bands of the Mer de Glace. In the anterior portion of the tube those sudden commotions were most intense; here buds of clond would sprout forth, and grow in a few seconds into perfect flower-like forms. The clond of iodide of isopropyl had a character of its own, and differed materially from all others that I had seen. A gorgeons mauve color was observed in the last twelve inches of the tube; the vapor of iodine was present and it may have been the sky-blue scattered by the precipitated particles which, mingling with the purple of the iodine, produced the mave. As in all other cases here adduced, the 
effects were proved to be due to the light; they never occurred in darkness.

The forms assumed by some of those actinic clouds, as I propose to call them, in consequence of rotations and other motions, due to differences of temperature, are perfectly astounding. I content myself here with a meager description of one more of them.

'The tube being filled with the sensitive mixture, the beam was sent through it, the lens at the same time being so placed as to produce a cone of very intense light. Two minutes elapsed before anything was visible; but at the end of this time a faint bluish cloud appeared to hang itself on the most concentrated portion of the beam.

Soon afterward a second cloud was formed five inches farther down the experimental tube. Both clouds were united by a slender cord of the same bluish tint as themselves.

As the action of the light continued, the first cloud gradually resolved itself into a series of parallel disks of exquisite delicacy, which rotated round an axis perpendicular to their surfaces, and finally blended to a screw surface with an inclined generatrix. This gradually changed into a filmy funnel, from the narrow end of which the "cord" extended to the cloud in advance. The latter also underwent slow but incessant modification. It first resolved itself into a series of strata resembling those of the electric discharge. After a little time, and through changes which it was difficult to follow, both clouds presented the appearance of a series of concentric funnels set one within the other, the interior ones being seen through the outer ones. Those of the distant clond resembled claret-glasses in shape. As many as six funnels were thus concentrically set together, the two series being united by the delicate cord of cloud already referred to. Other corls and slender tubes were afterward formed, which coiled themselves in delicate spirals around the funnels.

Rendering the light along the connecting-cord more intense, it diminished in thickness and became whiter; this was a consequence of the enlargement of its particles. The cord finally disappeared, while the funnels melted into two ghost-like films, shaped like parasols. They were barely visible, being of an exceerlingly delicate blue tint. 'They seemed woven of blue air. 'To compare them 
with cobweb or with gauze would be to liken them to something infinitely grosser than themselves.

In all cases a distant candle-flame, when looked at through the cloud, was sensibly undimmed.

2. ON THE BLUE COLOR OF THE SKY, AND THE POLARIZATION OF SKYLIGHT.*

1869.

After the communication to the Royal Society of the foregoing brief account of a new Series of Chemical Reactious produced by Light, the experiments upon this subject were continued, the number of substances thus acted on being considerably increased.

I now, however, beg to direct attention to two questions glanced at incidentally in the preceding pages - the blue color of the sky, and the polarization of skylight. Reserving the historic treatment of the subject for a more fitting occasion, I would merely mention now that these questions constitute, in the opinion of our most eminent authorities, the two great standing enigmas of meteorology. Indeed it was the interest manifested in them by Sir John Herschel, in a letter of singular speculative power, addressed to myself, that caused me to enter upon the consideration of these questions so soon.

The apparatus with which I work consists, as already stated, of a glass tube about a yard in length, and from $2 \frac{1}{2}$ to 3 inches internal diameter. The vapor to be examined is introduced into this tube in the manner already described, and upon it the condensed beam of the electric lamp is permitted to act, until the neutrality or the activity of the substance has been declared.

It has hitherto been my aim to render the chemical action of light upon vapors visible. For this purpose substances have been chosen, one at least of whose products of decomposition under light shall have a boiling point so high, that as soon as the substance is formed it shall be precipitated. By graduating the quantity of the vapor, this precipitation may be rendered of any degree of fine-

* In my "Lectures on light" (Longmans), the polarization of light will be found briefly, but, I trust, clearly explained. 
ness, forming particles distinguishable by the naked eye, or far beyond the reach of our highest microscopic powers. I have no reason to doubt that particles may be thus obtained, whose diameters constitute but a small fraction of the length of a wave of violet light.

In all cases when the vapors of the liquids employed are sufficiently attenuated, no matter what the liquid may be, the visible action commences with the formation of a blue cloud. But here I must guard myself against all misconception as to the use of this term. The "cloud" here referred to is totally invisible in ordinary daylight. 'To be seen, it requires to be surrounded by darkness, it only being illuminated by a powerful beam of light. This blue cloud differs in many important particulars from the finest ordiuary clouds, and might justly have assigned to it an intermediate position between such clouds and true vapor. With this explanation, the term "cloud," or "incipient cloud," or " actinic cloud," as I propose to employ it, cannot, I think, be misunderstood.

I had been endeavoring to decompose carbonic acid gas by light. A faint bluish cloud, due it may be, or it may not be, to the residue of some vapor previously employed, was formed in the experimental tube. On looking across this cloud through a Nicol's prism, the line of vision being horizontal, it was found that when the short diagonal of the prism was vertical, the quantity of light reaching the eye was greater than when the long diagonal was vertical. When a plate of tourmaline was held between the eye and the bluish cloud, the quantity of light reaching the eye when the axis of the prism was perpendicular to the axis of the illuminating beam, was greater than when the axis of the crystal and of the beam were parallel to each other.

'This was the result all round the experimental tube. Causing the crystal of tourmaline to revolve round the tube, with its axis perpendicular to the illuminating beam, the quantity of light that reached the eye was in all its positions a maximum. When the crystallographic axis was parallel to the axis of the beam, the quantity of light transmitted by the crystal was a minimum. From the illuminated bluish cloud, therefore, polarized light was discharged, the direction of maximum polarization being at right angles to 
the illuminating beam; the plane of vibration of the polarized light was perpendicular to the beam.*

'Thin plates of selenite or of quartz, placed between the Nicol and the actinic cloud, displayed the colors of polarized light, these colors being most vivid when the line of vision was at right angles to the experimental tube. The plate of selenite usually employed was a circle, thinnest at the center, and augmenting uniformly in thickness from the center outward. When placed in its proper position between the Nicol and the cloud, it exhibited a system of splendidly colored rings.

The cloud here referred to was the first operated upon in the manner iescribed. It may, however, be greatly improved upon by the choice of proper substances, and by the application, in proper quantities, of the substances chosen. Benzol, bisulphide of carbon, nitrite of amyl, nitrite of butyl, iodide of allyl, iodide of isopropyl, and many other substances may be employed. I will take the nitrite of butyl as illustrative of the means adopted to secure the best result, with reference to the present question.

And here it may be mentioned that a vapor, which when alone, or mixed with air in the experimental tube, resists the action of light, or shows but a feeble result of this action, may, when placed in proximity with another gas or vapor, exhibit vigorous, if not violent action. 'The case is similar to that of carbonic acid gas, which, diffused in the atmosphere, resists the decomposing action of solar light, but when placed in contignity with chlorophyll in the leaves of plants, has its molecules shaken asunder.

Dry air was permitted to bubble through the liquid nitrite of butyl, until the experimental tube, which had been previously exhausted, was filled with the mixed air and vapor. 'The visible action of light upon the mixture after fifteen minutes' exposure was slight. The tube was afterward filled with half an atmosphere of the mixed air and vapor, and a second half-atmosphere of air which had been permitted to bubble through fresh commercial hydrochloric acid. On sending the beam through this mixture, the tube,

* This is still an undecided point; but the probabilities are so much in its favor, and it is in my opinion so much preferable to have a physical image on which the mind can rest, that I do not hesitate to employ the phraseology in the text. 
for a moment, was optically empty. But the pause amounted ouly to a small fraction of a second, a dense cloud being immediately precipitated upon the beam.

This cloud began blue, but the advance to whiteness was so rapid as almost to justify the application of the term instantaneous. 'The dense clond, looked at perpendicularly to its axis, showed scarcely any signs of polarization. Looked at obliquely the polarization was strong.

The experimental tube being again cleansed and exhausted, the mixed air and nitrite-of-butyl vapor was permitted to enter it until the associated mercury column was depressed one-tenth of an inch. In other words, the air and vapor, united, exercised a pressure not exceeding one three hundredth of an atmosphere. Air, passed through a solution of hydrochloric acid, was then added, till the mercury column was depressed three inches. The condensed beam of the electric light was passed for some time through this mixture without revealing anything within the tube competent to scatter the light. Soon, however, a superbly blue cloud was formed along the track of the beam, and it continued blue sufficiently long to permit of its thorough examination. The light discharged from the cloud, at right angles to its own length, was at first perfectly polarized. It could be totally quenched by the Nicol. By degrees the cloud became of whitish blue, and for a time the selenite colors, obtained by looking at it normally, were exceedingly brilliant. 'The direction of maximum polarization was distinctly at right angles to the illuminating beam. This continued to be the case as long as the cloud maintained a decided blue color, and even for some time after the blue had changed to whitish blue. But, as the light continued to act, the cloud became coarser and whiter, particularly at its center, where it at length ceased to discharge polarized light in the direction of the perpendicular, while it continued to do so at both ends.

But the cloud which had thus ceased to polarize tice light emitted normally, showed vivid selenite colors when looked at obliquely, proving that the direction of maximum polarization changed with the texture of the cloud. This point shall receive further illustration subsequently.

A blue, equally rich and more durable, was obtained by employing the nitrite-of-butyl vapor in a still more attenuated condition. The instance here cited is representative. 
In all cases, and with all substances, the cloud formed at the commencement, when the precipitated particles are sufficiently fine, is blue, and it can be made to display a color rivaling that of the purest Italian sky. In all cases, moreover, this fine blue clond polarizes perfectly the beam which illuminates it, the direction of polarization enclosing an angle of 90 degrees with the axis of the illuminating beam.

It is exceedingly interesting to observe both the perfection and the decay of this polarization. For ten or fifteen minutes after its first appearance the light from a vividly illuminated actinic cloud, looked at perpendicularly, is absolutely quenched by a Nicol's prism with its longer diagonal vertical. But as the sky-blue is gradually rendered impure by the growth of the particles-in other words, as real clouds begin to be formed-the polarization begins to decay, a portion of the light passing through the prism in all its positions. It is worthy of note, that for some time after the cessation of perfect polarization, the residual light which passes, when the Nicol is in its position of minimum transmission, is of a gorgeous blue, the whiter light of the cloud being extinguished.* When the cloud texture has become sufficiently coarse to approximate to that of ordinary clouds, the rotation of the Nicol ceases to have any sensible effect on the quantity of light discharged normally.

The perfection of the polarization, in a direction perpendicular to the illuminating beam, is also illustrated by the following experiment: A Nicol's prism, large enough to embrace the entire beam of the electric lamp, was placed between the lamp and the experimental tube. A few bubbles of air, carried through the liquid nitrite of butyl were introduced into the tube and they were followerl by about three inches (measured by the mercurial gaug(*) of air which had passed through aqueous hydrochloric acid. Sending the polarized beam through the tube, I placed myself in front of it, my eye being on a level with its axis, my assistant occupying a similar position behind the tube. The short diagonal of the large Nicol was in the first instance vertical, the plane of vibration of the

* This shows that particles too large to polarize the blue, polarize perfectly light of lower refrangibility. 
emergent beam being therefore also vertical. As the light contirued to act, a superb blue cloud, visible to both my assistant and myself, was slowly formed. But this cloud, so deep and rich when looked at from the positions mentioned, utterly disappeared when looked at vertically downward or vertically upward. Reflection from the cloud was not possible in these directions. When the large Nicol was slowly turned round its axis, the eye of the observer being on the level of the beam, and the line of vision perpendicular to it, entire extinction of the light emitted horizontally occurred when the longer diagonal of the large Nicol was vertical. But now a vivid blue cloud was seen when looked at downward or upward. This truly fine experiment, which I contemplated making on my own account, was first definitely suggested by a remark in a letter addressed to me by Professor Stokes.

As regards the polarization of skylight, the greatest stumbling-block has hitherto been, that, in accordance with the law of Brewster, which makes the index of refraction the tangent of the polarizing angle, the reflection which produces perfect polarization would require to be made in air upon air; and indeed this led many of our most eminent men, Brewster himself among the number, to entertain the idea of aërial molecular reflection.* I have, however, operated upon substances of widely differ-

* "The cause of the polarization is evidently a reflection of the sun's light upon something. The question is on what? Were the angle of maximum polarization $76^{\circ}$, we should look to water or ice as the reflecting body, however inconceivable the existence in a cloudless atmosphere and a hot summer's day of unevaporated molecules (particles?) of water. But though we were once of this opinion, careful observation has satisfied us that $90^{\circ}$, or thereabouts, is the correct angle, and that therefore whatever be the body on which the light has been reflected, if polarized by a single reflection, the polarizing angle must be $45^{\circ}$, and the index of refraction, which is the tangent of that angle, unity; in other words, the reflection would r. quire to be made in air upon air!" (Sir John Herschel, " Meteorology," par. 233.)

Any particles, if small enough, will produce both the color and the polarization of the sky. But is the existence of small water-particles on a hot sumuer's day in the higher regions of our atmosphere inconceivable? It is to be remembered that the oxygen and nitrogen of the air behave as a vacuum to radiant heat, the exceedingly attenuated vapor of the higher atmosphere being therefore in practical contact with the cold of space. 
ent refractive indices, and therefore of very different polarizing angles as ordinarily defined, but the polarization of the beam, by the incipient cloud, has thus far proved itself to be absolutely independent of the polarizing angle. The law of Brewster does not apply to matter in this condition, and it rests with the undulatory theory to explain why. Whenever the precipitated particles are sufficiently fine, no matter what the substance forming the particles may be, the direction of maximum polarization is at right angles to the illuminating beam, the polarizing angle for matter in this condition being invariably 45 degrees.

Suppose our atmosphere surrounded by an enrelope impervious to light, but with an aperture on the sunward side through which a parallel beam of solar light could enter and traverse the atmosphere. Surrounded by air not directly illuminated, the track of such a beam would resemble that of the parallel beam of the electric lamp through an incipient cloud. 'The sunbeam would be blue, and it would discharge laterally light in precisely the same condition as that discharged by the incipient cloud. In fact, azure revealed by such a beam would be "to all intents and purposes" that which I have called a "blue cloud:" Conversely our " blue cloud" is, to all intents and purposes, an artificial sky.*

But, as regards the polarization of the sky, we know that not only is the direction of maximum polarization at right angles to the track of the solar beams, but that at certain angular distances, probably variable ones, from the sun, "neutral points," or points of no polarization, exist, on both sides of which the planes of atmospheric polarization are at right angles to each other. I have made various observations upon this subject which are reserved for the present; but, pending the more complete examination of

* The opinion of Sir John Herschel connecting the polarizations and the blue color of the sky, is verified by the foregoing results. "The more the subject [the polarization of skylight] is considered," writes this eminent philosopher, "the more it will be found beset with difficulties, and its explanation when arrived at will probably be found to carry with it that of the blue color of the sky itself, and of the great quantity of light it actually does send down to us." "We may observe, too," he adds, "that it is only where the purity of the sky is most absolute that the polarization is developed in its highest degree, and that where there is the sliglitest perceptible tendency to cirrus it is materially impaired." 'This applies word for word to our "incipient clouds." 
the question, the following facts bearing upon it may be submitted.

The parallel beam employed in these experiments tracked its way through the laboratory air, exactly as sunbeams are seen to do in the dusty air of London. I have reason to believe that a great portion of the matter thus floating in the laboratory air consists of organic particles, which are capable of imparting a perceptibly bluish tint to the air. These also showed, though far less vividly, all the effects of polarization obtained with the incipient clouds. The light discharged laterally from the track of the illuminating beam polarized, though not perfectly, the direction of maximum polarization being at right angles to the beam. At all points of the beam, moreover, throughout its entire length, the light emitted normally was in the same state of polarization. Keeping the positions of the Nicol and the selenite constant, the same colors were observed throughout the entire beam, when the line of vision was perpendicular to its length.

The horizontal column of air, thus illuminated, was 18 feet long, and could therefore be looked at very obliquely. I placed myself near the end of the beam, as it issued from the electric lamp, and, looking through the Nicol and selenite more and more obliquely at the beam, observed the colors fading until they disappeared. Augmenting the obliquity the colors appeared once more, but they were now complementary to the former ones.

Hence this beam, like the sky, exhibited a neutral point, on opposiue sides of which the light was polarized in planes at right angles to each other.

'Thinking that the action observed in the laboratory might be caused, in some way, by the vaporous fumes diffused in its air, I had the light removed to a room at the top of the Royal Institution. 'The track of the beam was seen very finely in the air of this room, a length of 14 or 15 feet being attainable. This bean exhibited all the effects observed with the beam in the laboratory. Even the uncondensed electric light falling on the floating matter showed, though faintly, the effects of polarization.

When the air was so sifted as to entirely remove the visible floating matter, it no longer exerted any sensible action upon the light, but behaved like a vacuuın. 'The light is scattered and polarized by particles, not by molecules or atoms. 
By operating upon the fumes of chloride of ammonium, the smoke of brown paper, and tobacco-smoke, I had varied and confirmed in many ways those experiments on neutral points, when my attention was drawn by Sir Charles Wheatstone to an important observation communicated to the Paris Academy in 1860 by Professor Govi, of Turin.* M. Govi had been led to examine a beam of light sent through a room in which were successively diffused the smoke of incense, and tobacco-smoke. His first brief communication stated the fact of polarization by such smoke; but in his second communication he announced the discovery of a neutral point in the beam, at the opposite sides of which the light was polarized in planes at right angles to each other.

But unlike my observations on the laboratory air, and unlike the action of the sky, the direction of maximum polarization in M. Govi's experiment enclosed a very small angle with the axis of the illuminating beam. 'The question was left in this condition, and I am not aware that M. Govi or any other investigator has pursued it further.

I had noticed, as before stated, that as the clouds formed in the experimental tube became denser, the polarization of the light discharged at right angles to the beam became weaker, the direction of maximum polarization becoming oblique to the beam. Experiments on the fumes of chloride of ammonium gave me also reason to suspect that the position of the neutral point was not constant, but that it varied with the density of the illuminated fumes.

The examination of these questions led to the following new and remarkable results: The laboratory being well filled with the fumes of incense, and sufficient time being allowed for their uniform diffusion, the electric beam was sent through the smoke. From the track of the beam polarized light was discharged; but the direction of maximum polarization, instead of being perpendicular, now enclosed an angle of only 12 degrees or 13 degrees with the axis of the beam.

A neutral point, with complementary effects at opposite sides of it, was also exhibited by the beam. The angle enclosed by the axis of the beam, and a line drawn from the

* "C'omptes Rendus," tome li. pp. 360 and 669. 
neutral point to the observer's eye measured in the first instance 66 degrees.

The windows of the laboratory were now opened for some minutes, a portion of the incense-smoke being permitted to escape. On again darkening the room and turning on the light, the line of vision to the neutral point was found to enclose, with the axis of the beam, an angle of 63 degrees.

The windows were again opened for a few minutes, more of the smoke being permitted to escape. Measured as before, the angle referred to was found to be 54 degrees.

This process was repeated three additional times; the neutral point was found to recede lower and lower down the beam, the angle between a line drawn from the eye to the neutral point and the axis of the beam falling successively from 54 degrees to 49 degreees, 43 degrees and 33 degrees.

The distances, roughly measured, of the neutral point from the lamp, corresponding to the foregoing series of observations, were these:

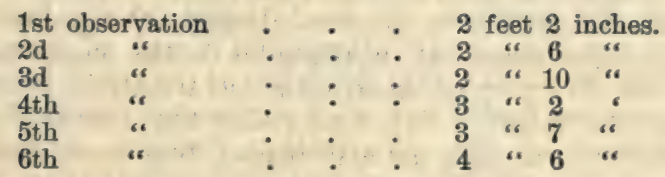

At the end of this series of experiments the direction of maximum polarization had again become normal to the beam.

The laboratory was next filled with the fumes of gunpowder. In five successive experiments, corresponding to five different densities of the gunpowder-smoke, the angles enclosel between the line of vision to the neutral point and the axis of the beam, were 63 degrees, 50 degrees, 47 degrees, 42 degrees, and 38 degrees respectively.

After the clouds of gunpowder had cleared away, the laboratory was filled with the fumes of common resin, rendered so dense as to be very irritating to the lungs. The direction of maximum polarization enclosed, in this case, an angle of 12 degrees, or thereabouts, with the axis of the beam. Looked at, as in the former instances, from a 
position near the electric lamp, no neutral point was observed throughout the entire extent of the beam.

When this beam was looked at normally through the selenite and Nicol, the ring-system, though not brilliant, was distinct. Keeping the eye upon the plate of selenite, and the line of vision perpendicular, the windows were opened, the blinds remaining undrawn. The resinous fumes slowly diminished, and as they did so the ringsystem became paler. It finally disappeared. Continuing to look in the same direction, the rings revived, but now the colors were complementary to the former ones. The neutral point had passed me in its motion down the beam, consequent upon the attenuation of the fumes of resin.

With the fumes of chloride of ammonium substantially the same results were obtained. Sufficient, however, has been here stated to illustrate the variability of the position of the neutral point.*

By a puff of tobacco-smoke, or of condensed steam, blown into the illuminated beam, the brilliancy of the selenite colors may be greatly enhanced. But with different clouds two different effects are produced. Let the ring-system observed in the common air be brought to its maximum strength, and then let an attenuated cloud of chloride of ammonium be thrown into the beam at the point looked at; the ring system flashes out with augmented brilliancy, but the character of the polarization remains unchanged. This is also the case when phosphorus or sulphur is burned underneath the beam, so as to cause the fine particles of phosphorus or of sulphur to rise into the light. With the sulphur-fumes the brilliancy of the colors is exceedingly intensified; but in none of these cases is there any change in the character of the polarization.

But when a puff of the fumes of hydrochloric acid, hydriodic acid, or nitric acid is thrown into the beam, there is a complete reversal of the selenite tints. Each of these clouds twists the plane of polarization 90 degrees, causing the center of the ring-system to change from black

* Brewster has proved the variability of the position of the neutral point for skylight with the sun's altitude, a result obviously connected with the foregoing experiments. 
to white, and the rings themselves to emit their complementary colors.*

Almost all liquids have motes in them sufficiently numerous to polarize sensibly the light, and very beautiful effects may be obtained by simple artificial devices. When, for example, a cell of distillea water is placed in front of the electric lamp, and a thin slice of the beam is permitted to pass through it, scarcely any polarized light is discharged, and scarcely any color produced with a plate of selenite. But if a bit of soap be agitated in the water above the beam, the moment the infinitesimal particles reach the light the liquid sends forth laterally almost perfectly polarized light; and if the selenite be employed, vivid colors flash into existence. A still more brilliant result is obtained with mastic dissolved in a great excess of alcohol.

The selenite rings, in fact, constitute an extremely delicate test as to the collective quantity of individually invisible particles in a liquid. Commencing with distilled water, for example, a thick slice of light is necessary to make the polarization of its suspended particles sensible. A much thinner slice suffices for common water; while, with Brücke's precipitated mastic, a slice too thin to produce any sensible effect with most other liquids, suffices to bring out vividly the selenite colors.

\section{THE SKY OF THE ALPS.}

The vision of an object always implies a differential action on the retina of the observer. The object is distinguished from surrounding space by its excess or defect of light in relation to that space. By altering the illumination, either of the object itself or of its environment, we alter the appearance of the object. Take the case of clouds floating in the atmosphere with patches of blue between them. Anything that changes the illumination of either alters the appearance of both, that appearance depending, as stated, upon differential action. Now the light of the sky, being polarized, may, as the reader of the foregoing

* Sir John Herschel suggested to me that this change of the polarization from positive to negative may indicate a clange from polarization by reflection to polarization by refraction. 'This thought repeatedly occurred to me while looking at the effects; but it will require much following up before it emerges into clearness. 
pages knows, be in great part quenched by a Nicol's prism, while the light of a common cloud, being unpolarized, cannot be thus extinguished. Hence the possibility of very remarkable variations, not only in the aspect of the firmament, which is really changed, but also in the aspect of the clouds, which have that firmament as a background. It is possible, for example, to choose clouds of such a depth of shade that when the Nicol quenches the light behind them, they shall vanish, being undistinguishable from the residual dull tint which outlives the extinction of the brilliancy of the sky. A cloud less deeply shaded, but still deep enough, when viewed with the naked eye, to appear dark on a bright ground, is suddenly changed to a white cloud on a dark ground by the quenching of the light behind it. When a reddish cloud at sunset chances to float in the region of maximum polarization, the quenching of the surrounding light causes it to flash with a brighter crimson. Last Easter eve the Dartmoor sky, which had just been cleansed by a snow-storm, wore a very wild appearance. Round the horizon it was of steely brilliancy, while reddish cumuli and cirri floated southward. When the sky was quenched behind them these floating masses seemed like dull embers suddenly blown upon; they brightened like a fire.

In the Alps we have the most magnificent examples of crimson clouds and snows, so that the effects just referred to may be here studied under the best possible conditions. On August 23, 1869, the evening Alpenglow was very fine, though it did not reach its maximum depth and splendor. The side of the Weisshorn seen from the Bel Alp, being turned from the sun, was tinted mauve; but I wished to observe one of the rose-colored buttresses of the mountain. Such a one was visible from a point a few hundred feet above the hotel. 'The Matterhorn also, though for the most part in shade, had a crimson projection, while a deep ruddy red lingered along its western shoulder. Four distant peaks and buttresses of the Dom, in addition to its dominant head-all covered with pure snow - were reddened by the light of sunset. The shoulder of the Alphubel was similarly colored, while the great mass of the Fletschorn was all aglow, and so was the snowy spine of the Monte Leone.

Looking at the Weisshorn through the Nicol, the glow 
of its protuberance was strong or weak according to the position of the prism. The summit also underwent striking changes. In one position of the prism it exhibited a pale white against a dark background; in the rectangular position it was a dark mauve against a light background. 'I'he red of the Matterhorn changed in a similar manner; but the whole mountain also passed through wonderful changes of definition. The air at the time was filled with a silvery haze, in which the Matterhorn almost disappeared. 'This could be wholly quenched by the Nicol, and then the mountain sprang forth with astonishing solidity and detachment from the surrounding air. The changes of the Dom were still more wonderful. A vast amount of light could be removed from the sky behind it, for it occupied the position of maximum polarization. By a little practice with the Nicol it was easy to render the extinction of the light, or its restoration, almost instantaneous. When the sky was quenched, the four minor peaks and buttresses, and the summit of the Dom, together with the shoulder of the Alphubel, glowed as if set suddenly on fire. This was immediately dimmed by turning the Nicol through an angle of 90 degrees. It was not the stoppage of the light of the sky behind the mountains alone which produced this startling effect; the air between them and me was highly opalescent, and the quenching of this intermediate glare augmented remarkably the distinctness of the mountains.

On the morning of August 24 similar effects were finely shown. At 10 A.M. all three mountains, the Dom, the Matterhorn, and the Weisshorn,were powerfully affected by the Nicol. But in this instance also, the line drawn to the Dom being very nearly perpendicular to the solar beams, the effects on this mountain were most striking. The gray summit of the Matterhorn, at the same time, could scarcely be distinguished from the opalescent haze around it; but when the Nicol quenched the haze, the summit became instantly isolated, and stood out in bold definition. It is to be remembered that in the production of these effects the only things changed are the sky behind, and the luminous haze in front of the mountains; that these are changed because the light ernitted from the sky and from the haze is plane polarized light, and that the light from the snows and from the mountains, being sensibly unpolar. 
ized, is not directly affected by the Nicol. It will also be understood that it is not the interposition of the haze as an opaque body that renders the mountains indistinct, but that it is the light of the haze which dims and bewilders the eye, and thus weakens the definition of objects seen through it.

The results have a direct bearing upon what artists call "aërial perspective." As we look from the summit of Mont Blanc, or from the lower elevation, at the serried crowd of peaks, especially if the mountains be darkly colored-coveled with pines, for example-every peak and ridge is separated from the mountains behind it by a thin blue haze which renders the relations of the mountains as to distance unmistakable. When this haze is regarded through the Nicol perpendicular to the sun's rays, it is in many cases wholly quenched, because the light which it emits in this direction is wholly polarized. When this happens, aërial perspective is abolished, and mountains very differently distant appear to rise in the same vertical plane. Close to the Bel Alp, for instance, is the gorge of the Massa, and beyond the gorge is a high ridge darkened by pines. This ridge may be projected upon the dark slopes at the opposite side of the Rhone valley, and between both we have the blue haze referred to, throwing the distant mountains far away. But at certain hours of the day the haze may be quenched, and then the Massa ridge and the mountains beyond the Rhone seem almost equally distant from the eye. The one appears, as it were, a vertical continuation of the other. The haze varies with the temperature and humidity of the atmosphere. At certain times and places it is almost as blue as the sky itself; but to see its color, the attention must be withdrawn from the mountains and from the trees which cover them. In point of fact, the haze is a piece of more or less perfect sky; it is produced in the same manner, and is subject to the same laws, as the firmament itself. We live in the sky, not under it.

'These points were further elucidated by the deportment of the selenite plate, with which the readers of the foregoing pages are so well acquainted. On some of the sunny days of August the haze in the valley of the Rhone, as looked at from the Bel Alp, was very remarkable. 'Toward evening the sky above the mountains opposite to my place of observation yielded a series of the most splendidly col- 
ored iris-rings; but on lowering the selenite until it had the darkness of the pines at the opposite side of the Rhone valley, instead of the darkness of space, as a background, the colors were not much diminished in brilliancy. I should estimate the distance across the valley, as the crow flies, to the opposite mountain, at nine miles; so that a body of air of this thickness can, under favorable circumstances, produce chromatic effects of polarization almost as vivid as those produced by the sky itself.

Again: the light of a landscape, as of most other things, consists of two parts; the one, coming purely from superficial reflection, is always of the same color as the light which falls upon the landscape; the other part reaches us from a certain depth within the objects which compose the landscape, and it is this portion of the total light which gives these objects their distinctive colors. The white light of the sun enters all substances to a certain depth, and is partly ejected by internal reflection; each distinet substance absorbing and reflecting the light, in accordance with the laws of its own molecular constitution. Thus the solar light is sifted by the landscape, which appears in such colors and variations of color as, after the sifting process, reach the observer's eye. Thus the bright green of grass, or the darker color of the pine, never comes to us alone, but is always mingled with an amount of light derived from-superficial reflection. A certain hard briliancy is conferred upon the woods and meadows by this superficially reflected light. Under certain circumstances, it may be quenched by a Nicol's prism, and we then obtain the true color of the grass and foliage. Trees and meadows, thus regarded, exhibit a richness and softness of tint which they never show as long as the superficial light is permitted to mingle with the true interior emission. The needles of the pines show this effect very well, large-leaved trees still better; while a glimmering field of maize exhibits the most extraordinary variations when looked at through the rotating Nicol.

Thoughts and questions like those here referred to took me, in August, 1869, to the top of the Aletschhorn. The effects described in the foregoing paragraphs were for the most, part reproduced on the summit of the mountain. I scanned the whole of the sky with my Nicol. Both alone, and in conjunction with the selenite, it pronounced the 
perpendicular to the solar beams to be the direction of maximum polarization. But at no portion of the firmament was the polarization complete. The artificial sky produced in the experiments recorded in the preceling pages could, in this respect, be rendered far more perfect than the natural one; while the gorgeous "residual blue" which makes its appearance when the polarization of the artificial sky ceases to be perfect, was strongly contrasted with the lack-luster hue which, in the case of the firmament, outlived the extinction of the brilliancy. With certain substances, however, artificially treated, this dull residue may also be obtained.

All along the are from the Matterhorn to Mont Blane the light of the sky immediately above the monntains was powerfully acted upon by the Nicol. In some cases the variations of intensity were astonishing. I have already said that a little practice enables the observer to shift the Nicol from one position to another so rapidly as to render the alternative extinction and restoration of the light immediate. When this was done along the arc to which I have referred, the alternations of light and darkness resembled the plity of sheet lightning behind the mountains. There was an element of awe connected with the suddenness with which the mighty masses, ranged along the line referred to, changed their aspect and definition under the operation of the prism.

[In the last edition of the "Fragments of Science" an essay on "Dust and Disease" followed here; but as almost al my writings on the "Germ Theory" are now collected in a single volume entitle 1 "Essays on the Floating Matter of the Air," "Dust and Disease" no longer appears in the "Fragments." In its place I venture to introduce a short article written early last year for an important American magazine.]

\section{CHAPTER V.}

\section{THE SKY.*}

Invited to write for the For um an article that would have brought me face to face with "problems of life and mind" for which I was at the moment unprepared, and unwilling to decline a request so courteously made, I

* From The Forum, February, 1888. 
offered, if the editor cured to accept it, to send him a contribution on the subject here presented.

I mentioned this subject, thinking that, in adảition to its interest as a fragment of "natural knowledge," it might permit of a glance at the workings of the scientific mind when engaged on the deeper problems which come before it. In the house of Science are many mansions, occupied by tenants of diverse kinds. Some of them execute with painstaking fidelity the useful work of observation, recording from day to day the aspects of Nature, or the indications of instruments devised to reveal her ways. Others there are who add to this capacity for observation a power over the language of experiment, by means of which they put questions to Nature, and receive from her intelligible replies. 'There is, again, a third class of minds, that cannot rest content with observation and experiment, whose love of causal unity tempts them perpetually to break through the limitations of the senses, and to seek beyond then the roots and reasons of the phenomena which the observer and experimenter record. To such spirits-adventurous and firm-we are indebted for our deeper knowledge of the methods by which the physical universe is ordered and ruled.

In his efforts to cross the common bourne of the known and the unknown, the effective force of the mau of science must depend, to a great extent, upon his acquired knowledge. But knowledge alone will not do; a stored memory will not suffice; inspiration must lend its aid. Scientific inspiration, however, is usually, if not always, the fruit of long reflection- of patiently "intending the mind," as Newton phrased it; and as Copernicus, Newton, and Darwin practiced it; until outer darkness yields a glimmer, which in due time opens out into perfect intellectual day. From some of his expressions it might be inferred that Newton scorned hypotheses; but he allowed them, nevertheless, an open avenue to his own mind. He propounded the famous corpuscular theory of light, illustrating it and defending it with a skill, power, and fascination which subsequently won for it ardent supporters among the best intellects of the world. This theory, moreover, was weighted with a supplementary hypothesis, which ascribed to the luminiferous molecules "fits of easy reflection and transmission," in virtue of which they were sometimes 
repelled from the surfaces of bodies and sometimes permitted to pass through. Newton may have scorned the levity with which hypotheses are sometimes framed; but he lived in an atmosphere of theory, which he, like all profound scientific thinkers, found to be the very breath of his intellectual life.

The theorist takes his conceptions from the world of fact, and refines and alters them to suit his needs. The sensation of sound was known to be produced by aërial waves impinging ou the auditory nerve. Air being a thing that could be felt, and its vibrations, by suitable treatment, made manifest to the eye, there was here a physical basis for the "scientific imagination" to build upon. Both Hooke and Huyghens built upon it with effect. By the illustrious astronomer last named the conception of waves was definitely transplanted from its terrestrial birthplace to a universal medium whose undulations could only be intellectually discerned. Huyghens did not establish the undulatory theory, but he took the first firm step toward establishing it. Laying this theory at the root of the phenomena of light, he went a good way toward showing that these phenomena are the necessary outgrowth of the conception.

By analysis and synthesis Newton proved the white light of the sun to be a skein of many colors. The cause of color was a question which immediately occupied his thoughts; and here, as in other cases, he freely resorted to hypothesis. He saw, with his mind's eye, his luminiferous corpuscles crossing the bodily eye, and imparting successive shocks to the retina behind. "To differences of "bigness" in the light-awakening molecules Newton ascribed the different color-sensations. In the undulatory theory we are also confronted with the question of color; and here again, to inform and guide us, we have the analogy of sound. Aërial waves of different lengths, or periods, produce notes of different pitch; and to differences of wave-length in that mysterious medium, the all-pervading ether, dilferences of color are ascribed. Hooke had already discoursed of "a very quick motion that causes light, as well as a more robust that causes heat." Newton had ascribed the sensation of red to the shock of his grossest, and that of violet to the shock of his finest luminiferous projectiles. Defining the one, and displacing the other of 
these notions, the wave-theory affirins red to be produced by the largest, and violet by the smallest waves of the visible spectrum. The theory of undulation had to encounter that fierce struggle for existence which all great changes of doctrine, scientific or otherwise, have had to endure. Mighty intellects, following the mightiest of them all, were arrayed against it. But the more it was discussed the more it grew in strength and favor, until it finally supplanted its formidable rival. No competent scientific man at the present day accepts the theory of emission, or refuses to accept the theory of undulation.

Boyle and Hooke had been fruitful experimenters on those beautiful iridescences known as the "colors of thin plates." The rich hues of the thin-blown soap-bubble, of oil floating on water, and of the thin layer of oxide on molten lead, are familiar illustrations of these iris color's. Hooke showed that all transparent films, if only thin enough, displayed such colors; and he proved that the particular color displayed depended upon the thickness of the film. Passing from solid and liquid films to films of air, he says: "Take two small pieces of ground and polished lookingglass plate, each about the bigness of a shilling; take these two dry, and with your forefingers and thumbs press them very hard and close together, and you shall find that when they approach each other very near, there will appear several irises or colored lines." Newton, bent on knowing the exact relation between the thickness of the film and the color it produced, varied Hooke's experiment. 'Taking two pieces of glass, the one plane and the other very slightly curved, and pressing both together, he obtained a film of air of gradually increasing thickness from the place of contact outward. As he expected, he found the place of contact surrounded by a series of colored circles, still known all over the world as " Newton's rings." The colors of his first circle, which immediately surrounded a black central spot, Newton called "colors of the first order;" the colors of the second circle, "colors of the second order," and so on. With unrivaled penetration and apparent success, he applied his theory of "fits" to the explanation of the "rings." Here, however, the only immortal parts of his labors are his facts and measarements; his theory has disappeared. It was reserved for the illustrious Thomas Young, a man of intellectual caliber resembling that of 
Newton himself, to prove that the rings were produced by the mutual action-in technical phrase, "interference"of the light waves reflected at the two surfaces of the film of air inclosed between the plane and convex glasses. 'The colors of thin plates were "residual colors"-survivals of the white light after the ravages of interference. Young soon translated the theory of "fits" into that of "waves;" the measurements pertaining to the former being so accurate as to render them immediately available for the purposes of the latter.

It is here that Newton's researches and opinions tonch the subject of this article. The color nearest to the black spot, in the experiment above described, was a faint blue"blue of the first order"-corresponding to the film of air when thinnest. If a solid or liquid film, of the thickness requisite to produce this color, were broken into bits and scattered in the air, Newton inferred that the tiny fragments would display the blue color. Tantamount to this, he considered, was the action of minute water-particles in the incipient stage of their condensation from aqueous vapor. Such particles suspended in our atmosphere ought, he supposed, to generate the serenest skies. Newton does not appear to have bestowed much thought upon this subject; for to produce the particular blue which he regarded as sky-blue, thin plates with parallel surfaces would be required. The notion that cloud-particles are hollow spheres, or vesicles, is prevalent on the Continent, but it never made any way among the scientific men of England. De Saussure thought that he had actually seen the clond-vesicles, and Faraday, as I learned from himself, believed that he had once confirmed the observation of the illustrions Alpine traveler. During my long acquaintance with the atmosphere of the Alps I have often sought for these aqueous bladders, but have never been able to find them. Clausius once published a profound essay on the color's of the sky. 'The assumption of small water drops, he proved, would lead to optical consequences entirely at variance with facts. For a time, therefore, he closed with the idea of vesicles, and endeavored to deduce from them the blue of the firmament and the morning and evening red.

It is not, however, necessary to invoke the blue of the first order to explain the color of the sky; nor is it necessary to impose upon condensing vapor the difficult, if not 
impossible, task of forming bladders, when it passes into the liquid condition. Let us examine the subject. Euude-Cologne is prepared by dissolving aromatic gums or resins in alcohol. Dropped into water, the scented liquid immediately produces a white cloudiness, due to the precipitation of the substances previously held in solution. 'The solid particles are, however, comparatively gross; but by diminishing the quantity of the dissolved gum, the precipitate may be made to consist of extremely minute particles. Brücke, for example, dissolved gum-mastic, in certain proportions, in alcohol, and carefully dropping his solution into a beaker of water, kept briskly stirred, he was able to reduce the precipitate to an extremely fine state of division. The particles of mastic can by no means be imagined as forming bladders. Still, against a dark ground-black velvet, for example-the water that contains them shows a distinctly blue color. The bluish color of many liquids is produced in a similar manner. Thin milk is an example. Blue eyes are also said to be simply turbid media. The rocks over which glaciers pass are finely ground and pulverized by the ice, or the stony emery imbedded in it; and the river which issues from the snout of every glacier is laden with suspended matter. When such glacier water is placed in a tall glass jar, and the heavier particles are permitted to subside, the liquid column, when viewed against a dark background, has a decidedly bluish tinge. 'The exceptional blueness of the lake of Geneva, which is fed with glacier water, may be due, in part, to particles small enough to remain suspended long after their larger and heavier companions have sunk to the bottom of the lake.

We need not, however, resort to water for the production of the color. We can liberate, in air, particles of a size capable of producing a blue as deep and pure as the azure of the firmament. In fact, artificial skies may be thus generated, which prove their brotherhood with the natural sky by exhibiting all its phenomena. There are certain chemical compounds-aggregates of molecules-the constituent atoms of which are readily shaken asunder by the impact of special waves of light. Probably, if not certainly, the atoms and the waves are so related to eich other, as regards vibrating period, that the wave-motion can accumulate until it becomes disruptive. A great number of substances might be mentioned whose vapors, when mixed, with 
air and subjected to the action of a solar or an electric beam, are thus decomposed, the products of decomposition hanging as liquid or solid particles in the beam which generates them. And here I must appeal to the inner vision already spoken of. Remembering the different sizes of the waves of light, it is not difficult to see that our minute particles are larger with respect to some waves than to others. In the case of water, for example, a pebble will intercept and reflect a larger fractional part of a ripple than of a larger wave. We have now to imagine light-undulations of different dimensions, but all exceedingly minute, passing through air laden with extremely small particles. It is plain that such particles, though scattering portions of all the waves, will exert their most conspicuous action upon the smallest ones; and that the color-sensation answering to the smallest waves-in other words, the color blue-will be predominant in the scattered light. This harmonizes perfectly with what we observe in the firmament. The sky is blue, but the blue is not pure. On looking at the sky through a spectroscope, we observe all the colors of the spectrum; blue is merely the predominant color. By means of our artificial skies we can take, as it were, the firmament in our hands and examine it at our leisure. Like the natural sky, the artificial one shows all the colors of the spectrum, but blue in excess. Mixing very small quantities of vapor with air, and bringing the decomposing luminous beam into action, we produce particles too small to shed any sensible light, but which may, and doubtless do, exert an action on the ultra-violet waves of the spectrum. We can watch these particles, or rather the space they occupy, till they grow to a size able to yield the firmamental azure. As the particles grow larger under the continued action of the light, the azure becomes less deep; while later on a milkiness, such as we often observe in nature, takes the place of the purer blue. Finally the particles become large enough to reflect all the light-waves, and then the suspended "actinic cloud" diffuses white light.

It must occur to the reader that even in the absence of definite clouds there are considerable variations in the hue of the firmament. Everybody knows, moreover, that as the sky bends toward the horizon, the purer blue is impaired. 'To measure the intensity of the color De Saussure invented a cyanometer, and Humboldt has given us a 
mathematical formula to express the diminution of the blue, in ares drawn east and west from the zenith downward. This diminution is a natural consequence of the predominance of coarser particles in the lower regions of the atmosphere. Were the particles which produce the purer celestial vault all swept away, we should, unless lielped by what has been called "cosmic dust," look into the blackness of celestial space. And were the whole atmosphere abolished along with its suspended matter, we should have the "blackness" spangled with steady stars; for the twinkling of the stars is caused by our atmosphere. Now, the higher we ascend, the more do we leave behind us the particles which scatter the light; the nearer, in fact, do we approach to that vision of celestial space mentioned a moment ago. Viewed, therefore, from the loftiest Alpine summits, the firmamental blue is darker than it is ever observed to be from the plains.

It is thus shown that by the scattering action of minute particles the blue of the sky can be produced; but there is yet more to be said upon the subject. Let the natural sky be looked at on a fine day through a piece of transparent Iceland spar cut into the form known as a Nicol prism. It may be well to begin by looking through the prism at a snow slope, or a white wall. Turning the prism round its axis, the light coming from these objects loes not undergo any sensible change. But when the prism is directed toward the sky the great probability is that, on turning it, variations in the amount of light reaching the eye will be observed. 'Testing various portions of the sky with due diligence, we at length discover one particular direction where the difference of illumination becomes a maximum. Here the $\mathrm{Nicol}$, in one position, seems to offer no impediment to the passage of the skylight, while, when turned through an arc of ninety degrees from this position, the light is almost entirely quenched. We soon discern that the particular line of vision in which this maximum difference is observed is perpendicular to the direction of the solar rays. The Nicol acts thus upon skylight because that light is polarized, while the light from the white wall or the white snow, being unpolarized, is not atfected by the rotation of the prism.

In the case of our manufactured sky not only is the azure of the firmament reproduced, but these phenomena 
of polarization are observed even more perfectly than in the natural sky. When the air-space from which our best artificial azure is emitted is examined with the Nicol prism, the blue light is found to be completely polarized at right angles to the illuminating beam. The artificial sky may, in fact, be employed as a second Nicol, between which and a prism held in the hand many of the beantiful chromatic phenomena observed in an ordinary polariscope may be reproduced.

Let us now complete our thesis by following the larger light-waves, which have been able to pass ainong the aërial particles with comparatively little fractional loss. Without going beyond inferential considerations, we can state what nuust occur. 'T'he action of the particles upon the solar light increases with the atmospheric distances traversed by the sun's rays. The lower the sun, therefore, the greater the action. The shorter waves of the spectrum being more and more withdrawn, the tendency is to give the longer waves an enhanced predominance in the transmitted light. The tendency, in other words, of this light, as the rays traverse ever-increasing distances, is more and more toward red. This, I say, might be stated as an inference, but it is borne out in the most impressive manner by facts. When the Alpine sun is setting, or, better still, some time after he has set, leaving the limbs and shoulder's of the mountains in shadow, while their snowy crests are bathed by the retreating light, the snow glows with a beauty and solemnity hardly equaled by any other natural phenomenon. So, also, when first illumined by the rays of the unrisen sun, the mountain heads, under farorable atmospheric conditions, shine like rubies. And all this splendor is evoked by the simple mechanism of miuute particles, themselves without color, suspended in the air. Those who referred the extraordinary succession of atmospheric glows, witnessed some years ago, to a vast and violent discharge of volcanic ashes, were dealing with "a true cause." "The fine floating residue of such ashes would, undoubtedly, be able to produce the effects ascribed to it. Still, the mechanism necessary to produce the morning and the evening red, though of variable efficiency, is always present in the atmosphere. I have seen displays, equal in magnificence to the finest of those above referred to, when there was no special volcanic outburst to which they could be referred. It was 
the long-continued repetition of the glaws which rendered the volcanic theory highly probable.

\section{CHAPTER VI.}

VOYAGE TO ALGERIA TO OBSERVE THE ECLIPSE.

1870.

The opening of the Eclipse Expedition was not propitious. Portsmouth, on Monday, December 5, 1870, was swathed by fog, which was intensified by smoke, and traversed by a drizzle of fine rain. At six P.M. I was on board the Urgent. On Tuesday morning the weather was too thick to permit of the ship being swung and her compasses calibrated. The admiral of the port, a man of very noble presence, came on board. Under his stimulus the energy which the weather had damped appeared to become more active, and soon after his departure we steamed down to Spithead. Here the fog had so far lightened as to enable the officers to swing the ship.

At three P.M. on T'uesday, December 6, we got away, gliding successively past Whitecliff Bay, Bembridge, Sandown, Shanklin, Ventnor, and St. Catherine's Lighthonse. On Wednesday morning we sighted the Isle of Ushant, on the French side of the Channel. 'The northern end of the island has been fretted by the waves into detached towerlike masses of rock of very remarkable appenrance. In the Channel the sea was green, and opposite Ushant it wils a brighter green. On Wednesday evening we committed ourselves to the bay of Biscay. The roll of the Atlantic was full, but not violent. There had been scarcely a gleam of sunshine throughout the day, but the cloud-forms were fine, and their apparent solidity impressive. On 'Thursday morning the green of the sea was displaced by a deep indigo blue. The whole of Thursday we steamed across the bay. We had little blue sky, but the clouds were again grand and varied-cirrus, stratus, cumulus, and nimbus, we had them all. Dusky hair-like trails were sometimes dropperl from the distant clouds to the sea. 'These were falling showers, and they sometimes occupied the whole horizon, 
while we steamed across the rainless circle which was thus surrounded. Sometimes we plunged into the rain, and once or twice, by slightly changing our course, avoided a heavy shower. From time to time perfect rainbows spanned the heavens from side to side. At times a bow would appear in fragments, showing the keystone of the arch midway in air, and its two buttresses on the horizon. In all cases the light of the bow could be quenched by a Nicol's prism, with its long diagonal tangent to the arc. Sometimes gleaming patches of the firmament were seen amid the clouds. When viewed in the proper direction, the gleam could be quenched by a Nicol's prism, a dark aperture being thus opened into stellar space.

At sunset on Thursday the denser clouds were fiercely fringed, while through the lighter ones seemed to issue the glow of a conflagration. On Friday morning we sighted Cape Finisterre-the extreme end of the arc which sweeps from Ushant round the bay of Biscay. Calm spaces of blue, in which floated quietly scraps of cumuli, were behind us, but in front of us was a horizon of portentous darkness. It continued thus threatening throughout the day. 'Toward evening the wind strengthened to a gale, and at dimner it was difficult to preserve the plates and dishes from destruction. Our thinned company hinted that the rolling had other consequences. It was very wild when we went to bed. I slumbered and slept, but after some time was rendered anxiously conscious that my body had become a kind of projectile, with the ship's side for a target. I gripped the edge of my berth to save myself from being thrown out. Outside, I could hear somebody say that he had been thrown from his berth, and sent spinning to the other side of the saloon. The screw labored violently amid the lurching; it incessantly quitted the water, and, twirling in the air, rattled against its bearings, causing the ship to shudder from stem to stern. At times the waves struck us, not with the soft impact which might be expected from a liquid, but with the sudden solid shock of battering-rams. "No man knows the force of water," said one of the officers, " until he has experienced a storm at sea." These blows followed each other at quicker intervals, the screw rattling after each of them, until, finally, the delivery of a heavier stroke than ordinary seemed to reduce the saloon to chaos. Furniture crashed, glasses raug, and 
alarmed inquiries immediately followed. Amid the noises I heard one note of forced laughter; it sounded very ghastly. Men tramped through the saloon, and busy voices were heard aft, as if something there had gone wrong.

I rose, and not without difficulty got into my clothes. In the after-cabin, under the superintendence of the able and energetic navigating lieutenant, Mr. Brown, a group of blue-jackets were working at the tiller-ropes. These had become loose, and the helm refused to answer the wheel. High moral lessons might be gained on shipboard, by observing what steadfast adherence to an object can accomplish, and what large effects are heaped up by the addition of infinitesimals. The tiller-rope, as the bluejackets strained in concert, seemed hardly to move; still it did more a little, until finally, by timing the pull to the lurching of the ship, the mastery of the rudder was obtained. I had previously gone on deck. Round the saloon-door were a few members of the eclipse party, who seemed in no mood for scientific observation. Nor did I; but I wished to see the storm. I climbed the steps to the poop, exchanged a word with Captain Toynbee, the only member of the party to be seen on the poop, and by his direction made toward a cleat not far from the wheel.* Round it I coiled my arms. With the exception of the men at the wheel, who stood as silent as corpses, I was alone.

I had seen grandeur elsewhere, but this was a new form of grandeur to me. The Urgent is long and narrow, and during our expedition she lacked the steadying influence of sufficient ballast. She was for a time practically rudderless, and lay in the trough of the sea. I could see the long ridges, with some hundreds of feet between their crests, rolling upon the ship perfectly parallel to her sides. As they approached, they so grew upon the eye as to render the expression "mountains high" intelligible. At all events, there was no mistaking their mechanical might, as they took the ship upon their shoulders, and swung her like a pendulum. The deck sloped sometimes at an angle which I estimated at over forty-five degrees; wanting my previous Alpine practice, I should have felt less confidence in my grip of the cleat. Here and there

* The cleat is a $\mathbf{T}$-shaped mass of metal employed for the fasten. ing of ropes. 
the long rollers were tossed by interference into heans of greater height. 'The wind caught their crests, and scattered them over the sea, the whole surface of which was seething white. The aspect of the clouds was a fit accompaniment to the fury of the ocean. The moon was almost full-at times concealed, at times revealed, as the scud flew wildly over it. These things appealed to the eye, while the ear was filled by the groaning of the screw and the whistle and boom of the storm.

Nor was the outward agitation the only object of interest to me. I was at once subject and object to myself, and watched with intense interest the workings of my own mind. The Urgent is an elderly ship. She had been built, I was told, by a contracting firm fo some foreign government, and had been diverted from her first purpose when converted into a troop-ship. She had been for some time out of work, and I had heard that one of her boilers, at least, needed repair. Our scanty but excellent crew, moreover, did not belong to the Urgent, but had been gathered from other ships. Our three lieutenants were also volunteers. All this passed swiftly through my mind as the steamer shook under the blows of the waves, and I thought that probably no one on board could say how much of this thumping and straining the Urgent would be able to bear. This uncertainty caused me to look steadily at the worst, and I tried to strengthen myself in the face of it.

But at length the helm laid hold of the water, and the ship was got gradually round to face the waves. The rolling diminished, a certain amount of pitching taking its place. Our speed had fallen from eleven knots to two. I went again to bed. After a space of calm, when we seemed crossing the vortex of a storm, heavy tossing recommenced. I was afraid to allow myself to fall asleep, as my berth was high, and to be pitched out of it might be attended with bruises, if not with fractures. From Friday at noon to Saturday at noon we accomplished sixty-six miles, or an average of less than three miles an hour. I overheard the sailors talking about thisstorm. The Urgent, according to those that knew her, had never previously experienced anything like it. *

* There is, it will be seen. a fair agreement between these impressions and those so vigorously described by a scientific corro. spondent of the limes. 
All through Saturday the wind, though somewhat sobered, blew dead against us. The atmospheric effects were exceedingly fine. 'The cunuli resembled mountains in shape, and their peaked summits shone as white as Alpine snows. At one place this resemblance was greatly strengthened by a vast area of cloud, uniformly illuminated, and lying like a névé below the peaks. From it fell a kind of clond-river strikingly like a glacier. The horizon at sunset was remarkable-spaces of brilliant green between clouds of fiery red. Ruinbows had been frequent throughout the day, and at night a perfectly continuous lunar low spauned the heavens from side to side. Its colors were feeble; but, contrasted with the black ground agaiust which it rested, its luninousness was extraordinary.

Sunday morning found us opposite to Lisbon, and at midnight we rounded Cape St. Vincent, where the lurching seemed disposed to recommence. 'Through the kindness of Lieutenant Walton, a cot had been slung for me. It hung between a tiller-wheel and a flue, and at one A.M. I was roused by the banging of the cot against its boundaries. But the wind was now behind us, and we went along at a speed of eleven knots. We felt certain of reaching Cadiz by three. But a new lighthouse came in sight, which some affirmed to be Cadiz Lighthonse, while the surrounding houses were declared to be those of Cadiz itself. Out of deference to these statements, the navigating lieutenant changed his course, and steered for the place. A pilot came on board, and he informed us that we were before the mouth of the Guadalquivir, and that the lighthouse was that of Cipiona. Cadiz was still some eighteen miles distant.

We steered toward the city, hoping to get into the harbor before dark. But the pilot who would have guided us had been snapped up by another vessel, and we did not get in. We beat about during the night, and in the morning found ourselves about fifteen miles from Cadiz. The sun rose behind the city, and we steered straight into the light. The three-towered cathedral stood in the midst, round which swarmed apparently a multitude of chimneystacks. A nearer approach showed the chimneys to be small turrets. A pilot was taken on board; for there is a dangerous shoal in the harbor. 'The appearance of the town as the sun shone upon its white and lofty walls was 
singularly beautiful. We cast anchor; some officials arrived and demanded a clean bill of health. We had none. They would have nothing to do with us; so the yellow quarantine flag was hoisted, and we waited for permission to land the Cadiz party. After some hours' delay the English consul and vice-consul came on board, and with them a Spanish officer ablaze with gold lace and decorations. Under slight pressure the requisite permission had been granted. We landed our party, and in the afternoon weighed anchor. T'hanks to the kindness of our excellent paymaster, I was here transferred to a more roomy berth.

Cadiz soon sank beneath the sea, and we sighted in succession Cape Trafalgar, Tarifa, and the revolving light of Ceuta. The water was very calm, and the moon rose in a quiet heaven. She swung with her convex surface downward, the common boundary between light and shadow being almost horizontal. A pillar of reflected light shimmered up to us from the slightly rippled sea. I had previously noticed the phosphorescence of the water, but tonight it was stronger than usual, especially among the foam at the bows. A backet let down into the sea brought up a number of the little sparkling organisms which caused the phosphorescence. I caught some of them in my hand. And here an appearance was observed which was new to most of us, and strikingly beautiful to all. Standing at the bow and looking forward, at a distance of forty or fifty yards from the ship, a number of luminons streaners were seen rushing toward us. On nearing the vessel they rapidly turner, like a comet round its perihelion, placed themselves side by side, and, in parallel trails of light, kept up with the ship. One of them placed itself right in front of the bow as a pioneer. These comets of the sea were joined at intervals by others. Sometimes as many as six at a time would rush at us, bend with extraordinary rapidity round a sharp curve, and afterward keep us company. I leaned over the bow, and scanned the streamers closely. 'The frontal portion of each of them revealed the outline of a porpoise. 'The rush of the creatures through the water had started the phosphorescence, every spark of which was converted by the motion of the retina into a line of light. Each porpoise was thus wrapped in a luminous sheath. 'The phosphorescence did not cease at 
the creature's tail, but was carried many porpoise-lengths behind it.

To our right we had the African hills, illuminated by the moon. Gibraltar Rock at length became visible, but the town remained long hidden by a belt of haze, through which at length the brighter lamps struggled. It was like the gradual resolution of a nebula into stars. As the intervening depth became gradually less, the mist vanished more and more, and finally all the lamps shone through it. They formed a bright foil to the somber mass of rock above them. The sea was so calm and the scene so lovely that $\mathrm{Mr}$. Huggins and myself stayed on deck till near midnight, when the ship was moored. During our walking to and fro a striking enlargement of the disk of Jupiter was observed, whenever the heated air of the funnel came between us and the planet. On passing away from the heated air, the flat dim disk would immediately shrink to a luminous point. The effect was one of visual persistence. The retinal image of the planet was set quivering in all azimuths by the streams of heated air, describing in quick succession minute lines of light, which summed themselves to a disk of sensible area.

At six o'clock next morning, the gun at the signal station on the summit of the rock, boomed. At eight the band on board the Trafalgar training-ship, which was in the harbor, struck up the national anthem; and immediately afterward a crowd of mite-like cadets swarmed up the rigging. After the removal of the apparatus belonging to the Gibraltar party we went on shore. Winter was in England when we left, but here we had the warmth of summer. The vegetation was luxuriant-palm trees, cactuses, and aloes, all ablaze with scarlet flowers. A visit to the governor was proposed, as an act of necessary courtesy, and I accompanied Admiral Ommaney and $\mathrm{Mr}$. Huggins to " the Convent," or Government House. We sent in our cards, waited for a time, and were then conducted by an orderly to his excellency. He is a fine old man, over six feet high, and of frank military bearing. He received us and conversed with us in a very genial manner. He took us to see his garden, his palms, his shaded promenarles, and his orange-trees loaded with fruit, in all of which he took manifest delight. Evidently "the hero of Kars" had fallen upon quarters after his own heart. He appeared 
full of good nature, and engaged us on the spot to dine with him that day.

We sought the town-major for a pass to visit the lines. While awaiting his arrival I purchased a stock of white glass bottles, with a view to experiments on the color of the sea. Mr. Huggins and myself, who wished to see the rock, were taken by Captain Salmond to the library, where a model of Gibraltar is kept, and where we had a useful preliminary lesson. At the library we met Colonel Maberly, a courteous and kindly man, who gave us good advice regarding our excursion. He sent an orderly with us to the entrance of the lines. The orderly handed us over to an intelligent Irishman, who was directed to show us everything that we desired to see, and to hide nothing from us. We took the "upper line," traversed the galleries hewn through the limestone; looked through the embrasures, which opened like doors in the precipice, toward the hills of Spain; reached St. George's hall, and went still higher, emerging on the summit of one of the noblest cliffs I have ever seen.

Beyond were the Spanish lines, marked by a line of white sentry-boxes; nearer were the English lines, less conspicuously indicated; and between both was the neutral ground. Behind the Spanish lines rose the conical hill called the Queen of Spain's Chair. The general aspect of the mainland from the rock is bold and rugged. Doubling back from the galleries, we struck upward toward the erest, reached the signal station, where we indulged in "shandy-gaff" and bread and cheese. Thence to O'Har's's 'Tower, the highest point of the rock. It was built by a former governor, who, forgetful of the laws of terrestrial curvature, thought he might look from the tower into the port of Cadiz. The tower is riven, and it may be climbed along the edges of the crack. We got to the top of it; thence descended the curious Mediterranean Stair-a zigzag, mostly of steps down a steeply falling slope, anid palmetto brush, aloes, and prickly pear.

Passing over the Windmill Hill, we were joined at the "Governor's Cottage" by a car, and drove afterward to the lighthouse at Europa Point. 'The tower was built, I believe, by Queen Adelaile, and it contains a fine dioptric apparatus of the first order, constructed by Messrs. Chance, of Birmingham. At the appointed hour we were at the Con- 
vent. During dinner the same genial traits which appeared in the morning were still more conspicuous. The freshness of the governor's nature showed itself best when he spoke of his old antagonist in arms, Mouravieff. Chivalry in war is consistent with its stern prosecution. These two men were chivalrous, and after striking the last blow became friends forever. Our kind and courteous reception at Gibraltar is a thing to be remembered with pleasure.

On December 15 we committed ourselves to the Mediterranean. 'The views of Gibraltar with which we are most acquainted represent it as a huge ridge; but its aspect, end on, both from the Spanish lines and from the other side, is truly noble. There is a sloping bank of sand at the back of the rock, which I was disposed to regard simply as the débris of the limestone. I wished to let myself down upon it, but had not the time. My friend Mr. Busk, however, assures me that it is silica, and that the same sand constitutes the adjacent neutral ground. There are theories afloat as to its having been blown from Sahara. The Mediterranean throughout this first day, and indeed throughout the entire voyage to Oran, was of a less deep blue than the Atlantic. Possibly the quantity of organisms may have modified the color. At night the phosphorescence was startling, breaking suddenly out along the crests of the waves formed by the port and starboard bows. Its strength was not uniform. Having flashed brilliantly for a time, it would in part subside, and afterward regain its vigor. Several large phosphorescent masses of weird appearance also floated past.

On the morning of the 16th we sighted the fort and lighthouse of Marsa el Kibir, and beyond them the white walls of Oran lying in the bight of a bay, sheltered by dominant hills. The sun was shining brightly; during our whole voyage we had not had so fine a day. 'The wisdom which had led us to choose Oran as our place of observation seemed demonstrated. A rather excitable pilot came on board, and he guided us in behind the mole, which had suffered much damage the previous year from an unexplained outburst of waves from the Mediterranean. Both port and bow anchors were cast in deep water. With three huge hawsers the ship's stern was made fast to three gun-pillars fixed iri the mole; and here for a time the Urgent rested from her labors. 
M. Janssen, who had rendered his name celebrated by his observations of the eclipse in India in 1868, when he showed the solar flames to be eruptions of incandescent hydrogen, was already encamped in the open country about eight miles from Oran. On December 2 he had quitted Paris in a balloon, with a strong young sailor as his assistant, had descended near the mouth of the Loire, seen M. Gambetta, and received from him encouragement and aid. On the day of our arrival his encampnent was visited by Mr. Huggins, and the kind and courteous engineer of the port drove me subsequently, in his own phaeton, to the place. It bore the best repute as regards freedom from haze and fog, and commanded an open outlook; but it was inconvenient for us on account of its distance from the ship. 'The place next in repute was the railway station, between two and three miles distant from the mole. It was inspected, but, being enclosed, was abandoned for an eminence in an adjacent garden, the property of Mr. Hinshelwood, a Scotchman who had settled some years previously as an esparto merchant in Oran.* He, in the most liberal manner, placed his grounds at the disposition of the party. Here the tents were pitched, on the Saturday, by Captain Salmond and his intelligent corps of sappers, the instruments being erected on the Monday under cover of the tents.

Close to the railway station runs a new loopholed wall of defense, through which the highway passes into the open country. Standing on the highway, and looking southward, about twenty yards to the right is a small bastionet, intended to carry a gun or two. Its roof I thought would form an admirable basis for my telescope, while the view of the surrounding country was unimpeded in all directions. The authorities kindly allowed me the use of this bastionet. Two men, one a blue-jacket named Elliot, and the other a marine named Hill, were placed at my disposal by Jientenant Walton; and, thus aided, on Monday morning I mounted my telescope. The instrument was new to me, and some hours of discipline were spent in mastering all the details of its manipulation.

Mr. Huggins joined me, and we visited together the Arab quarter of Oran. The flat-roofed houses appeared very

* Esparto is a kind of grass now much used in the manufacture of of paper. 
clean and white. The street was filled with loiterers, and the thresholds were occupied by picturesque groups. Some of the men were very fine. We saw many straight, manly fellows who must have been six feet four in height. 'They passed us with perfect indifference, evincing no anger, suspicion, or curiosity, hardly caring in fact to glance at us as we passed. In one instance only during my stay at Oran was I spoken to by an Arab. He was a tall, good-humored fellow, who came smiling up to me, and muttered something about "les Anglais." The mixed population of Oran is picturesque in the highest degree; the Jews, rich and poor, varying in their costumes as their wealth varies; the Arabs more picturesque still, and of all shades of complexion-the negroes, the Spaniards, the French, all grouped together, each race preserving its own individuality, formed a picture intensely interesting to me.

On Tuesday, the 20th, I was early at the bastionet. The night had been very squally. The sergeant of the sappers had taken charge of our key, and on Tuesday morning Elliot went for it. He brought back the intelligence that the tents had been blown down, and the instruments overturned. Among these was a large and valuable equatorial from the Royal Observatory, Greenwich. It seemed hardly possible that this instrument, with its wheels and verniers and delicate adjustments, could have escaped uninjured from such a fall. This, however, was the case; and during the day all the overturned instruments were restored to their places, and found to be in practical working order. This and the following day were devoted to incessant schooling. I had come out as a general stargazer, and not with the intention of devoting myself to the observation of any particular phenomenon. I wished to see the whole - the first contact, the advance of the moon, the successive swallowing up of the solar spots, the breaking of the last line of crescent by the lunar mountains into Bailey's beads, the advance of the shadow through the air, the appearance of the corona and prominences at the moment of totality, the radiant streamers of the corona, the internal structure of the flames, a glance through a polariscope, a sweep round the landscape with the naked eye, the reappearance of the solar limb through Bailey's beads, and, finally, the retreat of the lunar shadow through the air. 
I was provided with a telescope of admirable definition, mounted, aljusted, packed, and most liberally placed at my disposal by Mr. Warren De La Rue. The telescope grasped the whole of the sun, and a considerable portion of the space surrounding it. But it would not take in the extreme limits of the corona. For this I had lashed on to the large telescope a light but powerful instrument, constructed by Ross, and lent to me by Mr. Huggins. I was also furnished with an excellent binocular by Mr. Dallmeyer. In fact, no man could have been more efficiently supporterl. It required a strict parceling out of the interval of totality to embrace in it the entire series of observations. These, while the sun remained visible, were to be made with an unsilvered diagonal eye-piece, which reflected but a small fraction of the sun's light, this fraction being still further toned down by a dark glass. At the moment of totality the dark glass was to be removed, and a silver reflector pushed in, so as to get the maximum of light from the corona and prominences. The time of totality was distributed as follows:

1. Observe approach of shadow through the air: totality.

2. Telescope . . . 30 seconds.

3. Finder $\quad$. 30 seconds.

4. Double image prism . $\quad 15$ seconds.

5. Naked eye . . . 10 seconds.

6. Finder or binocular . . 20 seconds.

7. Telescope . . 20 seconds.

8. Observe retreat of shadow.

In our rehearsals Elliot stood beside me, watch in hand, and furnished with a lantern. He called out at the end of each interval, while I moved from telescope to finder, from finder to polariscope, from polariscope to naked eye, from naked eye back to finder, from finder to telescope, abandoning the instrument finally to observe the retreating shadow. All this we went over twenty times, while looking at the actual sun, and keeping him in the midale of the field. It was my object to render the repetition of the lesson so mechanical as to leave no room for flurry, forgetfulness, or excitement. Volition was not to be called upon, nor judgment exercised, but a well-beaten path of routine was to be followed. Had the opportunity occurred, I think the programme would have been strictly carried out. 
But the opportunity did not occur. For several days the weather had been ill-natured. We had wind so strong as to render the hawsers at the stern of the Urgent as rigid as iron, and to destroy the navigating lieutenant's sleep. We had clouds, a thunder-storm, and some rain. Still the hope was held out that the atmosphere would cleanse itself, and if it did we were promised air of extraordinary limpidity. Early on the 22d we were all at our posts. Spaces of blue in the early morning gave us some encouragement, but all depended on the relation of these spaces to the surrounding clouds. Which of them were to grow as the day advanced? 'The wind was high, and to secure the steadiness of my instrument I was forced to retreat behind a projection of the bastionet, place stones upon its stand, and, further, to avail myself of the shelter of a sail. My practiced men fastened the sail at the top, and loaded it with boulders at the bottom. It was tried severely, but it stood firm.

The clouds and blue spaces fought for a time with varying success. The sun was hidden and revealed at intervals, hope oscillating in synchronism with the changes of the sky. At the moment of first contact a dense cloud intervened; but a minute or two afterward the cloud had passed, and the encroachment of the black body of the moon was evident upon the solar disk. The moon marched onward, and I saw it at frequent intervals; a large group of spots were approached and swallowed up. Subsequently I caught sight of the lunar limb as it cut through the middle of a large spot. The spot was not to be distinguished from the moon, but rose like a mountain above it. The clonds, when thin, could be seen as gray scud drifting across the black surface of the moon; but they thickened more and more, and made the intervals of clearness scantier. During these moments I watched with an interest bordering upon fascination the march of the silver sickle of the sun across the field of the telescope. It was so sharp and so beautiful. No trace of the lunar limb could be observed beyond the sun's boundary. Here, indeed, it could only be relieved by the corona, which was utterly cut off by the dark glass. 'The blackness of the moon beyond the sun was, in fact, confounded with the blackness of space.

Beside me was Elliot with the watch and lantern, while Lieuteuant Archer, of the Royal Engineers, had the kind- 
ness to take charge of my notebook. I mentioned, and he wrote rapidly down, such things as seemed worthy of remembrance. Thus my hands and mind were entirely free; but it was all to no purpose. A patch of sunlight fell and rested upon the landscape some miles away. It was the only illuminated spot within view. But to the northwest there was still a space of blue which might reach us in time. Within seven minutes of totality another space toward the zenith became very dark. The atmosphere was, as it were, on the brink of a precipice, being charged with humidity, which required but a slight chill to bring it down in clouds. This was furnished by the withdrawal of the solar beams: the clouds did come down, covering up the space of blue on which our hopes had so long rested. I abandoned the telescope and walked to and fro in despair. As the moment of totality approached, the descent toward darkness was as obvious as a falling stone. I looked toward a distant ridge, where the darkness wonld first appear. At the moment a fan of beams, issuing from the hidden sun, was spread out over the southern heavens. Those beams are bars of alternate light and shade, produced in illuminated haze by the shadows of floating cloudlets of varying density. The beams are practically parallel, but by an effect of perspective they appear divergent, having the sun, in fact, for their point of convergence. The darkness took possession of the ridge referred to, lowered upon M. Janssen's observatory, passed over the southern heavens, blotting out the beams as if a sponge had been drawn across them. It then took successive possession of three spaces of blue sky in the southeastern atmosphere. I again looked toward the ridge. A glimmer as of day-dawn was behind it, and immediately afterward the fan of beams, which had been for more than two minutes absent, revived. The eclipse of $18 \% 0$ had ended, and, as far as the corona and flames were concerned, we had been defeated.

Even in the heart of the eclipse the darkness was by no means perfect. Small print could be read. In fact, the clonds which rendered the day a dark one, by scattering light into the shadow, rendered the darkness less intense than it would hare been had the atmosphere been without cloud. In the more open spaces I sought for stars, but could find none. There was a lull in the wind before and after totality, but during the totality the wind was strong. 
I waited for some time on the bastionet, hoping to get a glimpse of the moon on the opposite border of the sun, but in vain. The clouds continued, and some rain fell. The day brightened somewhat afterward, and, having packed all up, in the sober twilight $\mathrm{Mr}$. Crookes and myself climbed the heights above the fort of Vera Cruz. From this eminence we had a very noble view over the Mediterranean and the flanking African hills. The sunset was remarkable, and the whole outlook exceedingly fine.

The able and well-instructed medical officer of the Urgent, Mr. Goodman, observed the following temperatures during the progress of the eclipse:

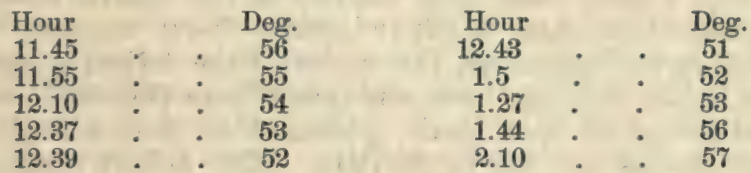

The minimum temperature occurred some minutes after totality, when a slight rain fell.

The wind was so strong on the $23 \mathrm{~d}$ that Captain Henderson would not venture out. Guided by Mr. Goodman, I visited a cave in a remarkable stratum of shellbreccia, and, thanks to my guide, secured specimens. Mr. Busk informs me that a precisely similar breccia is found at Gibraltar, at approximately the same level. During the afternoon, Admiral Ommaney and myself drove to the fort of Marsa el Kibir. 'The fortification is of ancient origin, the Moorish arches being still there in lecay, but the fort is now very strong. About four or five hundred fine-looking dragoons were looking after their horses, waiting for a lull to enable them to embark for France. One of their officers was wandering in a very solitary fashion over the fort. We had some conversation with him. He had been at Sedan, had been taken prisoner, but had effected bis escape. He shook his head when we spoke of the termination of the war, and prerlicted its long continuance. 'There was bitterness in his tone as he spoke of the charges of treason so lightly leveled against French commanders. The green waves raved round the promontory on which the fort stands, smiting the rocks, breaking into fosm, and jumping, after impact, to a 
height of a hundred feet and more into the air. As we returned our vehicle broke down through the loss of a wheel. 'The admiral went on board, while I remained long watching the agitated sea. 'The little horses of Oran well merit a passing word. 'Their speed and endurance, both of which are heavily drawn upon by their drivers, are extraordinary.

The wind sinking, we lifted anchor on the 24th. For some hours we went pleasantly along; but during the afternoon the storm revived, and it blew heavily against us all the night. When we came opposite the bay of Almeria, on the 25 th, the captain turned the ship, and steered into the bay, where, under the shadow of the Sierra Nevada, we passed Christmas night in peace. Next morning "a rose of lawn" rested on the snows of the adjacent mountains, while a purple haze was spread over the lower hills. I had no notion that Spain possessed so fine a range of mountains as the Sierra Nevada. The height is considerable, but the form also is such as to get the maximum of grandeur out of the height. We weighed anchor at eight A.M., passing for a time through shoal water, the bottom having been evidently stirred up. The adjacent land seemed eroded in a remarkable manner. It has its floods, which excavate these valleys and ravines, and leave those singular ridges behind. Toward evening I climbed the mainmast, and, standing on the cross-trees, saw the sun set amid a blaze of tiery clouds. The wind was strong and bitterly cold, and I was glad to slide back to the deck along a rope, which stretched from the mast-head to the ship's side. That night we cast anchor beside the mole of Gibraltar.

On the morning of the $2 \%$ th, in company with two friends, I drove to the Spanish lines, with the view of seeing the rock from that side. It is an exceedingly noble mass. 'The Peninsular and Oriental mail-boat had been signaled and had come. Heavy duties called me homeward, and by transferring myself from the Urgent to the mail-steamer I should gain three days. I hired a boat, rowed to the steamer, learned that she was to start at one, and returned with all speed to the Urgent. Making known to Captain Henderson my wish to get away, he expressed doubts as to the possibility of reaching the mailsteamer in time. With his accustomed kindness, he how- 
ever placed a boat at my disposal. Four harly fellows and one of the ship's officers jumped into it; my luggage, hastily thrown together, was tumbled in, and we were immediately on our way. We had nearly four miles to row in about twenty minutes; but we hoped the mail-boat might not be punctual. For a time we watched her anxiously; there was no motion; we came nearer, but the flags were not yet hauled in. The men put forth all their strength, animated by the exhortations of the officer at the helm. The roughness of the sea rendered their efforts to some extent nugatory: still we were rapidly approaching the steamer. At length she moved, punctual almost to the minute, at first slowly, but soon with quickened pace. We turned to the left, so as to cut across her bows. Five minutes' pull would have brought us up to her. The officer waved his cap and I my hat. "If they could only see us, they might back to us in a moment." But they did not see us, or if they did, they paid us no attention. I returned to the Urgent, discomfited, but grateful to the fine fellows who had wrought so hard to carry out my wishes.

Glad of the quiet, in the sober afternoon I took a walk toward Europa Point. The sky darkened and heavy squalls passed at intervals. Private theatricals were at the Convent, and the kind and courteous governor had sent cards to the eclipse party. I failed in my duty in not going. St. Michael's Cave is said to rival, if it does not outrival, the Mammoth Cave of Kentucky. On the 28th Mr. Crookes, Mr. Carpenter, and myself, guided by a military policenan who understood his work, explored the cavern. The mouth is about 1,100 feet above the sea. We zigzagged up to it, and first were led into an aperture in the rock, at some height above the true entrance of the cave. In this upper cavern we saw some tall and beautiful stalactite pillars.

The water drips from the roof charged with bicarbonate of lime. Exposed to the air, the carbonic acid partially escapes, and the sinıple carbonate of lime, which is hardly at all soluble in water, deposits itself as a solid, forming stalactites and stalagmites. Even the exposure of chalk or limestone water to the open air partially softens it. A specimen of the Redbourue water exposed by Professor's Graham, Miller, and Hofmann, in a shallow basin, fell from eighteen degrees to nine degrees of hardness. The 
softening process of Clark is virtually a hastening of the natural process. Here, however, instead of being permitted to evaporate, half the carbonic acid is appropriated by lime, the half thus taken up, as well as the remaining half, being precipitated. 'The solid precipitate is permitted to sink, and the clear supernatant liquid is limpid soft water.

We returned to the real mouth of St. Michael's Cave, which is entered by a wicket. 'The floor was somewhat muddy, and the roof and walls were wet. We soon found ourselves in the midst of a natural temple, where tall columns sprang complete from floor to roof, while incipient columns were growing to meet each other, upward and downward. The water which trickles from the stalactite, after having in part yielded up its carbonate of lime, falls upon the floor vertically underneath, and there builds the stalagmite. Consequently, the pillars grow from above and below simultaneously, along the same vertical. It is easy to distinguish the stalagmitic from the stalactitic portion of the pillars. The former is always divided into short segments by protuberant rings, as if deposited periodically, while the latter presents a uniform surface. In some cases the points of inverted cones of stalactite rested on the centers of pillars of stalagmite. The process of solidification and the consequent architecture were alike beautiful.

We followed our guide through various branches and arms of the cave, climbed and descended steps, halted at the edges of dark shafts and apertures, and squeezed ourselves through narrow passages. From time to time we halted, while Mr. Crookes illuminated with ignited magnesium wire, the roof, columns, dependent spears, and graceful drapery of the stalactites. Once, coming to a magnificent cluster of icicle-like spears, we helped ourselves to specimens. There was some difficulty in detaching the more delicate ones, their fragility was so great. A consciousness of vandalism, which smote me at the time, haunts me still; for, though our requisitions were moderate, this beauty ought not to be at all invaded. Pendent from the roof, in their natural habitat, nothing can exceed their delicate beanty; they live, as it were, surronnded by organic connections. In London they are curious, but not beantiful. Of gathered shells Emerson writes: 
I wiped away the weeds and foam,

And brought my sea-born treasures home:

But the poor, unsightly, noisome things

Had left their beauty on the shore,

With the sun, and the sand, and the wild uproar.

The promontory of Gibraltar is so burrowed with caverns that it has been called the Hill of Caves. They are apparently related to the geologic disturbances which the rock has undergone. The earliest of these is the tilting of the once horizontal strata. Suppose a force of torsion to act upon the promontory at its southern extremity near Europa Point, and suppose the rock to be of a partially yielding character; such a force would twist the strata into screw-surfaces, the greatest amount of twisting being endured near the point of application of the force. Such a twisting the rock appears to have suffered; but instead of the twist fading gradually and uniformly off, in passing from south to north, the want of uniformity in the material has produced lines of dislocation where there are abrupt changes in the amount of twist. Thus, at the northern end of the rock the dip to the west is nineteen degrees; in the Middle Hill, it is thirty-eight degrees; in the center of the South hill, or Sugar Loaf, it is fifty-seven degrees. At the southern extremity of the Sugar Loaf the strata are vertical, while farther to the south they actually turn over and dip to the east.

'The rock is thus divided into three sections, separated from each other by places of dislocation, where the strata are inuch wrenched and broken. These are called the Northern and Southern Quebrada, from the Spanish "Tierra Quebrada," or broken ground. It is at these places that the inland caves of Gibraltar are almost exclusively found. Based on the observations of Dr. Falconer and himself, an excellent and most interesting account of these caves, and of the human remains and works of art which they contain, was communicated by Mr. Busk to the meeting of the Congress of Prehistoric Archæology at Norwich, and afterward printed in the "Transactions" of the Congress.* Long subsequent to the operation of the

* In this essay Mr. Busk refers to the previous labors of Mr. Smith. of Jordan Hill, to whom we owe most of our knowledge of the geology of the rock. 
twisting force just referred to, the promontory under. went various changes of level. There are sea-terraces and layers of shell-breccia along its flanks, and numerous caves which, unlike the inland ones, are the product of marine erosion. 'The Ape's Hill, on the African side of the strait, Mr. Busk informs me, has undergone similar disturbances.*

In the harbor of Gibraltar, on the morning of our departure, I resumed a series of observations on the color of the sea. On the way out a number of specimens harl been collected, with a view to subsequent examination. But the bottles were claret bottles, of doubtful purity. At Gibraltar, therefore, I purchased fifteen white glass bottles, with ground glass stoppers, and at Cadiz, thanks to the friendly guidance of Mr. Cameron, I secured a dozen more. These seven-and-twenty bottles were filled with water, taken at different places between Oran and Spithead.

And here let me express my warmest acknowledgments to Captain Henderson, the commander of H.M.S. Urgent, who aided me in my observations in every pussibie way. Indeed, my thanks are due to all the officer's for their unfailing courtesy and help. The captain placed at my disposal his own cockswain, an intelligent fellow named 'Thorogood, who skillfully attached a cord to each bottle, weighted it with lead, cast it into the sea, and, after three successive rinsings, filled it under my eyes. The contact of jugs, buckets, or other vessels was thus avoided; and even the necessity of pouring out the water, afterward, through the dirty London air.

The mode of examination applied to these bottles has been already described. $\dagger$ The liquid is illuminated by a powerfully condensed beam, its condition being revealed through the light scattered by its suspended particles. "Care is taken to defend the eye from the access of all other light, and, thus defended, it becomes an organ of inconceivable delicacy." Were water of uniform density perfectly free from suspended matter, it would, in my

* No one can rise from the perusal of Mr. Busk's paper without a feeling of admiration for the principal discoverer and indefatigable explorer of the Gibraltar caves, the late Captain Frederick Brome.

†"Floating Matter of the Air," Art. "Dust and Disease," 
opinion, scatter no light at all. The track of a luminous beam could not be seen in such water. But "an amount of impurity so infinitesimal as to be scarcely expressible in numbers, and the individual particles of which are so small as wholly to elude the microscope, may, when examined by the method alluded to, produce not only sensible, but striking effects upon the eye."

The result of the examination of uineteen bottles filled at various places between Gibraltar and Spithead, are here tabulated:

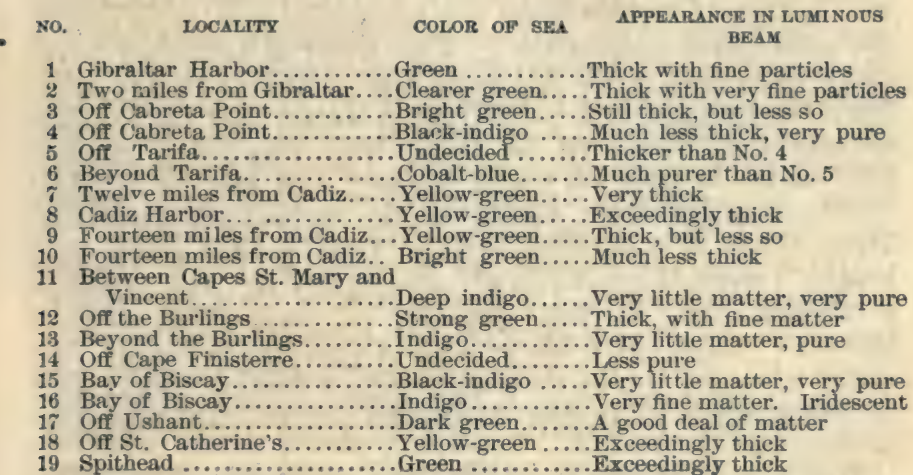

Here we have three specimens of water, described as green, a clearer green, and bright green, taken in Gibraltar harbor, at a point two miles from the harbor, and off Cabreta Point. The home examination showed the first to be thick with suspended matter, the second less thick, and the third still less thick. Thus the green brightened as the suspended matter diminished in amount.

Previous to the fourth observation our excellent navigating lieutenant, Mr. Brown, steered along the coast, thus avoiding the adverse current which sets in, through the strait, from the Atlantic to the Mediterranean. He was at length forced to cross the boundary of the Atlantic current, which was defined with extraordinary sharpness. On the one side of it the water was a vivid green, on the other a deep blue. Standing at the bow of the ship, a bottle could be filled with blue water, while at the same moment a bottle cast from the steru could be filled with green water. 
Two bottles were secured, one on each side of this remarkable boundarv. In the distance the Atlantic had the hue called ultra-marine; but looked fairly down upon, it was of almost inky blackuess-black qualified by a trace of indigo.

What change does the home examination here reveal? In passing to indigo, the water becomes suddenly augmenteri in purity, the suspended matter becoming suddenly less. Off Tarifa, the deep indigo disappears, and the sea is undecided in color. Accompanying this change, we have a rise in the quantity of suspended matter. Beyond Tarifa, we change to cobalt-blue, the suspended matter falling at the same time in quantity. This water is distinctly purer than the green. We approach Cadiz, and at twelve miles from the city get into yellow-green water; this the London examination shows to be thick with suspended matter. The same is true of Cadiz harbor, and also of a point fourteen miles from Cadiz in the homeward direction. Here there is a sudden change from yellow-green to a bright emerald-green, and accompanying the change a sudden fall in the quantity of suspended matter. Between Cape St. Mary and Cape St. Vincent the water changes to the deepest indigo, a further diminution of the suspended matter being the concomitant phenomenon.

We now reach the remarkable group of rocks called the Burlings, and find the water between the shore and the rocks a strong green; the home examination shows it to be thick with fine matter. Fifteen or twenty miles beyond the Burlings we come again into indigo water, from which the suspended matter has in great part disappeared. Off Cape Finisterre, about the place where the Captain went down, the water becomes green, and the home examination pronounces it to be thicker. Then we enter the bay of Biscay, where the indigo resumes its power, and where the home examination shows the greatly angmented purity of the water. A second specimen of water, taken from the bay of Biscay, held in suspension fine particles of a peculiar kind; the size of them was such as to render the water richly iridescent. It showed itself green, blue, or salmoncolored, according to the direction of the line of vision. Finally, we come to our last two bottles, the one taken opposite St. Catherine's lighthouse, in the Isle of Wight, 
the other at Spithead. The sea at both these places was green, and both specimens, as might be expected, were pronounced by the home examination to be thick with suspended matter.

Two distinct series of observations are here referred tothe one consisting of direct observations of the color of the sea, conducted during the voyage from Gibraltar to Portsmouth: the other carried out in the laboratory of the Royal Institution. And here it is to be noted that in the home examination I never knew what water was placed in my hands. The labels, with the names of the localities written upon them, had been tied up, all information regarding the source of the water being thus held back. The bottles were simply numbered, and not till all of them had been examined, and described, were the labels opened, and the locality and sea-color corresponding to the various specimens ascertained. The home observations, therefore, must have been perfectly unbiased, and they clearly establish the association of the green color with fine suspended matter, and of the ultramarine color, and more especially of the black-indigo hue of the Atlantic, with the comparative absence of such matter.

So much for mere observation; but what is the cause of the dark hue of the deep ocean?* A preliminary remark or two will clear our way toward an explanation. Color resides in white light, appearing when any constituent of the white light is withdrawn. The hue of a purple liquid, for example, is immediately accounted for by its action on a spectrum. It cuts out the yellow and green, and allows the red and blue to pass through. The blending of these two colors produces the purple. But while such a liquid attacks with special energy the vellow and green, it enfeebles the whole spectrum. By increasing the thickness of the stratum we may absorb the whole of the light. The color of a blue liquid is similarly accounted for. It first extinguished the red; then, as the thick-

* A note, written to me on October 22 , by my friend Canon Kingsley, contains the following reference to this point: "I have never seen the lake of Geneva, but I thought of the brilliant, dazaling dark blue of the mid Atlantic under the sunlight, and its black-blue under cloud, both so solid that one might leap off the sponson on to it with. out fear; this was to me the most wonderful thing which I saw on my voyages to and from the West Indies." 
ness angments, it attacks the orange, yellow, and green in succession; the blue alone finally remaining. But even it might be extinguished by a sufficient depth of the liquid.

And now we are prepared for a brief, but tolerably complete, statement of that action of sea-water upon light, to which it owes its larkness. The spectrum embraces three classes of rays - the thermal, the visual, and the chemical. These divisions overlap each other; the thermal rays are in part visual, the visual rays in part chemical, and vice versá. The vast body of thermal rays lie beyond the red, being invisible. These rays are attacked with exceeding energy by water. They are absorbed close to the surface of the sea, and are the great agents in evaporation. At the same time the whole spectrum suffers enfeeblement; water attacks all its rays, but with different degrees of energy. Of the visual rays, the red are first extinguished. As the solar beam plunges deeper into the sea, orange follows red, yellows follows orange, green follows yellow, and the various shades of blue, where the water is deep enough, follow green. Absolute extinction of the solar beam would be the consequence if the water were deep and uniform. If it contained no suspended matter, such water would be as black as ink. A reflected glimmer of ordinary light would reach us from its surface, as it would from the surface of actual ink; but no light, hence no color, would reach us from the body of the water.

In very clear and deep sea-water this condition is approximately fulfilled, and hence the extraordinary darkness of such water. The indigo, already referred to, is, I believe, to be ascribed in part to the suspended matter, which is never absent, even in the purest natural water; and in part to the slight reflection of the light from the limiting surfaces of strata of different densities. A modicum of light is thus thrown back to the eye, before the depth necessary to absolute extinction has been attained. An effect precisely similar occurs under the moraines of glaciers. The ice here is exceptionally compact, and, owing to the absence of the internal scattering common in bubbled ice, the light plunges into the mass, where it is extinguished, the perfectly clear ice presenting an appearance of pitchy blackness.*

* I learn from a correspondent that certain Welsh tarns, which are reputed bottomless, have this inky hue. 
'The green color of the sea has now to be accounter for; and here, again, let us fall back upon the sure basis of experiment. A strong white dinner-plate had a lead weight securely fastened to it. Fifty or sixty yards of strong hempen line were attached to the plate. My assistant, Thorogood, occupied a boat, fastened as usual to the davits of the Urgent, while I occupied a second boat nearer the stern of the ship. He cast the plate as a mariner heaves the lead, and by the time it reached me it had sunk a considerable depth in the water. In all cases the hue of this plate was green. Even when the sea was of the darkest indigo, the green was vivid and pronounced. I could notice the gradual deepening of the color as the plate sank, but at its greatest depth, even in indigo water, the color was still a blue-green.*

Other observations confirmed this one. The Urgent is a screw steamer, and right over the blades of the screw was an orifice called the screw-well through which one could look from the poop down upon the screw. The surfaceglimmer, which so pesters the eye, was here in a great measure removed. Midway down, a plank crossed the screw-well from side to side; on this I placed myself and olserved the action of the screw underneath. The eye was rendered sensitive by the moderation of the light; and, to remove still further all disturbing causes, Lieutenant Walton had a sail and tarpaulin thrown over the month of the well. Underneath this I perched myself on the plank and watched the screw. In an indigo sea the play of color was indescribably beautiful, and the contrast between the water, which had the screw blades, and that which had the bottom of the ocean, as a background, was extraordinary. 'The one was of the most brilliant green, the other of the deepest ultramarine. The surface of the water above the screw-blade was always ruffled. Liquid lenses were thus formed, by which the colored light was withdrawn from some places and concentrated upon others, the water flashing with metallic luster. The screw-blades in this case played the part of the dinner-plate in the former case, and there were other instances of a similar kind. The white bellies of porpoises showed the green hue, varying in inten-

* In no case, of course, is the green pure, but a mixture of green and blue. 
sity as the creatures swung to and fro between the surface and the deeper water. Foam, at a certain depth below the surface, was also green. In a rough sea the light which penetrated the summit of a wave sometimes reached the eye, a beautiful green cap being thus placed upon the wave, even in indigo water.

But how is this color to be connected with the suspended particles? 'Thus. 'Take the dinner-plate which showed so brilliant a green when thrown into indigo water. Suppose it to diminish in size, until it reaches an almost microscopic magnitude. It would still behave substantially as the larger plate, sending to the eye its modicum of green light. If the plate, instead of being a large coherent mass, were ground to a powder sufficiently fine, and in this condition diffused through the clear sea-water, it would also send green light to the eye. In fact, the suspended particles which the home examination reveals, act in all essential particulars like the plate, or like the screw-blades, or like the foam, or like the bellies of the porpoises. Thus I think the greenness of the sea is physically connected with the matter which it holds in suspension.

We reached Portsmouth on January 5, 18\%1. Then ended a voyage which, though its main object was not realized, has left behind it pleasant memories, both of the aspects of nature and the kindliness of men.

\section{CHAP'TER VII.}

NIAGARA.*

IT IS one of the disadvantages of reading books about natural scenery that they fill the mind with pictures, often exaggerated, often distorted, often blurred, and, even when well drawn, injurious to the freshness of first impressions. Such has been the fate of most of us with regard to the Falls of Niagara. There was little accuracy in the estimates of the first observers of the cataract. Startled by an exhibition of power so novel and so grand, emotion

* A Discourse delivered at the Royal Institution of Great Britain, April 4, 1873. 
leaped beyond the control of the judgment, and gave currency to notions which have often led to disappointment.

A record of a voyage in 1535 by a French mariner named Jacques Cartier, contains, it is said, the first printed allusion to Niagara. In 1603 the first map of the district was constructed by a Frenchman named Champlain. In 1648 the Jesuit Rageneau, in a letter to his superior at Paris, mentions Niagara as " a cataract of frightful height."* In the winter of 1678 and 1679 the cataract was visited by Father Hennepin, and described in a book dedicated "to the king of Great Britain." He gives a drawing of the waterfall, which shows that serious changes have taken place since his time. He describes it as "a great and prodigious cadence of water, to which the universe does not offer a parallel." The height of the fall, according to Hennepin, was more than 600 feet. "The waters," he says, "which fall from this great precipice do foam and boil in the most astonishing manner, making a noise more terrible than that of thunder. When the wind blows to the south its frightful roaring may be heard for more than fifteen leagues." The Baron la Hontan, who visited Niagara in $168 \%$, makes the height 800 feet. In 1721 Charlevois, in a letter to Madame de Maintenon, after referring to the exaggerations of his predecessors, thus states the result of his own observations: "For my part, after examining it ou all sides, I am inclined to think that we eannot allow it less than 140 or 150 feet"-a remarkably close estimate. At that time, viz., a hundred and fifty years ago, it had the shape $0:$ a horseshoe, and reasons will subsequently be given for holding that this has been always the form of the cataract, from its origin to its present site.

As regards the noise of the fall, Charlevois declares the accounts of his predecessors, which, I may say, are repeated to the present hour, to be altogether extravagant. He is perfectly right. The thunders of Niagara are formidable enough to those who really seek them at the base of the Horseshoe Fall; but on the banks of the river, and particularly above the fall, its silence, rather than ils noise,

* From an interesting little book presented to me at Brooklyn by its author, Mr. Holly, some of these data are derived: Hennepin, Kalm, Bakewell, Lyell, Hall, and others I have nyself consulted. 
is surprising. This arises, in part, from the lack of resonance; the surrounding country being flat, and therefore furnishing no echoing surfaces to reiniorce the shock of the water. 'The resonance from the surrounding rocks causes the Swiss Reuss at the Devil's Bridge, when full, to thunder more loudly than the Niagara.

On Friday, November 1,1872 , just before reaching the village of Niagara Falls, I caught, from the railway train, my first glimpse of the smoke of the cataract. Immediately after my arrival I went with a friend to the northern end of the American Fall. It may be that my mood at the time toned down the impression produced by the first aspect of this grand cascade; but $I$ felt nothing like disappointment, knowing, from old experience, that time and close acquaintanceship, the gradual interweaving of mind and nature, must powerfully influence my final estimate of the scene. After dinner we crossed to Goat Island, and, turning to the right, reached the southern end of the American Fall. The river is here studded with small islands. Crossing a wooden bridge to Luna Island, and clasping a tree which grows near its edge, I looked long at the cataract, which here shoots down the precipice like an avalanche of foam. It grew in power and beauty. 'The channel spanned by the wooden bridge was deep, and the river there doubled over the edge of the precipice, like the swell of a muscle, unbroken. The ledge here overhangs, the water being poured out far beyond the base of the precipice. A space, called the Cave of the Winds, is thus enclosed between the wall of rock and the falling water.

Goat Island ends in a sheer dry precipice, which connects the American and Horseshoe Falls. Midway between both is a wooden hut, the residence of the guide to the Cave of the Winds, and from the hut a winding staircase, called Biddle's Stair, descends to the base of the precipice. On the evening of $\mathrm{my}$ arrival I went down this stair, and wande'ed along the bottom of the cliff. One well-known factor in the formation and retreat of the cataract was immediately observed. A thick layer of limestone formed the upper portion of the cliff. This rested upon a bed of soft shale, which extender round the base of the cataract. 'The violent recoil of the water against this yielding substance crumbles it away, undermining the ledge above, which, unsupported, eventually breaks off, and produces the observed recession. 
At the southern extremity of the Horseshoe is a promontory, formed by the doubling back of the gorge excavated by the cataract, and into which it plunges. On the promontory stands a stone building, called the Terrapin Tower, the door of which had been nailed up because of the decay of the staircase within it. 'Through the kindness of Mr. Townsend, the superintendent of Goat Island, the door was opened for me. From this tower,at all hours of the day, and at some hours of the night, I watched and listened to the Horseshoe Fall. The river here is evidently much deeper than the American branch; and instead of bursting into foam where it quits the ledge, it bends solidly over, and falls in a continuous layer of the most vivid green. The tint is not uniform; long stripes of deeper hue alternating with bauds of brighter color. Close to the ledge over which the water rolls, foam is generated, the light falling upon which, and flashing back from it, is sifted in its passage to and fro, and changed from white to emerald-green. Heaps of superficial foam are also formed at intervals along the ledge, and are immediately drawn into long white striæ.* Lower down, the surface, shaken by the reaction from below, incessantly rustles into whiteness. The descent finally resolves itself into a rhythm, the water reaching the bottom of the fall in periodic gushes. Nor is the spray uniformly diffused throngh the air, but is wafted through it in successive veils of gauze-like texture. From all this it is evident that beauty is not absent from the Horseshoe Fall, but majesty is its chief attribute. The plunge of the water is not wild, but deliberate, rast and fascinating. From the Terrapin Tower, the adjacent arm of the Horseshoe is seen projected against the opposite one, midway down; to the imagiuation, therefore, is left the picturing of the gulf into which the cataract plunges.

The delight which natural scenery produces in some minds is difficult to explain, and the conduct which it prompts can hardly be fairly criticised by those who have never experienced it. It seems to me a deduction from the completeness of the celebrated Thomas Young, that he was unable to appreciate natural scenery. "He had

* The direction of the wind with reference to the course of a ship may be inferred with accuracy from the foam-streaks on the surface of the sea. 
really," says Dean Peacock, "no taste for life in the country: he was one of those who thought that no one who was able to live in London would be content to live elsewhere." Well, Dr. Young, like Dr. Johnson, had a right to his delights; but I can understand a hesitation to accept them, high as they were, to the exclusion of

That o'erflowing joy which Nature yields

To her true lovers.

To all who are of this mind, the strengthening of desire on my part to see and know Niagara Falls as far as it is possible for them to be seen and known, will be intelligible.

On the first evening of my visit, 1 met, at the head of Biddle's Stair, the guide to the Cave of the Winds. He was in the prime of manhood-large, well built, firm and pleasant in mouth and eye. My interest in the scene stirred up his, and made him communicative. Turning to a photograph, he described, by reference to it, a feat which he had accomplished some time previously, and which had brought him almost under the green water of the Horseshoe Fall. "Can you lead me there to-morrow?" I asked. He eyed me inquiringly, weighing, perhaps, the chances of a man of light build, and with gray in his whiskers, in such an undertaking. "I wish," I added, "to see as much of the fall as can be seen, and where you lead I will endeavor to follow." His scrutiny relaxed into a smile, and he said, "Very well; I shall be ready for you to-morrow."

On the morrow, accordingly, I came. In the hut at the head of Biddle's Stair I stripped wholly, and re-dressed according to instructions-drawing on two pairs of woolen pantaloons, three woolen jackets, two pairs of socks, and a pair of felt shoes. Even if wet, my guide assured me that the clothes would keep me from being chilled; and he was right. A suit and hood of yellow oilcloth covered all. Most laudable precautions were taken by the young assistant who helped to dress me to keep the water out; but his devices broke down immediately when severely tested.

We descended the stair; the handle of a pitchfork doing, in my case, the duty of an alpenstock. At the bottom, the guide inquired whether we should go first to the Cave of the Winds, or to the Horseshoe, remarking that the latter would try us most. I decided on getting the roughest done 
first, and he turned to the left over the stones. They were sharp and trying. The base of the first portion of the cataract is covered with huge boulders, obviously the ruins of the limestone ledge above. 'The water does not distribute itself uniformly among these, but seeks out channels through which it pours torrentially. We passed some of these with wetted feet, but without lifficulty. At length we came to the side of a more formidable current. My guide walked along its edge until he reached its least turbulent portion. Halting, he said, "This is our greatest difficulty; if we can cross here, we shall get far toward the Horseshoe."

He waded in. It evidently required all his strength to stealy him. The water rose above his loins, and it foamed still higher. He had to search for footing, amid unseen boulders, against which the torrent rose violently. He struggled and swayed, but he struggled successfully, and finally reached the shallower water at the other side. Stretching out his arm, he said to me, " Now come on." I looked down the torrent, as it rushed to the river below, which was seething with the tumult of the cataract. De Saussure recommended the inspection of Alpine dangers, with the view of making them familiar to the eye before they are encounterer; and it is a wholesome custom in places of difficulty to put the possibility of an accident clearly before the mind, and to decide beforehand what ought to be done should the accident occur. 'Thus wound up in the present instance, I entered the water. Even where it was not more than knee-deep, its power was manifest. As it rose around me, I sought to split the torrent by presenting a side to it; but the insecurity of the footing enabled it to grasp my loins, twist me fairly round, and bring its impetus to bear upon my back. Further struggle was impossible; and feeling my balance hopelessly gone, I turned, flung myself toward the bank just quitted, and was instantly, as expected, swept into shallower water.

'The oilcloth covering was a great incumbrance; it had been made for a much stouter man, and, stauding upright after my submersion, my legs occupied the center of two bags of water. My guide exhorted me to try again. Prudence was at my elbow, whispering dissuasion; but, taking everything into account, it appeared more immoral to retreat 
than to proceed. Instructed by the first misadventure, I once more entered the stream. Had the alpenstock been of iron it might have helped me; but, as it was, the tendency of the water to sweep it out of my hands rendered it worse than useless. I, however, clung to it by habit. Again the torrent rose and again I wavered; but, by keeping the left hip well against it, I remained upright, and at length grasped the hand of my leader at the other side. He laughed pleasantly. The first victory was gained, and he enjoyed it. "No traveler," he said, "was ever here before." Soon afterward, by trusting to a piece of driftwood which seemed firm, I was again taken off my feet, but was immediately caught by a protruding rock.

We clambered over the boulders toward the thickest spray, which soon became so weighty as to cause us to stagger under its shock. For the most part nothing could be seen; we were in the midst of bewildering tumult, lished by the water, which sounded at times like the cracking of innumerable whips. Underneath this was the deep resonant roar of the cataract. I tried to shield my eyes with my hands, and look upward; but the defense was useless. The guide continued to move on, but at a certain place he halted, desiring me to take shelter in his lee, and observe the cataract. The spray did not come so much from the upper ledge, as from the rebound of the shattered water when it struck the bottom. Hence the eyes could be protected from the blinding shock of the spray, while the line of vision to the upper ledges remained to some extent clear. On looking upward over the guide's shoulder I could see the water bending over the ledge, while the Terrapin Tower loomed fitfully through the intermittent spray-gusts. We were right under the tower. A little farther on the cataract, after its first plunge, hit a protuberauce some way down, and flew from it in a prodigious burst of spray; through this we staggered. We rounded the promontory on which the Terrapin Tower stands, and moved, amid the wildest commotion, along the arm of the Horseshoe, until the boulders failed us, and the cataract fell into the profound gorge of the Niagara river.

Here the guide sheltered me again, and desired me to look up; I did so, and could see, as before, the green gleam of the mighty curve sweeping over the upper ledge, and the fitful plunge of the water, as the spray between us 
and it alternately gathered and disappeared. An eminent friend of mine often speaks of the mistake of those physicians who regard man's ailments as purely chemical, to be met by chemical remedies only. He contends for the psychological element of cure. By agreeable emotions, he says, nervons currents are liberated which stimulate blood, brain, and viscera. 'The influence rained from ladies' eyes enables my friend to thrive on dishes which would kill him if eaten alone. A sanative effect of the same order I experienced amid thespray and thunder of Niagara. Quickened by the emotions there aroused, the blood sped exultingly through the arteries, abolishing introspection, clearing the heart of all bitterness, and enabling one to think with tolerance, if not with tenderness, on the most relentless and unreasonable foe. Apart from its scientific value, and purely as a moral agent, the play was worth the candle. My companion knew no more of me than that I enjoyed the wildness of the scene; but as I bent in the shelter of his large frame he said, "I should like to see you attempting to describe all this." He rightly thought it indescribable. 'The name of this gallant fellow was Thomas Conroy.

We returned, clambering at intervals up and down, so as to catch glimpses of the most impressive portions of the cataract. We passed under ledges formed by tabular masses of limestone, and through some curious openings formed by the falling together of the summits of the rocks. At length we found ourselves beside our enemy of the morning. Conroy halted for a minute or two, scanning the torrent thoughtfully. I said that, as a guide, heought to have a rope in such a place; but he retorted that, as no traveler had ever thought of coming there, he did not see the necessity of keeping a rope. He waded in. 'The struggle to keep himself erect was evident enough; he swayed, but recovered himself again and again. At length he slipped, gave way, did as I had done, threw himself toward the bank, and was sivept into the shallows. Standing in the stream near its elge, he stretched his arm toward me. I retained the pitchfork handle, for it had been useful among the boulders. By wading some way in, the staff could be made to reach him, and I proposed his seizing it. "If you are sure," he replied, "that, in case of giving way, you can maintain your grasp, then I will 
certainly hold you." Remarking that he might count on this, I waded in, and stretched the staff to my companion. It was firmly grasped by both of us. Thus helped, though its onset was strong, I moved safely across the torrent. All danger ended here. We afterward roamed sociably among the torrents and boulders below the Cave of the Winds. The rocks were covered with organic slime, which could not have been walked over with bare feet, but the felt shoes cffectually prevented slipping. We reached the cave and entered it, first by a wooden way carried over the boulders, and then along a narrow ledge, to the point eaten deepest in to the shale. When the wind is from the south, the falling water, I am told, can be seen tranquilly from this spot; but when we were there, a blinding hurricane of spray was whirled against us. On the evening of the same day, I went behind the water on the Canada side, which, after the experiences of the morning, struck me as an imposture.

Still even this latter is exciting to some nerves. Its effect upon himself is thus vividly described by $\mathrm{Mr}$. Bakewell, Jr.: "On turning a sharp angle of the rock, a sudden gust of wind met us, coming from the hollow between the fall and the rock, which drove the spray directly in our faces, with such force that in an instant we were wet through. When in the midst of this shower-bath the shock took away my breath: I turned back and scrambled over the loose stones to escape the conflict. 'The guide soon followed, and told me that I had passed the worst part. With that assurance I made a second attempt; but so wild and disordered was my imagination that when I had reached halfway I could bear it no longer." *

To complete my knowledge I desired to see the fall from the river below it, and long negotiations were necessary to secure the means of doing so. The only boat fit for the undertaking had been laid up for the winter; but this difficulty, through the kind intervention of $\mathrm{Mr}$. Townsend, was overcome. The main one was to secure oarsmen sufficiently strong and skillful to urge the boat where I wished it to be taken. The son of the owner of the boat, a finely built young fellow, but only twenty, and therefore not sufficiently hardened, was willing to go; and up the

* “Mag. of Nat. Hist.," 1830, pp. 121, 122. 
river, it was stated, there lived another man who could do anything with the boat which strength and daring could accomplish. He came. His figure and expression of face certainly indicated extraordinary firmness and power. On T'uesday, November 5th, we started, each of us being clad in oilcloth. The elder oarsman at ouce assumed a tone of authority over his companion, and struck immediately in amid the breakers below the American Fall. He hugged the cross freshets instead of striking out into the smoother water. I asked him why he did so, and he replied that they were directed ontward, not downward. The struggle, however, to prevent the bow of the boat from being turned by them, was often very severe.

The spray was in general blinding, but at times it disappeared and yielded noble views of the fall. 'The eilge of the cataract is crimped by indentations which exalt its beauty. Here and there, a little below the highest ledge, a secondary one juts out; the water strikes it and bursts from it in huge protuberant masses of foam and spray. We passed Goat Island, came to the Horseshoe, and worked for a time along its base, the boulders over which Conroy and myself had scrambled a few days previously lying between us and the cataract. A rock was before us, concealed and revealed at intervals, as the waves passed over it. Our leader tried to get above this rock, first on the ontside of it. 'The water, however, was here in violent motion. 'The men struggled fiercely, the older one ringing out an incessant peal of command and exhortation to the younger. As we were just clearing the rock, the bow came obliquely to the surge; the boat was turned suddenly round and shot with astonishing rapidity down the river. 'The men returned to the charge, now trying to get up between the half-concealed rock and the boulders to the left. But the torrent set in strongly through this channel. The tugging was quick and violent, but we made little way. At length, seizing a rope, the principal oarsman made a desperate attempt to get upon one of the boulders, hoping to be able to drag the boat through the channel; but it bumped so violently against the rock, that the man flung himself back and relinquished the attempt.

We returned along the base of the American Fall, running in and out among the currents which rushed from it laterally into the river. Seen from below the American 
Fall is certainly exquisitely beantiful, but it is a mere frill of adornment to its nubler neighbor the Horseshoe. At times we took to the river, from the center of which the Horseshoe Fall appeared especially magnificent. A streak of cloud across the neck of Mont Blanc can double its apparent height, so here the green sumnit of the cataract shining above the smoke of spray appeared lifted to an extraordinary elevation. Had Hennepin and La Hontan seen the fall from this position, their estimates of the height would have been perfectly excusable.

From a point a little way below the American Fall, a ferry crosses the river, in summer, to the Canadian side. Below the ferry is a suspension bridge for carriages and foot-passengers, and a mile or two lower down is the railway suspension bridge. Between ferry and bridge the river Niagara flows unruffled; but at the suspension bridge the bed steepens and the river quickens its motion. Lower down the gorge narrows, and the rapidity and turbulence increase. At the place called the "Whirlpool Rapids" I estimated the width of the river at 300 feet, an estimate confirmed by the dwellers on the spot. When it is remembered that the drainage of nearly half a continent is compressed into this space, the impetuosity of the river's rush may be imagined. Had it not been for Mr. Bierstädt, the distinguished photographer of Niagara, I should have quitted the place without seeing these rapids; for this, and for his agreeable company to the spot, I have to thank him. From the edge of the cliff above the rapids, we descended, " little, I confess, to a climber's disgust, in an " elevator," becuuse the effects are best seen from the water level.

'T'wo kinds of motion are here obviously active, a motion of translation and a motion of undulation-the race of the river through its gorge, and the great waves generated by its collision with, and rebound from, the obstacles in its way. In the middle of the river the rush and tossing are most violent; at all events, the impetuous force of the indivilual waves is here most strikingly displayed. Vast pyramidal heaps leap incessantly from the river, some of them with such energy as to jerk their summits into the air, where they hang momentarily suspended in crowds of liquid spherules. 'The sun shone for a few minutes. At times the wind, coming up the river, searched and sifted 
the spray, carrying away the lighter drops, and leaving the heavier ones behind. Wafted in the proper direction, rainbows appeared and disappeared fitfully in the lighter mist. In other directions the common gleam of the sunshine from the waves and their shattered crests was exquisitely beautiful. The complexity of the action wils still further illustrated by the fact, that in some cases, as if by the exercise of a local explosive force, the drops were shot radially from a particular center, forming around it a kind of halo.

The first impression, and, indeed, the current explanation of these rapids is, that the central bed of the river is cumbered with large boulders, and that the jostling, tossing, and wild leaping of the water there, are due to its impact against these obstacles. I doubt this explanation. At all events, there is another sufficient reason to be taken into account. Boulders derived from the adjacent cliffs visibly cumber the sides of the river. Against these the water rises and sinks rhythmically but violently, large waves being thus produced. On the generation of each wave, there is an immediate compounding of the wave-motion with the river-motion. The ridges which in still water would proceed in circular curves round the center of disturbance, cross the river obliquely, and the result is that at the center waves commingle, which have really been generated at the sides. In the first instance, we had a composition of wave-motion with river-motion; here we have the coalescence of waves with wares. Where crest and furrow cross each other, the motion is annulled; where furrow and furrow cross, the river is plowed to a greater depth; and where crest and crest aid each other, we have that astonishing leap of the water which breaks the cohesion of the crests, and tosses them shattered into the air. From the water level the cause of the action is not so casily seen, but from the summit of the cliff the lateral generation of the waves, and their propagation to the center, are perfectly obvious. If this explanation be correct, the phenomena observed at the Whirlpool Rapids form one of the grandest illustrations of the principle of interference. The Nile "cataract," Mr. Huxley informs me, offers more moderate examples of the same action.

At some distance below the Whirlpool Rapids we have the celebrated whirlpool itself. Here the river makes a 
sudden bend to the northeast, forming nearly a right angle with its previous direction. The water strikes the concave bank with great force, and scoops it incessantly away. A vast basin has been thus formed, in which the sweep of the river prolongs itself in gyratory currents. Bodies and trees which have come over the falls, are stated to circulate here for days without finding the outlet. From various points of the cliffs above, this is curiously hidden. 'The rush of the river into the whirlpool is obvious enough; and though you imagine the ontlet must be visible, if one existed, you cannot find it. 'Turning, however, round the bend of the precipice to the northeast, the outlet comes into view.

'The Niagara season was over; the chatter of sightseers had ceased, and the scene presented itself as one of holy seclusion and beauty. I went down to the river's edge, where the weird loneliness seemed to increase. The basin is enclosed by high and almost precipitous banks-covered, at the time, with russet woods. A kind of mystery attaches itself to gyrating water, due perhaps to the fact that we are to some extent ignorant of the direction of its force. It is said that at certain points of the whirlpool, pine trees are sucked down, to be ejected mysteriously elsewhere. The water is of the brightest emerald-green. The gorge through which it escapes is narrow, and the motion of the river swift though silent. The surface is steeply inclined, but it is perfectly unbroken. There are no lateral waves, no ripples with their breaking bubbles to raise a murmur; while the depth is here too great to allow the inequality of the bed to ruffle the surface. Nothing can be more beautiful than this sloping liquid mirror formed by the Niagara, in sliding from the whirlpool.

'The green color is, I think, correctly accounted for in the last Fragment. While crossing the Atlantic in 1872-73 I had frequent opportunities of testing the explanation there given. Lookerl properly down upon, there are portions of the ocean to which we should hardly ascribe a trace of blue; at the most, a mere hint of indigo reaches the eye. 'The water, indeed, is practically black, and this is an indication both of its depth and of its freedom from mechanically suspended matter. In small thicknesses water is sensibly transparent to all kinds of light; but, as the thicknees increases, the rays of low refrangibility are 
first absorbed, and after them the other rays. Where, therefore, the water is very deep and very pure, all the colors are absorbed, and such water ought to appear black, as no light is sent from itsinterior to the eye. 'The approximation of the Atlantic ocean to this condition is an indication of its extreme purity.

Throw a white pebble into such water; as it sinks it becomes greener and greener, and, before it disappears, it reaches a vivid blue-green. Break such a pebble into fragments, each of these will behave like the unbroken mass; grind the pebble to powder, every particle will yield its modicum of green; and if the particles be so fine as to remain suspended in the water, the scattered light will be a uniform green. Hence the greenness of shoal water. You go to bed with the black Atlantic around you. You rise in the morning, find it a vivid green, and correctly infer that you are crossing the bank of Newfoundland. Such water is found charged with fine matter in a state of mechanical suspension. The light from the bottom may sometimes come into play, but it is not necessary. A storm can render the water muddy, by rendering the particles too numerous and gross. Such a case occurred toward the close of my visit to Niagara. There had been rain and storm in the upper lake-regions, and the quantity of suspended matter brought down quite extinguished the fascinating green of the Horseshoe.

Nothing can be more superb than the green of the Atlantic waves, when the circumstances are favorable to the exhibition of the color. As long as a wave remains unbroken no color appears; but when the foam just doubles over the crest, like an Alpine snow-cornice, under the cornice we often see a display of the most exquisite green. It is metallic in its brilliancy. But the foam is necessary to its production. The foam is first illuminated, and it scatters the light in all directions; the light which passes through the higher portion of the wave alone reaches the eye, and gives to that portion its matchless color. The folding of the wave, producing as it does a series of longitudinal protuberances and furrows which act like cylindrical lenses, introduces variations in the intensity of the light, and materially enhances its beauty.

We have now to consider the genesis and proximate 
destiny of the Falls of Niagara. We may open our way to this subject by a few preliminary remarks upon erosion. Time and intensity are the main factors of geologic change, and they are in a certain sense convertible. A feeble force acting through long periods, and an intense force acting through short ones, may produce approximately the same results. 'To Dr. Hooker I have been indebted for' some specimens of stones, the first examples of which were pickell up by Mr. Hackworth on the shores of Lyell's bay, near Wellington, in New Zealand. They were described by Mr. 'Travers in the " Transactions of the New Zealand Institute." Unacquainted with their origin, you would certainly ascribe their forms to human workmanship. They resemble knives and spear-heads, being apparently chiseled off into facets, with as much attention to symmetry as if a tool, guided by human intelligence, had passed over them. But no human instrument has been brought to bear upon these stones. 'They have been wrought into their present shape by the wind-blown sand of Lyell's bay. 'I'wo winds are dominant here, and they in succession urged the sand against opposite sides of the stone; every little particle of sand chipped away its infinitesimal bit of stone, and in the end sculptured these singular forms.*

The Sphinx of Egypt is nearly covered up by the sand of the desert. The neck of the Sphinx is partly cut across,

* "These stones, which have a strong resemblance to works of luman art, occur in great abundance, and of various sizes, from halfan-inch to several inches in length. A large number were exhibited showing the various forms, which are those of wedges, knives, arrow-heads, etc., and all with sharp cutting edges.

"Mr. Travers explained that, notwithstanding their artificial appearance, these stones were formed by the cutting action of the wind-driven sand, as it passed to and fro over an exposed boulderbank. He gave a minute account of the manner in which the varieties of form are produced, and referred to the effect which the erosive action thus indicated would have on railway and other works executed on sandy tracts.

"Dr. Hector stated that although, as a group, the specimens on the table could not well be mistaken for artificial productions, still the forms are so peculiar, and the edges, in a few of them, so perfect, that if they were discovered associated with human works, there is no doubt that they would have been referred to the so-called 'stone period.'"-Hxtracted from the Minutes of the Wellington Philosophical Society, February 9, 1869. 
not, as I am assured by Mr. Huxley, by ordinary weathering, but by the eroding action of the fine sand blown against it. In these cases Nature furnishes us with hints which may be taken advantage of in art; and this action of sand has been recently turned to extraordinary account in the United States. When in Boston, I was taken by my courteous and helpful friend, Mr. Josiah Quincey, to see the action of the sand-blast. A kind of hopper containing fine silicious sand was connected with a reservoir of compressed air, the pressure being variable at pleasure. The hopper ended in a long slit, from which the sand was blown. A plate of glass was placed beneath this slit, and cansed to pass slowly under it; it came out perfectly depolished, with a bright opalescent glimmer, such as could only be produced by the most careful grinding. Every little particle of sand urged against the glass, having all its energy concentrated on the point of impact, formed there a little pit, the depolished surface consisting of innumerable hollows of this description.

But this was not all. By protecting certain portions of the surface, and exposing others, figures and tracery of any required form could be etched upon the glass. The figures of open iron-work could be thus copied; while wire-gauze placed over the glass produced a reticulated pattern. But it required no such resisting substance as iron to shelter the glass. The patterns of the finest lace could be thus reproduced; the delicate filaments of the lace itself offering a sufficient protection. All these effects have been obtained with a simple model of the sand-blast devised by my assistant. A fraction of a minute suffices to etch upon glass a rich and beautiful lace pattern. Any yielding substance may be employed to protect the glass. By diffusing the shock of the particle, such substances practically destroy the local erosive power. 'The hand can bear, without inconvenience, a sand-shower which would pulverize glass. Etchings executed on glass with suitable kinds of ink are accurately worked out by the sand-blast. In fact, within certain limits, the harder the surface, the greater is the concentration of the shock, and the more effectual is the erosion. It is not necessary that the sand should be the harder substance of the two; corundum, for example, is much harder than quartz; still, quartz-sand can not only depolish, but actually blow a hole through a plate of corun- 
dum. Nay, glass may be depolished by the impact of fine shot; the grains in this case bruising the glass, before they have time to flatten and turn their energy into heat.

And here, in passing, we may tie together one or two apparently unrelated facts. Supposing you turn on, at the lower part of a house, a cock which is fed by a pipe from a cistern at the top of the house, the column of water, from the cistern downward, is set in motion. By turning off the cock, this motion is stopped; and when the turning off is very sudden, the pipe, if not strong, may be burst by the internal impact of the water. By distributing the turning of the cock over half a second of time, the shock and and danger of rupture may be entirely avoided. We have here an example of the concentration of energy in time. The sand-blast illustrates the concentration of energy in space. The action of flint and steel is an illustration of the same principle. The heat required to generate the spark is intense; and the mechanical action, being moderate, must, to produce fire, be in the highest degree concentrated. This concentration is secured by the collision of hard substances. Calc-spar will not supply the place of flint, nor lead the place of steel, in the production of fire by collision. With the softer substances, the total heat produced may be greater than with the hard ones, but, to produce the spark, the heat must be intensely localized.

We can, however, go far beyond the mere depolishing of glass; indeed I have already said that quartz-sand can wear a hole through corundum. 'This leads me to express my acknowledgments to General Tilghman,* who is the inventor of the sand-blast. To his spontaneous kindness I am indebted for some beantiful illustrations of his process. In one thick plate of glass a figure has been worked out to a depth of three-eighths of an inch. A second plate, seveneighths of an inch thick, is entirely perforated. In a circu-

* The absorbent power, if I may use the phrase, exerted by the industrial arts in the United States, is forcibly illustrated by the rapid transfer of men like Mr. Tilghman from the life of the soldier to that of the civilian. (Yeneral McClellan, now a civil engineer, whom I had the honor of frequently meeting in New York, is a most eminent example of the same kind. At the end of the war, indeed, a million and a half of men were thus drawn, in an astonishingly short time, from military to civil life. 
lar plate of marble, nearly half an inch thick, open work of most intricate and elaborate description has been executed. It would probably take many days to perform this work by any ordinary process; with the sand-blast it was accomplished in an hour. So much for the strength of the blast; its delicacy is illustrated by this beautiful example of line engraving, etched on glass by means of the blast.

'This power of erosion, so strikingly displayed when sand is urged by air, renders us better able to conceive its action when urged by water. The erosive power of a river is vastly augmented by the solid matter carried along with it. Sand or pebbles, caught in a river vortex, can wear away the hardest rock; "potholes" and deep cylindrical shafts being thus produced. An extraordinary instance of this kind of erosion is to be seen in the Val Tournanche, above the village of this name. The gorge of Handeck has been thus cut out. Such waterfalls were once frequent in the valleys of Switzerland; for hardly any valley is without one or more transverse barriers of resisting material, over which the river flowing through the valley once fell as a cataract. Near Poutresina, in the Engadin, there is such a case; a hard gneiss being there worn away to form a gorge, through which the river from the Morteratsch glacier rushes. The barrier of the Kirchet above Meyringen is also a case in point. Behind it was a lake, derived from the glacier of the Aar, and over the barrier the lake poured its excess of water. Here the rock, being limestone, was in part dissolved; but added to this we had the action of the sand and gravel carried along by the water, which, on striking the rock, chipped it away like the particles of the sandblast. Thus, by solution and mechanical erosion, the great chasm of the Finsteraarschlucht was formed. It is demonstrable that the water which flows at the bottoms of such deep fissures once flowed at the level of their present edges, and tumbled down the lower faces of the barriers. Almost every valley in Switzerland furnishes examples of this kind; the untenable hypothesis of earthquakes, once so readily resorted to in accounting for these gorges, being now for the most part abandoned. To produce the cañons of western America, no other cause is needed than the integration of effects individually infinitesimal.

And now we come to Niagara. Soon after Europeans had taken possession of the country, the conviction appear's 
to have arisen that the deep channel of the river Niagara below the falls had been excavated by the cataract. In Mr. Bakewell's "Introduction to Geology," the prevalence of this belief has been referred to. It is expressed thus by Professor Joseph Henry in the "Transactions of the Albany Institute:" * "In viewing the position of the falls, and the features of the country round, it is impossible not to be impressed with the idea that this great natural raceway has been formed by the continued action of the irresistible Niagara, and that the falls, beginning at Lewiston, have, in the course of ages, worn back the rocky strata to their present site." The same view is adrocated by Sir Charles Lyell, by Mr. Hall, by M. Agassiz, by Professor Ramsay, indeed by most of those who have inspected the place.

A connected image of the origin and progress of the cataract is easily obtained. Walking northward from the village of Niagara Falls by the side of the river, we have to our left the deep and comparatively narrow gorge, through which the Niagara flows. The bounding cliffs of this gorge are from 300 to 350 feet high. We reach the whirlpool, trend to the northeast, and after a little time gradually resume our northward course. Finally, at abont seven miles from the present falls, we come to the edge of a declivity, which informs us that we have been hitherto walking on table-land. At some hundreds of feet below us is a comparatively level plain, which stretches to Lake Ontario. 'The declivity marks the end of the precipitous gorge of the Niagara. Here the river escapes from its steep mural boundaries, and in a widened bed pursues its way to the lake which finally receives its waters.

'The fact that in historic times, even within the memory of man, the fall has sensibly receded, prompts the question, How far has this recession gone? At what point did the ledge which thus continually creeps backward begin its retrograde course? 'To minds disciplined in such researches the answer has been, and will be-At the precipitous declivity which crossed the Niagara from Lewiston on the American to Queenston on the Canadian side. Over this transverse barrier the united affluents of all the upper lakes once poured their waters, and here the work of erosion be- 
gan. The dam, moreover, was demonstrably of sufficient height to cause the river above it to submerge Goat Island; and this would perfectly account for the finding by Sir Charles Lyell, Mr. Hall, and others, in the sand and gravel of the island, the same fluviatile shells as are now found in the Niagara river higher up. It would also account for those deposits along the sides of the river, the discovery of which enabled Lyell, Hall, and Ramsay to reduce to demonstration the populiur belief that the Niagara once flowed through a shallow valley.

The physics of the problem of excavation, which I made clear to my mind before quitting Niagara, are revealed by a close inspection of the present IIorseshoe Fall. We see evidently that the greatest weight of water bends over the very apex of the Horseshoe. In a passage in his excellent chapter on Niagara Falls, Mr. Hall alludes to this fact. Here we have the most copions and the most violent whirling of the shattered liquid; here the most powerful eddies recoil against the shale. From this portion of the fall, indeed, the spray sometimes rises without solution of continuity to the region of clouds, becoming gradually more attenuated, and passing finally through the condition of true cloud into invisible vapor, which is sometimes reprecipitated higher up. All the phenomena point distinctly to the center of the river as the place of greatest mechanical energy, and from the center the vigor of the fall gradually dies away toward the sides. The Horseshoe form, with the concavity facing downward, is an obvious and necessary consequence of this action. Right along the middle of the river the apex of the curve pushes its way backward, cutting along the center a deep and comparatively narrow groove, and draining the sides as it passes them.* Hence the remarkable discrepancy between the widths of the Niagara above and below the Horseshoe. All along its course, from Lewiston Heights to its present position, the form of the fall was probably that of a horseshoe; for this is merely the expression of the greater depth, and consequently greater excavating power, of the center of the river. 'The gorge, moreover, varies in width, as the depth of the

* In the discourse the excavation of the center and drainage of the sides action was illustrated by a model devised by my assistant, Mr. John Cottrell. 
center of the ancient river varied, being narrowest where that depth was greatest.

'The vast comparative erosive energy of the Horseshoe Fall comes strikingly into view when it and the American Fall are compared together. The American branch of the river is cut at a right angle by the gorge of the Niagara. Here the Horseshoe Fall was the real excavator. It cut the rock, and formed the precipice, over which the American Fall tumbles. But since its formation, the erosive action of the American Fall has been almost nil, while the Horseshoe has cut its way for 500 yards across the end of Goat Island, and is now doubling back to excavate its channel parallel to the length of the island. This point, which impressed me forcibly, has not, I have just learned, escaped the acute observation of Professor Ramsay.* The river bends; the Horseshoe immediately accommodates itself to the bending, and will follow implicitly the direction of the deepest water in the upper stream. The flexures of the gorge are determined by those of the river channel above it. Were the Niagara center above the fall sinuous, the gorge would obediently follow its sinuosities. Once suggested, no doubt geographers will be able to point out many examples of this action. The Zambesi is thought to present a great difficulty to the erosion theory, because of the sinuosity of the chasm below the Victoria Falls. But, assuming the basalt to be of tolerably uniform texture, had the river been examined before the formation of this sinuous channel, the present zigzag course of the gorge below the fall could, I am persuaded, have been predicted, while the sounding of the present river would enable us to predict the course to be pursued by the erosion in the future.

But not only has the Niagara river cut the gorge; it has carried away the chips of its own workshop. The shale, being probably crumbled, is easily carried away. But at the base of the fall we find the huge boulders alrealy described, and by some means or other these are removed

* His words are: "Where the body of water is small in the American Fall, the edge has only receded a few yards (where most eroded) during the time that the Canadian Fall has receded from the north corner of Goat Island to the innermost curve of the Horseshoe Fall."-Quarterly Journal of Geological Society, May, 1859. 


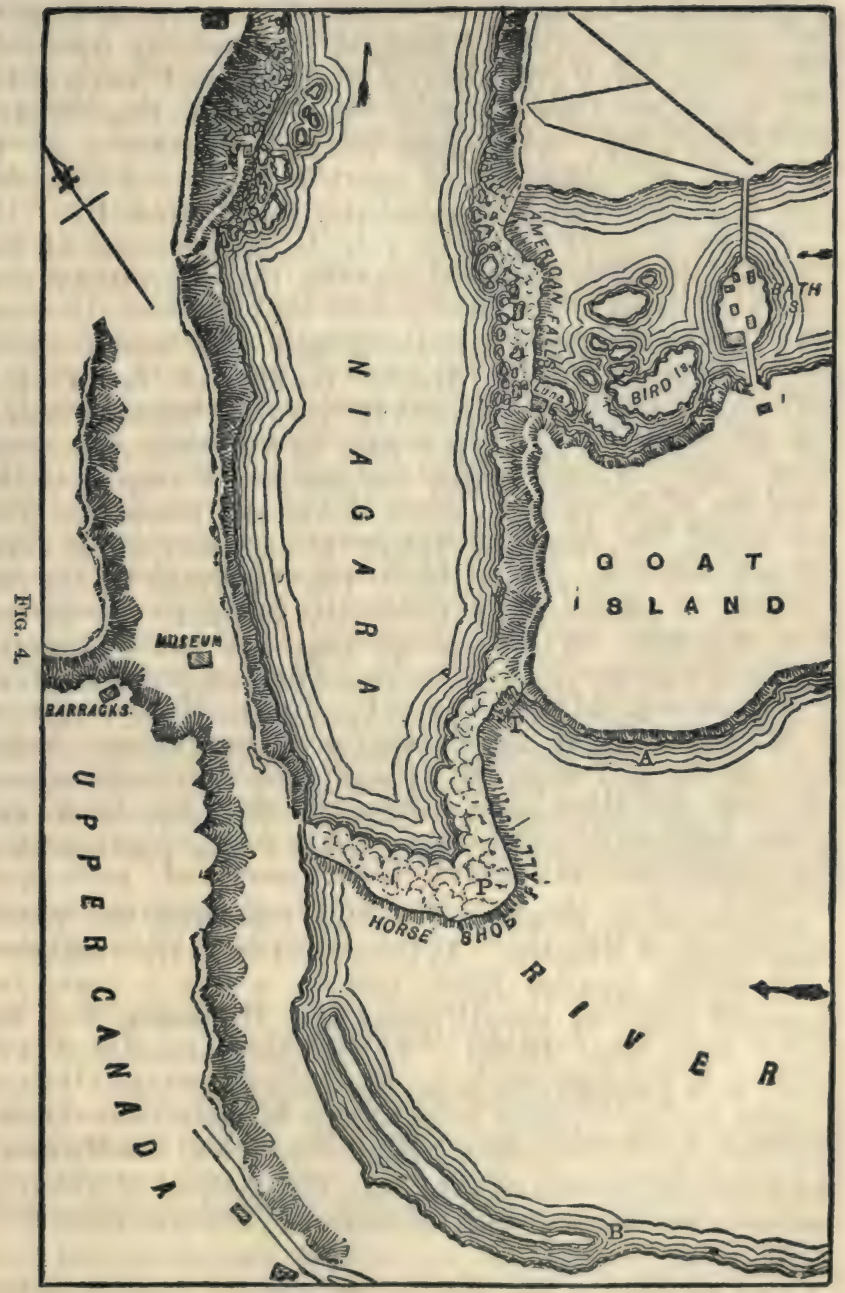


down the river. The ice which fills the gorge in winter, and which grapples with the boulders, has been regarded as the transporting agent. Probably it is so to some extent. But erosion acts without ceasing on the abutting points of the boulders, thus withdrawing their support and urging them gradually down the river. Solution also does its portion of the work. That solid matter is carried down is proved by the difference of depth between the Niagara river and Lake Ontario, where the river enters it. The depth falls from 72 feet to 20 feet, in consequence of the deposition of solid matter caused by the diminished motion of the river.*

'The annexed highly instructive map has been reduced from one published in Mr. Hall's "Geology of New York." It is based on surveys execnted in 1842, by Messrs. Gibson and Evershed. The ragged edge of the American Fall north of Goat Island marks the amount of erosion which it has been able to accomplish, while the Horseshoe Fall was cutting its way southward across the end of Goat Island to its present position. The American Fall is 168 feet high, a precipice cut down, not by itself, but by the Horseshoe Fall. The latter in 1842 was 159 feet high, and, as shown by the map, is already turning eastward, to excavate its gorge along the center of the upper river. $P$ is the apex of the Horseshoe, and T marks the site of the Terrapin 'Tower, with the promontory adjacent, round which I was conducted by Conroy. Probably since 184\% the Horseshoe has worked back beyond the position here assigned to it.

In conclusion, we may say a word regarding the proximate future of Niagara. At the rate of excavation assigned to it by Sir Charles Lyell, namely, a foot a year, five thousand years or so will carry the Horseshoe Fall far higher than Goat Island. As the gorge recedes it will lrain, as it has hitherto done, the banks right and left of it, thus leaving a nearly level terrace between Goat Island and the edge of the gorge. Higher up it will totally drain the American branch of the river; the channel of which in due time will become cultivable land. The American Fall

* Near the mouth of the gorge at Queenston, the depth, according to the Admiralty Chart, is 180 feet well within the gorge it is 132 feet. 
will then be transformed into a dry precipice, forming a simple continuation of the cliffy boundary of the Niagara gorge. At the place occupied by the fall at this moment we shall have the gorge enclosing a right angle, a second whirlpool being the consequence. To those who visit Niagara a few millenniums hence I leave the verification of this prediction. All that can be said is, that if the causes now in action continue to act, it will prove itseif literally true

\section{Postscript.}

A year or so after I had quitted the United States, a man sixty years of age, while engaged in painting one of the bridges which connect Goat Island with the Three Sisters, slipped through the rails of the bridge into the rapids, and was carried impetuously toward the Horseshoe Fall. He was urged against a rock which rose above the water, and with the grasp of desperation he clung to it. The population of the village of Niagara Falls was soon upon the island, and ropes were brought, but there was none to use them. In the midst of the excitement, a tall powerful young fellow was observed making his way silently through the crowd. He reached a rope; selected from the bystanders a number of men, and placed one end of the rope in their hands. The other end he fastened round himself, and choosing a point considerably above that to which the man clung, he plunged into the rapids. He was carried violently downward, but he canght the rock, secured the old painter and saved him. Newspaper's from all parts of the Union poured in upon me, describing this gallant act of my guide Conroy.

\section{CHAPTER VIII.}

\section{THE PARALLEL ROADS OF GLEN ROY.*}

THE FIRst published allusion to the Parallel Roads of Glen Roy occurs in the appendix to the third volume of Pennant's "Tour in Scotland," a work published in $17 \% 6$.

* A discourse delivered at the Royal Institution of Great Britain on June 9, 1876. 
"In the face of these hills," says this writer, "both sides of the glen, there are three roads at small distances from each other and directly opposite on each side. These roads have been measured in the complete parts of them, and found to be 26 paces of a man 5 feet 10 inches high. 'The two highest are pretty near each other, about 50 yards, and the lowest double that distance from the nearest to it. They are carried along the sides of the glen with the utmost regularity, nearly as exact as drawn with a line of rule and compass."

The correct heights of the three roads of Glen Roy are respectively $1,150,1,070$, and 860 feet above the sea. Hence a vertical distance of 80 feet separates the two highest, while the lowest road is 210 feet below the middle one.

These " roads" are usually shelves or terraces formed in the yielding drift which here covers the slopes of the mountains. They are all sensibly horizontal and therefore parallel. Pennant accepted as reasonable the explanation of them given by the country people in his time. They thought that the roads "were designed for the chase, and that the terraces were made after the spots were cleared in lines from wood, in order to tempt the animals into the open paths after they were roused, in order that they might come within reach of the bowmen who might conceal themselves in the woods above and below."

In these attempts of "the country people" we have an illustration of that impulse to which all scientific knowledge is due-the desire to know the causes of things; and it is a matter of surprise that in the case of the parallel roads, with their weird appearance challenging inquiry, this impulse did not make itself more rapidly and energetically felt. Their remoteness may perhaps account for the fact that until the year $181 \%$ no systematic description of them, and no scientific attempt at an explanation of them, appeared. In that year Dr. MacCulloch, who was then president of the Geological Society, presented to that Society a memoir, in which the roads were discussed, and pronounced to be the margins of lakes once embosomed in Glen Rov. Why there should be three roads, or why the lakes should stand at these particular levels, was left unexplained.

To Dr. MacCulloch succeeded a man, possibly not so 
learned as a geologist, but obviously fitted by nature to grapple with her facts and to put them in their proper setting. I refer to Sir 'Thomas Dick-Lauder, who presented to the Royal Society of Elinburgh, on the $2 d$ of March, 1818, his paper on the Parallel Roads of Glen Roy. In looking over the literature of this subject, which is now copious, it is interesting to observe the differentiation of minds, and to single out those who went by a kind of instinct to the core of the question, from those who erred in it, or who learnedly occupied themselves with its analogies, adjuncts, and details. There is no man, in my opinion, connected with the history of the subject, who has shown, in relation to it, this spirit of penetration, this force of scientific insight, more conspicuously than Sir Thomas Dick-Lauder. Two distinct mental processes are incolved in the treatment of such a question. Firstly, the faithful and sufficient observation of the data; and secondly, that higher mental process in which the constructive imagination comes into play, connecting the separate facts of observation with their common cause, and weaving them into an organic whole. In neither of these requirements did Sir 'Thomas Dick-Lauder fail.

Adjacent to Glen Roy is a valley caller Glen Gluoy, along the sides of which ran a single shelf, or terrace, formed obviously in the same manner as the parallel roads of Glen Roy. The two shelves on the opposing sides of the glen were at precisely the same level, and Dick-Lauder wished to see whether, and how, they became united at the head of the glen. He followed the shelves into the recesses of the mountains. The bottom of the valley, as it rose, came ever nearer to them, until finally, at the head of Glen Gluoy, he reached a col, or watershed, of precisely the same elevation as the road which swept round the glen.

The correct height of this col is $1,1 \% 0$ feet above the sea; that is to say, 20 feet above the highest road in Glen Roy.

From this col a lateral branch-valley-Glen Turrit-led down to Glen Roy. Our explorer descended from the col to the highest road of the latter glen, and pursued it exactly as he had pursued the road in Glen Gluoy. For a time it belted the mountain sides at a considerable height above the bottom of the valley; but this rose as he proceeded, coming ever nearer to the highest shelf, until finally he 
reached a col, or watershed, looking into Glen Spey, and of precisely the same elevation as the highest road of Glen Roy.

IIe then dropped down to the lowest of these roads, and followed it toward the mouth of the glen. Its elevation above the bottom of the valley gradually increased; not becanse the shelf rose, but because it remained level while the valley sloped downward. He found this lowest road doubling round the hills at the mouth of Glen Roy, and

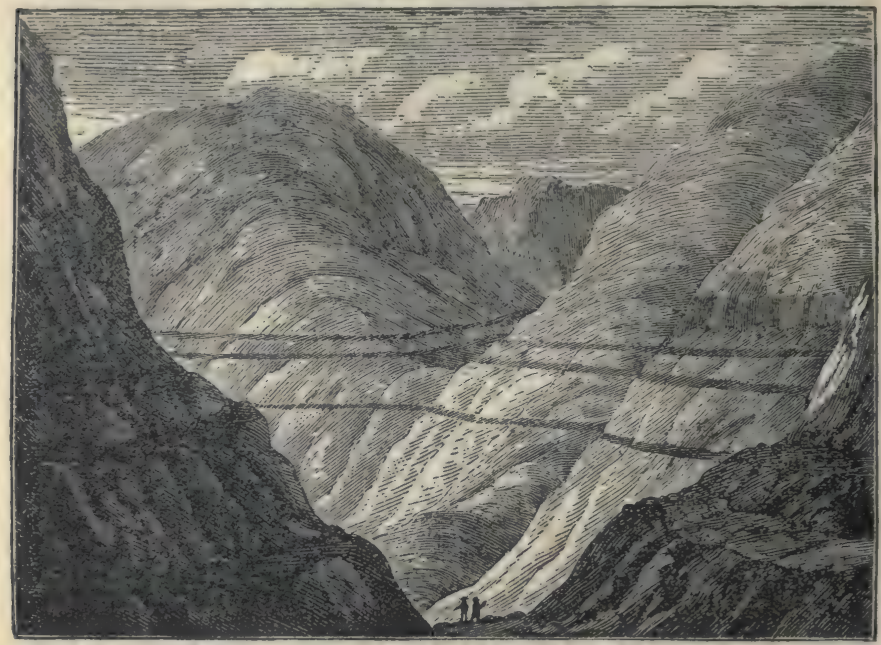

PARALLEL ROADS OF GLEN ROY.

After a Sketch by Sir Thomas Dick-LaUder.

running along the sides of the mountains which flank Glen Spean. He followed it eastward. The bottom of the Spean Valley, like the others, gradually rose, and therefore gradually approached the road on the adjacent mountain-side. He came to Loch Laggan, the surface of which rose almost to the level of the road, and beyond the head of this lake he found, as in the other two cases, a col, or watershed, at Makul, of exactly the same level as the single road in Glen Spean, which, it will be remembered, is a continuation of the lowest road in Glen Roy. 


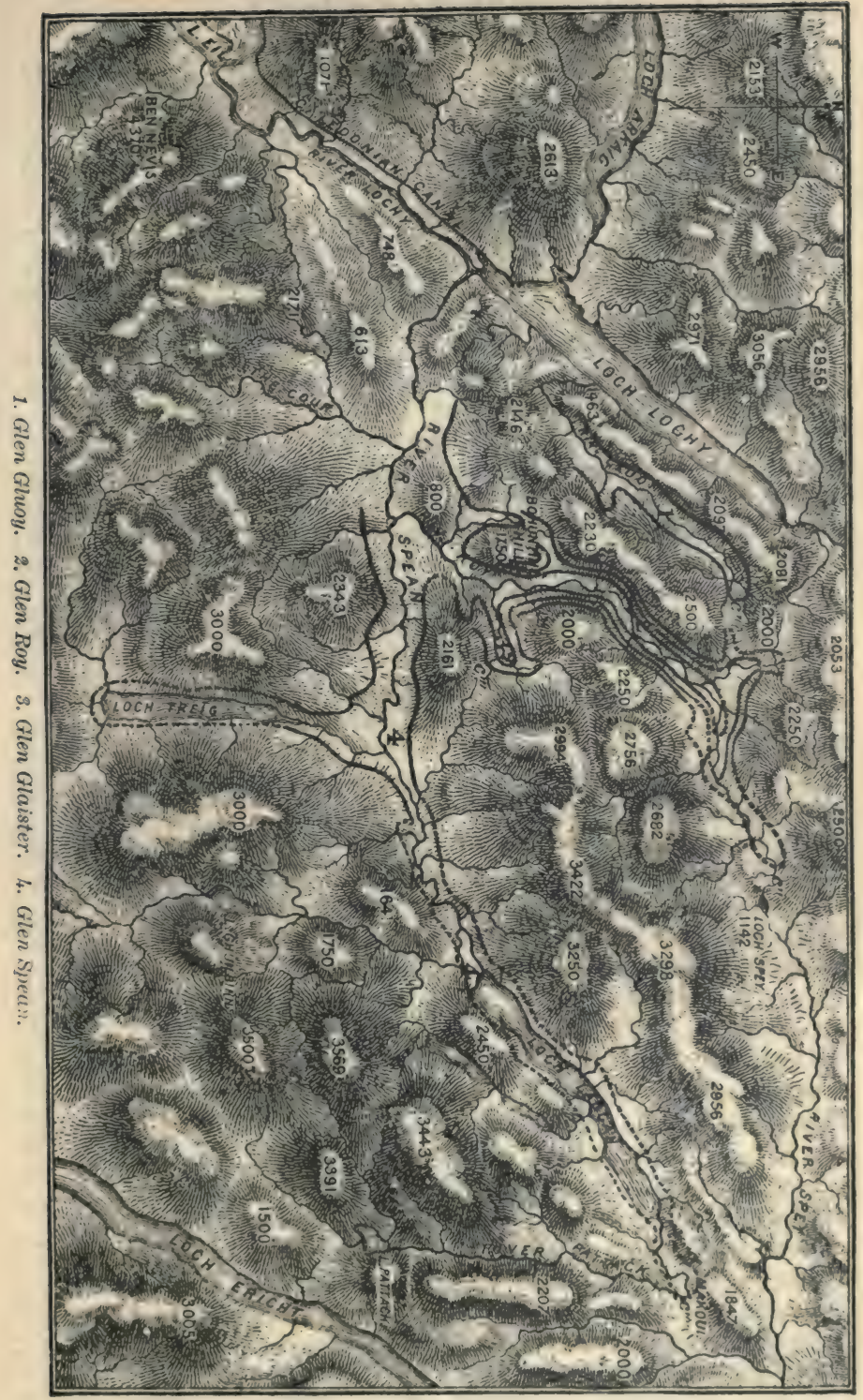


Here we have a series of facts of obvions significance as regards the solution of this problem. The effort of the mind to form a coherent image from such facts may be compared with the effort of the eyes to cause the pictures of a stereoscope to coalesce. For a time we exercise a certain strain, the object remaining vague and indistinct. Suddenly its varions parts seem to run together, the object starting forth in clear and definite relief. Such, I take it, was the effect of his ponderings upon the mind of Sir 'Thomas Dick-Lauder. His solution was this: Taking all their features into account, he was convinced that water only could have produced the terraces. But how had the water been collected? He saw clearly that, supposing the mouth of Glen Gluoy to be stopped by a barrier sufficiently high, if the waters from the mountains flanking the glen were allowed to collect, they would form behind the barrier a lake, the surface of which would gradually rise until it reached the level of the col at the head of the glen. T'he rising would then cease; the superfluous water of Glen Gluoy discharging itself over the col into Glen Roy. As long as the barrier stopping the month of Glen Gluoy continued high enough, we should have in that glen a lake at the precise level of its shelf, which lake, acting upon the loose drift of the flanking mountains, would form the shelf revealed by observation.

So much for Glen Gluoy. But suppose the mouth of Glen Roy also stopped by a similar barrier. Behind it also the water from the adjacent mountains would collect. 'The surface of the lake thus formed would gradually rise, until it had reaclied the level of the col which divides Glen Roy from Glen Spey. Here the rising of the lake would cease; its superabundant water being poured over the col into the valley of the Spey. This state of things would continue as long as a sufficiently high barrier remained at the mouth of Glen Roy. The lake thus dammed in with its surface at the level of the highest parallel road, would act, as in Glen Gluoy, upon the friable drift overspreading the mountains, and would form the highest road or terrace of Glen Roy.

And now let us suppose the barrier to be so far removed from the mouth of Glen Roy as to establish a connection between it and the upper part of Glen Spean, while the lower part of the latter glen still continued to be blocked up. Upper Glen Spean and Glen Roy would then be oc- 
cupied by a continuous lake, the level of which would obviously be determined by the col at the head of Loch Laggan. The water in Glen Roy would sink from the level it had previously maintained, to the level of its new place of escape. This new lake-surface would correspond exactly with the lowest parallel road, and it would form that road by its action upon the drift of the adjacent mountains.

In presence of the observed facts, this solution commends itself strongly to the scientific mind. The question next occur's, What was the character of the assumed barrier which stopped the glens? There are at the present moment vast masses of detritus in certain portions of Glen Spean, and of such detritus Sir Thomas Dick-Lauder imagined his barriers to have been formed. By some unknown convulsion, this detritus had been heaped up. But, once given, and once granted that it was subsequently removed in the manner indicated, the single road of Glen Gluoy and the highest and lowest roads of Glen Roy, would be explained in a satisfactory manner.

To account for the second or middle road of Glen Roy, Sir Thomas Dick-Lauder invoked a new agency. He supposed that at a certain point in the breaking down or waste of his dam, a halt occurred, the barrier holding its ground at a particular level sufficiently long to dam a lake rising to the height of, and forming the second road. 'This point of weakness was at once detected by Mr. Darwin, and adduced by him as proving that the levels of the cols did not constitute an essential feature in the phenomena of the parallel roads. Though not destroyed, Sir Thomas DickLauler's theory was seriously shaken by this argument, and it became a point of capital importance, if the facts permitted, to remove such source of weakness. This was done in 1847 by Mr. David Milne, now Mr. Milne-Home. On walking up Glen Roy from Roy Bridge, we pass the mouth of a lateral glen, called Glen Glaster, running eastward from Glen Roy. There is nothing in this lateral glen to attract attention, or to suggest that it sould have any conspicuous influence in the production of the parallel roads. Hence, probably, the failure of Sir Thomas DickLauder to notice it. But Mr. Milne-Home entered this glen, on the northern side of which the middle and lowest roads are fairly shown. The principal stream running through the glen turns at a certain point northward and 
loses itself among the hills too high to offer any outlet. But another branch of the glen turus to the southeast; and, following up this branch, Mr. Milne-Home reached a col, or watershed, of the precise level of the second Glen Roy road. When the barrier blocking the glens had been so far removed as to open this col, the water in Glen Roy would sink to the level of the second road. A new lake of diminished depth would be thus formed, the surplus water of which would escape over the Glen Glaster col into Glen Spean. The margin of this new lake, acting upon the detrital matter, would form the second road. The theory of Sir 'Thomas Dick-Lauder, as regards the part played by the cols, was re-riveted by this new and unexpected discovery.

I have referred to Mr. Darwin, whose powerful mind swayed for a time the convictions of the scientific world in relation to this question. His notion was-and it is a notion which very naturally presents itself-that the parallel roads were formed by the sea; that this whole region was once submerged and subsequently upheaved; that there were pauses in the process of upheaval, during which these glens constituted so many fiords, on the sides of which the parallel terraces were formed. This theory will not bear close criticism; nor is it now maintained by Mr. Darwin himself. It would not account for the sea being 20 feet higher in Glen Gluoy than in Glen Roy. It would not account for the absence of the second and third Glen Roy roads from Glen Gluoy, where the mountain flanks are quite as impressionable as in Glen Roy. It would not account for the absence of the shelves from the other mountains in the neighborhood, all of which would have been clasped by the sea hal the sea been there. Here then, and no doubt elsewhere, Mr. Darwin has shown himself to be fallible; but here, as elsewhere, he has shown himself equal to that discipline of surrender to evidence which girds his intellect with such unassailable moral strength.

But, granting the significance of Sir 'Thomas DickLauder's facts, and the reasonableness, on the whole, of the views which he has founded on them, they will not bear examination in detail. No such barriers of detritus as he assumed could have existed without leaving traces behind them; but there is no trace left. 'There is detritus enough in Glen Spean, but not where it is wanted. The 
two highest parallel roads stop abruptly at different points near the mouth of Glen Roy, but no remnant of the barrier against which they abutted is to be seen. It might be urged that the subsequent invasion of the valley by glaciers has swept the detritus away; but there have been no glaciers in these valleys since the disappearance of the lakes Professor Geikie has favored me with a drawing of Glen Spean "road" near the entrance to Glen 'Trieg. The road forms a shelf round a great mound of detritus which, had a glacier followed the formation of the shelf, must have been cleared away. Taking all the circumstances into account, you may, I think, with safety dismiss the detrital barrier as incompetent to account for the present condition of Glen Gluoy and Glen Roy.

Hypotheses in science, though apparently transcending experience, are in reality experience modified by scientific thought and pushed into an ultra experiential region. At the time that he wrote, Sir Thomas Dick-Lauder could not possibly have discerned the canse subsequently assigned for the blockage of these glens. A knowledge of the action of ancient glaciers was the necessary antecedent to the new explanation, and experience of this nature was not possessed by the distinguished writer just mentioned. The extension of Swiss glaciers far beyond their present limits, was first made known by a Swiss engineer named Venetz, who established, by the marks they had left behind them, their former existence in places which they had long forsaken. 'The subject of glacier extension was subsequently followed up with distinguished success by Charpentier, Studer, and others. With characteristic vigor Agassiz grappled with it, extending his observations far beyond the domain of Switzerland. He came to this country in 1840, and found in various places indubitable marks of ancient glacier action. Englanil, Scotland, Wales, and Ireland he proved to have once given birth to glaciers. He visited Glen Roy, surveyed the surrounding neighborhood, and pronounced, as a consequence of his investigation, the barriers which stopped the glens and produced the parallel roads to have been barriers of ice. 'To Mr. Jamieson, above all others, we are indebted for the thorough testing and confirmation of this theory.

And let me here say that Agassiz is only too likely to be misrated and misjudged by those who, though accurate within a limited sphere, fail to grasp in their totality the 
motive powers invoked in scientific investigation. True he lacked mechanical precision, but he abounded in that force and freshness of the scientific imagination which in some sciences, and probably in some stages of all sciences, are essential to the creator of knowledge. 'T'o Agassiz was given, not the art of the refiner, but the instinct of the discoverer, and the strength of the delver who brings ore from the recesses of the mine. That ore may contain its share of dross, but it also contains the precious metal which gives employment to the refiner, and without which his occupation would depart.

Let us dwell for a moment upon this subject of ancient glaciers. Under a flask containing water, in which a thermometer is immersed, is placed a Bunsen's lamp. 'The water is heated, reaches a temperature of 212 degrees, and then begins to boil. The rise of the thermometer then ceases, although heat continues to be poured by the lamp in to the water. What becomes of that heat? We know that it is consumed in the molecular work of vaporization. In the experiment here arranged, the steam passes from the flask through a tube into a second vessel kept at a low temperature. Here it is coudensed, and indeed congealed to ice, the second vessel being plunged in a mixture cold enough to freeze the water. As a result of the process we obtain a mass of ice. 'That ice has an origin very antithetical to its own character. Though cold, it is the child of heat. If we removed the lamp, there would be no steam, and if there vere no steam there would be no ice. The mere cold of the mixture surrounding the second vessel would not produce 1ce. 'The cold must have the proper material to work upon; and this material-aqueous vapor-is, as we here see, the direct product of heat.

It is now, I suppose, fifteen or sixteen years since I found myself conversing with an illustrious philosopher regarding that glacial epoch which the researches of Agassiz and other's had revealed. This profoundly thoughtful man maintained the fixed opinion that, at a certain stage in the history of the solar system, the sun's radiation had suffered diminution, the glacial epoch being a consequence of this solar chill. The celebrated French mathematician Poisson had another theory. Astronomers have shown that the solar system moves through space, and "the temperature of space" is a familiar expression 
with scientific men. It was considered probable by Poisson that our system, during its motion, had traversed portions of space of different temperatures; and that, during its passage through one of the colder regions of the universe, the glacial epoch occurred. Notions such as these were more or less current everywhere not many years ago, and I therefore thought it worth while to show how incomplete they were. Suppose the temperature of our planet to be reduced, by the subsidence of solar heat, the cold of space, or any other cause, say one hundred degrees. Four-andtwenty hours of such a chill would bring down as snow nearly all the moisture of our atmosphere. But this would not produce a glacial epoch. Such an epoch would require the long-continued generation of the material from which the ice of glaciers is derived. Mountain snow, the nutriment of glaciers, is derived from aqueous vapor raised mainly from the tropical ocean by the sun. The solar fire is as necessary a factor in the process as our lamp in the experiment referred to a moment ago. Nothing is easier than to calculate the exact amount of heat expended by the sun in the production of a glacier. It would, as I have elsewhere shown,* raise a quantity of cast iron five times the weight of the glacier not only to a white heat, but to its point of fusion. If, as I have already urged, instead of being filled with ice, the valleys of the Alps were filled with white-hot inetal, of quintuple the mass of the present glaciers, it is the heat and not the cold, that would arrest our attention and solicit our explanation. 'The process of glacier making is obviously one of distillation, in which the fire of the sun, which generates the vapor, plays as essential a part as the cold of the mountains which condenses it. $\dagger$

It was their ascription to glacier action that first gave the parallel roads of Glen Roy an interest in my eyes; and in 1867 , with a view to self-instruction, I made a solitary

\footnotetext{
* "Heat a Mode of Motion," fifth edition, chap. vi.: Forms of Water, $\$ \$ 55$ and 56 .

+In Lyell's excellent “ Principles of Geology," the remark occurs that " several writers have fallen into the strange error of supposing that the glacial period must have been one of ligher mean temperature than usual." 'The really strange error was the forgetfulness of the fact that without the heat the substance necessary to the production of glaciers would be wanting.
} 
pilgrimago to the place, and explored pretty thoroughly the roads of the principal glen. I traced the highest road to the col dividing Glen Roy from Glen Spey, and, thanks to the eivility of an ordnance surveyor, I was enabled to inspect some of the roads with a theodolite, and to satisfy myself regarding the common level of the shelves at opposite sides of the valley. As stated by Pennant, the width of the roads amounts sometimes to more than twenty yards; but near the head of Glen Roy the highest road ceases to have any width, for it runs along the face of a rock, the effect of the lapping of the water on the more friable portions of the rock being perfectly distinct to this hour. My knowledge of the region was, however, far from complete, and nine years had dimmed the memory even of the portion which had been thoroughly examined. Hence my desire to see the roads once more before venturing to talk to you about them. The Easter holidays of 1876 were to be devoted to this purpose; but at the last moment a telegram from Roy Bridge informed me that the roads were snowed up. Finding books and memories poor substitutes for the flavor of facts, I resolved subsequently to make another effort to see the roads. Accordingly last Thursday fortnight, after lecturing here, I packed up, and started (not this time alone) for the North. Next day at noon my wife and I found ourselves at Dalwhinnie, whence a drive of some five-and-thirty miles brought us to the excellent hostelry of Mr. Macintosh, at the mouth of Glen Roy.

We might have found the hills covered with mist, which would have wholly defeated us; but Nature was gooc-natured, and we had two successful working days among the hills. Guided by the excellent ordnance map of the region, on the Saturday morning we went up the glen, and on reaching the stream called Allt Bhreac Achaidh faced the hills to the west. At the watershed between Glen Roy and Glen Fintaig we bore northward, struck the ridge above Glen Gluoy, came in view of its road, which we persistently followed as long as it continued visible. It is a feature of all the roads that they ranish before reaching the cols over which fell the waters of the lakes which formed them. One reason doubtless is that at their upper ends the lakes were shallow, and incompetent on this account to raise wavelets of any strength to 
act upon the mountain drift. A second reason is that they were land-locked in the higher portions and protected from the southwesterly winds, the stillness of their waters causing them to produce but a feeble impression upon the mountain sides. From Glen Gluoy we passed down Glen Turrit to Glen Roy, and through it homeward, thus accomplishing two or three and twenty miles of rough and honest work.

Next day we thoroughly explored Glen Glaster, following its two roads as far as they were visible. We reached the col discovered by Mr. Milne-Home, which stands at the level of the middle road of Glen Roy. Thence we crossed southward over the mountain Creag Dhubh, and examined the erratic blocks upon its sides, and the ridges and mounds of moraine natter which cumber the lower flanks of the mountain. The observations of Mr. Jamieson upon this region, including the mouth of Glen Trieg, are in the highest degree interesting. We entered Glen Spean, and continued a search begun on the evening of our arrival at Roy Bridge-the search, namely, for glacier polishings and markings. We did not find them copious, but they are indubitable. One of the proofs most convenient for reference, is a great rounded rock by the roadside, 1,000 yards east of the milestone marked three-quarters of a mile from Roy Bridge. Farther east other cases occur, and they leave no doubt upon the mind that Glen Spean was at one time filled by a great glacier. To the disciplined eye the aspect of the mountains is perfectly conclusive on this point; and in no position can the observer more readily and thoroughly convince himself of this than at the head of Glen Glaster. The dominant hills here are all intensely glaciated.

But the great collecting ground of the glaciers which dammed the glens and produced the parallel roads, were the mountains south and west of Glen Spean. The monarch of these is Ben Nevis, 4,3\%0 feet high. The position of Ben Nevis and his colleagues, in reference to the vaporladen winds of the Atlantic, is a point of the first importance. It is exactly similar to that of Carrantual and the Macgillicuddy Reeks in the southwest of Ireland. These mountains are, and were, the first to encounter the southwestern Atlantic winds, and the precipitation, even at present, in the neighborhood of Killarney, is enormous. 
The winds, robbed of their vapor, and charged with the heat set free by its precipitation, pursue their direction obliquely across Ireland; and the effect of the drying process mav be understood by comparing the rainfall at Cahirciveen with that at Portarlington. As found by Dr. Lloyd, the ratio is as 59 to 21 -fifty-nine inches annually at Cahirciveen to tweuty-one at Portarlington. During the glacial epoch this vapor fell as snow, and the consequence was a system of glaciers which have left traces and evidences of the most impressive character in the region of the Killarney lakes. I hare referred in other places to the great glacier which, descending from the Reeks, moved through the Black Valley, took possession of the lake-basins, and left its traces on every rock and island emergent from the waters of the upper lake. They are all conspicuously glaciated. Not in Switzerland itself do we find clearer traces of ancient glacier action.

What the Macgillicuddy Reeks did in Ireland, Ben Nevis and the adjacent mountains did, and continue to do, in Scotland. We had an example of this on the morning we quitted Roy Bridge. From the bridge westward rain fell copiously, and the roads were wet; but the precipitation ceased near Loch Laggan, whence eastward the roads were dry. Measured by the gauge, the rainfall at Fort William is 86 inches, while at Laggan it is only 46 inches annually. The difference between west and east is forcibly brought out by observations at the two ends of the Caledonian canal. Fort William at the southwestern end has, as just stated, 86 inches, while Culloden, at its northeastern end, has only 24. To the researches of that able and accomplished meteorologist, Mr. Buchan, we are indebted for these and other data of the most interesting and valuable kind.

Adhering to the facts now presented to us, it is not difficult to restore in idea the process by which the glaciers of Lochaber were produced and the glens dammed by ice. When the cold of the glacial epoch began to invade the Scottish hills, the sun at the same time acting with sufficient power upon the tropical ocean, the vapors raised and drifted on to these northern mountains were more and more converted into snow. This slid down the slopes, and from every valley, strath, and corry, south of Glen Spean, glaciers were poured into that glen. The two great factors here 
brought into play are the nutrition of the glaciers by the frozen material above, and their consumption in the milder air below. For a period supply exceeded consumption, and the ice extended, filling Glen Spean to an ever-increasing height, and abutting against the mountains to the north of that glen. But why, it may be asked, should the valleys south of Glen Spean be receptacles of ice at a time when those north of it were receptacles of water? 'The answer is to be found in the position and the greater elevation of the mountains south of Glen Spean. 'They first received the loals of moisture carried by the Atlantic winds, and not until they had been in part dried, and also warmed by the liberation of their latent heat, did these winds touch the hills north of the Glen.

An instructive observation bearing upon this point is here to be noted. Had our visit been in the winter we should have found all the mountains covered; had it been in the summer we should have found the snow all gone. But happily it was at a season when the aspect of the mountains north and south of Glen Spean exhibited their relative powers as snow collectors. Scanning the former hills from many points of view, we were hardly able to detect a fleck of snow, while heavy swaths and patches loaded the latter. Were the glacial epoch to return, the relation indicated by this observation would cause Glen Spean to be filled with glaciers from the south, while the hills and valleys on the north, visited by warmer and drier winds, would remain comparatively free from ice. 'This flow from the south would be reinforced from the west, and as long as the supply was in excess of the consumption the glacier's would extend, the dams which closed the glens increasing in height. By and by supply and consumption becoming approximately equal, the height of the glacier barriers would remain constant. Then, if milder weather set in, consumption would be in excess, a lowering of the barriers and a retreat of the ice being the consequence. But for a long time the conflict between supply and consumption would continue, retarding indefinitely the disappearance of the barriers, and keeping the imprisoned lakes in the norther'n glens. But however slow its retreat, the ice in the long run would be forcel to yield. The dam at the mouth of Glen Roy, which probably entered the glen sufficiently far to block up Glen Glaster, would gradu- 
ally retreat. Glen Glaster and its col being opened, the subsidence of the lake eighty feet, from the level of the highest to that of the second parallel road, would follow as a consequence. I think this the most probable course of things, but it is also possible that Glen Glaster may have been blocked by a glacier from Glen Trieg. 'The ice dan continuing to retreat, at length permitted Glen Roy to commect itself with upper Glen Spean. A continuons lake then filled both the glens, the level of which, as already explained, was determined by the col at Makul, above the head of Loch Laggan. The last to yield was the portion of the glacier which derived nutrition from Ben Nevis, and probably also from the mountains north and south of Loch Arkaig. But it at length yielded, and the waters in the glens resumed the courses which they pursue to-day.

For the removal of the ice barriers no cataclysm is to be invoked; the gradual melting of the dam would produce the entire series of phenomena. In sinking from col to col the water would flow over a gradually melting barrier, the surface of the imprisoned lake not remaining sufficiently long at any particular level to produce a shelf comparable to the parallel roads. By temporary halts in the process of melting due to atmospheric conditions or to the character of the dam itself, or through local softuess in the drift, small pseudo-terraces would be formed which, to the perplexity of some observers, are seen upon the flanks of the glens to-day.

In presence then of the fact that the barriers which stopped these glens to a height, it may be, of 1,500 feet above the bottom of Glen Spean, have dissolved and left not a wreck behind; in presence of the fact, insisted on by Professor Geikie, that barriers of detritus would undoubtedly have been able to maintain themselves had they ever been there; in presence of the fact that great glaciers once most certainly filled these valleys - that the whole region, as provel by $\mathrm{Mr}$. Jamieson, is filled with the traces of their action; the theory which ascribes the parallel roads to lakes dammed by barriers of ice has, in my opinion, a degree of probability on its side which amounts to a practical demonstration of its truth.

Into the details of the terrace formation I do not enter. Mr. Darwin and Mr. Jamieson on the one side, and Sir John Lubbock on the other, deal with true causes The 
terraces, no doubt, are due in part to the descending drift arrested by the water, and in part to the fretting of the wavelets, and the rearrangement of the stirred detritus, along the belts of contact of lake and hill. The descent of matter must have been frequent when the drift was unbound by the rootlets which hold it together now. In some cases, it may be remarked, the visibility of the roads is materially augmented by differences of vegetation. 'The grass upon the terraces is not al ways of the same character as that above and below them, while on heather-covered hills the absence of the dark shrub from the roads greatly enhances their conspicuousuess.

The annexed sketch of a model (p. 173) will enable the reader to grasp the essential features of the problem and its solution. Glen Gluoy and Glen Roy are lateral valleys which open into Glen Spean. Let us suppose Glen Spean filled from $\mathrm{V}$ to $\mathrm{w}$ with ice of a uniform elevation of 1,500 feet above the sea, the ice not filling the upper part of that glen. The ice would thrust itself for some distance up the lateral valleys, closing all their mouths. The streams from the mountains right and left of Glen Gluoy would pour their waters into that glen, forming a lake, the level of which would be deternimed by the height of the col, at A, 1,170 feet above the sea. Over this col the water wonld flow into Glen Roy. But in Glen Roy it could not rise higher than 1,150 feet, the height of the col at $\mathbf{B}$, over which it would flow into Glen Spey.

'The water halting at these lavels for a sufficient time, would form the single road in Glen Gluoy and the highest road in Glen Roy. 'This state of things would continue as loug as the ice dam was sufficiently high to dominate the cols at $\mathrm{A}$ and $\mathrm{B}$; but when throngh change of climate the gradually sinking dam reached, in succession, the levels of thesecols, the water would then begin to flow over the dam instead of over the cols. Let us suppose the wasting of the ice to continue until a connection was established between Glen Roy and Glen Glaster, a common lake would then fill both these glens, the level of which would be determined by that of the col c, over which the water would pour for an indefinite period into Glen Spean. During this period the second Glen Roy road and the highest, road of Glen Glaster would be formed. 'The ice subsiding still further, a connection would eventually be 
established between Glen Roy, Glen Glaster, and the upper part of Glen Spean. A common lake would fill all three glens, the level of which would be that of the col D, over which for an indefinite period the lake would pour its water. During this period the lowest Glen Roy road, which is common also to Glen Glaster and Glen Spean, would be formed. Finally, on the disappearance of the ice from the lower part of Glen Spean the waters would flow down their respective valleys as they do to-day.

Reviewing our work, we find three considerable steps to have marked the solution of the problem of the Parallel Roads of Glen Roy. 'The first of these was taken by Sir Thomas Dick-Lauder, the second was the pregnant conception of Agassiz regarding glacier action, and the third was the testing and verification of this conception by the very thorongh researches of Mr. Jamieson. No circumstance or incident connected with this discourse gives me greater pleasure than the recognition of the value of these researches. They are marked throughout by unflagging industry, by novelty and acuteness of observation, and by reasoning power of a high and varied kind. These pages had been returned "for press" when I learned that the relation of Ben Nevis and his colleagnes to the vapor-laden winds of the Atlantic had not escaped Mr. Janieson. To him obviously the exploration of Lochaber, and the development of the theory of the Parallel Roads, has been a labor of love.

Thus ends our rapid survey of this brief episode in the physical history of the Scottish hills-brief, that is to say, in comparison with the immeasurable lapses of time through which, to produce its varied structure and appearances, our planet must have passed. In the survey of such a field two things are specially worthy to be taken into account-the widening of the intellectual horizon and the reaction of expanding knowledge upon the intellectual organ itself. At first, as in the case of ancient glaciers, through sheer want of capacity, the mind refuses to take in revealed facts. But by degrees the steady contemplation of these facts so strengthens and expanis the intellectual powers, that where truth once could not find an entrance it eventually finds a home.*

* The formation, connection, successive subsidence, and final disappearance of the glacial lakes of Lochaber were illustrated in the 
THE PARALLEL ROADS OW GLEN ROY.

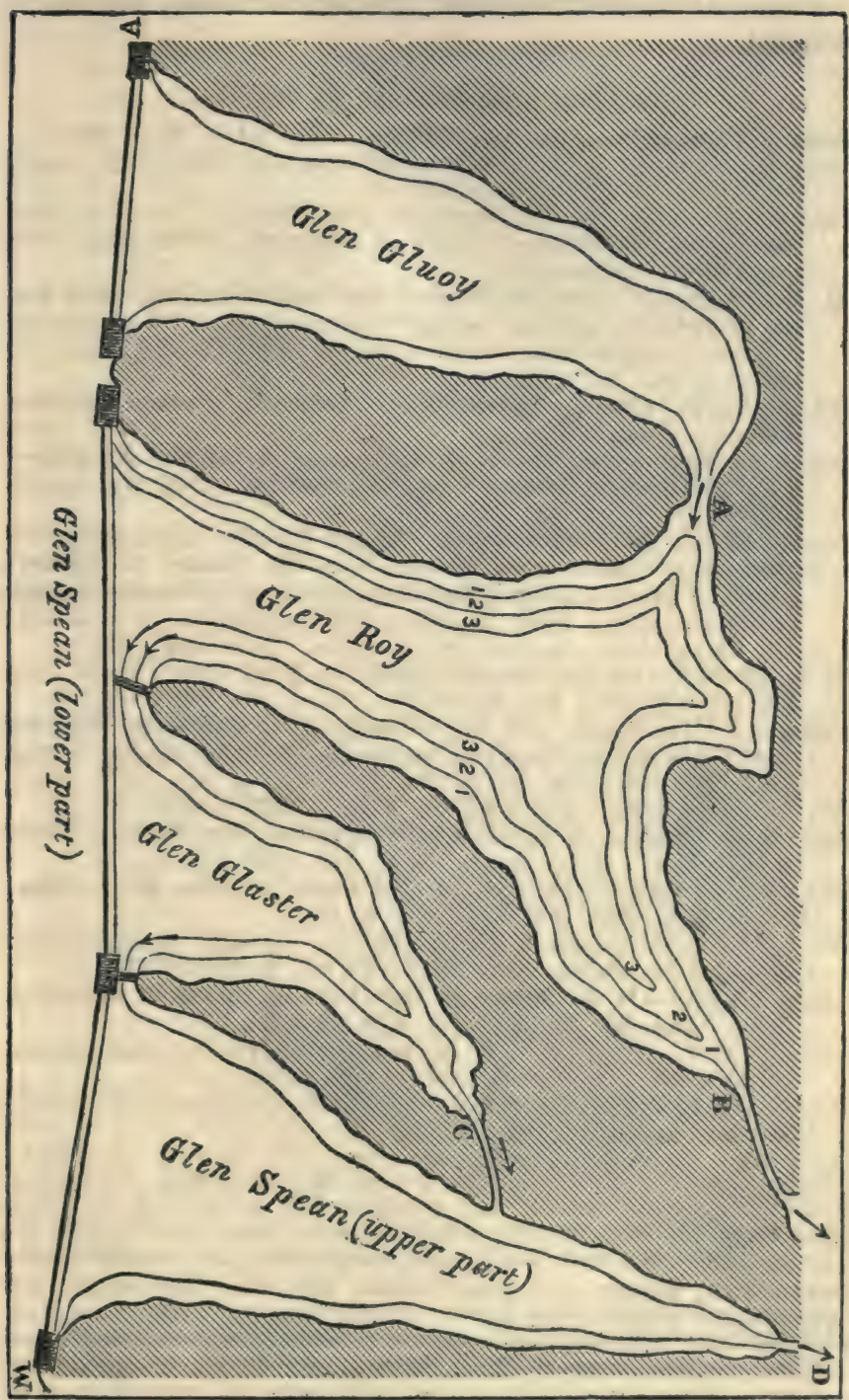


A map of the district, with the parallel roads shown, is annexed.

\section{LITERATURE OF THE SUBJECT.}

Thomas Pennant.-A tour in Scotland. Vol. iii. 1776, p. 394. John MacCuldoch.-On the Parallel Roads of Glen Roy. Geol. Soc. Trans. vol. iv. 1817 , p. 314.

'Thomas Lauder Dick (afterward Sir Thomas Dick-Lauder, Bart.).-On the Parallel Roads of Lochaber. Edin. Roy. Soc. Trans. 1818, vol. ix. p. 1.

Charles Darwin.-Observations on the Parallel Roads of Glen Roy, and of the other parts of Lochaber in Scotland, with an attempt to prove that they are of marine origin. Phil. Trans. 1839, vol. cxxix. p. 39.

Sir Charles Lxell.-Elements of Geology. Second edition, 1841.

Lovis AgAssIz. - The Glacial Theory and its Recent ProgressParallel Terraces. Edin. New Phil. Journal, 1842, vol. xxxiii. p. 236.

David Milne (afterward David Milne-Home).-On the Parallel Roads of Lochaber; with Remarks on the Change of Relative Levels of Sea and Land in Scotland, and on the Detrital Deposits in that Country. Edin. Roy. Soc. Trans. 1847, vol. xvi. p. 395.

Robert Chambers.-Ancient Sea Margins. Edinburgh, 1848.

H. D. Rogens. - On the Parallel Roads of Glen Roy. Royal Inst. Proceedings, 1861, vol. iii. p. 341.

Thomas F. Jamieson. - On the Parallel Roads of Glen Roy, and their Place in the History of the Glacial Period. Quart. Journal Geol. Soc. 1863 , vol. xix. p. 235.

Sir Charles Lyell. - Antiquity of Man. 1863, p. 253.

Rev. R. B. WAtson. - On the Marine Origin of the Parallel Roads of Glen Roy. Quart. Journ. Geol. Soc. 1865, vol. xxii. p. 9.

Sir John Lubbock.-On the Parallel Roads of Glen Roy. Quart. Journ. Geol. Soc. 1867 , vol. xxiv. p. 83.

Charles Babbage.-Observations on the Parallel Roads of Glen Roy. Quart. Journ. Geol. Soc. 1868, vol. xxiv. p. 273.

JaMes Nicol. - On the origin of the Parallel Roads of Glen Roy. 1869. Geol. Soc. Journal, vol. Xxv. p. 282.

JAMES NiCOL. - How the Parallel Roads of Glen Roy were formed. 1872. Geol. Soc. Journal, vol. xxviii. p. 237.

Major-General Sir Henry James, R. E.-Notes on the Parallel Roads of Lochaber. 4to. 1874.

discourse here reported by the model just described, constructed under the supervision of $\mathrm{my}$ assistant, Mr. John Cottrell. Glen Gluoy with its lake and road and the eataract over its col; Glen Roy and its three roads with their respective cataracts at the bead of Glen Spey, Glen (xlaster, and Glen Spean, were all represented. The successive shiftings of the barriers, which were formed of plate glass, brought each successive lake and its corresponding road into view, while the entire removal of the barriers caused the streams to flow down the glens of the modelas they flow down the real glens of today. 


\section{CHAPTER IX.}

\section{ALPINE SCULPTURE.}

\section{4.}

To ACcount for the conformation of the Alps, two hypotheses have been advanced, which may be respectively named the hypothesis of fracture and the hypothesis of erosion. 'The former assumes that the forces by which the mountains were elevated produced fissures in the earth's crust, and that the valleys of the Alps are the tracks of these fissures; while the latter maintains that the valleys have been cut out by the action of ice and water, the mountains themselves being the residual forms of this grand sculpture. I had heard the Via Mala cited as a conspicuous illustration of the fissure theory-the profound chasm thus named, and through which the HinterRhein now flows, could, it was alleged, be nothing else than a crack in the earth's crust. 'To the Via Mala I therefore went in 1864 to instruct myself upon the point in question.

The gorge commences about a quarter of an hour ahove Tusis; and, on entering it, the first impression certainly is that it must be a fissure. This conclusion in my case was modified as I advanced. Some distance up the gorge I found upon the slopes to my right quantities of rolled stones, evidently rounded by water-action. Still further up, and just before reaching the first bridge which spans the chasm, I found more rolled stones, associated with sand and gravel. Through this mass of detritus, fortunately, a vertical cutting had been made, which exhibited a section showing perfect stratification. 'There was no agency in the place to roll these stones, and to deposic these alternating layers of sand and pebbles, but the river which now rushes some hundreds of feet below them. At one period of the Via Mala's history the river must have run at this high level. Other evidences of water-action soon revealed themselves. From the parapet of the first bridge I could see the solid rock 200 feet above the bed of the river scooped and eroded.

It is staterl in the guille-books that the river, which usually runs along the bottom of the gorge, has been known almost to fill it during violent thunder-storms; and it may 
be urged that the marks of erosion which the sides of the chasm exhibit are due to those occasional floods. In reply to this, - it may be stated that even the existence of such floods is not well authenticated, and that if the supposition were trie, it would be an additional argument in favor of the cutting power of the river. For if floods operating at rare intervals could thus erode the rock, the same agency, acting without ceasing upon the river's bed, must certainly be competent to excavate it.

I proceeded upward, and frum a point near another bridge (which of them I did not note) had a fine view of a portion of the gorge. 'The river here runs at the bottom of a cleft of profound depth, but so narrow that it might be leaped across. That this cleft must be a crack is the impression first produced; but a brief inspection suffices to prove that it has been cut by the river. From top to bottom we have the unmistakable marks of erosion. This cleft was best seen on looking downward from a point near the bridge; but looking upward from the bridge itself, the evidence of aqueous erosion was equally convincing.

The character of the erosion depends upon the rock as well as upon the river. The action of water upon some rocks is almost purely mechanical; they are simply ground away or detached in sensible masses. Water, however, in passing over limestone, charges itself with carbonate of lime without damage to its transparency; the rock is dissolved in the water; and the gorges cut by water in such rocks often resemble those cut in the ice of glaciers by glacier streams. To the solubility of limestone is probably to be ascribed the fantastic forms which peaks of this rock usually assume and also the grottos and caverns which interpenetrate limestone formations. A rock capable of being thus dissolved will expose a smooth surface after the water has quitted it; and in the case of the Via Mala it is the polish of the surfaces and the curved hollows scooped in the sides of the gorge, which assure us that the chasm has been the work of the river.

About four miles from Tusis, and not far from the little village of Zillis, the Via Mala opens into a plain bounded by high terraces. It occurred to me the moment I saw it that the plain had been the bed of an ancient lake; and a farmer, who was ny temporary companion, immediately 
informed me that such was the tradition of the neighborhood. This man conversed with intelligence, and as I drew his attention to the rolled stones, which rest not only above the river, but above the road, and inferred that the river must once have been there to have rolled those stones, he saw the force of the evidence perfectly. In fact, in former times, and subsequent to the retreat of the great glaciers, a rocky barrier crossed the valley at this place, damming the river which came from the mountains higher up. A lake was thus formed which poured its waters over the barrier. Two actions were here at work, both tending to obliterate the lake-the raising of its bed by the deposition of detritus, and the cutting of its dam by the river. In process of time the cut deepened into the Via Mala; the lake was drained, and the river now flows in a definite channel through the plain which its waters once totally covered.

From Tusis I crossed to Tiefenkasten by the Schien Pass, and thence over the Julier Pass to Pontresina. 'There are three or four ancient lake-beds between 'Tiefenkasten and the summit of the Julier. They are all of the same type-a more or less broad and level valley-bottom, with a barrier in front through which the river has cut a passage, the drainage of the lake being the consequence. 'These lakes were sometimes dammed by barrier's of rock, sometimes by the moraines of ancient glaciers.

An example of this latter kind occurs in the Rosegg valley, about twenty minutes below the end of the Rosegg glacier, and about an hour from Pontresina. The valley here is crossed by a pine-covered moraine of the noblest dimensions; in the neighborhood of London it might be called a mountain. 'That it is a moraine, the inspection of it from a point on the Surlei slopes above it will convince any person possessing an educated eye. Where, moreover, the interior of the mound is exposed, it exhibits morainematter-detritus pulverized by the ice, with boulders entangled in it. It stretched quite across the valley, and at one time dammed the river up. But now the barrier is cut through, the stream having about one-fourth of the moraine to its right, and the remaining three-fourths to its left. Other moraines of a more resisting character hold their ground as barriers to the present day. In the Val di Campo, for example, about three-quarters of an hour from 
Pisciadello, there is a moraine composed of large boulders, which interrupt the course of a river and compel the water to fall over them in cascades. They have in great part resisted its action since the retreat of the ancient glacier which formed the moraine. Behind the moraine is a lakebed, now converted into a level meadow, which rests on a deep layer of mold.

At Pontresina a very fine and instructive gorge is to be seen. 'The river from the Morteratsch glacier rushes through a deep and narrow chasm which is spanned at one place by a stone bridge. The rock is not of a character to preserve smooth polishing; but the larger features of water-action are perfectly evirent from top to bottom. 'Those features are in part visible from the bridge, but still better from a point a little disiance from the bridge in the direction of the upper village of Pontresina. The hollowing out of the rock by the eddies of the water is here quite manifest. A few minutes' walk upward brings us to the end of the gorge; and behind it we have the usual indications of an ancient lake, and terraces of distinct water origin. From this position indeed the genesis of the gorge is clearly revealed. After the retreat of the ancient glacier, a transverse ridge of comparatively resisting material crossed the valley at this place. Over the lowest part, of this ridge the river flowed, rushing steeply down to join at the bottom of the slope the stream which issued from the Rosegg glacier. On this incline the water became a powerful eroding agent, and finally cut the channel to its present depth.

Geological writers of reputation assume at this place the existence of a fissure, the "washing out" of which resulted in the formation of the gorge. Now no examination of the bed of the river ever proved the existence of this fissure; and it is certain that water, particularly when charged with solid matter in suspension, can cut a channel through unfissured rock. Cases of deep cutting can be pointed out where the clean bed of the stream is exposed, the rock which forms the floor of the river not exhibiting a trace of fissure. An example of this kind on a small scale occurs near the Bernina Gasthans, about two hours from Pontresina. A little way below the junction of the two streams from the Bernina Pass and the Heuthal the river flows through a channel cut by itself, and 20 or 30 feet in 
depth. At some places the river-bed is covered with rolled stones; at other places it is bare, but shows no trace of fissure. 'The abstract power of water, if I may use the term, to cut through rock is demonstrated by such instances. But if water be competent to form a gorge without the aid of a fissure, why assume the existence of such fissures in cases like that at Pontresina? It seems far more philosophical to accept the simple and impressive history written on the walls of those gorges by the agent which produced them.

Numerous cases might be pointed out, rarying in magnitude, but all identical in kind, of barriers which crossed valleys and formed lakes having been eut through by rivers, narrow gorges being the consequence. One of the most famous examples of this kind is the Finsteraarschlucht in the valley of Hasli. Here the ridge called the Kirchet seems split across, and the river Aar rushes through the fissure. Behind the barrier we have the meadows and pastures of Imhof resting on the sediment of an ancient lake. Were this an isolated case, one night with an apparent show of reason conclude that the Finsteraarschlucht was produced by an earthquake, as some suppose it to have been; but when we find it to be a single sample of actions which are frequent in the Alps-when probably a hundred cases of the same kind, though different in magnitude, can be pointed out-it seems quite unphilosophical to assume that in each particular case an earthquake was at hand to form a channel for the river. As in the case of the barrier at Pontresina, the Kirchet, after the retreat of the Aar glacier, dammed the waters flowing from it, thus forming a lake, on the bed of which now stands the village of Imhof. Over this barrier the Aar tumbled toward Meyringen, cutting, as the centuries passed, its bed ever deeper, until finally it became deep enough to drain the lake, leaving in its place the alluvial plain, through which the river now flows in a definite channel.

In 1866 I subjected the Finsteraarschlucht to a close examination. The earthquake theory already adverted to was then prevalent regarding it, and I wished to see whether any evidences existed of aqueous erosion. Near the summit of the Kirchet is a signbosrl inviting the traveler to visit the Aarenschlucht, a narrow lateral gorge 
which runs down to the very bottom of the principal one. 'The aspect of this smaller chasm from bottom to top proves to demonstration that water had in former ages heen there at work. It is scooped, rounded, and polished, so as to render palpable to the most careless eye that it is a gorge of erosion. But it was regarding the sides of the great chasm that instruction was needed, and from its erlge nothing to satisfy me could be seen. I therefore stripped and waded into the river until a point was reached which commanded an excellent view of both sides of the gorge. 'The water was cutting cold, but I was repaid. Below me on the left-hand side was a jutting cliff which bore the thrust of the river and caused the Aar to swerve from its direct course. From top to bottom this cliff was polished, rounded and scooped. There was no room for doubt. The river which now runs so deeply down had once been above. It has been the delver of its own channel through the barrier of the Kirchet.

But the broad view taken by the advocates of the fracture theory is, that the valleys themselves follow the tracks of primeral fissures produced by the uphearal of the land, the cracks across the barriers referred to being in reality portions of the great cracks which formed the valleys. Such an argument, however, would virtually concede the theory of erosion as applied to the valleys of the Alps. The narrow gorges, often not more than twenty or thirty feet across, sometimes eren narrower, frequently occur at the bottom of broad valleys. Such fissures might enter into the list of accidents which gave direction to the real erosive agents which scooped the valley out; but the formation of the valley, as it now exists, could no more be ascribed to such cracks than the motion of the railway train could be ascribed to the finger of the engineer which turns on the steam.

These deep gorges ocenr, I believe, for the most part in limestone strata; and the effects which the merest driblet of water can produce on limestone are quite astonishing. It is not uncommon to meet chasms of considerable depth produced by small streams the beds of which are dry for a large portion of the year. Right and left of the larger gorges such secondary chasms are often found. The idea of time must, I think, be more and more included in our reasonings on these phenomena. Happily, the marks 
which the rivers have, in most cases, left behind them, and which refer, geologically considered, to actions oi yesterday, give us ground and courage to conceive what may be effected in geologic periods. 'Thus the modern portion of the Via Mala throws light upon the whole. Near Bergün, in the valley of the Albula, there is also a little Via Mala, which is not less significant than the great one. The river flows here through a profound limestone gorge, and to the very edges of the gorge we have the evidences of erosion. But the most striking illustration of water-action upon limestone rock that I have ever seen is the gorge at Pfäffers. Here the traveler passes along the side of the chasm midway between top and bottom. Whichever way he looks, backward or forward, upward or downward, toward the sky or toward the river, he meets 'everywhere the irresistible and impressive evidence that this wonderful fissure has been sawn through the mountain by the waters of the Tamina.

I have thus far confined myself to the consideration of the gorges formed by the cutting through of the rock-barriers which frequently cross the valleys of the Alps; as far as they have been examined by me they are the work of erosion. But the larger question still remains, To what action are we to ascribe the formation of the valleys themselves? This question includes that of the formation of the mountain-ridges, for were the valleys wholly filled, the ridges would disappear. Possibly no answer can be given to this question which is not beset with more or less of difficulty. Special localities might be found which would seem to contradict every solution which refers the conformation of the Alps to the operation of a single cause.

Still the Alps present features of a character sufficiently definite to bring the question of their origin within the sphere of close reasoning. That they were in whole or in part once beneath the sea will not be disputed; for they are in great part composed of sedimentary rocks which required a sea to form them. Their present elevation above the sea is due to one of those local changes in the shape of the earth which have been of frequent occurrence throughout geologic time, in some cases depressing the land, and in others causing the sea-bottom to protrude beyond its surface. Considering the inelastic character of its materials, the protuberance of the Alps could hardly have been pushed 
out without dislocation and fracture; and this conclusion gains in probability when we consider the foldings, contortions, and even reversals in position of the strata in many parts of the Alps. Such changes in the position of beds which were once horizontal could not have been effected without dislocation. Fissures would be produced by these chinges; and such fissures, the adrocates of the fracture theory contend, mark the positions of the valleys of the Alps.

Imagination is necessary to the man of science, and we could not reason on our present subject without the power of presenting mentally a picture of the earth's crust cracked and fissured by the forces which produced its upheaval. Imagination, however, must be strictly checked by reason and by observation. That fractures occurred cannot, I think, be doubted, but that the valleys of the Alps are thus formed is a conclusion not at all involved in the admission of dislocations. I never met with a precise statement of the manner in which the advocates of the fissure theory suppose the forces to have acted-whether they assume a general elevation of the region, or a local elevation of distinct ridges; or whether they assume local subsidences after a general elevation, or whether they would superpose upon the general upheaval minor and local upheavals.

In the absence of any distinct statement, I will assume the elevation to be general - that a swelling out of the earth's crust occurred here, sufficient to place the most prominent portions of the protuberance three miles above the sea-level. To fix the ideas, let us consider a circular portion of the crust, say one hundred miles in diameter, and let us suppose, in the first instance, the circumference of this circle to remain fixed, and that the elevation was confined to thespace within it. The upheaval would throw the crust into a state of strain; and, if it were inflexible, the strain must be relieved by fracture. Crevasses would thus intersect the crust. Let us now inquire what proportion the area of these open fissures is likely to bear to the unfissured crust. An approximate answer is all that is here required; for the problem is of such a character as to render minute precision unnecessary.

No one, I think, would affirm that the area of the fissures would be one-hundredth the area of the land. For 
let us consider the strain upon a single line drawn over the summit of the protuberance from a point on its rim to a point opposite. Regarding the protuberance as a spherical swelling, the length of the arc corresponiling to a chord of 100 miles and a versed sine of three miles is 100.24 miles; consequently the surface to reach its new position must stretch 0.24 of a mile, or be broken. A fissure or a number of cracks with this total width would relieve the strain; that is to say, the sum of the widths of all the cracks over the length of 100 miles would be 420 yards. If instead of comparing the willth of the fissures with the length of the lines of tension, we compared their areas with the area of the unfissured land, we should of course find the proportion much less. These considerations will help the imagination to realize what a small ratio the area of the open fissures must bear to the unfissured crust. They enable us to say, for example, that to assume the area of the fissures to be one-tenth of the area of the land would be quite absurd, while that the area of the fissures could be onehalf or more than one-half that of the land would be in a proportionate degree unthinkable. If we suppose the elevation to be due to the shrinking or subsidence of the land all round our assumed circle, we arrive equally at the conclusion that the area of the open fissures would be altogether insiguificant as compared with that of the unfissured crust.

To those who have seen them from a commanding elevation, it is needless to say that the Alps themselves bear no sort of resemblance to the picture which this theory presents to us. Instead of deep cracks with approximately vertical walls, we have ridges running into peaks, and gradually sloping to form valleys. Instead of a fissured crust, we have a state of things closely resembling the surface of the ocean when agitated by a storm. The valleys, instead of being much narrower than the ridges, occupy the greater space. A plaster cast of the Alps turned upside down, so as to invert the elevations and depressions, would exhibit blunter and broader mountains, with narrower valleys between them, than the present ones. The valleys that exist cannot, I think, with any correctness of language be called fissures. It may be urged that they originated in fissures: but even this is unproved, and, were it proved, the fissures would still play the subordinate part 
of giving direction to the agents which are to be regarded as the real sculptors of the Alps.

The fracture theory, then, if it regards the elevation of the Alps as due to the operation of a force acting throughout the entire region, is, in my opinion, utterly incompetent to account for the conformation of the country. If, on the other hand, we are compelled to resort to local disturbances, the manipulation of the earth's crust necessary to obtain the valleys and the mountains will, I imagine, bring the difficulties of the theory into very strong relief. Indeed an examination of the region from many of the more accessible eminences-from the Galenstock, the Grauhaupt, the Pitz Languard, the Monte Confinale-or, better still, from Mont Blanc, Monte Rosa, the Jungfrau, the Finsteraarhorn, the Weisshorn, or the Matterhorn, where local peculiarities are toned down, and the operations of the powers which really made this region what it is are alone brought into prominencemust, I imagine, convince every physical geologist of the inability of any fracture theory to account for the present conformation of the Alps.

A correct model of the mountains, with an nnexaggerated vertical scale, produces the same effect upon the mind as the prospect from one of the highest peaks. We are apt to be influenced by local phenomena which, though insignificant in view of the general question of Alpine conformation, are, with reference to our customary standards, vast and impressive. In a true model those local peculiarities disappear; for on the scale of a model they are too small to be visible; while the essential facts and forms are presented to the undistracted attention.

A minute analysis of the phenomena strengthens the conviction which the general aspect of the Alps fixes in the mind. We find, for example, numerous valleys which the most ardent plutonist would not think of ascribing to any other agency than erosion. That such is their genesis and history is as certain as that erosion produced the Chines in the Isle of Wight. From these indubitable cases of erosion -commencing, if necessary, with the small ravines which run down the flanks of the ridges, with their little working navigators at their bottoms - we can proceed, by almost insensible gradations, to the largest valleys of the Alps; and it would perplex the plutonist to fix upon the point at which fracture begius to play a material part. 
In ascending one of the larger valleys, we enter it where it is wide and where the eminences are gentle on either side. The flanking mountains become higher and nore abrupt as we ascend, and at length we reach a place where the depth of the valley is a maximum. Continuing our walk upward, we find ourselves flanked by gentler slopes, and finally emerge from the valley and reach the summit of an open col, or depression in the chain of mountains. This is the common character of the large valleys. Crossing the col, we descend alnng the opposite slope of the chain, and through the same series of appearances in the reverse order. If the valleys on both sides of the col were produced by fissures, what prevents the fissure from prolonging itself across the col? 'The case here cited is representative; and I am not acquainted with a single instance in the Alps where the chain has been cracked in the manner indicated. 'The cols are simply depressions, in many of which the unfissured rock can be traced from side to side.

The typical instance just sketched follows as a natural consequence from the theory of erosion. Before either ice or water can exert great power as an erosive agent, it must collect in sufficient mass. On the higher slopes and plateaus-in the region of cols-the power is not fully developed; but lower down tributaries unite, erosion is carried on with increased vigor, and the excavation gradually reaches a maximum. Lower still the elevations diminish and the slopes become more gentle; the cutting power gradually relaxes, until finally the eroding agent quits the mountains altogether, and the grand effects which it produced in the earlier portions of its course entirely disappear.

I have hitherto confined myself to the consideration of the broad question of the erosion theory as compared with the fracture theory; and all that I have been able to observe and think with reference to the subject leads me to adopt the formel. Under the term erosion I include the action of water, of ice, and of the atmosphere, including frost and rain. Water and ice, however, are the principal agents, and which of these two has produced the greatest effect it is perhaps impossible to say. 'T'wo years ago I wrote a brief note "On the Conformation of the Alps," *

* Phil. Mag. vol. xxiv. p. 169. 
in which I ascribed the paramount influence to glaciers. The facts on which that opinion was founded are, I think, unassailable; but whether the conclusion then announced fairly follows from the facts is, I confess, an open question.

The arguments which have been thus far urged against the conclusion are not convincing. Indeed, the idea of glacier erosion appears so daring to some minds that its boldness alone is deemed its sufficient refutation. It is, however, to be remembered that a precisely similar position was taken up by many excellent workers when the question of ancient glacier extension was first mooted. The idea was considered too hardy to be entertained; and the evidences of glacial action were sought to be explained by reference to almost any process rather than the true one. Let those who so wisely took the side of " boldness" in that discussion beware lest they place themselves, with reference to the question of glacier erosion, in the position formerly occupied by their opponents.

Looking at the little glaciers of the present day-mere pygmies as compared to the giants of the glacial epochwe find that from every one of them issues a river more or less voluminous, charged with the matter which the ice has rubbed from the rocks. Where the rocks are soft, the amount of this finely pulverized matter suspended in the water is very great. The water, for example, of the river which flows from Santa Catarina to Bormio is thick with it. The Rhine is charged with this matter, and by it has so silted up the Lake of Constance as to abolish it for a large fraction of its length. The Rhone is charged with it, and tens of thousands of acres of cultivable land are formed by the silt above the Lake of Geneva.

In the case of every glacier we have two agents at work - the ice exerting a crushing force on every point of its bed which bears its weight, and either rasping this point into powder or tearing it bodily from the rock to which it belongs; while the water which everywhere circulates upon the bed of the glacier continually washes the detritus away and leaves the rock clean for further abrasion Confining the action of glacier's to the simple rubbing away of the rocks, and allowing them sufficient time to act, it is not a matter of opinion, but a physical certainty, that they will 
scoop out valleys. But the glacier does more than abrade. Rocks are not homogeneous; they are intersected by joints and places of weakness, which divide them into virtually detached masses. A glacier is undoubtedly competent to root such masses bodily away. Indeed the mere à priori consideration of the subject proves the competence of a glacier to deepen its bed. Taking the case of a glacier 1,000 feet deep (and some of the older ones were probably three times this depth), and allowing 40 feet of ice to an atmosphere, we find that on every square inch of its bed such a glacier presses with a weight of $375 \mathrm{lbs}$., and on every square yard of its bed with a weight of 486,000 lbs. With a vertical pressure of this amount the glacier is urged down its valley by the pressure from behind. We can hardly, I think, deny to such a tool a power of excavation.

The retardation of a glacier by its bed has been referred to as proving its impotence as an erosive agent: but this very retardation is in some measure an expression of the magnitude of the erosive energy. Either the bed must give way, or the ice must slide over itself. We get indeed some idea of the crushing pressure which the moving glacier exercises against its bed from the fact that the resistance, and the effort to overcome it, are such as to make the upper layers of a glacier move bodily over the lower ones-a portion ouly of the total motion being due to the progress of the entire mass of the glacier down its valley.

The sudden bend in the valley of the Rhone at Martigny has also been regarded as conclusive evidence against the theory of erosion. "Why," it has been asked, "did not the glacier of the Rhone go straight forward instead of making this awkward bend?" But if the valley be a crack, why did the crack make this bend? The crack, I submit, had at least as much reason to prolong itself in a straight line as the glacier had. A statement of Sir Jolın Herschel with reference to another matter is perfectly applicable here: "A crack once produced has a tendency to run-for this plain reason, that at its momentary limit, at the point at which it has just arrived, the divellent force on the molecules there situated is counteracted only by half of the cohesive force which acted when there was no crack, viz. , the cohesion of the uncracked portion alone" 
("Proc. Roy. Soc." vol. xii. p. 678). To account, then, for the bend, the altherent of the fracture theory must assume the existence of some accident which turned the crack at right angles to itself; and he surely will permit the adherent of the erosion theory to make a similar assumption.

The influence of small accidents on the direction of rivers is beautifully illustrated in glacier streams, which are made to cut either straight or sinuons channels by causes apparently of the most trivial character. In his interesting paper "On the Lakes of Switzerland," M. Studer also refers to the bend of the Rhine at Sargans in proof that the river must there follow a pre-existing fissure. I made a special expedition to the place in 1864; and though it was plain that M. Studer had good grounds for the selection of this spot, I was unable to arrive at his conclusion as to the necessity of a fissure.

Again, in the interesting volume recently published by the Swiss Alpine Club, M. Desor informs us that the Swiss naturalists who met last year at Samaden visited the end of the Morteratsch glacier, and there convinced themselves that a glacier had no tendency whatever to imbed itself in the soil. I scarcely think that the question of glacier erosion, as applied either to lakes or valleys, is to be disposed of so easily. Let me record here my experience of the Morteratsch glacier. I took with me in 1864 a theodolite to Pontresina, and while there had to congratulate myself on the aid of my friend Mr. Hirst, who in $185 \%$ did such good service upon the Mer de Glace and its tributaries. We set out three lines across the Morteratsch glacier, one of which crossed the ice-stream near the wellknown hut of the painter Georgei, while the two others were staked out, the one above the hut and the other below it. Calling the highest line $\mathrm{A}$, the line which crossed the glacier at the hut B, and the lowest line $\mathrm{C}$, the following are the mean hourly motions of the three lines, deduced from nbservations which extended over several days. On each line eleven. stakes were fixed, which are designated by the figures $1,2,3$, etc. in the tables.

\section{Morteratsch Glacier, Line A.}

No. of Stake.

Hourly Motion.

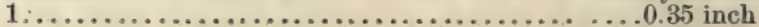

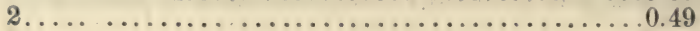

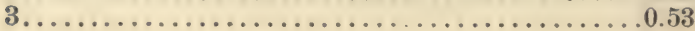


4.

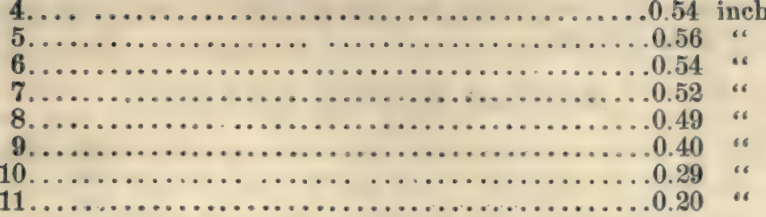

As in all other measurements of this kind, the retarding influence of the sides of the glacier is manifest: the center moves with the greatest velocity.

\section{Morteratsch Glacier, Line $B$.}

No. of Stake.

Hourly Motion.

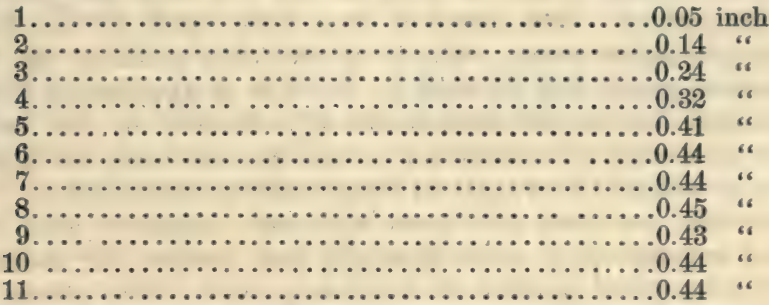

The first stake of this line was quite close to the edge of the glacier, and the ice was thin at the place, hence its slow motion. Crevasses prevented us from carrying the line sufficiently far across to render the retardation of the further side of the glacier fully evident.

\section{Morteratsch Glacier, Line $C$.}

No. of Stake.

Hourly Motion.

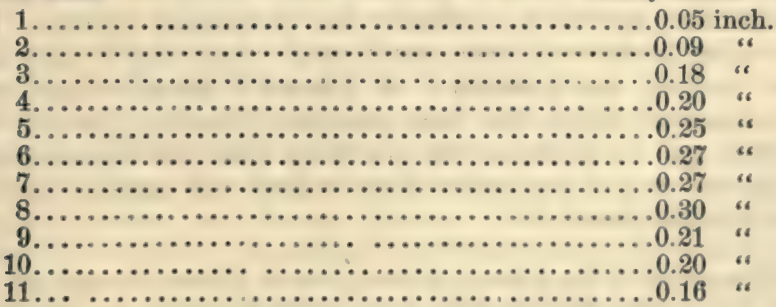

Comparing the three lines together, it will be observed that the velocity diminishes as we descend the glacier. In 
100 hours the maximum motion of the three lines respectively is as follows:

\section{Maximum Motion in 100 hours.}

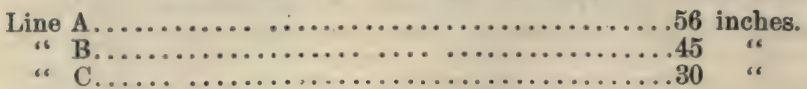

This deportment explains an appearance which must strike every observer who looks upon the Morteratsch from the Piz Languard, or from the new Bernina road. A metlial moraine runs along the glacier, commencing as a narrow streak, but toward the end the moraine extends in width, until finally it quite covers the terminal portion of the glacier. The cause of this is revealed by the foregoing measurements, which prove that a stone on the moraine where it is crossed by the line A approaches a seconil stone on the moraine where it is crossed by the line $\mathrm{C}$ with a velocity of twenty-six inches per one hundred hours. 'T'he moraine is in a state of longitudinal compression. Its materials are more and more squeezed together, and they must consequently move laterally and render the moraine at the terminal portion of the glacier wider than above.

'The motion of the Morteratsch glacier, then, diminishes as we descend. The maximum motion of the third line is thirty inches in one hundred hours, or seven inches a day - a very slow motion; and had we run a line nearer to the end of the glacier, the motion would have been slower still. At the end itself it is nearly insensible.* Now I submit that this is not the place to seek for the scooping power of a glacier. The opinion appears to be prevalent that it is the snout of a glacier that must act the part of plowshare; and it is certainly an erroneuus opinion. The scooping power will exert itself most where the weight and the motion are greatest. A glacier's snout often rests upon matter which has been scooped from the glacier's bed higher up. I therefore do not think that the inspection of what the end of a glacier does or does not accomplish can decide this question.

* The snout of the Aletsch Glacier has a diurnal :motion of less than two inches, while a mile or so above the snout the velocity is eighteen inches. The spreading out of the moraine is here very striking. 
The snout of a glacier is potent to remove anything against which it can fairly abut; and this power, notwithstanding the slowness of the motion, manifests itself at the end of the Morteratsch glacier. A hillock, bearing pine trees, was in front of the glacier when Mr. Hirst and myself inspected its end; and this hillock is being bodily removed by the thrust of the ice. Several of the trees are overturned; and in a few years, if the glacier continues its reputed advance, the mound will certainly be plowed away.

The question of Alpine conformation stands, I think, thus: We have, in the first place, great valleys, such as those of the Rhine and the Rhone, which we might conveniently call valleys of the first order. The mountains which flank these main valleys are also cut by lateral valleys running into the main ones, and which may be called valleys of the second order. When these latter are examined, smaller valleys are found running into them, which may be called valleys of the third order. Smaller ravines and depressions, again, join the latter, which may be called valleys of the fourth order, and so on until we reach streaks and cuttings so minute as not to merit the name of valleys at all. At the bottom of every valley we have a stream, diminishing in magnitude as the order of the valley ascends, carving the earth and carrying its materials to lower levels. We find that the larger valleys have been filled for untold ages by glaciers of enormous dimensions, always moving, grinding down and tearing away the rocks over which they passed. We have, moreover, on the plains at the feet of the mountains, and in enormous quantities, the very matter derived from the sculpture of the mountains themselves.

The plains of Italy and Switzerland are cumbered by the débris of the Alps. The lower, wider, and more level valleys are also filled to unknown depths with the materials derived from the higher ones. In the vast quantities of moraine-matter which cumber many even of the higher valleys we have also suggestions as to the magnitude of the erosion which has taken place. This moraine-matter, moreover, can only in small part have been derived from the falling of rocks upon the ancient glacier; it is in great part derived from the grinding and the plowing-out of the glacier itself. This accounts for the maguitude of 
many of the ancient moraines, which date from a period when almost all the mountains were covered with ice and snow, and when, consequently, the quantity of morainematter derived from the naked crests cannot have been considerable.

The erosion theory ascribes the formation of Alpine valleys to the agencies here briefly referred to. It invokes nothing but true causes. Its artificers are still there, though, it may be, in diminished strength; and if they are granted sufficient time, it is demonstrable that they are competent to produce the effects ascribed to them. And what does the fracture theory offer in comparison? From no possible application of this theory, pure and simple, can we obtain the slopes and forms of the mountains. Erosion must in the long run be invoked, and its power therefore conceded. The fracture theory infer's from the disturbances of the Alps the existence of fissures: and this is a probable inference. But that they were of a magnitude sufficient to produce the conformation of the Alps, and that they followed, as the Alpine valleys do, the lines of natural drainage of the country, are assumptions which do not appear to me to be justified either by reason or by observation.

There is a grandeur in the secular integration of small effects implied by the theory of erosion almost superior to that involved in the idea of a cataclysm. Think of the ages which must have been consumed in the execution of this colossal sculpture. The question may, of course, be pushed further. Think of the ages which the molten earth required for its consolidation. But these vaster epochs lack sublimity through our inability to grasp them. They bewilder us, but they fail to make a solemn impression. The genesis of the mountains comes more within the scope of the intellect, and the majesty of the operation is enhanced by our partial ability to conceive it. In the falling of a rock from a mountain-head, in the shoot of an avalanche, in the plunge of a cataract, we often see more impressive illustrations of the power of gravity than in the motions of the stars. When the intellect has to intervene, and calculation is necessary to the building up of the conception, the expansion of the feelings ceases to be proportioual to the magnitude of the phenomena. 
I will here record a few other measurements executed on the Rosegg glacier: the line was staked out across the trunk formed by the junction of the Rosegg proper with the 'Tschierva glacier, a short distance below the rocky promontory called Agaliogs.

\section{Rosegg Glacier.}

No. of Stake.

Hourly Motion.

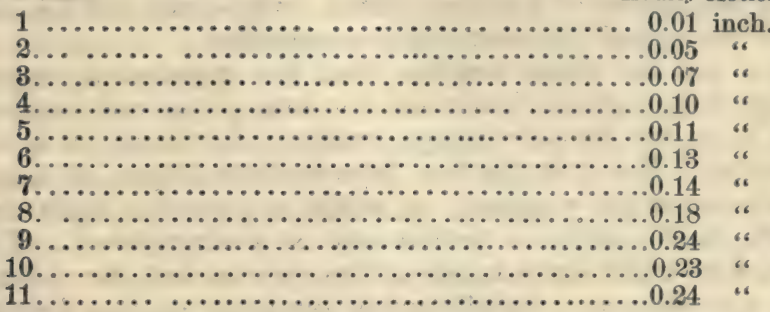

This is an extremely slowly moving glacier; the maximum motion hardly amounts to seven inches a day. Crevasses prevented us from continuing the line quite across the glacier.

\section{CHAP'TER X.}

\section{RECENT EXPERIMENTS ON FOG SIGNALS.*}

THE CARE of its sailors is one of the first duties of a maritime people, and one of the sailor's greatest dangers is his proximity to the coast at night. Hence the idea of warning him of such proximity by beacon-fires placed sometimes on natural eminences and sometimes on towers built expressly for the purpose. Close to Dover Castle, for example, stands an ancient Pharos of this description.

As our marine increased greater skill was invoked, and lamps reinforced by parabolic reflector's poured their light upon the sea. Several of these lamps were sometimes grouped together so as to intensify the light, which at a little distance appeared as if it emanated from a single source. This "catoptric" form of apparatus is still to some extent employed in our lighthouse-service, but for a long time past it has been more and more displaced by the great lenses devised by the illustrious Frenchman, Fresnel.

* A discourse delivered in the Royal Institution, March 22, 1878. 
In a first-class "dioptric " apparatus the light emanates from a lamp with several concentric wicks, the flame of which, being kindled by a very active draught, attains to great intensity. In fixed ligits the lenses refract the rays issuing from the lamp so as to cause them to form a luminous sheet which grazes the sea-horizon. In revolving lights the lenses gather up the rays into distinct beams, resembling the spokes of a wheel, which sweep over the sea and strike the eye of the mariner in succession.

It is not for clear weather that the greatest strengthening of the light is intended, for here it is not needed. Nor is it for densely foggy weather, for here it is ineffectual. But it is for the intermediate stages of hazy, snowy, or rainy weather, in which a powerful light can assert itself, while a feeble one is extinguished. The usual first-order lamp is one of four wicks, but Mr. Douglass, the able and indefatigable engineer of the Trinity House, has recently raised the number of the wicks to six, which produce a very noble flame. To Mr. Wigham, of Dublin, we are indebted for the successful application of gas to lighthouse illumination. In some lighthouses his power varies from 28 jets to 108 jets, while in the lighthouse of Galley Head three burners of the largest size can be employed, the maximum number of jets being 324 . These larger powers are invoked only in case of fog, the 28-jet burner being amply sufficient for clear weather. The passage from the small burner to the large, and from the large burner to the small, is made with ease, rapidity, and certainty. This employment of gas is indigenous to Ireland, and the Board of Trade has exercised a wise liberality in allowing every facility to Mr. Wigham for the development of his invention.

The last great agent employed in lighthouse illumination is electricity. It was in this Institution, beginning in 1831, that Faraday proved the existence and illustrated the laws of those induced currents which in our day have receired such astounding development. In relation to this subject Faraday's words have a prophetic ring. "I have rather," he writes in 1831, "been desirous of discovering new facts and new relations dependent on magneto-electric induction than of exalting the force of those already obtained, being assured that the latter would find their full development hereafter." The labors of Holmes, 
of the Paris Alliance Company, of Wilde, and of Gramme, constitute a brilliant fulfillment of this prediction.

But, as regards the augmentation of power, the greatest step hitherto made was independently taken a few years ago by Dr. Werner Siemens and Sir Charles Wheatstone. 'Through the application of their discovery a machine endowed with an infinitesimal charge of magnetism may, by a process of accumulation at compound interest, be caused so to eurich itself magnetically as to cast by its performance all the older machines into the shade. The light now before you is that of a small machine placed downstairs, and worked there by a minute steam-engine. It is a light of about 1,000 candles; and for it, and for the steam-engine that works it, our members are indebted to the liberality of Dr. William Siemens, who in the most generous manner has presented the machine to this Institution. After an exhaustive trial at the South Foreland, machines on the principle of Siemens, but of far greater power than this one, have been recently chosen by the Elder Brethren of the Trinity House for the two lighthouses at the Lizard Point.

Our most intense lights, including the six-wick lamp, the Wigham gas-light, and the electric light, being intended to aid the mariner in heavy weather, may be regarded, in a certain sense, as fog-signals. But fog, when thick, is intractable to light. The sun cannot penetrate it, much less any terrestrial source of illumination. Hence the necessity of employing sound-signals in dense fogs. Bells, gongs, horns, whistles, guns, and syrens have been used for this purpose; but it is mainly, if not wholly, with explosire signals that we have now to deal. 'The gun has been employed with useful effect at the North Stack, near Holyhear, on the Kish Bank near Dublin, at Lundy Island, and at other points on our coasts. During the long, laborious, and I venture to think memorable series of observations conducted under the auspices of the Elder Brethren of the Trinity House at the South Foreland in 1872 and 1873 , it was proved that a short $5 \frac{1}{2}$-inch howitzer, firing 3 lbs. of powder, yielded a louder report than a long 18-pounder firing the same charge. Here was a hint to be acted on by the Elder Brethren. The effectiveness of the sound depended on the shape of the gun, and as it could not be assumed that in the howitzer we had hit accident- 
ally upon the best possible sh:ıpe, arrangements were made with the War Office for the construction of a gun specially calculated to produce the loudest sound attainable from the combustion of $3 \mathrm{lbs}$. of powder. To prevent the unnecessary landward waste of the sound, the gun was furnished with a parabolic muzzle, intended to project the sound over the sea, where it was most needed. The construction of this gun was based on a searching series of experiments executed at Woolwich with small models, provided with muzzles of various kinds. A drawing of the gun is annexed (p. 197). It was constructed on the principle of the revolver, its various chambers being loaded and brought in rapid succession into the firing position. The performance of the gun proved the correctness of the principles on which its construction was based.

An incideutal point of some interest was decided by the earliest Woolwich experiments. It had been a widely spread opinion among artillerists, that a bronze gun produces a specially loud report. I doubted from the outset whether this would help us; and in a letter dated April $22 \mathrm{~d}, 18 \% 4$, I ventured to express myself thus: "'The report of a gun, as affecting an observer close at hand, is made up of two factors - the sound due to the shock of the air by the violently expanding gas, and the sound derived from the vibrations of the gun, which, to some extent, rings like a bell. 'This latter, I apprehend, will ủisappear' at considerable distances." The result of subsequent trial, as reported by General Campbell, is, "that the sonorous qualities of bronze are greatly superior to those of cast iron at short distances, but that the advantage lies with the baser metal at long ranges.”*

Coincident with these trials of guns at Woolwich, guncotton was thought of as a probably effective sound-producer. From the first, indeed, theoretic considerations caused me to fix my attention persistently on this substance; for the remarkable experiments of Mr. Abel, whiereby its rapidity of combustion and violently explosive energy are

* General Campbell assigns a true cause for this difference. The ring of the bronze gun represents so much energy withdrawn from the explosive force of the gunpowder. Further experiments would, however, be needed to place the superiority of the cast-iron gun at a distance beyond question. 
demonstrated, seemed to single it out as a substance eminently calculated to fulfill the conditions necessary to the production of an intense wave of sound. What those conditions are we shall now more particularly inquire, calling to our aid a brief but very remarkable paper, published by Professor Stokes in the Philosophical Magazine for 1868.

'The explosive force of gunpowder is known to depend on the sudden conversion of a solid body into an intensely heated gas. Now the work which the artillerist requires the expanding gas to perform is the displacement of the projectile, besides which it has to displace the air in front

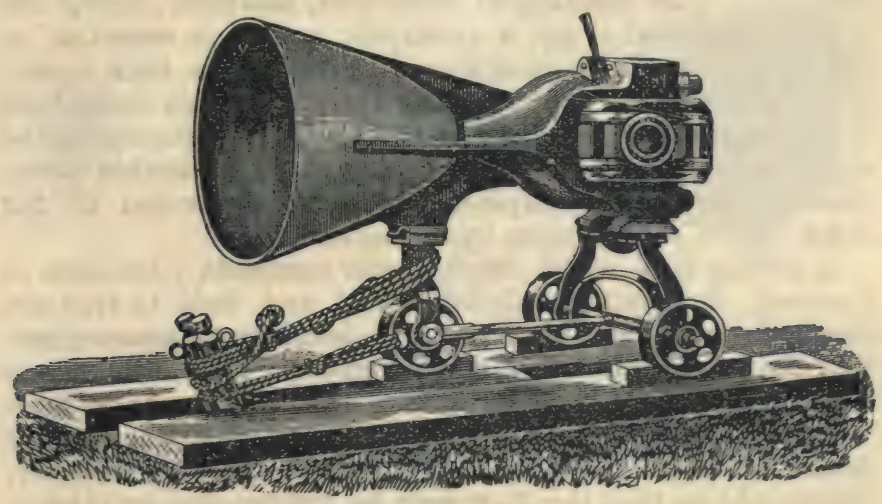

Fig. 6.

Breech-loading Fog-signal Gun, with Bell Mouth,* proposed by Major Maitland, R.A., Assistant Superintendent.

of the projectile, which is backed by the whole pressure of the atmosphere. Such, however, is not the work that we want our gunpowder to perform. We wish to transmute its energy not into the mere mechanical translation of either shot or air, but into vibratory motion. We want pulses to be formed which shall propagate themselves to vast distances through the atmosphere, and this requires a certain choice and management of the explosive material.

* The carriage of this gun has been modified in construction since this drawing was made. 
A sound-wave consists essentially of two parts-a condensation, and a rarefaction. Now air is a very mobile fluid, and if the shock imparted to it lack due promptness, the wave is not produced. Consider the case of a common clock pendulum, which oscillates to and fro, and which might be expected to generate corresponding pulses in the air. When, for example, the bob moves to the right, the air to the right of it might be supposed to be condensed, while a partial vacuum might be supposed to follow the bob. As a matter of fact, we have nothing of the kind. 'The air particles in front of the bob retreat so rapidly, and those behind it close so rapidly in, that no sound-pulse is formed. 'The mobility of hydrogen, moreover, being far' greater than that of air, a prompter action is essential to the formation of sonorous waves in hydrogen than in air. It is to this rapid power of readjustment, this refusal, so to speak, to allow its atoms to be crowded together or to be drawn apart, that Professor Stokes, with admirable penetration, refers the damping power, first described by Sir John Leslie, of hydrogen upon sound.

A tuning-fork which executes 256 complete vibrations in a second, if struck gently on a pad and held in free air, emits a scarcely audible note. It behaves to some extent like the pendulum bob just referred to. This feebleness is due to the prompt " reciprocating flow" of the air between the incipient condensations and rarefactions, whereby the formation of sound-pulses is forestalled. Stokes, however, has taught us that this flow may be intercepted by placing the edge of a card in close proximity to one of the corner's of the fork. An inmediate augmentation of the sound of the fork is the consequence.

The more rapid the shock imparted to the air, the greater is the fractional part of the energy of the shock converted into wave motion. And as different kinds of gunpowder vary considerably in their rapidity of combustion, it may be expected that they will also vary as producers of sound. 'This theoretic inference is completely verified by experiment. In a series of preliminary trials conducted at Woolwich on the 4 th of June, 1875, the sound-producing powers of four different kinds of powder were determined. In the order of the size of their grains they bear the names respectively of Fine-grain (F. G.), LargeGrain (L. G.), Rifle Large-grain (R. L. G.), and Pebble- 
powder (P.) (See annexed figures.) The charge in each case amounted to $4 \frac{1}{2} \mathrm{lbs}$; four 24-lb. howitzers being employed to fire the respective charges. There were eleven observers, all of whom, without a single dissentient, pronounced the sound of the fine-grain powder loudest of all. In the opinion of seven of the eleven the large-grain powder came next; seven also of the eleven placed the rifle large-grain third on the list; while they were again unanimous in pronouncing the pebble-powder the worst sound-producer. These differences are entirely due to differences in the rapidity of combustion. All who have witnessed the performance of the 80-ton gun must have
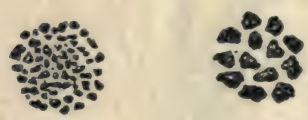

F.G.

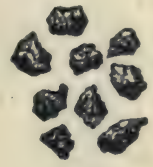

R.I.G.

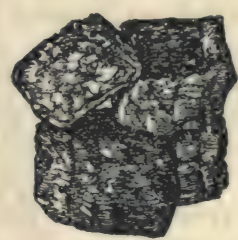

P.

FIG. 7.

been surprised at the mildness of its thunder. To avoid the strain resulting from quick combustion, the powder employed is composed of lumps far larger than those of the pebble-powder above referred to. In the long tube of the gun these lumps of solid matter gradually resolve themselves into gas, which on issuing from the muzzle imparts a kind of push to the air, instead of the sharp shock necessary to form the condensation of an intensely sonorous wave.

These are some of the physical reasons why gun-cotton might be regarded as a promising fog-signal. Firing it as we have been taught to do by Mr. Abel, its explosion is more rapid than that of gunpowder. In its case the air particles, alert as they are, will not, it might be presumed, be able to slip from condensation to rarefaction with a ratpidity sufficient to forestall the formation of the wave. On $\grave{a}$ priori grounds then, we are entitled to infer the effectiveness of gun-cotton, while in a great number of comparative experiments, stretching from 1874 to the present time, this inference has been verified in the most conclusive manner. 
As regards explosive material, and zealous and accomplished help in the use of it, the resources of Woolwich Arsenal have been freely placed at the disposal of the Elder Brethren. General Campbell, General Younghusband, Colonel Fraser, Colonel Maitland, and other officer's, have taken an active personal part in the investigation, and in most cases have incurred the labor of reducing and reporting on the observations. Guns of various forms and sizes have been inroked for gunpowder, while gun-cotton has been fired in free air and in the foci of parabolic reflectors.

On the $22 \mathrm{~d}$ of February, 18\%5, a number of small guns, cast specially for the purpose-some with plain, some with conical, and some with parabolic muzzles-firing $4 \mathrm{oz}$. of fine grain powder, were pitted against $4 \mathrm{oz}$. of gull-cotton detonated both in the open and in the focus of a parabolic reflector.* 'The sound produced by the gun-cotton, reinforced by the reflector, was nnanimously pronounced loudest of all. With equal unanimity, the gun-cotton detonated in free air was placed second in intensity. 'Though the same charge was used throughout, the guns differed notably among themselves, but none of them came up to the guncotton, either with or without the reflector. A second series, observed from a different distance on the same day, confirmed to the letter the foregoing result.

As a practical point, however, the comparative cost of gun-cotton and gunpowder has to be taken into account, though considerations of cost ought not to be stretched too far in cases involving the safety of human life. In the earlier experiments, where quantities of equal price were pitted against each other, the results were somewhat fluctuating. Indeed, the perfect manipulation of the gun-cotton required some preliminary discipline-promptness, certainty, and effectiveness of firing, augmenting as experience increased. As $1 \mathrm{lb}$. of gun-cotton costs as much as $3 \mathrm{lbs}$. of gunpowder, these quantities were compared together on the $22 d$ of February. 'The guns employed to discharge the gunpowder were a 12-lb. brass howitzer, a 24-lb. castiron howitzer, and the long 18-pounder employed at the South Foreland. 'The result was, that the 24-lb. howitzer, firing $3 \mathrm{lbs}$. of gunpowder, had a slight adrantage over $1 \mathrm{lb}$. of gun-cotton detonated in the open;

* For charges of this weight the reflector is of moderate size, and may be employed without fear of fracture. 
while the 12-lb. howitzer and the 18-pounder were both beaten by the gun-cotton. On the $2 \mathrm{~d}$ of May, on the other hand, the gun-cotton is reported as having been beaten by all the guns.

Meanwhile, the parabolic-muzzle gun, expressly intended for fog-signaling, was pushed rapidly forward, and on

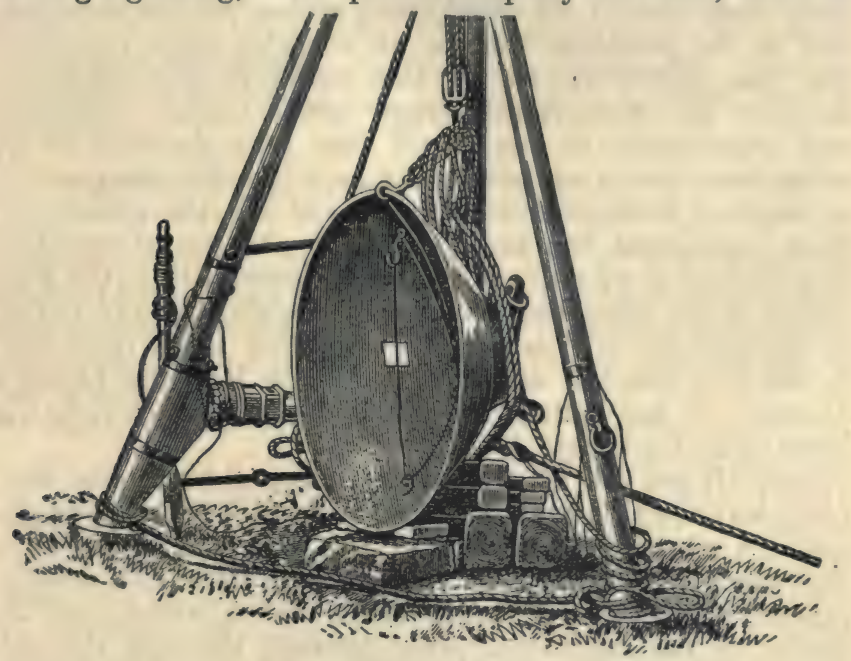

Fig. 8.

Gun-cotton Slab ( $1 \frac{1}{2} \mathrm{lb}$.) Detonated in the Focus of a Cast-iron Reflector.

March 22 and 23, 1876, its power was tested at Shoeburyness. Pitted against it were a 16-pounder, a $5 \frac{1}{2}$-inch howitzer, $1 \frac{1}{2} \mathrm{lb}$. of gun-cotton detonated in the focus of a reflector (see annexed figure), and $1 \frac{1}{2} \mathrm{lb}$. of gun-cotton detonated in free air. On this occasion nineteen different series of experiments were made, when the new experimental gun, firing a 3:lb. charge, demonstrated its superiority over all guns previonsly employed to fire the same charge. As regards the comparative merits of the gun-cotton fired in the open, and the gunpowder fired from the new gun, the mean values of their sound were the same. Fired in the focus of the reflector, the gun-cotton clearly dominated over all the other sound-producers. *

* The reflector was fractured by the explosion, but it did good service afterward, 
The whole of the observations here referred to were embraced by an angle of about 70 degrees, of which 50 degrees lay on the one side and 20 degrees on the other side of the line of fire. The shots were heard by eleven observers on board the Galatea, which took up positions varying from 2 miles to $13 \frac{1}{2}$ miles from the firing-point. In all these observations the reinforcing action of the reflector, and of the parabolic muzzle of the gun, came into play. But the reinforcement of the sound in one direction implies its withdrawal from some other direction, and accordingly it was found that at a distance of $5 \frac{1}{4}$ miles from the firing-point, and on a line including nearly an angle of 90 degrees with the line of fire, the gun-cotton in the open beat the new gun; while behind the station, at distances of $8 \frac{1}{2}$ miles and $13 \frac{1}{2}$ miles respectively, the guncotton in the open beat both the gun and the gun-cotton in the reflector. This result is rendered more important by the fact that the sound reached the Mucking Light, a distance of $13 \frac{1}{2}$ miles, against a light wind which was blowing at the time.

Most, if not all, of our ordinary sound-producers send forth waves which are not of uniform intensity throughout. A trumpet is loudest in the direction of its axis. The same is true of a gun. A bell, with its mouth pointed upward or downward, sends forth waves which are far denser in the horizontal plane passing through the bell than at an angular distance of 90 degrees from that plane. The oldest bellhangers must have been aware of the fact that the sides of the bell, and not its month, emitted the strongest sound, their practice being probably determined by this knowledge. Our slabs of gun-cotton also emit waves of different densities in different parts. It has occurred in the experiments at Shoeburyness that when the broad side of a slab was turned toward the suspending wire of a second slab six feet distant, the wire was cut by the explosion, while when the edge of the slab was turned to the wire this never occurred. To the circumstance that the broadsides of the slabs faced the sea is probably to be ascribed the remarkable fact observed on March 23d, that in two directions, not far removed from the line of fire, the gun-cotton detonated in the open had a slight advantage over the new gun.

Theoretic considerations rendered it probable that the 
shape and size of the exploding mass would affect the constitution of the wave of sound. I did not think large rectangular slabs the most favorable shape, and accordingly proposed cutting a large slab into fragments of lifferent sizes, and pitting them against each other. The differences between the sounds were by no means so great as the differences in the quantities of explosive material might learl one to expect. 'The mean values of eighteen series of observations made on board the Galatea, at distances varying from $1 \frac{3}{4}$ mile to 4.8 miles, were as follows:

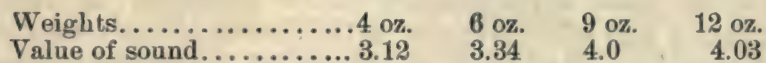

These charges were cut from a slab of dry gun-cotton about $1 \frac{3}{4}$ inch thick: they were squares and rectangles of the following dimensions: $4 \mathrm{oz}$., 2 inches by 2 inches; 6 oz., 2 inches by 3 inches; 9 oz., 3 inches by 3 inches; 12 oz., 2 inches by 6 inches.

The numbers under the respective weights express the recorded value of the sounds. They must be simply taken as a ready means of expressing the approximate relative intensity of the sounds as estimated by the ear. When we find a 9-oz. charge marked 4, and a 12-oz. charge marked 4.03, the two sounds may be regarded as practically equal in intensity, thus proving that an addition of 30 per cent. in the larger charges produces no sensible difference in the sound. Were the sounds estimated by some physical means, instead of by the ear, the values of the sounds at the distances recorded would not, in my opinion, show a greater advance with the increase of material than that indicated by the foregoing numbers. Subsequent experiments rendered still more certain the effectiveness, as well as the economy, of the smaller charges of gun-cotton.

It is an obvious corollary from the foregoing experiments that on our " nesses" and promontories, where the land is clasped on both sides for a considerable distance by the sea-where, therefore, the sound has to propagate itself rearward as well as forward - the use of the parabolic gun, or of the parabolic reflector, might be a disadvantage rather than an advantage. Here gun-cotton, exploded in the open, forms the most appropriate source of sound. This remark is especially applicable to such lightships as 
are intended to spread the sound all around them as from central foci. As a signal in rock lighthouses, where neither syren, steam-whistle, nor gun could be mounted; and as a handy fleet-signal, dispensing with the lumber of special signal-guns, the gun-cotton will prove invaluable. But in most of these cases we have the drawback that local damage may be done by the explosion. The lantern of the rock lighthouse might suffer from concussion near at hand, and though mechanical arrangements might be devised, both in the case of the lighthouse and of the ship's deck, to place the firing-point of the gun-cotton at a safe distance, no such arrangement could compete, as regards simplicity and effectiveness, with the expedient of a guncotton rocket. Had such a means of signaling existed at the Bishop's Rock lighthouse, the ill-fated Schiller might have been warned of her approach to danger ten, or it may be twenty miles before she reached the rock which wrecked her. Had the fleet possessed such a signal, instead of the ubiquitous but ineffectual whistle, the Iron Duke and Vanguard need never have came into collisor.

It was the necessity of providing a suitable signal for rock lighthouses, and of clearing obstacles which cast an acoustic shadow, that suggested the idea of the gun-cotton rocket to Sir Richard Collinson, deputy master of the 'Trinity House. His idea was to place a disk or short cylinder of gun-cotton in the head of a rocket, the ascensional force of which should be employed to carry the disk to an elevation of 1,000 feet or thereabouts, where by the ignition of a fuse associated with a detonator, the gun-cotton should be fired, sending its sound in all directions vertically and obliquely down upon earth and sea. The first attempt to realize this idea was made on July $18,18 \% 6$, at the firework manufactory of the Messrs. Brock, at Nunhead. Eight rockets were then fired, four being charged with $5 \mathrm{oz}$. and four with $7 \frac{1}{2} \mathrm{oz}$. of gun-cotton. They ascended to a great height, and exploded with a very loud report in the air. On July 2\%, the rockets were tried at Shoeburyness. 'The most noteworthy result on this occasion was the hearing of the sounds at the Mouse lighthouse, $8 \frac{1}{2}$ miles E. by S., and at the Chapman lighthouse, $8 \frac{1}{2}$ miles W. by N.; that is to say, at opposite sides of the firing point. It is worthy to remark that, in the case of the Chapman light- 
house, land and trees intervened between the firing-point and the place of observation. "This," as General Younghusband justly remarked at the time, " may prove to be a valuable consideration if it should be found necessary to place a signal station in a position whence the sea could not be freely observer." Inileed, the clearing of such obstacles was one of the objects which the inventor of the rocket had in view.

With reference to the action of the wind, it was thought desirable to compare the range of explosions produced near the surface of the earth with others produced at the elevation attainable by the gun-cotton rockets. Wind and weather, however, are not at our command; and hence one of the objects of a series of experiments conducted on December $13,18 \% 6$, was not fulfilled. It is worthy, however, of note that on this day, with smooth water and a calm atmosphere, the rockets were distinctly heard at a distance of 11.2 miles from the firing point. The quantity of guncotton employed was $7 \frac{1}{2}$ oz. On Thursday, March 8, 187\%, these comparative experiments of firing at high and low elevations were pushed still further. The gun-cotton near the ground consister of $\frac{1}{2}$-lb. disks, suspended from a horizontal iron bar about $4 \frac{1}{2}$ feet above the ground. The rockets carried the same quantity of gun-cotton in their heads, and the height to which they attained, as delermined by a theodolite, was from 800 to 900 feet. The day was cold, with occasional squalls of snow and hail, the direction of the sound being at right angles to that of the wind. Five series of observations were made on board the Vestal, at distances varying from 3 to 6 miles. The mean value of the explosions in the air exceeded that of the explosions near the ground by a small but sensible quantity. At Windmill Hill, Gravesend, however, which was nearly to leeward, and $5 \frac{1}{2}$ miles from the firing-point, in nineteen cases ont of twenty-four the disk fired near the ground was lourlest; while in the remaining five the rocket had the advantage.

Toward the close of the day the atmosphere became very serene. A few distant cumuli sailed near the horizon, but the zenith and a vast angular space all round it were absolutely free from cloud. From the deck of the Galatea a rocket was discharged, which reached a great elevation, and exploded with a loud report. Following this solid 
nucleus of sound was a continuous train of echoes, which retreated to a continually greater distanc e, dying gradually off into silence after seven seconds' duration. 'These echoes were of the same character as those so frequently noticed at the South Foreland in 18\%2-73, and called by me "aërial echoes."

On the 23d of March the experiments were resumed, the most noteworthy results of that day's observations being that the sounds were heard at Tillingham, 10 miles to the N. E.; at West Mersea, $15 \frac{3}{4}$ miles to the N. E. by E.; at Brightlingsea, $17 \frac{1}{2}$ miles to the N. E.; and at Clacton Wash, $20 \frac{1}{2}$ miles to the N. E. by $\frac{1}{2}$ E. The wind was blowing at the time from the S. E. Some of these sounds were produced by rockets, some by a 24-1b. howitzer, and some by an 8-inch Maroon.

In December, 1876, Mr.Gardiner, the managing director of the Cotton-powder Company, had proposed a trial of this material against the gun-cotton. The density of the cotton he urged was only 1.03, while that of the powder was 1.\%0. A greater quantity of explosive material being thus compressed into the same volume, Mr. Gardiner thought that a greater sonorous effect must be produced by the powder. $\Lambda$ t the instance of Mr. Mackie, who had previously gone very thoroughly into the subject, a committee of the Elder Brethren visited the cotton-powder manufactory, on the banks of the Swale, near Faversham, on the 16th of June, 18\%\%. 'The weights of cotton-powder employed were $2 \mathrm{oz} ., 8 \mathrm{oz}, 1 \mathrm{lb}$, , and $2 \mathrm{lbs}$., in the form of rockets and of signals fired a few feet above the ground. 'The experiments throughout were arranged and conducted by Mr. Mackie. Our desire on this occasion was to get as near to windward as possible, but the Swale and other obstacles limited our distance to $1 \frac{1}{2}$ mile. We stood here E. S. E. from the firing-point while the wind blew fresh from the N. E.

The cotton-powder yielded a very effective report. The rockets in general had a slight advantage over the same quantities of material fired near the ground. The loudness of the sound was by no means proportional to the quantity of the material exploled, $8 \mathrm{oz}$. yielding very nearly as loud a report as $1 \mathrm{lb}$. The "ä̈rial echoes," which invariably followed the explosion of the rockets, were loud and longcontinued. 
On the 17th of October, $187 \%$, another series of experiments with howitzers and rockets was carried out at Shoeburyness. 'The charge of the howitzer was 3 lbs. of L. G. powder. The charges of the rockets were 12 oz., 8 oz., 4 oz., and $2 \mathrm{oz}$. of gun-cotton respectively. 'The gun and the four rockets constituted a series, and eight series were fired during the afternoon of the 1\%th. The observations were made from the Vestal and the Galatea, positions being successively assumed which permitted the sound to reach the observers with the wind, against the wind, and across the wind. The distance of the Galatea varied from 3 to 7 miles, that of the Vestal, which was more restricted in her movements, being 2 to 3 miles. Briefly summed up, the result is that the howitzer, firing a 3-lb. charge, which it will be remembered was our best gun at the South Foreland, was beaten by the $12-0 z$. rocket, by the 8-oz. rocket, and by the 4-oz. rocket. The 2-oz. rocket alone fell behind the howitzer.

It is worth while recording the distances at which some of the sounds were heard on the day now referred to:

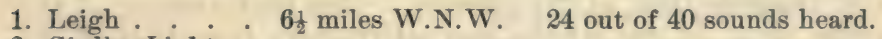

2. Girdler Light-

vessel . . 12 " S.E. by E. 5 "

3. Reculvers . . 17t " S.E. by S. 18

4. St. Nicholas . 20 " S.E. : 3

5. Epple Bay . . 22 " S.E. by E. 19

6. Westgate . . 23 " S.E. by E. 9

7. Kingsgate * . 25 “ S.E. by E. 8

The day was cloudy, with occasional showers of drizzling rain; the wind about $\mathrm{N}$. W. by $\mathrm{N}$. all day; at times squally, rising to a force of 6 or 7 and sometimes dropping to it force of 2 or 3 . 'The station at Leigh excepted, all these places were to le ward of Shoeburyness. At four other stations to leeward, varying in distance from $15 \frac{1}{2}$ to $24 \frac{1}{2}$ miles, nothing was heard, while at eleven stations to windward, varying from 8 to 26 miles, the sounds were also inaudible. It was found, indeed, that the sounds proceeding directly against the wind did not penetrate much beyond 3 miles.

On the following day, viz., October 18th, we proceeded to Dungeness with the view of making a series of strict comparative experiments with gun-cotton and cottonpowiler. Rockets containing $8 \mathrm{oz}, 4 \mathrm{oz}$, and $2 \mathrm{oz}$. of guncotton had been prepared at the Royal Arsenal; while 
others, containing similar quantities of cotton-powder, had been supplied by the Cotton-powder Company at Faversham. With these were compared the ordinary 18-pounder gun, which happened to be mounted at Dungeness, firing the usual charge of $3 \mathrm{lbs}$. of powder, and a syren.

From these experiments it appeared that the gun-cotton and cotton-powder were practically equal as producers of sound.

The effectiveness of small charges was illustrated in a very striking manner, only a single unit separating the numerical value of the 8-oz. rocket from that of the 2-oz. rocket. The former was recorded as 6.9 and the latter as 5.9 , the value of the 4-oz. rocket being intermediate between them. These results were recorded by a number of very practiced observers on board the Galatea. They were completely borne out by the observations of the Coastguard who marked the value of the 8-oz. rocket 6.1 , and that of the 2-oz. rocket 5.2. The 18-pounder gun fell far behind all the rockets, a result, possibly, to be in part ascribed to the imperfection of the powder. The performance of the syren was, on the whole, less satisfactory than that of the rocket. The instrument was worked, not by steam of 70 lbs. pressure, as at the South Foreland, but by compressed air, beginning with $40 \mathrm{lbs}$. and ending with $30 \mathrm{lbs}$. pressure. The trumpet was pointed to windward, and in the axis of the instrument the sound was about as effective as that of the 8-oz. rocket. But in a direction at right angles to the axis, and still more in the rear of this direction, the syren fell very sensibly behind even the 2-oz. rocket.

These are the principal comparative trials made between the gun-cotton rocket and other fog-signals; but they are not the only ones. On the $2 d$ of August, 18\% , for example, experiments were made at Lundy Island with the following results. At 2 miles distant from the firing-point, with land intervening, the 18-pounder, firing a 3-lb. charge, was quite unheard. Both the 4-oz. rocket and the 8-oz. rocket, however, reached an elevation which commancled the acoustic shadow, and yielded loud reports. When both were in view the rockets were still superior to the gun. On the 6th of August, at St. Ann's, the 4-oz. and 8-oz. rockets proved superior to the syren. On the 
Shambles light-vessel, when a pressure of 13 lbs. was employed to sound the syren, the rockets proved greatly superior to that instrument. Proceeding along the sea margin at Flamboro' Head, Mr. Edwards states that at a distance of $1 \frac{1}{4}$ mile, with the 18-pounder previously used as a fog-signal hidden behind the cliffs, its report was quite unheard, while the $4 \cdot \mathrm{oz}$. rocket, rising to an elevation which brought it clearly into view, yielded a powerful sound in the face of an opposing wind.

On the evening of February 9, 18\%\%, a remarkable series of experiments were made by Mr. Prentice at Stowmarket with the gun-cotton rocket. From the report with which he has kindly furnished me I extract the following particulars. 'The first column in the annexed statement contains the name of the place of observation, the second its distance from the firing-point, and the third the result observed:

Stoke Hill, Ipswich . . 10 miles Rockets clearly seen and sounds distinctly heard 53 seconds after the flash.

Melton . . . . 15 " Signals distinctly heard. Thought at first that sounds were reverberated from the sea.

Framlingham . . . 18 " Signals very distinctly heard, both in the open air and in a closed room. Wind in favor of sound.

Stratford. St. Andrews . 19 " Reports loud; startled pheasants in a cover close by.

Tuddenham. St. Martin 10 " Reports very loud; rolled away like thunder.

Christ Church Park. . . 11 " Report arrived a little more than a minute after Hlash.

Nettlestead Hall . . . 6 " Distinct in every part of observer's house. Very loud in the open air.

Bildestone. . . . . 6 “ Explosion very loud, wind against sound.

Nacton . . . . 14 " Reports quite distinct-mistaken by inhabitants for claps of thunder.

Aldboro' . . . . . 25 " Rockets seen through a very hazy atmosphere; a rumbling detonation heard.

Capel Mills . . . . 11 " Reports heard within and without the observer's house. Wind opposed to sound.

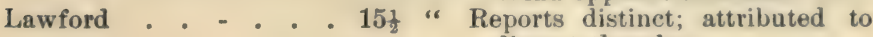
distant thunder. 
In the great majority of these cases, the direction of the sound enclosed a large angle with the direction of the wind. In gome cases, indeed, the two directions were at right angles to each other. It is needless to $\mathrm{dwell}$ for a moment on the advantage of possessing a signal commanding ranges such as these.

'Ihe explosion of substances in the air, after having been carried to a considerable elevation by rockets, is a familiar performance. In 1873, moreover, the Board of Trade proposed a light-and-sound rocket as a signal of distress, which proposal was subsequently realized, but in a form too elaborate anil expensive for practical use. The idea of a gun cotton rocket fit for signaling in fogs is, I believe, wholly due to Sir Richard Collinson, the deputy master of the Trinity House. Thanks to the skillful aid given by the authorities of IVoolwich, by Mr. Prentice, and Mr. Brock, that idea is now an accomplished fact; a signal of great power, handiness, and economy, being thus placed at the service of our mariners. Not only may the rocket be applied in association with lighthouses and lightships, but in the navy also it may be turned to important account. Soon after the loss of the Vanguard I ventured to urge upon an eminent naval officer the desirability of having an organized code of fog-signals for the fleet. $\mathrm{He}$ shook his head doubtingly, and referred to the difficulty of finding room for signal guns. The gun-cotton rocket completely surmounts this difficulty. It is manipulated with ease and rapidity, while its discharges may be so grouped and combined as to give a most important extension to the voice of the admiral in command. It is needless to add that at any point upon our coast, or upon any other coast, where its establishment might be desirable, a fog-signal station might be extemporized without difficulty.

I have referred more than once to the train of echoes which accompanied the explosion of gun-cotton in free air, speaking of them as similar in all respects to those which were described for the first time in my Report on Fog-signals, addressed to the Corporation of 'Trinity House in $18 \% 4$.* 'To these echoes I attached a funda-

* See also "Philosophical Transactions" for 1874, p. 183. 
mental significance. 'There was no visible reflecting surface from which they could come. On some days, with hardly a cloud in the air and hardly a ripple on the sea, they reached a magical intensity. As far as the sense of hearing could judge, they came from the body of the air in front of the great trumpet which produced them. The trumpet blasts were five seconds in duration, but long before the blast had ceased the echoes struck in, adding their strength to the primitive note of the trumpet. After the blast had ended the echoes continued, retreating further and further from the point of observation, and finally dying away at great distances. The echoes were perfectly continuous as long as the sea was clear of ships, "tapering" by imperceptible gradations into absolute silence. But when a ship happened to throw itself athwart the course of the sound, the echo from the broadside of the vessel was returned as a shock which rudely interrupted the continuity of the dying atmospheric music.

'These echoes have been ascribed to reflection from the crests of the sea-waves. But this hypothesis is negatived by the fact that the echoes were produced in great intensity and duration when no wares existed-when the sea, in fact, was of glassy smoothness. It has been also shown that the direction of the echoes depended not on that of waves, real or assumed, but on the direction of the axis of the trumpet. Causing that axis to traverse an arc of 210 degrees, and the trumpet to sound at various points of the arc, the echoes were always, at all events in calm weather, returned from that portion of the atmosphere toward which the trumpet was directed. They could not, under the circumstances, come from the glassy sea; while both their variation of direction and their perfectly continuous fall into silence, are irreconcilable with the notion that they came from fixed objects on the land. They came from that portion of the atmosphere into which the trumpet poured its maximum sound, and fell in intensity as the direct sound penetrated to greater atmospheric distances.

The day on which our latest observations were made was particularly fine. Before reaching Dungeness, the smoothness of the sea and the serenity of the air caused me to test the echoing power of the atmosphere. A single ship lay about half a mile distant between us and the land. The result of the proposed experiment was clearly foreseen. 
It was this. The rocket being sent up, it exploded at a great height; the echoes retreated in their usual fashion, becoming less and less intense as the distances of the invisible surfaces of reflection from the observers increased. About five seconds after the explosion, a single loud shock was sent back to us from the side of the ressel lying between us and the land. Obliterated for a moment by this more intense echo, the aërial reverberation continued its retreat, dying away into silence in two or three seconds afterward.*

I have referred to the firing of an $8-0 z$. rocket from the deck of the Galatea on March 8, 187\%, stating the duration of its echoes to be seven seconds. Mr. Prentice, who was present at the time, assured me that in his experiments similar echoes had been frequently heard of more than twice this duration. The ranges of his sounds alone would render this result in the highest degree probable.

To attempt to interpret an experiment which I have not had an opportunity of repeating, is an operation of some risk; and it is not without a conscionsness of this that I refer here to a result announced by Professor Joseph Henry, which he considers adverse to the notion of aërial echoes. He took the trouble to point the trumpet of a syren toward the zenith, and found that when the syren was sounded no echo was returned. Now the reflecting surfaces which give rise to these echoes are for the most .part due to differences of temperature between sea and air. If, through any cause, the air above be chilled, we have descending streams-if the air below be warmed, we hare ascending streams as the initial cause of atmospheric flocculence. A sound proceeding vertically does not cross the streams, nor impinge upon the reflecting surfaces, as does a sound proceeding horizontally across them. Aërial echoes, therefore, will not accompany the vertical sound as they accompany the horizontal one. The experiment, as I interpret it, is not opposed to the theory of these echoes which I have ventured to enunciate. But, as I have indicated, not oniy to see but to vary such an experiment is a necessary prelucie to grasping its full significance.

* The echoes of the gun fired on shore this day were very brief; those of the 12-oz. gun-cotton rocket were $12^{\prime \prime}$ and those of the 8-oz. cotton-powder rocket $11^{\prime \prime}$ in duration. 
In a paper published in the "Philosophical Transactions" for 18\%6, Professor Osborne Reynolds refers to these echoes in the following terms: "Without attempting to explain the reverberations and echoes which have been observed, I will merely call attention to the fact that in no case have I heard any attending the reports of the rockets, * although they seem to have been invariable with the guns and pistols. These facts suggest that the echoes are in some way connected with the direction given to the sound. They are caused by the voice, trumpets, and the syren, all of which give direction to the sound; but I am not aware that they have ever been observed in the case of a sound which has no direction of greatest intensity." The reference to the voice, and other references in his paper, cause me to think that, in speaking of echoes, Professor Osborne Reynolds and myself are dealing with different phenomena. Be that as it may, the foregoing observations render it perfectly certain that the condition as to direction here laid down is not necessary to the production of the echoes.

There is not a feature connected with the aërial echoes which cannot be brought out by experiments in the air of the laboratory. I have recently made the following experiment: A rectangle, $\mathrm{x}$ Y (p. 214), 22 inches by 12, was crossed by twenty-three brass tubes (half the number would suffice and only eleven are shown in the figure), each having a slit along it from which gas can issue. In this way twenty-three low flat flames were obtained. A sounding reed $a$ fixed in a short tube was placerl at one end of the rectangle, and a "sensitive flame," $\dagger f$ at some distance beyond the other end. When the reed sounded, the flame in front of it was violertly agitated, and roared boisterously. Turning on the gas, and lighting it as it issued from the slits, the air above the flames became so heterogeneous that the sensitive flame was instantly stilled, rising from a height of 6 inches to a height of 18 inches. Here we had the acoustic opacity of the air in front of the South Foreland strikingly imitated.t Turning off the gas,

* These carried $12 \mathrm{oz}$. of gunpowder, which has been found by Colonel Fraser to require an iron case to produce an effective explosion.

+ Fully described in my " Lectures on Sound," 3d edition, p. 227.

f "Lectures on Sound," 3d ed., p. 268. 
and removing the sensitive flame to $f^{\prime}$, some distance behind the reed, it burned there tranquilly, though the reed was sounding. Again lighting the gas as it issued from the brass tubes, the sound reflected from the heterogeneous

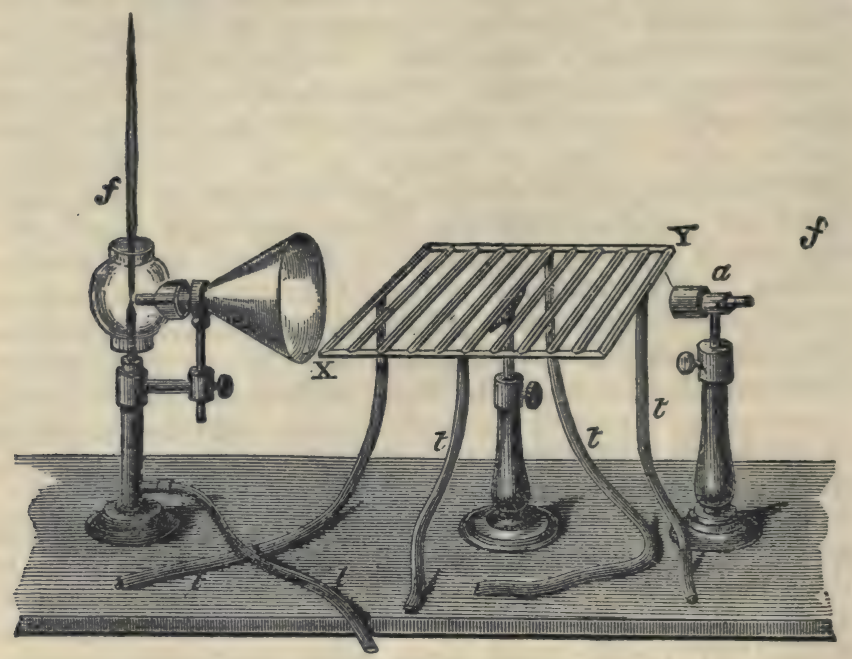

Fig. 9.

air threw the sensitive flame into violent agitation. Here we had imitated the aërial echoes heard when standing behind the syren-trumpet at the South Foreland. 'The experiment is extremely simple, and in the highest degree impressive.

The explosive rapidity of dynamite marks it as a substance specially suitable for the production of sound. At the suggestion of Professor Dewar, Mr. McRoberts has carried out a series of experiments on dynamite, with extremely promising results. Immediately after the delivery of the foregoing lecture I was informed that $\mathrm{Mr}$. Brock proposed the employment of dynamite in the Collinsou rocket, 


\section{CHAPTER XI.}

\section{ON THE STUDY OF PHYSICS.*}

I HOLD in my hand an uncorrected proof of the syllabus of this course of lectures, and the title of the present lecture is there stated to be "On the Importance of the Study of Physics as a Means of Education." The corrected proof, however, contains the title: "On the Importance of the Study of Physics as a Branch of Education." Small as this editorial alteration may seem, the two words suggest two radically distinct modes of viewing the subject before us. 'I'he term Education is sometimes applied to a single faculty or organ, and if we know wherein the education of a single faculty consists, this will help us to clearer notions regarding the education of the sum of all the faculties, or of the mind. When, for example, we speak of the education of the voice, what do we mean? There are certain membranes at the top of the windpipe which throw into vibration the air forced between them from the lungs, thus producing musical sounds. These membranes are, to some extent, under the control of the will, and it is found that they can be so modified by exercise as to produce notes of a clearer and more melodious character. This exervise we call the education of the voice. We may choose for our exercise songs new or old, festive or solemn; the education of the voice being the object aimed at, the songs may be regarded as the means by which this education is accomplished. I think this expresses the state of the case more clearly than if we were to call the songs a branch of education. Regarding also the education of the human mind as the improvement and delvopment of the mental faculties, I shall consider the study of Physics as a means toward the attainment of this end. From this point of view, I degrade Physics into an implement of culture, and this is my deliberate design.

The term Physics, as made use of in the present Lecture, refers to that portion of natural science which lies midway between astronomy and chemistry. 'The former' indeed, is Physics applied to " masses of enormous weight,"

* From a lecture delivered in the Royal Institution of Great Britain in the spring of 1854 . 
while the latter is Physics applied to atoms and molecules. The subjects of Physics proper are therefore those which lie nearest to human perception: light and heat, color, sound, motion, the loadstone, electrical attractions and repulsions, thunder and lightning, rain, snow, dew, and so forth. Our senses stand between these phenomena and the reasoning mind. We observe the fact, but are not satisfied with the mere act of observation: the fact must be accounted for-fitted into its position in the line of cause and effect. Taking our facts from Nature we transfer them to the domain of thought: look at them, compare them, observe their mutual relations and connections, and bringing them ever clearer before the mental eye, finally alight upon the cause which unites them. This is the last act of the mind, in this centripetal direction-in its progress from the multiplicity of facts to the central cause on which they depend. But, having guessed the cause, we are not yet contented. We set out from the center and travel in the other direction. If the guess be true, certain consequences must follow from it, and we appeal to the law and testimony of experiment whether the thing is so. Thus is the circuit of thought completed-from without inward, from multiplicity to unity, and from within ontward, from unity to multiplicity. In thus traversing both ways the line between cause and effect, all our reasoning powers are called into play. The mental effort involved in these processes may be compared to those exercises of the body which invoke the co-operation of every muscle, and thus confer upon the whole frame the benefits of healthy action.

The first experiment a child makes is a physical experiment: the suction-pump is but an imitation of the first act of every new-born infant. Nor do I think it calculated to lessen that infant's reverence, or to make him a worse citizen, when his riper experience shows him that the atmosphere was his helper in extracting the first draught from his mother's breast. The child grows, but is still an experimenter: he grasps at the moon, and his failure teaches him to respect distance. At length his little fingers acquire sufficient mechanical tact to lay hold of a spoon. He thrusts the instrument into his mouth, hurts his gums, and thus learns the impenetrability of matter. He lets the spoon fall, and jumpswith delight to 
hear it rattle against the table. The experiment made by accilent is repeated with intention, and thus the young student receives his first lessons upon sound and gravitation. There are pains and penalties, however, in the path of the enquirer: he is sure to go wrong, and Nature is just as sure to inform him of the fact. He falls down stairs, burns his fingers, cuts his hand, scalds his tongue, and in this way learns the conditions of his physical well being. This is Nature's way of proceeding, and it is wonderful what progress her pupil makes. His er:joyments for a time are physical, and the confectioner's shop occupies the foreground of human happiness; but the blossoms of a finer life are already beginning to unfold themselves, and the relation of cause and effect dawns upon the boy. He begin to see that the present condition of things is not final, but depends upon one that has gone before, and will be succeederl by another. He becomes a puzzle to himself; and to satisfy his newly awakened curiosity, asks all manner of inconvenient questions. The needs and tendencies of human nature express themselves through these early yearnings of the child. As thought ripens, he desires to know the character and causes of the phenomena presented to his observation; and unless this desire has been granted for the express purpose of having it repressed, unless the attractions of natural phenomena be like the blush of the forbidden fruit, conferred merely for the purpose of exercising our self-denial in letting them alone; we may fairly claim for the study of Physics the recognition that it answers to an impulse implanted by nature in the constitution of man.

A few days ago, a master of arts, who is still a young man, and therefore the recipient of a modern education, stated to me that until he had reached the age of twenty years he had never been taught anything whatever regarding natural phenomena, or natural law. Twelve years of his life previously had been spent exclusively among the ancients. 'The case, I regret to say, is typical. Now, we cannot, without prejudice to humanity, separate the present from the past. Thie nineteenth century strikes its roots into the centuries gone by, and draws nutriment from them. 'The world cannot afford to lose the record of any great deed or utterance; for such are prolific through- 
out all time. We cannot yield the companionship of our loftier brothers of antiquity-of our Socrates and Catowhose lives provoke us to sympathetic greatness across the interval of two thousand years. As long as the ancient languages are the means of access to the ancient mind, they must ever be of priceless value to humanity; but surely these avenues might be kept open without making such sacrifices as that above referred to, universal. We have conquered and possessed ourselves of continents of land, concerning which antiquity knew nothing; and if new continents of thought reveal themselves to the exploring human spirit, shall we not possess them also? In these latter days, the study of Physics has given us glimpses of the methods of Nature which were quite hidden from the ancients, and we should be false to the trust committed to us, if we were to sacrifice the hopes and aspirations of the present out of deference to the past.

The bias of my own education probably manifests itself in a desire I always feel to seize upon every possible opportunity of checking my assumptions and conclusions by experience. In the present case, it is true, your own consciousness might be appealed to in proof of the tendency of the human mind to inquire into the phenomena presented to it by the senses; but I trust you will excuse me if, instead of doing this, I take advantage of the facts which have fallen in my way through life, referring to your judgment to decide whether such facts are truly representative and general, and not merely individual and local.

At an agricultural college in Hampshire, with which I was connected for some time, and which is now converted into a school for the general education of youth, a society was formed among the boys, who met weekly for the purpose of reading reports and papers upon various subjects. The society had its president and treasurer; and abstracts of its proceedings were published in a little monthly periodical issuing from the school press. One of the most remarkable features of these weekly meetings was, that after the general business had been concluded, each member enjoyed the right of asking questions on any subject on which he desired information. 'The questions were either written out previously in a book, or, if a questiou happened to suggest itself during the meeting, it was 
written upon a slip of paper and handed in to the secretary, who afterward read ali the questions aloud. A number of teachers were usually present, and they and the boys made a common stock of their wisdom in furuishing replies. As might be expected from an assemblage of eighty or ninety boys, varying from eighteen to eight years old, many odd questions were proposed. To the mind which loves to detect in the tendencies of the young the instincts of humanity generally, such questions are not without a certain philosophic interest, and I have therefore thonght it not derogatory to the present course of lectures to copy a few of them, and to introduce them here. They run as follows:

What are the duties of the astronomer royal?

What is frost?

Why are thunder and lightning more frequent in summer than in winter?

What occasions falling stars?

What is the cause of the sensation called "pins and needles ?"

What is the cause of waterspouts?

What is the cause of hiccough?

If a towel be wetted with water, why does the wet portion become darker than before?

What is meant by Lancashire witches?

Does the dew rise or fall?

What is the principle of the hydraulic press?

Is there more oxygen in the air in summer than in winter?

What are those rings which we see round the gas and sun?

What is thunder?

How is it that a black hat can be moved by forming round it a magnetic circle, while a white hat remains stationary?

What is the cause of perspiration?

Is it true that men were once monkeys?

What is the difference between the soul and the mind?

Is it contrary to the rules of vegetarianism to eat eggs?

In looking over these questions, which were wholly unprompted, and have been copied almost at random from the book alluded to, we see that many of thein are suggested directly by natural objects, and are not such as had 
an interest conferred on them by previous culture. Now the fact is beyond the boy's control, and so certainly is the desire to know its cause. The sole question then is, whether this desire is to be gratified or not. Who created the fact? Who implanted the desire? Certainly not man. Who then will undertake to place himself between the desire and its fulfillment, and proclaim a divoree between them? 'Take, for example, the case of the wetted towel, which at first sight appears to be one of the most unpromising questions in the list. Shall we tell the proposer to repress his curiosity, as the subject is improper for him to know, and thus interpose our wisdom to rescue the boy from the consequences of a wish which acts to his prejudice? Or, recognizing the propriety of the question, how shall we answer it? It is impossible to answer it without reference to the laws of optics-without making the boy to some extent a natural philosopher. You may say that the effect is due to the reflection of light at the common surface of two media of different refractive indices. But this answer presupposes on the part of the boy a knowledge of what reflection and refraction are, or reduces you to the necessity of explaining them.

On looking more closely into the matter, we find that our wet towel belongs to a class of phenomena which have long excited the interest of philosophers. The towel is white for the same reason that snow is white, that foam is white, that pounded granite or glass is white, and that the salt we use at table is white. On quitting one medium and entering another, a portion of light is always reflected, but on this condition-the media must possess different refractive indices. 'Thus, when we immerse a bit of glass in water, light is reflected from the common surface of both, and it is this light which enables us to see the glass. But when a transparent solid is immersed in a liquid of the same refractive index as itself, it immediately disappears. I remember once dropping the eyeball of an ox into water; it vanished as if by magic, with the exception of the crystalline lens, and the surprise was so great as to cause a bystander to suppose that the vitreous humor had been instantly dissolved. This, however, was not the case, and a comparison of the refractive index of the humor with that of water cleared up the whole matter. 'I'he indices were identical, and hence the light pursued its way through both as if they formed one continuous mass. 
In the case of snow, powdered quartz, or salt, we have a transparent solid mixed with air. At every transition from solid to air, or from air to solid, a portion of light is reflected, and this takes place so often that the light is wholly intercepted. Thus from the mixture of two transparent bodies we obtain an opaque one. Now the case of the towel is precisely similar. 'The tissue is composed of semi-transparent regetable fibers, with the interstices between them filled with air; repeated reflection takes place at the limiting surfaces of air and fiber, and hence the towel becomes opaque like snow or salt. But if we fill the interstices with water, we diminish the reflection; a portion of the light is transmitted, and the darkness of the towel is due to its increased transparency. Thus the deportment of various minerals, such as hydrophane and tahasheer, the transparency of tracing paper used by engineers, and many other consiclerations of the highest scientific interest, ure involved in the simple inquiry of this nnsuspecting little boy.

Again, take the question regarding the rising or falling of the dew - a question long agitated, and finally set at rest by the beautiful researches of Wells. I do not think that any boy of average intelligence will be satisfied with the simple answer that the dew falls. He will wish to learn how you know that it falls, and, if acquainted with the notions of the middle ages, he may refer to the opinion of Father Laurus, that a goose egg filled in the morning with dew and exposed to the sun, will rise like a balloon-a swan's egg being better for the experiment than a goose egg. It is impossible to give the boy a clear notion of the beautiful phenomenon to which his question refers, with. out first making him acquainted with the radiation and conduction of heat. 'Take, for example, a blade of grass, from which one of these orient pearls is depending. During the day the grass, and the earth beneath it, possess a certain amount of warmth imparted by the sun; during a serene night, heat is radiated from the surface of the grass into space, and to supply the loss, there is a flow of heat from the earth to the blade. Thus the blade loses heat by radiation, and gains heat by conduction. Now, in the case before $\mathrm{ns}$, the pow er of radiation is great, whereas the power of conduction is small; the consequence is that the blade loses more than it gains, and hence becomes more 
and more refrigerated. The light vapor floating around the surface so cooled is condensed upon it, and there accumulates to form the little pearly globe which we call a dewdrop.

Thus the boy finds the simple and homely fact which addressed his senses to be the outcome and flower of the deepest laws. 'The fact becomes, in a measure, sanctified as in object of thought, and invested for him with a beauty for evermore. He thus learns that things which, at first sight, seem to stand isolated and without apparent brotherhood in Nature are organically united, and finds the detection of such analogies a source of perpetual delight. 'I'o enlist pleasure on the side of intellectual performance is a point of the utmost importance; for the exercise of the mind, like that of the body, depends for its value upon the spirit in which it is accomplished. Every physician knows that something more than mere mechanical motion is comprehended under the idea of healthful exercise-that, indeed, being most healthful which makes us forget all ulterior ends in the mere enjoyment of it. What, for example, could be substituted for the action of the playground, where the boy plays for the mere love of playing, and without reference to physiological laws; while kindly Nature accomplishes her ends unconsciously, and makes his very indifference beneficial to him. You may have more systematic motions, you may devise means for the more perfect traution of each particular muscle, but you cannot create the joy and gladness of the game, and where these are absent, the charm and the health of the exercise are gone. The case is similar with the education of the mind.

The study of Physics, as already intimated, consists of two processes, which are complementary to each otherthe tracing of facts to their causes, and the logical adrance from the cause to the fact. In the former process, called induction, certain moral qualities come into play. The first condition of success is patient industry, an honest receptivity, and a willingness to abandon all preconceived notions, however cherished, if they be found to contradict the truth. Believe me, a self-renunciation which has something lofty in it, and of which the world never hears, is often enacted in the private experience of the true votary of science. And if a man be not capable of this self-renun- 
ciation-this loyal surrender of himself to Nature and to fact, he lacks, in my opinion, the first mark of a true philosopher. 'Thus the earnest prosecutor of science, who does not work with the idea of producing a sensation in the world, who loves the truth better than the transitory blaze of to-day's fame, who comes to his task with a single eye, finds in that task an indirect means of the highest moral culture. And although the virtue of the act depends upon its privacy, this sacrifice of self, this upright determination to accept the truth, no matter how it may present itself - even at the hands of a scientific foe, if necessarycarries with it its own reward. When prejulice is put under foot and the stains of personal bias have been washed away -when a man consents to lay aside his vanity and to become Nature's organ-his elevation is the instant consequence of his humility. I should not wonder if my remarks provoked a smile, for they seem to indicate that I regard the man of science as a heroic, if not indeed an angelic, character; and cases may occur to you which indicate the reverse. You may point to the quarrels of scientific men, at their struggles for priority, to that unpleasant egotism which screams around its little property of discovery like a scared plover about its young. I will not deny all this; but let it be set down to its proper account, to the weaknessor, if you will-to the selfishness of Man, but not to the charge of Physical Science.

'The second process in physical investigation is deduction, or the advance of the mind from fixed principles to the conclusions which flow fiom them. 'The rules of logic are the formal statement of this process, which, however, was practiced by every healthy mind before ever such rules were written. In the study of Physics, induction and deduction are perpetually wedded to each other. The man observes, strips facts of their peculiarities of form, and tries to unite them by their essences; having effected this, he at once deduces, and thus checks his induction. Here the grand difference between the methods at present followed, and those of the ancients, becomes manifest. 'They were one-sided in these matters: they omitted the process of induction, and substituted conjecture for observation. 'Ihey could never, therefore, fulfill the mission of Man to "replenish the earth, and subdue it." 'The subjugation of Nature is only to be accomplished by the penetration of 
her secrets and the patient mastery of her laws. This not only enables us to protect ourselves from the hostile action of natural forces, but makes them our slaves. By the study of Physics we liave indeed opened to us treasuries of power of which antiquity never dreamed. But while we lorl it over Matter, we have thereby become better acquainted with the laws of Mind; for to the mental philosopher the study of Physics furnishes a screen against which the human spirit projects its own image, and thus becomes capable of self-inspection.

'Thus, then, as a means of intellectual culture, the study of Physics exercises and sharpens observation: it brings the most exhaustive logic into play: it compares, abstracts, and generalizes, and provides a mental scenery appropriate to these processes. The strictest precision of thought is everywhere enforced, and prudence, foresight, and sagacity are demanded. By its appeals to experiment, it continually checks itself, and thus walks on a foundation of facts. Hence the exercise it invokes does not end in a mere game of intellectual gymnastics, such as the ancients delighted in, but tends to the mastery of Nature. This gradual conquest of the external world, and the consciousness of angmented strength which accompanies it, render the study of Physics as delightful as it is importart.

With regard to the effect on the imagination, certain it is that the cool results of physical induction furnish conceptions which transcend the most daring flights of that faculty. 'Take for example the idea of an all-pervading ether which transmits a tingle, so to speak, to the finger ends of the universe every time a street lamp is lighted. The invisible billows of this ether can be measured with the same ease and certainty as that with which an engineer measures a base and two angles, and from these finds the distance across the Thames. Now it is to be confessed that there may be just as little poetry in the measurement of an ethereal undulation as in that of the river; for the intellect, during the acts of measurement and calculation, destroys those notions of size which appeal to the poetic sense. It is a mistake to suppose, with Dr. Young, that

An undevout astronomer is mad;

there being no necessary connection between a devout state of mind and the observations and calculations of a 
practical astronomer. It is not until the man withdraws from his calculation, as a painter from his work, and thus realizes the great idea on which he has been engaged, that imagination and wonder are excited. There is, 1 admit, a possible danger here. If the arithmetical processes of science be too exclusively pursued, they may impair the imagination, and thus the study of Physics is open to the same objection as philological, theological, or political studies, when carried to excess. But even in this case, the injury done is to the investigator himself: it does not reach the mass of mankind. Indeed, the conceptions furnished by his cold, unimaginative reckonings may furnish themes for the poet, and excite in the highest degree that sentiment of wonder which, notwithstanding all its foolish vagaries, tableturning included, I, for my part, should be sorry to see banished from the world.

I have thus far dwelt upon the study of Physies as an agent of intellectual culture; but like other things in Nature, this study subserves more than a single end. The colors of the clouds delight the eye, and, no doubt, accomplish moral purposes also, but the self-same clouds hold within their fleeces the moisture by which our fields are rendered fruitful. The sunbeams excite our interest and invite our investigation; but they also extend their beneficent influences to our fruits and corn, and thus accomplish not only intellectual ends, but minister, at the same time, to our material necessities. And so it is with scientific research. While the love of science is a sufficient incentive to the pursuit of science, and the investigator, in the prosecution of his inquiries, is raised above all material considerations, the results of his labors may exercise a potent influence upon the physical condition of the community. This is the arrangement of Nature, and not that of the scientific investigator himself; for he usually pursues his object without regard to its practical applications.

And let him who is dazzled by such applications-who sees in the steam-engine and the electric telegraph the highest embodiment of human genius and the only legitimate object of scientific research, beware of jrescribing conditions to the investigator. Let him beware of attempting to substitute for that simple love with which the votary of science pursues his task, the calculations of what he is pleased to call utility. 'The professed utilitarian is unfor- 
tunately, in most cases, the very last man to see the occult sources from which useful results are derived. He admires the flower, but is ignorant of the conditions of its growth. The scientific man must approach Nature in his own way; for if you invade his freedom by your so-called practical considerations, it may be at the expense of those qualities on which his success as a discoverer depends. Let the selfstyled practical man look to those from the fecundity of whose thoughts he, and thousands like him, have sprung into existence. Were they inspired in their first inquiries by the calculations of utility? Not one of them. 'They were often forced to live low and lie hard, and to seek compensation for their penury in the delight which their favorite pursuits afforded them. In the words of one well qualified to speak upon this subject, "I say not merely look at the pittance of men like John Dalton, or the voluntary starvation of the late Graff; but compare what is considered as competency or affluence by your Faradays, Liebigs, and Herschels, with the expected results of a life of successful commercial enterprise: then compare the amount of mind put forth, the work done for society in either case, and you will be constrained to allow that the former belong to a class of workers who, properly speaking, are not paid, and cannot be paid for their work, as indeed it is of a sort to which no payment could stimulate."

But while the scientific investigator, standing upon the frontiers of human knowledge, and aiming at the conquest of fresh soil from the surrounding region of the unknown, makes the discovery of truth his exclusive object for the time, he cannot but feel the deepest interest in the practical application of the truth discovered. 'There is something enmobling in the triumph of Mind over Matter. A part even from its uses to society, there is something elevatiug in the idea of Man having tamed that wild force which flashes through the telegraphic wire, and made it the minister of his will. Our attainments in these directions appear to be commensurate with our needs. We had alrealy subdued horse and mule, and obtained from them all the service which it was in their power to render: we must either stand still, or find more potent agents to execute our purposes. At this point the steam-engine appears. These are still new things; it is not long since we struck into the scientific methods which have produced these results. We 
cannot for an instant regard them as the final achievements of Science, but rather as an earnest of what she is yet to do. They mark our first great advances upon the dominion of Nature. Animal strength fails, but here are the forces which hold the world together, and the instincts and successes of Man assure him that these forces are his when he is wise enough to command them.

As an instrument of intellectual culture, the study of Physics is profitable to all; as bearing upon special functions, its value, though not so great, is still more tangible. Why, for example, should members of parliament be ignorant of the subjects concerning which they are called upon to legislate? In this land of practical physics, why should they be unable to form an independent opinion upon a physical question? Why should the nember of a parliamentary committee be left at the mercy of interested disputants when a scientific question is discussed, until he deems the nap a blessing which rescues him from the bewilderments of the committee-room? 'I'he education which does not supply the want here referred to, fails in its duty to England. With regard to our working people, in the ordinary sense of the term working, the study of Physics would, I imagine, be profitable, not only as a means of intellectual culture, but also as a moral influence to woo them from pursuits which now degrale them. A man's reformation oftener depends upon the indirect, than upon the direct action of the will. The will must be exerted in the choice of employment which shall break the force of temptation by erecting a barrier against it. The drunkard, for example, is in a perilous condition if he content himself merely with saying, or swearing, that he will avoid strong drink. His thoughts, if not attracted by another force, will revert to the public house, and to rescue him permanently from this, you must give him an equivalent.

By investing the objects of hourly intercourse with an interest which prompts reflection, new enjoyments would be opened to the workingman, and every one of these would be a point of force to protect him against temptation. Besides this, our factories and our foundries present an extensive field of observation, and were those who work in them rendered capable, by previous culture, of observing what they see, the results might be incalculable. Who 
can say what intellectual Samsons are at the present moment toiling with closed eyes in the mills and forges of Manchester and Birmingham? Grant these Samsons sight, and you multiply the chances of discovery, and with them the prospects of national advancement. In our multitudinous technical operations we are constantly playing with forces our ignorance of which is often the cause of our destruction. There are agencies at work in a iocomotive of which the maker of it probably never dreamed, but which nevertheless may be sufficient to convert it into an engine of death. When we reflect on the intellectual condition of the people who work in our coal mines, those terrific explosions which occur from time to time need not astonish us. If these men possessed sufficient physical knowledge, from the operatives themselves would probably emanate a system by which these shocking accidents might be avoided. Possessed of the knowledge, their personal interests would furnish the necessary stimulus to its practical application, and thus two ends would be served at the same time-the elevation of the men and the diminution of the calamity.

Before the present Course of Lectures was publicly announced, I had many misgivings as to the propriety of my taking a part in them, thinking that my place might be better filled by an older and more experienced man. To my experience, however, such as it was, I resolved to adhere, and I have therefore describer things as they revealed themselves to my own eyes, and have been enacted in my own limited practice. There is one mind common to us all; and the true expression of this mind, even in small particulars, will attest itself by the response which it calls forth in the convictions of my hearers. I ask your permission to proceed a little further in this fashion, and to refer to a fact or two in addition to those already cited, which presented themselves to my notice during my brief career as a teacher in the college already alluded to. The facts, though extremely humble, and deviating in some slight degree from the strict subject of the present discourse, may yet serve to illustrate an educational principle.

One of the duties which fell to my share was the instruction of a class in mathematics, and I usually found that Euclid and the ancient geometry generally, when properly and sympathetically addressed to the understand- 
ing, formed a most attractive study for youth. But it was my habitual practice to withrdraw the boys from the routine of the book, and to appeal to their self-power in the treatment of questions not comprehended in that routine. At first, the change from the beaten track usually excited aversion: the youth felt like a child amid strangers; but in $n$ o single instance did this feeling continue. When utterly disheartened, I have encouraged the boy by the anecdote of Newton, where he attributes the difference between him and other men mainly to his own patience; or of Mirabeau, when he ordered his servant, who had stated something to be impossible, never again to use that blockhead of a word. 'Thus cheered, the boy has returned to his task with a smile, which perhaps had something of doubt in it, but which, nevertheless, evinced a resolution to try again. I have seen his eye brighten, and, at length, with a pleasure of which the ecstasy of Archimedes was but a simple expansion, heard him exclaim, "I have it, sir." 'The consciousness of self-power, thus awakened, was of immense value; and, animated by it, the progress of the class was astonishing. It was often my custom to give the boys the choice of pursuing their propositions in the book, or of trying their strength at others not to be found there. Never in a single instance was the book chosen. I was ever ready to assist when help was needful, but my offers of assistance were habitually declined. 'The boys had tasted the sweets of intellectual conquest and demanded victories of their own. 'Their diagrams were scratched on the walls, cut into the beams upon the playground, and numberless other illustrations were afforded of the living interest they took in the subject. For my own part, as far as experience in teaching goes, I was a mere fledgling-knowing nothing of the rules of pedagogics, as the Germans name it; but adhering to the spirit indicated at the commencement of this discourse, and endeavoring to make geometry a means rather than a branch of education. 'The experiment was successful, and some of the most delightful hours of my existence have been spent in marking the vigorous and cheerful expansion of mental power, when appealed to in the manner here described.

Our pleasure was enhanced when we applied our mathematical knowledge to the solution of physical problems. Many objects of hourly contact had thus a new interest and 
significance imparted to them. The swing, the see-saw, the tension of the giant-stride ropes, the fall and rebound of the football, the advantage of a small boy over a large one when turning short, particularly in slippy weather; all became subjects of investigation. A lady stands before a looking-glass, of her own height; it was required to know how much of the glass was really useful to her? We learned with pleasure the economic fact that she might dispense with the lower half and see her whole figure notwithstanding. It was also pleasant to prove by mathematics, and verify by experiment, that the angulai velocity of a reflected beam is twice that of the mirror which reflects it. From the hum of a bee we were able to determine the number of times the insect flaps its wings in a second. Following up our researches upon the pendulum, we learned how Colonel Sabine had made it the means of determining the figure of the earth; and we were also startled by the inference which the pendulum enabled us to draw, that if the diurnal velocity of the earth were seventeen times its present amount, the centrifugal force at the equator would be precisely equal to the force of gravitation, so that an inhabitant of those regions would then have the same tendency to fall upward as downward. All these things were sources of wonder and delight to us: and when we remembered that we were gifted with the powers which had reached such results, and that the same great field was ours to work in, our hopes arose that at some future day we might possibly push the subject a little further, and add our own victories to the conquests already won.

I ought to apologize to you for dwelling so long upon this subject; but the days spent among these young philosophers made a deep impression on me. I learned among them something of myself and of human nature, and obtained some notion of a teacher's vocation. If there be one profession in England of paramount importance, I believe it to be that of the schoolmaster; and if there be a position where selfishness and incompetence do most serious mischief, by lowering the moral tone and exciting irreverence and cunning where reverence and noble truthfulness ought to be the feelings evoked, it is that of the principal of a school. When a man of enlarged heart and mind comes among boys--when he allows his spirit to 
stream through them, and observes the operation of his own character evidenced in the elevation of theirs-it would be idle to talk of the position of such a man being honorable. It is a blessed position. 'The man is a blessing to himself and to all around him. Such men, I believe, are to be found in England, and it behoves those who busy themselves with the mechanics of education at the present day, to seek them out. For no matter what means of culture may be chosen, whether physical or philological, success must ever mainly depend upon the amount of life, love, and earnestness, which the teacher himself brings with him to his vocation.

Let me again, and finally, remind you that the claims of that science which fiuds in me to-rlay its unripened advocate, are those of the logic of Nature upon the reason of her child-that its disciplines, as an agent of culture, are based upon the natura' relations subsisting between Man and the universe of which he forms a part. On the one side, we have the apparently lawless shifting of phenomena; on the other side, mind, which requires law for its equilibrium, and through its own indestructible instincts, as well as through the teachings of experience, knows that these phenomena are reducible to law. To chasten this apparent chaos is a problem which man has set before him. The world was built in order: and to us are trusted the will and power to discern its harmonies, and to make them the lessons of our lives. From the cradle to the grave we are surrounded with objects which provoke inquiry. Descending for a moment from this high plea to considerations which lie closer to us as a nationas a land of gas and furuaces, of steam and electricity: as a land which science, practically applied, has made great in peace and mighty in war: I ask you whether this "land of old and just renown" has not a right to expect from her institutions a culture more in accordance with her present needs than that supplied by declension and conjugation? And if the tendency should be to lower the estimate of science, by regarding it exclusively as the instrument of material prosperity, let it be this high mission of our universities to furnish the proper counterpoise by pointing out its nobler uses-lifting the national mind to the contemplation of it as the last development of that "increasing purpose" which runs through the ages and widens the thoughts of men. 


\section{CHAP'IER XII.}

\section{ON CRYSTALLINE AND SLATY CLEAVAGE。*}

WHEN the student of physical science has to investigate the character of any natural force, his first care must be to purify it from the mixture of other forces, and thus study its simple action. If, for example, he wishes to know how a mass of liquid would shape itself if at liberty to follow the bent of its own molecular forces, he must see that these forces have free and undisturbed exercise. We might perhaps refer him to the dewdrop for a solution of the question; but here we have to do, not only with the action of the molecules of the liquid upon each other, but also with the action of gravity upon the mass, which pulls the drop downward and elongates it. If he would examine the problem in its purity, he must do as Plateau has done, detach the liquid mass from the action of gravity; he would then find the shape to be a perfect sphere. Natural processes come to us in a mixed manner, and to the uninstructed mind are a mass of unintelligible confusion. Suppose half a dozen of the best musical performers to be placed in the same room, each playing his own instrument to perfection, but no two playing the same tune; though each individual instrument might be a source of perfect music, still the mixture of all would produce mere noise. Thus it is with the processes of nature, where mechanical and molecular laws intermingle and create apparent confusion. Their mixture constitutes what may be called the noise of natural laws, and it is the vocation of the man of science to resolve this noise into its components, and thus to detect the underlying music.

The necessity of this detachment of one force from all other forces is nowhere more strikingly exhibited than in the phenomena of crystallization. Here, for example, is a solution of common sulphate of soda or Glauber salt. Looking into it mentally, we see the molecules of that liquid, like disciplined squadrons under a governing eye, arrunging themselves into battalions, gathering round distinct centers, and forming themselves into solid masses,

* From a discourse delivered in the Royal Institution of Great Britain, June 6, 1856. 
which after a time assume the visible shape of the crystal now held in my hand. I may, like an ignorant meddler wishing to hasten matters, introduce confusion into this order. This may be done by plunging a glass rod in to the vessel; the consequent action is not the pure expression of the crystalline forces; the molecules rush together with the confusion of an unorganized mob, and not with the stealy accuracy of a disciplined host. In this mass of bismuth also we have an example of confused crystallization; but in the crucible behind me a slower process is going on: here there is an architect at work "who makes no chips, no din," and who is now building the particles into crystals, similar in shape and structure to those beautiful masses which we see upon the table. By permitting alum to crystallize in this slow way, we obtain these perfect octahedrons; by allowing carbonate of lime to crystallize, nature produces these beatiful rhomboids; when silica crystallizes, we have formed these hexagonal prisms capped at the ends by pyramids; by allowing saltpeter to crystallize we have these prismatic masses, and when carbon crystallizes, we have the diamond. If we wish to obtain a perfect crystal we must allow the molecular forces free play; if the crystallizing mass be permitted to rest upon a surface it will be flattened, and to prevent this a small crystal must be so suspended as to bə surrounded on all sides by the liquid, or, if it rest upon the surface, it must be turned daily so as to present all its faces in succession to the working builder.

In building up crystals these little atomic bricks often arrange themselves into layers which are perfectly parallel to each other, and which can be separated by mechanical means; this is called the cleavage of the crystal. The crystal of sugar I hold in my hand has thus far escaped the solvent and abrading forces which sooner or later determine the fate of sugar-candy. I readily discover that it cleaves with peculiar facility in one direction. Again I lay my knife upon this piece of rocksalt, and with a bluw cleave it in one direction. Laying the knife at right angles to its former position, the crystal cleaves again; and finally placing the knife at right angles to the two former positions, we find a third cleavage. Rocksalt cleaves in three directions and the resulting solid is this perfect cube, which may be brokeu up into any number of smaller cubes. 
Iceland spar also cleaves in three directions, not at right angles, but oblique to each other, the resulting solid being a rhomboid. In each of these cases the mass cleaves with equal facility in all three directions. For the sake of completeness I may say that many crystals cleave with unequal facility in different directions: heavy spar presents an example of this kind of cleavage.

'Turn we now to the consideration of some other phenom. ena to which the term cleavage may be applierl. Beech, deal, and other woods cleave with facility along the fiber, and this cleavage is most perfect when the edge of the axe is laid across the rings which mark the growth of the tree. If you look at this bundle of hay severed from a rick, you wili see a sort of cleavage in it also; the stalks lie in horizontal planes, and only a small force is required to separate them laterally. But we cannot regard the cleavage of the tree as the same in character as that of the hayrick. In the one case it is the molecules arranging themselves according to organic laws which produce a cleavable structure, in the other case the easy separation in one direction is due to the mechanical arrangement of the coarse sensible stalks of hay.

'This sandstone rock was once a powder held in mechanical suspension by water. 'The powder' was composed of two distinct parts, fine grains of sand and small plates of mica. Imagine a wide strand covered by a tide, or an estuary with water which holds such powder in suspension: how will it sink? 'The rounded grains of sand will reach the bottom first, because they encounter least resistance, the mica afterward, and when the tide recedes we have the little plates shining like spangles upon the surface of the sand. Each successive tide brings its charge of mixed powder, deposits its duplex layer day after day, and finally masses of immense thickness are piled up, which by preserving the alternations of sand and mica, tell the tale of their formation. 'Take the sand and mica, mix them together in water, and allow them to subside; they will arrange themselves in the manner indicated, and by repeating the process you can actually build up a mass which shall be the exact counterpart of that presented by nature. Now this structure cleaves with readiness along the planes in which the particles of mica are strewn. Specimens of such a rock sent to me from Halifax, and other masses from the 
quarries of Over Darwen in Lancashire, are here before you. With a hammer and chisel I can cleave them into flags; indeed these flags are employed for roofing purposes in the districts from which the specimens have come, and receive the name of "slatestone." But you will discern without a word from me, that this cleavage is not a crystalline cleavage any more than that of a hayrick is. It is molar, not molecular.

This, so far as I am aware of, has never been imagined, and it has been agreed among geologists not to call such splitting as this cleavage at all, but to restrict the term to a phenomenon of a totally different character.

'Those who have visited the slate quarries of Cumberland and North Wales will have witnessed the phenomenon to which 1 refer. We have long drawn our supply of roofingslates from such quarries; schoolboys ciphered on these slates, they were used for tombstones in churchyards, and for billiard-tables in the metropolis; but not until a comparatively late period did men begin to inquire how their wonderful structure is produced. What is the agency which enables us to split Honister Crag, or the cliffs of Snowdon, into laminæ from crown to base? 'This question is at the present moment one of the great difficulties of geologists, and occupies their attention perhaps more than any other. You may wonder at this. Looking into the quarry of Penrhyn, you may be disposed to offer the explanation I heari given two years ago. "These planes of cleavage," said a friend who stood beside me on the quarry's edge, "are the planes of stratification which have been lifted by some convulsion into an almost vertical position." But this was a mistake, and indeed here lies the grand difficulty of the problem. The planes of cleavage stand in most cases at a ligh angle to the bedding. Thanks to Sir Roderick Murchison, I am able to place the proof of this before you. Here is a specimen of slate in which both the planes of eleavage and of bedding are distiuctly marked, one of them making a large angle with the other. This is common. The cleavage of slates then is not a question of stratification; what then is its cause?

In an able and elaborate essay published in 1835, Professor Sodgwick proposed the theory that cleavage is due to the action of crystalline or polar forces subsequent to the consolidation of the rock. "We may affirm," he says, 
"that no retreat of the parts, no contraction of dimensions in passing to a solid state, can explain such phenomena. They appear to me only resolvable on the supposition that crystalline or polar forces acted upon the whole mass simultaneously in one direction and with adequate force." And again, in another place: "Crystalline forces have rearranged whole mountain masses, producing a beautiful crystalline cleavage, passing alike through all the strata."** 'The utterance of such a man struck deep, as it ought to do, into the minds of geologists, and at the present day there are few who do not entertain this view, either in whole or in part. $\nmid$ The boldness of the theory, indeed, has, in some cases, caused speculation to run riot, and we have books published on the action of polar forces and geologic magnetism, which rather astonish those who know something about the subject. According to this theory whole districts of North Wales and Cumberland, mountains included, are neither more nor less than the parts of a gigantic crystal. These masses of slate were originally fine mud, composed of the broken and abraded particles of older rocks. They contain silica, alumina, potash, soda, and mica mixed mechanically together. In the course of ages the mixture became consolidated, and the theory before us assumes that a process of crystallization afterward rearranged the particles and developed in it a single plane of cleavage. Though a bold, and I think inadmissible, stretch of analogies, this hypothesis has done good service. Right or wrong, a thoughtfully uttered theory has a dynamic power which operates against intellectual stagnation; and even by provoking opposition is eventually of service to the cause of truth. It would, however, have been remarkable if, among the ranks of geologists them-

* Transactions of the Geological Society, ser. ii., vol. iii., p. 477.

t In a letter to Sir Charles Lyell, dated from the Cape of Good Hope, February 20, 1836, Sir John Herschel writes as follows: "If rocks have been so heated as to allow of a commencement of crystallization, that is to say, if they have been heated to a point at which the particles can begin to move among themselves, or at least on their own axes, some general law must then determine the position in which these particles will rest on cooling. Probably that position will have some relation to the direction in which the heat escapes. Now when all or a majority of particles of the same nature have a general tendency to one position, that must of course determine a cleavage plane." 
selves, men were not found to seek an explanation of slatecleavage involving a less hardy assumption.

The first step in an inquiry oi this kind is to seek facts. 'This has been done, and the labors of Daniel Sharpe (the late president of the Geological Society, who, to the loss of science and the sorrow of all who knew him, has so suddenly been taken away from us), Mr. Henry Clifton Sorby. and others, have furnisined us with a body of facts associated with slaty cleavage, and having a most important bearing upon the question.

Fossil shells are found in these slate-rocks. I have here several specimens of such shells in the actual rock, and occupying various positions in regard to the cleavage planes. TThey are squeezed, distorted, and crushed; in all cases the distortion leads to the inference that the rock which contains these shells has been subjected to enormous pressure in a direction at right angles to the planes of cleavage. The shells are all flattened and spread out in these planes. Compare this fossil trilobite of normal proportions with these others which have suffered distortion. Some have lain across, some along, and some oblique to the cleavage of the slate in which they are found; but in all cases the distortion is such as required for its production a compressing force acting at right angles to the planes of cleavage. As the trilobites lay in the mud, the jaws of a gigantic vise appear to have closed upon them and squeezed them into the shapes you see.

We sometimes find a thin layer of coarse gritty material, between two layers of finer rock, through which and across the gritty layer pass the planes of lamination. The coarse layer is found bent by the pressure into sinuosities like a contorted ribbon. Mr. Sorby has described a striking case of this kind. 'This crumpling can be experimentally imitated; the amount of compression might, moreover, be roughly estimated by supposing the contorted bed to be stretched out, its length measured and compared with the shorter distance into which it has been squeezed. We find in this way that the yielding of the mass has been considerable.

Let me now direct your attention to another proof of pressure; you see the varying colors which indicate the berlding on this mass of slate. 'The dark portion is gritty, being composed of comparatively coarse particles, which, 
owing to their size, shape and gravity, sink first and constitute the bottom of each layer. Gradually, from botton. to top the coarseness diminishes, and near the upper surface we have a layer of exceedingly fine grain. It is the fine mud thus consolidated from which are derived the German razol-stones, so much prized for the sharpening of surgical instruments. When a bed is thin, the fine-grain slate is permitted to rest upon a slab of the coarse slate in contact with it; when the fine bed is thick, it is cut into slices which are cemented to pieces of ordinary slate, and thus rendered stronger. The mud thus deposited is, as might be expected, often rolled up into nodular masses, carried forward, and deposited among coarser material by the rivers from which the slate-mud has subsided. Here are such nodules enclosed in sandstone. Everybody, moreover, who has ciphered upon a school-slate must remember the whitish-green spots which sometimes dotted the surface of the slate, and over which the pencil usually slid as if the spots were greasy. Now these spots are composed of the finer mud, and they could not, on account of their fineness, bite the pencil like the surrounding gritty portions of the slate. Here is a beautiful example of these spots: you observe them, on the cleavage surface, in broad round patches. But turn the slate edgeways and the section of each nodule is seen to be a sharp oval with its longer axis parallel to the cleavage. This instructive fact has been adduced by Mr. Sorby. I have made excursions to the quarries of Wales and Cumberland, and to many of the slate yards of London, and found the fact general. Thus we elevate a common experience of our boyhood into evidence of the highest significance as regards a most important geological problem. From the magnetic deportment of these slates, I was led to infer that these spots contain a less amount of iron than the surrounding dark slate. An analysis was made for me by Mr. Hambly in the laboratory of Dr. Percy at the School of Mines with the following result:

ANALYsis of SLATE.

Dark Slate, two analyses.

1. Percentage of iron

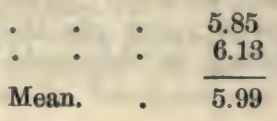


Whitish Green Slate.
1. Percentage of iron
3.24
2.

\begin{tabular}{lll}
- & $\quad$ & 3.24 \\
Mean . & 3.12 \\
\hline & 3.18
\end{tabular}

According to these analyses the quantity of iron in the dark slate immediately adjacent to the greenish spot is nearly double the quantity contained in the spot itself. This is about the proportion which the magnetic experiments suggested.

Let me now remind you that the facts brought before you are typical - each is the representative of a class. We have seen shells crtished, the trilobites squeezed, beds contorted, nodules of greenish marl flattened; and all these sources of independent testimony point to one and the same conclusion, namely, that slate-rocks have been subjected to enormous pressure in a direction at right angles to the planes of cleavage.

In reference to Mr. Sorby's contorted bed, I have said that by supposing it to be stretched out and its length measured, it would give us an idea of the amount of yielding of the mass above and below the bed. Such a measurement, however, would not give the exact amount of yielding. I hold in my hand a specimen of slate with its bedding marked upon it; the lower portions of each layer being composed of a comparatively coarse gritty material something like what you may suppose the contorted bed to be composed of. Now, in crossing these gritty portions, the cleavage turns, as if tending to cross the bedding at another angle. When the pressure began to act, the intermediate bed, which is not entirely unyielding, suffereu longitudinal pressure; as it bent, the pressure became gralually more transverse, and the direction of its cleavage is exactly such as you would infer from an action of this kind-it is neither quite across the bed, nor yet in the same direction as the cleavage of the slate above and below it, but intermediate between both. Supposing the cleavage to be at right angles to the pressure, this is the direction which it ought to take across these more unyielding strata.

Thus we have established the concurrence of the phenomeua of cleavage and pressure-that they accompany each other; but the question still remains, Is the pressure suffi- 
cient to account for the cleavage? A single geologist, as far as I am aware, answers boldly in the affirmative. 'This geologist is Sorby, who has attacked the question in the true spirit of a physical investigator. Call to mind the cleavage of the flags of Halifax and Over Darwen, which is callsed by the interposition of layers of mica between the gritty strata. Mr. Sorby finds plates of mica to be also a constituent of slate-rock. He asks himself, what will be the effect of pressure upon a mass containing such plates confusedly mixed up in it? It will be, he argues, and he argues rightly, to place the plates with their flat surfaces more or less perpendicular to the direction in which the pressure is exerted. He takes scales of the oxide of iron, mixes them with a fine powder, and on squeezing the mass finds that the tendency of the scales is to set themselves at right angles to the line of pressure. Along the planes of weakuess produced by the scales the mass cleaves.

By tests of a different character from those applied by Mr. Sorby, it might be shown how true his conclusion isthat the effect of pressure on elongated particles, or plates, will be such as he describes it. But while the scales must be regarded as a true cause, I should not ascribe to them a large share in the production of the cleavage. I believe that even if the plates of mica were wholly absent, the cleavage of slate-rocks would be much the same as it is at present.

Here is a mass of pure white wax; it contains no mica particles, no scales of iron, or anything analogous to them. Here is the selfsame substance submitted to pressure. I would invite the attention of the eminent geologists now before me to the structure of this wax. No slate ever exhibited so clean a cleavage; it splits into laminæ of surpassing tenuity, and proves at a single stroke that pressure is sufficient to produce cleavage, and that this cleavage is independent of intermixed plates or scales. I have purposely mixed this wax with elongated particles, and am unable to say at the present moment that the cleavage is sensibly affected by their presence-if anything, I should say they rather impair its fineness and clearness than promote it.

'The finer the slate is the more perfect will be the resemblance of its cleavage to that of the wax. Compare the surface of the wax with the surface of this slate from Bor- 
rodale in Cumberland. You have precisely the same features in both; you see flakes clinging to the surfaces of each, which have been partially torn away in cleaving. Let any close observer compare these two effects, he will, I am persuaded, be led to the conclusion that they are the product of a common cause.*

But you will ask me how, according to my view, does pressure produce this remarkable result? This may be stated in a very few words.

'There is no such thing in nature as a body of perfectly homogeneous structure. I break this clay which seems so uniform, and find that the fracture presents to my eyes innumerable surfaces along which it has given way, and it has yielded along those surfaces because in them the cohesion of the mass is less than elsewhere. I break this marble, and even this wax, and observe the same result; look at the mud at the bottom of a dried pond; look at some of the ungraveled walks in Kensington Gardens on drying after rain--they are cracked and split, and other circumstances being equal, they crack and split where the cohesion is a minimum. Take then a mass of partially consolidated mud. Such a mass is divided and subdivided by interior surfaces along which the sohesion is comparatively small. Penetrate the mass in idea, and you will see it composed of numberless irregular polyhedra bounded by surfaces of weak cohesion. Imagine such a mass subjected to pressure-it vields and spreads out in the direction of least resistance; $\nmid$ the little polyhedra become converted into laminæ, separated from each other by surfaces of weak cohesion, and the infallible result will be a tendency to cleave at right angles to the line of pressure.

* I have usually softened the wax by warming it, kneaded it with the fingers, and pressed it between thick plates of glass previously wetted. At the ordinary summer temperature the pressed wax is soft, and tears rather than cleaves; on this account I cool my compressed specimens in a mixture of pounded ice and salt, and when thus cooled they split cleanly.

+ It is scarcely necessary to say that if the mass were squeezed equally in all directions no laminated structure could be produced; it must have room to yield in a lateral direction. Mr. Warren De la Rue informs me that he once wished to obtain white-lead in a fine granular state, and to accomplish this be first compressed it. The mold was conical, and permitted the lead to spread out a little laterally. The lamination was as perfect as that of slate, and it quite defeated him in his effort to obtain a granular powder. 
Further, a mass of dried mud is full of cavities and fis. sures. If you break dried pipe-clay you see them in great numbers, and there are multitudes of them so small that you cannot see them. A flattening of these cavities must take place in squeezed mud, and this must to some extent facilitate the cleavage of the mass in the direction indicated.

Althongh the time at my disposal has not permitted me duly to develop these thoughts, yet for the last twelve months the subject has presented itself to me almost daily under one aspect or another. I have never eaten a biscuit during this period without remarking the cleavage developed by the rolling-pin. You have only to break a biscuit across, and to look at the fracture, to see the laminated structure. We have here the means of pushing the analogy further. I invite you to compare the structure of the slate, which was subjected to a high temperature during the conflagration of Mr. Scott Russell's premises, with that of a biscuit. Air or vapor within the slate has caused it to swell, and the mechanical structure it reveals is precisely that of a biscuit. During these inquiries I have received much instruction in the manufacture of puff-paste. Here is some such paste baked under my own superintendence. The cleavage of our hills is accidental cleavage, but this is cleavage with intention. The volition of the pastrycook has entered into its formation. It has been his aim to preserve a series of surfaces of structural weakness, along which the dough divides into layers. Puff-paste in preparation must not be handled too much; it ought, moreover, to be rolled on a cold slab, to perevent the butter from melting, and diffusing itself, thus rendering the paste more homogeneous and less liable to split. Puff-paste is, then, simply an exaggerated case of slaty cleavage.

The principle here enunciated is so simple as to be almost trivial; nevertheless, it embraces not only the cases mentioned, but, if time permitted, it might be shown you that the principle has a much wider range of application. When iron is taken from the puddling furnace it is more or less spongy, an aggregate in fact of small nodules: it is at a welding heat, and at this temperature is submitted to the process of rolling. Bright smooth bars are the result. But notwithstanding the high heat the nodules do not 
perfectly blend together. The process of rolling draws them into fibers. Here is a mass acted upon by dilute sulphuric acid, which exhibits in a striking manner this fibrous structure. The experiment was made by my friend Dr. Percy, without any reference to the question of cleavage.

Break a piece of ordinary iron and you have a granular fracture; beat the iron, you elongate these granules, and finally render the mass fibrous. Here are pieces of rails along which the wheels of locomotives have slidden; the granules have yielded and become plates. They exfoliate or come off in leaves; all these effects belong, I believe, to the great class of phenomena of which slaty cleavage forms the most prominent example.*

We have now reached the termination of our task. You have witnessed the phenomena of crystallization, and have had placed before you the facts which are found associated with the cleavage of slate rocks. Such facts, as expressed by Helmholtz, are so many telescopes to our spiritual vision, by which we can see backward through the night of antiquity, and discern the forces which have been in operation upon the earth's surface

\section{Ere the lion roared,}

Or the eagle soared.

From evidence of the most independent and trustworthy character, we come to the conclusion that these slaty masses have been subjected to enormous pressure, and by the sure method of experiment we have shown-and this is the only really new point which has been brought before you-how the pressure is sufficient to produce the cleavage. Expanding our field of view, we find the selfsame law, whose footsteps we trace amid the crags of Wales and Cumberland. extending into the domain of the pastrycook and ironfounder; nay, a wheel cannot roll over the half-dried mad of our streets without revealing to us more or less of the features of this law. Let me say, in conclusion, that the spirit in which this problem has been attacked by geologists, indicates the dawning of a new day for their science. 'The great intellects who have labored at geology, and who have raised it to its present pitch of grandeur, were compelled to

\footnotetext{
* For some further observations on this subject by Mr. Sorby and myself, see Philosophical Maguzine for August, 1856.
} 
deal with the subject in mass; they harl no time to look after details. But the desire for more exact knowledge is increasing; facts are flowing in which, while they leave untouched the intrinsic wonders of geology, are gradually supplanting by solid truths the uncertain speculations which beset the subject in its infancy. Geologists now aim to imitate, as far as possible, the conditions of nature, and to produce her results; they are approaching more and more to the domain of physics, and I trust the day will soon come when we shall interlace our friendly arms across the common boundary of our sciences, and pursue our respective tasks in a spirit of mutual helpfulness, encouragement and goodwill.

[I would now lay more stress on the lateral yielding, referred to in the note at the bottom of page 241 , accompanied as it is by tangential sliding, than I was prepared to do when this lecture was given. 'This sliding is, I think, the principal cause of the planes of weakness, both in pressed wax and slate rock. J. T. 18\%1.]

\section{CHAPTER XIII.}

\section{ON PARAMAgNeTIC AND DIAMAgNeTIC FORCES.*}

The Notion of an attractive force, which draws bodies toward the center of the earth, was entertained by Anaxagoras and his pupils, by Democritus, Pythagoras, and Kipicurus; and the conjectures of these ancients were renewed by Galileo, Huyghens, and others, who stated that bodies attract each other as a magnet attracts iron. Kepler applied the notion to bodies beyond the surface of the earth, and affirmed the extension of this force to the most distant stars. Thus it would appear, that in the attraction of iron by a magnet originated the conception of the force of gravitation. Nevertheless, if we look closely at the matter, it will be seen that the magnetic force possesses characters strikingly distinct from those of the force which holds the universe together. The theory of gravitation is, that every particle of matter attracts

* Abstract of a discourse delivered in the Royal Institution, February 1, 1856. 
every other particle; in magnetism also we have attraction, but we have always, at the same time, repulsion, the final effect being due to the difference of these two forces. A body may be intensely acted on by a magnet, and still no motion of translation will follow, if the repulsion be equal to the attraction. Previous to magnetization, a dipping needle, when its center of gravity is supported, stands accurately level; but, after magnetization, one end of it, in our latitude, is pulled toward the north pole of the earth. The needle, however, being suspender from the arm of a fine balance, its weight is found unaltered by its magnetization. In like manner, when the needle is permitted to float upon a liquid, and thus to follow the attraction of the north magnetic pole of the earth, there is no motion of the mass toward that pole. The reason is known to be, that although the marked end of the needle is attracted by the north pole, the unmarked end is repelled by an equal force, the two equal and opposite forces neutralizing each other.

When the pole of an ordinary magnet is brought to act upon the swimming needle, the latter is attracted-the reason being that the attracted end of the needle being nearer to the pole of the magnet than the repelled end, the force of attraction is the more powerful of the two. In the case of the earth, its pole is so distant that the length of the needle is practically zero. In like manner, when a piece of iron is presented to a magnet, the nearer parts are attracted, while the more distant parts are repelled; and because the attracted portions are nearer to the magnet than the repelled ones, we have a balance in favor of attraction. Here, then, is the special characteristic of the magnetic force, which distinguishes it from that of gravitation. The latter is a simple unpolal' force, while the former is duplex or polar. Were gravitation like magnetism, a stone would no more fall to the ground than a piece of iron toward the north magnetic pole: and thus, however rich in consequences the supposition of Kepler and others may have been, it is clear that a force like that of magnetism would not be able to transact the business of the universe.

The object of this discourse is to inquire whether the force of diamagnetism, which manifests itself as a repulsion of certain bodies by the poles of a magnet, is to 
be ranged as a polar force, beside that of magnetism; or as an unpolar force beside that of gravitation. When a cylinder of soft iron is placed within a wire helix, and surrounded by an electric current, the antithesis of its two ends, or, in other words, its polar excitation, is at once manifesterl by its action upon a magnetic needle; and it may be asked why a cylinder of bismuth may not be substituted for the cylinder of iron, and its state similarly examined. The reason is, that the excitement of the bismuth is so feeble, that it would be quite masked by that of the helix in which it is enclosed; and the problem that now meets us is, so to excite a diamagnetic body that the pure action of the body upon a magnetic needle may be observed, unmixed with the action of the body used to excite the diamagnetic.

How this has been effected may be illustrated in the following manner: When through an upright helix of covered copper wire, a voltaic current is sent, the top of the helix attracts, while its bottom repels, the same pole

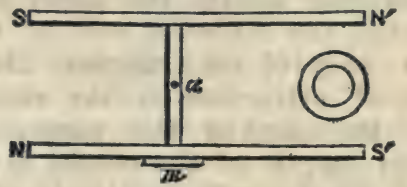

Fig. 10.

of a magnetie needle; its central point, on the contrary, is neutral, and exhibits neither attraction nor repulsion. Such a helix is caused to stand between the two poles $\mathbf{N}^{\prime} \mathbf{s}^{\prime}$ of an astatic system.* 'The two magnets $\mathrm{S} \mathrm{N}^{\prime}$ and $\mathrm{s}^{\prime} \mathrm{N}$ are united by a rigid cross piece at their centers, and are suspended from the point $a$, so that both magnets swing in the same horizontal plane. It is so arranged that the pole's $\mathrm{N}^{\prime} \mathrm{S}^{\prime}$ are opposite to the central or neutral point of the helix, so that when a current is sent through the latter, the magnets, as before explained, are unaffected. Here, then, we have an excited helix which itself has no action upon the magnets, and we are thus enabled to examine the action of a body placed within the helix and excited by it,

* The reversal of the poles of the two magnets, which were of the same strength, completely annulled the action of the earth as a magnet. 
undisturbed by the influence of the latter. The helix being 12 inches high, a cylinder of soft iron 6 inches long, suspended from a string and passing over a pulley, can be raised or lowered within the helix. When it is so far sunk that its lower end rests upon the table, the upner end finds itself between the poles $\mathbf{N}^{\prime} \mathbf{s}^{\prime}$ of the astatic system. The iron cylinder is thus conrerted into a strong magnet, attracting one of the poles, and repelling the other, and consequently deflecting the entire astatic system. When the cylinder is raised so that the upper end is at the level of the top of the helix, its lower end comes between the poles $\mathrm{N}^{\prime} \mathrm{s}^{\prime}$; and a deflection opposed in direction to the former one is the immediate consequence. To render these deflections more easily visible, a mirror $m$ is attached to the system of magnets; a beam of light thrown upon the mirror being reflected and projected as a bright disk against the wall. The distance of this image from the mirror being considerable, and its angular motion double that of the latter, a very slight motion of the magnet is sufficient to produce a displacement of the image through several yards.

This, then, is the principle of the beautiful apparatus* by which the investigation was conducted. It is manifest that if a second helix be placed between the poles $\mathbf{s} \mathbf{N}$ with a cylinder within it, the action upon the astatic magnet may be exalted. This was the arrangement made use of in the actual inquiry. Thus to intensify the feeble action, which it is here our object to seek, we have in the first place neutralized the action of the earth upon the magnets, by placing them astatically. Secondly, by making use of two cylinders, and permitting them to act simultaneously on the four poles of the magnets, we have rendered the leflecting force four times what it would be, if only a single pole were used. Finally the whole apparatus was enclosed in a suitable case which protected the magnets from air-currents, and the deflections were read off throngh a glass plate in the case, by means of a telescope and scale placed at a considerable distance from the instrument.

A pair of bismuth cylinders was first examined. Sending

* Devised by Prof. W. Weber, and constructed by M. Leyser, of Leipsic. 
a current through the helices, and observing that the magnets swung perfectly free, it was first arranged that the bismuth cylinders within the helices had their central or neutral points opposite to the poles of the magnets. All being at rest the number on the scale marked by the cross wire of the telescope was 5\%2. The cylinders were then moved, one up, the other down, so that two of their ends were brought to bear simultaneously upon the magnetic poles: the magnet moved promptly, and after some oscillations* came to rest at the number $61 \%$; thus moving from a smaller to a larger number. The other two ends of the bars were next brought to bear upon the magnet: a prompt deflection was the consequence, and the fiual position of equilibrium was 526: the movement being from a larger to a smaller number. We thus observe a manifest polar action of the bismuth cylinders upon the magnet; one pair of ends reflecting it in one direction, and the other pair deflecting it in the opposite direction.

Substituting for the cylinders of bismuth thin cylinders of iron, of magnetic slate, of sulphate of iron, carbonate of iron, protochloride of iron, red ferrocyanide of potassium, and other magnetic bodies, it was found that when the position of the magnetic cylinders was the same as that of the cylinders of bismuth, the deflection produced by the former was always opposed in direction to that produced by the latter; and hence the disposition of the force in the diamagnetic body must have been precisely antithetical to its disposition in the magnetic ones.

But it will be urged, and indeed has been urged against this inference, that the deflection produced by the bismuth cylinders may be due to induced currents excited in the metal by its motion within the helices. In reply to this objection, it may be stated, in the first place, that the deflection is permanent, and cannot therefore be due to induced currents, which are only of momentary duration. It has also been urged that such experiments onght to be made with other metals, and with better conductors than bismuth; for if due to currents of induction, the better the conductor the more exalted will be the effect. This requirement was complied with.

* To lessen these a copper damper was made use of. 
Cylinders of antimony were substituted for those of bismuth. 'This metal is a better conductor of electricity, but less strongly diamagnetic than bismuth. If therefore the action referred to be due to induced currents we ought to have it greater in the case of antimony than with bismuth: but if it springs from a true diamagnetic polarity, the action of the bismuth ought to exceed that of the antimony. Experiment proves this to be the case. Hence the deflection produced by these metals is due to their diamagnetic, and not to their conductive capacity. Copper cylinders were next examined: here we have a metal which conducts electricity fifty times better than bismuth, but its diamagnetic power is nearly null; if the effects be due to induced currents we ought to have them here in an enormously exaggerated degree, but no sensible deflection was produced by the two eylinders of copper.

It has also been proposed by the opponents of diamagnetic polarity to coat fragments of bismuth with some insulating substance, so as to render the formation of induced currents impossible, and to test the question with cylinders of these fragments. This requirement was also fulfilled. It is only necessary to reduce the bismuth to powder and expose it for a short time to the air to cause the particles to become so far oxidized as to render them perfectly insulating. The insulating power of the powder was exhibited experimentally; nevertheless, this powder, enclosed in glass tubes, exhibited an action scarcely less powerful than that of the massive bismuth cylinders.

But the most rigid proof, a proof admitted to be conclusive by those who have denied the antithesis of magnetism and diamagnetism, remains to be stated. Prisms of the same heavy glass as that with which the diamagnetic force was discovered, were substituted for the metallic cylinders, and their action upon the magnet was proved to be precisely the same in kind as that of the cylinders of bismuth. 'The inquiry was also extended to other' insulators: to phosphorus, sulphır, niter, calcareous spar, statuary marble, with the same invariable result: each of these substances was proved to be polar, the disposition of the force being the same as that of bismuth and the reverse of that of iron. When a bar of iron is set ereet, its lower end is known to be a north pole, and its upper end a south pole, in virtue of the earth's induction. A marble 
statue, on the contrary, has its feet a south pole, and its head a north pole, and there is no doubt that the same remark applies to its living archetype; each man walking over the earth's surface is a true diamagnet, with its poles the reverse of those of a mass of magnetic matter of the same shape and position.

An experiment of practical value, as affording a ready estimate of the different conductive powers of two metals for electricity, was exhibited in the lecture, for the purpose of proving experimentally some of the statements made in reference to this subject. A cube of bismuth was suspended by a twisted string between the two poles of an electromagnet. 'The cube was attached by a short copper wire to a little square pyramid, the base of which was horizontal, and its sides formed of four small triangular pieces of looking-glass. A beam of light was suffered to fall upou this reflector, and as the reflector followed the motion of the cube the images cast from its sides followed each other in succession, each describing a circle about thirty feet in diameter. As the velocity of rotation augmented, these images blended into a continuous ring of light. At a particular instant the electro-magnet was excited, currents were evolved in the rotating cube, and the strength of these currents, which increases with the conductivity of the cube for electricity, was practically estimated by the time required to bring the cube and its associated mirrors to a state of rest. With bismuth this time amounter to a score of seconds or more: a cube of copper, on the contrary, was struck almost instantly motionless when the circuit was established.

\section{CHAPTER XIV.}

\section{PHYSICAL BASIS OF SOLAR CHEMISTRY.*}

OMitTing all preface, attention was first drawn to an experimental arrangement intended to prove that gaseous bodies radiate heat in different degrees. Near a double screen of polished tin was placed an ordinary ring gasburner, and on this was placed a hot copper ball, from

* From a discourse delivered at the Royal Institution of Great Britain, June 7, 1861. 
which a column of heated air ascended. Behind the screen, but so situated that no ray from the ball could reach the instrument, was an excellent thermo-electric pile, connected by wires with a very delicate galvanometer. The pile was known to be an instrument whereby heat is applied to the generation of electric currents; the strength of the current being an accurate measure of the quantity of the heat. As loug as both faces of the pile are at the same temperature, no current is produced; but the slightest difference in the temperature of the two faces at once declares itself by the production of a current, which, when carried through the galvanometer, indicates by the deflection of the needle both its strength and its direction.

The two faces of the pile were in the first instance brought to the same temperature; the equilibrium being shown by the needle of the galvanometer standing at zero. The rays emitted by the current of hot air already referred to were permitted to fall upon one of the faces of the pile; and an extremely slight movement of the needle showed that the radiation from the hot air, though sensible, was extremely feeble. Connected with the ring-burner was a holder containing oxygen gas; and by turning a cock, a strean of this gas was permitted to issue from the burner, strike the copper ball, and ascend in a heated column in front of the pile. The result was, that oxygen showed itself, as a radiator of heat, to be quite as feeble as atmospheric air.

A second holder containing olefiant gas was then connected with the ring-burner. Oxygen and air had alrearly flowed over the ball and cooled it in some degree. Hence the olefiant gas labored under a disadvantage. But on permitting the gas to rise from the ball, it casts an amount of heat against the adjacent face of the pile sufficient to impel the needle of the galvanometer almost to ninety degrees. This experiment proved the vast difference between two equally invisible gases with regard to their power of emitting radiant heat.

The converse experiment was now performed. The thermo-electric pile was removed and placed between two cubes filled with water kept in a state of constant ebullition; and it was so arranged that the quantities of heat falling from the cubes on the opposite faces of the pile 
were exactly equal, thus neutralizing each other. The needle of the galvanometer being at zero, a sheet of oxygen gas was caused to issue from a slit between one of the cubes and the adjacent face of the pile. If this sheet of gas possessed any sensible power of intercepting the thermal rays from the cube, one face of the pile being deprived of the heat thus intercepted, a difference of temperature between its two faces would instantly set in, and the result would be declared by the galvanometer. The quantity absorbed by the oxygen under those circumstances was too feeble to affect the galvanometer; the gas, in fact, proved perfectly transparent to the rays of heat. It had but a feeble power of radiation: it had an equally feeble power of absorption.

The pile remaining in its position, a sheet of olefiant gas was caused to issue from the same slit as that through which the oxygen had passed. No one present could see the gas; it was quite invisible, the light went through it as freely as through oxygen or air; but its effect upon the thermal rays emanating from the cube was what might be expected from a sheet of metal. A quantity so large was cut off, that the needle of the galvanometer, promptly quitting the zero line, moved with energy to its stops. Thus the olefiant gas, so light and clear and perrious to luminous rays, was proved to be a most potent destruyer of the rays emanating from an obscure source. The reciprocity of action established in the case of oxygen comes out here; the good radiator is found by this experiment to be the good absorber.

This result, now exhibited before a public audience for the first time, was typical of what had been obtained with gases generally. Going through the entire list of gases and vapors in this way, we find radiation and absorption to be as rigidly associated as positive and negative in electricity, or as north and south polarity in magnetism. So that if we make the number which expresses the absorptive power the numerator of a fraction, and that which expresses its radiative power the denominator, the result would be, that on account of the numerator and denominator varying in the same proportion, the value of that fraction would always remain the same, whatever might be the gas or vapor experimented with.

But why should this reciprocity exist? What is the 
meaning of absorption? what is the meaning of radiation? When you cast a stone into still water, rings of waves surround the place where it falls; motion is radiater on all sides from the center of disturbance. When a hammer strikes a bell, the latter vibrates; and sound which is nothing more than an undulatory motion of the air, is radiated in all directions. Modern philosophy reduces light and heat to the same mechanical category. A luminous body is one with its atoms in a state of vibration; a hot body is one with its atoms also vibrating, but at a rate which is incompetent to excite the sense of vision; and, as a sounding body has the air around it, through which it propagates its vibrations, so also the luminous or heated body has a medium, called ether, which accepts its motions and carries them forward with inconceivable relocity. Radiation, then, as regards both light and heat, is the transference of motion from the vibrating body to the ether in which it swillgs: and, as in the case of sound, the motion imparted to the air is soon transferred to surrounding objects, against which the aërial undulations strike, the sound being in technical language, absorbed; so also with regard to light and lieat, absorption consists in the transference of motion from the agitated ether to the molecules of the absorbing body.

The simple atoms are found to be bad radiators; the compound atoms good ones: and the higher the degree of complexity in the atomic grouping, the more potent, as a general rule, is the radiation and absorption. Let us get definite ideas here, however gross, and purify them afterward by the process of abstraction. Imagine our simple atoms swinging like single spheres in the ether; they cannot create the swell which a group of them uniter to form asystem can produce. An oar runs freely edgeways through the water, and imparts far less of its motion to the water than when its broad flat side is brought to bear upon it. In our present language the oar, broad side vertical, is a good radiator; broad side horizontal, it is a bail radiator. Conversely the waves of water, impinging upon the flat face of the oar-blade, will impart a greater amount of motion to it than when impinging upon the erge. In the position in which the nar raliates well, it also absorbs well. Simple atoms glide through the ether without much resistance; compound ones encounter resistance, and hence 
yield up more speedily their motion to the ether. Mix oxygen and nitrogen mechanically, they absorb and radiate a certain amount of heat. Cause these gases to combine chemically and form nitrous oxide, both the absorption and radiation are thereby augmented hundreds of times!

In this way we look with the telescope of the intellect into atomic systems, and obtain a conception of processes which the eye of sense can never reach. But gases and vapors possess a power of choice as to the rays which they absorb. 'They single out certain groups of rays for destruction, and allow other groups to pass unharmed. This is best illustrated by a famous experiment of Sir David Brewster's, modified to suit present requirements. Into a glass cylinder, with its ends stopped by disks of plate-glass, a small quantity of nitrous acid gas is introduced, the presence of the gas being indicated by its rich brown color. The beam from an electric lamp being sent through two prisms of bisulphide of carbon, a spectrum seven feet long and eighteen inches wide is cast upon the screen. Introducing the cylinder containing the nitrous acid into the path of the beam as it issues from the lamp, the splendid and continuous spectrum becomes instantly furrowed by numerous dark bands, the rays answering to which are intercepted by the nitric gas, while the light which falls upon the intervening spaces is permitted to pass with comparative impunity.

Here also the principle of reciprocity, as regards radiation and absorption, holds good; and could we, without otherwise altering its physical character, render that nitrous gas luminous, we should find that the very rays which it absorbs are precisely those which it would emit. When atmospheric air and other gases are brought to a state of intense incandescence by the passage of an electric spark, the spectra which we obtain from them consist of it series of bright bands. But such spectra are produced with the greatest brilliancy when, instead of ordinary gases, we make use of metals heated so highly as to volatilize them. 'This is easily done by the voltaic current. A capsule of carbon filled with mercury, which formed the positive electrode of the electric lamp, has a carbon point brought down upon it. On separating the one from the other, a brilliant are containing the mercury in a volatilized condition passes between them. The spectrum of this 
arc is not continuous like that of the solid carbon points, but consists of a series of vivid bands, each corresponding in color to that particular portion of the spectrum to which its rays belong. Copper gives its system of bands; zinc gives its system; and brass, which is an alloy of copper and zinc, gives a spectrum made up of the bands belonging to both metals.

Not only, however, when metals are united like zinc and copper to form an alloy, is it possible to obtain the bands which belong to them. No matter how we may disguise the metal-allowing it to unite with oxygen to form an oxide, and this again with an acid to form a salt; if the heat applied be sufficiently intense, the bands belonging to the metal reveal themselves with perfect definition. Into holes drilled in a cylinder of retort carbon, pure culinary salt is introduced. When the carbon is made the positive electrode of the lamp, the resultant spectrum shows the brilliant yellow lines of the metal sodium. Similar experiments made with the chlorides of strontium, calcium, lithium, ${ }^{*}$ and other metals, give the bands due to the respective metals. When different salts are mixed together, and rammed into holes in the carbon, a spectrum is obtained which contains the bands of them all.

The position of these bright bands never varies, and each metal has its own system. Hence the competent observer can infer from the bands of the spectrum the metals which prorluce it. It is a language addressed to the eye instead of the ear; and the certainty would not be augmented if each metal possessed the power of audibly calling out, "I am here!" Nor is this language affected by distance. If we find that the sun or the stars give us the bands of our terrestrial metals, it is a declaration on the part of these orbs that such metals enter into their composition. Does the sun give us any such intimation? Does the solar spectrum exhibit bright lines which we

* The vividness of the colors of the lithium spectrum is extraordinary; the spectrum, moreover, contained a blue band of indescribable splendor. It was thought by many, during the discourse, that I had mistaken strontium for lithium, as this blue band had never before been seen. I have obtained it many times since; and ny friend Dr. Miller, having kindly analyzed the substance made use of, pronounces it pure chloride of lithium.-J. T. 
might compare with those produced by our terrestrial metals, and prove either their identity or difference? No. The solar spectrum, when closely examined, gives us a multitude of fine dark lines instead of bright ones. They were first noticed by Dr. Wollaston, but were multiplied and investigated with profound skill by Fraumhofer, and named after him Fraunhofer's lines. 'They had been long a standing puzzle to philosophers. The bright lines yielded by metallic vapors had been also known to us for years; but the connection between both classes of phenomena was wholly unknown, until Kirchhoff, with admirable acuteness, revealed the secret, and placed it at the same time in our power to chemically analyze the sun.

We have now some difficult work before us. Hitherto we have been delighted by objects which addressed themselves as much to our æsthetic taste as to our scientific faculty; we have ridden pleasantly to the base of the final cone of Etna, and must now dismount and march through ashes and lava, if we would enjoy the prospect from the summit. Our problem is to connect the dark lines of Fraunhofer with the bright ones of the metals. 'The white beam of the lamp is refracted in passing through our two prisms, but its different components are refracted in different degrees, and thus its colors are drawn apart. Now the color depends solely upon the rate of oscillation of the atoms of the luminous body; red light being produced by one rate, blue light by a much quicker rate, and the colors between red and blue by the intermediate rates. The solid incandescent coal-points give us a continuous spectrum; or in other words they emit rays of all possible periods between the two extremes of the spectrum. Color, as many of you know, is to light what pitch is to sound. When a violin-player presses his finger on a string he makes it shorter and tighter, and thus, causing it to vibrate more speedily, heightens the pitch. Imagine such a player to move his fingers slowly along the string, shortening it gradually as he draws his bow, tine note would rise in pitch by a regular gradation; there would be no gap intervening between note and note. Here we have the analogue to the continuous spectrum, whose colors insensibly blend together without gap or interruption, from the red of the lowest pitch to the violet of the highest. But suppose the player, instead of gradually shortening his string, to press 
his finger on a certain point, and to sound the corresponding note; then to pass on to another point more or less distant, and sound its note; then to another, and so on, thus sounding particular notes separated from each other by gaps which correspond to the intervals of the string passed over; we should then have the exact analogue of a spectrum composed of separate bright bands with intervals of darkness between them. But this, though a perfectly true and intelligible analogy, is not sufficient for our purpose; we must look with the mind's eye at the oscillating atoms of the volatilized metal. Figure these atoms as connected together by springs of a certain tension, which, if the atoms are squeezed together, push them again asunder, and if the atoms are drawn apart, pull them again together, causing them, before coming to rest, to quiver for a certain time at a certain definite rate determined by the strength of the spring. Now the volatilized metal which gives us one bright band is to be figured as having its atoms united by springs all of the same tension, its vibrations are all of one kind. The metal which gives us two bands may be figured as having some of its atoms united by springs of one tension, and others by springs of a different tension. Its vibrations are of two distinct kinds; so also when we have three or more bands we are to figure as many distinct sets of springs, each capable of vibrating in its own particular time and at a different rate from the others. If we seize this idea definitely, we shall have no difficulty in dropping the metaphor of springs, and substituting for it mentally the forces by which the atoms act upon each other. Having thus far cleared our way, let us make another effort to advance.

A heavy ivory ball is here suspended from a string, I blow against this ball; a single puff of my breath moves it a little way from its position of rest; it swings back toward me, and when it reaches the limit of its swing I puff again. It now swings further; and thus by timing the puffs I can so accumulate their action as to produce oscillations of large amplitude. The ivory ball here has absorbed the motion which my breath coinmunicated to the air. I now bring the ball to rest. Suppose, instead of the breath, a wave of air to strike against it, and that this wave is followed by a series of others which succeed each other exactly in the same intervals as my puffs; it is obvious that 
these waves would communicate their motion to the ball and cause it to swing as the puffs did. And it is equally manifest that this would not be the case if the impulses of the waves were not properly timed; for then the motion imparted to the pendulum by one wave would be neutralized by another, and there could not be the accumulation of effect obtained when the periods of the waves correspond with the periods of the pendulum. So much for the particular impulses absorbed by the pendulum. But if such a pendulum set oscillating in air could produce wares in the air, it is evident that the waves it would produce would be of the same period as those whose motions it would take up or absorb most completely, if they struck against it.

Perhaps the most curious effect of these timed impulses ever described was that observed by a watchmaker, named Ellicott, in the year 1741. He left two clocks leaning against the same rail; one of them, which we may call A, was set going; the other B, not. Some time afterward he found, to his surprise, that $B$ was ticking also. The pendulums being of the same length, the shocks imparted by the ticking of $\mathrm{A}$ to the rail against which both clocks rested were propagated to $B$, and were so timed as to set $B$ going. Other curious effects were at the same time observed. When the pendulums differed from each other a certain amount, $\mathrm{A}$ set $\mathrm{B}$ going, but the reaction of $\mathrm{B}$ stopped A. Then B set A going, and the reaction of A stopped B. When the periods of oscillation were close to each other, but still not quite alike, the clocks mutually controlled each other, and by a kind of compromise they ticked in perfect unison.

But what has all this to do with our present subject? The varied actions of the universe are all modes of motion; and the vibration of a ray claims strict brotherhood with the vibrations of our pendulum. Suppose ethereal waves striking upon atoms which oscillate in the same periods as the waves, the motion of the waves will be absorbed by the atoms; suppose we send our beam of white light through a sodium flame, the atoms of that flame will be chiefly affected by those undulations which are synchronous with their own periods of vibration. There will be on the part of those particular rays a transference of motion from the agitated ether to the atoms of the volatilized metal, which, as already defined, is absorption. 
The experiment justifying this conclusion is now for the first time to be made before a public audience. I pass a beam through our two prisms, and the spectrum spreads its colors upon the screen. Between the lamp and the prisin I interpose a snapdragon light. Alcohol and water are here mixed with common salt, and the metal dish that holds them is heated by a spirit-lamp. 'T'he vapor from the mixture ignites and we have a monochromatic flame. Through this flame the beam from the lamp is now passing; and observe the result upon the spectrum. You see a shady band cut out of the yellownot very dark, but sufficiently so to be seen by everybody present.

But let me exalt this effect. Placing in front of the electric lamp the intense flame of a large Bunsen's burner, a platinum capsule containing a bit of sodium less than a pea in magnitude is plunged into the flame. The sodium soon volatilizes and burns with brilliant incandescence. The beam crosses the flame, and at the same time the yellow band of the spectrum is clearly and sharply cut out, a band of intense darkness occupying its place. On withdrawing the sodium, the brilliant yellow of the spectrum takes its proper place, while the reintroduction of the flame causes the band to reappear.

Let me be more precise: The yellow color of the spectrum extends over a sensible space, blending on one side with the orange and on the other with the green. The term "yellow band" is therefore somewhat indefinite. This vagueness may be entirely remored. By dipping the carbon-point used for the positive electrode into a solution of common salt, and replacing it in the lamp, the bright yellow band produced by the sodium vapor stands out from the spectrum. When the sodium flame is caused to nct upon the beam it is that particular yellow band that is obliterated, an intensely black streak occupying its place.

An additional step of reasoning leads to the conclusion that if, instead of the flame of sodium alone, we were to introduce into the path of the beam a flame in which lithium, strontium, magnesium, calcium, etc., are in a state of volatilization, each metallic vapor would cut out a system of bands, corresponding exactly in position with the bright bands of the same metallic vapor. The 
light of our electric lamp shining through such a composite flame would give us a spectrum cut up by dark lines, exactly as the solar spectrum is cut up by the lines of Fraunhofer.

Thus by the combination of the strictest reasoning with the most conclusive experiment, we reach the solution of one of the grandest of scientific problems-the constitution of the sun. 'The sun consists of a nucleus surrounded by flaming atmosphere. The light of the nucleus would give us a continuous spectrum, like that of our common carbon-points; but having to pass through the photosphere, as our beam had to pass through the flame, those rays of the nucleus which the photosphere can itself emit are absorbed, and shaded spaces, corresponding to the particular rays absorbed, occur in the spectrum. Abolish the solar nucleus, and we should have a spectrum showing a bright line in the place of every dark line of Fraunhofer. These lines are therefore not absolutely dark, but dark by an amount corresponding to the difference between the light of the nucleus intercepted by the photosphere, and the light which issues from the latter.

The man to whom we owe this noble generalization is Kirchhoff, professor of natural philosophy in the University of Heidelberg; ${ }^{*}$ but, like every other great discovery, it is compounded of various elements. Mir. Talbot observed the bright lines in the spectra of colored flames. Sixteen years ago Dr. Miller gave drawings and descriptions of the spectra of various colored flames. Wheatstone, with his accustomed ingenuity, analyzed the light of the electric spark, and showed that the metals between which the spark passed determined the bright bands in the spectrum of the spark. Masson published a prize essay on these bands; Van der Willigen, and more recently Plücker, have given us beantiful drawings of the spectra, obtained from the discharge of Ruhmkorff's coil. But none of these distinguished men betrayed the least knowledge of the connection between the bright bands of the metals and the dark lines of the solar spectrum. The man who came nearest to the philosophy of the subject was Angström. In a paper translated from Poggendorff's Annalen by myself, and published in the Philosophical Magazine

* Now professor in the University of Berlin. 
for 1855 , he indicates that the rays which a body absorbs are precisely those which it can emit when rendered luminous. In another place, he speaks of one of his spectra giving the general impression of a reversal of the solar' spectrum. Foucault, Stokes, and Thomson, have all been very close to the discovery; and, for my own part, the examination of the radiation and absorption of heat by gases and vapors, some of the results of which I placed before you at the commencement of this discourse, would have led me in 1859 to the law on which all Kirchhoff's speculations are founded, had not an accident withdrawn me from the investigation. But Kirchhoff's claims are unaffected by these circumstances. True, much that I have referred to formed the necessary basis of his discovery; so did the laws of Kepler furnish to Newton the basis of the theory of gravitation. But what Kirchhoff has done carries us far beyond all that had before been accomplished. He has introduced the order of law amid a vast assemblage of empirical observations, and has ennobled our previous knowledge by showing its relationship to some of the most sublime of natural phenomena.

\section{CHAPTER XV.}

\section{ELEMENTARY MAGNETISM.}

A LECTURE TO SCEOOLMASTERS.

WE HAVE no reason to believe that the sheep or the dog, or indeed any of the lower animals, feel an interest in the laws by which natural phenomena are regulated. A herd may be terrified by a thunderstorm; birds may go to roost, and cattle return to their stalls, during a solar eclipse; but neither birds nor cattle, as far as we know, ever think of inquiring into the causes of these things. It is otherwise with man. The presence of natural objects, the occurrence of natural events, the varied appearances of the universe in which he dwells, penetrate beyond his organs of sense, and appeal to an inner power of which the senses are the mere instruments and excitants. No fact is to him either original or final. He cannot limit himself to tise contemplation of it alone, but endeavors to ascertain 
its position in a series to which uniform experience assures him it must belong. He regards all that he witnesses in the present as the efflux and sequence of something that has gone before, and as the source of a system of events which is to follow. The notion of spontaneity, by which in his ruder state he accounted for natural events, is abandoned; the idea that Nature is an aggregate of independent parts also disappears, as the connection and mutual depender:ce of physical power's become more and more manifest: until he is finally led to regard Nature as an organic whole-as a body each of whose members sympathizes with the rest, changing, it is true, from age to age, but changing without break of continuity in the relation of cause and effect.

'The system of things which we call Nature is, however, too vast and various to be studied first-hand by any single mind. As knowledge extends there is always a tendency to subdivide the field of investigation. Its various parts are taken up by different minds, and thus receive a greater amount of attention than could possibly be bestowed on them if each investigator aimed at the mastery of the whole. The centrifugal form in which knowledge, as a whole, advances, spreading ever wider on all sides, is due in reality to the exertions of individuals, each of whom directs his efforts, more or less, along a single line. Accepting, in many respects, his culture from his fellow-men-taking it from spoken words or from written books-in some one direction, the student of Nature ought actually to touch his work. He may otherwise be a distributor of knowledge, but not a creator, and he fails to attain that vitality of thought, and correctness of judgment, which direct and habitual contact with natural truth can alone impart.

One large department of the system of Nature which forms the chief subject of my own studies, and to which it is my duty to call your attention this evening, is that of physics, or natural philosophy. This term is large enough to cover the study of Nature generally, but it is usually restricted to a department which, perhaps, lies closer to our perceptions than any other. It deals with the phenomena and laws of light and heat-with the phenomena and laws of magnetism and electricity - with those of sound-with the pressures and motions of liquirls and gases, whether at rest or in a state of translation or of undulation. 'The 
science of mechanics is a portion of natural philosophy, though at present so large as to need the exclusive attention of him who would cultivate it profoundly. Astronomy is the application of physics to the motions of the heavenly bodies, the rastuess of the field causing it, however, to be regarded as a department in itself. In chemistry physical agents play important parts. By heat and light we cause atoms anil molecules to unite or to fall asunder. Electricity exerts a similar power. Through their ability to separate nutritire compounds into their constituents, the solar beams build up the whole vegetable world, and by it the animal world. The touch of the selfsame beams causes hydrogen and chlorine to unite with sudden explosion, and to form by their combination a powerful acid. Thus physics and chemistry intermingle. Physical agents are, however, employed by the chemist as a means to an end; while in physics proper the laws and phenomena of the agents themselves, both qualitative and quantitative, are the primary objects of attention.

My duty here to-night is to spend an hour in telling how this subject is to be studied, and how a knowledge of it is to be imparted to others. From the domain of physics, which would be unmanageable as a whole, I select as a sample the subject of magnetism. I might readily entertain you on the present occasion with an account of what natural philosophy has accomplished. I might point to those applications of science of which we hear so much in the newspapers, and which are so often mistaken for science itself. I might, of course, ring changes on the steam-engine and the telegraph, the electrotype and the photograph, the medical applications of physics, and the various other inlets by which scientific thought filters into practical life. That would be easy compared with the task of informing you how you are to make the study of physics the instrument of your pupil's culture; how you are to possess its facts and make them living seeds which shall take root and grow in the mind, and not lie like dearl lumber in the storehouse of memory. This is a task much heavier than the mere recounting of scientific achievements; and it is one which, feeling my own want of time to execute it aright, I might well hesitate to accept.

But let me sink excuses, and attack the work before me. First and foremost, then, I would advise you to get a 
knowledge of facts from actual observation. Facts looked at directly are vital; when they pass into words half the sap is taken out of them. You wish, for example, to get a knowledge of magnetism; well, provide yourself with a good book on the subject, if you can, but do not be content with what the book tells you; do not be satisfied with its descriptive woodcuts; see the operations of the force yourself. Half of our book writers describe experiments which they never made, and their descriptions often lack both force and truth; but no matter how clever or conscientious they may be, their written words cannot supply the place of actual observation. Every fact has numerous radiations, which are shorn off by the man who describes it. Go, then, to a philosophical instrument maker, and give a shilling or half a erown for a straight bar-magnet, or, if you can afford it, purchase a pair of theii); or get a smith to cat a length of ten inches from a bar of steel an inch wide and half an inch thick; file its ends smoothly, harden it, and get somebody like myself to magnetize it. Procure some darning needles, and also a little unspun silk, which will give you a suspending fiber void of torsion. Make a little loop of paper, or of wire, and attach your fiber to it. Do it neatly. In the loop place a darningneerlle, and bring the two ends or poles, as they are called, of your bar-magnet successively up to the ends of the needle. Both the poles, you find, attract both ends of the needle. Replace the needle by a bit of annealed iron wire; the same effects ensue. Suspend successively little rods of lead, copper, silver, brass, wood, glass, ivory, or whalebone; the magnet produces no sensible effect upon any of the substances. You thence infer a special property in the case of steel and iron. Multiply your experiments, however, and you will find that some other substances, besides iron and steel, are acted upon by your magnet. A rod of the metal nickel, or of the metal cobalt, from which the blue color used by painters is derived, exhibits powers similar to those observed with the iron and steel.

In studying the character of the force you may, however, confine yourself to iron and steel, which are always at hand. Make your experiments with the darning-needle over and over again; operate on both ends of the needle; try both ends of the magnet. Do not think the work dull; you are conversing with Nature, and must acquire over her lan- 
guage a certain grace and mastery, which practice can alone impart. Let overy movement be male with care, and avoid slovenliness from the outset. Experiment, as I have said, is the language by which we address Nature, and through which she sends her replies; in the use of this language a lack of straightforwardness is as possible, and as prejudicial, as in the spoken language of the tongue. If, therefore, you wish to become acquainted with the truth of Nature, you must from the first resolve to deal with her sincerely.

Now remove your needle from its loop, and draw it from eye to point along one of the ends of the magnet; resuspend it, and repeat your former experiment. You now find that each extremity of the magnet attracts one end of the needle, and repels the other. The simple attraction observed in the first instance, is now replaced by a dual force. Repeat the experiment till you have thoroughly observed the ends which attract and those which repel each other.

Withdraw the magnet entirely from the vicinity of your needle, and leave the latter freely suspended by its fiber. Shelter it as well as you can from currents of air, and if you have iron buttons on your coat, or a steel penknife in your pocket, beware of their action. If you work at night, beware of iron candlesticks, or of brass ones with iron rods inside. Freed from such disturbances, the needle takes up a certain determinate position. It sets its length nearly north and south. Draw it aside and let it go. After several oscillations it will again coine to the same position. If you have obtained your magnet from a philosophical instrument maker, you will see a mark on one of its ends. Supposing, then, that you drew your needle along the end thus marked, and that the point of your needle was the last to quit the magnet, you will find that the point turns to the south, the eye of the needle turning toward the north. Make sure of this, and do not take the statement on my authority.

Now take a second darning-neelle like the first, and magnetize it in precisely the same manner; freely suspencled it also will turn its eye to the north and its point to the south. Your next step is to examine the action of the two needles which you have thus magnetized upon each other.

Take one of them in your hand, and leave the other sus- 
pended; bring the eye-end of the former near the eye-end of the latter; the suspended needle retreats: it is repellerl. Make the same experiment with the two points; you obtain the same result, the suspended needle is repelled. Now canse the dissimilar ends to act on each other-you have attraction-point attracts eye, and eye attracts point. Prove the reciprocity of this action by removing the suspended needle, and putting the other in its place. Yon obtain the same result. 'The attraction, then, is mutual, and the repulsion is mutual. You have thus demonstrated in the clearest manner the fundamental law of magnetism, that like poles repel, and that unlike poles attract each other. You may say that this is all easily understood without doing; but do it, and your knowledge will not be confined to what I have uttered here.

I have said that one end of your bar-magnet has a mark upon it; lay several silk fibers together, so as to get sufficient strength, or employ a thin silk ribbon, and form a loop large enough to hold your magnet. Suspend it; it turns its marked end toward the north. 'This marked end is that which in England is called the north pole. If a common smith has made your magnet, it will be convenient to determine its north pole yourself, and to mark it with a file. Vary your experiments by cansing your magnetized darning-neerlle to attract and repel your large magnet; it is quite competent to do so. In magnetizing the needle, I have supposed the point to be the last to quit the marked end of the magnet; the point of the needle is a south pole. The end which last quits the magnet is always opposed in polarity to the end of the magnet with which it has been last in contact.

Yon may perhaps learn all this in a single hour; but spend several at it, if necessary; and remember, unlerstanding it is not sufficient: you must obtain a mannal aptitude in addressing Nature. If you speak to your fellow-man you are not entitled to use jargon. Bad experiments are jargon addressed to Nature, and just as much to be deprecated. Manual dexterity in illustrating the interaction of magnetic poles is of the utmost importance at this stage of your progress; and you must not neglect attaining this power over your implements. As you proceed, moreover, you will be tempted to do more than I can possibly suggest. 'Thoughts will occur to you 
which you will endeavor to follow out: questions will arise which you will try to answer. The same experiment may be twenty different things to twenty people. Having witnessed the action of pole on pole. through the air, you will perhaps try whether the magnetic power is not to be screened off. You use plates of glass, wood, slate, pasteboard, or gutta-percha, but find them all pervious to this wondrous force. One magnetic pole acts upon another through these bodies as if they were not present. Should you ever become a patentee for the regulation of ships' compasses, you will not fall, as some projectors have done, into the error of screening off the magnetism of the ship by the interposition of such substances.

If you wish to teach a class you must contrive that the effects which you have thus far witnessed for yourself shall be witnessed by twenty or thirty pupils. And here your private ingenuity must come into play. You will attach bits of paper to your needles, so as to render their movements visible at a distance, denoting the north and south poles by different colors, say green and red. You may also improve upon your darning-needle. Take a strip of sheet steel, heat it to vivid redness and plunge it into cold water. It is thereby hardened; rendered, in fact, almost as brittle as glass. Six inches of this, magnetized in the manner of the darning-needle, will be better able to carry your paper indexes. Having secured such a strip, you proceed thus:

Magnetize a small sewing-needle and determine its poles; or, break half an inch, or an inch, off your magnetized darning-needle and suspend it by a fine silk fiber. The sewing-needle, or the fragment of the darning-needle, is now to be used as a test-needle, to examine the distribution of the magnetism in your strip of steel. Hold the strip upright in your left hand, and cause the test-needle to approach the lower end of your strip; one end of the test-needle is attracted, the other is repelled. Raise your needle along the strip; its oscillations, which at first were quick, become slower; opposite the middle of the strip they cease entirely; neither end of the needle is attracted; abore the middle the test-needle turns suddenly round, its other end being now attracted. Go through the experiment thoroughly: you thus learn that the entire lower half of the strip attracts one end of the needle, while the entire 
upper half attracts the opposite end. Supposing the north end of your little neecile to be that attracted below, you infer that the entire lower half of your magnetized strip exhibits south magnetism, while the entire upper half exhibits north magnetism. So far, then, you have determined the distribution of maguetism in your strip of steel.

You look at this fact, you think of it; in its suggestiveness the value of an experiment chiefly consists. The thought naturally arises: "What will occur if I break my strip of steel across in the middle? Shall I obtain two magnets each possessing a single pole?" Try the experiment; break your strip of steel, and test each half as you tested the whole. 'The mere presentation of its two ends in succession to your test-needle suffices to show that you have not a magnet with a single pole-that each half possesses two poles with a neutral point between them. And if you again break the half into two other halves, you will find that each quarter of the original strip exhibits precisely the same magnetic distribution as the whole strip. You may continue the breaking process: no matter how small your fragment may be, it still possesses two opposite poles and a nentral point between them. Well, your hand ceases to break where breaking becomes a mechanical impossibility; but does the mind stop there? No: you follow the breaking process in idea when you can no longer realize it in fict; your thoughts wander amid the very atoms of your steel, and you conclude that each atom is a magnet, and that the force exerted by the strip of steel is the mere summation, or resultant, of the force of its ultimate particles.

Here, then, is an exhibition of power which we can call forth at pleasure or cause to disappear. We magnetize our strip of steel by drawing it along the pole of a magnet; we can demagnetize it, or reverse its magnetism, by properly drawing it along the same pole in the opposite direction. What, then, is the real nature of this wondrous change? What is it that takes place among the atoms of the steel when the substance is magnetized? 'The question learls us beyond the region of sense, and into that of imagination. 'This faculty, indeed, is the divining-rod of the man of science. Not, however, an imagination which catches its creations from the air, but one informed and inspired by facts; 
capable of seizing firmly on a physical image as a principle, of discerning its consequences, and of devising means whereby these forecasts of thought may be brought to an experimental test. If such a principle be adequate to account for all the phenomena-if from an assumed cause the observed acts necessarily follow, we call the assumption a theory, and, once possessing it, we can not only revive at pleasure facts already known, but we can predict others which we have never seen. 'Thus, then, in the prosecution of physical science, our powers of observation, memory, imagination, and inference, are all drawn upon. W̌e observe facts and store them up; the constuctive imagination broods upon these memories, tries to discern their interdependence and weave them to an organic whole. The theoretic principle flashes or slowly dawns upon the mind; and then the deductive faculty interposes to carry out the principle to its logical consequences. A perfect theory gives dominion over natural facts; and even an assumption which can only partially stand the test of a comparison with facts, may be of eminent use in enabling us to connect and classify groups of phenomena. The theory of magnetic fluids is of this latter character, and with it we must now make ourselves familiar.

With the view of stamping the thing more firmly on your minds, I will make use of a strong and vivid image. In optics, red and green are called complementary colors; their mixture produces white. Now I ask you to imagine each of these colors to possess a self-repulsive power; that red repels red, that green repels green; but that red attracts green and green attracts red, the attraction of the dissimilar colors being equal to the repulsion of the similar ones. Imagine the two colors mixed so as to produce white, and suppose two strips of wood painted with this white; what will be their action upon each other? Suspend one of them freely as we suspended our darningneedle, and bring the other near it; what will occur? The red component of the strip you hold in your hand will repel the red component of your suspended strip; but then it will attract the green, and the forces being equal, they neutralize each other. In fact, the least reflection shows you that the strips will be as indifferent to each other as two unmagnetized darning-neelles would be uncler the same circumstances. 
But suppose, instead of mixing the colors, we painted one half of each strip from center to end red, and the other half green, it is perfectly manifest that the two strips would now behave toward each other exactly as our two magnetized darning-needles-the red end would repel the red and attract the green, the green would repel the green and attract the red; so that, assuming two colors thus related to each other, we could by their mixture produce the neutrality of an unmagnetized body, while by their separation we could produce the duality of action of magnetized bodies.

But you have already anticipated a defect in my con. ception; for if we break one of our strips of wood in the middle we nave one half entirely red, and the other entirely green, and with these it would be impossible to imitate the action of our broken magnet. How, then, must we modify our conception? We must evidently suppose each molecule of the wood painted green on one face and red on the opposite one. The resultant action of all the atoms would then exactly resemble the action of a magnet. Here also, if the two opposite colors of each atom could be caused to mix so as to produce white, we should have, as before, perfect neutrality.

For these two self-repellent and mutually attractive colors, substitute in your minds two invisible self-repellent and mutually attractive fluids, which in ordinary steel are mixed to form a neutral compound, but which the act of magnetization separates from each other, placing the opposite fluids on the opposite face of each molecule. You have then a perfectly distinct conception of the celebrated theory of magnetic fluids. The strength of the magnetism excited is supposed to be proportional to the quantity of neutral fluid decomposed. According to this theory nothing is actually transferred from the exciting magnet to the excited steel. The act of magnetization consists in the forcible separation of two fluids which existed in the steel before it was magnetized, but which then neutralized each other by their coalescence. And if you test your magnet, after it has excited a hundred pieces of steel, you will find that it has lost no force-no more, incleed, than I should lose, had my words such a magnetic influence on your minds as to excite in them a strong resolve to study natural philosophy. I should rather be the 
gainer by my own utterance, and by the reaction of your fervor. The magnet also is the gainer by the reaction of the body which it magnetizes.

Look now to your excited piece of steel; figure each molecule with its opposed fluids spread over its opposite faces. How can this state of things be permanent? 'The fluids, by hypothesis, attract each other; what then, keeps them apart? Why do they not instantly rush together across the equator of the atom, and thus neutralize each other? 'To meet this question philosopher's have been obliged to infer the existence of a special force, which holds the fluids asunder. 'They call it coercive force; and it is found that those kinds of steel which offer most resistance to being magnetized-which require the greatest amount of " coercion" to tear their fluids usunder-are the very ones which offer the greatest resistance to the reunion of the fluids, after they have been once separated. Such kinds of steel are most suited to the formation of permanent magnets. It is manifest, indeed, that without coercive force a permanent magnet would not be at all possible.

Probably long before this you will have dipped the end of your magnet among iron filings, and observed how they cling to it; or into a nail-box, and found how it drags the nails after it. I know very well that if you are not the slaves of routine, you will have by this time dous many things that I have not told you to do, and thus multiplied yourexperience beyond what I have indicated. You are almost sure to have caused a bit of iron to hang from the end of your magnet, and you have probably succeeded in causing a second bit to attach itself to the first, a third to the second; until finally the force has become too feeble to bear the weight of more. If you have operated with nails, you may have observed that the points and edges hold together with the greatest tenacity; and that a bit of iron clings more firmly to the corner of your magnet that to one of its flat surfaces. In short, you will in all likelihood have enriched your experience in many ways without any special direction from me.

Well, the magnet attracts the nail, and the nail attracts a second one. This proves that the nail in contact with the magnet has had the magnelic quality developed in it by that contact. If it be withdrawn from the magnet its 
power to attract its fellow nail ceases. Contact, however, is not necessary. A sheet of glass or paper, or a space of air, may exist between the magnet and the nail; the latter is still magnetized, though not so forcibly as when in actual contact. The nail thus presented to the magnet is itself a temporary magnet. That end which is turned toward the magnetic pole has the opposite magnetism of the pole which excites it; the end most remote from the pole has the same magnetism as the pole itself, and between the two poles, the nail, like the magnet, possesses a magnetic equator.

Conversant as you now are with the theory of magnetic fluids, you have already, I doubt not, anticipated me in imagining the exact condition of an iron nail under the influence of the matgnet. You picture the iron as possessing the neutral fluid in abundance; you picture the magnetic pole, when brought near, decomposing the fluid; repelling the fluid of a like kind with itself, and attracting the unlike fluid; thus exciting in the parts of the iron nearest to itself the opposite polarity. But the iron is incapable of becoming a permanent magnet. It only shows its virtue as long as the magnet acts upon it. What, then, does the iron lick which the steel possesses? It lacks coercive force. Its fluids are separated with ease; but, once the separating cause is removed, they flow together again, and neutrality is restored. Imagination must be quite nimble in picturing these changes-able to see the fluids dividing and reuniting, according as the magnet is brought near or withdrawn. Fixing a definite pole in your mind, you must picture the precise arrangement of the two fluids with reference to this pole, and be able to arouse similar pictures in the minds of your pupils. You will cause them to place magnets and iron in various positions, and describe the exact magnetic state of the iron in each particular case. The mere facts of magnetism will have their interest immensely augmented by an acquaintance with the principles whereon the facts depend. Still, while you use this theory of magnetic fluids to track out the phenomena and link them together, you will not forget to tell your pupils that it is to be regarded as a symbol merely - a symbol, moreover, which is incompetent to cover all the facts, * but which does good practi-

* This theory breaks down when applied to diamagnetic bodies which are repelled by magnets. Like soft iron, such bodies are 
cal service while we are waiting for the actual truth to become known.

The state of excitement into which iron is thrown by the influence of a magnet is sometimes called " magnetization by influence." More commonly, however, the magnetism is said to be "induced" in the iron, and hence this mode of magnetizing is called "magnetic induction." Now there is nothing theoretically perfect in Nature: there is no iron so soft as not to possess a certain amount of coercive force, and no steel so hard as not to be capable, in some degree, of magnetic induction. The quality of steel is in some measure possessed by iron, and the quality of iron is shared in some degree by steel. It is in virtue of this latter fact that the unmagnetized darning-needle was attracted in your first experiment; and from this you may at once deduce the consequence that, after the steel has been magnetized, the repulsive action of a magnet must be always less than its attractive action. For the repulsion is opposed by the inductive action of the magnet on the steel, while the attraction is assisted by the same inductive action. Make this clear to your minds, and verify it by your experiments. In some cases you can actually make the attraction due to the temporary magnetism overbalance the repulsion due to the permanent magnetism, and thus cause two poles of the same kind apparently to attract each other. When, however, good hard magnets act on each other from a sufficient distance, the inductive action practically vanishes, and the repulsion of like poles is sensibly equal to the attraction of unlike ones.

I dwell thus long on elementary principles, because they are of the first importance, and it is the temptation of this age of unhealthy cramming to neglect them. Now follow me a little farther. In examining the distribution of magnetism in your strip of steel you raised the needle slowly from bottom to top, and found what we called a neutral point at the center. Now does the magnet really exert no influence on the pole pi'esented to its center? Let us see.

Let $\mathrm{s} \mathrm{N}$, fig. 11 , be our magnet, and let $n$ represent a particle of north magnetism placed exactly opposite the middle of the magnet. Of course this is an imagituary case,

thrown into a state of temporary excitement, in virtue of which they are repelled; but any attempt to explain such a repulsion by the decomposition of a fluid will demonstrate its own futility. 
as you can never in reality thus detach your north magnetism from its neighbor. But supposing us to have done so, what would be the action of the two poles of the magnet on $n$ ? Your reply will of course be that the pole sattracts $n$ while the pole $\mathrm{N}$ repels it. Let the magnitude and direction of the attraction be expressed by the line $n m$, and the magnitude and direction of the repulsion by the line $n 0$. Now, the particle $n$ being equally distant from s and $\mathrm{N}$, the line $n$, expressing the repulsion, will be equal to $m n$, which expresses the attraction. Acted upon by two such forces, the particle $n$ must evidently move in the direction $n p$, exactly midway between $m n$ and $n o$. Hence you see that although there is no tendency of the particle $n$ to move toward the magnetic equator, there is a tendency on its part to move parallel to the magnet. If, instead of a particle of north magnetism, we placed a particle of south magnetism opposite to the magnetic equator, it would evi-

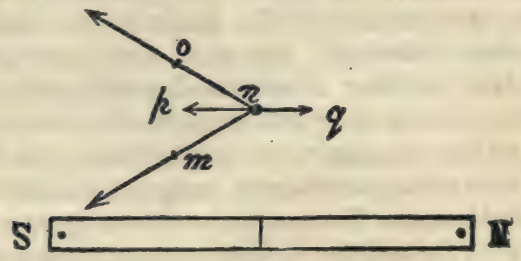

FIG. 11.

dently be urged along the line $n q$; and if, instead of two separate particles of magnetism we place a little magnetic needle, containing both north and south magnetism, opposite the magnetic equator, its south pole being urged along $n q$, and its north along $n p$, the little needle will be compelled to set itself parallel to the magnet $\mathrm{s}$. . Make the experiment, and satisfy yourselves that this is a true deduction.

Substitnte for your magnetic needle a bit of iron wire, devoid of permanent magnetism, and it will set itself exactly as the needle does. Acted upon by the magnet, the wire, as you know, becomes a magnet and behaves as such; it will turn its north pole toward $p$, and south pole toward $q$, just like the needle.

But supposing you shift the position of your particle of north magnetism, and bring it nearer to one end of 
your magnet than to the other; the forces acting on the particle are no longer equal; the nearest pole of the magnet will act more powerfully on the particle than the more distant one. Let $\mathrm{s} N$, fig. 12 , be the magnet, and $n$ the particle of north magnetism, in its new position. It is repelled by $\mathbf{N}$, and attracted by $\mathrm{s}$. Let the repulsion be represented in maguitude and direction by the line $n o$, and the attraction by the shorter line $n m$. The resultant of these two forces will be found by completing the parallelogram $m n 0 p$, and drawing its diagonal $n p$. Along $n p$, then, a particle of north maguetism would be urged by the simultaneous action of $\mathrm{S}$ and $\mathrm{N}$. Substituting a particle of south magnetism for $n$, the same reasoning would lead to the conclusion that the particle would be urged along $n q$. If we place at $n$ a short magnetic needle, its north pole will be urged along $n p$, its south pole along $n q$, the only position possible to the

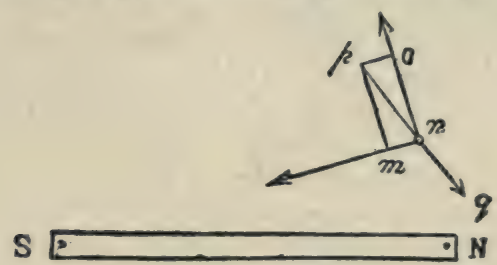

FIG. 12.

needle, thus acted on, being along the line $p q$, which is no longer parallel to the magnet. Verify this deduction by actual experiment.

In this way we might go round the entire magnet; and, considering its two poles as two centers from which the force enamates, we could, in accordance with ordinary mechanical principles, assign a definite direction to the magnetic needle at every particular place. And substituting, as before, a bit of iron wire for the magnetic needle, the positions of both will be the same.

Now, I think, without further preface, you will be able to comprehend for yourselves, and explain to others, one of the most interesting effects in the whole domain of magnetism. Iron filings you know are particles of iron, irregular in shape, being longer in some directions than in others. For the present experiment, moreover, instead of 
the iron filings very small scraps of thin iron wire might be employed. I place a sheet of paper over the magnet; it is all the better if the paper be stretched on a wooden frame, as this enables us to keep it quite lerel. I scatter the filings, or the scraps of wire, from a sieve upon the paper, and tap the latter gently, so as to liberate the particles for a moment from its friction. 'The magnet acts on the filings through the paper, and see how it arringes them! 'They embrace the magnet in a series of beatiful curves, which are technically called " magnetic curves," or "lines of magnetic force." Does the meaning of these lines yet flash upon you? Set your magnetic needle, or your suspended bit of wire, at any point of one of the curves, and you will find the direction of the needle, or of the wire, to be exactly that of the particle of iron, or of the magnetic curve, at that point. Go round and round the magnet; the direction of your needle always coincides with the direction of the curve on which it is placed. 'These, then, are the lines along which a particle of south magnetism, if you could detach it, would move to the north pole, and a bit of north magnetism to the south pole. They are the lines along which the decomposition of the neutral fluid takes place. In the case of the magnetic needle, one of its poles being urged in one direction, and the other pole in the opposite direction, the needle must necessarily setitself as a tangent to the curve. I will not seek to simplify this subject further. If there be anything obscure or confused or incomplete in my statement, you ought now, by patient thought, to be able to clear away the obscurity, to reduce the confusion to order, and to supply what is needed to render the explanation complete. Do not quit the subject until you thoroughly unclerstand it; and if you are then able to look with your mind's eye at the play of forces around a magnet, and see distinctly the operation of those forces in the production of the magnetic curves, the time which we have spent together will not have been spent in rain.

In this thorough manner we must master our materials, reason upon them, and, by determined study, attain to clearness of conception. Facts thus dealt with exercise an expansive force upon the intellect-they widen the mind to generalization. We soon recognize a brotherhood between the larger phenomena of Nature and the minute 
FIG. 13.

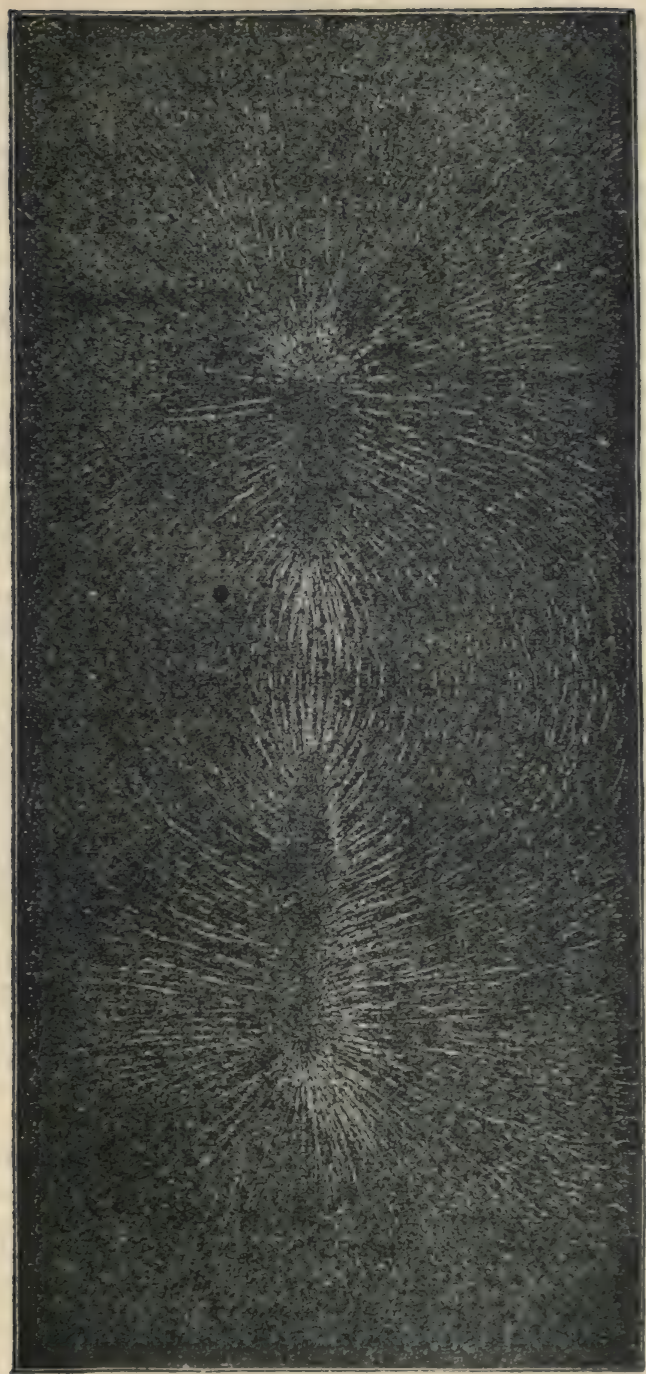

MAGNETIC LINES OF FORCE.

From a Photograph by Professor Mayer. 
effects which we have observed in our private chambers. Why, we inquire, does the magnetic neerle set north and south? Evidently it is compelled to do so by the earth; the great globe which we inherit is itself a magnet. Let us learn a little more about it. By means of a bit of wax or otherwise, attach the end of your silk fiber to the middle point of your magnetic needle; the needle will thus be uninterfered with by the paper loop, and will enjoy to some extent a power of "dipping" its point, or its eye, below the horizon. Lay your bar-magnet on a table, and hold the needle over the equator of the magnet. The needle sets horizontal. Move it toward the north end of the magnet; the south end of the needle dips, the dip augmenting as you approach the north pole, over which the needle, if free to move, will set itself exactly vertical. Move it back to the center, it resumes its horizontality; pass it on toward the south pole, its north end now dips, and directly over the south pole the needle becomes vertical, its north end being now turned downward. Thus we learn that on the one side of the magnetic equator the north end of the needle dips; on the other side the south end dips, the dip varying from nothing to ninety degrees. If we go to the equatorial regions of the earth with a suitably suspended needle we shall find there the position of the needle horizontal. If we sail north one end of the needle dips; if we sail south the opposite end dips; and over the north or south terrestrial magnetic pole the needle sets vertical. The south magnetic pole has not yet been found, but Sir James Ross discovered the north magnetic pole on June 1, 1831. In this manner we establish a complete parallelism between the action of the earth and that of an ordinary magnet.

'The terrestrial magnetic poles ilo not coincide with the geographical ones; nor does the earth's magnetic equator quite coincide with the geographical equator. The direction of the magnetic needle in London, which is called the magnetic meridian, encloses an angle of 24 degrees with the astronomical meridian, this angle being called the Declination of the needle for Loudon. 'The north pole of the needle now lies to the west of the true meridian; the declination is westerly. In the year 1660, however, the declination was nothing, while before that time it was easterly. All this proves that the earth's magnetic constituents are gradually changing their distribution. 'This 
change is very slow: it is therefore called the secular change, and the observation of it has not yet extended over a sufficient period to enable us to guess, even approximately, at its laws.

Having thus discovered, to some extent, the secret of the earth'£ magnetic power, we can turn it to account. In the line of "dip" I hold a poker formed of good soft iron. The earth, acting as a magnet, is at this moment constraining the two fluids of the poker to separate, making the lower end of the poker a north pole, and the upper end a south pole. Mark the experiment: When the knob is uppermost, it attracts the north end of a magnetic needle; when undermost it attracts the south end of a magnetic needle. With such a poker repeat this experiment and satisfy yourselves that the fluids shift their position accurding to the manner in which the poker is presented to the earth. It has already been stated that the softest iron possesses a certain amount of coercive force. The earth, at this moment, finds in this force an antagonist which opposes the decomposition of the neutral fluid. The component fluids may be figured as meeting an amount of friction, or possessing an amount of adhesion, which prevents them from gliding over the molecules of the poker. Can we assist the earth in this case? If we wish to remove the residue of a powder from the interior surface of a glass to which the powder clings, we invert the glass, tap it, loosen the hold of the powder, and thus enable the force of gravity to pull it down. So also by tapping the end of the poker we loosen the adhesion of the magnetic fluids to the molecules and enable the earth to pull them apart. But, what is the consequence? The portion of fluid which has been thus forcibly dragged over the molecules refuses to return. when the poker has been removed from the line of dip; the iron, as you see, has become a permanent magnet. By reversing its position and tapping it again we reverse its magnetism. A thoughtful and competent teacher will know how to place these remarkable facts before his pupils in a manner which will excite their interest. By the use of sensible images, more or less gross, he will first give those whom he teaches definite conceptions, purifying these conceptions afterward, as the minds of his pupils become more capable of abstraction. By thus giving them a distinct substratum for their reasonings, he will 
confer upon his pupils a profit and a joy which the mere exhibition of facts without principles, or the appeal to the bodily senses and the power of memory alone, could never inspire.

As an expansion of the note at p. 272, the following extract may find a place here:

"It is well known that a voltaic current exerts an attractive force upon a second current, flowing in the same direction; and that when the directions are opposed to each other the force exerted is a repulsive one. By coiling wires into spirals, Ampère was enabled to make them produce all the phenomena of attraction and repulsion exhibited by magnets, and from this it was but a step to his celebrated theory of molecular currents. He supposed the molecules of a magnetic body to be surrounded by such currents, which, however, in the natural state of the body mutually neutralized each other, on account of their confused grouping. The act of magnetization he supposed to consist in setting these molecular currents parallel to each other; and, starting from this principle, he reduced all the phenomena of magnetism to the mutual action of electric currents.

"If we reflect upon the experiments recorded in the foregoing pages from first to last, we can bardly fail to be convinced that diamagnetic bodies operated on by magnetic forces possess a polarity 'the same in kind as, but the reverse in direction of that acquired by magnetic bodies.' But if this be the case, how are we to conceive the physical mechanism of this polarity? According to Coulomb's and Poisson's theory, the act of magnetization consists in the decomposition of a neutral magnetic fluid; the north pole of a magnet, for example, possesses an attraction for the south fluid of a piece of soft iron submitted to its influence, draws the said fluid toward it, and with it the material particles with which the fluid is associated. To account for diamagnetic phenomena this theory seems to fail altogether: according to it, indeed, the oft-used phrase, " a north pole exciting a north pole, and a south pole a south pole,' involves a contradiction. For if the north fluid be supposed to be attracted toward the influencing north pole, it is absurd to suppose that its presence there could produce repulsion. The theory of Ampère is equally at a loss to explain diamagnetic action; for if we suppose the particles of bismuth surrounded by molecular currents, then, according to all that is known of electro-dynamic laws, these currents would set themselves parallel to, and in the same direction as those of the magnet, and hence attraction, and not repulsion, would be the result. The fact, however, of this not being the case, proves that these molecular currents are not the mechanism by which diamagnetic induction is effected. The consciousness of this, I doubt not, drove $\mathbf{M}$. Weber to the assumption that the phenomena of diamagnetism are produced by molecular currents, not directed, but actually excited in the bismuth by the magnet. Such induced currents would, according to known laws, have a direction opposed to those of the inducing magnet; and hence would produce the phenomena of repulsion. To carry 
out the assumption here made, $\mathbf{M}$. Weber is obliged to suppose that the molecules of diamagnetic bodies are surrounded by channels, in which the induced molecular currents, once excited, continue to flow without resistance." *-Diamagnetism and Magne-crystallic Action, p. 136-7.

\section{CHAPTER XVI.}

oN FORCE. $\nmid$

A SPHERE of lead was suspended at a height of 16 feet above the theater floor of the Royal Institution. It was liberated, and fell by gravity. That weight required a second to fall to the floor from that elevation; and the instant before it touched the floor it had a velocity of 32 feet a second. That is to say, if at that instant the earth were annihilated, and its attraction annulled, the weight would proceed through space at the uniform velocity of 32 feet a second.

If instead of being pulled downward by gravity, the weight be cast upward in opposition to gravity, then, to reach a height of 16 feet it must start with a velocity of 32 feet a second. This velocity imparted to the weight by the human hand, or by any other mechanical means, would carry it to the precise height from which we saw it fall.

Now the lifting of the weight may be regarded as so much mechanical work performed. By means of a ladder placed against the wall, the weight might be carried up to a height of 16 feet; or it might be drawn up to this height by means of a string and pulley, or it might be suddenly jerked up to a height of 16 feet. The amount of work done in all these cases, as far as the raising of the weight is concerned, would be absolutely the same. The works done at one and the same place, and neglecting the small change of gravity with the height, depends solely upon two things; on the quantity of matter lifted, and on the height to which it is lifted. If we call the quantity or mass of matter $m$, and the height through which it is lifted $h$, then the product of $m$ into $h$, or $m h$, expresses, or is proportional to the amount of work done.

Supposing, instead of imparting a velocity of 32 feet a

* In assuming these non-resisting channels M. Weber, it must be admitted, did not go beyond the assumptions of Ampè re.

† A discourse delivered in the Royal Institution, June 6, 1862. 
second we impart at starting twice this velocity. To what height will the weight rise? You might be disposed to answer, "To twice the height;" but this would be quite incorrect. Instead of twice 16 , or 32 feet, it would reach a height of four times 16 , or 64 feet. So also, if we treble the starting velocity, the weight would reach nine times the height; if we quadruple the speed at starting, we attain sixteen times the height. Thus, with a fourfold velocity of 128 feet a second at starting, the weight would attain an elevation of 256 feet. With a sevenfold velocity at starting, the weight would rise to 49 times the height, or to an elevation of 784 feet.

Now the work done-or, as it is sometimes called, the mechanical effect-other things being constant, is, as before explained, proportional to the height, and as a double velocity gives four times the height, a treble velocity nine times the height, and so on, it is perfectly plain that the mechanical effect increases as the square of the velocity. If the mass of the body be represented by the letter $m$, and its velocity by $v$, the mechanical effect would be proportional to or represented by $m v^{2}$. In the case considered, I have supposed the weight to be cast upward, being opposed in its flight by the resistance of gravity; but the same holds true if the projectile be sent into water, mud, earth, timber, or other resisting material. If, for example, we double the velocity of a cannon ball we quadruple its mechanical effect. Hence the importance of augmenting the velocity of a projectile, and hence the philosophy of Sir William Armstrong in using a large charge of powder in his recent striking experiments.

The measure then of mechanical effect is the mass of the body multiplied by the square of its velocity.

Now in firing a ball against a target the projectile, after collision, is often found hot. Mr. Fairbairn informs me that in the experiments at Shoeburyness it is a common thing to see a flash, even in broad daylight, when the ball strikes the target. And if our lead weight be examined after it has fallen from a height it is also founil heated. Now here experiment and reasoning lead us to the remarkable law that, like the mechanical effect, the amount of heat generated is proportional to the product of the mass into the square of the velocity. Double your mass, other things being equal, and you double your amount of heat; 
double your velocity, other things remaining equal, and you qualruple your amount of heat. Here then we have common mechanical motion destroyed and heat produced. When a violin bow is drawn across a string, the sound produced is due to motion imparted to the air, and to produce that motion muscular force has been expended. We may here correctly say, that the mechanical force of the arm is converted into music. In a similar way we say that the arrested motion of our descending weight, or of the cannon ball, is converted into lieat. The mode of motion changes, but motion still continues; the motion of the mass is converted into a motion of the atoms of the miss; and these small motions, communicated to the nerves, produce the sensation we call heat.

We know the amount of heat which a given amount of mechanical force can develop. Our lead ball, for example, in falling to the earth generated a quantity of heat sufficient to raise its own temperature three-fifths of a Fahrenheit degree. It reached the earth with a velocity of 32 feet a second, and forty times this velocity would be small for a rifle bullet; multiplying three-fifths by the square of 40 , we find that the amount of heat developed by collision with the target would, if wholly concentrated in the lead, raise its temperature 960 degrees. 'This would be more than sufficient to fuse the lead. In reality, however, the heat developed is divided between the lead and the body against which it strikes; nevertheless, it would be worth while to pay attention to this point, and to ascertain whether rifle bullets do not, under some circumstances, show sigus of fusion.*

From the motion of sensible masses, by gravity and other means, we now pass to the motion of atoms toward each other by chenical affinity. A collodion balloon filled with a mixture of chlorine and hydrogen being hung in the focus of a parabolic mirror, in the focus of a second mirror 20 feet distant a strong electric light was suddenly generated; the instant the concentrated light fell upon the balloon, the gases within it exploded, hydrochloric acid being the result. Here the atoms virtually fell together, the amount of heat produced showing the

* Eight years subsequently this surmise was proved correct. In the Franco.German War signs of fusion were observed in the case of bullets impinging on bones, 
enormous force of the collision. The burning of charcoal in oxygen is an old experiment, but it has now a significance beyond what it used to have; we now regard the act of combination on the part of the atoms of oxygen and coal as we regard the clashing of a falling weight against the earth. The heat produced in both cases is referable to a common cause. A diamond, which burns in oxygen as a star of white light, glows and burns in consequence of the falling of the atoms of oxygen against it. And could we measure the velocity of the atoms when they clash, and could we find their number and weights, multiplying the weight of each atom by the square of its velocity, and adding all together, we should get a number representing the exact amount of heat developed by the union of the oxygen and carbon.

'Thus far we have regarded the heat developed by the clashing of sensible masses and of atoms. Work is expeuded in giving motion to these atoms or masses, and heat is developed. But we reverse this process daily, and by the expenditure of heat execute work. We can raise a weight by heat; and in this agent we possess an enormous store of mechanical power. A pound of coal produces by its combination with oxygen an amount of heat which, if mechanically applied, wonld suffice to raise a weight of 100 lbs. to a height of 20 miles above the earth's surface. Conversely, 100 lbs. falling from a height of 20 miles, and striking against the earth, would generate an amount of heat equal to that developed by the combustion of a pound of coal. Wherever work is done by heat, heat disappears. A gun which fires a ball is less heated than one which fires blank cartridge. The quantity of heat communicated to the boiler of a working steam-engine is greater than that which could be obtained from the recondensation of the steam, after it had done its work; and the amount of work performed is the exact equivalent of the amount of heat lost. Mr. Smyth informed us in his interesting discourse, that we dig annually 84 millions of tons of coal from our pits. The amount of mechanical force represented by this quantity of coal seems perfectly fabulous. The combustion of a single pound of coal, supposing it to take place in a minute, would be equivalent to the work of 300 horses; and if we suppose 108 millions of horses working day and night with unimpaired strength, for a year, their united 
energies would enable them to perform an amount of work just equivalent to that which the annual produce of our coal-fields would be able to accomplish.

Comparing with ordinary gravity the force with which oxygen and carbon unite together, chemical affinity seems almost infinite. But let us give gravity fair play by permitting it to act throughout its entire range. Place a body at such a distance from the earth that the attraction of our planet is barely sensible, and let it fall to the earth from this distance. It would reach the earth with a final velocity of 36,747 feet a second; and on collision with the earth the body would generate about twice the amount of heat, generated by the combustion of an equal weight of coal. We have stated that by falling through a space of 16 feet our lead bullet would be heated three-fifths of a degree; but a body falling from an infinite distance has already used up 1,299,999 parts out of $1,300,000$ of the earth's pulling power, when it has arrived within $\mathbf{3 6}$ feet of the surface; on this space only one one million three hundred thousandths of the whole force is exerted.

Let us now turn our thoughts for a moment from the earth to the sun. The researches of Sir John Herschel and M. Pouillet have informed us of the annual expenditure of the sun as regards heat; and by an easy calculation we ascertain the precise amount of the expenditure which falls to the share of our planet. Out of 2,300 million parts of light and heat the earth receives one. The whole heat emitted by the sun in a minute would be competent to boil 12,000 millions of cubic miles of ice-cold water. How is this enormous loss male good-whence is the sun's heat derived, and by what means is it maintained? No combustion-no chemical affinity with which we are acquainted, would be competent to produce the temperature of the sun's surface. Besides, were the sun a burning body merely, its light and heat would speedily come to an end. Supposing it to be a solid globe of coal, its combustion would only cover 4,600 years of expenditure. In this short time it would burn itself out. What agency then can produce the temperature and maintain the outlay? We have already regarded the case of a body falling from a great distance toward the earth and found that the heat generater by its collision would be twice that produced by the combustion of an equal weight 
of coal. How much greater must be the heat developed by a body falling against the sun! The maximum velocity with which a body can strike the earth is about seven miles in a second; the maximum velocity with which it can strike the sun is 390 miles in a second. And as the heat developed by the collision is proportional to the square of the velocity destroyeu, an asteroid falling into the sun with the above velocity would generate about 10,000 times the quantity of heat produced by the combustion of an asteroid of coal of the same weight.

Have we any reason to believe that such bodies exist in space, and that they may be raining down upon the sun? The meteorites flashing through the air are small planetary borlies, drawn by the earth's attraction. They enter our atmosphere with planetary velocity, and by friction against the air they are raised to incandescence and caused to emit light and heat. At certain seasons of the year they shower down upon us in great numbers. In Boston 240,000 of them were observed in nine hours. There is no reason to suppose that the planetary system is limited to "vast masses of enormous weight;" there is, on the contrary, reason to believe that space is stocked with smaller masses, which obey the same laws as the larger ones. That lenticular envelope which surrounds the sun, and which is known to astronomers as the Zodiacal light, is probably a crowd of meteors; and moving as they do in a resisting medium, they must continually approach the sun. Falling into it, they would produce enormous heat, and this would constitute a source from which the annual loss of heat might be made good. The sun, according to this hypothesis, would continually grow larger; but how much larger? Were our moon to fall into the sun, it would develop an amount of heat sufficient to cover one or two years' loss; and were our earth to fall into the sun a century's loss would be made good. Still, our moon and our earth, if distributed over the surface of the sun, would utterly vanish from perception. Indeed, the quantity of matter competent to produce the required effect, would, during the range of history, cause no appreciable angmentation in the sun's magnitude. The augmentation of the sun's attractive force would be more sensible. However this hypothesis may fare as a representant of what is going on in nature, it certainly shows 
how a sun might be formed and maintained on known thermo-dyuamic principles.

Our earth moves in its orbit with a velocity of 68,040 miles an hour. Were this motion stopped, an amount of heat would be developed sufficient to raise the temperature of a globe of lead of the same size as the eirth 384,000 degrees of the centigrade thermometer. It has been prophesied that " the elements shall melt with fervent heat." 'The earth's own motion embraces the conditions of fulfilment; stop that motion and the greater part, if not the whole, of our planet would be reduced to vapor. If the earth fell into the sun, the amount of heat developed by the shock wonld be equal to that dereloped by the combustion of a mass of solid coal 6,435 times the earth in size.

There is one other consideration connected with the permanence of our present terrestrial conditions, which is well worthy of our attention. Standing upon one of the London bridges, we observe the current of the Thames reversed, and the water poured upward twice a day. The water thus moved rubs against the river's bed, and heat is the consequence of this friction. The heat thus generated is in part radiated into space and lost, as far as the earth is concerned. What supplies this incessant loss? 'The earth's rotation. Let us look a little more closely at the matter. Imagine the moon fixed, and the earth turning like a wheel from west to east in its diurnal rotation. Suppose a high mountain on the earth's surface approaching the earth's meridian; that mountain is, as it were, laid hold of by the moon; it forms a kind of handle by which the earth is pulled more quickly round. But when the meridian is passed the pull of the moon on the mountain would be in the opposite direction, it would tend to diminish the velocity of rotation as much as it previously augmented it; thus the action of all fixed bodies on the earth's surface is neutralized. But suppose the mountain to lie always to the east of the moon's meridian, the pull then would be always exerted against the earth's rotation, the velocity of which would be diminished in a degree corresponding to the strength of the pull. The tidal wave occupies this position - it lies always to the east of the moon's meridian. The waters of the ocean are in part dragged as a brake along the surface of the earth; and as a brake they must dimin- 
ish the velocity of the earth's rotation.* Supposing then that we turn a mill by the action of the tide, and produce heat by the friction of the millstones; that heat has an origin totally different from the heat produced by another mill which is turned by a mountain stream. The former is produced at the expense of the earth's rotation, the latter at the expense of the sun's radiation.

The sun, by the act of vaporization, lifts mechanically all the moisture of our air, which when it condenses falls in the form of rain, and when it freezes falls as snow. In this solid form it is piled upon the Alpine heights, and furnishes materials for glaciers. But the sun again interposes, liberates the solidified liquid, and permits it to roll by grarity to the sea. The mechanical force of every river in the world as it rolls toward the ocean, is drawn from the heat of the sun. No streamlet glides to a lower level without having been first lifted to the elevation from which it springs by the power of the sun. The energy of winds is also due entirely to the same power.

But there is still another work which the sun performs, and its connection with which is not so obvious. Trees and regetables grow upon the earth, and when burned they give rise to heat, and hence to mechanical energy. Whence is this power derived? You see this oxide of iron, produced by the falling together of the atoms of iron and oxygen; you cannot see this transparent carbonic acid gas, formed by the falling together of carbon and oxygen. The atoms thus in close union resemble our lead weight while resting on the earth; but we can wind up the weight and prepare it for another fall, and so these atoms can be wound up and thus enabled to repeat the process of combination. In the building of plants carbonic acid is the material from which the carbon of the plant is derived; and the solar beam is the agent which tears the atoms asunder, setting the oxygen free, and allowing the carbon to aggregate in woody fiber. Let the solar rays fall upon a surface of sand; the sand is heated, and finally radiates away as much heat as it receives; let the same beams fall upon a forest, the quantity of heat given back is less than the forest receives; for the energy of a portion of the sunbeams is invested in building the trees. Without the sun

* Kant surmised an action of this kind. 
the reduction of the carbouic acid cannot be effected, and an amount of sunlight is consumed exactly equivalent to the molecular work done. 'Thus trees are formed; thus the cotton on which Mr. Bazley discoursed last Friday is produced. I ignite this cotton, and it flames; the oxygen again unites with the carbon; but an amount of heat equal to that produced by its combustion was sacrificed by the sun to form that bit of cotton.

We cannot, however, stop at vegetable life, for it is the source, mediate or immediate, of all animal life. The sun severs the carbon from its oxygen and builds the vegetable; the animal consumes the vegetable thus formed, a reunion of the severed elements takes place, producing animal heat. The process of building a vegetable is one of winding up; the process of building an auimal is one of running down. 'The warmth of our bodies, and every mechanical energy which we exert, trace their lineage directly to the sun. 'The fight of a pair of pugilists, the motion of an army, or the lifting of his own body by an Alpine climber up a mountain slope, are all cases of mechanical energy drawn from the sun. A man weighing 150 pounds has 64 pounds of muscle; but these, when dried, reduce themselves to 15 pounds. Doing an ordinary day's work, for eighty days, this mass of muscle would be wholly oxidizerl. Special urgans which do more work would be more quickly consumed: the heart, for example, if entirely unsustained, would be oxidized in about a week. Take the amount of heat due to the direct oxidation of a given weight of food; less heat is developed by the oxidation of the same amount of food in the working animal frame, and the missing quantity is the equivalent of the mechanical work accomplished by the muscles.

I might extend these considerations; the work, indeed, is done to my hand-but I am warned that you have been already kept too long. To whom then are we indebted for the most striking generalizations of this evening's discourse? They are the work of a man of whom you have scarcely ever heard-the published labors of a German doctor, named Mayer. Without external stimulus, and pursuing his profession as town physician in Heilbronn, this man was the first to raise the conception of the interaction of heat and other natural forces to clearness in his own mind. And yet he is scarcely ever heard of, and 
even to scientific men his merits are but partially known. Led by his own beautiful researches, and quite independent of Mayer, Mr. Joule published in 1843 his first paper on the "Mechanical Value of Heat;" but in 1842 Mayer" had actually calculated the mechanical equivalent of heat from data which only a man of the rarest penetration could turn to account. In 1845 he published his memoir on "Organic Motion," and applied the mechanical theory of heat in the most fuarless and precise manner to vital processes. He also embraced the other natural agents in his chain of conservation. In $1853 \mathrm{Mr}$. Waterston proposed, independently, the meteoric theory of the sun's heat, and in 1854 Professor William 'Thomson applied his admirable mathematical powers to the developnent of the theory; but six years previously the subject had been handled in a masterly manner by Mayer, and all that I have said about it has been derived from him. When we consider the circumstances of Mayer's life, and the period at which he wrote, we cannot fail to be struck with astonishment at what he has accomplished. Here was a man of genius working in silence, animated solely by a love of his subject, and arriving at the most important results in advance of those whose lives were entirely devoted to natural philosophy. It was the accident of bleeding a feverish patient at Java in 1840 that led Mayer to speculate on these subjects. He noticed that the venous blood in the tropics was of a brighter red than in colder latitudes, and his reasoning on this fact led him into the laboratory of natural forces, where he has worked with such signal ability and success. Well, you will desire to know what has become of this man. His mind, it is alleged, gave way; it is sail he became insane, and he was certainly sent to a lunatic asylum. In a biographical dictionary of his country it is stated that he died there, but this is incorrect. He recovered; and, I believe, is at this moment a cultivator of vineyards in Heilbronn.

June 20, 1862.

While pre paring for publication my last course of lectures on Heat, I wished to make myself acquainted with all that Dr. Mayer had done in connection with this subject. I accordingly wrote to two gentlemen who above all others 
seemed likely to give me the information which I needed.* Both of them are Germans, and both particularly distinguished in connection with the Dynamical 'Theory of Heat. Each of them kindly furnished me with the list of Mayer's publications, and one of them [Clausius] was so friendly as to order them from a bookseller, and to send them to me. 'This friend, in his reply to my first letter regarding Mayer, stated his belief that I should not find anything very important in Mayer's writings; but before forwarding the memoirs to me he read them himself. His letter accompanying them contains the following words: "I must here retract the statement in my last letter, that you would not find much matter of importance in Mayer's writings: I am astonished at the multitude of beantiful and correct thoughts which they contain;" and he goes on to point out various important subjects, in the treatment of which Mayer had anticipated other eminent writers. My other friend, in whose own publications the name of Mayer repeatedly occurs, and whose papers contaiuing these references were trauslated some years ago by myself, was, on the 10th of last month, unacquainted with the thoughtful and beautiful essay of Mayer's, entitled "Beiträge zur Dynamik des Himmels," and in 1854, when Professor William 'Thomson developed in so striking a manner the meteoric theory of the sun's heat, he was certainly not aware of the existence of that essay, though from a recent article in "Macmillan's Magazine" I infer that he is now aware of it. Mayer's physiological writings have been referred to by physiologists - by Dr. Carpenter, for example -in terms of honoring recognition. We have hitherto, indeed, obtained fragmentary glimpses of the man partly from physicists and partly from physiologists; but his total merit has never yet been recognized as it assuredly would have been had he chosen a happier mode of publication. I do not think a greater disservice could be done to a man of science, than to overstate his claims: such overstatement is sure to recoil to the disadvantage of him in whose interest it is made. But when Mayer's opportunities, achievements, and fate are taken into account, $\mathrm{I}$ do not think that I shall be deeply blamed for attempting to place him in that honorable position, which I believe to be his due. 
Hele, however, are the titles of Mayer's papers, the perusal of which will correct any error of julgment into which I may have fallen regarding their author. "Bemerkungen über die Kräfte der unbelebten Natur," Liebig's "Annalen," 1842, Vol. 42, p. 231; "Die Organische Bewegung in ihrem Zusammenhange mit dem Stoffwechsel," Heilbronn, 1845̃; "Beiträge zur Dynamik des Himmels," Heilbronn, 1848; "Bemerkungen über das Mechanische Equivalent der Wärme," Heilbronn, 1851.

In Memoriam.-Dr. Julius Robert Mayer died at Heilbroun on March 20, 1878, aged 63 years. It gives me pleasure to reflect that the great position which he will forever occupy in the annals of science was first virtually assigned to him in the foregoing discourse. He was subsequently chosen by acclamation a member of the French Academy of Sciences; and he received from the Royal Society the Copley medal-its highest reward.*

November, 1878.

At the meeting of the British Association at Glasgow in 1876 -that is to say, more than fourteen years after its delivery and publication-the foregoing lecture was made the cloak for an unseemly personal attack by Professor 'I'ait. The anger which found this uncourteous rent dates from 1863, + when it fell to my lot to maintain, in opposition to him and a more eminent colleague, the position which in 1862 I had assigned to Dr. Mayer. In those days Professor 'I'ait denied to Mayer all originality, and he has since, I regret to say, never missed an opportunity, however small, of carping at Mayer's claims. The action of the Academy of Sciences and of the Royal Society summarily disposes of this detraction, to which its object, during his lifetime, never vouchsafed either remonstrance or reply.

Some time ago Professor 'Tait published a volume of lectures entitled "Recent Advances in Physical Science," which I have reason to know has evoked an amount of censure far beyond that hitherto publicly expressed. Many

* See “The Copley Medalist for 1871," p. 479.

† See "Philosophical Magazine" for this and the succeeding years. 
of the best heads on the continent of Europe agree in their rejection and condemnation of the historic portions of this book. In March last it was subjected to a brief but pungent critique by Du Bois-Reymond, the celebrated perpetual secretary of the Academy of Sciences in Berlin. Du BoisReymond's address was on "National Feeling," and his critique is thus wound up: "The author of the 'Lectures' is not, perhaps, sufficiently well acquainted with the history on which he professes to throw light, and on the later phases of which he passes so unreserved ( $s$ hroff) a judgment. He thus exposes himself to the suspicionwhich, unhappily, is not weakened by his other writings - that the fiery Celtic blood of his country occasionally runs away with him, converting him for the time into a scientific Chauvin. Scientific Chauvinism," adds the learned secretary, "from which German investigators have hitherto kept free, is more reprehensible (gehössig) than political Chauvinism, inasmuch as self-control (sittliche Haltung) is more to be expected from men of science, than from the politically excited mass." *

In the case before this "expectation" would, I fear, be doomed to disappointment. But Du Bois-Reymond and his countrymen must not accept the writings of Professor Tait as representative of the thought of England. Surely no nation in the world has more effectually shaken itself free from scientific Chauvinism. From the day that Davy, on presenting the Copley medal to Arago, scornfully brushed aside that spurious patriotism which would run national boundaries throngh the free domain of science, chivalry toward foreigners has been a guiding principle with the Royal Society

On the more private amenities indulged in by Professor 'Tait, I do not consider it necessary to say a word.

* Festrede, delivered before the Academy of Sciences of Berlin, in celebration of the birtluday of the emperor and king, March 28, 1878. 


\section{CHAPTER XVII.}

\section{CONTRIBUTIONS TO MOLECULAR PHYSICS. *}

HaviNG on previous occasions dwelt upon the enormous differences which exist among gaseous bollies both as regards their power of absorbing and emitting radiant heat, I have now to consider the effect of a change of aggregation. When a gas is condensed to a liquid, or a liquid congealed to a solid, the molecules coalesce, and grapple with each other by forces which are insensible as long as the gaseous state is maintained. But, even in the solid and liquid conditions, the luminiferous ether still surrounds the molecules: hence, if the acts of radiation and absorption depend on them individually, regardless of their state of aggregation, the change from the gaseous to the liquid state ought not materially to affect the radiant and absorbent power. If, on the contrary, the mutual entanglement of the molecules by the force of cohesion be of paramount influence, then we may expect that liquids will exhibit a deportment toward radiant heat altogether different from that of the vapors from which they are derived.

'The first part of an inquiry conducted in 1863-64 was devoted to an exhaustive examination of this question. 'Twelve different liquids were employed, and five different layers of each, varying in thickness from $0.0 \%$ of an inch to 0.27 of an inch. The liquids were enclosed, not in glass vessels, which would have materially modified the incident heat, but between plates of transparent rock-salt, which only slightly affected the radiation. The source of heat throughout these comparative experiments consister of a platinum wire, raised to incandescence by an electric current of unvarying strength. The quantities of radiant heat absorbel and transmitted by each of the liquids at the respective thicknesses were first determined. 'The vapor's of these liquids were subsequently examined, the quantities of vapor employed being rendered proportional to the quantities of liquid previously traversed by the radiant heat. The result was that, for heat from the same source, the order of absorption of liquids and of their vapors proved

* A discourse delivered at the Royal Institution, March 18, 1864 supplementing, though of prior date, the Rede Lecture on Radiation. 
absolutely the same. There is no known exception to this law; so that, to determine the position of a vapor as an absorber or a radiator, it is only necessary to determine the position of its liquid.

'This result proves that the state of aggregation, as far at all events as the liquid stage is concerned, is of altogether subordinate moment-a conclusion which will probably prove to be of cardinal importance in molecular physics. On one important and contesterl point it has a special bearing. If the position of a liquid as an absorber and radiator determine that of its vapor, the position of water fixes that of aqueous vapor. Water has been compared with other liquids in a multitude of experiments, and it has been found, both as a radiant and as an absorbent, to transcend them all. 'Thus, for example, a layer of bisulphide of carbon 0.02 of an inch in thickness absorbs 6 per cent., and allows 94 per cent. of the radiation from the red-hot platinum spiral to pass through it; benzol absorbs 43 and transmits $5 \%$ per cent. of the same radiation; alcohol absorbs 67 and transmits 33 per cent., and alcohol, as an absorber of radiant heat, stands at the head of all liquids except one. The exception is water. A layer of this substance, of the thickness above given, absorbs 81 per cent., and permits only 19 per cent. of the radiation to pass through it. Had no single experiment ever been made upon the vapor of water, its vigorous action upon radiant heat might be inferred from the deportment of the liquid.

The relation of absorption and radiation to the chemical constitution of the radiating and absorbing substances was next briefly considered. For the six substances in the list of liquids examined, the radiant and absorbent powers augment as the number of atoms in the compound molecule augments. Thus, bisulphide of carbon has 3 atoms, chloroform 5, iodide of ethyl 8 , benzol 12 , and amylene 15 atoms in their respectire molecules. The order of their power as radiants and absorbents is that here indicated, bisulplide of carbon being the feeblest and amylene the strongest of the six. Alcohol, however, excels benzol as an absorber, though it has but 9 atoms in its molecule; but, on the other hand, its molecule is rendered more complex by the introduction of a new element. Benzol contains carbon and hyllogen, while alcohol contains carbon, hydrogen and oxygen. 'Thus, not only does atomic multi- 
tude come into play in absorption and radiation--atomic complexity must also be taken into account. 1 would recommend to the particular attention of chemists the molecule of water; the deportment of this substance toward radiant heat being perfectly anomalous, if the chemical formula at present ascribed to it be correct.

Sir William Herschel made the important discovery that, beyond the limits of the red end of the solar spectrum, rays of high heating power exist which are incompetent to excite vision. 'The discovery is capable of extension. Dissolving iodine in the bisulphide of carbon, a solution is obtained which entirely intercepts the light of the most brilliant flames, while to the ultra-red rays of such flames the same iodine is found to be perfectly diathermic. The transparent bisulphide, which is highly pervious to invisible heat, exercises on it the same absorption as the perfectly opaque solution. A hollow prism filled with the opaque liquid being placed in the path of the beam from an electric lamp, the light-spectrum is completely intercepted, but the heat-spectrum may be received upon a screen and there examined. Falling upon a thermo-electric pile, its invisible presence is shown by the prompt deflection of even a coarse galvanometer.

What, then, is the physical meaning of opacity and transparency as regards light and radiant heat? The visible rays of the spectrum differ from the invisible ones simply in period. The sensation of light is excited by waves of ether shorter and more quickly recurrent than the non-visual waves which fall beyond the extreme red. But why should iodine stop the former and allow the latter to pass? 'The answer to this question no doubt is, that the intercepted waves are those whose periods of recurrence coincide with the periods of oscillation possible to the atoms of the dissolved iodine. The elastic forces which keep these atoms apart compel them to vibrate in definite periods, and, when these periods synchronize with those of the ethereal waves, the latter are absorbed. Briefly defined, then, transparency in liqnids, as well as in gases, is synonymous with discord, while opacity is synonymous with accord, between the periods of the waves of ether and those of the molecules on which they impinge.

According to this view transparent and colorless substances owe their transparency to the dissonance existing 
between the oscillating periods of their atoms and those of the waves of the whole visible spectrum. From the prevalence of transparency in compound bodies, the general discord of the vibrating periods of their atoms with the light-giving waves of the spectrum may be inferred; while their synchronism with the ultra-red periods is to be inferred from their opacity to the ultra-red rays. Water illustrates this in a most striking manner. It is highly transparent to the luminous rays, which proves that its atoms do not readily oscillate in the periods which excite vision. It is highly opaque to the ultra-red undulations, which proves the synchronism of its vibrating periods with those of the longer waves.

If, then, to the radiation from any source water shows itself eminently or perfectly opaque, we may infer that the atoms whence the radiation emanates oscillate in ultrared periods. Let us apply this test to the radiation from a flame of hydrogen. This flame consists mainly of incandescent aqueous vapor, the temperature of which, as calculated by Bunsen, is 3,259 degrees C., so that, if the penetrative power of radiant heat, as generally supposed, augment with the temperature of its source, we may expect the radiation from this flame to be copiotisly transinitted by water. While, however, a layer of the bisulphide of earbon 0.07 of an inch in thickness transmits 72 per cent. of the incident radiation, and while every other liquid examined transmits more or less of the heat, a layer of water of the above thickness is entirely opaque to the radiation from the hydrogen flame. Thus we establish accord between the periods of the atoms of cold water and those of aqueous vapor at a temperature of 3,259 degrees C. But the periods of water have already been proved to be ultra-red -hence those of the hydrogen flame must be sensibly ultra-red also. The absorption by dry air of the heat emitted by a platinum spiral raised to incandescence by electricity is insensible, while that by the ordinary undried air is 6 per cent. Substituting for the platinum spiral a hydrogen flame, the absorption by dry air still remains insensible, whlle that of the undried air rises to 20 per cent. of the entire radiation. The temperature of the hydrogen flame is, as stater, 3,259 degrees C.; that of the aqueuns vapor of the air 20 degrees C. Suppose, then, the temperature of aqueous vapor to rise from 20 degrees 
C. to 3,259 degrees C., we must conclude that the angmentation of temperature is applied to an increase of amplitude or width of swing, and not to the introduction of quicker periods into the radiation.

The part played by aqueous vapor in the economy of nature is far more wonderful than has been hitherto supposed. To nourish the vegetation of the earth the actinic and luminous rays of the sun must penetrate our atmosphere; and to such rays aqueous vapor is eminently transparent. The violet and the ultra-violet rays pass through it with freedom. 'To protect vegetation from destructive chills the terrestrial rays must be checked in their transit toward stellar space; and this is accomplished by the aqueous vapor diffused through the air. This substance is the great moderator of the earth's temperature, bringing its extremes into proximity, and obviating contrasts between day and night which would render life insupportable. But we can advance beyond this general statement, now that we know the radiation from aqueous vapor is intercepted, in a special degree, by water, and, reciprocally, the radiation from water by aqueous vapor; for it follows from this that the very act of nocturnal refrigeration which produces the condensation of aqueous vapor at the surface of the earth-giving, as it were, a varnish of water to that surface -imparts to terrestrial radiation that particular character which disqualifies it from passing through the earth's atmosphere and losing itself in space.

And here we come to a question in molecular physics which at the present moment occupies attention. By allowing the violet and ultra-violet rays of the spectrum to fall upon sulphate of quinine and other substances, Professor Stokes has changed the periods of those rays. Attempts have been made to produce a similar result at the other end of the spectrum-to convert the ultra-red periorls into periods competent to excite vision-but hitherto with. out success. Such a change of period, I agree with Dr. Miller in believing, occurs when the limelight is produced by an oxyliydrogen flame. In this common experiment there is an actual breaking up of long periods into short ones-a true rendering of unvisual periods visual. The change of refrangibility here effected differs from that of Professor Stokes; firstly, by its being in the opposite direction-that is, from a lower refrangibility to a higher; and, 
secondly, in the circumstance that the lime is heated by the collision of the molecules of aqueous vapor, before their heat has assumed the radiant form. But it cannot be doubted that the same effect would be produced by radiant heat of the same periods, provided the motion of the ether could be rendered sufficiently intense.* The effect in principle is the same, whether we consider the lime to be struck by a particle of aqueous vapor oscillating at a certain rate, or by a particle of ether oscillating at the same rate.

By plunging a platinum wire into a hydrogen flame we canse it to glow, and thus introduce shorter periods into the raliation. These, as already stated, are in discord with the atomic vibrations of water; hence we may infer that the transmission through water will be rendered more copious by the introduction of the wire into the flame. Experiment proves this conclusion to be true. Water, from being opaque, opens a passage to 6 per cent. of the radiation from the spiral. A thin plate of colorless glass, moreover, transmits 58 per cent. of the radiation from the hydrogen flame; but when the flame and spiral are employed, 78 per cent. of the heat is transmitted.

For an alcohol flame Knoblanch and Melloni found glass to be less transparent than for the same flame with a platinum spiral immersed in it; but Melloni afterward showed that the result was not general-that black glass and black mica were decidedly more diathermic to the radiation from the pure alcohol flame. Melloni did not explain this, but the reason is now obvious. The mica and glass owe their blackness to the carbon diffused through them. This carbon, as first proved by Melloni, is in some measure transparent to the ultra-red rays, and I have myself succeeded in transmitting between 40 and 50 per cent. of the radiation from a hydrogen flame through a layer of carbon whish in tercepted the light of an intensely brilliant flame. The products of combustion of alcohol are carbonic acid and aqueous vapor, the heat of which is almost wholly ultra-red. For this radiation, then, the carbon is in a considerable degree transparent, while for the radiation from the platinum spiral, it is in a great measure opaque. The platinum wire, therefore, which augmented the radiation through the pure glass, angmented the absorption of the black glass and mica.

* This was soon afterward accomplished. See pp. 35, 36 . 
No more striking or instructive illustration of the influence of coincidence could be adduced than that furnished by the radiation from a carbonic oxide flame. Here the product of combustion is carbonic acid; and on the radiation from this flame even the ordinary carbonic acid of the atmosphere exerts a powerful effect. A quantity of the gas, only one-thirtieth of an atmosphere in density, contained in a polished brass tube four feet long, intercepts 50 per cent. of the radiation from the carbonic oxide flame. For the heat emitted by lampblack, olefiant gas is a far morè powerful absorber than carbonic acid; in fact, for such heat, with one exception, carbonic acid is the most feeble absorber to be found among the compound gases. Moreover, for the radiation from a hydrogen flame olefiant gas possesses twice the absorbent power of carbonic acid, while for the radiation from the carbonic oxide flame, at a common pressure of one inch of mercury, the absorption by carbonic acid is more than twice that of olefiant gas. Thus we establish the coincidence of period between carbonic acid at a temperature of 20 degrees $\mathrm{C}$. and carbonic acid at a temperature of over 3,000 degrees C., the periods of oscillation of both the incandescent and the cold gas belonging to the ultra-red portion of the spectrum.

It will be seen from the foregoing remarks and experiments how impossible it is to determine the effect of temperature pure and simple on the transmission of radiant heat if different sources of heat be employed. Throughout such an examination the same oscillating atoms ought to be retained. This is done by heating a platinum spiral by an electric current, the temperature meanwhile varying between the widest possible limits. Their comparative opacity to the ultra-red rays shows the general accord of the oscillating periods of the vapors referred to at the commencement of this lecture with those of the ultra-red undulations. Hence, by gradually heating a platinum wire from darkness up to whiteness, we ought gradually to augment the discord between it and these vapors, and thus augment the transmission. Experiment entirely confirms this conclusion. Formic ether, for example, absorbs 45 per cent. of the radiation from a platinum spiral heated to barely visible redness; 32 per cent. of the radiation from the same spiral at a red heat; 26 per cent. of the radiation from a white-hot spiral, and only 21 per cent. when the 
spiral is brought near its point of fusion. Remarkable cases of inversion as to transparency also occur. For barely visible redness formic ether is more opaque than sulphuric; for $u$ bright red heat both are equally transparent; while, for a white heat, and still more for a higher temperature, sulphuric ether is more opaque than formic. This result gives us a clear view of the relationship of the two substances to the luminiferous ether. As we introduce waves of shorter period the sulphuric ether augments most rapidly in opacity; that is to say, its accord with the shorter waves is greater than that of the formic. Hence we may infer that the atoms of formic ether oscillate, on the whole, more slowly than those of sulphuric ether.

When the source of heat is a Leslie's cube coated with lampblack and filled with boiling water, the opacity of formic ether in comparison with sulphuric is very decided. With this source also the positions of chloroform and iodide of methyl are inverted. For a white-hot spiral, the absorption of chloroform vapor being 10 per cent., that of iodide of methyl is 16 ; with the blackened cube as source, the absorption by chloroform is 22 per cent., while that by the iodide of methyl is only 19. This inversion is not the result of temperature merely; for when a platinnm wire, heated to the temperature of boiling water, is employed as a source, the iodide continues to be the most powerful absorber. All the experiments hitherto made go to prove that from heated lampblack an emission takes place which synchronizes in an especiai manner with chloroform. For the cube at 100 degrees C., coated with lampblack, the absorption by chloroform is more than three times that by bisulphide of carbon; for the raliation from the most luminous portion of a gas-flame the absorption by chloroform is also considerably in excess of that by bisulphide of carbon; while, for the flame of a Bunsen's burner, from which the incandescent carbon particles are removed by the free admixture of air, the absorption by bisulphide of carbon is nearly twice that by chloroform. The removal of the carbon particles more than doubles the relative transparency of the chloroform. 'T'esting, moreover, the radiation from various parts of the same flame, it was found that for the blue base of the flame the bisulphicle of carbon was most opaque, while for all other parts of the flame the 
chloroform was most opaque. For the radiation from a very small gas-flame, consisting of a blue base and a small white tip, the bisulphide was also most opaque, and its opacity very decidedly exceeded that of the chloroform when the source of heat was the flame of bisulphide of carbun. Comparing the radiation from a Leslie's cube coated with isinglass with that from a similar cube coated with lampblack, at the common temperature of 100 degrees C., it was found that, out of eleven vapors, all but one absorbed the radiation from the isinglass most powerfully; the single exception was chloroform.

It is worthy of remark that whenever, through a change of source, the position of a vapor as an absorber of radiant heat was altered, the position of the liquid from which the vapor was derived underwent a similar change.

It is still a point of difference between eminent investigators whether radiant heat, up to a temperature of 100 degrees C., is monochromatic or not. Some affirm this; some deny it. A long series of experiments enables me to state that probably no two substances at a temperature of $100 \mathrm{de}-$ grees C. emit heat of the same quality. 'The heat emitted by isinglass, for example, is different from that emitted by lampblack, and the heat emitted by cloth, or paper, differs from both. It also a subject of discussion whether rock-salt is equally diathermic to all kinds of calorific rays; the differences affirmed to exist by some investigators being ascribed by others to differences of incidence from the various sources employed. MM. de la Provostaye and Desains maintain the former view, Melloni and M. Knoblauch maintain the latter. I tested this point without changing anything but the temperature of the source; its size, distance, and surroundings remaining the same. 'The experiments proved rock-salt to be colored thermally. It is more opaque, for example, to the radiation from a barely visible spiral than to that from a white-hot one.

In regard to the relation of radiation to conduction, if we define radiation, internal as well as external, as the communication of motion from the ribrating atoms to the ether, we may, I think, by fair theoretic reasoning, reach the conclusion that the best radiators ought to prove the worst conductors. A broad consideration of the subject shows at once the general harmony of this conclusion with observed facts. Organic substances are all excellent radi- 
ators; they are also extremely bad conductors. The moment we pass from the metals to their compounds we pass from good conductors to bad ones, and from bad radiators to good ones. Water, among liquids, is probably the worst conductor; it is the best radiator. Silver, among solids, is the best conductor; it is the worst radiator. 'The excellent researches of MM. de la Provostaye and Desains furnish a striking illustration of what I am inclined to regard as a natural law-that those atoms which transfer the greatest amount of motion to the ether, or, in other words, radiate most powerfully, are the least competent to comınunicate motion to each other, or, in other words, to propagate by conduction readily.

\section{CHAPTER XVIII.}

\section{LIFE AND LETTERS OF FARADAY.}

$18 \% 0$.

UNDERTAKEN and executed in a reverent and loving spirit, the work of Dr. Bence Jones makes Faraday the virtual writer of his own life. Everybody now knows the story of the philosopher's birth; that his father was a smith; that he was born at Newington Butts in 1791; that he ran along the London pavements, a bright-eyed errand boy, with a load of brown curls upon his head and a packet of newspapers under his arm; that the lad's master was a bookseller and bookbinder-a kindly man, who became attached to the little fellow, and in due time male him his apprentice without fee; that during his apprenticeship he found his appetite for knowlelge provoked and strengthened by the books he stitched and covered. Thus he grew in wisdom and stature to his year of legal manhood, when he appears in the volumes before us as a writer of letters, which reveal his occupation, acquirements, and tone of mind. His correspondent was Mr. Abbott, a member of the Society of Friends, who, with a forecast of his correspondent's greatness, preserved his letters and produced them at the proper time.

In later years Faraday always carried in his pocket a blank card, on which he jotted down in pencil his thoughts and memoranda. He made his notes in the laboratory. in the theater, and in the streets. 'This distrust of his mem- 
ory reveals itself in his first letter to Abbot. To a proposition that no new inquiry should be started between them before the old one had been exhaustively discussed, Faraday objects. "Your notion," he says, "I can hardly allow, for the following reason: ideas and thoughts spring up in my mind which are irrevocably lost for want of noting at the time." Gentle as he seemed, he wished to have his own way, and he had it throughout his life. Differences of opinion sometimes arose between the two friends, and then they resolutely faced each other. "I accept your offer to fight it out with joy, and shall in the battle of experience cause not pain, but, I hope, pleasure." Faraday notes his own impetuosity, and incessantly checks it. There is at times something almost mechanical in his self-restraint. In another nature it would have hardened into mere "correctness" of conduct; but his overflowing affections prevented this in his case. The habit of self-control became a second nature to him at last, and lent serenity to his later years.

In October, 1812, he was engaged by a Mr. De la Roche as a journeyman bookbinder; but the situation did not suit him. His master appears to have been an austere and passionate man, and Faraday was to the last degree sensitive. All his life he continued so. He suffered at times from dejection; and a certain grimness, too, pervaded his moods. "At present," he writes to Abbott, "I am as serious as you can be, and would not scruple to speak a truth to any human being, whatever repugnance it might give rise to. Being in this state of mind, I should have refrained from writing to you, did I not conceive from the general tenor of your letters that your mind is, at proper times, occupied upon serious subjects to the exclusion of those that are frivolous." Plainly he had fallen into that stern Puritan mood, which not oniy crucifies the affections and lusts of him who harbors it, but is often a cause of disturbed digestion to his friends.

About three months after his engagement with De la Roche, Faraday quitted him and bookbinding together. He had heard Davy, copied his lectures, and written to him, entreating to be released from Trade, which he hated, and enabled to pursue Science. Davy recognized the merit of his corresponilent, kept his eye upon him, and, when occasion offered, drove to his door and sent in a letter, 
offering him the post of assistant in the laboratory of the Royal Institution. He was engaged March 1, 1813, and on the 8 th we find him extracting the sugar from beetroot. He joined the City Philosophical Society which had been founded by Mr. Tatum in 1808. "The discipline was very sturdy, the remarks very plain, and the results most valuable." Faraday derived great profit from this little association. In the laboratory he had a discipline sturdier still. Both Davy and himself were at this time frequently cut and bruised by explosions of chloride of nitrogen. One explosion was so rapid "as to blow my hand open, tear away a part of one nail, and make my fingers so sore that I cannot use them easily." In another experiment " the tube and receiver were blown to pieces, I got a cut on the head, and Sir Humphry a bruise on his hand." And again speaking of the same substance, he says, "when put in the pump and exhausted, it stood for a moment, and then exploded with a fearful noise. Both Sir H. and I had masks on, but I escaped this time the best. Sir H. had his face cut in two places about the chin, and a violent blow on the forehead struck through a considerable thickness of silk and leather." It was this same substance that blew out the eye of Dulong.

Over and over again, even at this early date, we can discern the quality which, compounded with his rare intel. lectual power, made Faraday a great experimental philosopher. This was his desire to see facts, and not to rest contented with the descriptions of them. He frequently pits the eye against the ear, and affirms the enormous superiority of the organ of vision. Late in life I have heard him say that he could never fully understand an experiment until he had seen it. But he did not confine himself to experiment. $\mathrm{He}$ aspired to be a teacher, and reflected and wrote upon the method of scientific exposition. "A lecturer," he observes, "should appear easy and collected, undaunted and unconcerned:" still " his whole behavior should evince respect for his audience." These recommendations were afterward in great part embodied by himself. I doubt his "unconcern," but his fearlessness was often manifested. It used to rise within him as a wave, which carried both him and his andience along with it. On rare occasions also, when he felt himself and his subject hopelessly unintelligible, he suddenly evoked a 
certain recklessness of thought, and, without halting to extricate his bewildered followers, he would dash alone through the jungle into which he had unwittingly led them; thus saving them from ennui by the exhibition of a vigor which, for the time being, they could neither share nor comprehend.

In October, 1813, he quitted England with Sir Humphry and Lady Davy. During his absence he kept a journal, from which copious and interesting extracts have been made by Dr. Bence Jones. Davy was considerate, preferring at times to be his own servant rather than impose on Faralay duties which he disliked. But Lady Davy was the reverse. She treated him as an underling; he chafed under the treatment, and was often on the point of return. ing home. They halted at Geneva. De la Rive, the elder, had known Davy in 1799, and, by his writings in the "Bibliothèque Britannique," had been the first to make the English chemist's labor's known abroal. He welcomed Dary to his country residence in 1814 . Both were sportsmen, and they often went out shooting together. On these occasions Faraday charged Davy's gun while De la Rive charged his own. Once the Genevese philosopher found himself by the side of Faraday, and in his frank and genial way entered into conversation with the young man. It was evident that a person possessing such a charm of manner and such high intelligence could be no mere servant. On inquiry De la Rive was somewhat shocked to fincl that the soidisant domestique was really préparateur in the laboratory of the Royal Institution; and he immediately proposed that Faraday thenceforth should join the masters instead of the servants at their meals. 'To this Davy, probably out of weak deference to his wife, objected; but an arrangement was come to that Faraday thenceforward should have his food in his own room. Rumor states that a dinner in honor of Faraday was given by De la Rive. This is a delusıon; there was no such banquet; but Faraday never forgot the kindness of the friend who saw his merit when he was a mere gargon de laboratoire.*

* While confined last autumn at Geneva by the effects of a fall in the Alps, my friends, with a kindness I can never forget, did all that friendship could suggest to render my captivity pleasant to me. M. De la Rive then wrote out for me the full account, of which the foregoing is a condensed abstract. It was at the desire of 
He returned in 1815 to the Royal Institution. Here he helped Davy for years; he worked also for himself, and lectured frequently at the City Philosophical Society. He took lessons in elocution, happily without damage to his natural force, earnestuess, and grace of delivery. He was never pledged to theory, and he changed in opinion as knowledge advanced. With him life was growth. In those early lectures we hear him say, "In knowledge, that man only is to be contemned and despised who is not in a state of transition." And again: "Nothing is more difficult and requires more cantion than philosophical deduction, nor is there anything more adrerse to its accuracy than fixity of opinion." Not that he was wafted about by every wind of doctrine; but that he united flexibility with his strength. In striking contrast with this inteliectual expansiveness was his fixity in religion, but this is a subject which cannot be discussed here.

Of all the letters published in these volumes none possess a greater charm than those of Faraday to his wife. Here, as Dr. Bence Jones truly remarks, " he laid open all his mind and the whole of his character, and what can be made known can scarcely fail to charm every one by its loveliness, its truthfulness, and its earnestness." Abbott and he sometimes swerved into word-play about love; but up to 1820 , or thereabonts, the passion was potential merely. Faraday's journal indeed contains entries which show that he took pleasure in the assertion of his contempt for love; but these very entries became links in his destiny. It was through them that he became acquainted with one who inspired him with a feeling which only ended with his life. His biographer has given us the means of tracing the varying moods which preceded his acceptance. 'T'hey reveal more than the common alternations of light and gloom; at one moment he wishes that his flesh might melt and that he might become nothing; at another he is intoxicated with hope. The impetuosity of his character was then unchastened by the discipline to which it was subjected in after years. The very strength of his passion proved for a time a bar to its advance, suggesting, as it did, to the conscientious mind of Miss Barnard, doubts of

Dr. Bence Jones that I asked him to do so. The rumor of a banquet at (ieneva illustrates the tendency to substitute for the youth of 1814 the Faraday of later years. 
her capability to return it with adequate force. But they met again and again, and at each successive meeting he found his hearen clearer, until at length he was able to say, “Not a monent's alloy of this erening's happiness occurred. Everything was delightful to the last moment of my stay with my companion, because she was so." The turbulence of doubt subsided, and a calm and elevating confidence took its place. "What can I call myself," he writes to her in a subsequent letter, " to convey most perfectly my affection and love for you? Can I or can truth say more than that for this world I am yours?" Assuredly he made his profession good, and no fairer light falls upon his character than that which reveals his relations to his wife. Never, I believe, existed a manlier, purer, steadier love. Like a burning diamond, it continued to shed, for six-and-forty years, its white and smokeless glow.

Faraday was married on June 12, 1821; and up to this date Davy appears throughout as his friend. Soon afterward, however, disunion occurred between them, which, while it lasted, must have given Faraday intense pain. It is impossible to doubt the honesty of conviction with which this subject has been treated by Dr. Bence Jones, and there may be facts known to him, but not appearing in these volumes, which justify his opinion that Dary in those days had become jealous of Faraday. This, which is the prevalent belief, is also reproduced in an excellent article in the March number of " Fraser's Magazine." But the best analysis I can make of the data fails to present Davy in this light to me. The facts, as I regard them, are briefly these.

In 1820, Oersted of Copenhagen made the celebrated discovery which connects electricity with magnetism, and immediately afterward the acute mind of Wollaston percelved that a wire carrying a current ought to rotate round its own axis under the influence of a magnetic pole. In 1821 he tried, but failed, to realize this result in the laboratory of the Royal Institution. Faraday was not present at the moment, but he came in immediately afterward and heard the conversation of Wollaston and Davy about the experiment. He had also heard a rumor of a wager that Dr. Wollaston would eventually succeed.

This was in April. In the autumn of the same year Faraday wrote a history of electro-magnetism, and repeated 
for himself the experiments which he described. It was while this instructing himself that he succeded in causing a wire, carrying an electric current, to rotate round a magnetic pole. This was not the result sought by Wollaston, but it was closely related to that result.

'The strong tendency of Faraday's mind to look upon the reciprocal actions of natural forces gave birth to his greatest discoveries; and we, who know this, should be justified in concluding that, even had Wollaston not preceded him, the result would have been the same. But in judging Davy we ought to transport ourselves to his time, and carefully exclude from our thoughts and feelings that noble subsequent life, which would render simply impossible the ascription to Faraday of anything unfair. It would be unjust to Davy to put our knowledge in the place of his, or to credit him with data which he could not have possessed. Rumor and fact had connected the name of Wollaston with these supposed interactions between magnets and currents. When, therefore, Faraday in October published his successful experiment, withont any allusion to Wollaston, general, though really ungrounded, criticism followed. I say ungrounded because, firstly, Faraday's experiment was not that of Wollaston, and secondly, Faraday, before he published it, had actually called upon Wollaston, and not finding him at home, did not feel himself anthorized to mention his name.

In December, Faraday published a second paper on the same subject, from which, through a misapprehension, the name of Wollaston was also omitted. Warburton and others thereupon affirmed that Wollaston's ideas had been appropriated without acknowledgment, and it is plain that Wollaston himself, though cutious in his utterance, was also hurt. Censure grew till it became intolerable. "I hear," writes Faraday to his friend Stodart, "every day more and more of these sounds, which, thongh only whispers to me, are, I suspect, spoken aloud among scientific men." He might have written explanations and defenses, but he went straighter to the point. He wisheil to see the principals face to face-to plead his cause before them personally. There was a certain vehemence in his desire to do this. He saw Wollaston, he saw Davy, he saw Warburton; and I am inclined to think that it was the irresistible candor and truth of character which these 
viva voce defenses revealed, as much as the defenses themselves, that disarmed resentment at the time.

As regards Davy, another cause of dissension arose in 1823. In the spring of that year Furaday analyzed the hydrate of chlorine, a substance once believed to be the element chlorine, but proved by Davy to be a compound of that olement and water. 'The analysis was looked over by Davy, who then and there suggested to Faraday to heat the hydrate in a closed glass tube. This was done, the substance was decomposed, and one of the products of decomposition was proved by Faraday to be chlorine liquefied by its own pressure. On the day of its discovery he communicated this result to Dr. Paris. Davy, on being informed of it, instantly liquefied another gas in the same way. Having struck thus into Faraday's inquiry, ought he not to have left the matter in Faraday's hands? I think he ought. But, considering his relation to both Faraday and the hydrate of chlorine, Davy, I submit, may be excused for thinking differently. A father is not always wise enough to see that his son has ceased to be a boy, and estrangement on this account is not rare; nor was Davy wise enough to discern that Faraday had passed the mere assistant stage, and become a discoverer. It is now hard to avoid magnifying this error. But hat Faraday died $o l^{\circ}$ ceased to work at this time, or had his subsequent life been devoted to money-getting, instead of to research, would anybody now dream of ascribing jealousy to Davy? Assuredly not. Why should he be jealous? His reputation at this time was almost without a parallel; his glory was with. out a cloud. He had added to his other discoveries that of Earaday, and after having been his teacher for seven years, his language to him was this: "It gives me great pleasure to hear that you are comfortable at the Royal Institution, and I trust that you will not only do somethiue good and honorable for yourself, but also for science." This is not the language of jealousy, potential or actual. But the chlorine business introduced irritation and anger, to which, and not to any ignobler motive, Davy's opposition to the election of Faraday to the Royal Society is, I am persuaded, to be ascribed.

'These matters are touched upon with perfect candor, and becoming consideration, in the volumes of Dr. Bence Jones; but in "society" they are not always so handled. 
Here a name of noble intellectual associations is surrounded by injurious rumors which I would willingly scatter forever. The pupil's magnitude, and the splendor of his position, are too great and absolute to need as a foil the humiliation of his master. Brothers in intellect, Dary and Faraday, however, could never have become brothers in feeling; their characters were too unlike. Davy loved the pomp and circumstance of fame; Faraday the inner conscionsuess that he had fairly won renown. 'They were both proud men. But with Davy pride projected itself into the outer world; while with Faraday it became a steadying and dignifying inward force. In one great particular they agreed. Each of them could have turned his science to immense commercial profit, but neither of them did so. 'The noble excitement of research, and the delight of discovery, constituted their reward. I commend them to the reverence which great gifts greatly exercised ought to inspire. They were both ours; and through the coming centuries England will be able to point with just pride to the possession of such men.

The first volume of the "Life and Letters" reveals to us the youth who was to be father to the man. Skillful, aspiring, resolute, he grew steadily in knowledge and in power. Consciously or unconsciously, the relation of Action to Reaction was ever present to Fararlay's mind. It had been fostered by his discovery of Magnetic Rotations, and it planted in him more daring ideas of a similar kind. Magnetism he knew could be eroked by electricity, and he thought that electricity, in its turn, onght to be capable of evolution by magnetism. On August 29, 1831, his experiments on this subject began. He had been fortified by previous trials, which, though failures, had begotten instincts directing him toward the truth. He, like every strong worker, might at times miss the outward object, but he always gained the inner light, elucation, and expansion. Of this Faraday's life was a constant illustration. By November he had discovered and colligated a multitude of the most wonderful and unexpected phenomena. He had generated currents by currents; currents by magnets, permanent and transitory; and he afterward generated curreuts by the earth itself. Arago's "Magnetism of Rotation," 
which had for years offered itself as a challenge to the best scientific intellects of Europe, now fell into his hands. It proved to be a beantiful, but still special, illustration of the great principle of Magneto-electric Induction. Nothing equal to this latter, in the way of pure experimental inquiry, had previously been achieved.

Electricities from various sources were next examined, and their differences and resemblances revealed. He thus assured himself of their substantial identity. He then took up Conduction, and gave many striking illustrations of the influence of Fusion on Conducting Power. Renouncing professional work, from which at this time he might have derived an income of many thousands a year, he poured his whole momentum into his researches. He was long entangled in Electro-chemistry. The light of law was for a time obscured by the thick umbrage of novel facts; but he finally energed from his researches with the great principle of Definite Electro-chemical Decomposition in his hands. If his discovery of Magneto-electricity may be ranked with that of the pile by Volta, this new discovery may almost stand beside that of Definite Combining Proportions in Chemistry. He passed on to Static Electricity -its Conduction, Induction, and mode of Propagation. He discovered and illustrated the principle of Inductive Capacity; and, turning to theory, he asked himself how electrical attractions and repulsions are transmitted. Are they, like gravity, actions at a distance, or do they require a medium? If the former, then, like gravity, they will act in straight lines; if the latter, then, like sound or light, they may turn a corner. Faraday held-and his views are gaining ground-that his experiments proved the fact of curvilinear propagation, and hence the operation of a medium. Others denied this; but none can deny the profound and philosophic chiracter of his leading thought. * The first volume of the Researches contains all the papers here referred to.

Faraday had heard it stated that henceforth physical discoveries would be made solely by the aid of mathematics; that we had our data, and needed only to work deductively. Statements of a similar character crop ont from time to time

* In a very remarkable paper published in Poggendorff's “ Annalen" for 1857, Werner Siemens accepts and develops Faraday's theory of Molecular Induction. 
in our day. 'They arise from an imperfect acquaintance with the nature, present condition, and prospective vastness of the field of physical inquiry. 'The tendency of natural science doubtless is to bring all physical phenomena under the dominion of mechanical laws; to give them, in other words, mathematical expression. But our approach to this result is asymptotic; and for ages to come-possibly for all the ages of the human race-Nature will find room for both the philosophical experimenter and the mathematician. Faraday entered his protest against the fore. going statement by labeling his investigations "Experimental Researches in Electricity." 'They were completed in 1854, and three volumes of them have been published. For the sake of reference, he numbered every paragraph, the last number being 3,362. In 1859 he collected and published a fourth volume of papers, under the title, "Experimental Researches in Chemistry and Physics." Thus did this apostle of experiment illustrate its power, and magnify his office.

The second volume of the Researches embraces memoirs on the Electricity of the Gymnotus; on the Source of Power in the Voltaic Pile; on the Electricity evolved by the Friction of Water and Steam, in which the phenomena and principles of Sir William Armstrong's Hydro-electric machine are described and developed; a paper on Magnetic Rotations, and Faraday's letter's in relation to the controversy it aroused. The contribution of most permanent value here, is that on the Source of Power in the Voltaic Pile. By it the Contact 'Theory, pure and simple, was totally overthrown, and the necessity of chemical action to the maintenance of the current demonstrated.

The third volume of the Researches opens with a memoir entitled "The Magnetization of Light," and the "Illumination of Magnetic Lines of Force." It is difficult even now to affix a definite meaning to this title; but the discovery of the rotation of the plane of polarization, which it aunounced, seems pregnant with great results. The writings of William 'Thomson on the theoretic aspects of the discovery; the excellent electro-dynamic measurements of Wilhelm Weber, which are molels of experimental completeness and skill; Weber's labors in conjunction with his lamented friend Kohlransch-above all, the researches of Clerk Maxivell ou the Electro-magnetic 'Theory of Light- 
point to that wonderful and mysterious medium, which is the vehicle of light and radiant heat, as the probable basis also of magnetic and electric phenomena. The hope of such a counection was first raised by the discovery here referred to.* Faraday himself seemed to cling with particular affection to this discovery. He felt that there was more in it than he was able to unfold. He predicted that it would grow in meaning. with the growth of science. 'This it has done; this it is doing now. Its right interpretation will probably mark an epoch in scientific history.

Rapidly following it is the discovery of Diamagnetism, or the repulsion of matter by a magnet. Brugmans had shown that bismuth repelled a magnetic needle. Here he stopped. Le Bailliff proved that antimony did the same. Here he stopped. Seebeck, Becquerel, and others, also touched the discovery. 'These fragmentary gleams excited a momentary curiosity and were almost forgotten, when Faraday independently alighted on the same facts: and, instead of stopping, made them the inlets to a new and vast region of research. The value of a discovery is to be measured by the intellectual action it calls forth; and it was Faraday's good fortune to strike such lodes of scientific truth as give occupation to some of the best intellects of our age.

'The salient quality of Faraday's scientific character reveals itself from beginning to end of these volumes; a union of ardor and patience-the one prompting the attack, the other holding him on to it, till defeat was final or victory assured. Certainty in one sense or the other was necessary to his peace of mind. "The right method of investigation is perhaps incommunicable; it depends on the individual rather than on the system, and the mark is missed when Faraday's researches are pointed to as merely

* A letter addressed to me by Professor Weber on March 18th last contains the following reference to the connection here mentioned: "Die Hoffnung einer solchen Combination ist durch Faraday's Entdeckung der Drehung der Polarisationsebene durch magnetische Directionskraft zuerst, und sodann durch die Uebereinstimuung derjenigen Geschwindigkeit, welche das Verhältniss der electrodynamischen Einheit zur electro-statischen ausdrückt, mit der Geschwindigkeit des Lichts angeregt worden; und mir scheint von a!len Versuchen, welche zur Verwirklichung dieser Hoffnung gemacht worden sind, das von Herrn Maxwell gemachte am erfolgreichsten." 
illustrative of the power of the inductive philosophy. The brain may be filled with that philosophy; but without the energy and insight which this man possessed, and which with him were persoual and distinctive, we should never rise to the level of his achievements. His power is that of individual genius, rather than of philosophical method; the energy of a strong soul expressing itself after its own fashion, and acknowledging no mediator between it and Nature.

The second volume of the "Life and Letters," like the first, is a historic treasury as regards Faraday's work and character, and his scientific and social relations. It contains letters from Humboldt, Herschel, Hachette, De la Rive, Dumas, Liebig, Melloni, Becquerel, Oersted, Plücker, Du Bois-Reymoud, Lord Melbourne, Prince Louis Napoleon, and many other distinguished men. I notice with particular pleasure a letter from Sir John Herschel, in reply to a sealed packet addressed to him by Faraday, but which he had permission to open if he pleased. The packet referred to one of the many unfulfilled hopes which spring up in the minds of fertile investigators:

"Go on and prosper, 'from strength to strength,' like a victor marching, with assured step to further conquests; and be certain that no voice will join more heartily in the peans that already begin to rise, and will speedily swell into a shout of triumpl, astounding even to yourself, than that of J. F. W. Herschel."

Faraday's behavior to Melloni in 1835 merits a word of notice. The young man was a political exile in Paris. He had newly fashioned and applied the thermo-electric pile, and had obtained with it results of the greatest importance. But they were not appreciated. With the sickuess of disappointed hope Melloni waited for the report of the commissioners, appointed by the Academy of Sciences to examine the primier. At length he published his researches in the "Annales de Chimie." They thus fell into the hands of Faraday, who, discerning at once their extraordinary merit, obtained for their author the Rumford Medal of the Royal Society. A sum of money always accompanies this medal; and the pecuniary help was, at this time, even more essential than the mark of homor to the young refugee. Melloni's gratitude was boundless: 
"Et rous, monsieur," he writes to Faraday, "qui appartenez à une société à laquelle je n'arais rien offert, vous qui me connaissiez à peine de nom; vous n'avez pas demandé si j’avais des ennemis faibles ou puissants, ni calculé quel en était le nombre; mais vous àvez parlé pour l'opprimé étranger, pour celui qui n'avait pas le moindre droit à tant de bienveillance, et vos paroles ont été accueillies favorablement par des collègues consciencieux! Je reconnais bien lá des hommes dignes de leur noble mission, les véritable représentants de la science d'un pays libre et généreux."

Within the prescribed limits of this article it would be impossible to give even the slenderest summary of Faraday's correspondence, or to carve from it more than the merest fragments of his character. His letters, written to Lord Melbourne and others in 1836, regarding his pension, illustrate his uncompromising independence. The prime minister had offended him, but assuredly the apology demanded and given was complete. I think it certain that, notwithstanding the very full account of this transaction given by Dr. Bence Jones, motives and influences were at work which even now are not entirely revealed. The minister was bitterly attacked, but he bore the censure of the press with great dignity. Faraday, while he disavowed having either directly or indirectly furnished the matter of those attacks, did not publicly exonerate the primier. The Hon. Caroline Fox had proved herself Faraday's ardent friend, and it was she who had healed the breach between the philosopher and the minister. She manifestly thought that Faraday ought to have come forward in Lord Melbourne's defense, and there is a flavor of resentment in one of her letters to him on the subject. No doubt Faraday had good grounds for his reticence, but they are to me unknown.

In 1841 his health broke down utterly, and he went to Switzerland with his wife and brother-in-law. His bodily vigor soon revived, and he accomplished feats of walking respectable even for a trained mountaineer. The published extracts from his Swiss journal contain many beautiful and touching allusions. Amid references to the tints of the Jungfran, the blue rifts of the glaciers, and the noble Niesen towering over the lake of Thun, we come upon the charming little scrap which I have elsewhere quoted: "Clout-nail making goes on here rather considerably, and is a very neat and pretty operation to observe. I love a 
smith's shop and anything relating to smithery. My father was a smith." This is from his journal; but he is unconsciously speaking to somebody-perhaps to the world.

His description of the Stanbbach, Giessbach, and of the scenic effects of sky and mountain, are all fine and sympathetic. But amid it all, and in reference to it all, he tells his sister that " true enjoyment is from within, not from without." In those days Agassiz was living under a slab of gneiss on the glacier of the Aar. Faraday met Forbes at the Grimsel, and arranged with him an excursion to the "Hôtel des Neuchtelâois;" but indisposition put the project out.

From the Fort of Ham, in 1843, Faraday received a letter addressed to him by Prince Louis Napoleon Bonaparte. He read this letter to me many years ago, and the desire, shown in various ways by the French emperor, to turn modern science to account, has often reminded me of it since. At the age of thirty-five the prisoner of Ham speaks of "rendering his captivity less sad by studying the great discoveries" which science owes to Faraday; and he asks a question which reveals his cast of thought at the time: "What is the most simple combination to give to a voltaic battery, in order to produce a spark capable of setting fire to powder under water or under ground?" Should the necessity arise, the French emperor will not lack at the outset the best appliances of modern science; while we, I fear, shall have to learn the magnitude of the resources we are now neglecting amid the pangs of actual war.*

One turns with renewed pleasure to Faraday's letters to his wife, published in the second volume. Here surely the loving essence of the man appears more distinctly than anywhere else. From the house of Dr. Percy, in Birmingham, he writes thus:

"Here-even here-the moment I leave the table, I wish I were with you IN QUIET. Oh, what happiness is ours! My runs into the world in this way only serve to make me esteem that happiness the more."

* The "science" has since been applied, with astonishing effect, by those who had studied it far more thoroughly than the emperor of the French. We also, I aın happy to think, have improved the time since the above words were written [1878]. 
And again:

"We have been to a grand conversazione in the town hall, and I have now returned to my room to talk with you, as the pleasantest and happiest thing that I can do. Nothing rests me so much as communion with you. I feel it even now as I write, and catch myself saying the words aloud as I write them." Take this, moreover, as indicative of his love for Nature:

" After writing, I walk out in the evening hand in hand with my dear wife to enjoy the sunset; for to me who love scenery, of all that I have seen or can see, there is none surpasses that of heaven. A glorious sunset brings with it a thousand thoughts that delight me."

Of the numberless lights thrown upon him by the "Life and Letters" some fall upon his religion. In a letter to Jady Lovelace, he describes himself as belonging to "a very sinall and despised sect of Christians, known, if known at all, as Sandemanians, and our hope is founded on the faith that is in Christ." He adds: "I do not think it at all necessary to tie the study of the natural sciences and religion together, and in my intercourse with my fellowcreatures, that which is religious, and that which is philosophical, have ever been two distinct things." He saw clearly the danger of quitting his moorings, and his science acted indirectly as the safeguard of his faith. For his investigations so filled his mind as to leave no room for skeptical questionings, thus shielding from the assaults of philosophy the creed of his youth. His religion was constitutional and hereditary. It was implied in the eddies of his blood and in the tremors of his brain; and, however its outward and visible form might have changed, Faraday would still have possessed its elemental constituents-awe, reverence, truth, and love.

It is worth inquiring how so profoundly religious a mind, and so great a teacher, would be likely to regard our present discussions on the subject of education. Faraday would be a "secularist" were he now alive. He had no sympathy with those who contemn knowledge unless it be accompanied by dogma. A lecture delivered before the City Philosophical Society in 1818, when he was twentysix years of age, expresses the views regarding education which he entertained to the end of his life. "First, then," he says, "all theological considerations are banished from 
the society, and of course from my remarks; and whatever I may say has no reference to a future state, or to the means which are to be adopted in this world in anticipation of it. Next, I have no intention of substituting anything for religion, but I wish to take that part of human nature which is independent of it. Morality, philosophy, commerce, the varions institutions and habits of society, are independent of religion, and may exist either with or without it. 'They are always the same, and can dwell alike in the breasts of those who, from opinion, are entirely opposed in the set of principles they include in the ter'm religion, or in those who have none.

"'T'o discriminate more closely, if possible, I will observe that we have no right to judge religious opinions; but the human nature of this evening is that part of man which we have a right to judge. And I think it will be found on examination, that this humanity-as it may perhaps be called-will accord with what I have before described as being in our own hands so improvable and perfectible."

In an old jourual I find the following remarks on one of my earliest dinner's with Faraday: "At two o'clock he came down for me. He, his niece, and myself, formed the party. 'I never give dinners,' he said. 'I don't know how to give dinners, and I never dine out. But I should not like my friends to attribute this to a wrong cause. I act thus for the sake of securing time for work, and not through religious motives, as some imagine.' He said grace. I am almost ashamed to call his prayer a 'saying of grace.' In the language of Scripture, it might be described as the petition of a son, into whose heart God had sent the Spirit of His Son, and who with absolute trust asked a blessing from his father. We dined on roast beef, Yorkshire pudding, and potatoes; drank slierry, talked of research and its requirements, and of his habit of keeping himself free from the distractions of society. He was bright and joyful-boy-like, in fact, though he is now sixty-two. His work excites admiration, but contact with him warms and elevates the heart. Here, surely, is a strong man. I love strength; but let me not forget the example of its union with modesty, tenderness, and sweetness, in the character of Faraday."

Faraday's progress in discovery, and the salient points of his character, are well brought out by the wise choice 
of letters and extracts published in the volumes before us. I will not call the labor's of the biographer final. So great a character will challenge reconstruction. In the coming time some sympathetic spirit, with the requisite strength, knowledge, and solvent power, will, I doubt not, render these materials plastic, give them more perfect organic form, and send through them, with less of interruption, the currents of Faraday's life. "He was too good a man," writes his present biographer, "for me to estimate rightly, and too great a philosopher for me to understand thoroughly." That may be: but the reverent affection to which we owe the discovery, selection, and arrangement of the materials here placed before us, is probably a surer guide than mere literary skill. The task of the artist who may wish in future times to reproduce the real though unobtrusive grandeur, the purity, beauty, and childlike simplicity of him whom we have lost, will find his chief treasury already provided for him by Dr. Bence Jones' labor of love.

\section{CHAPTER XIX.}

\section{THE COPLEY MEDALIST OF $18 \%$.}

ThiRTY years ago Electro-magnetism was looked to as a motive power, which might possibly compete with steam. In the centers of industry, such as Manchester, attempts to investigate and apply this power were numerous. This is shown by the scientific literature of the time. Among others Mr. James Prescot Joule, a resident of Manchester, took up the subject, and, in a series of papers published in Sturgeon's "Annals of Electricity" between 1839 and 1841, described various attempts at the construction and perfection of electro-magnetic engines. The spirit in which Mr. Joule pursued these inquiries is revealed in the following extract: "I am particularly anxious," he says, "to communicate any new arrangement in order, if possible, to forestall the monopolizing designs of those who seem to regard this most interesting subject merely in the light of pecuniary speculation." He was naturally led to investigate the laws of electro-magnetic attractions, and in 1840 he announced the important principle that the attractive force exerted by two electro-magnets, or by an electro- 
magnet and a mass of annealed iron, is directly proportional to the square of the strength of the magnetizing current; while the attraction exerted between an electromagnet and the pole of a permanent steel magnet varies simply as the strength of the current. These investigations were conducted independently of, though a little subsequently to the celebrated inquiries of Henry, Jacobi, and Lenz and Jacobi, on the same subject.

On December 17, 1840, Mr. Joule communicated to the Royal Society a paper on the production of heat by Voltaic electricity. In it he announced the law that the calorific effects of equal quantities of transmitted electricity are proportional to the resistance overcome by the current, whit tever may be the length, thickness, shape, or character of the metal which closes the circuit; and also proportional to the square of the quantity of transmitted electricity. 'This is a law of primary importance. In another paper, presented to, but declined by the Royal Society, he confirmed this law by new experiments, and materially extended it. He also executerl experiments on the heat consequent on the passage of Voltaic electricity through electrolytes, and found, in all cases, that the heat evolved by the proper action of any Voltaic current is proportional to the square of the intensity of that current, multiplied by the resistance to conduction which it experiences. From this law he deduced a number of conclusions of the highest importance to electro-chemistry.

It was during these inquiries, which are marked throughout by rare sagacity and originality, that the great idea of establishing quantitative relations between Mechanical Energy and Heat arose and assumed definite form in his mind. In $1843 \mathrm{Mr}$. Joule read before the meeting of the British Association at Cork a paper "On the Calorific Effects of Magneto-Electricity, and on the Mechanical Value of Heat." Even at the present day this memoir is tough reading, and at the time it was written it must have appeared hopelessly entangled. 'This, I should think, was the reason why Faraday advised Mr. Joule not to submit the paper to the Royal Society. But its drift and results are sumined up iti these memorable words by the author, written some time subsequently: "In that paper it was demonstrated experimentally, that the mechanical power exerted in turning a magneto-electric machine is couverted 
into the heat evolved by the passage of the currents of induction through its coils; and, on the other hand, that the motive power of the electro-magnetic engine is obtained at the expense of the heat due to the chemical reaction of the battery by which it is worked."* It is needless to dwell upon the weight and importance of this statement.

Considering the imperfections incidental to a first determination, it is not surprising that the "mechanical values of heat," deduced from the different series of experiments published in 1843, varied widely from each other. The lowest limit was 58\%, and the highest 1,026 foot-pounils, for one degree Fahrenheit of temperature.

One noteworthy result of his inquiries, which was pointed out at the time by Mr. Joule, had reference to the exceedingly small fraction of the heat actually converted into useful effect in the steam-engine. The thoughts of the celebrated Julius Robert Mayer, who was then engaged in Germany upon the sanıe question, had moved independently in the same groove; but to his labors due reference will be made on a future occasion. $\downarrow$ In the memoir now reierred to, Mr. Joule also announced that he had proved heat to be evolved during the passage of water through narrow tubes; and he deduced from these experiments an equivalent of $7 \% 0$ foot-pounds, a figure remarkably near the one now accepted. A detached statement regarding the origin and convertibility of animal heat strikingly illustrates the penetration of Mr. Joule, and his mastery of principles, at the period now referred to. A friend had mentioned to him Haller's hypothesis, that animal heat might arise from the friction of the blood in the veins and arteries. "It is unquestionable," writes $\mathrm{Mr}$. Joule, "that heat is produced by such friction; but it must be understood that the mechanical force expeniler in the friction is a part of the force of affinity which causes the venous blood to unite with oxygen, so that the whole heat of the system must still be referred to the chemical changes. But if the animal were engaged in turning a piece of machinery, or in ascending a nonntain, I apprehend that in proportion to the muscular effort put forth for the purpose, a diminution of the heat evolved in the system by a given chemical action would be experienced." 
The italics in this memorable passage, written, it is to be remembered, in 1843, are Mr. Joule's own.

The concluding paragraph of this British association paper equally illustrates his insight and precision, regarding the nature of chemical and latent heat. "I had," he writes, "endeavored to prove that when two atoms combine together, the heat evolved is exactly that which would have been evolved by the electrical current due to the ehemical action taking place, and is therefore proportional to the intensity of the chemical force causing the atoms to combine. I now venture to state more explicitly, that it is not precisely the attraction of affinity, but rather the mechanical force expended by the atoms in falling toward one another, which determines the intensity of the cnrrent, and, consequently, the quantity of heat evolved; so that we have a simple hypothesis by which we may explain why heat is evolved so freely in the combination of gases, and by which indeed we may account 'latent heat' as a mechanical power, prepared for action, as a watch-spring is when wound up. Suppose, for the sake of illustration, that $8 \mathrm{lbs}$. of oxygen and $1 \mathrm{lb}$. of hydrogen were presented to one another in the gaseous state, and then exploded; the heat evolved would be about 1 degree Fahr. in 60,000 lbs. of water, indicating a mechanical force, expended in the combination, equal to a weight of about 50,000,000 lbs. raised to the height of one foot. Now if the oxygen and hydrogen could be presented to each other in a liquid state, the heat of combination wonld be less than before, because the atoms in combining would fall through less space." No words of mine are needed to point ont the commanding grasp of molecular physics, in their relation to the mechanical theory of heat, implied by this statement.

Perfectly assured of the importance of the principle which his experiments aimed at establishing, Mr. Joule did not rest content with results presenting such discrepancies as those above referred to. He resorted in 1844 to entirely new methods, and made elaborate experiments on the thermal changes produced in air during its expansion: firstly against a pressure, and therefore performing work; secondly. against no pressure, and therefore performing no work. He thus established anew the relation between the heat consumed and the work done. From five different series of experiments he deduced five different mechanical 
equivalents; the agreement between them being far greater than that attained in his first experiments. The mean of them was 802 foot-pounds. From experiments with water agitated by a paddle-wheel, he deduced, in 1845, an equivalent of 890 foot-pounds. In $184 \%$ he again operated upon water and sperm oil, agitated them by a paddle-wheel, determined their elevation of temperature, and the mechanical power which produced it. From the one he derivel an equivalent of 781.5 foot-pounds; from the other an equivalent of 782.1 foot-pounds. The mean of these two very close determinations is 781.8 footpounds.

By this time the labors of the previous ten years had made Mr. Joule completely master of the conditions essential to accuracy and success. Bringing his ripened experience to bear upon the subject, he executed in 1849 a series of 40 experiments on the friction of water, 50 experiments on the friction of mercury, and 20 experiments on the friction of plates of cast iron. He deduced from these experiments our present mechanical equivalent of heat, justly recognized all over the world as “Joule's equivalent."

'There are labors so great and so pregnant in consequences, that they are most highly praised when they are most simply stated. Such are the labors of Mr. Joule. 'They constitute the experimental foundation of a principle of incalculable moment, not only to the practice, but still more to the philosophy of Science. Since the days of Newton, nothing more important than the theory, of which Mr. Joule is the experimental demonstrator, has been enunciated.

I have omitted all reference to the numerous minor papers with which Mr. Joule has enriched scientific literature. Nor have I alluded to the important investigations which he has conducted jointly with Sir William 'Thomson. But sufficient, I think, nas been here said to show that, in conferring upon Mr. Joule the highest honor of the Royal Society, the Council paid to genius not only a well-won tribute, but one which had been fairly earned twenty years previously.*

* Lord Beaconsfield has recently honored himself and England by bestowing an annual pension of $200 l$. on Dr. Joule. 


\section{CHAPTER XX.}

\section{THE COPLEY MEDALIST OF $18 \% 1$.}

Dr. Julius Robert MAYer was educated for the medical profession. In the summer of 1840, as he himself informs us, he was at Java, and there observed that the venous blood of some of his patients had a singularly bright red color. The observation riveted his attention; he reasoned upon it, and came to the conclusion that the brightness of the color was due to the fact that a less amount of oxidation sufficed to keep up the temperature of the body in a hot climate than in a cold one. The darkness of the venous blood he regarded as the visible sign of the energy of the oxidation.

It would be trivial to remark that accidents such as this, appealing to minds prepared for them, have often led to great discoveries. Mayer's attention was thereby drawn to the whole question of animal heat. Lavoisier had ascribed this heat to the oxidation of the food. "One great principle," says Mayer, " of the physiological theory of combustion, is that under all circumstinces the same amount of fuel yields, by its perfect combustion, the same amount of heat; that this law holds good even for vital processes; and that hence the living body, notwithstanding all its enigmas and wonders, is incompetent to generate heat out of nothing."

But beyond the power of generating internal heat, the animal organism can also generate heat outside of itself. A blacksmith, for example, by hammering can heat a nail, and a savage by friction can warm wood to its point of ignition. Now, unless we give up the physiological axiom that the living body cannot create heat out of nothing, "we are driven," says Mayer, "to the conclusion that it is the total heat generated within and without that is to be regarded as the true calorific effect of the matter oxidized in the body."

From this, again, he inferred that the heat generated externally must stand in a fixed relation to the work expended in its production. For, supposing the organic processes to remain the same; if it were possible, by the mere alteration of the apparatus, to generate difierent amounts of heat by the same amount of work, it would follow that the 
oxidation of the same amount of material would sometimes yield a less, sometimes a greater, quantity of heat. "Hence," says Mayer, "that a fixed relation subsists between heat and work, is a postulate of the physiological theory of combustion."

This is the simple and natural account, given subsequently by Mayer himself. of the course of thought started l,y his observation in Java. But the conviction once formed, that au unalterable relation subsists between work and heat, it was inevitable that Mayer should seek to express it numerically. It was also inevitable that a mind like his, having raised itself to clearness on this important point, should push forward to consider the relationship of natural forces generally. At the beginuing of 1842 his work had made considerable progress; but he had become physician to the town of Heilbroun, and the duties of his profession limited the time which he could devote to purely scientific inquiry. He thought it wise, therefore, to secure himself against accident, and in the spring of 1842 wrote to Liebig, asking him to publish in his "Anualen" a brief preliminary notice of the work then accomplished. Liebig did so, and Dr. Mayer's first paper is contained in the May number of the "Annalen" for 1842.

Mayer had reached his conclusions by reflecting on the complex processes of the living body; but his first step in public was to state definitely the physical principles on which his physiological deductions were to rest. He begins, therefore, with the forces of inorganic nature. He finds in the universe two systems of causes which are not mutually convertible-the different kirds of matter and the different forms of force. 'The first quality of both he aftirms to be indestructibility. A force cannot become nothing, nor can it arise from nothing. Forces are convertible but not destructible. In the terminology of his time, he then gives clear expression to the ideas of potential and dynamic energy, illustrating his point by a weight resting upon the earth, suspended at a height above the earth, and actually falling to the earth. He next fixes his attention on cases where motion is apparently destroyed, without producing other motion; on the shock of inelastic bodies, for example. Under what form loes the ranished motion maintain itself? Experiment alone, says Mayer, can help us here. He warms water by stirring it; he refers 
to the force expender in overcoming friction. Motion in both cases disappears; but heat is generated, and the quantity generated is the equivalent of the motion destroyed. " Our locomotives," he observes with extraordinary sagacity, "may be compared to distilling apparatus: the heat beneath the boiler passes into the motion of the train, and is again deposited as heat in the axles and wheels."

A numerical solution of the relation between heat and work was what Mayer aimed at, and toward the end of his first paper he makes the attempt. It was known that a definite amount of air, in rising one degree in temperature, can take up two different amounts of heat. If its volume be kept constant, it takes up one amount: if its pressure be kept constant, it takes up a different amount. 'These two amoun ts are called the specific heat under constant volume and under constant pressure. The ratio of the first to the second is as 1: 1.421. No man, to my knowledge, prior to Dr. Mayer, penetrated the significance of these two numbers. He first saw that the excess 1.421 was not, as then universally supposed, heat actually lodged in the gas, but heat which had been actually consumed by the gas in expanding against pressure. 'The amount of work here performed was accurately known, the amount of heat consuined was also accurately known, and from these data Mayer determined the mechanical equivalent of heat. Even in this first paper he is able to direct attention to the enormons discrepancy between the theoretic power of the fuel consumed in steam-engines, and their useful effect.

'Though this paper contains but the germ of his further labors, I think it may be safely assumed that, as regards the mechanical theory of heat, this obscure Heilbronn physician, in the year 1842, was in advance of all the scientific mell of the time.

Having, by the publication of this paper, secured himself against what he calls "Eventualitäten," he devoter every hour of his spare time to his studies, and in 1845 published a memoir which far transcends his first one in weight and fullness, and, indeed, marks an epoch in the history of science. 'The title of Mayer's first puper' was, "Remarks on the Forces of Inorganic Nature." 'The title of his second great essay was, "Organic Motion in its Connection with Nutrition." In it he expancis and illustrates the physical principles laid down in his first brief 
paper. He goes fully through the calculation of the mechanical equivalent of heat. He calculates the performances of steam-engines, and finds that $100 \mathrm{lbs}$. of coal, in a good working engine, produce only the same amount of heat as 95 lbs. in an unworking one; the 5 missing lbs. having been converted into work. He determines the useful effect of gunpowder, and finds nime per cent. of the force of the consumed charcoal invested on the moving ball. He records observations on the heat generated in water agitated by the pulping-engine of a paper manufactory, and calculates the equivalent of that heat in horsepower. He compares chemical combination with mechanical combination - the union of atoms with the union of falling bodies with the earth. He calculates the velocity with which a body starting at an infinite distance would strike the earth's surface, and finds that the heat generated by its collision would raise an equal weight of water 17,356 degrees C. in temperature. He then determines the thermal effect which would be produced by the earth itself falling into the sun. So that here, in 1845, we have the germ of that meteoric theory of the sun's heat which Mayer developed with such extraordinary ability three year's afterward. He also points to the almost exclusive efficacy of the sun's heat in producing mechanical motions upon the earth, winding up with the profound remark, that the heat developed by friction in the wheels of our wind and water mills comes from the sun in the form of vibratory motion; while the heat produced by mills driven by tidal action is generated at the expense of the earth's axial rotation.

Having thus, with firm step, passed through the powers of inorganic nature, his next object is to bring his principles to bear upon the phenomena of vegetable and animal life. Wood and coal can burn; whence come their heat, and the work produciole by that heat? From the immeasurable reservoir of the sun. Nature has proposed to herself the task of storing up the light which streams earthward from the sun, and of casting into a permanent form the most fugitive of all powers. Tho this end she has overspread the earth with organisms which, while living, take in the solar light, and by its consumption generate forces of another kind. These organisms are plants. The vegetable world, indeed, constitutes the instrument where- 
by the wave-motion of the sun is changed into the rigid form of chemical tension, and thus prepared for future use. With this prevision, as shall subsequently be shown, the existence of the human race itself is inseparably connected. It is to be observed that Mayer's utterances are far from being anticipated by vague statements regarding the "stimulus" of light, or regarding coal as "bottled sunlight." He first saw the full meaning of De Saussure's ubservation as to the reducing power of the solar rays, and gave that observation its proper place in the doctrine of conservation. In the leaves of a tree, the carbon and oxygen of carbonic acid, and the hydrogen and oxygen of water, are forced asunder at the expense of the sun, and the amount of power thus sacrificed is accurately restored by the combustion of the tree. 'The heat and work potential in our coal strata are so much strength withdrawn from the sun of former ages. Mayer lays the axe to the root of the notions regarding "vital force" which were prevalent when he wrote. With the plain fact before us that in the absence of the solar rays plants cannot perform the work of reduction, or generate chemical tensions, it is, he conteuds, incredible that these tensions should be caused by the mystic play of the vital force. Such an hypothesis would cut off all investigation; it would land us in a chaos of unbridled fantasy. "I count," he says, " therefore, upon your agreement with me when I state, as an axiomatic truth, that during vital processes the conver. sion only, and never the creation of matter or force occurs."

Having cleared his way through the vegetable world, as he had previously done through inorganic nature, Mayer passes on to the other organic kingdom. The physical forces collected by planis become the property of animals. Animals consume vegetables, and cause them to reunite with the atmospheric oxygen. Animal heat is thus produced; and not ouly animal heat, but animal motion. There is no indistinctness about Mayer here; he grasps his subject in all its details, and reduces to figures the concomitants of muscular action. A bowler who imparts to an 8-1b. ball a velocity of thirty feet, consumes in the act one-tenth of a grain of carbon. A man weighing 150 lbs., who lifts his own bouly to a height of eight feet, corisumes in the act one grain of carbon. In climbing a mountain 
10,000 feet high, the consumption of the same man would be $2 \mathrm{oz} .4$ drs. 50 grs. of carbon. Boussingiult had determined experimentally the addition to be made to the food of horses when actively working, and Liebig had determined the addition to be made to the food of men. Employing the mechanical equivalent of heat, which he had previously calculated, Mayer proves the additional food to be amply sufficient to cover the increased oxiclation.

But he does not content himself with showing, in a general way, that the human body burns according to definite laws, when it performs mechanical work. He seeks to determine the particular portion of the body consumed, and in doing so executes sume noteworthy calculations. The muscles of a laborer $150 \mathrm{lbs}$. in weight weigh 64 lbs.; but when perfectly desiccated they fall to $15 \mathrm{lbs}$. Were the oxidation corresponding to that laborer's work exerted on the muscles alone, they would be utterly consumed in eighty days. The heart furnishes a still more striking example. Were the oxidation necessary to sustain the heart's action exerted upon its uwn tissue, it would be utterly consumed in eight days. And if we confine our attention to the two ventricles, their action would be sufficient to consume the associated muscular tissue in $3 \frac{1}{2}$ days. Here, in his own words, emphasized in his own way, is Mayer's pregnant conclusion from these calculations: "The muscle is only the apparatus by means of which the conversion of the force is effected; but it is not the substance consumed in the production of the mechanical effect." He calls the blood "the oil of the lamp of life;" it is the slow-burning fluid whose chemical force, in the furnace of the capillaries, is sacrificed to produce animal motion. 'I'his was Mayer's conclusion twenty-six year's ago. It was in complete opposition to the scientific conclusions of his time; but eminent investigators have since amply verified it.

Thus, in baldest outline, I have sought to give some notion of the first half of this marvelous essay. 'The second half is so exclusively physiological that I do not wish to meddle with it. I will only add the illustration empioyed by Mayer to explain the action of the nerves upon the muscles. As an engineer, by the motion of his finger in opening a valve or loosing a detent, can liberate an amount of mechanical motion almost infinite compared 
with its exciting cause, so the nerres, acting upon the muscles, can unlock an amount of activity, wholly out of proportion to the work done by the nerves themselves.

As regards these questions of weightiest import to the science of physiology, Dr. Mayer, in 18t5, was assuredly far in advance of all living men.

Mayer grasped the mechanical theory of heat with commanding power, illustrating it and applying it in the most diverse clomains. He began, as we have seen, with physical principles; he determined the numerical relation between heat and work; he revealed the source of the energies of the vegetable world, and showed the relationship of the heat of our fires to the solar heat. He followed the energies which were potential in the vegetable. up to their local exhaustion in the animal. But in 1845 a new thought was forced upon him by his calculations. He then, for the first time, drew attention to the astounding amount of heat generated by gravity where the force has sufficient distance to act through. He proved, as I have before stated, the heat of collision of a body falling from an infinite distance to the earth, to be sufficient to raise the temperature of a quantity of water, equal to the falling body in weight, 17,356 degrees $\mathrm{C}$. He also found, in 1845 , that the gravitating force between the earth and sun was competent to generate an amount of heat equal to that obtainable from the combustion of 6,000 times the weight of the earth of solid coal. With the quickness of genius he saw that we had here a power sufficient to produce the enormous temperature of the sun, and also to account for the primal molten condition of our own planet. Mayer shows the utter inalequacy of chemical forces, as we know them, to produce or maintain the solar temperature. He shows that were the sun a lump of coal it would be utterly consumed in 5,000 years. He shows the difficulties attending the assumption that the sun is a cooling body; for, supposing it to possess even the high specific heat of water, its temperature would fall 15,000 degrees in 5,000 years. He finally concludes that the light and heat of the sun are maintained by the constant impact of meteoric matter. I never ventured an opinion as to the truth of this theory; that is a question which may still have to be fought ont. But I refer to it as an illustration of the force of genius with which Mayer followed the mechanical theory of heat 
through all its applications. Whether the meteoric theory be a matter of fact or not, with him abides the honor of proving to demonstration that the light and heat of suns and stars may be originated and maintained by the collisions of cold planetary matter.

It is the man who with the scantiest data could accomplish all this in six short years, and in the honrs snatched from the duties of an arduous profession, that the Royal Society, in 18\%1, crowned with its highest honor.

Comparing this brief history with that of the Copley Medalist of $18 \%$, the differentiating influence of " environment," on two minds of similar natural cast and endowment, comes out in an instructive manner. Withdrawn from mechanical appliances, Mayer fell back upon reflection, selecting with marvelous sagacity, from existing physical data, the single result on which could be founded a calculation of the mechanical equiralent of heat. In the midst of mechanical appliances, Joule resorted to experiment, and laid the broad and firm foundation which has secured for the mechanical theory the acceptance it now enjoys. A great portion of Joule's time was occupied in actual manipulation; freed from this, Mayer had time to follow the theory into its most abstruse and impressive applications. With their places reversed, however, Joule might have become Mayer, and Mayer might have become Joule.

It does not lie within the scope of these brief articles to enter upon the developments of the Dynamical Theory accomplished since Joule and Mayer executed their memorable labors.

\section{CHAPTER XXI.}

\section{DEATH BY LIGHTNING.}

PEOPLe in general imagine, when they think at all about the matter, that an impression upon the nerves-a blow, for example, or the prick of a pin-is felt at the moment it is inflicted. But this is not the case. The seat of sensation being the brain, to it the intelligence of muy impression made upon the nerves has to be transmitted 
before this impression can become manifest as consciousness. 'The transmission, moreover, requires time, and the consequence is, that a wound inflicted on a portion of the body distant from the brain is more tardily appreciated than one inflicted adjacent to the brain. By an extremely ingenious experimental arrangement, Helmholtz has determined the velocity of this nervous transmission, and finds it to be about eighty feet a second, or less than one-thirteenth of the velocity of sound in air. If, therefore, a whale forty feet long were wounded in the tail, it would not be conscious of the injury till half a second after the wound had been inflicted.* But this is not the only ingredient in the delay. There can scarcely be a doubt that to every act of consciousness belongs a determinate molecular arrangement of the brain-that every thought or feeling has its physical correlative in that organ; and nothing can be more certain than that every physical change, whether molecular or mechanical, requires time for its accomplishment. So that, besides the interval of transmission, a still further time is necessary for the brain to put itself in order-for its molecules to take up the motions or positions necessary to the completion of consciousness. Helmholtz considers that one-tenth of a second is demanded for this purpose. Thus, in the case of the whale above supposed, we have first half a second consumed in the transmission of the intelligence through the sensor nerves to the head, one-tenth of a second consumed by the brain in completing the arrangements necessary to consciousness, and, if the velocity of transmission through the motor be the same as that through the sensor nerves, half a second in sending a command to the tail to defend itself. Thus one second and a tenth would elapse before un impression made upon its caudal nerves could be responded to by a whale forty feet long.

Now, it is quite conceivable that an injury might be inflicted so rapidly that within the time required by the brain to complete the arrangements necessary to consciousness, its power of arrangement might be destroyed. In such a case, though the injury might be of a nature to cause

* A most admirable lecture on the velocity of nervous transmission has been published by Dr. Du Bris-Reymond in the "Proceedings of the Royal Institution" for 1866, vol. iv., p. 575. 
death, this would occur without pain. Death in this case would be simply the sudden negation of life, without any intervention of consciousness whatever.

'The time required for a rifle bullet to pass clean through a man's head may be roughly estimated at a thousandth of a second. Here, therefore, we should have no room for sensation, and death would be painless. But there are other actions which far transcend in rapidity that of the rifle bullet. A flash of lightning cleaves a clond, appearing and disappearing in less than a hundred thousandth of a second, and the velocity of electricity is such as would carry it in a single second over a distance almost equal to that which separates the earth and moon. It is well known that a luminous impression once made upon the retina endures for about one-sixth of a second, and that this is the reason why we see a continuous band of light when a glowing coal is caused to pass rapidly through the air. A body illuminated by an instantaneous flash continues to be seen for the sixth of a second after the flash has become extinct; and if the body thus illuminated be in motion, it appears at rest at the place where the flash falls upon it. When a color-top with differently colored sectors is caused to spin rapidly the colors blend together. Such a top, rotating in a dark room and illuminated by an electric spark, appears motionless, each distinct color being clearly seen. Professor Dove has found that a flush of lightning produces the same effect. During a thunderstorm he put a color-top in exceedingly rapid motion, and found that every flash revealed the top as a motionless object with its colors distinct. If illuminated solely by a flash of lightning, the motion of all bodies on the earth's surface would, is Dove has remarked, appear suspended. A cannon ball, for example, would have its flight apparently arrested, and would seem to hang motionless in space as long as the luminous impression which revealed the ball remained upon the eye.

If, then, a rifle bullet move with sufficient rapidity to destroy life without the interposition of sensation, much more is a flash of lightning competent to produce this effect. Accordingly, we have well-authenticated cases of people being struck senseless by lightning who, on recovery, had no memory of pain. The following circumstantial case is described by Hemmer: 
On June 30, 1788, a soldier in the neighborhood of Mannheim, being overtaken by rain, placed himself under a tree, beneath which a woman had previously taken shelter. He looked upward to see whether the branches were thick enough to afford the required protection, and, in doing 8o, was struck by lightning, and fell senseless to the earth. 'The woman at his side experienced the shock in her foot, but was not struck down. Some hours afterward the man revived, but remembered nothing about what had occurred, save the fact of his looking up at the branches. This was his last act of consciousness, and he passed from the conscious to the unconscious condition without pain. The visible marks of a lightuing stroke are usually insignificant: the hair is sometimes burned; slight wounds are observed; while, in some instances, a red streak marks the track of the discharge over the skin.

Under ordinary circumstances, the discharge from a small Leyden jar is exceedingly unpleasant to me. Some time ago I happened to stand in the presence of a numerous audience, with a battery of fifteen large Leyden jars charged beside me. Through some awkwardness on my part, I touched a wire leading from the battery, and the discharge went through my body. Life was absolutely blotted out for a very sensible interval, without a trace of pain. In a second or so consciousuess returned; I vaguely discerned the audience and apparatus, and, by the help of these external appearances, immediately concluded that I had received the battery discharge. 'The intellectual consciousness of my position was restored with exceeding rapidity, but not so the optical consciousness. To prevent the audience from being alarmed, I observed that it had often been my desire to receive accidentally such a shock, and that my wish had at length been fulfilled. But, while making this remark, the appearance which my body presented to my eyes was that of a number of separate pieces. The arms, for example, were detached from the trunk, and seemed suspended in the air. In fact, memory and the power of reasoning appeared to be complete long before the optic nerve was restored to healthy action. But what I wish chiefly to dwell upon here is, the absolute painlessness of the shock; and there cannot, I think, be a doubt that, to a person struck dead by lightning, the passage from life to death occurs without consciousness being in the least 
degree implicated. It is an abrupt stoppage of sensation, unaccompanied by a pang.

\section{CHAPTER XXII. \\ SCIENCE AND THE " SPIRITS."}

THEIR refusal to investigate "spiritual phenomena" is often urged as a reproach against scientific men. I here propose to give a sketch of an attempt to apply to the "phenome:ia" those methods of inquiry which are found available in dealing with natural truth.

Some years ago, when the spirits were particularly active in this country, Faraday was invited, or rather entreated, by one of his friends to meet and question them. He had, however, already made their acquaintance, and did not wish to renew it. I had not been so privileged, and he therefore kindly arranged a transfer of the invitation to me. The spirits themselves named the time of meeting, and I was conducted to the place at the day and hour appointed.

Absolute unbelief in the facts was by no means my condition of mind. On the contrary, I thought it probable that some physical principle, not evident to the spiritualists themselves, might underlie their manifestations. Extraordinary effects are produced by the accumulation of small impulses. Galileo set a heavy pendulum in motion by the well-timed puffs of his breath. Ellicot set one clock going by the ticks of another, even when the two clocks were separated by a wall. Preconceived notions can, moreover, ritiate, to an extraordinary degree, the testimony of even veracious persons. Hence my desire to witness those extraordinary phenomena, the existence of which seemed placed beyond a doubt by the known veracity of those who had witnessed and described them. The meeting took place at a private residence in the neighborhood of London. My host, his intelligent wife, and a gentleman who may be called X., were in the house when I arrived. I was informed that the " medium" had not yet made her appearance; that she was sensitive, and might resent suspicion. It was therefore requested that the tables and chairs should be examined before her arrival, in order to be assured that 
there was no trickery in the furniture. This was done; and I then first learned that my hospitable host had arranged that the séance should be a dinner-party. 'This was to me an unusual form of investigation; but I accepted it, as one of the accidents of the occasion.

The " medium" arrived - a delicate-looking young lady, who appeared to have suffered much from ill-health. I took her to dinner and sat close beside her. Facts were absent for a considerable time, a series of rery wonderful narratives supplying their place. The duty of belief on the testimony of witnesses was frequently insisted on. X. appeared to be $\mathbf{a}$ chosen spiritual agent, and told us many surprising things. He affirmed that, when he took a pen in his hand, an influence ran from his shoulder downward, and impelled him to write oracular sentences. I listened for a time, offering no observation. "And now," continued $X$., "this power has so risen as to reveal to me the thoughts of others. Only this morning I told a friend what he was thinking of, and what he intended to do during the day." Here, I thought, is something that can be at once tested. I said immediately to X.: "If you wish to win to your cause an apostle, who will proclaim your principles to the world from the housetop, tell me what I am now thinking of." X. reddened, and did not tell me my thought.

Some time previously I had visited Baron Reichenbach, in Vienna, and I now asked the young lady who sat beside me, whether she conld see any of the curious things which he describes-the light emitted by crystals, for example? Here is the conversation which followed, as extracted from my notes, written on the day following the séance.

Medium._." Oh, yes; but I see light around all bodies." I.- " Even in perfect darkness?"

Medium._- "Yes; I see luminous atmospheres round all people. The atmosphere which surrounds $\mathrm{Mr}$. R. C. would fill this room with light."

I. - "You are aware of the effects ascribed by Baron Reichenbach to magnets?"

Medium._- Yes; but a magnet makes me terribly ill."

1.- "Am I to understand that, if this room were perfectly dark, you could tell whether it contained a magnet, without being informed of the fact?"

Medium.- "I should know of its presence on entering the room." 


\section{I._- How?"}

Medium._- I should be rendered instantly ill."

I._- How do you feel to-day?"

Medium._" "Particularly well; I have not been so well for months."

I._ "Then, may I ask you whether there is, at the present moment, a magnet in my possession?"

The young lady looked at me, blushed, and stammered:

" No; I am not en rapport with yon."

I sat at her right hand, and a left-hand pocket, within six inches of her person, contained a magnet.

Our host here deprecated discussion, as it "exhausted the medium." 'The wonderful narratives were resumed; but I had narratives of my own quite as wonderful. 'I'hese spirits, indeed, seemed clumsy creations, compared with those with which my own work had made me familiar. I therefore began to match the wonders related to me by other wonders. A lady present discoursed on spiritual atmospheres, which she could see as beautiful colors when she closed her eyes. I professed niyself able to see similar colors, and, more than that, to be able to see the interior of my own eyes. 'The medium affirmed that she could see actual waves of light coming from the sun. I retorted that men of science could tell the exact number of waves emitted in a second, and also their exact length. The merlium spoke of the performances of the spirits on musical instruments. I said that such performance was gross, in comparisun with a kind of music which had been discovered some time previously by a scientific man. Standing at a distance of twenty feet from a jet of gas, he could command the flame to emit a melorious note; it wonld obey, and continue its song for hours. So loud was the music emitted by the gas-flame, that it might be heard by an assembly of a thousand people. These were acknowledged to be as great marvels as any of those of spiritdom. The spirits were then consulted, and I was pronounced to be a first-class medium.

During this conversation a low knocking was heard from time to time under the table. These, I was told, were the spirits' knocks. I was informed that one knock, in answer to a question, meant "No;" that two knocks meant "Not yet;" and that three knocks meant "Yes." In answer to a question whether I was a medium, the response was three 
brisk and vigorous knocks. I noticed that the knocks issued from a particular locality, and therefore requested the spirits to be good enough to answer from another corver of the table. 'They did not comply; but I was assured that they would do it, and much more, by and by. The knocks continuing, I turned a wine glass upside down, and placed my ear upon it, as upon a stethoscope. 'The spirits seemed disconcerted by the act; they lost their playfulness, and did not recover it for a considerable time.

Somewhat weary of the proceedings, I once threw myself back against my chair and gazed listlessly out of the window. While thus engaged, the table was rudely pushed. Attention was drawn to the wine, still oscillating in the glasses, and I was asked whether that was not convincing. I readily granted the fact of motion, and began to feel the delicacy of my position. There were several pairs of arms upon the table, and several pairs of legs under it; but how was I, without offense, to express the conviction which I really entertained? To ward off the difficulty, I again turned a wine glass upside down and rested my ear upon it. The rim of the glass was not level, and my hair, on touching it, caused it to vibrate, and produce a peculiar buzzing sound. A perfectly candid and warm-hearted old gentleman at the opposite side of the table, whom I may call A., drew attention to the sound, and expressed his entire belief that it was spiritual. I, however, informed him that it was the moving hair acting on the glass. The explanation was not well received; and $X$. , in a tone of severe pleasantry, demanded whether it was the hair that had moved the table. The promptness of my negative probably satisfied him that my notion was a very different one.

The superhuman power of the spirits was next dwelt upon. The strength of man, it was stated, was unavailing in opposition to theirs. No human power could prevent the table from moving when they pulled it. During the evening this pulling of the table occurred, or rather was attempted, three times. 'Twice the table moverl when my attention was withdrawn from it; on a third occasion, I tried whether the act could be provoked by an assumed air of inattention. Grasping the table firmly between my knees, I threw myself back in the chair, and waited, with 
eyes fixed on vacancy, for the pull. It came. For some seconds it was pull spirit, hold muscle; the muscle, however, prevailed, and the table remained at rest. Up to the present moment this interesting fact is known only to the particular spirit in question and myself.

A species of mental scene-painting, with which my own pursuits had long rendered me familiar, was employed to figure the changes and distribution of spiritual power. The spirits, it was alleged, were provided with atmospheres, which combined with and interpenetrated each other, and considerable ingenuity was shown in demonstrating the necessity of time in effecting the adjustment of the atmospheres. A rearrangement of our positions was proposed and carried out; and soon afterward my attention was drawn to a scarcely sensible vibration on the part of the table. Several persons were leaning on the table at the time, and I asked permission to touch the medium's hand. "Oh! I know I tremble," was her reply. Throwing one leg across the other, I accidentally nipperl a muscle, and produced thereby an involuntary vibration of the free leg. This vibration, I knew, must be communicated to the floor, and thence to the chairs of all present. I therefore intentionally promoted it. My attention was promptly drawn to the motion; and a gentleman beside me, whose value as a witness I was particularly desirous to test, expressed his belief that it was out of the compass of human power to produce so strange a tremor. "I believe," he added, earnestly, "that it is entirely the spirits' work." "So do I," added, with heat, the candid and warm-hearted old gentleman A. " "IVhy, sir," he continued, "I feel them at this moment shaking my chair." I stopped the motion of the leg. "Now, sir," A. exclaimed, "they are gove." I began again, and $\mathrm{A}$. once more affirmed their presence. I conld, however, notice that there were doubters present, who did not quite know what to think of the manifestations. I saw their perplexity; and, as there was sufficient reason to believe that the disclosure of the secret would simply provoke anger, I kept it to myself.

Again a period of conversation intervened, during which the spirits became animated. The evening was confessedly a dull one, but matters appeared to brighten toward its close. The spirits were requested to spell the name by which I was known in the heavenly world, Our host com- 
monced repeating the alphabet, and when he reached the letter " $\mathrm{P}$ " a knock was hearl. He began again, and the spirits knocked at the letter " 0 ." I was puzzled, but waited for the eud. The next letter knocked down was "E." I laughed, and remarked that the spirits were going to make a poet of me. Admonishel for my levity, I was informed that the frame of mind proper for the occasion ought to have been superinduced by a perusal of the Bible immediately before the séance. 'The spelling, however, went on, and sure enough I came out a poet. But matters did not end here. Our host continued his repetition of the alphabet, and the next letter of the name proved to be "O." Here was manifestly an unfinished word, and the spirits were apparently in their most communicative mood. The knocks came from under the table, but no person present evinced the slightest desire to look under it. I asked whether I might go underneath; the permission was granted; so I crept under the table. Some tittered; but the candid old A. exclaimed, "He has a right to look into the very dregs of it, to convince himself." Having pretty well assured myself that no sound could be produced under the table without its origin being revealed, I requested our host to continue his questions. He did so, but in vain. He adopted a tone of tender entreaty; but the "dear spirits" had become dumb dogs, and refused to be entreated. I continued under that table for at least a quarter of an hour, after which, with a feeling of despair as regards the prospects of humanity never before experienced, I regained my chair. Once there, the spirits resumed their loquacity, and dujbed me "Poet of Science."

'This, then, is the result of an attempt made by a scientific man to look into these spiritual phenomena. It is not encouraging; and for this reason. The present promoters of spiritual phenomena divide themselves into two classes, one of which needs no demonstration, while the other is beyond the reach of proof. 'The victims like to believe, and they do not like to be undeceived. Science is perfectly powerless in the presence of this frame of mind. It is, moreover, a state perfectly compatible with extreme intellectual subtlety and a capacity for devising hypotheses which only require the hardihood engendered by strong conviction, or by callous mendacity, to render them impregnable. The logical feebleness of science is not suffi- 
ciently borne in mind. It keeps down the weed of superstition, not by logic but by slowly rendering the mental soil unfit for its cultivation. When science appeals to uniform experience, the spiritualist will retort, "How do you know that a uniform experience will continue uniform? You tell me that the sun has risen for six thousand years: that is no proof that it will rise to-morrow; within the next twelve hours it may be puffed out by the Almighty." Taking this ground, a man may maintain the story of "Jack and the Beanstalk" in the face of all the science in the world. You urge, in vain, that science has given us all the knowledge of the universe which we now possess, while spiritualism has added nothing to that knowleclge. 'The drugged soul is beyond the reach of reason. It is in vain that impostor's are exposed, and the special demou cast out. He has but slightly to change his shape, return to his house, and find it "empty, swept, ancl garnished."

Since the time when the foregoing remarks were written I have been more than once among the spirits, at their own invitation. They do not improve on acquaintance. Surely no baser delusion ever obtained dominance over the weak mind of man.

In the bright sky they perceived an illuminator; in the allencircling firmament an embracer; in the roar of thunder and in the violence of the storm they felt the presence of a shouter and of furious strikers; and out of the rain they created an Indra, or giver of rain.-MAX MüLLER.

\section{CHAP'TER XXIII.}

REFLECTIONS ON PRAYER AND NATURAL LAW. 1861.

AMID the apparent confusion and caprice of natural phenomena, which roused emotions hostile to calm investigation, it must for ages have seemed hopeless to seek for law or orderly relation; and before the thought of law dawned upon the unfolding human mind these otherwise inexplicable effects were referred to personal agency. In the fall of a cataract the savage saw the leap of a spirit, and the echoed thunder-peal was to him the hammer-clang of an exasperated god. Propitiation of these terrible powers 
was the consequence, and sacrifice was offered to the demons of earth and air.

But observation tends to chasten the emotions and to check those structural efforts of the intellect which have emotion for their base. One by one natural phenomena came to be associated with their proximate causes; the illea of direct personal volition mixing itself with the economy of nature retreating more and more. Many of us fear this change. Our religious feelings are dear to us, and we look with suspicion and dislike on any philosophy, the apparent tendency of which is to dry them up. Probably every change from ancient savagery to our present enlightenment has excited, in a greater or less degree, fears of this kind. But the fact is, that we have not yet determined whether its present form is necessary to the life and warmth of religious feeling. We may err in linking the imperishable with the transitory, and confound the living plant with the decaying pole to which it clings. My object, however, at present is not to argue, but to mark a temlency. We have ceased to propitiate the powers of nature-ceased even to pray for things in manifest contradiction to natural laws. In Protestant countries, at least, I think it is conceded that the age of miracles is past.

At an auberge near the foot of the Rhone glacier, I met, in the summer of 1858, an athletic young priest, who, after a solid breakfast, including a bottle of wine, informed me that he had come up to "bless the mountains." 'This was the annual custom of the place. Year by year the Highest was entreated, by official intercessors, to make such meteorological arrangements as should ensure food and shelter for the flocks and herds of the Valaisians. A diversion of the Rhone, or a deepening of the river's bed, would, at the time I now mention, have been of incalculable benefit to the inhabitants of the valley. But the priest would have shrunk from the idea of asking the Omniputent to open a new channel for the river, or to cause a portion of it to flow over the Grimsel pass, and down the valley of Oberhasli to Brientz. 'This he would have deemed a miracle, and he did not come to ask the Creator to perform miracles, but to do something which he manifestly thought lay quite within the bounds of the natural and nou-miraculous. A Protestant gentleman who wus present at the time smiled at this recital. He had no 
faith in the priest's blessing; still, he deemed his prayer different in kind from a request to open a new river-cut, or to cause the water to flow uphill.

In a similar manner the same Protestant gentleman would doubtless smile at the honest 'Tyrolese priest, who, when he feared the bursting of a glacier dain, offered the sacrifice of the Mass upon the ice as a means of averting the calamity. 'That poor man did not expect to convert the ice into adamant, or to strengthen its texture, so as to enable it to withstand the pressure of the water; nor did he expect that his sacrifice would cause the stream to roll back upon its source and relieve him, by a miracle, of its presence. But beyond the boundaries of his knowledge lay a region where rain was generated, he knew not how. He was not so presumptnous as to expect a miracle, but he firmly believed that in yonder cloud-land matters could be so arranged, without trespass on the miraculous, that the stream which threatened him and his people should be caused to shrink within its proper bounds.

Both these priests fashioned that which they did not understund to their respective wants and wishes. In their case imagination came into play, uncontrolled by a knowledge of law. A similar state of mind was long prevalent among mechanicians. Many of these, among whom were to be reckoned men of consummate skill, were occupied a century ago with the question of perpetual motion. They aimed at constructing a machiue which should execute work without the expenditure of power, and some of them went mad in the pursuit of this object. The faith in such a consummation, involving, as it did, immense personal profit to the inventor, was extremely exciting, and every attempt to destroy this faith was met by bitter resentment on the part of those who held it. Gradually, however, as men became more and more acquainted with the true functions of machinery, the dream dissolved. The hope of getting work out of mere mechanical combinations disappeared: but still there remained for the speculator a cloud-land denser than that which filled the imagination of the Tyrolese priest, and out of which he still hoped to evolve perpetual motion. There was the mystic store of chemic force, which noboly understood; there were heat and light, elestricity and magnetism, all competent to produce mechanical motion.* Here, then, was the mine in

* See Helmholtz: "Wechselwirkung der Naturkräfte." 
which our gem must be sought. A modified and more refined form of the ancient faith revived; and, for anght I know, a remnant of sanguine desiguers may at the present moment be engaged on the problem which like-minded men in former ages left unsolved.

And why should a perpetual motion, even under modern conditions, be impossible? The answer to this question is the statement of that great generalization of modern science, which is known under the name of the Conservation of Energy. This principle asserts that no power can make its appearance in nature without an equivalent expenditure of some other power; that natural agents are so related to each other as to be inutually convertible, but that no new agency is created. Light rums into heat; heat into electricity; electricity into magnetism; magnetism into mechanical force; and mechanical force again into light and heat. The Proteus changes, but he is ever the same; and his changes in nature, supposing no miracle to supervene, are the expression, not of spontaneity, but of physical necessity. A perpetual motion, then, is deemed impossible, because it demands the creation of energy, whereas the principle of Conservation is-no creation, but infinite conversion.

It is an old remark that the law which molds a tear also rounds a planet. In the application of law in nature the terms great and small are unknown. Thus the principle referred to teaches 1 s that the Italian wind, gliding over the crest of the Matterhorn, is as firmly ruled as the earth in its orbital revolution round the sun; and that the fall of its vapor into clouds is exactly as much a matter of necessity as the return of the seasons. The dispersion, therefore, of the slightest mist by the special volition of the Eternal, would be as much a miracle as the rolling of the Rhone over the Grimsel precipices, down the valley of Hasli to Meyringen and Brientz.

It seems to me quite beyond the present power of science to demonstrate that the "Tyrolese priest, or his colleague of the Rhone valley, asked for an "impossibility" in praying for good weather; but Science can demonstrate the incompleteness of the knowledge of nature which limited their prayers to this narrow ground; and she may lessen the number of instances in which we " ask amiss," by showing that we sometimes pray for the performance of a miracle 
when we do not intend it. She does assert, for example, that without a disturbance of natural law, quite as serious as the stoppage of an eclipse, or the rolling of the river Niagara up the Falls, no act of humiliation, individual or national, could call one shower from heaven, or deflect toward us a single beam of the sun.

Those, therefore, who believe that the miraculous is still active in nature, may, with perfect consistency, join in our periodic prayers for fair weather and for rain: while those who hold that the age of miracles is past, will, if they be consistent, refuse to join in these petitions. And these latter, if they wish to fall back upon such a justification, may fairly urge that the latest conclusions of science are in perfect accordance with the doctrine of the Master himself, which manifestly was that the distribution of natural phenomena is not affected by moral or religious causes. "He maketh His sun to rise on the evil and on the good, and sendeth rain on the just and on the unjust." Granting "the power of Free Will in man," so strongly claimed by Professor Mansel in his admirable defense of the belief in miracles, and assuming the efficacy of free prayer to produce changes in external nature, it necessarily follows that natural laws are more or less at the mercy of man's volition, and no conclusion founded on the assumed permanence of those laws would be worthy of confidence.

It is a wholesome sign for England that she numbers among her clergy men wise enough to understand all this, and courageous enough to act up to their knowledge. Such men do service to public character, by encouraging a manly and intelligent conflict with the real causes of disease and scarcity, instead of a delusive reliance on supernatural aid. But they have also a value beyond this local and temporary one. They prepare the public mind for changes, which though inevitable, could hardly, without such preparation, be wrought without violence. Iron is strong; still, water in crystallizing will shiver an iron envelope, and the more unyielding the metal is, the worse for its safety. There are in the world men who would encompass philosophic speculation by a rigid envelope, hoping thereby to restrain it, hut in reality giving it explosive force. In England, thanks to men of the stamp to which I have alluded, scope is gradualiy given to thought for changes of aggregation, 
and the envelope slowly alters its form, in accordance with the necessities of the time.

The proximate origin of the foregoing slight article, and probably the remoter origin of the next following one, was this. Some years ago, a day of prayer and humiliation, on account of a bad harvest, was appointed by the proper religious authorities; but certain clergy men of the Church of England, doubting the wisdom of the demonstration, declined to join in the services of the day. For this act of nonconformity they were severely censured by some of their brethren. Rightly or wrongly, my sympathies were on the side of these men; and, to lend them a helping hand in their struggle against odds, I inserted the foregoing chapter in a little book entitled " Mountaineering in 1861." Some time subsequently I received from a gentleman of great weight and distinction in the scientific world, and, I believe, of perfect orthodoxy in the religious one, a note directing my attention to an exceedingly thoughtful article on Prayer and Cholera in the Pall Mall Gazette. My eminent correspondent deemed the article a fair answer to the remarks made by me in 1861. I, also, was struck by the temper and ability of the article, but I could not deem its arguments satisfactory, and in a short note to the editor of the Pall Mall Gazette I ventured to state so much. This letter elicited sume very able replies, and a second leading article was also devoted to the subject. In answer to all I risked the publication of a second letter, and soon afterward, by an extremely courteous note from the editor, the discussion was closed.

'Though thus stopped locally, the discussion flowed in other directions. Sermons were preached, essays were published, articles were written, while a copious correspondence occupied the pages of some of the religious newspapers. It gave me sincere pleasure to notice that the discussion, save in a few cases where natural coarseness had the upper hand, was conducted with a ninimum of vituperation. The severity shown was hardly more than sufficient to demonstrate earnestness, while gentlemanly feeling was too predominant to permit that earnestness to contract itself to bigotry or to clothe itself in abuse. It was probably the memory of this discussion which caused another excellent friend of mine to recommend to my perusal the exceedingly able work which in the next article I have endeavored to review. 


\section{CHAPTER XXIV.}

\section{MIRACLES AND SPECIAL PROVIDENCES. * $186 \%$.}

Mr. Mozley's book belongs to that class of writing of which Butler may be taken as the type. It is strong, genuine argument about difficult matters, fairly tracing what is difficult, fairly trying to grapple, not with what appears the gist and strong point of a question, but with what really at bottom is the knot of it. It is a book the reasoning of which may not satisfy every one. ... But we think it is a book for people who wish to see a great subject handled on a scale which befits it, and with a perception of its real elements. It is a book which will have attractions for those who like to see a powerful mind applying itself, without shrinking or holding back, without trick or reserve or show of any kind, as a wrestler closes body to body with his antagonist, to the strength of an adverse and powerful argument.-Times, Tuesday, June 5, 1866.

We should add, that the faults of the work are wholly on the surface and in the arrangement; that the matter is as solid and as logical as that of any book within recent memory, and that it abounds in striking passages, of which we have scarcely been able even to give a sample. No future arguer against miracles can afford to pass it over. -Saturday Review, September 15, 1866.

IT Is my privilege to enjoy the friendship of a select number of religious men, with whom I converse frankly upon theological subjects, expressing without disguise the notions and opinions I entertain regarding their tenets, and hearing in return these notions and opinions subjected to criticism. I have thus far found them liberal and loving men, patient in hearing, tolerant in reply, who know how to reconcile the duties of courtesy with the earnestness of debate. From one of these, nearly a year ago, I received a note, recommending strongly to my attention the volume of "Bampton Lectures" for 1865, in which the question of miracles is treated by Mr. Mozley. Previous to receiving this note, $I$ had in part made the acquaintance of the work through an able and elaborate review of it in the Times. The combined effect of the letter and the review was to make the book the companion of my summer tour in the Alps. There, during the wet and snowy days which were only too prevalent in 1866, and during the days of rest interpolated between days of toil, I made myself more thoroughly conversant with $\mathrm{Mr}$.

* Fortnightly Review, New Series, vol. i., p. 645. 
Mozley's volume. I found it clear and strong-an intellectual tonic, as bracing and pleasant to my mind as the keen air of the mountains was to my body. From time to time I jotted down thoughts regarding it, intending afterward to work them up into a coherent whole. Other duties, however, interfered with the complete carrying out of this intention, and what I wrote last summer I now publish, not hoping to be able, within any reasonable time, to render my defense of scientific method more complete.

Mr. Mozley refers at the outset of his task to the move. ment against miracles which of late years has taken place, and which determined his choice of a subject. He acquits modern science of having had any great share in the production of this movement. The objection against miracles, he says, does not arise from any minute knowlerlge of the laws of nature, but simply becanse they are opposed to that plain and obvious order of nature which everybody sees. 'The present movement is, he thinks, to be ascribed to the greater earnestness and penetration of the present age. Formerly miracles were accepted without question, because without reflection; but the exercise of the "historic imagination" is a characteristic of our own time. Men are now accustomed to place before themselves vivid images of historic facts; and when a miracle rises to view, they halt before the astounding occurrence, and, realizing it with the same clearness as if it were now passing before their eyes, they ask themselves, "Can this have taken place?" In some instances the effort to answer this question has ler to a disbelief in miracles, in others to a strengthening of belief. The aim of Mr. Mozley's lectures is to show that the strengthening of belief is the logical result which ought to follow from the examination of the facts.

Attempts have been made by religious men to bring the Scripture miracles within the scope of the order of nature, but all such attempts are rejected by Mr. Mozley as utterly futile and wide of the mark. Regarling miracles as a necessary accompaniment of a revelation, their evidential value in his eyes depends entirely upon their deviation from the order of nature. Thus deriating, they suggest and illustrate a power higher than nature, a "personal will;" and they commend the person in whom this power is vested as a messenger from on high. Withont these credentials such a messenger would have no right to demand 
belief, even were his assertions rogarding his dirine mission backed by a holy life. Nor is it by miracles a!one that the order of nature is, or may be, disturbed. 'The material universe is also the arena of "special providences." Under these two heads Mr. Mozley distributes the total preternatural. One form of the preternatural may shade in to the other, as one color passes into another in the rainbow; but, while the line which divides the specially providential from the miraculous cannot be sharply drawn, their distinction broadly expressed is this: that while a special providence can only excite surmise more or less probable, it is "the nature of a miracle to give proof, as distinguished from mere surmise, of Divine design."

Mr. Mozley adduces various illustrations of what he regards to be special providences, as distinguished from miracles. " 'The death of Arius," he says, "was not miraculous, because the coincidence of the death of a heresiarch taking place when it was peculiarly advantageous to the orthodox faith .... was not such as to compel the inference of extraordinary Divine agency; but it was a special providence, because it carried a reasonable appearance of it. 'The miracle of the Thundering Legion was a special providence, but not a miracle, for the same reason, because the coincidence of an instantaneous fall of rain, in answer to prayer, carried some appearance, but not proof, of preternatural agency." The eminent lecturer's remarks on this heal brought to my recollection certain narratives published in Methodist magazines, which I used to read with avidity when a boy. The general title of these exciting stories, if I remember right, was " The Providence of God asserted," and in them the most extraordinary escapes from peril were recounted and ascribed to prayer, while equally wonderful instances of calamity were adduced as illus. trations of Divine retribution. In such magazines, or elsewhere, I found recorded the case of the celebrated Samuel Hick, which, as it illustrates a whole class of special providences approaching in conclusiveness to miracles, is worthy of mention here. It is related of this holy man that, on one occasion, flour was lacking to make the sacramental bread. Grain was present, and a windmill was present, but there was no wind to grind the corn. With faith undoubting, Samuel Hick prayed to the Lord of the winds; the sails turned, the corn was ground, after which 
the wind ceased. According to the canon of the Bampton lecturer, this, though carrying a strong appearance of an immediate exertion of Divine energy, lacks by a hair's breadth the quality of a miracle. For the wind might hare arisen, and might have ceased, in the ordinary course of nature. Hence the occurrence did not "compel the inference of extraordinary Divine agency." In like manner Mr. Mozley considers that " the appearance of the cross to Constantine was a miracle, or a special providence, according to what account of it we ausopt. As only a meteoric appearance in the shape of a cross it gave some token of preternatural agency, but not full evidence."

In the Catholic canton of Switzerland where I now write, and still more among the pious 'Tyrolese, the mountains are dotted with shrines, containing offerings of all kinds, in acknowledgment of special mercies-legs, feet, arms, and hands - of gold, silver, brass, and wood, according as worldly possessions enabled the grateful heart to express its indebtedness. Most of these offerings are made to the Virgin Mary. They are recognitions of "special providences," wronght through the instrumentality of the Mother of God. Mr. Mozley's belief, that of the Methodist chronicler, and that of the 'Tyrolese peasant, are substantially the same. Each of them assumes that nature instead of flowing ever onward in the uninterrupted rhythm of cause and effect, is mediately ruled by the free human will. As regards direct action upon natural phenomena, man's wish and will, as expressed in prayer, are confessedly powerless; but prayer is the trigger which liberates the divine power, and to this extent, if the will be free, man, of course, comnands nature.

Did the existence of this belief depend solely upon the material benefits derived from it, it could not, in my opinion, list a decade. As a purely objective fact, we should soon see that the distribution of natural phenomena is unaffected by the merits or the demerits of men; that the law of gravitation crushes the simple worshipers of Ottery St. Mary, while singing their hymns, just as surely as if they were engaged in a midnight brawl. The hold of this belief upon the human mind is not due to outward verification, but to the inner warmth, force, and elevation with which it is commonly assuciated. It is plain, however, that these feelings may exist under the most various forms. They 
are not limiter to Church of England Protestantism-they are not even limited to Christianity. Though less refined, they are certainly not less strong in the heart of the Methodist and the Tyrolese peasant than in the heart of $\mathrm{Mr}$. Mozley. Indeed, those feelings belong to the primal powers of man's nature. A "skeptic" may have them. They find vent in the battle-cry of the Moslem. 'They take hue and form in the hunting-grounds of the Red Indian; and raise all of them, as they raise the Christian, upon a wave of victory, above the terrors of the grave.

'The character, then, of a miracle, as distinguished from a special providence, is that the former furnishes proof, while in the case of the latter we have unly surmise. Dissolve the element of doubt, and the alleged fact passes from the one class of the preternatural into the other. In other words, if a special providence could be proved to be a special providence, it would cease to be a special providence and become a miracle. There is not the least cloudiness about Mr. Mozley's meaning here. A special providence is a doubtful miracle. Why, then, not call it so? 'The term employed by Mr. Mozley conveys no negative suggestion, whereas the negation of certainty is the peculiar churacteristic of the thing intended to be expressed. There is an apparent unwillingness on the part of the lecturer to call a special providence what his own definition makes it to be. Instead of speaking of it as a doubtful miracle, he calls it " an invisible miracle." $\mathrm{He}$ speaks of the point of contact of supernatural power with the chain of causation being so high up as to be wholly, or in part, out of sight, whereas the essence of a special providence is the uncertainty whether there is any contact at all, either high or low. By the use of an incorrect term, however, a grave danger is avoided. For the idea of duubt, if kept systematically before the mind, would soon be fatal to the special providence, considered as a means of edification. The term employed, on the contrary, invites and encourages the trust which is necessary to supplement the evidence.

This inner trust, though at first rejected by Mr. Mozley in favor of external proof, is subsequently called upon to do momentous duty in regard to miracles. Whenever the evidence of the miraculous seems incommensurate with the fact which it has to establish, or rather when the fact is so 
amazing that hardly any evidence is sufficient to establish it, Mr. Mozley invokes "the affections." They must urge the reason to accept the conclusion, from which unaided it recoils. The affections and emotions are eminently the court of appeal in matters of real religion, which is an affair of the heart; but they are not, I submit, the court in which to weigh allegations regarding the credibility of physical facts. These must be judged by the dry light of the intellect alone, appeals to the affections being reserved for cases where moral elevation, and not historic conviction, is the aim. It is, moreover, because the result, in the case under consideration, is deemed desirable that the affections are called upon to back it. If undesirable, they would, with equal right, be called upon to act the other way. Even to the disciplined scientific mind this would be a dangerous doctrine. A farorite theory-the desire to establish or avoid a certain result-can so warp the mind as to destroy its powers of estimating facts. I have known men to work for years under a fascination of this kind, unable to extricate themselves from its fatal influence. They had certain data, but not, as it happened, enough. By a process exactly analogous to that invoked by Mr. Mozley, they supplemented the data, and went wrong. From that hour their intellects were so blinded to the perception of adverse phenomena that they nerer reached truth. If, then, to the disciplined scientific mind, this incongruous mixture of proof and trust be fraught with danger, what must it be to the indiscriminate audience which Mr. Mozley addresses? In calling upon this agency he acts the part of Frankenstein. It is a monster thus evoked that we see stalking abroad, in the degrading spiritualistic phenomena of the present day. Again, I say, where the aim is to elerate the mind, to quicken the moral sense, to kindle the fire of religion in the soul, let the affections by all means be invoked; but they must not be permitted to color our reports, or to influence our acceptance of reports of occurrences in exterual nature. 'Testimony as to natural facts is worthless when wrapped in this atmosphere of the affections; the most earnest subjective truth being thus rendered perfectly compatible with the most astounding objective error.

There are questions in judging of which the affections or sympathies are often our best guides, the estimation of 
moral goodness being one of these. But at this precise point, where they are really of use, Mr. Mozley excludes the affections and demands a miracle as a certificate of character. He will not accept any other evidence of the perfect goodness of Christ. "No outward life and conduct," he says, "however irreproachable, could prove II is perfect sinlessness, because goodness depends upon the inward motive, and the perfection of the inward motive is not proved by the outward act." But surely the miracle is an outward act, and to pass from it to the inner motive imposes a greater strain upon logic than that involved in on ordinary methods of estimating men. There is, at least, moral congruity between the outward goodness and the imner life, but there is no such congruity between the miracle and the life within. The test of moral goodness laid down by Mr. Mozley is not the test of John, who says, "He that doeth righteousness is righteous;" nor is it the test of Jesus: "By their fruits ye shall know them: do men gather grapes of thorns, or figs of thistles?" But it is the test of another: "If thou be the Son of God, command that these stones be made bread." For my own part, I prefer the attitude of Fichte to that of Mr. Mozley. "The Jesus of John," says this noble and mighty thinker, "knows no other God than the True God, in whom we all are, and live, and may be blessed, and out of whom there is only Death and Nothingness. And," continues Fichte, " he appeals, and rightly appeals, in support of this truth, not to reasoning, but to the inward practical sense of truth in man, not even knowing any other proof than this inward testimony, "If any man will do the will of Him who sent $\mathrm{Me}$, he shall know of the doctrine whether it be of God.'" Accepting Mr. Mozley's test, with which alone I am now dealing, it is evident that, in the demonstration of moral goorlness, the quantity of the miraculous comes into play. Had Christ, for exaniple, limited himself to the conversion of water into wine, He would have fallen short of the performance of Jannes and Jambres; for it is a smaller thing to convert one liquid into another than to convert a dead rod into a living serpent. But Jannes and Jambres, we are informed, were not good. Hence, if Mr. Mozley's test be a true one, a point must exist, on the one side of which miraculous power demonstrates goodness, while on the other side it does not. How is this "point 
of contrary flexure" to be determined? It must lie somewhere between the magicians and Moses, for within this space the power passed from the diabolical to the Divine. But how to mark the point of passage-how, out of a purely quantitative difference in the visible manifestation of power, we are to infer a total inversion of quality -it is extremely difficult to see. Moses, we are informed, produced a large reptile; Jannes and Jambres produced a small one. I do not possess the intellectual faculty which would enable me to infer, from those data, either the goodness of the one or the badness of the other; and in the highest recorded manifestations of the miraculous I am equally at a loss. Let us not play fast and loose with the iniraculous; either it is a demonstration of goodness in all cases or in none. If Mr. Mozley accepts Christ's goodness as transcendent, because He did such works as no other man did, he ought, logically speaking, to accept the works of those who, in His name, had cast out devils, as demonstrating a proportionate goodness on their part. But it is people of this class who are consigned to everlasting fire prepared for the devil and his angels. Such zeal as that of Mr. Mozley for miracles tends, I fear, to eat his religion up. The logical threatens to stifle the spiritual. 'The truly religious soul needs no miraculous proof of the goodness of Christ. The words addressed to Matthew at the receipt of custom requirel no niracle to produce obedience. It was by no stroke of the supernatural that Jesus caused those sent to seize Him to go backward and fall to the ground. It was the sublime and holy effluence from within, which needed no prodigy to commend it to the reverence even of his foes.

As regards the function of miracles in the founding of a religion, Mr. Mozley institutes a comparison between the religion of Christ and that of Mohammed; and he derides the latter as "irrational " because it does not profess to adduce miracles in proof of its supernatural origin. But the religion of Mohammed, notwithstanding this drawback, has thriven in the world, and at one time it held sway over larger populations than Christianity itself. The spread and influence of Christianity are, however, brought forward by Mr. Mozley as "a permanent, enormous, and incalculable practical result" of Christian miracles; and he makes use of this result to strengthen his plea for the miraculous. 
II is logical warrant for this proceeding is not clear. It is the method of science, when a phenomenon presents itself, toward the production of which several elements may contribute, to exclude them one by one, so as to arrive at length at the truly effective canse. Heat, for example, is associated with a phenomenon; we exclude heat, but the phenomenon remains: hence, heat is not its cause. Magnetism is associated with a phenomenon; we exclude magnetism, but the phenomenon remains: hence, magnetism is not its cause. 'Thus, also, when we seek the cause of a diffusion of a religion-whether it be due to miracles, or to the spiritual force of its founders-we exclude the miracles, and, finding the result unchanged, we infer that miracles are not the effective cause. 'This important experiment Mohammedanism has made for us. It has lived and spread without miracles; and to assert, in the face of this, that Christianity has spread because of miracles, is, I submit, opposed both to the spirit of science and the common sense of mankind.

The incongruity of inferring moral goodness from miraculous power has been dwelt upon above; in another particular also the strain put by Mr. Mozley upon miracles is, I think, more than they can bear. In consistency with his principles, it is difficult to see how he is to draw from the miracles of Christ any certain conclusion as to His Divine nature. He dwells very forcibly on what he calls "the argument from experience," in the demolition of which he takes obvious delight. He destroys the argument, and repeats it, for the mere pleasure of again and again knocking the breath ont of it. Experience, he urges, can only deal with the past; and the moment we attempt to project experience a hair's breadth beyond the point it has at any moment reached, we are condemned by reason. It appears to me that when he infer's from Christ's miracles a Divine and altogether superhuman energy, Mr. Mozley places himself precisely under this condenmation. For what is his logical ground for concluding that the miracles of the New Testament illustrate Divine power? May. they not be the result of expanded human power? A miracle he defines as something impossible to man. But how does he know that the miracles of the New 'Testament are impossible to man? Seek as he may, he has absolutely no reason to adduce save this-that man has never hitherto accomplished such 
things. But does the fact that man has never raised the dead prove that he $c$ an never raise the dead? "Assuredly not," must be Mr. Mozley's reply; "for this would be pushing experience beyond the limit it has now reachedwhich I pronounce unlawful." Then a period may come when man will be able to raise the dead. If this be conceded-and I do not see how Mr. Mozley can avoid the concession-it destroys the necessity of inferring Christ's divinity from His miracles. He, it may be contended, anterated the humanity of the future; as a mighty tidal wave leaves high upon the beach a mark which by and isy becomes the general level of the ocean. Turn the matter as you will, no other warrant will be found for the all-important conclusion that Christ's miracles demonstrate divine power, than an argument which has been stigmatized by Mr. Mozley as a "rope of sand"-the argument from experience.

The learned Bampton lecturer would be in this position, even had he seen with his own eyes every miracle recorded in the New Testament. But he has not seen these miracles; and his intellectual plight is therefore worse. He accepts these miracles on testimony. Why does he believe that testimony? How does he know that it is not delusion; how is he sure that it is not even fraud? He will answer, that the writing bears the marks of - sobriety and truth: and that in many cases the bearers of this message to mankind sealed it with their blood. Granted with all my heart; but whence the value of all this? Is it not solely derived from the fact that men, as we know them, do not sacrifice their lives in the attestation of that which they know to be untrue? Does not the entire value of the testimony of the apostles depend ultimately upon our experience of human nature? It appears, then, that those said to have seen the miracles based their inferences from what they saw on the argument from experience; and that Mr. Mozley bases his belief in their testimony on the same argument. The weakness of his conclusion is quadrupled by this double insertion of a principle of belief, to which he flatly denies rationality. His reasoning, in fact, cuts two ways-if it destroys our trust in the order of nature, it far more effectually abolishes the basis on which Mr. Mozley seeks to found the Christian religion. 
Over this argument, from experience, which at bottom is his argument, Mr. Mozley rides roughshod. 'There is it dash of scorn in the energy with which he tramples on it. Probably some previous writer had male too much of it, and thus invited his powerful assault. Finding the difficulty of belief in miracles to rise from their being in contrailiction to the order of nature, he sets himself to examine the grounds of our belief in that order. With a vigor of logic rarely equaled, and with a confidence in its conclusions never surpassed, he disposes of this belief in a manner calculated to startle those who, without due examination, had come to the conclusion that the order of nature was secure.

What we mean, he says, by our belief in the order of nature, is the belief that the future will be like the past. 'There is not, according to Mr. Mozley, the slightest rational basis for this belief.

"That any cause in nature is more permanent than its existing and known effects, extending further, and about to produce other and more instances besides what it has produced already, we have no evidence. Let us imagine," he continues, "the occurrense of a particular physical phenomenon for the first time. Upon that single occurrence we should have but the very faintest expectation of another. If it did occur again, once or twice, so far from counting on another occurrence, a cessation would occur as the most natural event to us. But let it continue one hundred times, and we should find no hesitation in inviting persons from a distance to see it; and if it occurred every day for years, its occurrence would be a certainty to us, its cessation a marvel. . . What ground of reason can we assign for an expectation that any part of the course of nature will be the next moment what it has been up to this moment, i.e., for our belief in the uniformity of nature? None. No demonstrative reason can be given, for the contrary to the recurrence of a fact of nature is no contradiction. No probable reason can be given; for all probable reasoning respecting the course of nature is founded upon this presumption of likeness, and therefore cannot be the foundation of it. No reason can be given for this belief. It is without a reason. It rests upon no rational grounds and can be traced to no rational principle."

“Everything," Mr. Mozley, however, adds, "depends upon this belief, every provision we make for the future, every safeguard and caution we employ against it, all calculation, all adjustment of means to ends, supposes this belief; and yet this belief has no more producible reason for it than a speculation of fancy. . . . It is necessary, 
all-important for the purposes of life, but solely practical, and possesses no intellectual chiracter.... The proper function," continues Mr. Mozley, "of the inductive principle, the argument from experience, the belief in the order of nature-by whatever phrase we designate the same instinct-is to operate as a practical basis for the affairs of life and the carrying on of human society." 'To sum up, the belief in the order of nature is general, but it is " an unintelligent impulse, of which we can give no rational account." It is inserted into our constitution solely to induce us to till our fields, to raise our winter fuel, and thus to meet the future on the perfectly gratuitous supposition that it will be like the past.

"'Thus, step by step," says Mr. Mozley, with the emphasis of a man who feels his position to be a strong one, " has philosophy loosened the connection of the order of nature with the ground of reason, befriending in exact proportion as it has done this the principle of miracles." For " this belief not having itself a foundation in reason, the ground is gone upon which it conld be maintained that miracles, as opposed to the order of nature, are opposed to reason." When we regard this belief in connection with science, "in which connection it receives a more imposing name, and is called the inductive principle," the result is the same. "The inductive principle is only this unreasoning impulse applied to a scientifically ascertained fact. . . Science has led up to the fact; but there it stops, and for converting this fact into a law, a totally unscientific principle comes into play, the same as that which generalizes the commonest observation of nature."

The eloquent pleader of the cause of miracles passes over without a word the results of scientific investigation, as proving anything rational regarding the principles or method by which such results have been achieved. Here, as elsewhere, he declines the test, "By their fruits shall ye know them." Perhaps our best way of proceeding will be to give one or two examples of the mode in which men of science apply the unintelligent impulse with which $\mathrm{Mr}$. Mozley credits them, and which shall show, by illustration, the surreptitious method whereby they climb from the region of facts to that of laws.

Before the sixteenth century it was known that water 
rises in a pump; the effect being then explained by the maxim that " Nature abhor's a vacuum." It was not known that there was any limit to the height to which the water would ascend, until, on one occasion, the gardeners of Florence, while attempting to raise water to a very great elevation, found that the column ceased at a height of thirty-two feet. Beyond this all the skill of the pumpmaker could not get it to rise. The fact was brought to the notice of Galileo, and he, soured by a world which had not treated his science over kindly, is said to have twitted the philosophy of the time by remarking that nature evidently abhorred a vacuum only to a height of thirtytwo feet. Galileo, however, did not solve the problem. It was taken up by his pupil Torricelli, to whom, after due pondering, the thought occurred, that the water might be forced in to the tube by a pressure applied to the surface of the liquid outside. But where, under the actual circumstances, was such a pressure to be found? After much reflection, it flashed upon Torricelli that the atmosphere might possibly exert this pressure; that the impalpable air might possess weight, and that a column of water thirtytwo feet high might be of the exact weight necessary to hold the pressure of the atmosphere in equilibrium.

There is much in this process of pondering and its results which it is impossible to analyze. It is by a kind of inspiration that we rise from the wise and sedulous conterplation of facts to the principles on which they depend. The mind is, as it were, a photographic plate, which is gradually cleansed by the effort to think rightly, and which, when so cleansed, and not before, receives impressions from the light of truth. This passage from facts to principles is called induction; and induction, in its highest form, is, as I have just stated, a kind of inspiration. But, to make it sure, the inward sight must be shown to be in accordance with outward fact. 'To prove or disprove the induction, we must resort to deduction and experiment.

Torricelli reasoned thus: If a column of water thirty-two feet high holds the pressure of the atmosphere in equilibrium, a shorter column of a heavier liquid ought to do the same. Now, mercury is thirteen times heavier than water; hence, if my induction be correct, the atmosphere ought to be able to sustain only thirty inches of mercury. Here, then, is a deduction which can be immediately sub- 
mitted to experiment. Torricelli took a glass tube a yard or so in length, closed at one end and open at the other, and filling it with mercury, he stopped the open end with his thumb, and inverted it into a basin filled with the liquid metal. One can imagine the feeling with which Torricelli removed his thumb, and the delight he experiencel on finding that his thought had forestalled a fact never before revealed to human eyes. 'The column sank, but it ceased to sink at a height of thirty inches, leaving the Torricellian vacuum overhead. From that hour the theory of the pump was established.

The celebrated Pascal followed Torricelli with another deduction. He reasoned thus: if the mercurial column be supported by the atmosphere, the higher we ascend in the air, the lower the column ought to sink, for the less will be the weight of the air overhead. He caused a friend to ascend the Puy de Dôme, carrying with him a barometric column; and it was found that during the ascent the column sank, and that during the subsequent descent the column rose. Between the time here referred to and the present, millions of experiments have been made upon this subject. Every village pump is an apparatus for such experiments. In thousands of instances, moreover, pumps have refused to work; but on examination it has infallibly been found that the well was dry, that the pump required priming, or that some other defect in the apparatus accounted for the anomalous action. In every case of the kind the skill of the pump-maker has been found to be the true remedy. In no case has the pressure of the atmosphere ceased; constancy, as regards the lifting of pump-water, has been hitherto the demonstrated rule of nature. So also as regards Pascal's experiment. His experience has been the universal experience ever since. Men have climbed mountains, and gone up in balloons; but no deviation from Pascal's result has ever been observed. Barometers, like pumps, have refused to act; but instead of indicating a1:y suspension of the operations of nature, or any interference on the part of its Author with atmospheric pressure, examisation has in every instance fixed the anomaly upon the instruments themselves. It is this welding, then, of rigid logic to verifying fact that Mr. Mozley refers to an "unreasoning impulse."

Let us now briefly consider the case of Newton.

Before 
his time men had occupied themselves with the problem of the solar system. Kepler had deduced, from a vast mass of observations, those general expressions of planetary motion known as "Kepler's laws." It had been observed that a magnet attracts iron; and by one of those flashes of inspiration which reveal to the human mind the vast in the minute, the general in the particular, it had been inferred, that the force by which bodies fall to the earth might also be an attraction. Newton pondered all these things. He looked, as was his wont, into the darkness until it becume entirely luminous. How this light arises we camnot explain; but, as a matter of fact, it does arise. Let me remark here, that this kind of pondering is a process with which the ancients could have been but imperfectly acquainted. They, for the most part, found the exercise of fantasy more pleasant than careful observation, and subsequent brooding over facts. Hence it is, that when those whose education has been derived from the ancients speak of " the reason of man," they are apt to omit from their conception of reason one of its most important factors. Well, Newton slowly marshaled his thoughts, or rather they came to him while he "intended his mind," rising like a series of intellectual births out of chaos. He made this idea of attraction his own. But, to apply the idea to the solar system, it was necessary to know the magnitude of the attraction, and the law of its variation with the distance. His conceptions first of all passed from the action of the earth as a whole, to that of its constituent particles. And persistent thought brought more and more clearly out the final conclusion, that every particle of matter attracts every other particle with a force varying inversely as the square of the distance between the particles.

Here we have the flower and ontcome of Newton's induction; and how to verify it, or to disprove it, was the next question. The first step of the philosopher in this direction was to prove, mathematically, that if this law of attraction be the true one; if the earth be constituted of particles which obey this law; then the action of a sphere equal to the earth in size on a body outside of it, is the same as that which would be exerted if the whole mass of the splsere were contracted to a point at its center. Practically speaking, then, the center of the earth is the point 
from which distances must be measured to bodies attracted by the earth.

From experiments executed before his time, Newton knew the amount of the earth's attraction at the earth's surface, or at a distance of $\$, 000$ miles from its center. His object now was to measure the attraction at a greater distance, and thus to determine the law of its diminution. But how was he to find a body at a sufficient distance? He had no balloon? and eveu if he had, he knew that any height to which he could attain would be too small to enable him to solve his problem. What did he do? He fixed his thoughts upon the moon-a body 240,000 miles, or sixty times the earth's radius, from the earth's center. He virtually weighed the moon, and found that weight to be one thirty-six hundred th of what it would be at the earth's surface. This is exactly what his theory required. I will not $d$ well here upon the pause of Newton after his first calcula tions, or speak of his self-denial in withholding them because they did not quite agree with the observations then at his command. Newton's action in this matter is the normal action of the scientific mind. If it were otherwise-if scientific men were not accustomed to demand verification -if they were satisfied with the imperfect while the perfect is attainable, their science, instead of being, as it is, a fortress of adamant, would be a house of clay, ill-fitted to bear the buffetings of the theologic storms to which it is periodically exposed.

Thus we see that Newton, like Torricelli, first pondered his facts, illuminated them with persistent thought, and finally divined the character of the force of gravitation. But, having thus traveled inward to the principle, he reversed his steps, carried the principle outward, and justified it by demonstrating its fitness to external nature.

And here, in passing, I wonld notice a point which is well worthy of attention. Kepler had deduced his laws from observation. As far back as those observations extended, the planetary motions had obeyed these laws; and neither Kepler nor Newton entertained a doubt as to their continuing to obey them. Year after year, as the ages rolled, they believer that those laws would continue to illustrate themselves in the heavens. But this was not sufficient. The scientific mind can find no repose in the mere registration of sequence in nature. The further 
question intrudes itself with resistless might, Whence comes the sequence? What is it that binds the consequent to its antecedent in nature? 'The truly scientific intellect never can attain rest until it reaches the forces by which the observed succession is produced. It was thus with Torricelli; it was thus with Newton; it is thus pre-eminently with the scientific man of to-day. In common with the most ignorant, he shares the belief that spring will succeed winter, that summer will succeed spring, that autumn will succeed summer, and that winter will succeed autumn. But he knows still further-and this knowledge is essential to his intellectual repose-that this succession, besides being permanent, is, under the circumstances, necessary; that the gravitating force exerted between the sun and a revolving sphere, with an axis inclined to the plane of its orbit, must produce the observed succession of the seasons. Not until this relation between forces and phenomena has been established, is the law of reason rendered concentric with the law of nature; and not until this is effected does the mind of the scientific philosopher rest in peace.

The expectation of likeness, then, in the procession of phenomena, is not that on which the scientific mind founds its belief in the order of nature. If the force be permanent the phenomena are necessary, whether they resemble or do not resemble anything that has gone before. Hence, in judging of the order of nature, our inquiries eventually relate to the permanence of force. From Galileo to Newton, from Newton to our own time, eager eyes have been scanning the heavens, and clear heads have been pondering the phenomena of the solar system. The same eyes and minds have been also observing, experimenting, and reflecting on the action of gravity at the surface of the earth. Nothing has occurred to indicate that the operation of the law has for a moment been suspended; nothing has ever intimated that nature has been crossed by spontaneous action, or that a state of things at any time existed which could not be rigorously deduced from the preceding state.

Given the distribution of matter, and the forces in operation, in the time of Galileo, the competent mathematician of that day conld predict what is now occurring in our own. We calculate eclipses in advance, and find our cal- 
culations true to the second. We determine the dates of those that have occurred in the early times of history, and find calculation and history in harmony. Anomalies and perturbations in the planets have been over and over again observer; but these, instead of demonstrating any inconstancy on the part of natural law, have invariably been reduced to consequences of that law. Instead of referring the perturbations of Uranus to any interference on the part of the Author of nature with the law of gravitation, the question which the astronomer proposed to himself was, "How, in accordance with this law, can the perturbation be produced?" Guided by a principle, he was enabled to fix the point of space in which, if a mass of matter were placed, the observed perturbations would follow. We know the result. The practical astronomer turned his telescope toward the region which the intellect of the theoretic astronomer had already explored, and the planet now named Neptune was found in its predicted place. A very respectable outcome, it will be admitted, of an impulse which "rests upon no rational grounds, and can be traced to no rational principle;" which possesses " no intellectual character;" which "philosophy" has uprooted from "the ground of reason," and fixed in that "large irrational department" discovered for it, by Mr. Mozley, in the hitherto unexplored wilderness of the human mind.

The proper function of the inductive principle, or the belief in the order of nature, says Mr. Mozley, is " to act as a practical basis for the affairs of life, and the carrying on of human society." But what, it may be asked, has the planet Neptune, or the belts of Jupiter, or the whiteness about the poles of Mars, to do with the affairs of society? How is society affected by the fact that the sun's atmosphere contains solium, or that the nebula of Orion contains hydrogen gas? Nineteen-twentieths of the force employed in the exercise of tre inductive principle, which, reiterates Mr. Mozley, is " purely practical," have been expended upon subjects as unpractical as these. What practical interest has society in the fact that the spots on the sun have a decennial period, and that when a magnet is closely watched for half a century, it is found to perform small motions which synchronize with the appearance and disappearance of the solar spots? And yet, I doubt not, Sir Edward Sabine would deem a life of intellectual toil 
amply rewarded by being privileged to solve, at its close, these infinitesimal motions.

'The inductive principle is founded in man's desire to know-a desire arising from his position among phenomena which are reducible to order by his intellect. The material universe is the complement of the intellect; and, without the study of its laws, reason could never have awakened to the higher forms of self-consciousness at all. It is the Non-ego through and by which the Ego is endowed with self-discernment. We hold it to be an exercise of reason to explore the meaning of a universe to which we stand in this relation, and the work we have accomplished is the proper commentary on the methods we have pursued. Before these methods were adopted the unbridled imagination roamed through nature, putting in the place of law the figments of superstitious dread. For thousands of years witchcraft, and magic, and miracles, and special providences, and Mr. Mozley's " distinctive reason of man," had the world to themselves. They made worse than nothing of it-worse, I say, because they let and hindered those who might have made something of it. Hence it is, that during a single lifetime of this era of " unintelligent impulse," the progress in knowledge is all but infinite as compared with that of the ages which preceded our's.

'The believers in magic and miracles of a couple of centuries ago had all the strength of Mr. Mozley's present logic on their side. 'They had done for themselves what he rejoices in having so effectually done for us-cleared the ground of the belief in the order of nature, and declared magic, miracles, and witcheraft to be matter's for "ordinary evidence" to decide. "The principle of miracles" thus "befiriended" had free scope, and we know the result. Lacking that rock-barrior of natural knowledge which we now possess, keen jurists and cultivated men were hurried on to deeds, the bare recital of which makes the blood run cold. Skilled in all the rules of human evidence, and versed in all the arts of cross-examination, these men, nevertheless, went systematically astray, and committed the deadliest wrongs against humanity. And why? Because they could not put Nature into the witness-box, and question her-of her voiceless "testimony" they knew nothing. In all cases between man and man, their judg- 
ment was to be relied on; but in all cases between man and nature, they were blind leader's of the blind.*

Mr. Mozley concedes that it would be no great result if miracles were only accepted by the ignorant and superstitious, "because it is easy to satisfy those who do not inquire." But he does consider it "a great result" that they have been accepted by the educated. In what sense educated? Like those statesmen, jurists, and church dignitaries whose education was unable to save them from the frightful errors glanced at above? Not even in this sense; for the great mass of Mr. Mozley's educater people had no legal training, and must have been absolutely defenseless against delusions which could set even that training at nanght. Like nine-tenths of our clergy at the present day, they were versed in the literature of Greece, Rome, and Judea; but as regards a knowledge of nature, which is here the one thing needful, they were "noble savages," and nothing more. In the case of miracles, then, it behoves us to understanil the weight of the negative, before we assign a value to the positive; to comprehend the depositions of nature, before we attempt to measure, with them, the evidence of men. We have only to open our eyes to see what honest and even intellectual men and women are capable of, as to judging evidence, in this nineteenth century of the Christian era, and in latitude fiftytwo degrees north. 'The experience thus gained ought, I imagine, to influence our opinion regarding the testimony of people inhabiting a sunnier clime, with a richer imagination, and without a particle of that restraint which the discoveries of physical science have imposed upon mankind.

Having thus submitted Mr. Mozley's views to the examination which they challenged at the hands of a student of nature, I am unwilling to quit his book without expressing

* “In 1664 two women were hung in Śuffolk, under a sentence of Sir Matthew Hale, who took the opportunity of declaring that the reality of witchcraft was unquestionable; 'for first, the scriptures had affirmed so much; and secondly, the wisdon of all nations had provided laws against such persons, which is an argument of their confidence of such a crime.' Sir Thomas Browne, who was a great physician as well as a great writer, was called as a witness, and swore 'that he was clearly of opinion that the persons were bewitched.' "--Lecky's History of Rationalism, vol. i., p. 120. 
my admiration of his genius, and my respect for his character. Though barely known to him personally, his recent death affected me as that of a friend. With regard to the style of his book, I heartily subseribe to the description with which the Times winds up its able and appreciative review. "It is marked throughout with the most serious and earnest conviction, but is withont a single word from first to last of asperity or insinuation against opponents; and this not from any deficiency of feeling as to the importance of the issue, but from a deliberate and resolutely maintained self-control, and from an overruling, everpresent sense of the duty, on themes like these, of a more than judicial calmness."

['To the argument regarding the quantity of the miraculous, introduced at page 355, Mr. Mozley has done me the honor of publishing a reply in the seventh volume of the Contemporary Review. -J. T.]

\section{ADDITIONAL REMARKS ON MIRACLES.}

Among the scraps of manuscript, written at the time when Mr. Mozley's work occupied my attention, I find the following reflections:

With regard to the influence of modern science which Mr. Mozley rates so low, one obvious effect of it is to enhance the magnitude of many of the recorded miracles, and to increase proportionably the difficulties of belief. 'The ancients knew but little of the vastness of the universe. The Rev. Mr. Kirkman, for example, has shown what inadequate notions the Jews entertained regarding the "firmament of lieaven;" and Sir George Airy refers to the case of a Greek philosopher who was persecuted for hazarding the assertion, then deemed monstrous, that the sun might be as large as the whole country of Greece. The concerns of a universe, regarded from this point of view, were much more commensurate with man and his concerns than those of the universe which science now reveals to us; and hence that to suit man's purposes, or that in compliance with his prayers, changes should occur in the order of the universe, was more easy of belief in the ancient world that it can be now. In the very magnitude which it 
assigns to natural phenomena, science has augmented the distance between them and man, and increased the popular belief in their orderly progression.

As a natural consequence the demand for evidence is more exacting than it used to be, whenever it is affirmed that the order of nature has been disturbed. Let us take as an illustration the miracle by which the victory of Joshua over the Amorites was rendered complete. In this case the sun is reported to have stood still for "about a whole day" upon Gibeon, and the moon in the valley of Ajalon. An Englishman of average education at the present day would naturally demand a greater amount of evidence to prove that this occurrence took place, than would have satisfied an Israelite in the age succeeding that of Joshua. For to the one, the miracle probably consisted in the stoppage of a fiery ball less than a yard in diameter, while to the other it would be the stoppage of an orb fourteen hundred thousand times the earth in size. And even accepting the interpretation that Joshua dealt with what was apparent merely, but that what really occurred was the suspension of the earth's rotation, I think the right to exercise a greater reserve in accepting the miracle, and to demand stronger evidence in support of it than that which would have satisfied an ancient Israelite, will still be conceded to a man of science.

'There is a scientific as well as an historic imagination; and when, by the exercise of the former, the stoppage of the earth's rotation is clearly realized, the erent assumes proportions so vast, in comparison with the result to be obtained by it, that belief reels under the reflection. 'The energy here involved is equal to that of six trillions of horses working for the whole of the time employed by Joshua in the destruction of his foes. The amount of power thus expended would be sufficient to supply every individual of an army a thousand times the strength of that of Joshua, with a thousand times the fighting power of each of Joshua's soldiers, not for the few hours necessary to the extinction of a handful of Amorites, but for millions of years. All this wonder is silently passed over by the sacred historian, manifestly because he knew nothing about it. Whether, therefore, we consider the miracle as purely evidential, or as a practical means of vengeance, the same lavish squaudering of energy stares us in the face. If 
evidential, the energy was wasted, because the Israelites knew nothing of its amount; if simply destructive, then the ratio of the quantity lost to the quantity employed, may be inferred from the foregoing figures.

'T'o other miracles similar remarks apply. Transferring our thoughts from this little sand-grain of an earth to the immeasurable heavens, where countless worlds with freights of life probably revolve unseen, the very suns which warm them being barely visible across abysmal space; reflecting that beyond these sparks of solar fire suns innumerable may burn, whose light can never stir the optic nerve at all; and bringing these reflections face to face with the idea of the Builder and Sustainer of it all showing Himself in a burning bush, exhibiting His hinder parts, or behaving in other familiar ways ascribed to Him in the Jewish Scriptures, the incongruity must appear. Did this credulous prattle of the ancients about iniracles stand alone; were it not associated with words of imperishable wisdom, and with examples of moral grandeur unmatched elsewhere in the history of the human race, both the miracles and their "evidences" would have long since ceased to be the transmitted inheritance of intelligent men. Influenced by the thoughts which this universe inspires, well may we exclaim in David's spirit, if not in David's words: " When I consider the heavens, the work of thy fingers, the moon, and the stars, which thou hast ordained; what is man that thou shouldst be mindful of him, or the son of man that thou shouldst so regard him?"

If you ask me who is to limit the outgoings of Almighty power, my answer is, Not I. If you should urge that if the Builder and Maker of this universe chose to stop the rotation of the earth, or to take the form of a burning bush, there is nothing to prevent Him from doing so, I am not prepared to contradict you. I neither agree with you nor differ from you, for it is a subject of which I know nothing. But I observe that in such questions regarding Almighty power, your inquiries relate, not to that power as it is actually displayed in the universe, but to the power of your own imagination. Your question is, not has the Omnipotent done so and so? or is it in the least degree likely that the Omnipotent should do so and so? but, is my imagination competent to picture a Being able and willing to do so and so? I am not prepared to deny your 
competence. To the human mind belongs the fuculty of enlarging and diminishing, of distorting and combining, indefinitely, the objects revealed by the senses. It can imagine a mouse as large as an elephant, an elephant as large as a mountain, and a mountain as high as the stars. It can separate congruities aud unite incongruities. We see a fish and we see a woman; we can drop one half of each, and unite in idea the other two halves to a mermaid. We see a horse and we see a man; we are able to drop one half of each, and unite the other two halves to a centaur. Thus also the pictorial representations of the Deity, the bodies and wings of cherubs and seraphs, the hoofs, horns, and tail of the evil one, the joys of the blessed, and the torments of the damned, have been elaborated from materials furvished to the imagination by the senses. It behoves you and me to take care that our notions of the Power which rules the universe are not mere fanciful or iguorant enlargements of human power. The capabilities of what you call your reason are not denied. By the exercise of the faculty here adrerted to, you can picture to yourself a Being able and willing to do any and every conceivable thing. You are right in saying that in opposition to this power science is of no avail-that it is " a weapon of air." 'The man of science, however, while accepting the figure, would probably reverse its application, thinking it is not science which is here the thirg of air, but that unsubstantial pageant of the imagination to which the solidity of science is opposed.

\section{CHAPTER XXV.}

\section{ON PRAYER AS A FORM OF PHYSICAL ENERGY.}

Prayer as a means to effect a private end is theft and meanness.EMERBON.

'THE EDITOR of the Contemporary Review is liberal enough to grant me space for some remarks upon a subject, which, though my relation to it was simply that of a vehicle of transmission, has brought down upon me a considerable amount of animadversion.

It may be interesting to some of my readers if I glance at a few cases illustrative of the history of the human 
mind, in relation to this and kindred questions. In the fourth century the belief in Antipodes was deemed unscriptural and heretical. The pious Lactantius was as angry with the people who held this notion as my censors are now with me, and quite as unsparing in his denunciations of their "Monstrosities." Lactantius was irritated because, in his mind, by education and habit, cosmogony and religion were indissolubly associated, and, therefore, simultaneously disturbed. In the early part of the seventeenth century the notion that the earth was fixed, and that the sun and stars revolved round it daily, was interwoven with religious feeling, the separation then attempted by Galileo rousing the animosity and kindling the persecution of the Church. Men still living can remember the indignation excited by the first revelations of geology regarding the age of the earth, the association between chronology and religion being for the time indissoluble. In our day, however, the best-informed theologians are prepared to admit that our views of the universe and its Auchor are not impaired, but improved, by the abandoument of the Mosaic account of the creation. Look, finally, at the excitement caused by the publication of the "Origin of Species," and compare it with the calm attendant on the appearance of the far more outspoken, and, from the old point of view, more impious, "Descent of Mau.”

'I'hus religion survives after the removal of what had been long considered essential to it. In our day the Antipodes are accepted; the fixity of the earth is given up; the period of creation and the reputed age of the world are alike dissipated; evolution is looked upon without terror; and other changes have occurred in the same direction too numerous to be dwelt upon here. In fact, from the earliest times to the present, religion has been undergoing a process of purification, freeing itself slowly and painfully from the physical errors which the active but uninformed intellect mingled with the aspirations of the soul. Some of us think that a final act of purification is needed, while others oppose this notion with the confidence and the warmth of ancient times. 'The bone of contention at present is the physical value of prayer. It is not $\mathrm{my}$ wish to excite surprise, much less to draw forth protest, by the employment of this phrase. I would simply ask any 
intelligent person to look the problem honestly in the face, and then to say whether, in the estimation of the great body of those who sincerely resort to it, prayer does not, at all events upon special occasions, invoke a power which checks and augments the descent of rain, which changes the force and direction of winds, which affects the growth of corn and the health of men and cattle-a Power, in short, which, when appealed to under pressing circumstances, produces the precise effects caused by physical energy in the ordinary course of things. 'To any person who deals sincerely with the subject, and refuses to blur his moral vision by intellectual subtleties, this, I think, will appear a true statement of the case.

It is under this aspect alone that the scientific student, so far as I represent him, has any wish to meddle with prayer. Forced upon his attention as a form of physical energy, or as the equivalent of such energy, he claims the right of subjecting it to those methods of examination from which all our present knowledge of the physical universe is derived. And if his researches lead him to a conclusion adverse to its claims-if his inquiries rivet him still closer to the philosophy implied in the words, " $\mathrm{He}$ maketh his sun to shine on the evil and on the good, and sendeth rain upon the just and upon the unjust"-he contends only for the displacement of prayer, not for its extinction. He simply says, physical nature is not its legitimate domain.

This conclusion, moreover, must be based on pure physical evidence, and not on any inherent unreasonableness in the act of prayer. The theory that the system of nature is under the control of a Being who changes phenomena in cornpliance with the prayers of men, is, in my opinion, a perfectly legitimate one. It may of course be rendered futile by being associated with conceptions which contradict it; but such conceptions form no necessary part of the theory. It is a matter of experience that an earthly father, who is at the same time both wise and tender, listens to the requests of his children, and, if they do not ask amiss, takes pleasure in granting their requests. We know also that this compliance extends to the alteration, within certain limits, of the current of events on earth. With this suggestion offered by experience, it is no departure from scientific method to place behind natural phenomena a 
Universal Futher, who, in answer to the prayers of His children, alters the currents of those phenomena. Thus far 'Theology and Science go hand in hand. 'The conception of an ether, for example, trembling with the waves of light, is suggested by the ordinary phenomena of wavemotion in water and in air; and in like manner the conception of personal volition in nature is suggested by the ordinary action of man upon earth. I therefore urge no impossibilities, though 1 am constantly charged with doing so. I do not even urge inconsistency, but, on the contrary, frankly admit that the theologian has as good a right to place his conception at the root of phenomena as I have to place mine.

But without verification a theoretic conception is a mere figment of the intellect, and I am sorry to find us parting company at this point. 'The region of theory, both in science and theology, lies behind the world of the senses, but the verification of theory occurs in the sensible world. To check the theory we have simply to compare the deductions from it with the facts of observation. If the deductions be in accordance with the facts, we accept the theory: if in opposition, the theory is given up. A single experiment is frequently devised, by which the theory must stand or fall. Of this character was the determination of the velocity of light in liquids, as a crucial test of the Emission Theory. According to it, light traveled faster in water than in air; according to the Undulatory 'Theory, it traveled faster in air than in water. An experiment suggested by Arago, and executed by Fizeau and Foucault was conclusive against Newton's theory.

But while science cheerfully submits to this ordeal, it seems impossible to devise a mode of verification of their theories which does not rouse resentment in theological minds. Is it that, while the pleasure of the scientific man culminates in the demonstrated harmony between theory and fact, the highest pleasure of the religious man has been already tasted in the very act of praying, prior to verification, any further effort in this direction being a mere disturbance of his peace? Or is it that we have before us a residue of that mysticism of the middle ages, so almirably described by Whewell-that "practice of referring things and events not to clear and distinct notions, not to general rules capable of direct verification, but to 
notions vague, distant, and vast, which we cannot bring into contact with facts; as when we connect natural events with moral and historic causes." "Thus," he continues, "the character of mysticism is that it refers particulars, not to generalizations, homogeneous and immediate, but to such as are heterogeneous and remote; to which we must add, that the process of this reference is not a calm act of the intellect, but is accompanied with a glow of enthusiastic feeling."

Every feature here depicted, and some more questionable ones, have shown themselves of late; most conspicnously, I regret to say, in the "leaders" of a weekly journal of considerable influence, and one, on many grounds, entitled to the respect of thoughtful men. In the correspondence, however, published by the same journal, are to be found two or three letters well calculated to correct the temporary flightiness of the journal itself.

It is not my habit of mind to think otherwise than solemnly of the feeling which prompts prayer. It is a power which I should like to see guided, not extinguished - levoted to practicable objects instead of wasted upon air. In some form or other, not yet evident, it may, as alleged, be necessary to man's highest culture. Certain it is that, while I rank many persons who resort to prayer low in the scale of being-natural foolishness, bigotry, and intolerance being in their case intensified by the notion that they have access to the ear of God-I regard others who employ it, as forming part of the very cream of the earth. The faith that adds to the folly and ferocity of the one is turned to enduring sweetness, holiness, abounding charity, and self-sacrifice by the other. Religion, in fact, varies with the nature upon which it falls. Often unreasonable, if not contemptible, prayer, in its purer forms, hints at disciplines which few of us can neglect without moral loss. But no good can come of giving it a delusive value, by claiming for it a power in physical nature. It may strengthen the heart to meet life's losses, and thus indirectly promote physical well-being, as the digging of Asop's orchard brought a treasure of fertility greater than the golden treasure sought. Such indirect issues we all arlmit; but it would be simply dishonest to affirm that it is such issues that are always in view. Here, for the present, I must end. I ask no space to reply to those railer's who 
make such free use of the terms insolence, outrage, pro. fanity, and blasphemy. They obviously lack the sobriety of mind necessary to give accuracy to their statements, or to render their charges worthy of serious refutation.

\section{CHAPTER XXVI.}

\section{VITALITY.}

THE ORIGIN, growth, and energies of living things are subjects which have always engaged the attention of thinking men. To account for them it was usual to assume a special agent, free to a great extent from the limitations observed among the powers of inorganic nature. This agent was called vital force; and, under its influence, plants and animals were supposed to collect their materials and to assume determinate forms. Within the last few years, however, our ideas of vital processes have undergone profound modifications; and the interest, and even disquietude, which the change has excited are amply evidenced by the discussions and protests which are now common, regarding the phenomena of vitality. In tracing these phenomena through all their modifications, the most advanced philosophers of the present day declare that they ultimately arrive at a single source of power, from which all vital energy is derived; and the disquieting circumstance is that this source is not the direct fiat of a supernatural agent, but a reservoir of what, if we do not accept the creed of Zoroaster, must be regarded as inorganic force. In short, it is considered as proved that all the energy which we derive from plants and animals is drawn from the sun.

A few years ago, when the sun was affirmed to be the source of life, nine ont of ten of those who are alarmed by the form which this assertion has latterly assumed would have assented, in a general way, to its correctness. Their assent, however, was more poetic than scientific, and they were by no means prepared to see a rigid mechanical signification attached to their words. This, however, is the peculiarity of modern conclusions-that there is no creative energy whatever in the vegetable or animal organism, but that all the power which we obtain from the 
muscles of man and animals, as much as that which we develop by the combustion of coal or wood, has been produced at the sun's expense. The sun is so much the colder that we may have our fires; he is also so much the colder that we may have our horse-racing and Alpine climbing. It is, for example, certain that the sun has been chilled to an extent capable of being accurately expressed in numbers, in order to furnish the power which lifted this year a certain number of tourists from the vale of Chamouni to the summit of Mont Blanc.

'To most minds, however, the energy of light and heat presents itself as a thing totally distinct from ordinary mechanical energy. Either of them can nevertheless be derived from the other. Wood can be raised by friction to the temperature of ignition; while by properly striking a piece of iron a skillful blacksmith can cause it to glow. 'T'hus, by the rude agency of his hammer, he generates light and heat. 'This action, if carried far' enough, would produce the light and heat of the sun. In fact, the sun's light and heat have actually been referred to the fall of meteoric matter upon his surface; and whether the sun is thus supported or not, it is perfectly certain that he might be thus supportel. Whether, moreover, the whilom molten condition of our planet was, as supposed by eminent men, due to the collision of cosmic masses or not, it is perfectly certain that the molten condition might be thus brought about. If, then, solar light and heat can be produced by the impact of dead matter, and if from the light and heat thus produced we can derive the energies which we have been accustomed to call vital, it indubitably follows that vital energy may have a proximately mechanical origin.

In what sense, then, is the sun to be regarded as the origin of the energy derivable from plants and animals? Let us try to give an intelligible answer to this question. Water may be raised from the sea-level to a high elevation, and then permitted to descend. In descending it may be made to assume various forms - to fall in cascades, to spurt in fountains, to boil in eddies, or to flow tranquilly along a uniform bed. It may, moreover, be caused to set complex machinery in motion, to turu millstones, throw shuttles, work saws and hammers, and drive piles. But every form of power here indicated would be derived from the original power expended in raising the water to the height 
from which it fell. There is no energy generated by the machinery: the work performed by the water in descending is merely the parceling out and distribution of the work expended in raising it. In precisely this sense is all the energy of plants and animals the parceling out and distribution of a power originally exerted by the sun. In the case of the water, the source of the power consists in the forcible separation of a quantity of the liquid from a low level of the earth's surface, and its elevation to a higher position, the power thus expended being returned by the water in its descent. In the case of vital phenomena, the source of power consists in the forcible separation of the atoms of compound substances by the sun. We name the force which draws the water earthward " gravity," and that which draws atoms together " chemical affinity;" but these different names must not mislead us regarding the qualitative identity of the two forces. 'They are both attractions; and, to the intellect, the falling of carbon atoms against oxygen atoms is not more difficult of conception than the falling of water to the earth.

The building up of the vegetable, then, is effected by the sun, through the reduction of chemical compounds. The phenomena of animal life are more or less complicated reversals of these processes of reduction. We eat the vegetable, and we breathe the oxygen of the air; and in our bodies the oxygen, which had been lifted from the carbon and hydrogen by the action of the sun, again falls toward them, producing animal heat and developing animal forms. Through the most complicated phenomena of vitality this law runs: the vegetable is produced while a weight rises, the animal is produced while a weight falls. But the question is not exhausted here. The water employed in our first illustration generates all the motion displayed in its descent, but the form of the motion depends on the character of the machinery interposed in the path of the water. In a similar way, the primary action of the sun's rays is qualified by the atoms and molecules among which their energy is distributed. Molecular forces determine the form which the solar energy will assume. In the separation of the earbon and oxygen this energy may be so conditioned as to result in one case in the formation of a cabbage, and in another case in the formation of an oak. So also, as regards the reunion of the carbon and the oxygen, the 
molecular machinery through which the combining energy acts may, in one case, weave the texture of a frog, while in another it may weave the texture of a man.

'The matter of the animal body is that of inorganic nature. 'I'here is no sulstance in the animal tissues which is not primarily derived from the rocks, the water, and the air. Are the forces of organic matter, then, different in kind from those of inorganic matter? The philosophy of the present day negatives the question. It is the compounding, in the orgauic world, of forces belonging equally to the inorganic, that constitutes the mystery and the miracle of vitality. Every portion of every animal body may be reduced to purely inorganic matter. A perfect reversal of this process of reduction would carry us from the inorganic to the organic; and such a reversal is at least conceivable. The tendency, indeed, of modern science is to break down the wall of partition between organic and inorganic, and to reduce both to the operation of forces which are the same in kind, but which are differently compounded.

Consider the question of personal identity, in relation to that of molecular form. Thirty-four years ago, Mayer of Heilbronn, with that power of genius which breathes large meanings into scanty facts, pointed out that the blood was "the oil of the lamp of life," the combustion of which sustains muscular action. The muscles are the machinery by which the dynamic power of the blood is brought into play. 'Thus the blood is consumed. But the whole body, though more slowly than the blood, wastes also, so that after a certain number of years it is entirely renewed. How is the sense of personal identity maintained across this flight of molecules? 'To man, as we know him, matter is necessary to consciousness; but the matter of any period may be all changed, while consciousness exhibits no solution of continuity. Like changing sentinels, the oxygen, hydrogen, and carbon that depart, seem to whisper their secret to their comrades that arrive, and thus, while the Non-ego shifts, the Ego remains the same. Constancy of form in the grouping of the molecules, and not constancy of the molecules themselves, is the correlative of this constancy of perception. Life is a wave which in no two consecutive moments of its existence is composed of the same particles.

Supposing, then the molecules of the human body, 
instead of replacing others, and thus renewing a pre-existing form, to be gathered first hand from nature and put together in the same relative positions as those which they occupy in the body. Supposing them to have the selfsame forces and distribution of forces, the selfsame motions and distribution of motions-would this organized concourse of molecules stand before us as a sentient thinking being? 'There seems no valid reason to believe that it would not. Or, supposing a planet carved from the sun, set spinning round an axis, and revolving round the sun at a distance from him equal to that of our earth, would one of the consequences of its refrigeration be the development of organic forms? I lean to the affirmative. Structural forces are certainly in the mass, whether or not those forces reach to the extent of forming a plant or an animal. In an amorphous drop of water lie latent all the marvels of crystalline force; and who will set limits to the possible play of molecules in a cooling planet? If these statements startle, it is because matter has been defined and maligned by philosophers and theologians, who were equally unaware that it is, at bottom, essentially mystical and trauscendental.

Questions such as these derive their present interest in great part from their audacity, which is sure, in due time, to disappear. And the sooner the public dread is abolished with reference to such questions the better for the cause of truth. As regards knowledge, physical science is polar. In one sense it knows, or is destined to know, everything. In another sense it knows nothing. Science understands much of this intermediate phase of things that we call nature, of which it is the product; but science knows nothing of the origin or destiny of nature. Who or what made the sun, and gave his rays their alleged power? Who or what male and bestowed upon the ultimate particles of matter their wondrous power of varied interaction? Science does not know: the mystery, though pushed back remains unaltered. To many of us who feel that there are more things in heaven and earth than are dreamed of in the present philosophy of science, but who have been also taught, by baffled efforts, how vain is the attempt to grapple with the Inscrutable, the ultimate frame of mind is that of Goethe: 
Who dares to name His name,

Or belief in Him proclaim,

Veiled in mystery as He is, the All-enfolder?

Gleams across the mind His light,

Feels the lifted soul His might,

Dare it then deny His reign, the All-upholder?

\section{CHAPTER XXVII.}

\section{MATTER AND FORCE.*}

As I rode through the Schwarzwald, I said to myself: That little fire which glows star-like across the dark-growing moor, where the sooty smith bends over his anvil, and thou hopest to replace thy lost horseshoe-is it a detached, separated speck, cut off from the whole Universe; or indissolubly joined to the whole? Thou fool, tha smithy fire was primarily kindled at the Sun; is fed by air that circulates from before Noah's Deluge, from beyond the Dogstar; therein, with Iron Force, and Coal Force, and the far stranger Force of Man, are cunning affinities and battles and victories. of Force brought about; it is a little ganglion, or nervous center, in the great vital system of Immensity. Call it, if thou wilt, an unconscious Altar, kindled on the bosom of the All.... Detached, separated! I say there is no such separation: nothing hitberto was ever stranded, cast aside; but all, were it only a withered leaf, works together with all; is borne forward on the bottomless, shoreless flood of action, and lives through perpetual metamorphoses.-CARLYLE.

IT Is the custom of the professors in the Royal School of Mines in London to give courses of evening lectures every year to workingmen. The lecture-room holds 600 people; and tickets to this amount are disposed of as quickly as they can be handed to those who apply for them. So desirons are the workingmen of London to attend these lectures, that the persons who fail to obtain tickets always bear a large proportion to those who succeed. Indeed, if the lecture-room could hold 2,000 instead of 600, I do not doubt that every one of its benches would be occupied on these occasions. It is, moreover, worthy of remark that the lectures are but rarely of a character which conld help the workingman in his daily pursuits. The information acquired is hardly ever of a nature which admits of being turned into money. It is, therefore, a pure desire for

* A Lecture delivered to the workingmen of Dundee, September 5,1867 , with additions. 
knowledge, as a thing good in itself, and without regard to its practical application, which animates the hearers of these lectures.

It is also my privilege to lecture to another audience in London, composed in part of the aristocracy of rank, while the audience just referred to is composed wholly of the aristocracy of labor. As regards attention and courtesy to the lecturer, neither of these audiences has anything to learn of the other; neither can claim superiority over the other. It would not, perhaps, be quite correct to take those persons who flock to the School of Mines as average samples of their class; they are probably picked menthe aristocracy of labor, as I have just called them. At all events, their conduct demonstrates that the essential qualities of what we in England understand by a gentleman are confined to no class; and they have of ten raised in iny mind the wish that the gentlemen of all classes, artisans as well as lords, could, by some process of selection, be sifted from the general mass of the community, and caused to know each other better.

When pressed some months ago by the Council of the British Association to give an evening lecture to the workingmen of Dundee, nyy experience of the workingmen of London naturally rose to my mind; and, though heavily weighted with other duties, I could not bring myself to decline the request of the Council. Hitherto, the evening discourses of the Association have been delivered before its members and associates alone. But after the meeting at Nottingham, last year, where the workingmen, at their own request, were addressed by our late president, Mr. Grove, and by my excellent friend, Professor Huxley, the idea arose of incorporating with all subsequent meetings of the Association an audress to the workingmen of the town in which the meeting is held. A resolution to that effect was sent to the Committee of Recommendations; the Committee supported the resolution; the Council of the Association ratified the decision of the Committee; and here I am to carry out to the best of my ability their united wishes.

Whether it be a consequence of long-continued development, or an endowment conferred once for all on man at his creation, we find him here gifted with a mind curious 
to know the causes of things, and surrounded by objects which excite its questionings, and raise the desire for an explanation. It is related of a young prince of one of the Pacific islands, that when he first saw himself in a lookingglass, he ran round the glass to see who was standing at the back. And thus it is with the general human intellect, as regards the phenomena of the external world. It wishes to get behind and learn the causes and connections of these phenomena. What is the sun, what is the earth, what should we see if we came to the edge of the earth and lookel over? What is the meaning of thunder and lightning, of hail, rain, storm, and snow? Such questions presented themselves to early men, and by and by it was discovered that this desire for knowledge was not implanted in vain. After many trials it became evident that man's capacities were, so to speak, the complement of nature's facts, and that, within certain limits, the secret of the universe was open to the human understanding. It was found that the mind of man had the power of penetrating far beyond the boundaries of his fivesenses; that the things which are seen in the material world depend for their action upon things unseen; in short, that besides the phenomena which address the senses, there are laws and principles and processes which do not address the senses at all, but which must be, and can be, spiritually discerned.

'I'o the subjects which require this discernment belong the phenomena of molecular force. But to trace the genesis of the notions now entertained upon this subject, we have to go a long way back. In the drawing of a bow, the darting of a javelin, the throwing of a stone-in the lifting of burdens, and in personal combats, even savage man became acquainted with the operation of force. Ages of discipline, moreover, taught him foresight. He laid by at the proper season stores of food, thus obtaining time to look about him, and to become an observer and inquirer. 'I'wo things which he noticed must have profoundly stirred his curiosity. He found that a kind of resin dropped from a certain tree possessed, when rubbed, the power of drawing light bodies to itself, and of causing them to cling to it; and he had also found that a particular stone exerted a similar power over a particular kind of metal. I allude, of course, to electrified amber, and to the loadstone, or natural magnet, and its power to attract particles of iron. Pre- 
vious experience of his own muscles had enabled our early inquirer to distinguish between a push and a pull. Augmented experience showed him that in the case of the magnet and the amber pulls and pushes-attractions and repulsions-were also exerted; and, by a kind of poetic transfer, he applied to things external to himself, conceptions derived from himself. The magnet and the rubbed amber were credited with pushing and pulling,or, in other words, with exerting force.

In the time of the great Lord Bacon the margin of these pushes and pulls was vastly extended by Dr. Gilbert, a man probably of firmer scientific fiber, and of finer insight, than Bacon himself. Gilbert proved that a multitude of other bodies, when rubbed, exerted the power which, thousands of years previonsly, had been observed in amber. In this way the notion of attraction and repulsion in external nature was rendered familiar. It was a matter of experience that bodies, between which no visible link or connection existed, possessed the power of acting upon each other; and the action came to be technically called "action at a distance."

But out of experience in science there grows something finer than mere experience. Experience furnishes the soil for plants of higher growth; and this observation of action at a distance provided material for speculation upon the largest of problems. Bodies were observed to fall to the earth. Why should they do so? The earth was proved to revolve round the sun; and the moon to revolve round the earth. Why should they do so? What prevents them from flying straight off into space? Supposing it were ascertained that from a part of the earth's rocky crust a firmly fixed and tightly stretched chain started toward the sun, we mighi be inclined to conclude that the earth is held in its orbit by the chain-that the sun twirls the earth around him, as a boy twirls round his head a bullet at the end of a string. But why should the chain be needed? It is a fact of experience that bodies can attract each other at a distance, without the intervention of any chain. Why should not the sun and earth so attract each other? and why should not the fall of bodies from a height be the result of their attraction by the earth? Here then we reach one of those higher speculations which grow out of the fruitful soil of observation. Having started with 
the savage, and his sensations of muscular force, we pass on to the observation of force exerted between a magnet and rubbed amber and the bodies which they attract, rising, by an unbroken growth of ideas, to a conception of the force by which sun and planets are held together.

'This idea of attraction between sun and planets had become familiar in the time of Newton. He set himself to examine the attraction: and here, as elsewhere, we find the speculative mind falling back for its materials upon experience. It had been observed, in the case of magnetic and electric bodies, that the nearer they were brought together the stronger was the force exerted between them; while, by increasing the distance, the force diminished until it became insensible. Hence the inference that the assumed pull between the earth and the sun would be influenced by their distance asunder. Guesses had been made as to the exact manner in which the force varied with the distance; but Newton supplemented the guess by the severe test of experiment and calculation. Comparing the pull of the earth upon a body close to its surface, with its pull upon the moon, 240,000 miles away, Newton rigidly established the law of variation with the distance. But on his way to this result Newton found room for other conceptions, some of which indeed, constituted the necessary stepping-stones to his result. The one which here concerns us is, that not only does the sun attract the earth, and the earth attract the sun as wholes, but every particle of the sun attracts every particle of the earth, and the reverse. His conclusion was, that the attraction of the masses was simply the sum of the attractions of their constituent particles.

This result seems so obvious that you will perhaps wonder at my dwelling upon it; but it really marks a turning point in our notions of force. You have probably heard of certain philosophers of the ancient world named Democritus, Epicurus, and Lucretius. These men adopted, developed, and diffused the doctrine of atoms and molecules, which found its consummation at the hands of the illustrious John Dalton. But the Greek and Roman philosophers I have named, and their followers, up to the time of Newton, pictured their atoms as falling and flying through space, hitting each other, and clinging together by imaginary hooks and claws. 'They inissed the centra' 
idea that atoms and molecules could come together, not by being fortuitously knocked against each other, but by their own mutual attractions. This is one of the great steps taken by Newton. He familiarized the world with the conception of molecular force.

Newton, you know, was preceded by a grand fellow named John Kepler-a true workingman-who, by analyzing the astronomical observations of his master, Tycho Brahe, had actually found that the planets moved as they are now known to move. Kepler knew as much about the motion of the planets as Newton did; in fact, Kepler taught Newton and the world generally the facts of planetary motion. But this was not enough. The question arose-Why should the facts be so? This was the great question for Newton, and it was the solution of it which renders his name and fame immortal. Starting from the principle that every particle of matter in the solar system attracts every other particle by a force which varies as the inver'se square of the distance between the particles, he proved that the planetary motions must be what observation makes them to be. He showed that the moon fell toward the earth, and that the planets fell toward the sun, through the operation of the same force that pulls an apple from its tree. This all-pervading force, which forms the solder of the material universe, and the conception of which was necessary to Newton's intellectual peace, is called the force of gravitation.

Graritation is a purely attractive force, but in electricity and magnetism, repulsion had been always seen to accompany attraction. Electricity and magnetism are double or polar forces. In the case of magnetism, experience soon pushed the mind beyond the bounds of experience, compelling it to conclude that the polarity of the magnet was resident in its molecules. I hold a magnetized strip of steel by its center, and find that one half of the strip attracts, and the other half repels, the north end of a magnetic needle. I break the strip in the middle, find that this half, which a moment ago attracted throughout its entire length the north pole of a magnetic needle, is now divided into two new halves, one of which wholly attracts, and the other of which wholly repels, the north pole of the needle. The half proves to be as perfect a magnet as the whole. You may break this half and go on till further 
breaking becomes impossible through the very smallness of the fragments; the smallest fragment is found endowed with two poles, and is, therefore, a perfect magnet. But you cannot stop here: you imagine where you cannot $e x$ periment; and reach the conclusion entertained by all scientific men, that the magnet which you see and feel is an assemblage of molecular-magnets which you cannot see and feel, but which, as before stated, must be intellectually discerned.

Magnetism then is a polar force; and experience hints that a force of this kind may exert a certain structural power. It is known, for example, that iron filings strewn round a magnet arrange themselves in definite lines, called, by some, " magnetic curves," and, by others "lines of magnetic force." Over two magnets now before me is spread a sheet of paper. Scattering iron filings over the paper, polar force comes into play, and every particle of the iron responds to that force. We have a kind of architectural effort-if I may use the term-exerted on the part of the iron filings. Here then is a fact of experience which, as you will see immediately, furnishes further material for the mind to operate upon, rendering it possible to attain intellectual clearness and repose, while speculating upon apparently remote phenomena.

The magnetic force has here acted upon particles visible to the eye. But, as already stated, there are numerous processes in nature which entirely elude the eye of the body, and must be figured by the eye of the mind. The processes of chemistry are examples of these. Long thinking and experimenting has led philosophers to conclude that matter is composed of atoms from which, whether separate or in combination, the whole material world is built up. The air we breathe, for example, is mainly a mechanical mixture of the atoms of oxygen and nitrogen. The water we drink is also composed of oxygen and hydrogen. But it differs from the air in this particular, that in water the oxygen and hydrogen are not mechanically mixed, but chemically combined. The atoms of oxygen and those of hydrogen exert enormous attractions on each other, so that when brought into sufficient proximity they rush together with an almost incredible force to form a chemical compound. But powerful as is the force with which these atoms lock themselves together, we have the 
means of tearing them asunder, and the agent by which we accomplish this may here receive a few moments? attention.

Into a vessel containing acidulated water I dip two strips of metal, the one being zinc and the other platinum, not permitting them to touch each other in the liquid. I connect the two upper ends of the strips by a piece of copper wire. 'I'he wire is now the channel of what, for want of a better name, we call an "electric current." What the inner change of the wire is we do not know, but we do kuow that a change has occurred, by the external effects produced by the wire. Let me show you one or two of these effects. Before you is a series of ten vessels, each with its pair of metals, and I wish to get the added force of all ten. The arrangement is culled a voltaic battery. I plunge a piece of copper wire among these iron filings; they refuse to cling to it. I employ the selfsame wire to connect the two ends of the battery, and subject it to the same test. The iron filings now crowd round the wire and cling to it. I interrupt the current, and the filings immediately fall; the power of attraction continues only so long as the wire connects the two ends of the battery.

Here is a piece of similar wire, overspun with cotton, to prevent the contact of its various parts, and formed into a coil. I make tlie coil part of the wire which connects the two ends of the voltaic battery. By the attractive force with which it has become suddenly endowed, it now empties this tool-box of its iron nails. I twist a covered copper wire round this common poker; connecting the wire with the two ends of the voltaic battery, the poker is instantly transformed into a strong magnet. 'T'wo flat spirals here are suspended facing each other, about six inches apart. Sending a current through both spirals, they clash sudilenly together; reversing what is called the direction of the current in one of the spirals, they fly asunder. All these effects are due to the power which we name an electric current, and which we figure as flowing through the wire when the voltaic circuit is complete.

By the same agent we tear asunder the locked atoms of a chemical compound. Into this small cell, containing water, dip two thin wires. A magnified image of the cell is thrown upon the screen before you, and you see plainly the images of the wires. From a small battery I send an 
electric current from wire to wire. Bubbles of gas rise immediately from each of them, and these are the two gases of which the water is composed. 'The oxygen is always liberatenl on the one wire, the hydrogen on the other. 'The gases may be collected either separately or mixed. I place upon my hand a soap bubble filled with the mixture of both gases. Applying a taper to the bubble, a lond explosion is heard. The atoms have rushed together with detonation, and without injury to my hand, and the water from which they were extracted is the result of their reunion.

One consequence of the rushing together of the atoms is the development of heat. What is this heat? Here are two ivory balls suspended from the same point of support by two short strings. I draw them thus apart and then liberate them. They clash together, but, by virtue of their elasticity, they quickly recoil, and a sharp vibratory rattle succeeds their collision. This experiment will enable you to figure to your mind a pair of clashing atoms. We have in the first place, a motion of the one atom toward the other-a motion of translation, as it is usually calledthen a recoil, and afterward a motion of vibration. To this vibratory motion we give the name of heat. Thus, three things are to be kept before the mind-first, the atoms themselves; secondly, the force with which they attract each other; and thirdly, the motion consequent upon the exertion of that force. This motion must be tigured first as a motion of translation, and then as a motion of vibration, to which latter we give the name of heat. For some time after the act of combination this motion is so violent as to prevent the molecules from coming together, the water being maintained in a state of vapor. But as the vapor cools, or in other words loses its motion, the molecules coalesce to form a liquid.

And now we approach a new and wonderful display of force. As long as the substance remains in a liquid or vaporous condition, the play of this force is altogether masked and hidden. But as the heat is gradually withdrawn, the molecules prepare for new arrangements and combinations. Solid crystals of water are at length formed, to which we give the familiar name of ice. Looking at these beautiful edifices and their internal structure, the 
pondering mind has forced upon it the question, How are they built up? We have obtained clear conceptions of polar force; and we infer from our broken magnet that polar force may be resident in the molecules or smallest particles of matter, and that by the play of this force structural arrangement is possible. What, in relation to our present question, is the natural action of a mind furnished with this knowledge? It is compelled to transcend experience, and endow the atoms and molecules of which crystals are built with definite poles whence issue attractions and repulsions. In virtue of these forces some poles are drawn together, while some retreat from each other; atom is added to atom, and molecule to molecule, not boisterously or fortuitously, but silently and symmetrically, and in accordance with laws more rigid than those which gnide a human builder when he places his materials together. Imagine the bricks and stones of this town of Dundee endowed with structural power. Imagine them attracting and repelling, and arranging themselves into streets and houses and Kinnaird Halls-would not that be wonderful? Hardly less wonderful is the play of force by which the molecules of water build themselves into the sheets of ice which every winter roof your ponds and lakes.

If I could show you the actual progress of this molecular architecture, its beanty would delight and astonish you. A reversal of the process of crystallization may be actually shown. The molecules of a piece of ice may be taken asunder before your eyes; and from the manner in which they separate you may to some extent infer the manner in which they go together. When a beam is sent from our electric lamp through a plate of glass, a portion of the beam is intercepted and the glass is warmerl by the portion thus retained within it. When the beam is sent throngh a plate of ice, a portion of the beam is also absorber; but instead of warming the ice, the intercepted heat melts it internally. It is to the delicate, silent action of this beam within the ice that I now wish to direct your attention. Upon the screen is thrown a magnified image of the slab of ice: the light of the beam passes freely through the ice without melting it, and enables us to form the image; but the heat is in great part intercepted, and that heat now applies itself to the work of internal liquefaction. Select- 
ing certain points for attack, round about those points the beam works silently, undoing the crystalline architecture, and reducing to the freedom of liquidity molecules which had been previously locked in a solid embrace. 'The liquefied spaces are rendered visible by strong illumination. Observe those six-petaled flowers breaking out over the white surface, and expanding in size as the action of the beam continues. 'These flowers are liquefieil ice. Under' the action of the heat the molecules of the crystals fall asunder, so as to leave behind them these exquisite forms, We have here a process of demolition which clearly reveals the reverse process of construction. In this fashion, and in strict accordance with this hexangular type, every ice molecule takes its place upon our ponds and lakes during the frosts of winter. 'To use the language of an American poet, "the atoms march in tune," moving to the music of law, which thus renders the commonest substance in nature a miracle of beauty.

It is the function of science, not as some think tu divest this universe of its wonder and mystery, but, as in the case before us, to point out the wonder and the mystery of common things. Those fern-like forms, which on a frosty morning overspread your window paues, illustrate the action of the same force. Breathe upon such a pane before the fires are lighted, and reduce the solid crystalline film to the liquid condition; then watch its sabsequent resolidification. You will see it all the better if you look at it through a common magnifying glass. After you have ceased breathing, the film, abandoned to the action of its own forces, appears for a moment to be alive. Lines of motion run through it; molecule closes with molecule, until finally the whole film passes from the state of liquidity, through this state of motion, to its final crystalline repose.

I can show you something similar. Over a piece of perfectly clean glass I pour a little water in which certain crystals have been dissolved. A film of the solution clings to the glass. By means of a microscope and a lamp, an image of the plate of glass is thrown upon the screen. The beam of the limp, besiles illuminating the glass, also heats it; evaporation sets in, and at a certain moment, when the solution has become supersaturated, splendid branches of crystal shoot out over the screen. A dozen square feet 
of surface are now covered by those beautiful forms. With another solution we obtain crystalline spears, feathered right and left by other spears. From distant nuclei in the middle of the field of view the spears shoot with magical rapidity in all directions. The film of water on a window pane on a frosty morning exhibits effects quite as wonderful as these. Latent in these formless solutions, latent in every drop of water, lies this marvelous structural power, which only requires the withdrawal of opposing forces to bring it into action.

'The clear liquid now held up before you is a solution of nitrate of silver-a compound of silver and nitric acid. When an electric current is sent through this liquid the silver is severed from the acid, as the hydrogen was separated from the oxygen in a former experiment; and I would ask you to observe how the metal behaves when its molecules are thus successively set free. The image of the cell, and of the two wires which dip into the liquid of the cell, are now clearly shown upon the screen. Let us close the circuit, and send the current through the liquid. From one of the wires a beantiful silver tree commences immediately to sprout. Branches of the metal are thrown out, and umbrageous foliage loads the branches. You have here a growth, apparently as wonderful as that of any vegetable, perfected in a minute before your eyes. Substituting for the nitrate of silver acetate of lead, which is a compound of lead and acetic acid, the electric current severs the lead from the acid, and you see the metal slowly branching into exquisite metallic ferms, the fronds of which, as they become too heavy, break from their roots and fall to the bottom of the cell.

These experiments show that the common matter of our earth - " brute matter," as Dr. Young, in his " Night Thoughts," is pleased to call it-when its atoms and molecules are permitted to bring their forces into free play, arranges itself, under the operation of these forces, into forms which rival in beauty those of the vegetable world. And what is the vegetable world itself, but the result of the complex play of these molecular forces? Here, as elsewhere throughout nature, if matter moves it is force that moves it, and if a certain structure, vegetable or mineral, is produced, it is througb the operation of the forces exerted between the atoms and molecules. 
The solid matter of which our lead and silver trees were formed was, in the first instance, disguised in a transparent liquid; the solid matter of which our woods and forests are composed is also, for the most part, disguised in a transparent gas, which is mixed in small quantities with the air of our atmosphere. This gas is formed by the union of carbon and oxygen, and is called carbonic acid gas. The carbonic acid of the air being subjected to an action somewhat analogous to that of the electric current in the case of our lead and silver solutions, has its carbon liberated and deposited as wooly fiber. The watery vapor of the air is subjected to similar action; its hydrogen is liberated from its oxygen, and lies down side by side with the carbon in the tissues of the tree. The oxygen in both cases is permitted to wander away into the atmosphere. But what is it in nature that plays the part of the electric current in our experiments, tearing asunder the locked atoms of carbon, oxygen, and hydrogen? The rays of the sun. The leaves of plants which absorb both the carbonic acid and the aqueous vapor of the air, answer to the cells in which our decompositions took place. And just as the molecular attractions of the silver and the lead found expression in those beautiful branching forms seen in our experiments, so do the molecular attractions of the liberated carbon and hydrogen find expression in the architecture of grasses, plants, and trees.

In the fall of a cataract and the rush of the wind we have examples of mechanical power. In the combinations of chemistry and in the formation of crystals and vegetables we have examples of molecular power. You have learned how the atoms of oxygen and hydrogen rush together to form water. I have not thought it necessary to dwell upon the mighty mechanical energy of their act of combination; but it may be said, in passing, that the clashing together of $1 \mathrm{lb}$. of hydrogen and $8 \mathrm{lbs}$. of oxygen to form $9 \mathrm{lbs}$. of aqueous vapor, is greater than the shock of a weight of 1,000 tons falling from a height of 20 feet against the earth. Now, in order that the atoms of oxygen and hydrogen should rise by their mutual attractions to the velocity corresponding to this enormous mechanical effect, a certain distance must exist, between the particles. It is in rushing over this that the velocity is attained. 
This idea of distance between the attracting atoms is of the highest importance in our conception of the system of the world. For the matter of the world may be classified under two distinct heads: atoms and molecules which have already combined and thus satisfied their mutual attractions, and atoms and molecules which have not yet combined, and whose mutual attractions are, therefore, unsatisfied. Now, as regards motive power, we are entirely dependent on atoms and molecules of the latter kind. Their attractions can produce motion, because sufficient distance intervenes between the attracting atoms, and it is this atomic motion that we utilize in oul machines. 'Thus we can get power out of oxygen and hydrogen by the act of their union; but once they are combined, and once the vibratory motion consequent on their combinations has been expended, no further power can be got out of their mutual attraction. As dynamic agents they are dead. The materials of the earth's crust consist for the most part of substances whose atoms have already closed in chemical union-whose mutual attractions are satisfied. Granite, for instance, is a widely diffused substance; but granite consists, in great part, of silicon, oxygen, potassium, calcium, and aluminum, whose atoms united long ago, and are therefore dead. Limestone is composed of carbon, oxygen, and a metal called calcium, the atums of which have already closed in chemical union, and are therefore finaliy at rest. In this way we might go over nearly the whole of the materials of the earth's crust, and satisfy ourselves that though they were sources of power in ages past, and long before any creature appeared on the earth capable of turning their power to account, they are sources of power no longer. And here we might halt for a moment to remark on that tendency, so prevaler, in the world, $t_{1}$ regard everything as made for human use. Those who entertain this notion, hold, I think, an overweening opinion of their own importance in the system of uature. Flowers bloomed before men saw them, and the quantity of power wasted before man could utilize it is all but infinite compared with what now remains. We are truly heirs of all the ages; but as honest men it behooves us to learn the extent of our inheritance, and as brave ones not to whimper if it should prove less than we had supposed. The healthy attitude of mind with reference to this sub- 
ject is that of the poet, who, when asked whence came the rhodori, joyfully acknowledged his brotherhood with the flower:

Why thou wert there, $O$ rival of the rose!

I never thought to ask, I never knew,

But in my simple ignorance supposed

The selfsame power that brought me there brought you.*

A few exceptions to the general state of union of the molecules of the earth's crust-vast in relation to us, but trivial in comparison to the total store of which they are the residue-still remain. They constitute our main sources of motive power. By far the most important of these are our beds of coal. Distance still intervenes between the atoms of carbon and those of atmospheric oxygen, across which the atoms may be urged by their mutual attractions: and we can utilize the motion thus produced. Once the carbon and the oxygen have rushed together, so as to form car'bonic acid, their mutual attractions are satisfied; and, while they continue in this condition, as dynamic agents they are dead. Our woods and forests are also sources of mechanical energy, because they have the power of uniting with the atmospheric oxygen. Passing from plants to animals, we find that the source of motive power just referred to is also the source of muscular power. A horse call perform work, and so can a man; but this work is at bottom the molecular work of the transmuted food and the oxygen of the air. We inhale this vital gas, and bring it into sufficiently close proximity with the carbon and the hydrogen of the body. These unite in obedience to their mutual attractions; and their motion toward each other, properly turned to account by the wonderful mechanism of the body, becomes muscular motion.

One fundamental thought pervades all these statements: there is one tap root from which they all spring. 'This is the ancient maxim that out of nothing nothing comes; that neither in the organic world nor in the inorganic is power produced without the expenditure of power; that neither in the plant nor in the animal is there a creation of force or motion. 'Trees grow, and so do men and horses; and here we have new power incessantly introduced upon the eartl. But its source, as I have already stated, is the 
sun. It is the sun that separates the carbon from the oxygen of the carbonic acid, and thus enables them to recombine. Whether they recombine in the furnace of the steam-engine or in the animal body, the origin of the power they produce is the same. In this sense we are all "souls of fire and children of the sun." But, as remarked by Helmholtz, we must be content to share our celestial pedigree with the meanest of living things.

Some estimable persons, here present, very possibly shrink from accepting these statements; they may be frightened by their apparent tendency toward what is called materialism-a word which, to many minds expresses something very dreadful. But it ought to be known and avowed that the physical philosopher, as such, must be a pure materialist. His inquiries deal with matter and force, and with them alone. And whatever be the forms which matter and force assume, whether in the organic world or the inorganic, whether in the coal-beds and forests of the earth, or in the brains and muscles of men, the physical philosopher will make good his right to investigate them. It is perfectly vain to attempt to stop inquiry in this direction. Depend upon it, if a chemist by bringing the proper materials together, in a retort or crucible, could make a baby, he would do it. There is no law, moral or physical, forbidding him to do it. At the present moment there are, no doubt, persons experimenting on the possibility of producing what we call life out of inorganic materials. Let them pursue their studies in peace; it is only by such trials that they will learn the limits of their own powers and the operation of the laws of matter and force.

But while thus making the largest demand for freedom of investigation-while I consider science to be alike powerful as an instrument of intellectual culture and as a ministrant to the material wants of men; if you ask me whether it has solved, or is likely in our day to solve, the problem of this universe, I must shake my head in doubt. You remember the first Napoleon's question, when the savans who accompanied him to Egypt discussed in his presence the origin of the universe, and solved it to their own apparent satisfaction. He looked aloft to the starry heavens, and said, "It is all very well, gentlemen; but who made these?" That question still remains unanswered, and science makes no attempt to answer it. As far as I 
can see, there is no quality in the human intellect which is fit to be applied to the solntion of the problem. It entirely transcends us. The mind of man may be compared to a musical instrument with a certain range of notes, beyond which in both directions we have an infinitude of silence. The phenomena of matter and force lie within our intellectual range, and as far as they reach we will at all hazards push our inquiries. But behind, and above, and around all, the real mystery of this universe lies unsolved, and, as far as we are concerned, is incapable of solution. Fashion this mystery as you will, with that I have nothing to do. But let your conception of it not be an unworthy one. Invest that conception with your highest and holiest thought, but be careful of pretending to know more about it than is given to man to know. Be careful, above all things, of professing to see in the phenomena of the material world the evidences of Divine pieasure or displeasure. Doubt those who would deduce from the fall of the tower of Siloam the anger of the Lord against those who were crushed. Doubt equally those who pretend to see in cholera, cattle-plague, and bad harvests, evidences of Divine anger. Doubt those spiritual guides who in Scotland have lately propounded the monstrous theory that the depreciation of railway scrip is a consequence of railway traveling on Sundays. Let them not, as far as you are concerned, libel the system of nature with their ignorant hypotheses. Looking from the solitudes of thought into this highest of questions, and seeing the puerile attempts often made to solve it, well might the mightiest of living Scotchmen-that strong and earnest soul, who has made every soul of like nature in these islands his debtor-well, I say, might your noble old Carlyle scornfully retort on such interpreters of the ways of God to men:

The Builder of this universe was wise,

He formed all souls, all systems, planets, particles;

The plan he formed his worlds and Fons by,

Was-Heavens! - was thy small nine-and-thirty articles! 


\section{CHAPTER XXVIII. \\ SCIENTIFIC MATERIALISM.* 1868。}

Here, indeed, we arrive at the barrier which needs to be perpetually pointed out; alike to those who seek materialistic explanations of mental phenomera, and to those who are alarmed lest such explanations may be found. The last class prove by their fear, almost as much as the first prove by their hope, that they believe Mind may possibly be interpreted in terms of Matter; whereas many whom they vituperate as materialists are profoundly convinced that there is not the remotest possibility of so interpreting them.-HERBERT SPENCER.

ThE celebrated Fichte, in his lectures on the "Vocation of the Scholar," insisted on a culture which should be not one-sided, but all-sided. The scholar's intellect was to expand spherically, and not in a single direction only. In one direction, however, Fichte required that the scholar should apply himself directly to nature, become a creator of knowledge, and thus repay, by original labors of his own, the immense debt he owed to the labors of others. It was these which enabled him to supplement the kuowledge derived from his own researches, so as to render his culture rounded and not one-sided.

As regards science, Fichte's idea is to some extent illustrated by the constitution and labors of the British Association. We have here a body of men engaged in the pursuit of Natural Knowledge, but variously engaged. While sympathizing with each of its departments, and supplementing his culture by knowledge drawn from all of them, each student among us selects one subject for the exercise of his own original faculty-one line, along which he may carry the light of his private intelligence a little way into the darkness by which all knowledge is surrounded. Thus, the geologist deals with the rocks; the biologist with the conditions and phenomena of life; the astronomer with stellar masses and motions; the mathematician with the relations of space and number; the chemist pursues his atoms; while the physical investigator has his own large field in optical, thermal, electrical, acoustical, and other phenomena. The British Association then, as a

* President's Address to the Mathematical and Physical Section of the British Association at Norwich. 
whole, faces physical nature on all siles, and pushes knowledge centrifugally ontward, the sum of its labors constituting what Fichte might call the sphere of natural knowledge. In the meetings of the association it is found necessary to resolve this sphere into its component parts. which take concrete form under the respective letters of our Sections.

Mathematics and Physics have been long accustomed to coalesce, and here they form a single section. No matter how subtle a natural phenomenon may be, whether we observe it in the region of sense, or follow it into that of imagination, it is in the long run reducible to mechanical laws. But the mechanical data once guessed or given, mathematics are all-powerfulas an instrument of deduction. The command of Geometry over the relations of space, and the far-reaching power which Analysis confers, are potent both as means of physical discovery, and of reaping the entire fruits of discovery. Indeed, without mathematics, expressed or implied, our knowledge of physical science would be both friable and incomplete.

Side by side with the mathematical method we have the method of experiment. Here from a starting-point furnished by his own researches or those of others, the investigator proceeds by combining intuition and rerification. He ponders the knowledge he possesses, and tries to push it further; he guesses, and checks his guess; he conjectures, and coufirms or explodes his conjecture. These guesses and conjectures are by no means leaps in the dark; for knowledge once gained casts a faint light beyond its own immediate boundaries. There is no discovery so limited as not to illuminate something beyond itself. The force of intellectual penetration into this penumbral region which surrounds actual knowlelge is not, as some seem to think, dependent upon method, but upon the genius of the investigator. There is, however, no genius so gifted as not to need control and verification. The profoundest minds know best that nature's ways are not at all times their ways, and that the brightest flashes in the world of thought are incomplete until they have been proved to have their counterparts in the world of fact. Thus the vocation of the true experimentalist may be defined as the continued exercise of spiritual insight, and its incessant correction and realization. His experinents constitute a body, of which his purified intuitions are, as it were, the soul. 
Partly through mathematical and partly through experimental research, physical science has, of late years, assumed a momentous position in the world. Both in a material and in an intellectual point of view it has produced, and it is destined to produce, immense changesvast social ameliorations, and vast alterations in the popular conception of the origin, rule, and governance of natural things. By science, in the physical world, miracles are wrought, while philosophy is forsaking its ancient metaphysical channels, and pursuing others which have been opened, or indicated by scientific research. This must become more and more the case as philosophical writers become more deeply imbued with the methods of science, better acquainted with the facts which scientific men have established, and with the great theories which they have elaborated.

If you look at the face of a watch, you see the hour and minute-hands, and possibly also a second-hand, moving over the graduated dial. Why do these hands move? and why are their relatire motions such as they are observed to be? These questions cannot be answered without opening the wateh, mastering its various parts, and ascertaining their relationship to each other. When this is done, we find that the observed motion of the hands follows of necessity from the inner mechanism of the watch when acted upon by the force invested in the spring. The motion of the hands may be called a phenomenon of art, but the case is similar with the phenomena of nature. These also have their inner mechanism and their store of force to set that mechanism going. The ultimate problem of physical science is to reveal this mechanism, to discern this store, and to show that from the combined action of both, the phenomena of which they coustitute the basis, must, of necessity, flow.

I thought an attempt to give you even a brief and sketchy illustration of the manner in which scientific thinker's regard this problem would not be uninteresting to you on the present occasion; more especially as it will give me oecasion to say a word or two on the tendencies and limits of modern science; to point out the region which men of science claim as their own, and where it is futile to oppose their advance; and also to define, if possible, the bourne between this and that other region, to which the question- 
ings and yearnings of the scientific intellect are directed in vain.

But here your tolerance will be needed. It was the American Emerson, I think, who said that it is hardly possible to state any truth strongly, without apparent injustice to some other truth. 'Truth is often of a dual character, taking the form of a magnet with two poles; and many of the differences which agitate the thinking part of mankind are to be traced to the exclusiveness with which partisan reasoners dwell upon one half of the duality, in forgetfulness of the other. 'The proper course appears to be to state both halves strongly, and allow each its fair share in the formation of the resultant conviction. But this waiting for the statement of the two sides of a question implies patience. It implies a resolution to suppress indignation, if the statement of the one half should clash with our convictions; and to repress equally undue elation, if the half-statement should happen to chime in with our views. It implies a determination to wait calmly for the statement of the whole, before we pronounce judgment in the form of either acquiescence or dissent.

This premised, and I trust accepted, let us enter upon our task. There have been writer's who affirmed that the pyramids of Egypt were natural productions; and in his early youth Alexander von Humboldt wrote a learned essay with the express object of refuting this notion. We now regard the pyramids as the work of men's hands, aided probably by machinery of which no record remains. We picture to ourselves the swarming workers toiling at those vast erections, lifting the inert stones, and, guided by the volition, the skill, and possibly at times by the whip of the architect, placing them in their proper positions. 'The blocks, in this case, were moved and posited by a power external to themselves, and the final form of the pyramid expresser the thought of its human builder.

Let us pass from this illustration of constructive power to another of a different kind. When a solution of common salt is slowly evaporated, the water which holds the salt in solution disappears, but the salt itself remains behind. At a certain stage of concentration the salt can no longer retain the liquid form; its particles, or molecules, as they are called, begin to deposit themselves as minute solids-so miuute, indeed, as to defy all microscopic power. As evap- 
oration continues, solidification goes on, and we finally obtain through the clustering together of innumerable molecules, a finite crystalline mass of a definite form. What is this form? It sometimes seems a mimicry of the architecture of Egypt. We have little pyramids built by the salt, terrace above terrace from base to apex, forming a series of steps resembling those up which the traveler in Egypt is dragged by his guides. 'The human mind is as little disposed to look without questioning at these pyramidal salt-crystals, as to look at the pyramids of Egypt, without inquiring whence they came. How, then, are those salt-pyramids built up?

Guided by analogy, you may, if you like, suppose that, swarming among the constituent molecules of the salt, there is an invisible population, controlled and coerced by some invisible master, placing the atomic blocks in their positions. This, however, is not the scientific idea, nor do I think your good sense will accept it as a likely one. The scientific idea is, that the molecules act upon each other without the intervention of slave labor; that they attract each other, and repel each other, at certain definite points, or poles, and in certain definite directions; and that the pyramidal form is the result of this play of attraction and repulsion. While, then, the blocks of Egypt were laid down by a power external to themselves, these molecular blocks of salt are self-posited, being fixed in their places by the inherent forces with which they act upon each other.

I take common salt as an illustration, because it is so familiar to us all; but any other crystalline substance would answer my purpose equally well. Everywhere, in fact, throughout inorganic nature, we have this formative power, as Fichte would call it-this structural energy ready to come into play, and build the ultimate particles of matter into definite shapes. The ice of our winters, and of our polar regions, is its handiwork, and so also are the quartz, feldspar, and mica of our rocks. Our chalk-beds are for the most part composed of minute shells, which are also the product of structural energy; but behind the shell, as a whole, lies a more remote and subtle formative act. These shells are built up of little crystals of calc-spar, and, to form these crystals, the structural force had to deal with the intangible molecules of carbonate of lime. This tendency on the part of matter to organize itself, to grow into 
shape, to assume definite forms in obedience to the definite action of force, is, as I have said, all-pervading. It is in the ground on which you tread, in the water you drink, in the air you breathe. Incipient life, as it were, manifests itself throughout the whole of what we call inorganic nature.

The forms of the minerals resulting from this play of polar forces are various, and exhibit different degrees of complexity. Men of science avail themselres of all possible means of exploring their molecular architecture. For this purpose they employ in turn, as agents of exploration, light, heat, magnetism, electricity, and sound. Polarized light is especially useful and powerful here. A beam of such light, when sent in among the molecules of a crystal, is acted on by them, and from this action we infer with more or less clearness the manner in which the molecules are arranged. That differences, for example, exist between the inner structure of rocksalt and that of crystallized sugar or sugar-candy, is thus strikingly revesled. 'These actions often display themselves in chromatic phenomena of great splendor, the play of molecular force being so regulated as to cause the removal of some of the colored constituents of white light, while others are left with increased intensity behind.

And now let us pass from what we are accustomed to regard as a dead mineral, to a living grain of corn. When this is examined by polarized light, chromatic phenomena similar to those noticed in crystals are observed. And why? Because the architecture of the grain resembles that of the crystal. In the grain also the molecules are set in definite positions, and in accordance with their arrangement they act upon the light. But what has built together the molecules of the corn? Regarding crystalline architecture, I have already said that you mav, if you please, consider the atoms and molecules to be placed in position by a Power external to themselves. The same hypothesis is open to you now. But if in the case of crystals you have rejected this notion of an external architect, I think you are bound to reject it in the case of the grain, and to conclude that the molecules of the corn, also, are posited by the forces with which they act upon each other. It would be poor philosophy to invoke an external agent in the one case, and to reject it in the other. 
Instead of cutting our grain of corn into slices and subjecting it to the action of polarized light, let us place it in the earth, and subject it to a certain degree of warmth. In other words, let the molecules, both of the corn and of the surrounding earth, be kept in that state of agitation which we call heat. Under these circumstances, the grain and the substances which surround it interact, and a definite molecular architecture is the result. A bud is formed; this bud reaches the surface, where it is exposed to the sun's rays, which are also to be regarded as a kind of vibratory motion. And as the motion of common heat, with which the grain and the substances surrounding it were first endowed, enabled the grain and these substances to exercise their mutual attractions and repulsions, and thus to coalesce in definite forms, so the specific motion of the sun's rays now enables the green bud to feed upon the carbonic acid and the aqueous vapor of the air. The bud appropriates those constituents of both for which it has an elective attraction, and permits the other constituent to return to the atmosphere. Thus the architecture is carried on. Forces are active at the root, forces are actire in the blade, the matter of the air and the matter of the atmosphere are drawn upon, and the plant algments in size. We have in succession the stalk, the ear, the full corn in the ear; the cycle of molecular action being completed by the production of grains, similar to that with which the process began.

Now there is nothing in this process which necessarily eludes the conceptive or imagining power of the human mind. An intellect the same in kind as our own would, if only sufficiently expanded, be able to follow the whole process from beginning to end. It would see every molecule placed in its position by the specific attractions and repulsions exerted between it and other molecules, the whole process, and its consummation, being an instance of the play of molecular force. Given the grain and its environment, with their respective forces, the purely human intellect might, if sufficiently expanded, trace out ${ }^{\circ}$ priori every step of the process of growth, and, by the application of purely mechanical principles, demonstrate that the cycle must end, as it is seen to end, in the reproduction of forms like that with which it began. A necessity rules here, similar to that which rules the planets in their circuits round the sun. 
You will notice that I am stating the truth strongly, as at the beginning we agreed it should be stated. But I must go still further, and affirm that in the eye of science the animal body is just as much the product of molecular force as the chalk and the ear of corn, or as the crystal of salt or sugar. Many of the parts of the body are obviously mechanical. 'Take the human heart, for example, with its system of valves, or take the exquisite mechanism of the eye or hand. Animal heat, moreover, is the same in kind as the heat of a fire, being produced by the same chemical process. Animal motion, too, is as certainly derived from the food of the animal, as the motion of 'Trevethyck's walking-engine from the fuel in its furnace. As regards matter, the animal body creates nothing; as regards force, it creates nothing. Which of you by taking thought can add one cubit to his stature? All that has been said, then, regarding the plant, may be restated with regard to the animal. Every particle that enters into the composition of a nerve, a muscle, or a bone has been placed in its position by molecular force. And unless the existence of law in these matters be denied, and the element of caprice introduced, we must conclude that, given the relation of any molecule of the borly to its environment, its position in the body might be determined mathematically. Our difficulty is not with the quality of the problem, but with its complexity; and this difficulty might be met by the simple expansion of the faculties we now possess. Given this expansion, with the necessary molecular data, and the chick might be deduced as rigorously and as logically from the egg, as the existence of Neptune from the disturbances of Uranus, or as conical refraction from the undulatory theory of light.

You see I am not mincing matters, but avowing nakedly what many scientific thinker's more or less distinctly believe. 'The formation of a crystal, a plant, or an animal, is, in their eyes, a purely mechanical problem, which differs from the problems of ordinary mechanics, in the smallness of the masses, and the complexity of the processes involved. Here you have one half of our dual truth; let us now glance at the other half. Associated with this wonderful mechanism of the animal body we have phenomena no less certain than those of physics, but between which and the mechanism we discern no necessary connection. A man, 
for example, can say "I feel," "I think," "I love;" but how does consciousness infuse itself into the problem? The human brain is said to be the organ of thought and feeling: when we are hurt, the brain feels it; when we ponder, or when our passions or affections are excited, it is through the instrumentality of the brain. Let us endeavor to be a little more precise here. I hardly imagine there exists a profound scientific thinker, who has reflecterl upon the subject, unwilling to admit the extreme probability of the hypothesis, that for every fact of consciousness, whether in the domain of sense, thought, or emotion, a definite molecular condition, of motion or structure, is set up in the brain; or who would be disposed even to deny that if the motion, or structure, be induced by internal causes instead of external, the effect on consciousness will be the same? Let any nerve, for example, be thrown by morbid action into the precise state of motion which would be communicated to it by the pulses of a heated body, surely that nerve will declare itself hot-the mind will accept the subjective intimation exactly as if it were objective. The retina may be excited by purely mechanical means. A blow on the eye causes a luminous flash, and the mere pressure of the finger on the external ball produces a star of light, which Newton compared to the circles on a peacock's tail. Disease makes people see visions and dream dreams; but, in all such cases, conld we examine the organs implicated, we should, on philosophical grounds, expect to find them in that precise molecular condition which the real objects, if present, would superinduce.

The relation of physics to consciousness being thus invariable, it follows that, given the state of the brain, the corresponding thought or feeling might be inferred: or, given the thought or feeling, the corresponding state of the brain might be inferred. But how inferred? It woulil be at bottom not a case of logical inference at all, but of empirical association. You may reply, that many of the inferences of science are of this character-the inference, for example, that an electric current, of a given direction, will deflect a magnetic needle in a definite way. But the cases liffer in this, that the passage from the current to the needle, if not demonstrable, is conceivable, aud that we entertain no doubt as to the final mechanical solution of the problem. But the passige from the physics of the 
brain to the corresponding facts of consciousness is inconceirable as a result of mechanics. Granted that a definite thought, and a definite molecular action in the brain, occur simultaneously; we do not possess the intellectual organ, nor apparently any rudiment of the organ, which would enable us to pass, by a process of reasoning, from the one to the other. They appear together, but we do not know why. Were our minds and senses so expanded, strengthened, and illuminated, as to enable us to see and feel the very molecules of the brain; were we capable of following all their motions, all their groupings, all their electric discharges, if such there be; and were we intimately acquainted with the corresponding states of thought and feeling, we should be as far as ever from the solution of the problem, "How are these physical processes connected with the facts of consciousness?" The chasm between the two classes of phenomena would still remain intellectually impassable. Let the consciousness of love, for example, be associated with a right-handed spiral motion of the molecules of the brain, and the consciousness of hate with a left-handed spiral motion. We should then know, when we love, that the motion is in one direction, and, when we hate, that the motion is in the other; but the "WHY?" would remain as unanswerable as before.

In affirming that the growth of the body is mechanical, and that thought, as exercised by us, has its correlative in the physics of the brain, I think the position of the "Materialist" is stated, as far as that position is a tenable one. I think the materialist will be able finally to maintain this position against all attacks; but I do not think, in the present condition of the human mind, that he can pass beyond this position. I do not think he is entitled to say that his molecular groupings, and motions, explain everything. In reality they explain nothing. 'The utmost he call affirm is the association of two classes of phenomena, of whose real bond of union he is in absolute ignorance. 'The problem of the connection of body ana soul is as insoluble, in its morlern form, as it was in the pre-scientific ages. Phosphorus is known to enter into the composition of the human brain, and a trenchant German writer has exclaimed, "Ohne Phosphor, kein Gedanke!" That may or may not be the case; but even if we knew it to be the case, the knowlerlge would not lighten our darkness. On 
both sides of the zone here assigned to the materialist he is equally helpless. If you ask him whence is this "Matter" of which we have been discoursing-who or what divided it into molecules, who or what impressed upon them this necessity of running into organic forms-he has no answer. Science is mute in reply to these questions. But if the materialist is confounded and science rendered dumb, who else is prepared with a solution? 'Io whom has this arm of the Lord been revealed? Let us lower our heads, and acknowledge our igmorance, priest and philosopher, one and all.

Perhaps the mystery may resolve itself into knowledge at some future day. 'The process of things upon this earth has been one of amelioration. It is a long way from the Iguanodon and his contemporaries, to the President and Members of the British Association. And whether we regard the improvement from the scientific or from the theological point of view-as the result of progressive development, or of successive exhibitions of creative energy -neither view entitles us to assume that man's present faculties end the series, that the process of amelioration ends with him. A time may therefore come when this ultra-scientific region, by which we are now enfolded, may offer itself to terrestrial, if not to human, investigation. Two-thirds of the rays emitted by the sun fail to arouse the sense of vision. The rays exist, but the visual organ requisite for their translation into light does not exist. Aud so from this region of darkness and mystery which surrounds us, rays may now be darting, which require but the development of the proper intellectual organs to translate them into knowledge as far surpassing ours as ours surpasses that of the wallowing reptiles which once held possession of this planet. Meanwhile the mystery is not without its uses. It certainly may be made a power in the human soul; but it is a power which has feeling, not knowledge, for its base. It may be, will be, and I hope is turned to account, both in steadying and strengthening the intellect, and in rescuing man from that littleness to which, in the struggle for existence, or for precedence in the world, he is continually prone. 
Musings on the Matterhorn, July 2\%, 1868.

Hacked and hurt by time, the aspect of the mountain from its higher crags saddened me. Hitherto the impression it made was that of savage strength; here we had inexorable decay. But this notion of decay implied a reference to a period when the Matterhorn was in the full strength of mountainhood. 'Thought naturally ran back to its remoter origin and sculpture. Nor did thought halt there, but wandered on throngh molten worlds to that nebulous haze which philosophers have regarded, and with good reason, as the proximate source of all material things. I tried to look at this universal cloud, containing within itself the prediction of all that has since occurred; I tried to imagine it as the seat of those forces whose action was to issue in solar and stellar systems, and all that they involve. Did that formless fog contain potentially the sadness with which I regarded the Matterhorn? Did the thought which now ran back to it simply return to its primeval home? If so, had we not better recast our definitions of matter and force? for, if life and thought be the very flower of both, any definition which omits life and thought must be inadequate, if not untrue. Are questions like these warranted? Why not? If the final goal of man has not been yet attained; if his development has not been yet arrested, who can say that such yearnings and questionings are not necessary to the opening of a finer vision, to the budding and the growth of diviner powers? When I look at the heavens and the earth, at my own body, at my strength and weakness, even at these ponderings, and ask myself, Is there no being or thing in the universe that knows more about these matters than I do; what is my answer? Supposing our theologic schemes of creation, condemnation, and redemption to be dissipated; and the warmth of denial which they excite, and which, as a motive force, can match the warmth of affirmation, dissipated at the same time; would the undeflected human mind return to the meridian of absolute nentrality as regards these ultraphysical questions? Is such a position one of stable equilibrium? The channels of thought being already formed, such are the questions, without replies, which could run athwart conscionsness during a ten minutes' halt upon the weathered crest of the Matterhorn. 


\section{CHAPTER XXIX.}

\section{AN ADDRESS TO STUDENTS. *}

Self-reverence, self-knowledge, self-control,

These three alone lead life to sovereign power,

Yet not for power (power of herself

Would come uncalled for), but to live by law,

Acting the law we live by without fear;

And, because right is right, to follow right

Were wisdom in the scorn of consequence.

TEN YBON.

THERE is an idea regarding the nature of man which modern philosophy has sought, and is still sceking, to raise into clearness; the idea, namely, of secular growth. Man is not a thing of yesterday; nor lo I imagine that the slightest controversial tinge is imparted into this aldress when I say that he is not a thing of six thousand years ago. Whether he came originally from stocks or stones, from nebulous gas or solar fire, I know not; if he had any such origin the process of his transformation is as inscrutable to you and me as that of the grand old legend, according to which "the Lord God formed man of the dust of the ground, and breathed into his nostrils the breath of life; and man became a living soul." But however obscure man's origin may be, his growth is not to be denied. Here a little and there a little added through the ages have slowly transformel him from what he was into what he is. The doctrine has been held that the mind of the child is like a sheet of white paper, on which by education we can write what characters we please. This doctrine assuredly needs qualification and correction. In physics, when an external force is applied to a body with a view of affecting its inner texture, if we wish to predict the result, we must know whether the external force conspires with or opposes the internal forces of the body itself; and in bringing the influence of education to bear upon the new-born man his inner powers also must be taken into account. He comes to us as a bundle of inherited capacities and tendencies, labeled "from the indefinite past to the indefinite future;" and he makes his transit from the one to the other

* Delivered at University College, London, Session 1868.69. 
through the enlucation of the present time. The object of that education is, or ought to be, to provide wise exercise for his capacities, wise direction for his tendencies, and through this exercise and this direction to furnish his mind with such knowledge as may contribute to the usefulness, the beanty, and the nobleness of his life.

How is this discipline to be secured, this knowledge imparted? Two rival methods now solicit attention-the one organized and equipped, the labor of centuries having been expended in bringing it to its present state of perfection; the other, more or less chaotic, but becoming daily less so, and giving signs of enormous power, both as a source of knowledge and as a means of discipline. These two methods are the classical and the scientific method. I wish they were not rivals; it is only bigotry and shortsightedness that make them so; for assuredly it is possible to give both of them fair play. Though hardly anthorized to express an opinion upon the subject, I nevertheless hold the opinion that the proper study of a language is an intellectual discipline of the highest kind. If I except discussions on the comparative merits of Popery and Protestantism, English grammar was the most important discipline of $m y$ boybood. The piercing through the involverl and inverterl sentences of "Paradise Lost;" the linking of the verb to its often distant nominative, of the relative to its distant antecedent, of the agent to the object of the transitive verb, of the preposition to the noun or pronoun which it governed, the study of variations in mood and tense, the transpositions often necessary to bring out the true grammatical structure of a sentence-all this was to my young mind a discipline of the highest value, and a source of unflagging delight. How I rejoiced when I found a great author tripping, and was fairly able to pin him to a corner from which there was no escape! Ás I speak, some of the sentences which exercised me when a boy rise to my recollection. For instance, "He that hath ears to hear, let him hear;" where the "He" is left, as it were. floating in mid air without any verb to support it. I speak thus of English because it was of real value to me. I do not speak of other languages becanse their educational vilue for me was almost insensible. But knowing the value of English so well, I should be the last to deny, or even to doubt, the high discipline involved in the proper study of Latin and Greek. 
That study, moreover, has other merits and recommendations. It is, as I have said, organized and systematized by long-continued use. It is an instrument wielded by some of our best intellects in the education of youth; and it can point to results in the achievements of our foremost men. What, then, has science to offer which is in the least degree likely to compete with such a system? I cannot better reply than by recurring to the grand old story from which I have already quoted. Speaking of the world and all that therein is, of the sky and the stars around it, the ancient writer says, "And God saw all that he had made, and behold it was very good." It is the body of things thus described which science offers to the study of man. There is a very renowned argument much prized and much quoted by theologians, in which the universe is compared to a watch. Let us deal practically with this comparison. Supposing a watch-maker, having completed his instrument, to be so satisified with his work as to call it very good, what would you understand him to mean? You would not suppose that he referred to the dial-plate in front and the chasing of the case behind, so much as to the wheels and pinions, the springs and jeweled pivots of the works within-to those qualities and powers, in short, which enable the watch to perform its work as a keeper of time. With regard to the knowledge of such a watch he would be a mere ignoramus who would content himself with outward inspection. I do not wish to say one severe word here to-day, but I fear that many of those who are very loud in their praise of the works of the Lord know them only in this ontside and superficial way. It is the inner works of the universe which science reverently uncovers; it is the study of these that she recommends as a discipline worthy of all acceptation.

The ultimate problem of physies is to reduce matter by analysis to its lowest condition of divisibility, and force to its simplest manifestations, and then by synthesis to construct from these elements the world as it stands. We are still a long way from the final solution of this problem; and when the solution comes, it will be more one of spiritual insight than of actual observation. But though we are still a long way from this complete intellectual mastery of nature, we have conquered rast regions of it, have learned their polities and the play of their powes's. We live upon a 
ball of 8,000 miles in diameter, swathed by an atmosphere of unknown height. 'This ball has been molten by heat, chilled to a solid, anil sculptured by water. It is made up of substances possessing distinctive properties and modes of action, which offer problems to the intellect, some profitable to the child, others taxing the highest powers of the philosopher. Our native suhere turns on its axis, and revolves in space. It is one of a band which all do the same. It is illuminated by a sun which, though nearly a hundred millions of miles distant, can be brought virtually into our closets and there subjected to examination. It has its winds and clouds, its rain and frost, its light, heat, sound, electricity, and magnetism. And it has its vast kingdoms of animals and vegetables. 'T'o a most amazing extent the human mind has conquered these things, and revealed the logic which runs through them. Were they facts only, without logical relationship, science might, as a means of discipline, suffer in comparison with language. But the whole body of phenomena is instinct with law; the facts are hung on principles, and the value of physical science as a means of discipline consists in the motion of the intellect, both inductively and deductively, along the lines of law marked out by phenomena. As regards the discipline to which I have alrealy referred as derivable from the study of languages - that, and more, is involved in the study of physical science. Indeed, I believe it would be possible so to limit and arrange the study of a portion of physics as to render the mental exercise involved in it almost qualitatively the same as that involved in the unraveling of a language.

I have thus far confined myself to the purely intellectual side of this question. But man is not all intellect. If he were so, science would, I believe, be his proper nutriment. But he feels as well as thinks; he is receptive of the sublime and beautiful as well as of the true. Indeed, I believe that even the intellectual action of a complete man is, consciously or unconsciously, sustained by an undercurrent of the emotions. It is vain to attempt to separate the moral and emotional from the intellectual. Let a man but observe himself, and he will, if I mistake not, find that in nine cases out of ten the emotions constitute the motive force which pushes his intellect into action. 'T'he reading of the works of two men, neither of them imbuel 
with the spirit of modern science-neither of them, indeed, friendly to that spirit-has placed me here to-day. 'These men are the English Carlyle and the American Emerson. I must ever gratefully remember that through three long cold German winters Carlyle placed me in my tub, even when ice was on its surface, at five o'clock every morning - not slavishly, but cheerfully, meeting each day's studies with a resolute will, determined whether victor or vanquished not to shrink from difficulty. I never should have gone throngh Analytical Geometry and the Calculus had it not been for those men. I never should have become a physical investigator, and hence without them I should not have been here to-day. They told me what I ought to do in a way that caused me to do it, and all my consequent intellectual action is to be traced to this purely moral source. To Carlyle and Emerson I ought to add Fichte, the greatest representative of pure idealism. 'These three unscientific men made me a practical scientific worker. They called out "Act!" I hearkened to the summons, taking the liberty, however, of determining for myself the direction which effort was to take.

And I may now cry "Act!" but the potency of action must be yours. I may pull the trigger, but if the gun be not charged there is no result. We are creators in the intellectual world as little as in the physical. We may remove obstacles, and render latent capacities active, but we cannot suddenly change the nature of man. The "new birth" itself implies the pre-existence of a character which requires not to be created but brought forth. You cannot by any amount of missionary labor suddenly transform the savage into the civilized Christian. 'The improvement of man is secular-not the work of an hour or of a day. But though indubitably bound by our organizations, no man knows what the potentialities of any human mind may be, requiring only release to be brought into action. There are in the mineral world certain crystals-certain forms, for instance, of fluor-spar, which have lain darkly in the earth for ages, but which nevertheless have a potency of light locked up within them. In their case the potential has never become actual-the light is in fact held back by a molecular detent. When these crystals are warmed, the detent is lifted, and an outflow of light immediately begins. I know not how many of you may be in the condition of 
this fluor-spar. For aught I know, every one of you may be in this condition, requiring but the proper agent to be applied-the proper word to be spoken-to remove a detent, and to render you conscious of light and warmth within yourselves and sources of both to others.

The circle of human nature, then, is not complete without the arc of the emotions. The lilies of the field have a value for us beyond their botanical ones-a certain lightening of the heart accompanies the declaration that "Solomon in all his glory was not arrayed like one of these." 'The sound of the village bell has a value beyond its acoustical one. The setting sun has a value beyond its optical one. The starry heavens, as you know, had for Immanuel Kant a value beyond their astronomical one. I think it very desirable to keep this horizon of the emotions open, and not to permit either priest or philosopher to draw down his shutters between you and it. Here the dead languages, which are sure to be beaten by science in the purely intellectual fight, have an irresistible claim. They stupplement the work of science by exalting and refining the resthetic faculty, and must on this account be cherished by all who desire to see human culture complete. 'There must be a reason for the fascination which these languages have so long exercised upon powerful and elevated minds a fascination which will probably continue for men of Greek and Roman mold to the end of time.

In connection with this question one very obvious danger besets many of the more earnest spirits of our day-the danger of haste in endeavoring to give the feelings repose. We are distracted by systems of theology and philosophy which were taught to us when young, and which now excite in us a hunger and a thirst for knowledge not proved to be attainable. There are periods when the judgment onght to remain in suspense, the data on which a decision might be based being absent. 'This discipline of suspending the judgment is a common one in science, but not so common as it ought to be elsewhere. I walked down Regent Street some time ago with a man of great gifts and acquirements, discussing with him various theological questions. I could not accept his views of the origin and destiny of the universe, nor was I prepared to enunciate any definite views of $\mathrm{my}$ own. He turned to me at length and said, "You surely must have a theory of the universe." 
That I should in one way or another have solved this mystery of mysteries seemed to my friend a matter of course. "I have not even a theory of magnetism" was my reply. We ought to learn to wait. We ought assuredly to pause before closing with the advances of those expounders of the ways of God to men, who offer us intellectual peace at the modest cost of intellectual life.

The teachers of the world ought to be its best men, and for the present at all events such men must learn self-trust. By the fullness and freshness of their own lives and utterences they must awaken life in others. The hopes and terrors which influenced our fathers are passing away, and our trust henceforth must rest on the innate strength of man's moral nature. And here, I think, the poet will have a great part to play in the future culture of the world. 'To him, when he rightly understands his mission, and does not flinch from the tonic discipline which it assuredly demands, we have a right to look for that heightening and brightening of life which so many of us need. To him it is given for a long time to come to fill those shores which the recession of the theologic tide has left exposed. Void of offense to science, he may freely deal with conceptions which science shuns, and become the illustrator and interpreter of that Power which as

\section{“Jehovah, Jove, or Lord,"}

has hitherto filled and strengthened the human heart.

Let me utter one practical word in conclusion-take care of your health. There have been men who by wise attention to this point might have risen to any eminencemight have made great discoveries, written great poems, commanded armies, or ruled states, but who by unwise neglect of this point have come to nothing. Imagine Hercules as oarsman in a rotten boat; what can he do there but by the very force of his stroke expedite the ruin of his craft? Take care then of the timbers of your boat, and avoid all practices likely to introduce either wet or dry rot among them. And this is not to be accomplished by desultory or intermittent efforts of the will, but by the formation of habits. The will no doubt has sometimes to put forth its strength in order to crush the special temptation. But the formation of right habits is essential to your per- 
manent security. They diminish your chance of falling when assailed, and they angment your chance of recovery when overthrown.

\section{CHAP'TER XXX.}

\section{SCIENTIFIC USE OF THE IMAGINATION.*}

"If thou would'st know the mystic song

Chaunted when the sphere was young,

Aloft, abroad the pæan swells,

Oh wise man, hear'st thou half it tells?

To the open ear it sings

The early genesis of things;

Of tendency through endiess ages

Of star-dust and star-pilgrimages,

Of rounded worlds, of space and time,

Of the old floods' subsiding slime,

Of chemic matter, force and form,

Of poles and powers, cold, wet, and warm.

The rushing metamorphosis

Dissolving all that fixture is,

Melts things that be to things that seem,

And solid nature to a dream."

EMERSON.

“Was wär' ein Gott der nur von aussen stiesse,

Im Kreis das All am Finger laufen liesse

Ihm ziemt's, die Welt im Innern zu bewegen,

Natur in Sich, Sich in Natur zu hegen."

Goethe.

"Lastly, physical investigation, more than anything besides, helps to teach us the actual value and right use of the Imagination-of that wondrous faculty, which, left to ramble uncontrolled, leads us astray into a wilderness of perplexities and errors, a land of mists and shadows; but which, properly controlled by experience and reflection, becomes the noblest attribute of man; the source of poetic genius, the instrument of discovery in Science, without the aid of which Newton would never have invented fluxions, nor Davy lave decomposed the earths and alkalies, nor would Columbus have found another continent."-Address to the Royal Society by its President sir Benjamin Brodie, November 30, 1859.

I CARRIED with me to the Alps this year the burden of this evening's work. Save from memory I hakl no direct aid upon the mountains; but to spur up the emotions, on

* Discourse delivered before the British Association at Liverpool, September 16, 1870. 
which so much depends, as well as to nourish indirectly the intellect and will, I took with me four works, comprising two volumes of poetry, Goethe's "Farbenlehre," and the work on "Logic" recently published by Mr. Alexander Bain. In Goethe, so noble otherwise, I chiefly noticed the self-inflicted hurts of genius, as it broke itself in vain against the philosophy of Newton. Mr. Bain I found, for the most part, learned and practical, shining generally with a dry light, but exhibiting at times a flush of emotional strength, which proved that even logicians share the common fire of humanity. He interested me most when he became the mirror of my own condition. Neither intellectually nor socially is it good for man to be alone, and the sorrows of thought are more patiently borne when we find that they have been experienced by another. From certain passages in his book I could infer that $\mathrm{Mr}$. Bain was no stranger to such sorrows. Speaking for example of the ebb of intellectual force, which we all from time to time experience, Mr. Bain says: "The uncertainty where to look for the next opening of discovery brings the pain of conflict and the debility of indecision." These words have in them the true ring of personal experience. The action of the investigator is periodic. He grapples with a subject of inquiry; wrestles with it, and exhausts, it may be, both himself and it for the time being. He breathes a space, and then renews the struggle in another field. Now this period of halting between two investigations is not always one of pure repose. It is often a period of doubt and discomfort-of gloom and ennui. "The uncertainty where to look for the next opening of discovery brings the pain of conflict and the debility of indecision." It was under such conditions that I had to equip myself for the hour and the ordeal that are now come.

The disciplines of common life are, in great part, exercises in the relations of space, or in the mental grouping of bodies in space; and, by such exercises, the public mind is, to some extent, prepared for the reception of physical conceptions. Assuming this preparation on your part, the wish gradually grew within me to trace, and to enable you to trace, some of the more occult features and operations of Light and Color. I wished, if possible, 
to take you beyond the boundary of mere observation, into a region where things are intellectually discerned, and to show you there the hiddeu mechanism of optical action.

But how are those hidden things to be revealed? Philosophers may be right in affirming that we cannot transcend experience: we can, at all events, carry it a long way from its origin. We can magnify, diminish, qualify, and combine experiences, so as to render them fit for purposes entirely new. In explaining sensible phenomena, we habitually form mental images of the ultra-sensible. There are Tories even in science who regard Imagination as a faculty to be feared and avoided rather than employed. They have observed its action in weak vessels, and are unduly impressed by its disasters. But they might with equal justice point to exploded boilers as an argument against the use of steam. With accurate experiment and observation to work upon, Imagination becomes the architect of physical theory. Newton's passage from a falling apple to a falling moon was an act of thie prepared imagination, without which the "laws of Kepler" could never have been traced to their foundations. Out of the facts of chemistry the constructive imagination of Dalton formed the atomic theory. Davy was richly endowed with the imaginative faculty, while with Faraday its exercise was incessant, precerling, accompanying and guiding all his experiments. His strength and fertility as a discoverer is to be referred in great part to the stimulus of his imagination. Scientific men fight shy of the word because of its ultra-scientific connotations; but the fact is that without the exercise of this power, our knowledge of nature would be a mere tabulation of co-existences and sequences. We should still believe in the succession of day and night, of summer and winter; but the conception of Force would vanish from our universe; causal relations would disappear, and with them that seience which is now binding the parts of natnre to an organic whole.

I should like to illustrate by a few simple instances the use that scientific men have already made of this power of imagination, and to indicate afterward some of the further uses that they are likely to make of it. Let us.begin with the rudimentary experiences. Observe the falling of heavy rain-drops into a tranquil pond. Each drop as it strikes 
the water becomes a center of disturbance, from which : series of ring-ripples expand outward. Gravity and inertia are the agents by which this wave-motion is produced, and a rough experiment will suffice to show that the rate of propagation does not amount to a foot a second. A series of slight mechanical shocks is experienced by a body plunged in the water, as the warelets reach it in succession. But a finer motion is at the same time set up and propagated. If the head and ears be immersed in the water, as in an experiment of Franklin's, the tick of the drop is heard. Now, this sonorous impulse is propagated, not at the rate of a foot, but at the rate of 4,700 feet a second. In this case it is not the gravity but the elasticity of the water that comes into play. Every liquid particle pushed against its neighbor delivers up its motion with extreme rapidity, and the pulse is propagated as a thrill. The incompressibility of water, as illustrated by the famous Florentine experiment, is a measure of its elasticity; and to the possession of this property, in so high a degree, the rapid transmission of a sound-pulse through water is to be ascribed.

But water, as you know, is not necessary to the conduction of sommi; air is its most common velicle. And you know that when the air possesses the particular density and elasticity corresponding to the temperature of freezing water, the velocity of sound in it is 1,090 feet a second. It is almost exactly one-fourth of the relocity in water; the reason being that though the greater weight of the water tends to diminish the velocity, the enormous molecular elasticity of the liquid far more than atones for the disadvantage due to weight. By rarious contrivances we can compel the vibrations of the air to declare themselves; we know the length and frequency of the sonorous waves, and we have also obtained great mastery over the various methods by which the air is thrown into vibration. We know the phenomena and laws of vibrating rods, of organpipes, strings, membranes, plates, and bells. We can abolish one sound by another. We know the physical meaning of music and noise, of harmony and discord. In short, as regards sound in general, we have a very clear notion of the external physical processes which correspond to onr sensations.

In the phenomena of sound, we travel a very little way 
from downright sensible experience. Still the imagination is to some extent exercised. The bodily eye, for example, cannot see the condensations and rarefactions of the waves of sound. We construct them in thought, and we believe as firmly in their existence as in that of the air itself. But now our experience is to be carried into a new region, where a new use is to be made of it. Having mastered the cause and mechanism of sound, we desire to know the canse and mechanism of light. We wish to extend our inquiries from the anditory to the optic nerve. There is in the human intellect a power of expansion-I might almost call it a power of creation-which is brought into play by the simple brooding upon facts. The legend of the spirit brooding over chaos may have originated in experience of this power. In the case now before us it has manifested itself by transplanting into space, for the purposes of light, an adequately modified form of the mechanism of sound. We know intimately whereon the velocity of sound depends. When we lessen the density of the aërial medium, and preserve its elasticity constant, we augment the velocity. When we heighten the elasticity, and keep the density constant, we also augment the velocity. A small density, therefore, and a great elasticity, are the two things necessary to rapid propagation. Now light is known to move with the astounding velocity of 186,000 miles a second. How is such a velocity to be obtained? By boldly diffusing in space a medium of the requisite tenuity and elasticity.

Let us make such a medium our starting-point, and, endowing it with one or two other necessary qualities, let us handle it in accordance with strict mechanical laws. Let us then carry our results from the world of theory into the world of sense, and see whether our deductions do not issue in the very phenomena of light which ordinary knowledge and skilled experiment reveal. If in all the multiplied varieties of these phenomena, including those of the most remote and entangled description, this fundamental conception always bring us face to face with the truth; if no contradiction to our deductions from it be found in external nature, but on all sides ag!eement and verification; if, moreover, as in the case of Conical Refraction and in other cases, it actnally forces upon our attention phenomena which no eye had previously seen, and which no 
mind had previously imagined-such a conception, mus' we think, be something more than a mere figment of the scientific fancy. In forming it, that composite and creative power, in which reason and imagination are united, has, we believe, led us into a world not less real than that of the senses, and of which the world of sense itself is the suggestion and, to a great extent, the outcome.

Far be it from me, however, to wish to fix you immovably in this or in any other theoretic conception. IVith all our belief of it, it will be well to keep the theory of a luminiferous ether plastic and capable of change. You may, moreover, urge that, although the phenomena occur as if the medium existed, the absolute demonstration of its existence is still wanting. Far be it from me to deny to this reasoning such validity as it may fairly claim. Let us endeavor by means of analogy to form a fair estimate of its force. You believe that in society you are surrounded by reasonable beings like yourself. You are, perhaps, as firmly convinced of this as of anything. What is your warrant for this conviction? Simply and solely this: your fellow creatures behave as if they were reasonable; the hypothesis, for it is nothing more, accounts for the facts. 'Io take an eminent example: you believe that our president is a reasonable being. Why? There is no known method of superposition by which any one of us can apply himself intellectually to any other, so as to demonstrate coincidence as regards the possession of reason. If, therefore, you hold our president to be reasonable, it is because he behaves as if he were reasonable. As in the case of the ether, beyond the "as if" you cannot go. Nay, I should not wonder if a close comparison of the data on which both inferences rest caused many respectable persons to conclude that the ether had the best of it.

This universal medium, this light-ether as it is called, is the vehicle, not the origin, of wave-motion. It receives and transmits, but it does not create. Whence does it derive the motions it conveys? For the most part from luminous bodies. By the motion of a luminous body I do not mean its sensible motion, such as the flicker of a candle, or the shooting out of red prominences from the limb of the sun. I mean an intestine motion of the atoms or molecules of the luminous body. But here a certain reserve is 
necessary. Many chemists of the present day refuse to speak of atoms and molecules as real things. Their caution leals them to stop short of the clear, sharp, mechanically intelligible atomic theory enunciated by Dalton, or anv form of that theory, and to make the doctrine of " $\mathrm{mul}$. tiple proportions" their intellectual bourne. I respect the caution, though I think it is here misplaced. 'I'he chemists who recoil from these notious of atoms and molecules accept, without hesitation, the Undulatory 'Theory of Light. Like you and me they one and all believe in an ether and its light-producing waves. Let us consider what this belief involves. Bring your imaginations once more into play, and figure a series of sound-waves passing through air. Follow them up to their origin, and what do you there find? A definite, tangible, vibrating body. It may be the vocal chords of a human being, it may be an organ-pipe, or it may be a stretched string. Follow in the same manner a train of ether-waves to their source; remembering at the same time that your ether is matter, dense, elastic, and capable of motions subject to, and determined by, mechanical laws. What then do you expect to find as the source of a series of ether-waves? Ask your imagination if it will accept a vibrating multiple proportion-a numerical ratio in a state of oscillation? I do not think it will. You cannot crown the edifice with this abstraction. The scientific imagination, which is here authoritative, demands, as the origin and cause of a series of ether-waves, a particle of vibrating matter quite as definite, though it may be excessively minute, as that which gives origin to a musical sound. Such a particle we name an atom or a molecule. I think the intellect, when focused so as to give definition without penumbral haze, is sure to realize this image at the last.

With the view of preserving thought continuous throughout this discourse, and of preventing either failure of knowledge or of memory, from causing any rent in our picture, I liere propose to run rapidly over a bit of ground which is prohably familiar to most of you, but which I am anxious to make familiar to you all. 'The waves generated in the ether by the swinging atoms of luminous bodies are of different lengths and amplitudes. The amplitude is the 
width of swing of the individual particles of the waves. In water-waves it is the vertical height of the crest above the trough, while the length of the wave is the horizontal distance between two consecutive crests. 'The aggregate of waves emitted by the sun may be broadly divided into two classes: the one class competent, the other incompetent, to excite vision. But the light-producing waves differ markedly among thenselves in size, form, and force. The Jength of the largest of these waves is about twice that of the smallest, but the amplitude of the largest is probably a hundred times that of the smallest. Now the force or energy of the wave, which, expressed with reference to sensation, means the intensity of the light, is proportional to the square of the amplitude. Hence the amplitude being one-hundiedfold, the energy of the largest light-giving waves would be ten-thousandfold that of the smallest. This is not improbable. I use these figures not with a view to numerical accuracy, but to give you definite ideas of the differences that probably exist among the light-giving waves. And if we take the whole range of solar radiation into account-its non-visual as well as its visual waves-I think it probable that the force, or energy, of the largest wave is more than a million times that of the smallest.

Turned into their equivalents of sensation, the different light-waves produce different colors. Red, for example, is produced by the largest waves, violet by the smallest, while green is produced by a wave of intermediate length and amplitude. On entering from air into a more highly refracting substance, such as glass or water, or the sulphide of carbon, all the waves are retarded, but the smallest ones most. This furnishes a means of separating the different classes of waves from each other; in other words, of analyzing the light. Sent through a refracting prism, the waves of the sun are turned aside in different degrees from their direct course, the red least, the violet most. They are virtually pulled asunder, and they paint upon a white screen placed to receive them "the solar spectrum." Strictly speaking, the spectrum embraces an infinity of colors; but the limits of language, and of our powers of distinction, cause it to be divided into seven segments: rer, orange, yellow, green, blue, indigo, violet. These are the seven primary or prismatic colors.

Separately, or mixed in various proportions, the solar 
waves yield all the colors observed in nature and employed in art. Collectively, they give us the impression of whiteness. Pure unsifted solar light is white; and, if all the wave constituents of such light be reduced in the same proportion, the light, though diminished in intensity, will still be white. The whiteness of suow with the sun shining upon it, is barely tolerable to the eye. The same snow under an overcast firmament is still white. Such a firmament enfeebles the light by reflecting it upward; and when we stand above a cloud-field-on an Alpine summit, for instance, or on the top of Snowdon-and see, in the proper direction, the sun shining on the clouds below us, they appear dazzlingly white. Ordinary clouds, in fact, divide the solar light impinging on them into two parts-a reflected part and a transmitted part, in each of which the proportions of wave motion which produce the impression of whiteness are sensibly preserved.

It will be understood that the condition of whiteness would fail if all the waves were diminished equally, or by the same absolute quantity. 'They must be reduced proportionately, instead of equally. If by the act of reflection the waves of red light are split into exact halves, then, to preserve the light white, the waves of yellow, orange, green, and blue, must also be split into exact halves. In short, the reduction must take place, not by absolutely equal quantities, but by equal fractional parts. In white light the preponderance, as regards energy, of the larger over the smaller waves inust always be immense. Were the case otherwise, the visual correlative, blue, of the smaller waves would have the upper hand in our sensations.

Not only are the waves of ether reflected by clouds, by solide, and by liquids, but when they pass from light air to dense, or from dense air to light, a portion of the wavemotion is always reflected. Now our atmosphere changes continually in density from top to bottom. It will help our conceptions if we regard it as made up of a series of thin concentric layer's, or shells of air, each shell being of the same density throughont, a small and sudden change of density occurring in passing from shell to shell. Light would be reflected at the limiting surfaces of all these shells, and their action would be practically the same as that of the real atmosphere. And now I would ask your imagiuation to picture this act of reflection. What nust 
become of the reflected light? The atmospheric layers turn their convex surfaces toward the sun; they are so many convex mirrors of feeble power; and you will immediately perceive that the light regularly reflected from these surfaces cannot reach the earth at all, but is dispersed in space. Light thus reflected cannot, therefore, be the light of the sky.

But, though the sun's light is not reflected in this fashion from the aërial layers to the earth, there is indubitable evidence to show that the light of our firmament is scattered light. Proofs of the most cogent description could be here adduced; but we need only consider that we receive light at the same time from all parts of the hemisphere of heaven. The light of the firmment comes to us across the direction of the solar rays, and even against the direction of the solar rays; and this lateral and opposing rush of wave-motion can ouly be due to the rebound of the waves from the air itself, or from something suspended in the air. It is also evislent that, unlike the action of clouds, the solar light is not reflected by the sky in the proportions which produce white. The sky is blue, which indicates an excess of the shorter waves. In accounting for the color of the sky, the first question suggested by analogy would undoubtedly be, Is not the air blue? 'The blueness of the air has, in fact, been given as a solution of the blueness of the sky. But how, if the air be blue, can the light of sunrise and sunset, which travels through vast distances of air, be yellow, orange, or even red? 'The passage of white solar light through a blue medium conld by no possibility redien the light. 'The hypothesis of a blue air is therefore untenable. In fact the agent, whatever it is, which sends us the light of the sky, exercises in so doing a dichroitic action. 'The light reflected is blue, the light transmitterl is orange or red. A marked distinction is thus exhibitel between the matter of the sky, and that of an ordinary clond, which exercises no such dichroitic action.

By the scientific use of the imagination we may hope to penetrate this mystery. The cloul takes no note of size on the part of the waves of ether, but reflects them all alike. It exercises no selective action. Now the cause of this may be that the cloud particles are so large, in comparison with the waves of ether, as to reflect them all indifferently. A broad cliff reflects an Atlantic roller aș 
easily as a ripple producerl by a sen-bird's wing; and in the presence of large reflecting surfaces, the existing differences of magnitude among the waves of ether may disappear. But supposing the reflecting particles, instead of being very large, to be very small in comparison with the size of the waves. In this case, instead of the whole wave being fronterl and thrown back, a small portion only is shivered off. 'The great mass of the wave passes over such a particle without reflection. Scatter, then, a handful of such minute foreign particles in our atmosphere, and set imagination to watch their action upon the solar waves. Waves of all sizes impinge upon the particles, and you see at every collision a portion of the impinging wave struck off; all the waves of the spectrum, from the extreme red to the extreme violet, being thus acted upon.

Remembering that the red waves stand to the blue much in the relation of billows to ripples, we have to consider whether those extremely small particles are competent to scatter all the waves in the same proportion. If they be not-and a little reflection will make it clear that they are not-the production of color must be an incident of the scattering. Largeness is a thing of relation; and the smaller the wave, the greater is the relative size of any particle on which the wave impinges, and the greater also the ratio of the portion scattered to the total wave. A pebble, placed in the way of the ring-ripples produced by heavy raindrops on a tranquil pond, will scatter a large fraction of each ripple, while the fractional part of a larger watve thrown back by the same pebble might be infinitesimal. Now we have already male it clear to our minds that to preserve the solar light white, its coustituent proportions must not be altered; but in the act of division performed by these very small particles the proportions are altered; an undue fraction of the smaller waves is scattered by the particles, and, as a consequence, in the scattered light, blue will be the predominant color. 'The other color's of the spectrum must, to some extent, be associated with the blue. They are not absent, but deficient. We ought, in fact, to have them all, but in diminishing proportions, from the violet to the red.

We have here presented a case to the imagination, and, assuming the undulatory theory to be a reality, we have, I think, fairly reasoned our way to the conclusion, that 
were particles, small in comparison to the sizes of the ether waves, sown in our atmosphere, the light scattered by those particles would be exactly such as we observe in our azure skies. When this light is analyzed, all the colors of the spectrum are found, and they are found in the proportions indicated by our conclusion. Blue is not the sole, but it is the predomiuant color.

Let us now turn our attention to the light which passes unscattered among the particles. How must it be finally affected? By its successive collisions with the particles the white light is more and more robbed of its shorter waves; it therefore loses more and more of its due proportion of blue. The result may be anticipated. 'The transmitted light, where short distances are involved, will appear yellowish. But as the sun sinks toward the horizon the atmospheric distances increase, and consequently the number of the scattering particles. They abstract in succession the violet, the indigo, the blue, and even disturb the proportions of green. The transmitted light under such circumstances must pass from yellow through orange to red. 'This also is exactly what we find in nature. 'Thus, while the reflected light gives us at noon the deep azure of the Alpine skies, the transmitted light ives us at sunset the warm crimson of the Alpine snows. The phenomena certainly occur as if our atmosphere were a medium rendered slightly turbid by the mechanical sus. pension of exceedingly small foreign particles.

Here, as before, we encounter our skeptical "as if." It is one of the parasites of science, ever at hand, and ready to plant itself and sprout, if it can, on the weak points of our philosophy. But a strong constitution defies the parasite, and in our case, as we question the phenomena, probability grows like growing health, until in the end the malady of doubt is completely extirpated. The first question that naturally arises is this: Can small particles be really proved to act in the manner indicated? No doubt of it. Each one of you can submit the question to an experimental test. Water will not dissolve resin, but spirit will dissolve it; and when spirit holding resin in solution is dropped into water, the resin immediately separates in solid particles, which render the water milky. 'The coarseness of this precipitate depends on the quantity of the dissolved resin. You can cause it to separate either 
in thick clots or in exceedingly fine particles. Professor Brücke has given us the proportions which produce particles particularly suited to our present purpose. One gramme of clean mastic is dissolved in eighty-seven grammes of absolute alcohol, and the transparent solntion is allowed to drop inco a beaker containing clear water, kept briskly stirred. An exceedingly fine precipitate is thus formed, which declares its presence by its action upon light. Placing a dark surface behind the beaker, and permitting the light to fall into it from the top or front, the medium is seen to be distinctly blue. It is not perhaps so perfect a blue as may be seen on exceptional days among the Alps, but it is a very fair sky-blue. A trace of soap in water gives a tint of blue. London, and I fear Liverpool, milk makes an approximation to the same color, through the operation of the same canse; and Helmholtz has irreverently disclosed the fact that the deepest blue eye is simply a turbid medium.

The action of turbid media upon light was illustrated by Goethe, who, though unacquainted with the undulatory theory, was led by his experiments to regard the firmament as an illuminated turbid medium, with the darkness of space behind it. He describes glasses showing a bright yellow by transmitted, and a beantiful biue by reflected, light. Professor Stokes, who was probably the first to discern the real nature of the action of small particles on the waves of ether, * describes a glass of a similar kind. $\uparrow$ Capital specimens of such glass are to be found at Salviati's, in St. James' Street. What artists call "chill" is no doubt an effect of this description. Through the action of minute particles, the browns of a picture often present the appearance of the bloom of a plum. By rubbing the varnish with a silk hand kerchief optical continuity

* This is inferred from conversation. I am not aware that Professor Stokes has published anything upon the subject.

+ This glass, by reflected light, had a color "strongly resembling that of a decoction of horse-chestnut bark." Curiously enough, Goethe refers to this very decoction: "Man nelume einen Streifen friscluer Rinde von der Rosskastanie, man stecke denselben in ein Glas Wasser, und in der kirzesten Zeit werden wir das vollkom. menste Himmelblau entstehen sehen."-Goethe's Werke, B. xxix. p. 24 . 
is established and the chill disappears. Some yeurs ago I witnessed Mr. Hirst experimenting at Zermatt on the turbid water of the Visp. When kept still for a day or so, the grosser matter sank, but the finer particles remainea suspended, and gave a distinctly blue tinge to the water. The blueness of certain Alpine lakes has been shown to be in part due to this cause. Professor Roscoe has noticed several striking cases of a similar kind. In a very remarkable paper the late Principal Forbes showed that steam issuing from the safety-valve of a locomotive, when favorably observed, exhibits at a certain stage of its condensation the colors of the sky. It is blue by reflected light, and orange or red by transmitted light. 'The same effect, as pointed out by Guethe, is to some extent exhibited by peatsmoke. More than ten years ago, I amused myself by observing, on a calm day at Killarney, the straight smokecolumns rising from the cabin-chimneys. It was easy to project the lower portion of a column against a dark pine, and its upper portion against a bright clond. The smoke in the former case was blue, being seen mainly by reflected light; in the latter case it was reddish, being seen mainly by transmitted light. Such smoke was not in exactly the condition to give us the glow of the Alps, but it was a step in this direction. Brücke's fine precipitate above referred to looks yellowish by transmitted light; but, by duly strengthening the precipitate, you may render the white light of noon as ruby-colored as the sun, when seen through Liverpool smoke, or upon Alpine horizons. I do not, however, point to the gross smoke arising from coal as an illustration of the action of small particles, becanse such smoke soon absorbs and destroys the waves of blue, instead of sending them to the eyes of the observer.

'These multifarious facts, and numberless others which cannot now be referred to, are explained by reference to the single principle, that, where the scattering particles are small in comparison to the ethereal waves, we have in the reflected light a greater proportion of the smaller waves, and in the transmitted light a greater proportion of the larger waves, than existed in the original white light. 'The consequence, as regards sensation, is that in the one case blue is predominant, and in the other orange or red. Our best microscopes can readily reveal objects not more than one-fifty-thousandth of an inch in diameter. This is less 
than the length of a wave of red light. Indeed a first-rate microscope would enable us to discern objects not exceeding in diameter the length of the smallest waves of the visible spectrum.* $\mathrm{By}$ the microscope, therefore, we can test our particles. If they be as large as the light-wares they will infallibly be seen; and if they be not so seen, it is because they are smaller. Some months ago I placed in the hands of our president a liquid cuntaining Brücke's precipitate. The liquid was milky blue, and Mr. Huxley applied to it his highest microscopic power. He satisfied me that had particles of even one-one-hundred-thonsand th of an inch in diameter existed in the liquid, they could not have escaped detection. But no particles were seen. Under the microscope the turbid liquid was not to be distinguished from distilled water. $†$

But we have it in our power to imitate, far more closely than we have hitherto done, the natural conditions of this problem. We can generate, in air, artificial skies, and prove their perfect identity with the natural one, as regards the exhibition of a number of wholly unexpecter phenomena. By a continuous process of growth, moreover, we are able to connect sky-matter, if I may use the term, with molecular matter on the one side, and with molar matter, or matter in sensible masses, on the other. In illustration of this, I will take an experiment suggested by some of my own researches, and described by M. Morren of Marseilles at the Exeter meeting of the British Association. Sulphur and oxygen combine to form sulphurous acid gas, two atoms of oxygen and one of sulphur constituting the molecule of sulphurous acid. It has been recently shown that waves of ether issuing from a strong source, such as the sun or the electric light, are competent to shake asunder the atoms of gaseous molecules. I A chemist would call this, "decomposition" by light; but it belsooves us, who are examining the power and function of the imagination,

* Dallinger and Drysdale have recently measured cilia one-twohundred-thousandth of an inch in diameter. 1878.

† Like Dr. Burdon Sanderson's "pyrogen," the particles of mastic passed without sensible hindrance, through filtering-paper. By such filtering no freedom frou suspended particles is secured. The application of a condensed beam to the filtrate renders this at once evident.

† See "New Chemical Reactions Produced by Light," vol. i. 
to keep constantly before us the physical images which underlie our terms. Therefore I say, sharply and definitely, that the components of the molecules of sulphurous acid are shaken asunder by the ether-waves. Enclosing sulphurous acid in a suitable vessel, placing it in a dark room, and sending through it a powerful beam of light, we at first see nothing: the vessel containing the gas seems as empty as a vacuum. Soon, however, along the track of the beam a beautiful sky-blue color is observed, which is due to light scattered by the liberated particles of sulphur. For a time the blue grows more intense; it then becomes whitish; and ends in a more or less perfect white. When the action is continued long enough, the tube is filled with a dense cloud of sulplur particles, which by the application of proper means may be rendered individually visible.*

Here, then, our ether-waves untie the bond of chemical affinity, and liberate a body-sulphur-which at ordinary temperatures is a solid, and which therefore soon becomes an object of the senses. We have first of all the free atoms of sulphur, which are incompetent to stir the retina sensibly with scattered light. But these atoms gradually coalesce and form particles, which grow larger by continual accretion, until after a minute or two they appear as skymatter. In this condition they are individually invisible; but collectively they send an amount of wave-motion to the retina, sufficient to produce the firmamental blue. The particles continue, or may be caused to continue, in this condition for a considerable time, during which no microscope can cope with them. But they grow slowly larger, and pass by insensible gradations into the state of cloud, when they can no longer elude the armed eye. Thus, without solution of continuity, we start with matter in the atom, and end with matter in the mass; sky-matter being the middle term of the series of transformations.

Instead of sulphurous acid, we might choose a dozen other substances, and produce the same effect with all of them. In the case of some-probably in the case of all-it is possible to preserve matter in the firmamental condition

* M. Morren was mistaken in supposing that a modicum of sulphurous acid, in the drying tubes, had any share in the production of the "actinic clouds" described by me. A beautiful case of molecular instability in the presence of light is furnished by peroxide of chlorine as proved by Professor Dewar. 1878. 
for fifteen or twenty minutes under the continual operation of the light. During these fifteen or twenty minutes the particles constantly grow larger, without ever exceeding the size requisite to the production of the celestial blue. Now when two vessels are placed before us, each containing sky-matter, it is possible to state with great distinctness which vessel contains the largest particles. 'The eye is very sensitive to differences of light, when, as in our experiments, it is placed in comparative darkness, and the wavemotion thrown against the retina is small. 'T'he larger particles declare themselves by the greater whiteness of their scattered light. Call now to mind the observation, or effort at observation, made by our president, when he failed to distinguish the particles of mastic in Brücke's medium, and when you have done this, please follow me. A beam of light is permitted to act upon a certain vapor. In two minutes the azure appears, but at the end of fifteen minutes it has not ceased to be azure. After fifteen minutes its color, and some other phenomena, pronounce it to be a blue of distinctly smaller particles than those sought for in vain by Mr. Huxley. These particles, as already stated, must have been less than a hundred thousandth of an inch in diameter. And now I want you to consider the following question: Here are particles which have been growing continually for fifteen minutes, and at the end of that time are demonstrably smaller than those which defied the microscope of $\mathrm{Mr}$. Huxley. What must have been the size of these particles at the beginning of their growth? What notion can you form of the magnitude of such particles? The distances of stellar space give 118 simply a bewildering sense of vastness, without leaving any distinct impression on the mind; and the magnitudes with which we have here to do bewilder us equally in the opposite direction. We are dealing with infinitesimals, compared with which the test objects of the microscope are literally immense.

Small in mass, the vastness in point of number of the particles of our sky may be inferred from the continuity of its light. It is not in broken patches, nor at scattered points, that the heavenly azure is revealed. T'o the observer on the summit of Mont Blanc, the blue is as uniform and coherent as if it formed the surface of the most close grained solid. A marble dome would not exhibit a 
stricter continuity. And Mr. Glaisher will inform you, that if our hypothetical shell were lifted to twice the height of Mont Blanc above the earth's surface, we should still have the azure overhead. By day this light quenches the stars; even by moonlight it is able to exclude from vision all stars between the fifth and the eleventh magnitude. It may be likened to a noise, and the feebler stellar radiance to a whisper drowned by the noise.

What is the nature of the particles which shed this light? The celebrated De la Rive ascribes the haze of the Alps in fine weather to floating organic germs. Now the possible existence of germs in such profusion has been held up as an absurdity. It has been affirmed that they would darken the air, and on the assumed impossibility of their existence in the requisite numbers, without invasion of the solar light, an apparently powerful argument has been based by believers in spontaneous generation. Similar arguments have been used by the opponents of the germ theory of epidemic disease, who have triumphantly challenged an appeal to the microscope and the chemist's balance to decide the question. Such arguments, however, are founded on a defective acquaintance with the powers and properties of matter. Without committing myself in the least to De la Rive's notion, to the doctrine of spontaneous generation, or to the germ theory of disease, I would simply draw attention to the demonstrable fact, that, in the atmosphere here, we have particles which defy both the microscope and the balance, which do not darken the air, and which exist, nevertheless, in multitude sufficient to reduce to insignificance the Israelitish hyperbole regarding the sands upon the seashore.

The varying judgments of men on these and other questions may perhaps be, to some extent, accounted for by that doctrine of Relativity which plays so important a part in philosophy. This doctrine affirms that the impressions made upon us by any circumstance, or combination of circumstances, depend upon our previous state. 'Two travelers upon the same height, the one having ascended to it from the plain, the other having descended to it from a higher elevation, will be differently affected by the scene around them. To the one nature is expanding, to the other it is contracting, and impressions which have two 
such different antecedent states are sure to differ. In our scientific julgments the law of relativity may also play an important part. To two men, one educated in the school of the senses, having mainly occupied himself with observation; the other educated in the school of imagination as well, and exercised in the conceptions of atoms and molecules to which we have so frequently referred, a bit of matter, say one fifty-thousandth of an inch in diameter, will present itself differently. 'The one descenls to it from his molar heights, the other climbs to it from his molecular lowlands. 'To the one it appears small, to the other large. So, also, as regurds the appreciation of the most minute forms of life revealed by the microscope. To one of the men these naturally appear conterminous with the ultimate particles of matter; there is but a step from the atom to the organism. The other discerns numberless organic gradations between both. Compared with his atoms, the smallest vibrios and bacteria of the microscopic field are as behemoth and leviathan. The law of relativity may to some extent explain the different attitudes of two such persous with regard to the question of spontaneous generation. An amount of evidence which satisfies the one entirely fails to satisfy the other: and while to the one the last bold defense and startling expansion of the doctrine by Dr. Bastian will appear perfectly conclusive, to the other it will present itself as merely imposing a labor of demolition on subsequent investigators.*

Let me suy here that many of our physiological observers appear to form a very inadequate estimate of the distance which separates the microscopic from the molecular limil, and that as a consequence, they sometimes employ a phraseology calculated to mislead. When, for example, the contents of a cell are described as perfectly homogeneous or as absolutely structureless, because the nicroscope fails to discover any structure; or when two structures are pronounced to be without difference, because the microscope can discover none, then, I think the microscope begins to play a inischievous part. A little consideration will make it plain that the microscope can have no voice in the question of germ structure. Distilled water is more perfectly

* When these words were uttered I dicl not imagine that the chief labor of demolition would fall upon myself. 1878. 
homogeneous than any possible organic germ. What is it that causes the liquid to cease contracting at 39 degrees Fahr., and to expand until it freezes? We have here a structural process of which the microscope can take no rote, nor is it likely to do so by any conceivable extension of its powers. Place distilled water in the field of an electro-magnet, and bring a microscope to bear upon it. Will any change be observed when the magnet is excited? Absolutely none; and still profound and complex changes have occurred. First of all, the particles of water have been rendered diamagnetically polar; and secondly, in virtue of the structure impressed upon it by the magnetic whirl of its molecules, the liquid twists a ray of light in a fashion perfectly determinate both as to quantity and direction.

Have the diamond, the amethyst, and the conntless other crystals formed in the laboratories of nature and of man no structure? Assuredly they have; but what can the microscope make of it? Nothing. It cannot be too distinctly borne in mind that between the microscopic limit, and the true molecular limit, there is room for infinite permutations and combinations. It is in this region that the poles of the atoms are arranged, that tendency is giren to their powers; so that when these poles and powers have free action, proper stimulus, and a suitable environment, they determine, first the germ, and afterward the complete organism. This first marshaling of the atoms, on which all subsequent action depends, baffles a keener power than that of the microscope. When duly pondered, the complexity of the problem raises the doubt, not of the power of our instrument, for that is nil, but whether we ourselves possess the intellectual elements which will ever enable us to grapple with the ultimate structural energies of nature.*

* "In using the expression 'one sort of living substance' I must guard against being supposed to mean that any kind of living protoplasm is homogeneous. Hyaline though it may appear, we are not at present able to assign any limit to its complexity of structure." Burdon Sanderson, in the "British Medical Journal," January 16, 1875.

We have here scientific insight, and its correlative caution. In fact Dr. Sanderson's important researches are a continued illustration of the position laid down above. 
In more senses than one Mr. Darwin has drawn heavily upon the scientific tolerance of his age. He has drawn heavily upon time in his development of species, and he has drawn adventurously upon matter in his theory of pangenesis. Accorling to this theory, a germ, already microscopic, is a world of minor germs. Not only is the organism as a whole wrapped up in the germ, but every organ of the organism has there its special seed. This, I say, is an adventurnus draft on the power of matter to divide itself and distribute its forces. But, unless we are perfectly sure that he is overstepping the bounds of reason, that he is unwittingly sinning against observed fact or demonstrated law - for a mind like that of Darwin can never sin wittingly against either fact or law-we ought, I think, to be cautious in limiting his intellectual horizon. If there be the least doubt in the matter, it ought to be given in favor of the freedom of such a mind. To it a vast possibility is in itself a dynamic power, though the possibility may never be drawn upon. It gives me pleasure to think that the facts and reasonings of this discourse tend rather toward the justification of Mr. Darwin, than toward his condemnation; for they seem to show the perfect competence of matter and force, as regards divisibility and distribution, to bear the heaviest strain that he has hitherto imposed upon them.

In the case of Mr. Darwin, observation, imagination, and reason combined have run back with wonderful sagacity and success over a certain length of the line of biological succession. Guided by analogy, in his "Origin of Species" he placed at the root of life a primordial germ, from which he conceived the amazing variety of the organisms now upon the earth's surface might be deduced. If this hypothesis were even true, it would not be final. 'The human mind would infallibly look behind the germ, and however hopeless the attempt, would inquire in to the history of its genesis. In this dim twilight of conjecture the searcher welcomes every gleam, and seeks to angment his light by indirect incidences. He studies the methods of nature in the ages and the worlds within his reach, in order to shape the course of speculation in antecedent ages and worlds. And though the certainty possessed by experimental inquiry is here shut out, we are not left entirely without guidance. From the examination of the solar 
system, Kant and Laplace came to the conclusion that its various bodies once formed parts of the same undislocated mass; that matter in a nebulous form preceded matter in its present form; that as the ages rolled away, heat was wasted, condensation followed, planets were detached; and that finally the chief portion of the hot cloud reached, by self-compression, the magnitude and density of our sun. The earth itself offers evilence of a fiery origin; and in our day the hypothesis of Kant and Laplace receives the independent countenance of spectrum analysis, which proves the same substances to be common to the earth and sun.

Accepting some such view of the construction of our system as probable, a desire immediately arises to connect the present life of our planet with the past. We wish to know something of our remotest ancestry. On its first detachment from the central mass, life, as we understand it, could not have been present on the earth. How, then, did it come there? The thing to be encouraged here is a reverent freedom-a freedom preceded by the hard discipline which checks licentiousness in speculationwhile the thing to be repressed, both in science and out of it, is dogmatism. And here I am in the hands of the meeting-willing to end, but ready to go on. I have no right to intrude upon you, unasked, the unformed notions which are floating like clouds, or gathering to more solid consistency, in the modern speculative scientific mind. But if you wish me to speak plainly, honestly, and undisputatiously, I am willing to do so. On the present occasion-

You are ordained to call, and I to come.

Well, your answer is given, and I obey your call.

'T'wo or three years ago, in an ancient London college, I listened to a discussion at the end of a lecture by a very remarkable man. Three or four hundred clergymen were present at the lecture. The orator began with the civilization of Egypt in the time of Joseph; pointing out the very perfect organization of the kingdom, and the possession of chariots, in one of which Joseph rode, as proving a long antecedent period of civilization. He then passed on to the mud of the Nile, its rate of angmentation, its present thickness, and the remains of human handiwork found 
therein: thence to the rocks which bound the Nile valley, and which teem with organic remains. Thus in his own clear way he cansed the illea of the world's age to expand itself indefinitely before the minds of his andience, and he contrasted this with the age usually assigned to the world. During his discourse he seemed to be swimming against a stream, he manifestly thought that he was opposing a general conviction. He expected resistance in the subsequent discussion; so did I. But it was all a mistake; there was no adverse current, no opposing conviction, no resistance: merely here and there a half-humorous, but unsuccessful attempt to entangle him in his talk. The meeting agreed with all that had been said regarding the antiquity of the earth and of its life. They had, indeed, known it all long ago, and they rallied the lecturer for coming among them with so stale a story. It was quite plain that this large body of clergymen, who were, I shonld say, to be ranked among the finest samples of their class, had entirely given up the ancient landmarks, and trunsported the conception of life's origin to an indefinitely distunt past.

'This leads us to the gist of our present inquiry, which is this: Dives life belong to what we call matter, or is it an independent principle inserted into matter at some suitable epoch-say when the physical conditions became such as to permit of the development of life? Iet us put the question with the reverence due to a faith and culture in which we all were cradled, and which are the unleniable historic antecedents of our present enlightenment. I say, let us put the question reverently, but let us also put it clearly and definitely. There are the strongest grounds for believing that during a certain period of its history the earth was not, nor was it fit to be, the theater of life. Whether this was ever a nebulous period, or merely a molten period, loes not signify much; and if we revert to the nebulous condition, it is because the probabilities are raally on its side. Our question is this: Did creative energy panse until the nebulous matter hal condensed, until the earth had been detached, until the solar fire had sofar withlrawn from the earth s vicinity as to permit a romst to gather romml the planet? Did it wait nntil the air was isolated; until the seas were formed; until evaporation, condensation and the descent of rain had be- 
gun; until the eroding forces of the atmosphere had weathered and decomposed the molten rocks so as to form soils; until the sun's rays had become so tempered by distance, and by waste, as to be chemically fit for the decompositions necessary to vegetable life? Having waited through these æons unti? the proper conditions had set in, did it send the fiat forth, "Let there be Life?" These questions define a hypothesis not without its difficulties, but the dignity of which in relation to the world's knowledge was demonstrated by the nobleness of the men whom it sustained.

Modern scientific thought is called upon to decide between this hypothesis and another; and public thought generally will afterward be called upon to do the same. But, however the convictions of individuals here and there may be influenced, the process must be slow and secular which commends the hypothesis of Natural Evolution to the public mind. For what are the core and essence of this hypothesis? Strip it naked, and you stand face to face with the notion that not alone the more ignoble forms of animalcular or animal life, not alone the nobler forms of the horse and lion, not alone the exquisite and wonderful mechanism of the human body, but that the human mind itself-emotion, intellect, will, and all their phenomena-were once latent in a fiery cloud. Surely the mere statement of such a notion is more than a refutation. But the hypothesis would probably go even farther than this. Many who hold it would probably assent to the position that, at the present moment, all our philosophy, all our poetry, all our science, and all our art-Plato, Shakspeare, Newton, and Raphael-are potential in the fires of the sun. We long to learn something of our origin. If the Evolution hypothesis be correct, even this unsatisfied yearning must have come to us across the ages which separate the primeval mist from the consciousuess of to-day. I do not think that any holder of the Evolution hypothesis would say that I overstate or overstrain it in any way. I merely strip it of all vagueness, and bring before you, unclothed and unvarnished, the notions by which it must stand or fall.

Surely these notions represent an absurdity too monstrous to be entertained by any sane mind. But why are such notions absurd, and why shouid sanity reject them? 
The law of Relativity, of which we have previously spoken, may find its application here. 'These Erolution notions are absurd, monstrous, and fit only for the intellectual gibbet, in relation to the ideas concerning matter which were drilled into us when young. Spirit and matter lave ever been presented to us in the rudest contrast, the one as all-noble, the other as all-vile. But is thiscorrect? Upon the answer to this question all deponds. Supposing that, instend of having the foregoing antithesis of spirit and matter presented to our youthful minds, we had been taught to regard them as equally worthy, and equally wonderful; to consider them, in fact, as two opposite faces of the selfsame mystery. Supposing that in youth we had been impregnated with the notion of the poet foethe, instead of the notion of the poet Young, and tamght to look upon matter, not as "brute matter," but as the "living garment of God;" do you not think that under these altered circumstances the law of Relativity might have had an ontcome different from its present one? Is it not probable that our repugnance to the idea of primeval union between spirit and matter might be considerably abaterl? Without this total revolution of the notions now prevalent, the Evulution hypothesis muststand condemued; but in many profoundly thoughtful minds such a revolution has already taken place. 'They degrale neither member of the mysterious duality referred to; but they exalt one of them from its abasement, and repeal the divorce hitherto existing between them. In substance, if not in worls, their position as regards the relation of spirit and matter is: "What God hath joined together, let not man put asunder."

You have been thus led to the outer rim of speculative science, for beyond the nebula scientific thought has never hitherto ventured. I have tried to state that which I considered ought, in fairness, to be outspoken. I neither think this Evolution hypothesis is to be flouted away contemptuously, nor that it ought to be denounced as wicked. It is to be brought before the bar of disciplined reason, and there justifierl or comlemmerl. I tet us hearken to thuse who wisely support it, and to those who wisely oppose it; and let us tolerate those, whose name is legion, who try fool. ishly to do either of these things. The only thing out of place in the discussion is dogmatism on eicher side. Fear 
not the Evolution hypothesis. Steady yourselves, in its presence, upon that faith in the ultimate triumph of truth which was expressed by old Gamaliel when he said: "If it be of God, ye cannot overthrow it; if it be of man, it will come to naught." Under the fierce light of scientific inquiry, it is sure to be dissipated if it possess not a core of truth. Trust me, its existence as a hypothesis is quite compatible with the simultaneous existence of all those virtues to which the term "Christian" has been applied. It does not solve-it does not profess to solve-the ultimate mystery of this universe. It leaves, in fact, that mystery untouched. For, granting the nebula and its potential life, the question, whence they came, would still remain to baffle and bewilder us. At bottom, the hypothesis does nothing more than "transport the conception of life's origin to an indefinitely distant past."

Those who hold the doctrine of Evolution are by no means ignorant of the uncertainty of their data, and they only yield to it a provisional assent. 'They regard the nebular hypothesis as probable, and, in the utter absence of any evidence to prove the act illegal, they extend the method of nature from the present into the past. Here the observed uniformity of nature is their only guide. Within the long range of physical inquiry, they have never discerned in nature the insertion of caprice. 'Throughout this range, the laws of physical and intellectual continuity have run side by side. Having thus determined the elements of their curve in a world of observation and experiment, they prolong that curve into an antecedent world, and accept as probable the unbroken sequence of development from the nebula to the present time. You never hear the really philosophical defenders of the doctrine of Uniformity speaking of impossibitities in nature. They never say, what they are constantly charged with saying, that it is impossible for the Builder of the universe to alter His work. Their business is not with the possible, but the actual-not with a world which might be, but with a world that $i s$. This they explore with a courage not unmixed with reverence, and according to methorls which, like the quality of a tree, are tested by their fruits. They have but one desire-to know the truth. They have but one fear-to believe a lie. And if they know the strength of science, and rely upon it with unswerving trust, they 
also know the limits beyond which science ceases to be strong. 'They best know that questions offer themselves to thonght, which science, as now prosecuted, has not even the tendency to solve. They have as little fellowship with the atheist who says there is no God, as with the theigt who professes to know the mind of God. "Two things," said Immanuel Kant, “ fill me with awe: the starry heavens and the sense of moral responsibility in man." And in his hours of health and strength and sanity. when the stroke of action has ceased, and the pause of reflection has set in, the scientific investigator finds himself overshadowed by the same awe. Breaking contact with the hampering details of earth, it associates him with a Power which gives fullness and tone to his existeuce, but which he can neither analyze nor comprehend.

\section{CHAPTER XXXI.}

\section{THE BELFAST ADDRESS.*}

There is one frod supreme over all gods, diviner than mortals,

Whose form is not like unto man's, and as unlike his nature;

But vain mortals imagine that gods like themselves are begotten,

With human sensutions and voice and corporeal uembers;

So, if oxeu or lims liad hands and could work in man's fashion.

Arid trace out with chisel or brush their conception of liodhead,

Then would horses depict gods like horses, and oxen like oxen,

Each kind the divine with its own form and nature endowing.

Xenuruanes of Coloruon (six centuries B. C.), Supernatural Religion, vol. i., p. 76.

Section 1.-An impulse inherent in primeval man turned his thoughts and questionings betimes toward the sources of natural phenomena. The same impulse, inherited and intensified, is the spur of scientific action today. Determined by it, by a process of abstraction from experience we form physical theories which lie beyond the pule of experience, but which satisfy the desire of the mind to see every uatural occurrence resting upon a cause. In forming their notions of the origin of things, our earliest historic (and doubtless, we might add, our prehistoric) ancestors pursued, as far as their intelligence permitted,

* Delivered before the British Association on Wednesday evening. Angust 19, 1874. 
the same course. They also fell back upon experience; but with this difference-that the particular experiences which furnished the warp and woof of their theories were drawn, not from the study of nature, but from what lay much closer to them-the obserration of men. Their theories accordingly took an anthropomorphic form. To supersensual beings, which, "however potent and invisible, were nothing but a species of human ereatures, perhaps raised from among mankind, and retaining all human passions and appetites,"* were handed over the rule and governance of natural phenomena.

Tested by observation and reflection, these early notions failed in the long run to satisfy the more penetrating intellects of our race. Far in the depths of history we find men of exceptional power differentiating themselves from the crowd, rejecting these anthropomorphic notions, and seeking to connect natural phenomena with their physical principles. But, long prior to these purer efforts of the understanding, the merchant had been abroad, and rendered the philosopher possible; commerce had been developed, wealth amassed, leisure for travel and speculation secured, while races educated under different conditions, and therefore differently informed and endowed, had been stimulated and sharpened by mutual contact. In those regions where the commercial aristocracy of ancient Greece mingled with their eastern neighbors, the sciences were born, being nurtured and developed by free-thinking and courageous men. The state of things to be displaced may be gathered from a passage of Euripides quoted by Hume. "There is nothing in the world; no glory, no prosperity. The gods toss all into confusion; mix everything with its reverse, that all of us, from our ignorance and uncertainty, may pay them the more worship and reverence." Now as science demands the radical extirpation of caprice, and the absolute reliance upon law in nature, there grew with the growth of scientific notions, a desire and determination to sweep from the field of theory this mob of gods and demons, and to place natural phenomena on a basis more congruent with themselves.

The problem which had been previously approached from above, was now attacked from below; theoretic effort

* Hume, " Natural History of Religion." 
passed from the super to the sub-sensible. It was folt that to construct the universe in idea, it was necessary to have some notion of its constitnent parts-of what Lucretius subsequently called the "First Beginnings." Abstracting again from experience, the leaders of scientific speculation reached at length the pregnant doctrine of atoms and molecules, the latest developments of which were set forth with such power and clearness at the last meeting of the British Association. 'Thought, no doubt, had long hovered about this doctrine before it attained the precision and completeness which it assumed in the mind of Democritus, * a philosopher who may well for a moment arrest our attention. "Few great men," says Lange, a non-materialist, in his excellent "History of Materialism," to the spirit and to the letter of which I am equally indebted, "have been so despitefully used by history as Democritus. In the distorted images sent down to 118 through unscientific traditions, there remains of him almost nothing but the name of 'the langhing philosopher,' while figures of inmeasurably smaller significance spread themselves out at full length before us." Lange speaks of Bacon's high appreciation of Democritus-for ample illustrations of which I am indebted to my excellent friend Mr. Spedding, the learned editor and biographer of Bacon. It is evident, indeed, that Bavon considererl Democritus to be a man of weightier metal than either Plato or Aristotle, though their philosophy "was noised and celebrated in the schools, amid the din and pomp of professors." It was not they, but Genseric and Attila and the barbarians, who destroyed the atomic philosophy. “For, at a time when all human learning had suffered shipwreck, these planks of Aristotelian and Platonic philosophy, as being of a lighter and more inflated substance, were preserved and came down to us, while things more solid sank and almost passed into oblivion."

The son of a wealthy father, Democritus devoted the whole of his inherited fortune to the culture of his mind. He traveled everywhere; visited Athens when Sucrates and Plato were there, but quittel the city without making himself known. Indeed, the dialectic strife in which Socrates so much delighted, hal no charm for Democritus, 
who held that "the man who readily contradicts, and uses many words, is unfit to learu anything truly right." He is said to have discovered and educated Protagoras the Sophist, being struck as much by the manner in which he, being a hewer of wood, tied up his faggots, as by the sagacity of his conversation. Democritus returued poor from his travels, was supported by his brother, and at length wrote his great work entitled " Diakosmos," which he read publicly before the people of his native town. He was honored by his countrymen in various ways, and died serenely at a great age.

The principles enunciated by Democritus reveal his uncompromising antagonism to those who deduced the phenomena of nature from the caprices of the gods. They are briefly these: 1. From nothing comes nothing. Nothing that exists can be destroyed. All changes are due to the combination and separation of inolecules. 2. Nothing happens by chance; every occurrence has its cause, from which it follows by necessity. 3. The only existing things are the atoms and empty space; all else is mere opiniun. 4. The atoms are infinite in number and infinitely various in form; they strike together, and the lateral motions and whirlings which thus arise are the beginnings of worlds. 5 . The varieties of all things depend upon the varieties of their atoms, in number, size and aggregation. 6. The soul consists of fine, smooth, round atoms, like those of fire. 'These are the most mobile of all: they interpenetrate the whole body, and in their motions the phenomena of life arise.

The first five propositions are a fair general statement of the atomic philosophy, as now held. As regards the sixth, Democritus made his finer atoms do duty for the nervous system, whose functions were then unknown. 'The atoms of Democritus are individually without sensation; they combine in obedience to mechanical laws; and not only organic forms, but the phenomena of sensation and thought, are the result of their combination.

'That great enigma, "the exquisite adaptation of one part of an organism to another part, and to the conditions of life," more especially the construction of the human body, Democritus made no attempt to solve. Empedocles, a man of more fiery and poetic nature, introluced the notion of love and hate among the atoms, to account for 
their combination and separation; and bolder than Democritus, he struck in with the penetrating thought, linked, however, with some wild speculation, that it lay in the very nature of those combinations which were suited to their ends (in other words, in harmony with their environment) to maintain themselves, while unfit combinations, having no proper habitat, nust rapidly disappear. Thus, more than two thousand years ago, the doctrine of the "survival of the fittest," which in our day, not on the basis of vague conjecture, but of positive knowledge, has been raised to such extraordinary siguificance, had received at all events purtial enunciation."

Epicurus, $\uparrow$ said to be the son of a poor schoolmaster at Sumos, is the next dominant figure in the history of the atomic philosophy. He mastered the writings of Democritus, heard lectures in Athens, went back to Samos, and subsequently wandered through various countries. He finally returned to Athens, where he bought a garden, and surrounded himself by pupils, in the midst of whom he lived a pure and serene life, and died a peaceful death. Democritus looked to the soul as the ennobling part of man; even beauty, without understanding, partook of animalism. Epicurus also rated the spirit above the body; the pleasure of the body being that of the moment, while the spirit could draw upon the future and the past. His philosophy was almost identical with that of Democritus; but he never quoted either friend or foe. One main object of Epicurus was to free the world from superstition and the fear of death. Death he treated with indifference. It merely robs us of sensation. As long as we are, death is not; and when death is, we are not. Life has no more evil for him who lias made up his mind that it is no evil not to live. He adored the gods, but not in the ordinary fashion. The idea of divine power, properly purified, he thought an elevating one. Still he taught, "Not he is godless who rejects the gods of the crowd, but rather he who accepts them." The gods were to him eternal and immortal beings, whose blessedness excluded every thought of care or occupation of any kind. Nature pursues her course in accordance with everlasting laws, the gols never interfering. 'They haunt

* See "Lange," 2d edit., p. 28.

+ Burn 34: B. C. 


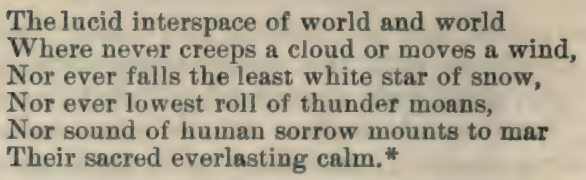

Lange considers the relation of Epicurus to the gods subjective; the indication, probably, of an ethical requirement of his own nature. We cannot read history with open eyes, or study human nature to its depths, and fail to discern such a requirement. Man never has been, and he never will be, satisfied with the operations and products of the understanding alone; hence physical science cannot cover all the demands of his nature. But the history of the efforts made to satisfy these demands might be broadly described as a history of errors-the error, in great part, consisting in ascribing fixity to that which is fluent, which varies as we vary, being gross when we are gross, and becoming, as our capacities widen, more abstract and sublime. On one great point the mind of Epicurus was at peace. He neither sought nor expected, here or hereafter, any personal profit from his relation to the gods. And it is assuredly a fact, that loftiness and serenity of thought may be promoted by conceptions which involve no idea of profit of this kind. "Did I not believe," said a great man $\nmid$ to me once, "that an Intelligence is at the heart of things, my life on earth would be intolerable." The utterer of these words is not, in my opinion, rendered less, but more noble by the fact that it was the need of ethical harmony here, and not the thought of personal happiness hereafter, that prompted his observation.

There are persons, not belonging to the highest intellectual zone, nor yet to the lowest, to whom perfect clearness of exposition suggests want of depth. They find comfort and edification in an abstract and learned phraseology. To such people Epicurus, who spared no pains to rid his style of every trace of haze and turbidity, appeared, on this very account, superficial He had, however, a disciple who thought it no unworthy occupation to spend his days and nights in the effort to reach the clearness of his master, and to whom the Greek philosopher is mainly indebted for

* Tennyson's “Lucretius.” - † Carlyle. 
the extension and perpetuation of his fame. Some two centuries after the death of Epicurus, Lncretius * wrote his great poen, "On the Nature of Things," in which he, a Roman, developed with extraordinary ardor the philosophy of his Greek predecessor. He wishes to win over his friend Memnius to the school of Epicurus; and although he has no rewards in a future life to offer, although his object appears to be a purely negative one, he add resses his friend with the heat of an apostle. His object, like that of his great forerunuer, is the destruction of superstition; and considering that men in his day trembled before eqvery natural event as a direct monition from the gools, and that everlasting torture was also in prospect, the freedom aimed at by Lueretius might be deemed a positive good. "This terror," he says. "and darkness of mind, must be dispelled, not by the rays of the sun and glittering shafts of day, but by the aspect and the law of nature." He refutes the notion that anything can come out of nothing, or that what is once begotten can be recalled to nothing. The first beginnings, the atoms, are indestructible, and into them all things can be resolved at last. Bodlies are partly atoms and partly combinations of atoms; but the atoms nothing can quench. They are strong in solid singleness, and, by their denser combination, all things can be closely packed and exhibit enduring strength. He denies that matter is infinitely divisible. We come at length to the atoms, without which, as an imperishable substratum, all order in the generation and development of things would be destroyed.

The mechanical shock of the atoms being, in his view, the all-sufficient cause of things, he combats the notion that the constitation of nature has been in any way determined by intelligent design. The interaction of the atoms thronghout infinite time rendered all manner of combinations possible. Of those, the fit ones persisted, while the unfit ones disappeared. Not after sage deliberation did the atoms station themselves in their riglit places, nor did they bargain what motions they should assume. From all eternity they have been driven together, and, after trying motions and unions of every kind, they fell at length into the arrangements out of which this 
system of things has been evolved. "If you will apprehend and keep in mind these things, Nature, free at once, and rid of her haughty lords, is seen to do all things spontaneously of herself, without the meddling of the gods." *

To meet the objection that his atoms cannot be seen, Lucretius describes a violent storm, and shows that the invisible particles of air act in the same way as the visible particles of water. We perceive, moreover, the different smells of things, yet never see them coming to our nostrils. Again, clothes hung up on a shore which waves break upon, become moist, and then get dry if spread out in the sun, though no eye can see either the approach or the escape of the water-particles. A ring, worn long on the finger, becomes thinner; a water-drop hollows out a stone; the plowshare is rubbed away in the field; the street-pavement is worn by the feet; but the particles that disappear at any moment we cannot see. Nature acts though invisible particles. That Lucretius had a strong scientific imagination the foregoing references prove. A fine illustration of his power iu this respect, is his explanation of the apparent rest of bodies whose atoms are in motion. He employs the image of a flock of sheep with skipping lambs, which, seen from a distance, presents simply a white patch upon the green hill, the jumping of the individual lambs being quite invisible.

+ His vaguely grand conception of the atoms falling eternally through space suggested the nebular hypothesis to Kant, its first propounder. Far beyond the limits of our visible world are to be found atoms innumerable, which have never been united to form bodies, or which, if once united, have been again dispersed-falling silently through immeasurable intervals of time and space. As everywhere throughout the All the same conditions are repeated, so must the phenomena be repeated also. Above us, below us, beside us, therefore, are worlds without end; and this, when considered, must dissipate every thonght of a deflection of the universe by the gods. The worlds come and go, attracting new atoms out of limitless space, - or dispersing their own particles. The reputed death of

* Monro's translation. In his criticism of this work (C'ontemporary Revievo, 1867) Dr. Hayman does not appear to be aware of the really sound and subtile observations on which the reasoning of Lucretius, though erroneous, sometimes rests. 
Lucretius, which forms the basis of Mr. Tennyson's noble poem, is in strict accordance with his philosophy, which was severe and pure.

Section 2.-Still earlier than these three philosophers, and during the centuries between the first of them and the last, the human intellect was active in other fields than theirs. Pythagoras had founded a school of mathematics, and made his experiments on the harmonic intervals. The Sophists had run through their career. At Athens had appeared Socrates, Plato, and Aristotle, who ruined the Sophists, and whose yoke remains to some extent unbroken to the present hour. Within this period also the school of Alexandria was founded, Euclid wrote his "Elements" and made some advance in optics. Archimedes had propounded the theory of the lever, and the principles of hydrostatics. Astronomy was immensely enriched by the discoveries of Hipparchus, who was followed by the historically more celebrated Ptolemy. Anatomy had been made the basis of scientific medicine; and it is said by Draper * that rivisection had begun. In fact, the science of ancient Greece had already cleared the world of the fantastic images of divinities operating capriciously through natural phenomena. It had shaken itself free from that fruitless scrutiny "by the internal light of the mind alone," which had rainly sought to transcend experience, and to reach a knowledge of ultimate causes. Instead of accidental observation, it had introduced observation with a purpose; instruments were employed to aid the senses; and scientific method was rendered in a great measure complete by the union of Iuduction and Experiment.

What, then, stopped its victorious advance? Why was the scientific intellect compelled, like an exhausted soil, to lie fallow for nearly two millenniums, before it could regather the elements necessary to its fertility and strength? Bacon has already let us know one cause; Whewell ascribes this stationary period to four causes - obscurity of thought, servility, intolerance of disposition, enthusiasm of cemper; and he gives striking examples of each. $\nmid$ But these char-

* "Histury of the Intellectual Development of Furope," p. 295.

f "History of the Inductive sicierces," vol. i. 
acteristics must have had their antecedents in the circumstances of the time. Rome, and the other cities of the empire, had fallen into moral putrefaction. Christianity had appeared, offering the Gospel to the poor, and by moderation, if not asceticism of life, practically protesting against the profligacy of the age. 'The sufferings of the early Christians, and the extraordinary exaltation of mind which enabled them to triumph over the diabolical tortures to which they were subjected, * must have left traces not easily effaced. They scomed the earth, in view of that " building of God, that house not made with hands, eternal in the heavens." The Scriptures which ministered to their spiritual needs were also the measure of their Science. When, for example, the celebrated question of Antipodes came to be discussed, the Bible was with many the ultimate court of appeal. Angustine, who flourished A.D. 400, would not deny the rotundity of the earth; but he would deny the pussible existence of inhabitants at the other side, "because no such race is recorded in Scripture among the descendants of Adam." Archbishop Boniface was shocked at the assumption of a "world of human beings out of the reach of the means of salvation." Thus reined in, Science was not likely to make much progress. Later on, the political and theological strife between the church and civil governments, so powerfully depicted by Draper, must have doue much to stifle investigation.

Whewell makes many wise and brave remarks regarding the spirit of the middle ages. It was a menial spirit. The seekers after natural knowledge had forsaken the fountain of living waters, the direct appeal to nature by observation and experiment, and given themselves up to the remanipulation of the notions of their predecessors. It was a time when thought had become abject, and when the acceptance of mere authority led, as it always does in science, to intellectual death. Natural events, instead of being traced to physical, were referred to moral causes; while an exercise of the phantasy, almost as degrading as the spiritualism of the present day, took the place of scientific speculation. Then came the mysticism of the middle ages, magic, alchemy, the Neoplatonic philosophy, with its visionary though sublime abstractions, which caused

* Described with terrible vividness in Renan's “Antichrist." 
men to look with shame upon their own bodies, as hindrances to the absorption of the creature in the blessedness of the Creator. Finally cume the scholastic philosophy, a fusion, according to Lange, of the least mature notions of Aristotle with the Christianity of the West. Intellectual immobility was the result. As a traveler without a compass in a fog may wander long, imagining he is making way, and find limself after hours of toil at his starting-point, so the schoolmen, having "tied and untied the same knots, and formed and dissipated the same clouds," * found themselves at the end of centuries in their old position.

With regard to the influence wielded by Aristotle in the middle ages, and which, to a less extent, he still wields, I would ask permission to make one remark. When the human mind has achieved greatness and given evidence of extraordinary power ia one domain, there is a tendency to credic it with similar power in all other domains. 'Thus theologians have found comfort and assurance in the thought that Newton dealt with the question of revelation -forgetful of the fact that the very devotion of his powers, through all the best years of his life, to a totally different class of ideas, not to speak of any natural disqualification, tended to render him less, instead of more competent to deal with theological and historic questions. Guethe, starting from his established greatness as a poet, and indeed from his positive discoveries in natural history, produced a profound impression among the painters of Germany, when he published his "Farbenlehre," in which he endeavored to overthrow Newton's theory of colors. This theory he deemed so obviously absurd, that he considered its author a charlatan, and attacked him with a corresponding vehemence of language. In the domain of natural history, Goethe had male really considerable discoveries; and we have high authority for assuming that, had he devoted himself wholly to that sile of science, he might have reached an eminence comparable with that which he attained as a poet. In sharpness of observation, in the detection of analogies apparenuly remote, in the classification and organization of facts according to the analogies discerned, Goethe possessed extraordinary powers. T'hese 
elements of scientific inquiry fall in with the disciplines of the poet. But, on the other hand, a mind thus richly endowed in the direction of natural history may be almost shorn of endowment as regards the physical and mechanical sciences. Goethe was in this condition. He could not formulate distinct mechanical conceptions; he could not see the force of mechanical reasoning; and in regions where such reasoning reigns supreme, he became a mere ignis fatuus to those who followed him.

I have sometimes permitted myself to compare Aristotle with Goethe-to credit the Stagirite with an almost superhuman power of amassing and systematizing facts, but to consider him fatally defective on that side of the mind, in respect to which incompleteness has been just ascribed to Goethe. Whewell refers the errors of Aristotle not to a neglect of facts, but to "a neglect of the idea appropriate to the facts; the idea of Mechanical cause, which is Force, and the substitution of vague or inapplicable notions, involving only relations of space or emotions of wonder." 'I'his is doubtless true; but the word "neglect" implies mere intellectual misdirection, whereas in Aristotle, as in Goethe, it was not, I believe, misdirection, but sheer natural incapacity which lay at the root of his mistakes. As a physicist, Aristotle displayed what we should consider some of the worst of attributes in a modern physical investigator-indistinctness of ideas, confusion of inind, and a confident use of language which led to the delusive notion that he had really mastered his subject, while he had, as yet, failed to grasp even the elements of it. He put words in the place of things, subject in the place of object. He preached Induction without practicing it, inverting the true order of inquiry, by passing from the general to the particular, instead of from the particular to the general. He made of the universe a closed sphere, in the center of which he fixed the earth, proving from general principles, to his own satisfaction and to that of the world for near two thousand years, that no other unirerse was possible. His notions of motion were entirely muphysical. It was natural or unnatural, better or worse, calm or violent-no real mechanical conception regarding it lying at the bottom of his mind. He affirmed that a vacuum could not exist, and proved that if it did motion in it would be impossible. He deternined à priori how many species of animals must 
exist, and showed on general principles why animals must haresuch and such parts. When an eminent contemporary philusopher, who is far removed from errors of this kind, remembers these abuses of the a priori method, he will be able to make allowance for the jealuusy of physicists as to the acceptance of so-called it priori truths. Aristotle's errors of detail, as shown by Eucken and Lange, were grave and numerous. He affirmed that only in man we hal the beating of the heart, that the left side of the body was colder than the right, that men have more teeth than women, and that there is an empty space at the back of every man's head.

'There is one essential quality in physical conceptions, which was entirely wanting in those of Aristotle and his followers-a capability of being placed as coherent pictures before the mind. The Germans express the act of picturing by the word rorstellen, and the picture they call a Forstellung. We have no word in English which comes nearer to our requirements than Imaginalion; and, taken with its proper limitations, the word answers very well. But it is tainter by its associations, and therefore objectionable to some minds. Compare, with reference to this capacity of mental presentation, the case of the Aristotelian, who refers the ascent of water in a pump to Nature's abhorrence of a racuum, with that of Pascal when he proposed to solve the question of atmospheric pressure by the ascent of the Puy de Dôme. In the one case the terms of the explanation refuse to fall into place as a physical image; in the other the image is distinct, the descent and rise of the barometer being clearly figured beforehand as the balancing of two varying and opposing pressures.

SEction 3.-During the drought of the middle ages in Christentom, the Arabian intellect, as forcibly shown by braper, was active. With the intrusion of the Moors into Spain, order, learning and refinement took the place of their opposites. When smitten with disease, the Christian peasant resorted to a shrine, the Moorish one to an instructed pligsician. The Arabs encouraged translations from the Greek philosophers, but not from the Greek poets. They turned in disgust " from the lewiness of our classical mythology, and denonnced us an unpardonablo blasphemy all connection between the impure Olympian 
Jove and the Most High God." Draper traces still further than Whewell the Arab elements in our scientific terms. He gives examples of what Arabian men of science accomplished, dwelling particularly on Alhazen, who was the first to correct the Platonic notion that rays of light are emitted by the eye. Alhazen discovered atmospheric refraction, and showed that we see the sun and the moon after they have set. He explained the enlargement of the sun and moon, and the shortening of the vertical diameters of both these bodies when near the horizon. He was aware that the atmosphere decreases in density with increase of elevation, and actually fixed its height at fifty-eight and one-half miles. In the "Book of the Balance of Wisdom," he sets forth the connection between the weight of the atmosphere and its increasing density. He slows that a body will weigh differently in a rare and dense atmosphere, and he considers the force with which plunged bodies rise through heavier media. He understood the doctrine of the center of gravity, and applied it to the investigation of balances and steelyards. He recognized gravity as a force, though he fell into the error of assuming it to diminish simply as the distance, and of making it purely terrestrial. He knew the relation between the velocities, spaces, and times of falling bodies, and had distinct ideas of capillary attraction. He improved the hydrometer. The determinations of the densities of bodies, as given by Alhazen, approach very closely to our own. "I join," says Draper, "in the pious prayer of Alhazen, that in the day of judgment the All-Merciful will take pity on the soul of Abur-Raihän, because he was the first of the race of men to construct a table of specific gravities." If all this be historic truth (and I have entire confidence in Dr. Draper), well may he "deplore the systematic manner in which the literature of Europe has contrived to put ont of sight our scientific obligations to the Mahommedans." *

The strain upon the mind during the stationary period toward ultra-terrestrial things, to the neglect of problems close at hand, was sure to provoke reaction. But the reaction was gradual; for the ground was dangerous, and a power was at hand competent to crush the critic who went too far. To elude this power, and still allow opportunity 
for the expression of opinion, the doctrine of "twofold truth" was invented, accorling to which an opinion might be held " theologically," and the opposite opinion "philosophically." * Thus, in the thirteenth century, the creation of the world in six days, and the unchangeableness of the individual soul, which had been so distinctly affirmed by St. Thomas Aquiuas, were both denied philosophically, but admitted to be true as articles of the Catholic faith. IV hen Protagoras utterei the maxim which bronght upon him so much vituperation, that "opposite assertions are equally true," he simply meant to affirm men's differences to be so great, that what was subjectively true to the one might be subjectively untrue to the other. T'ie great Sophist never meint to play fast and loose with the truth by saying that one of two opposite assertions, made by the same individual, could possibly escape being a lie. It was not "sophistry," but the dread of theologic vengeance, that generated this double dealing with conviction; and it is astonishing to notice what lengths were allowed to men who were alroit in the use of artifices of this kind.

Toward the close of the stationary period a word-weariness, if I may so express it, took more and more possession of men's minds. Christendom hal become sick of the School Philosophy and its verbal wastes, which led to no issue, but left the intellect in everlasting haze. Here and there was heard the voice of one impatiently crying in the wilderness, "Not unto Aristotle, not unto subtle hypothesis, not unto church, Bible, or blind tradition, must we turn for a knowledge of the universe, but to the direct investigation of nature by observation and experiment." In 1543 the epoch-marking work of Copernicus on the paths of the heavenly bodies appeared. The total crush of Aristotle's closed universe, with the earth at its center, followed as a consequence, and "The earth moves!" bocame a kind of watchword among intellectual freemen. Copernicus was canon of the church of Frauenburg in the diocese of Ermeland. For three-and-thirty years he hail withdrawn himself from the worlil, and levoted himself to the consolidation of his great sclieme of the solar system. He made its blocks eternal; and even to those who foared it, and desired its overthrow, it was so ohriously strong, 
that they refrained for a time from medilling with it. In the last year of the life of Copernicus his book appearerl: it is said that the old man receired a copy of it a few days before his death, and then departed in peace.

The Italian philosopher, Giordano Bruno, was one of the earliest converts to the new astronomy. Taking Lucretius as his exemplar, he revived the notion of the infinity of worlds; and, combining with it the doctrine of Copernicus, reached the sublime generalization that the fixed stars are suns, scattered numberless through space, and accompanied by satellites, which bear the same relation to them that our earth does to our sun, or our moon to our earth. This was an expansion of transcenclent import; but Brumo came closer than this to our present line of thought. Struck with the problem of the generation and maintenance of organisms, and duly pondering it, he came to the conclusion that Nature, in lier productions, does not imitate the technic of man. Her process is one of unraveling and unfolding. The infinity of forms under which matter appeurs was not imposed upon it by an external artificer; by its own intrinsic force and virtue it brings these forms forth. Matter is not the mere naked, empty capacity which philosophers have pictured her to be, but the universal mother, who brings forth all things as the fruit of her own womb.

This outspoken man was originally a Dominican monk. He was accusel of heresy and had to fly, seeking refuge in Geneva, Paris, England, and Germany. In 1592 he fell into the hands of the Inquisition at Venice. He was imprisoned for many years, tried, degraded, excommunicated, and handed over to the civil power, with the request that he should be treated gently, and "without the shedding of blood." This meant that he was to be burnt; and burnt accordingly he was, on February 16, 1600. 'To escape a similar fate Galileo, thirty-three years afterward, abjured upon his knees, with his hands upon the holy Gospels, the heliocentric doctrine, which he knew to be true. After Galileo came Kepler, who from his German home defied the ultramontane power. He traced ont from pre-existing observations the laws of planetary motion. Materials were thus prepared for Newton, who bound those empirical laws together by the principle of gravitation. 
Section 4.-In the seventeenth century Bacon and Descartes, the restorers of philosophy, appeared in sucession. Differently educated and endowed, their philosophie tendencies were different. Bacon held fast to Induction, believing firmly in the existence of an external world, and making collectenl experiences the basis of all knowledge. The mathematical studies of Descartes gave him a bias towaril deduction; and his fundamental principle was much the satme as that of Protagoras, who made the individual man the measure of all things. "I think, therefore I am," said Descartes. Only his own identity was sure to him; and the full development of this system would have led to an idealism, in which the onter world would have been resolsed into a mere phenomenon of consciousness. Gassencli, one of Descartes contemporaries, of whom we shall hear more presently, quickly pointed out that the fact of personal existence would be proved as well by reference to any other act, as to the act of thinking. I eat, therefore $I \mathrm{am}$, or I love, therefore I am, would be quite as conclusive. Lichtenberg, indeed, showed that the very thing to be proved was inevitably postulated in the first two words, "I think;" and it is plain that no inference from the postulate could, by any possibility, be stronger than the postulate itself.

But Descartes deviated strangely from the idealisn implied in his fundamental principle. He was the first to reduce, in a manner eminently capable of bearing the test of mental presentation, vital phenomena to purely mechanical principles. Through fear or love, Descartes was a good churchman; he accordingly rejected the notion of an atom, because it was absurd to suppose that God, if He so pleased, could not divide an atom; he puts in the place of the atoms small round particles, and light splinters, ont of which he builds the organism. He sketches with marvelous physical insight a machine, with water for its motire power, which shall illustrate vital actions. He has made clear to his mind that such a machine would be competent to carry on the processes of digestion, nutrition, growth, respiration, and the beating of the heart. It would be competent to accept impressions from the external sense, to store them up in imagination and memory, to go through the internal morements of the appetites and passions, and the external movements of the limbs. He deduces these 
functions of his machine from the mere arrangements of its organs, as the movement of a clock, or other automaton, is deduced from its weights and wheels. "As far as these functions are concerned," he says, " it is not necessary to conceive any other vegetative or sensitive soul, nor any other principle of motion or of life, than the blood and the spirits agitated by the fire which burns continually in the heart, and which is in nowise different from the fires existing in inanimate bodies." Had Descartes been acquainted with the steam-engine, he would have taken it, instead of a fall of water, as his motive power. He would have shown the perfect analogy which exists between the oxidation of the food in the body, and that of the coal in the furnace. He would assuredly have anticipated Mayer in calling the blood which the heart diffuses, "the oil of the lamp of life," deducing all animal motions from the combustion of this oil, as the motions of a steam-engine are deduced from the combustion of its coal. As the matter stands, however, and considering the circumstances of the time, the boldness, clearness, and precision, with which Descartes grasped the problem of vital dynamics constitute a marvelous illustration of intellectual power.*

During the middle ages the doctrine of atoms had to all appearance vanished from àiscussion. It probably held its ground among sober-minded and thougitful men, though neither the church nor the world was prepared to hear of it with tolerance. Once, in the year 1348, it received distinct expression. But retractation by compulsion immediately followed; and, thus discouraged, it slumbered till the seventeenth century, when it was revived by a cuntemporary and friend of Hobbes of Malmesbury, the orthodox Catholic provost of Digne, Gassendi. But, before stating his relation to the Epicurean doctrine, it will be well to say a few words on the effect, as regards science, of the general introduction of monotheism among European nations.

"Were men," says Hume, "led into the apprehension of invisible intelligent power by contemplation of the works of Nature, they could never possibly entertain any

* See Huxley's admirable "Essay on Descartes." “Lay Sermons," pp. $364,365$. 
conception but of one single Being, who bestowed existence and order on this vast machine, and adjusted all its parts to one regular system." Referring to the condition of the heathen, who soes a god behind every natural event, thus peopling the world with thousands of beings whose caprices are incalculable, Lange shows the impussibility of any compromise between such notions and those of science, which proceeds on the assumption of never-changing law and causality. "But," he continues, with characteristic penetration, "when the great thought of one fod, acting as a unit upon the universe, has been seized, the connection of things in accordance with the law of cause and effect is not only thinkable, but it is a necessary consequence of the assumption. For when I see ten thousand wheels in motion, and know, or believe, that they are all driven by one motive power, then I know that I have before me a mechanism, the action of every part of which is determined by the plan of the whole. So much being assumed, it follows that I may investigate the structure of that machine, and the various motions of its parts. For the time being, therefore, this conception renclers scientific action free." In other words, were a capricious God ut the circumference of every wheel and at the end of every lever, the action of the machine would be incalculable by the methods of science. But the actions of all its parts being rigidly determined by their connections and relations, and these being brought into play by a single motive power, then though this last prime mover may elude me, I am still able to comprehend the muchinery which it sets in motion. We have here a conception of the relation of Nature to its Author, which seems perfectly acceptable to some minds, but perfectly intolerable to others. Newton and Boyle lived and worked happily under the influence of this conception; Goethe rejected it with vehemence, and the same repugnance to accepting it is manifest in Cariyle.*

'The analytic and synthetic tendencies of the human

- Boyle's model of the universe was the Strashurg clock with an outside Artificer. Goethe, on the other hand, sang

"Ihm zient's die Welt in Innern au bewegen, Natur in sieh, sich in Natur zu hegen."

See also Ciarlyle, "Past and Present," chap. v. 
mind are traceable throughout history, great writers ranging themselves sometimes on the one side, sometimes on the other. Men of warm feelings, and minds open to the elevating impressions produced by nature as a whole, whose satisfaction, therefore, is rather ethical than logical, lean to the synthetic side; while the analytic harmonizes best with the more precise and more mechanical bias which seeks the satisfaction of the understanding. Some form of pantheism was usually adopted by the one, while a detached Creator, working more or less after the manner of men, was often assumed by the other. Gassendi, as sketched by Lange, is hardly to be ranked with either. Having formally acknowledged God as the great first cause, he immediately dropped the idea, applied the known laws of mechanics to the atoms, and deduced from them all vital phenomena. He defended Epicurus, and dwelt upon his purity, both of doctrine and of life. True, he was a heathen, but so was Aristotle. Epicurus assailed superstition and religion, and rightly, because he did not know the true religion. He thought that the gods neither rewarded nor punished, and he adored them purely in consequence of their completeness: here we see, says Gassendi, the reverence of the child, instead of the fear of the slave. 'The errors of Epicurus shall be corrected, and the body of his truth retained. Gassendi then proceeds, as any heathen might have done, to build up the world, and all that therein is, of atoms and molecules. God, who created earth and water, plants and animals, produced in the first place a definite number of atoms, which constituted the seed of all things. 'Then began that series of combinations and decompositions which now goes on, and which will continue in future. The principle of every change resides in matter. In artificial productions the moving principle is different from the material worked upon; but in nature the agent works within, being the most active and mobile part of the material itself. 'Thus this bold ecclesiastic, withont incurring the censure of the church or the world, contrives to outstrip Mr. Darwin. The same cast of mind which caused him to detach the Creator from his universe led him also to detach the soul from the body, though to the body he ascribes an influence so large as to render the soul almost unnecessary. 'The aberrations of reason were, in his view, an affair of the material brain. Mental disease 
is bruin-disease; but then the immortal reason sits apart, and cannot be touched by the disease. The errors of madness are those of the instrument, not of the performer.

It may be more than a mere result of edncation, connecting itself, probably, with the deeper mental structure of the two men, that the idea of Gassendi, above enunciated, is substantially the same as that expressed by Professor Clerk Maxwell, at the close of the very able lecture delivered by him at Bradford in 1873. According to both philosophers, the atoms, if I understand aright, are prepareal materials, which, formed once for all by the Etwrnal, produce by their subsequent interaction all the phenomena of the material world. There seems to be this difference, however, between Gassendi and Maxwell. 'The one postulates, the other infers his first cause. In his "manufactured articles," us he calls the atoms, Professur Maxwell finds the basis of an induction, which enables him to scale philosophic heights considered inaccessible by Kant, and to take the logical step from the atoms to their Maker.

Accepting here the leadership of Kant, I doubt the legitimacy of Maxwell's logic; but it is impossible not to feel the ethic glow with which his lecture conoludes. There is, moreover, a very noble strain of eloquence in his description of the steadfastness of the atoms: "Natural causes, as we know, are at work, which tenil to modify, if they do not at length destroy, all the arrangements and dimensions of the earth and the whole solar system. But though in the course of ages catastrophes have occurred and may yet occur in the heavens, thongh ancient systems may be dissolved and new systems evolved out of their ruins, the molecules out of which these systems are builtthe foundation stones of the material universe-remain unbroken and unworn."

The atomic doctrine, in whole or in part, was entertained by Bacon, Deseartes, IIobbes, Locke, Newton, Boyle, and their successors, until the chemical law of multiple proportions enabled Dalton to confer upon it an entirely new gignificance. In our day there are secessions from the theory, but it still stamis firm. Loschmidt, Stones, and Sir William Thomson have sought to determine the sizes of the atoms, or rather (o) fix the limits between which their sizes lie; while the disconres of Williamson and Maxwell delivered in Bradford in 1873 illustrute the present 
hold of the doctrine upon the foremost scientifie minds. In fact, it may be doubted whether, wanting this fundamental conception, a theory of the material universe is capable of scientific statement.

Section 5.-Ninety years subsequent to Gassendi the doctrine of bodily instruments, as it may be called, assumed immense importance in the hands of Bishop Butler, who, in his famous " Analogy of Religion," developed, from his own point of view, and with consummate sagacity, a similar idea. The bishop still influences many superior minds; and it will repay us to dwell for a moment on his views. He draws the sharpest distinction between our real selres and our bodily instruments. He does not, as far as I remember, use the word soul, possibly because the term was so hacknered in his day, as it harl been for many generations previously. But he speaks of "living powers," " perceiring or percipient powers," " moving agents," " ourselves," in the same sense as we should employ the term soul. He dwells upon the fact that limbs may be removed, and mortal diseases assail the body, the mind, almost up to the moment of death, remaining clear. He refers to sleep and to swoon, where the "living powers" are suspended but not destroyed. He considers it quite as easy to conceive of existence out of our bodies as in them; that we may animate a succession of bodies, the dissolution of all of them having no more tendency to dissolve our real selves, or "deprive us of living faculties-the faculties of perception and action-than the dissolution of any foreign matter which we are capable of receiving impressions from, or making use of for the common occasions of life." This is the key of the bishop's position: "our organized bodies are no more a part of ourselves than any other matter around us." In proof of this he calls attention to the use of glasses, which "prepare objects" for the "percipient power" exactly as the eye does. The eye itself is no more percipient than the glass; is quite as much the instrument of the true self, and also as foreigu to the true self, as the glass is. "And if we see with our eyes only in the same manner as we do with glasses, the like may justly be concluded from analogy of all our senses."

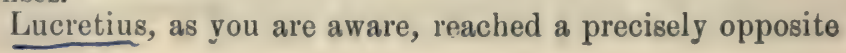


conclusion: and it certainly would bo interesting, if not profitable, to us all, to hear what he would or conld urge in opposition to the reasoning of the bishop. As a brief discussion of the point will enable us to see the isearings of an important question, I will here permit a disciple of Lucretius to try the strength of the bishop's position, ant then allow the bishop to retaliate, with the view of rolling back, if he cun, the difliculty upon Lucretius.

The argument might proceed in this fashion:-

- Subjected to the test of mental presentation (Vorstelluny), your views, most honored prelate, would offer to many minds a great, if not an insuperable difticulty. You speak of 'living powers,' 'percipient or perceiving powers,' and 'ourselves;' but can you form a mental picture of any of these, apart from the organism through which it is supposed to act? 'Test yourself honestly, and see whether you possess any faculty that would enable you to form such a conception. The true self has a local habitation in each of 118 ; thus localized, must it not possess a form? If so, what form? Have you ever for a moment realized it? When a leg is amputated the body is divided into two parts; is the true self in both of them or in one? Thomas Aquinas might say in both; bnt not you, for you appeal to the consciousness associated with one of the two parts, to prove that the other is foreign matter. Is consciousness, then, a necessary element of the true self? If 80, what do you say to the case of the whole body being deprived of consciousness? If not, then on what grounds do you deny any portion of the true self to the severed limb? It seems very singular that, from the beginning to the end of your admirable book (and no one admires its sober strength more than I (Jo), you never once mention the brain or nervous system. You begin at one end of the bolly, and show that its parts may be removed without prejudice to the perceiving power. What if you begin at the other end, and remove, instead of the leg, the brain? The body, as before, is divided into two parts; but both are now in the same predicament, and neither can be appealed to to prove that the other is foreign matter. Or. instead of going 80 far as to remove the brain itself, let a certain portion of its bony covering be removel, and lot a rhythmic series of pressures and relaxations of pressure be applied to the soft substance. At every pressure 'the 
faculties of perception and of action' vanish; at every relaxation of pressure they are restored. Where, during the intervals of pressure, is the perceiving power? I once had the discharge of a large Leylen battery passed unexpectedly through me: I felt nothing, but was simply blotted out of conscious existence for a sensible interval. Where was my true self during that interval? Men who have recovered from lightning-stroke have been much longer in the same state; and indeed in cases of ordinary concussion of the brain, days may elapse daring which no experience is registered in consciousness. Where is the man himself during the period of insensibility? You may say that I beg the question when I assume the man to have been unconscious, that he was really conscions all the time, and has simply forgotten what had occurred to him. In reply to this, I can only say that no one need shrink from the worst tortures that superstition ever invented, if only so felt and so remembered. I do not think your theory of instruments goes at all to the bottom of the matter. A telegraph operator has his instruments, by means of which he converses with the world; our bodies possess a nervons system, which plays a similar part between the perceiving power and external things. Cut the wires of the operator, break his battery, demagnetize his needle; by this means you certainly sever his connection with the world; but, inasmuch as these are real instruments, their destruction does not touch the man who uses them. The operator survives, and he knows that he survives. What is there, I would ask, in the himan system that answers to this conscious survival of the operator when the battery of the brain is so disturbed as to produce insensibility, or when it is destroyed altogether?

"Another consideration, which you may regard as slight, presses upon me with some force. The brain may change from health to disease, and through such a change the most examplary man may be converted into a debanchee or a murderer. My very noble and approved good master had, as you know, threatenings of lewdness introduced into his brain by his jealous wife's philter; and sooner than permit himself to run even the risk of yielding to these base promptings he slew himself. How could the hand of Lucretius have been thus turned against himself if the real Lucretius remained as before? Can the brain or cau it 
not act in this distempered way without the intorvention of the immortal reason? If it can, then it is a prime mover which requires only healthy regulation to render it reasonably self-ucting, and there is no apparent need of your immortal reason at all. If it camnot, then the immortal reason, by its mischievous activity in operating upou a broken instrument, must have the credit of committing every imaginable extravagance and crime. I think, if you will allow me to say so, that the gravest consequences are likely to flow from your estimate of the body. 'To regard the brain as you would a staff or an eyeglass-to shut your eyes to all its mystery, to the perfect correlation of its condition and our conscionsness, to the fact that a slight excess or defect of blood in it produces the very swoon to which you refer, and that in relation to it our meat, and drink, and air, and exercise, have a perfectly transcendental value and significance-to forgot all this does, I think, open a way to innumerable errors in our habits of life, and may possibly, in some cases, initiate and foster that very diseuse, and consequent mental ruin, which a wiser appreciation of this mysterious organ would have avoided.

I can imagine the bishop thoughtful after hearing this argument. He was not the man to allow anger to mingle with the consideration of a point of this kind. After due reflection, and having strengthened himself by that honest contemplation of the facts which was habitual with him, and which includes the desire to give even adverse reasonings their due weight, I can suppose the bishop to proceed thus: "You will renember that in the "Analogy of Religion," of which you have so kindly spoken, I did not profess to prove anything absolutely, and that I over and over again acknowledged and insisted on the smallness of our knowledge, or rather the depth of our ignorance, as regurds the whole system of the universe. My object was to show my deistical friends, who set forth so eloquently the beanty and beneficence of Nature and the Ruler thereof, while they had nothing bat scorn for the 8o-called absurdities of the Christian scheme, that they were in no better condition than we were, and that, for every difficulty found upon our side, quite as great a difficulty was to be found upon theirs. I will now, with your permission, adopt a similar line of argument. You are a Lucretian, and from 
the combination and separation of insensate atoms deduce all terrestrial things, including organic forms and their phenomena. Let me tell you in the first instance how far I am prepared to go with you. I admit that you can build crystalline forms out of this play of molecular force; that the diamond, amethyst, and snow-star are truly wonderful structures which are thus produced. I will go further and acknowledge that even a tree or flower might in this way be organized. Nay, if you can show me an animal without sensation, I will concede to you that it also might be put together by the suitable play of molecular force.

"Thus far our way is clear, but now comes my difficulty. Your atoms are individually without sensation, much more are they without intelligence. May I ask you, then, to try your hand upon this problem. 'Take your' dead hydrogen atoms, your dead oxygen atoms, your dead carbon atoms, your dead nitrogen atoms, your dead phosphorus atoms, and all the other atoms, dead as grains of shot, of which the brain is formed. Imagine them separate and sensationless; observe them rumning together and forming all imaginable combinations. 'This, as a purely mechanical process, is seeable by the mind. But can you see, or dream, or in any way imagine, how out of that mechanical act, and from these individually dead atoms, sensation, thought, and emotion are to rise? Are you likely to extract Homer out of the rattling of dice, or the Differential Calculus out of the clash of billiard-balls? I am not all bereft of this Vorstellungs-Kraft of which you speak, nor am I, like so many of my brethren, a mere vacuum as regards scientific knowledge. I can follow a particle of musk until it reaches the olfactory nerve; I can follow the waves of sound until their tremors reach the water of the labyrinth, and set the otoliths and Corti's fibers in motion; I can also visualize the waves of ether as they cross the eye and hit the retina. Nay more, I am able to pursue to the central organ the motion thus imparted at the periphery, and to see in idea the very molecules of the brain thrown into tremors. My insight is not baffled by these physical processes. What baffles and bewilders me is the notion that from those physical tremors things so ntterly incongruous with them as sensation, thought, and emotion can be derived. You may say, or think, that 
this issue of consciousness from the clash of atoms is not more incongruous than the flash of light from the union of oxygen and hydrogen. But I beg to say that it is. For such incongruity as the flush possesses is that which I now force upon your attention. The "flash" is an affair of consciuusness, the objective counterpart of which is a vibration. It is a flash only by your interpretation. Iou are the cause of the apparent incongruity; and you are the thing that puzzles me. I need not remind you that the great Leibnitz felt the diffeulty which I feel; and that to get rid of this monstrous deduction of life from death he displaced your atoms by his monals, which were more or less perfect mirrors of the universe, and out of the summation and integration of which he supposed all the phenomena of life-sentient, intellectual, and emotionalto arise.

"Your difficulty, then, as I see you are ready to admit, is quite as great as mine. You cannot satisfy the human understanding in its demand for logical contiauity between molecular processes and the phenomena of conscionsness. This is a rock on which Materialism must inevitably split whenever it pretonds to be a complete philosophy of life. What is the moral, my Lucretian? You and $P$ are not likely to indulge in ill-temper in the discussion of these great topics, where we see so much room for honest differences of opinion. But there are people of less wit or more bigotry (I say it with humility), on both sides, who are ever ready to mingle anger and vituperation with such discussions. There are, for example, writers of note and influence at the present day, who are not ashamed publicly to assume the "deep personal sin" of a great logician to be the cause of his unbelief in a theologic dogma.* And there are others who hold that we, who cherish our noble Bible, wrought as it has been into the constitution of our forefathers, and by inheritance into us, must necessarily be hypocritical and insincere. Let us disarow and discountenance such people, cherishing the unswerving faith that

* This is the aspect under which the late editor of the "Dublin Review" presonted to his remders the memory of John Stuart Mill. I can only say, that I would as soon take uy chance in the other world, in the company of the " unbeliever." gus in that of his Jesuis detractor. In Dr. Wird we lasve an exanple of a wholesusue and vigorous nature, soured and perverted by a poisonous creed. 
what is good and true in both our arguments will be preserved for the benefit of humanity, while all that is bad or false will disappear."

I hold the bishop's reasoning to be unanswerable, and his liberality to be worthy of imitation.

It is worth remarking that in one respect the bishop was a product of his age. Long previous to his day the nature of the soul had been so favorite and general a topic of discussion, that, when the students of the Italian universities wished to know the leanings of a new professor, they at once requested him to lecture upon the soul. About the time of Bishop Butler the question was not only agitated but extended. It was seen by the clear-witted men who entered this arena, that many of their best arguments applied equally to brutes and men. The bishop's arguments were of this character. He saw it, admitted it, took the consequence, and boldly embraced the whole auimal world in his scheme of immortality.

Section 6.-Bishop Butler accepted with unwavering trust the chronology of the Old Testament, describing it as "confirmed by the natural and civil history of the world, collected from common historians, from the state of the earth, and from the late inventions of arts and sciences." 'These words mark progress; and they must seem somewhat hoary to the bishop's successors of to-day. It is hardly necessary to inform you that since his time the domain of the naturalist has been immensely extender -the whole science of geology, with its astounding revelations regarding the life of the ancient earth, having been created. The rigidity of old conceptions has been relaxerl, the public mind being rendered gradually tolerant. of the idea that notfor six thousand, nor for sixty thousani, nor for six thousand thousand, but for æons embracing untold millions of years, this earth has been the theater of life and death. The riddle of the rocks has been read by the geologist and palæontologist, sub-cambrian depths to the deposits thickening over the sea-bottoms of to-day. And upon the leares of that stone book are, as you know, stamped the characters, plainer and surer than those formed by the ink of history, which carry the mind back into abysses of past time, compared with which the periods which satisfied Bishop Butler ceise to have a visual angle. 
The lode of discovery once struck, those petrified form: in which life was at one time active, increased to multitudes and demanded classification. They were grouped in genera, species, and rarieties, according to the degree of similarity subsisting between them. 'Thus confusion was avoided, each object being found in the pigeon-hole appropriated to it and to its fellows of similar morphological or physiological cbaracter. The general fact soon became evident that none but the simplest forms of life lie lowest down; that, as we climb higher among the superimposed strata, more perfect forms appear. The change, however, from form to form was not continnons, but by steps-8ome small, some great. "A seetion," says Mr. Huxley, " a hundred feet thick will exhibit at different heights a dozen species of Ammonite, none of which passes beyond the particular zone of limestone, or clay, into the zone below it, or into that above it." In the presence of such facts it was not possible to avoid the question: IIave these forms, showing, thongh in broken stages, and with many irregularities, this unmistakable general advance, being subjected to no continuous law of growth or variation? Had our education been purely scientific, or had it been sufficiently detached from influences which, howerer ennobling in another domain, have al ways proved hindrances and delusions when introduced as factors into the domain of physics, the scientific mind never could have swerved from the search for a law of growth, or allowed itself to accept the anthropomorphism which regarded each successire stratum as a kind of mechanie's bench for the manufacture of new species out of all relation to the old.

Biasel, however, by their previous education, the great majority of naturalists invoked a special creative act to account for the appearance of each new group of organisms. Doubtless numbers of them were clear-headed enough to see that this was no explanation at all-that, in poini of fact, it was an attempt, by the introduction of a greater difficulty, to account for a less. But, haring nothing to offer in the way of explanation, they for the most part held their pence. Still the thoughts of reflecting men naturally and necessarily simmered rotind the question. De Maillet, a contemporary of Newton, has licen bronglit into notice by Prufessor Huxley as one who "had a notion of the modifiability of living forms," The 
late Sir Benjamin Brodie, a man of highly philosophic mind, often drew my attention to the fact that, as early as 1794, Charles Darwin's grandfather was the pioneer of Charles Darwin.* In 1801, and in subsequent years, the celebrated Lamarck, who, through the vigorous exposition of his views by the author of the "Vestiges of Creation," rendered the public mind perfectly familiar with the idea of evolution, endeavored to show the development of species out of changes of habit and external condition. In 1813 Dr. Wells, the founder of our present theory of Dew, read before the Royal Society a paper in which, to use the words of Mr. Darwin, "he distinctly recognizes the principle of natural selection; and this is the first recognition that has been indicated." 'The thoroughness and skill with which Wells pursued his work, and the obvious independence of his character, rendered him long ago a favorite with me; and it gave me the liveliest pleasure to alight upon this additional testimony to his penetration. Professor Grant, Mr. Patrick Matthew, Von Buch, the author of the "Vestiges," D'Halloy, and others, by the enunciation of opinions more or less clear and correct, showed that the question had been fermenting long prior to the year 1858, whn Mr. Darwin and Mr. Wallace simultaneously, but independently, placed their closely concurrent views before the Linnean Society. $\downarrow$

These papers were followed in 1859 by the publication of the first edition of the "Origin of Species." All great things come slowly to the birth. Coperuicus, as I informed you, pondered his great work for thirty-three years. Newton for nearly twenty years kept the idea of Gravitation before his mind; for twenty years also he dwelt upon his discovery of Fluxions, and doubtless would have continued to make it the object of his private thought, had he not found Leibuitz upon his track. Darwin for twoand-twenty year's pondered the problem of the origin of species, and doubtless he would have continued to do so had

\section{* "Zoonomia," vol. i. pp. 500-510.}

†In 1855 Mr. Herbert Spencer ("Principles of Psychology," $2 \mathrm{~d}$ edit. vol. i. p. 465 ) expressed " the belief that life under all its forms has arisen by an unbroken evolution, and through the instrumentality of what are called natural causes." This was my belief also at that time. 
he not found Wallace upon his track.* $\mathbf{A}$ concentrated, but full and powerful, epitome of his labors was the consequence. The book was by no means an easy one; and probably not one in every score of those who then attacked it, had read its pages through, or were competent to grasp their signifieance if they had. I do not say this merely to discredit them: for there were in those days some really eminent scientific men, entirely raiserl above the heat of popular prejudice, and willing to accept any conclusion that science had to offer, provided it was duly backed by fact and argument, who entirely mistook Mr. Darwin's views. In fact, the work needed an expounder, and it found one in Mr. Huxley. I know nothing more admirable in the way of scientific exposition than those early articles of his on the origin of species. He swept the curve of discussion through the really significant points of the subject, enriehed his exposition with profound original remarks and reflections, often summing up in a single pitliy sentence an argument which a less compact mind woulit have spread over pages. But there is one impression male by the book itself which no exposition of it, however luminous, can consey; and that is the impression of the rast amount of labor, both of observation and of thought, implied in its production. Let 118 glance at its principles.

It is conceded on all hands that what are called "varieties" are continually produced. The rule is probably withont exception. No chick, or child, is in all respects and particulars the counterpart of its brother and sister; and in such differences we have "variety" incipient. No naturalist conld tell how far this variation could be carrien; but the great mass of them held that never, by any amount of internal or external change, nor by the mixture of both, could the offspring of the same progenitor so far deviate from each other as to constitute different species. The function of the experimental philosopher is to combine the conditions of Nature and to produce her results; and this was the method of Darwin. $\downarrow$ He made himself acquainted

* The behavior of Mr. Wallace in rolation to this subject lias ineen dignified in the lighest degree.

† The first stop only woward experimental domonstration has been

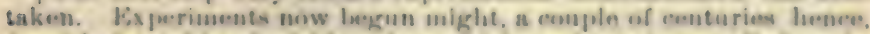
furnish data of incalendable value, which ouglit to toes supplieel wo the sevence of the future. 
with what could, without any manner of doubt, be done in the way of producing variation. He associated himself with pigeon-fanciers-bought, begged, kept, and observed every breed that he could obtain. 'Though derived from a common stock, the diversities of these pigeons were such that " a score of them might be chosen which, if shown to an ornithologist, and he were told that they were wild birils, would certainly be ranked by him as well-defined species." The simple principle which guides the pigeonfancier, as it does the cattle-breeder, is the selection of some variety that strikes his funcy, and the propagation of this variety by inheritance. With his eye still directed to the particular appearance which he wishes to exaggerate, he selects it as it reappears in successive broods, and thus adds increment to increment until an astonishing amount of divergence from the parent type is effected. The breeder in this case does not produce the elements of the variation. He simply observes them, and by selection adds them together until the required result has been obtained. "No man," says Mr. Darwin, "would ever try to make a fantail till he saw a pigeon with a tail developed in some slight degree in an unusual manner, or a pouter until he saw a pigeon with a crop of unusual size." 'Thus nature gives the hint, man acts upon it, and by the law of inheritance exaggerates the deviation.

Having thus satisfied himself by indubitable facts that the organization of an animal or of a plant (for precisely the same treatment applies to plants) is to some extent plastic, he passes from variation under domestication to variation under nature. Hitherto we have dealt with the adding together of small changes by the conscious selection of man. Can Nature thus select? Mr. Darwin's answer is "Assuredly she can." The number of living things produced is far in excess of the number that can be supported; hence at some period or other of their lives there must be a struggle for existence. And what is the infallible result? If one organism were a perfect copy of the other in regard to strength, skill, and agility, external conditions would decide. But this is not the case. Here we have the fact of variety offering itself to nature, as in the former instance it offered itself to man; and those varieties which are least competent to cope with surrounding conditions will infallibly give way to those that 
are most competent. 'T'o use a familar proverb, the weakest goes to the wall. But the triumpliant fraction again breeds to orer-production, transmitting the qualities which secured its maintenance, but transmining them in different degrees. The struggle for food again supervenes, and those to whom the farorable quality has been transmitted in excess, will triumph as before.

It is easy to see that we have here the addition of increments favorable to the individual, still more rigorously carried out than in the case of domestication; for not only wre unfarorable specimens not selected by nature, but they are destroyed. This is what Mr. Darwin calls "Natural selection," which acts by the preservation and accumulation of small inherited modifications, each profitable to the preserved being. With this idea he interpenetrates and leavens the rast store of facts that he and others have col. lected. We cannot, without shutting our eyes through fear or prejudice, fail to see that Darwin is liere dealing. not with imaginary, but with true causes; nor can we fail to discern what vast modifications may be produced by natural selection in periods sufficiently long. Each individual increment may resemble what mathematicians call a "differential" (a quantity indefinitely small); but definite and great changes may obviously be produced by the integration of these infinitesimal quantities, through practically infinite time.

If Darwin, like Bruno, rejects the notion of creative power, acting after human fashion, it certainly is not becanse he is unacquainted with the numberless exquisite adaptations on which this notion of a supernatural Artificer has been founded. His book is a repository of the most startling facts of this description. 'Take the marvelous observation which he cites from Dr. Krüger, where a bucket, with an aperture serving as a spout, is formed in an orchid. Bees visit the flower: in eager search of material for their combs, they push each other in to the bucket, the drenched ones escaping from their involuntary bath by the spout. Here they rub their backs against the riscid stigma of the flower and obtain glue; then against the pollen-mases, which are thus stuck to the back of the bee and carried away. "When the bee, so provided, flies to another flower or to the same flower a second time, and is pushed by its comrades into the bucket, and then crawls out by the 
passage, the pollen-mass upon its back necessarily comes first into contact with the viscid stigma," which takes up the pollen; and this is how that orchid is fertilized. $\mathrm{Or}$ take this other case of the Catasetum. "Bees visit these flowers in order to gnaw the labellum; in doing this they inevitably touch a long, tapering, sensitive projection. 'This, when touched, transmits a sensation or vibration to a certain membrane, which is instantly ruptured, setting free a spring, by which the pollen-mass is shot forth like an arrow in the right direction, and adheres by its viscid extremity to the back of the bee." In this way the fertilizing pollen is spread abroad.

It is the mind thus stored with the choicest materials of the teleologist that rejects teleology, seeking to refer these wonders to natural causes. They illustrate, according to him, the method of nature, not the "technic" of a manlike Artificer. The beauty of flowers is due to natural selection. Those that distinguish themselves by vividly contrasting colors from the surrounding green leaves are most readily seen, most frequently visited by insects, most often fertilized, and hence most favored by natural selection. Colored berries also readily attract the attention of birds and heasts, which feed upon them, spread their manured seeds abroad, thus giving trees and shrubs possessing such berries a greater chance in the struggle for existence.

With profound analytic and synthetic skill, Mr. Darwin investigates the cell-making instinct of the hive-bee. His method of dealing with it is representative. He falls back from the more perfectly to the less perfectly developed instinct-from the hive-bee to the humble-bee, which uses its own cocoon as a comb, and to classes of bees of intermediate skill, endeavoring to show how the passage might be gradually made from the lowest to the lighest. The saving of wax is the most important point in the economy of bees. T'welve to fifteen pounds of dry sugar are said to be needed for the secretion of a single pound of wax. The quantities of nectar necessary for the wax must therefore be vast; and every improvement of constructive instinct which results in the saving of wax is a direct profit to the insect's life. The time that would otherwise be devoted to the making of wax, is devoted to the gatliering and storing of honey for winter food. Mr. Darwin passes from 
the humble-bee with its rude cells, through the Melipona with its more artistia cells, to the hive-bee with its astonishing architecture. The bees place themselves at equal distances apart upon the wax, swoep and excarate equal spheres round the selected points. The spheres intersect, and the planes of intersection are built up with thin laminas. Hexagonal cells are thus formed. This mole of treating such questions is, as I have said, representative. The expositor habitually retires from the more perfect and complex, to the less perfect and simple, and carries you with him through stages of perfecting-alds increment to increment of intinitesimal change, and in this way gradually breaks down your reluctance to almit that the exquisite climax of the whole could be a result of natural selection,

Mr. Darwin shirks no difficulty; and, saturated as the sub. jeet was with his own thought, he musi have known, better than his critics, the weakness as well as the strength of his theory. This of course would be of little arail were his object a temporary dialectic victory, insteal of the establishment of a truth which he means to be everlasting. But he takes no pains to disguise the weaknoss he has discerned; nay, he takes every pains to bring it into the strongest light. His vast resources enable him to rope with objections started by himself and others, so as to leave the final impression upon the reader's mind that, if they be not completely answered, they certainly are not fatal. Their negative force being thus destroyed, you are free to be influenced by the vast positive mass of evidence he is ahle to bring before you. This largeness of knowledge, and readiness of resource, render Mr. Darwin the most terrible of antagonists. Accomplished naturalists have leveled heavy and sustained critioisms against him-not always with the view of fairly weighing his theory, but with the express intention of exposing its weak points only. 'This does not irritate him. He treats every objection with a soberness and thoronghness which even Bishop Butler might be proud to imitate, surrounding each fact with its appropriate detail, placing it in its proper relations, and usually giving it a gignificance which, as long as it was hept isolated, faled to appear. This is done withont a trace of ill-temper. He mores over the subject with the passionless strength of a glacier; and the grinding of the 
rocks is not always without a counterpart in the logical pulverization of the objector. But though in handling this mighty theme all passion has been stillerl, there is an emotion of the intellect, incident to the discernment of new truth, which often colors and warms the pages of $\mathrm{Mr}$. Darwin. His success has been great; and this implies not only the solidity of his work, but the preparedness of the public mind for such a revelation. On this head, a remark of Agassiz impressed me more than anything else. Sprung from a race of theologians, this celebrated man combated to the last the theory of natural selection. One of the many times I had the pleasure of meeting him in the United States was at Mr. Winthrop's beautiful residence at Brookline, near Boston. Rising from luncheon, we all halted as if by common consent, in front of a window, and continued there a discussion which had been started at table. The maple was in its autumn glory, and the exquisite beauty of the scene outside seemed, in my case, to interpenetrate without disturbance the intellectual action. Earnestly, almost sadly, Agassiz turned, and said to the gentlemen standing round, "I confess that I was not prepared to see this theory received as it has been by the best intellects of our time. Its success is greater than I could have thought possible."

Section \%.- In our day grand generalizations have been reached. The theory of the origin of species is but one of them. Another, of still wider grasp and more radical significance, is the doctrine of the Conservation of Energy, the ultimate philosuphical issues of which are as yet but dimly seen-that doctrine which "binds nature fast in fate," to an extent not hitherto recognized, exacting from every antecedent its equivalent consequent, from every consequent its equivalent antecedent, and bringing vital as well as physical phenomena under the dominion of that law of causal connection which, so far as the human understanding has yet pierced, asserts itself everywhere in nature. Long in advance of all definite experiment upon the subject, the constancy and indestructibility of matter had been affirmed; and all subsequent experience justified the affirmation. Mayer extended the attribute of indestructibility to energy, applying it in the first instance to 
inorganic, * and afterward with profound insight to organic nature. The vegetable world, though drawing all its nutriment from invisible sources, was proved incompetent to generate anew either matter or force. Its matter is for the most part transmuted gas; its force transformed solar force. The animal world was proved to be equally uncreative, all its motive energies being referred to the combustion of its food. The activity of each animal, as a whole, was proved to be the transferred activity of its molecules. The muscles were shown to be stores of mer-hanical energy, potential until unlocked by the nerves, and then resulting in muscular contractions. The specd at which messages fly to and fro along the nerves was determineal by Helmholtz, and found to be, not, as had been previnusly supposed, equal to that of light or electricity, but less than the speed of sound-less even than that of an eagle.

This was the work of the physicist: then came the conquests of the comparative anatomist and physiologist, revealing the structure of every animal, and the function of every organ in the whole biological series, from the lowest zoophyte up to man. The nerrous system had been made the object of profound and continued study, the wonderful and, at bottom, entirely mysterious controlling power which it exercises over the whole organism, physical and mental, being recognized more and more. Thought conld not be kept back from a subject 80 profoundly suggestive. Besides the physical life dealt with by Mr. Darwin, there is a psychical life presenting similar gradations, and asking equally for a solution. How are the different grades and orders of Mind to be accounted for? What is the principle of growth of that mysterious power which on our planet culminates in Reason? These are questions which, though not thrusting themselves so furcibly upon the attention of the general public, had not only occupied many reflecting minds, but had been formally bronched by one of them before the "Origin of Species" nppeared.

With the mass of materials furnished by the physicist and physiologist in his hands, Mr. Herbert Spencer. twenty years ago, sought to graft upon this basis a system

- Dr. Berthold has shown that Jeilnita had sound viows rogarding the conservation of energy in inorganie nature. 
of psychology; and two years ago a second and greatly amplified edition of his work appeared. Those who have occupied themselves with the beautiful experiments of Plateau will remember that when two spherules of olive-oil suspended in a mixture of alcohol and water of the same density as the oil, are brought together, they do not immediately unite. Something like a pellicle appears to be formed around the drops, the rupture of which is immediately followed by the coalescence of the globules into one. There are organisms whose vital actions are almost as purely physical as the coalescence of such drops of oil. They come into contact and fuse themselves thus together. From such organisms to others a shade higher, from these to others a shade higher still, and on through an ever-ascending series, Mr. Spencer conducts his argument. There are two obvious factors to be here taken into account-the creature and the medium in which it lives, or, as it is often expressed, the organism and its environ. ment. Mr. Spencer's fundamental principle is, that between these two factors there is incessant interaction. The organism is played upon by the environment, and is modified to meet the requirements of the environment. Life he defines to be "a continuous adjustment of internal relations to external relations."

In the lowest organisms we have a kind of tactual sense diffused over the entire body; then, through impressions from without and their corresponding adjustments, special portions of the surface become more responsive to stimuli than others. 'The senses are nascent, the basis of all of them being that simple tactual sense which the sage Democritus recognized 2,300 years ago as their common progenitor. The action of light, in the first instance, appears to be a mere disturbance of the chemical processes in the animal organism, similar to that which occurs in the leaves of plants. By degrees the action becomes localized in a few pigment-cells, more sensitive to light than the surrounding tissue. The eye is incipient. At first it is merely capable of revealing differences of light and shade produced by bodies close at hand. Followed, as the interception of the light commonly is, by the contact of the closely adjacent opaque body, sight in this condition becomes a kind of "anticipatory touch." "The adjustment continues; a slight bulging out of the epidermis over the 
pigment-granules supervenes, A lens is incipient, ant, through the operation of infinite adjustments, at length reaches the perfection that it displays in the hawk and eagle. So of the other aenses; they are special differentiations of a tissue which was originally raguely sensitive all over.

With the development of the senses, the adjustments between the organism and its environment gralually extend in space, a multiplication of experiences and a corresponding morlification of cunduct being the result. The aljustments also extend in time, covering continually greater intervals. Along with this extension in space and time the aljustments also increase in speciality and conplesity, passing through the various grades of binte life, and prolonging themselres into the domain of reason. Very striking are Mr. Spencer's remarks regarding the influence of the sense of touch upon the development of intelligence. This is, so to say, the mother-tongue of all the sensers, into which they inust be translated to be of service to the organism. Hence its importance. The parrot is the most intelligent of birds, and its tactual power is also greatest. From this sense it gets knowleilge, unattainable by birls which cannot employ their feet as hands. The elephant is the most sagacious of quadrupeds-its tactual range and skill, and the consequent multiplication of experiences, which it owes to its wonderfully alaptable trunk, being the basis of its sagacity. Feline animals, for a similar canse, are more sagacious than hoofed animals-atonement being to some extent male in the case of the horse, by the possession of sensitire prehensile lips. In the l'rimates the evolution of intellect and the evolution of tactual ap)penuages go hand in hand. In the most iutelligent anthropoid apes we find the tactual range and delicacy greatly augmented, new avenues of knowlerge being thus opened to the animal. Man crowng the edifice here, not only in virtue of his own inanipulatory power, but through the enormons extension of his range of experience, by the invention of instruments of precision, which sorve as supplemental senses and supplemental limbs. 'T'se reciprocil action of these is finely desoribed and illustrated. That chastened intellectual emotion to which I have roferred in connection with Mr. Darwin, is not absent in Mr. Spencer. His illustrations possess at times excevoling vivillicess and 
force; and from his style on such occasions it is to be inferred that the ganglia of this Apostle of the Understanding are sometimes the seat of a nascent poetic thrill.

It is a fact of supreme importance that actions, the performance of which at first requires even painful effort and deliberation, may, by habit, be rendered antomatic. Witiless the slow learning of its letter's by a child, and the subsequent facility of reading in a man, when each group) of letters which forms a word is instantly, and withunt effort, fused to a single perception. Instance the billiardplayer, whose muscles of hand and eye, when he reaches the perfection of his art, are unconsciously co-ordinated. Instance the musician, who, by practice, is enabled to fuse a multitude of arrangements, auditory, tactual and muscular, into a process of automatic manipulation. Combiuing such facts with the doctrine of hereditary transmission, we reach a theory of linstinct. A chick, after coming ont of the egg, balances itself correctly, runs about, picks up food, thus showing that it possesses a power of directing its movements to definite ends. How did the chick learn this very complex co-ordination of eyes, muscles, and beak? It has not been individually taught; its personal experience is $n i l$; but it has the benefit of ancestral experience. In its inherited organization are registered the powers which it displays at birth. So also as regards the instinct of the hive-bee, already referred to. 'The distance at which the insects stand apart when they sweep their hemispheres and build their cells is "organically remembered." Man also carries with him the physical texture of his ancestry, as well as the inherited intellect bound up with it. 'The defects of intelligence during infancy and youth are probably less due to a lack of individual experience, than to the fact that in early life the cerebral organization is still incomplete. The period necessary for completion varies with the race, and with the individual. As a round shot outstrips the rifled bolt on quitting the muzzle of the gun, so the lower race, in childhood, may outstrip the higher. But the higher eventually overtakes the lower, and surpasses it in range. As regards individuals, we do not always find the precocity of youth prolonged to mental power in maturity; while the dulluess of boyhood is sometimes strikingly contrasted with the intellectual energy of after years. Newton, when a boy, was weakly, and he 
showed no particular aptitude at school; but in lis eighteenth year he went to Cambridge, and soon afterward astonished his teachers by his power of dealing with geometrical problems. During his quiet youth his brain was slowly preparing itself to be the organ of those energies which he subsequently displayed.

By myriad blows (to use a Lacretian phrase) the image I and superseription of the external world are stamped as states of consciousness upon the organism, the depth of " the impression depending on the number of the blows. When two or more phenomena occur in the environment invariably together, they are stamped to the same depth or to the same relief, and indissolubly connected. And here we come to the threshold of a great question. Seeing that he could in no way rid himself of the conscionsness of Space and Time, Kant assumed them to be necessary "forms of intuition," the molds and shapes into which our intuitions are thrown, belonging to ourselves, and without objective existence. With unexpected power and success, Mr. Spencer brings the hereditary experience theory, as he holds it, to bear upon this question. "If there exist certain external relations which are experienced by all organisms at all instants of their waking lives-relations which are absolutely constant and unirersal-there will be established answering internal relations, that are absolutely constant and universal. Such relations we lave in those of Space and Time. As the substratum of all other relations of the Non-ego, they must be re. sponded to by conceptions that are the substrata of all other relations in the Ego. Being the constant and infinitely repeated elements of thonght, they must become the antomatic elements of thought-the elements of thought which it is impossible to get rid of-the "forms of intuition."

Thronghout this application and extension of Hartley's and Mill's "Law of Inseparable Association," Mlr. Spencer stands upon his own ground, invoking, insteal of the experiences of the individual, the registered experiences of the race. His overthrow of the restriction of exjerience to the indiridnal is, I think, complete. That restriction ignores the power of organizing experienco, furnished at the outset to each individual; it ignores the different degrees of this power possessed by different races, and by 
different individuals of the same race. Were there not in the human brain a potency antecedent to all experience, a dog or a calf ought to be as capable of education as a man. 'These predetermined internal relations are independent of the experiences of the individual. The human brain is the "organized register of infinitely numerous experiences received during the evolution of life, or rather during the evolution of that series of organisms through which the human organism has been reached. The effects of the most uniform and frequent of these experiences have been successively bequeathed, principal and interest, and have slowly mounted to that high intelligence which lies latent in the brain of the infant. Thus it happens that the European inherits from twenty to thirty cubic inches more of brain than the Papuan. Thus it happens that faculties, as of music, which scarcely exist in some inferior races, become congenital in superior ones. 'Thus it happens that out of savages unable to count up to the number of their fingers, and speaking a language containing only nouns and verbs, arise at length our Newtons and Shakspeares."

SECTION 8. - At the outset of this address it was stated that physical theories which lie beyond experience are derived by a process of abstraction from experience. It is instructive to note from this point of view the successive introduction of new conceptions. The idea of the attraction of gravitation was preceded by the observation of the attraction of iron by a magnet, and of light bodies by rubbed amber. The polarity of magnetism and electricity also appealed to the senses. It thus became the substratum of the conception that atoms and molecules are endowed with attractive and repellent poles, by the play of which definite forms of crystalline architecture are produced. Thus molecular force becomes structural.* It required no great boldness of thought to extend its play into organic nature, and to recognize in molecular force the agency by which both plants and animals are built up. In this way, out of experience arise conceptions which are wholly ultra-experiential. None of the atomists of antiquity had any notion of this play of molecular polar force, but they had experience of gravity, as manifested by fall-

* See Art. on Matter and Force, or "Lectures on Light," No. III. 
ing bodies. Abstracting from this, they permitted their utoms to fall eternally through empty space. Democritus assumed that the larger atoms moved more rapidly than the smaller ones, which they therefore could overtake, and with which tney could combine. Epicurus, holding that empty space could offer no resistance to motion, aseribed to all the atoms the same velocity; but he seems to have overlooked the consequence that under such circumstanees the atoms could never combine. Lucretius cut the knot by quitting the domain of physics al together, and causing the atoms to move together by a kind of volition.

Was the instinct utterly at fault which caused Lucretins thus to swerve from his own principles? Diminishing gralually the number of progenitors, Mr. Darwin comes at length to one "primordial form;" but he does not say, so far as I remember, how he supposes this form to have been introduced. He quotes with satisfaction the words of a celebrated author und divine who had " gradually learned to see that it was just as noble a conception of the Deity to believe He createrl a few original forms, capable of selfdevelopment into other and needful forms, as to believe He required a fresh act of creation to supply the voids caused by the action of his laws." What Mr. Darwin thinks of this view of the introduction of life, I do not know. But the anthropomorphism, which it seemed his object to set aside, is as firmly associated with the creation of a few forms as with the creation of a multitude. We need clearness and thoroughness here. Two courses and two only are possible. Either let 11 open our doors freely to the conception of creative acts, or abandoning them, let us radically change our notions of Matter. If we look at matter as pictured by Democritus, and as defined for generations in our scientific text-books, the notion of conscious life coming out of it cannot be formed by the mind. 'The argument placed in the month of Bishop Butler suffices, in my opinion, to crush all such materialism as this. Those, however, who framed these definitions of malter were but partial students. They were not biólogists, but mathematicians, whose labors referred only to such accidents and properties of matter as could be expressed in their formulas. Their seience was mechanical science, not the science of life. With matter in its wholeness they never denlt; and, denuded by their 
imperfect definitions, "the gentle mother of all" became the object of her children's dread. Let us reverently, but honestly, look the question in the face. Divorced from matter, where is life? Whatever our faith may say, our knowledge shows them to be indissolubly joined. Every meal we eat, and every cup we drink, illustrates the mysterious control of Mind by Matter.

On tracing the line of life backward, we see it approaching more and more to what we call the purely physical condition. We come at length to those organisms which I have compared to drops of oil suspended in a mixture of alcohol and water. We reach the protogenes of Haeckel, in which we have " a type distinguishable from a fragment of albumen only by its finely granular character." Can we pause here? We break a magnet, and find two poles in each of its fragments. We continue the process of breaking; but, however small the parts, each carries with it, though enfeebled, the polarity of the whole. And when we can break no longer, we prolong the intellectual vision to the polar molecules. Are we not urged to do something similar in the case of life? Is there not a temptation to close to some extent with Lucretius, when he affirms that " Nature is seen to do all things spontaneonsly of herself without the meddling of the gods?" or with Bruno, when he declares that Matter is not " that mere empty capacity which philosophers have pictured her to be, but the universal mother who brings forth all things as the fruit of her own womb?" Believing, as I do, in the continuity of nature, I cannot stop abruptly where our microscopes cease to be of use. Here the vision of the mind authoritatively supplements the vision of the eye. By a necessity engendered and justified by science I cross the boundary of the experimental evidence, ${ }^{*}$ and discern in that Matter which we, in our ignorance of its latent powers, and notwithstanding our professed reverence for its Creator, have hitherto covered with opprobrium, the promise and potency of all terrestrial Life.

If you ask me whether there exists the least evidence to prove that any form of life can be developed out of matter, without demonstrable antecedent life, my reply is that evidence considered perfectly conclusive by many has

* This mode of procedure was not invented in Belfast. 
been adiluced: and that were some of us who have pondered this question on follow a very common example, and accept testimony because it falls in with our belief, we also shonlil eagerly close with the evidence referred to. But there is in the true man of science a desire stronger than the wish to have his beliefs upheld; namely, the desire to have them true. And this stronger wish causes him w reject the most plasusible support, if he has reason to suspect that it is ritiated by error. Those to whom I refer as having studied this question, believing the evidence offered in faror of "spontaneons generation" to be thus vitiated, cannot accept it. They know full well that the chemist now prepares from inorganic matter a vast array of substances, which were some time ago regarded as the sole products of vitality. Tliey are intimately aequainted with the structural power of matter, as evidenced in the phenomena of crystallization. They can justify scientifieally their beticf in its potency, under the proper conditions, to produce organisms. Bnt, in reply to your question, they will frankly admit their inability to point to any satisfactory experimental proof that life cau bo developed, save from demoustrab!e antecedent life. As already indicated, they draw the line from the lighest organisms through fowor ones domn to the lowest; and it is the prolougation of this line by the intellect, bejond the rauge of the senses, that leads them to the conclusion which Bruno so boldiy enunciated."

The "materialism" here professed may bo vastly different from what you suppose, and I therefore crave your gracious patience to the end. "The question of an external world," says J. \$. Mill, “ is the great batle-ground of metaphysics." $t$ Mr. Mill himself reduces external phenomena to "possibilities of sensation." Kant, as we have seen, male time and space "forms" of our own intuitions. Fichte, having first by the inexorable logic of his understanding prosed himself to bo a mere link in that chain of eterinal cansation which holds so rigidly in mature, riolently broke the chain by making nature, and all that it inherits, an apparition of the mind.! And it is

" Bruno was a " l'antlueist," not an "Atheist " or a "Materialist."

f "Esamination of Hamilton," p. 104.

‡." Bestimmung des Menschen." 
by no means easy to combat such notions. For when I say "I see you," and that there is not the least doubt about it, the obvious reply is, that what I am really conscious of is an affection of my own retina. And if I urge that my sight can be checked by touching you, the retort would be that I am equally transgressing the limits of fact; for what I am really conscious of is, not that you are there, but that the nerves of my hand have undergone a change. All we hear, and see, and touch, and taste, and smell, are, it would be urged, mere variations of our own condition, beyond which, even to the extent of a hair's breadth, we cannot go. 'That anything answering to our impressions exists outside of ourselves is not a fact, but an inference, to which all validity would be denied by an idealist like Berkeley, or by a skeptic like Hume. Mr. Spencer takes another line. With him, as with the uneducated man, there is no doubt or question as to the existence of an external world. But he differs from the uneducated, who think that the world really is what consciousness represents it to be. Our states of consciousness are mere symbols of an outside entity which produces them and determines the order of their succession, but the real nature of which we can never know.* In fact, the whole process of evolution is the manifestation of a Power absolutely inscrutable to the intellect of man. As little in our day as in the days of Job can man by searching find this Power out. Considered fundamentally, then, it is by the operation of an insoluble mystery that life on earth is evolved, species differentiated, and mind unfolded, from their prepotent elements in the immeasurable past.

* In a paper, at once popular and profound, entitled " Recent Progress in the Theory of Vision," contained in the volume of lectures by Helmholtz, published by Longmans, this symbolism of our states of consciousness is also dwelt upon. The impressions of sense are the mere signs of exterual things. In this paper Helmboltz contends strongly rgainst the view that the consciousness of space is inborn; and he evidently doubts the power of the chick to pick up grains of corn without preliminary lessons. On this point, he says, further experiments are needed. Such experiments have been since made by Mr. Spalding, aided, I believe, in some of his observations by the accomplished and deeply lamented Lady Amberly; and they seem to prove conclusively that the chick does not need a single moment's tuition to enable it to stand, run, govern the muscles of its eyes, and peck. Helmholtz, however, is contending against the notion of pre-established harmony; and I am not aware of his views as to the organization of experiences of race or breed. 
The strength of the doctrine of evolution consists, not in an experimental demonstration (for the subject is hardiy accessible to this mole of proof), but in its general harmony with scientitic thonght. From contrast, moreover, it derives enormous relative cogency. On the one sile we have a theory (if it could with any propriety be so called) derived, as were the theories referred to at the beginning of this adilress, not from the study of nature, but from the observation of men-a theory which converts the Power whose garment is seen in the visible universe into an Artificer, fashioned after the human molel, and acting by broken efforts as man is seen to act. On the other side we have the conception that all we see around us, and all we feel within us--the phenomena of physical nature as well as those of the human mind-have their unsearchable roots in a cosmical life, if I dare apply the term, an infinitesimal span of which is offered to the investigation of man. And even this span is only knowable in part. We can trace the development of a nervous system, and correlate with it the parallel phenomena of sensation and thought. We see with undonbting certainty that they go land in hand. But we try to soar in a vacuum the moment we seek to comprehend the connection between them. An Arehimedean fulcrum is here required which the human mind cannot command; and the effort to solve the problem-to borrow a comparison from an illustrious friend of mineis like that of a man trying to lift himself by his own waistband. All that has been said in this discourse is to be taken in connection with this fundamental truth. When " nascent senses" are sploken of, when "the differentiation of a tissue at first vaguely sensitive all over" is spoken of, and when these possessions and processers are associated with "the modification of an organism by its environment," the same parallelism, withont contuce, or even approach to contact, is implied. Man the object is separated by an improssable gulf from man tho subjert. 'There is no motor energy in the human intellect to carry it without logical rupture, from the one to the other.

Sectios 9.- The doctrine of evolntion derives man, in his totality, from the interaction of organism and environment through countless ageg past. The hunan understauding, for example-that faculty which Mr. Spencer 
has turned so skillfully round upon its own antecedentsis itself a result of the play between organism and environment through cosmic ranges of time. Never, surely, did prescription plead so irresistible a claim. But, then it comes to pass that, over and above his understanding, there are many other things appertaining to man, whose prescriptive rights are quite as strong as those of the understanding itself. It is a result, for example, of the play of organism and environment that sugar is sweet, and that aloes are bitter; that the smell of henbane differs from the perfume of a rose. Such facts of consciousness (for which, by the way, no adequate reason has ever been rendered) are quite as old as the understanding; and many other things can boast an equally ancient origin. Mr. Spencer at one place refers to that most powerful of passions-the amatory passion-as one which, when it first occurs, is antecedent to all relative experience whatever; and we may press its claim as being at least as ancient, and as valid, as that of the understanding itself. 'Then there are such things woven into the texture of man as the feeling of awe, reverence, wonder-and not alone the sexual love just referred to, but the love of the beautiful, physical, and moral, in nature, poetry, and art. There is also that deep set feeling, which since the earliest dawn of history, and probably for ages prior to all history, incorporater itself in the religions of the world. You, who have escaper from these religions into the high-and-dry light of the intellect, may deride them; but in so doing you deride aceidents of form merely, and fail to touch the immovable basis of the religions sentiment in the nature of man. 'T'o yield this sentiment reasonable satisfaction is the problem of problems at the present hour. And grotesque in relation to scientific culture as many of the religions of the world have been and are-dangerous, nay, destructive, to the dearest privileges of freemen as some of them undoubtedly have been, and would, if they could, be againit will be wise to recognize them as the forms of a force, mischievous if permitted to intrude on the region of objective kuovlealge, over which it holds no command, but capable of adding, in the region of poetry and emotion, inward completeness and dignity to man.

Feeling, I say again, dates from as old an origin and as high a source as intelligence, and it equally demands its 
range of play. The wise teacher of humanity will recognize the necessity of meeting this demand, rather than of resisting it on account of errors and absurlities of form. What we shoald resist, at all hazards, is the attempt made in the past, and now repeated, to found upon this elemental bias of man's nature a system which should exercise despotic sway over his intellect. I have no fear of such a consummation. Science has alrealy to some extent leavened the world; it will leaven it more and more. I should look upon the mild light of seience breaking in upon the minds of the youth of Ireland, and strengthening gralually to the perfect day, as a surer check to any intellectual or spiritual trranny which may threaten this island, than the laws of princes or the swords of emperors. We fought and won our battle even in the midlle ages: should we doubt the issue of another conflict with our broken foe?

The impregnable position of science may be described in a few words. We claim, and we shall wrest from theology, the entire domain of cosmological theory. All schemes and systems which thus infringe upon the domain of science must, in so far as they do this, submit to its control, and relinquish all thought of controlling it. Acting otherwise proved always disastrous in the past, and it is simply fatuous to-day. Every system which would escape the fate of an organism too rigid to adjust itself to its environment, must be plastic to the extent that the growth of knowledge demands. When this truth has been thoronghly taken in, rigility will be relaxed, exclusiveness diminished, thisgs now deemed essential will be dropned, and elements now rejected will be assimilated. The lifting of the life is the essential point; and as long as dogmatism, fanaticism, and intolerunce are kept out, various modes of leverage may be employed to raise life to a higher level.

Seience itself not unfrequently lerives motive power from an ultra-scientific source. Some of its greatest discoverien have been male umler the the stimulus of a nonscientific ideal. This was the case among the ancients, and it has been so among ourselves. Mayer, Joule, and Colling, whose names are associated with the greatest of modern generalizations, were thus influenced. With his usual insiglit, bange at one pluce remarks, that " it is not always the objectively correct and intelligible that helps man most, or leals most quickly to the fullest and truest 
knowledge. As the sliding body upon the brachystochrone reaches its end sooner than by the straighter road of the inclined plane, so, through the swing of the ideal, we often arrive at the naked truth more rapidly than by the processes of the understanding." Whewell speaks of enthusiasm of temper as a hindrance to science; but he means the enthusiasm of weak heads. There is a strong and resolute enthusiasm in which science finds an ally; and it is to the lowering of this fire, rather than to the diminution of intellectual insight, that the lessening productiveness of men of science, in their mature years, is to be ascribed. Mr. Buckle sought to detach intellectual achievement from moral force. He gravely erred, for without moral force to whip it into action, the achievement of the intellect would be poor indeed.

It has been said by its opponents that science divorces itself from literature; but the statement, like so many others, arises from lack of knowledge. A glance at the less technical writings of its leaders-of its Helmholtz, its Huxley, and its Du Bois-Reymond-would show what breadth of literary culture they command. Where among modern writers can you find their superiors in clearness and vigor of literary style? Science desires not isolation, but freely combines with every effort toward the bettering of man's estate. Single-handed, and shpported, not by outward sympathy, but by inward force, it has built at least one great wing of the many-mansioned home which man in his totality demands. And if rough walls and protruding rafter-ends indicate that on one side the edifice is still incomplete, it is only by wise combination of the parts required, with those already irrevocably built, that we can hope for completeness. There is no necessary incongruity between what has been accomplished and what remains to be done. The moral glow of Socrates, which we all feel by ignition, has in it nothing incompatible with the physics of Anaxagoras which he so much scorned, but which he would hardly scorn to-day. And here I am reminded of one among us, hoary, but still strong, whose prophet-voice some thirty years ago, far more than any other of this age, unlocked whatever of life and nobleness lay latent in its most gifted minds-one fit to stand beside Socrates or the Maccabean Eleazar, and to dare and suffer all that they suffered and dared-fit, as he once said of 
Fichte, " to have been the teacher of the Stoa, and to have discoursed of Beauty and Virtue in the groves of Academe." With a capacity to grasp physical principles which his friend Goethe did not possess, and which even total lack of exercise has not been able to reduce to atrophy, it is the world's loss that he, in the vigor of his years, did not open his mind and sympathies to science, and make its conclusions a portion of his message to mankind. Marvelously endowed as he was-equally equipped on the side of the heart and of the understanding - he might have done much toward teaching us how to reconcile the claims of both, and to enable them in coming times to dwell together, in unity of spirit and in the bond of peace.

And now the end is come. With more time, or greater strength and knowledge, what has been here said might hare been better said, while worthy matters, here omitted, might have received fit expression. But there would have been no material deviation from the views set forth. As regarls myself, they are not the growth of a day; and as regards you, I thought you ought to know the environment which, with or without your consent, is rapidly surrounding you, and in relation to which some adjustment on your part may be necessary. A hint of Hamlet's, however, teaches us how the troubles of common life may be ended; and it is perfectly possible for you and me to purchase intellectual peace at the price of intellectual death. The world is not without refuges of this description; nor is it wanting in persons who seek their shelter, and try to persuade others to do the same. The unstable and the weak have yielded and will yield to this persnasion, and they to whom repose is sweeter than the truth. But I would exhort you to refuse the offered shelter, and to scorn the base repose-to accept, if the choice be forced upon you, commotion before stagnation, the breezy leap of the torrent before the foetid stillness of the swamp. In the course of this address I have tonched on debatable questions, and led you over what will be deemed dangerons ground-and this partly with the view of telling you that, as regurds these questions, science claims unrestricted right of search. It is trot to the point to suy that the riews of Dincretius and Bruno, of Darwin and Spencer, may be wrong. Hore I should agree with you, deeming it indeed 
certain that these views will undergo modification. But the point is, that, whether right or wrong, we claim the right to discuss them. For science, however, no exclusive claim is here made; you are not urged to erect it into an idol. The inexorable advance of man's understanding in the path of knowledge, and those unquenchable claims of his moral and emotional nature, which the understanding can never satisfy, are here equally set forth. 'The world embraces not only a Newton, but a Shakespeare -not only a Boyle, but a Raphael-not only a Kant, but a Beethoven-not only a Darwin, but a Carlyle. Not in each of these, but in all, is human nature whole. They are not opposed, but supplementary-not mutually exclusive, but reconcilable. And if, unsatisfied with them all, the human mind, with the yearning of a pilgrim for his distant home, will still turn to the mystery from which it has emerged, seeking so to fashion it as to give unity to thought and faith; so long as this is done, not only without intolerance or bigotry of any kind, but with the enlightened recognition that ultimate fixity of conception is here unattainable, and that each succeeding age must be held free to fashion the mystery in accordance with its own needs-then, casting aside all the restrictions of materialism, I would affirm this to be a field for the noblest exercise of what, in contrast with the knowing faculties, may be called the creative faculties of man. Here, however, I touch a theme too great for me to handle, but which will assuredly be handled by the loftiest minds, when you and I, like streaks of morning cloud, shall have melted into the infinite azure of the past.

\section{CHAPTER XXXII.}

APOLOGY FOR THE BELFAST ADDRESS. 1874.

THE WORLD has been frequently informed of late that I have raised up against myself a host of enemies; and considering, with few exceptions, the deliverances of the Press, and more particularly of the religious Press, I am forced to admit that the statement is only too true. I derive some comfort, nevertheless, from the reflection of Diogenes, transmitted to us by Plutarch, that "he who 
would be saved must have good friends of violent encmies; and that he is best off who possesses both." 'This " best" condition, I have reason to believe, is mine.

lieflecting on the fraction I have read of recent remonstrances, appeals, menaces, and judgments-covering not only the world that now is, but that which is to come-I have noticed with mournful interest how trivially men seem to be influenced by what they call their religion, and how potently by that "nature" which it is the alleged province of religion to eralieate or subdue. From fair and manly argument, from the tenderest and holiest srmpatly on the part of those who desire my eternal good. I pass by many gradations, through deliberate unfairness, to a spirit of bitterness, which desires with a fervor inexpressible in worls my eternal ill. Now, were religion the potent factor, we might expect a homogeneous utterance from those professing a common creed, while, if human nature be the really potent factor, we may expect utterances as heterogeneous as the characters of men. As a matter of fact we have the latter; suggesting to my mind that the common religion, professed and defended by these different people, is merely the accidental conduit through which they pour their own tempers, lofty or low, courteous or vulgar, mild or ferocious, as the case may be. Pureabuse, however, as serving no good end, I have, wherever possible, deliberately aroided reading, wishing, indeed, to keep not only hatred, malice, and uncharitableness, but esen every trace of irritation, far away from my side of a discussion which demands not only good-temper, but largeness, clearness, and many-sidedness of mind, if it is to guice us to even provisional solutions.

It has been stated, with many variations of note and comment, that in the address as subsequently published by Messrs. Longmans I have retracted opinions uttered at Belfast. A Roman Catholic writer is specially strong upon this point. Startled by the deep chorus of dissent which my "dazzling fallacies" have evoked, I am now trying to retreat. This be will by no means tolerate. "It is too late now to seek to hide from the eyes of mankind one foul blot, one ghastly deformity. Professor Tyudall has himself told us how and where this aldress of his was composed. It was writtun amoug the glaciers and the solitudes of the Swiss mountains. It was no hasty, hurried, 
crude production; its every sentence bore marks of thought and care."

My critic intends to be severe: he is simply just. In the "solitudes" to which he refers I worked with deliberation, endeavoring even to purify my intellect by disciplines similar to those enjoined by his own church for the sanctification of the soul. I tried, moreover, in my ponderings to realize not only the lawful, but the expedient; and to permit no fear to act upon my mind, save that of uttering a single word on which I could not take my stand, either in this or in any other world.

Still my time was so brief, the difficulties arising from my isolated position were so numerous, and my thought and expression so slow, that, in a literary point of view, I halted, not only behind the ideal, but behind the possible. Hence, after the delivery of the address, I went over it with the desire, not to revoke its principles, but to improve it verbally, and above all to remove any word which might give color to the notion of "crudeness, hurry, or" haste."

In connection with the charge of atheism my critic refers to the preface to the second issue of the Belfast Address: "Christian men," I there say, "are proved by their writings to hare their hours of weakness and of doubt, as well as their hours of strength and of conviction; and men like myself share, in their own way, these variations of mood and tense. Were the religious moods of many of my assailants the only alteruative ones, I do not know how strong the claims of the doctrine of "Material Atheism" upon my allegiance might be. Probably they would be very strong. But, as it is, I have noticed during years of self-observation that it is not in hours of clearness and vigor that this doctrine commends itself to my mind; that in the presence of stronger and healthier thought it ever dissolves and disappears, as offering no solution of the mystery in which we dwell, and of which we form a part."

With reference to this honest and reasonable utterance my censor exclaims, "This is a most remarkable passage. Much as we dislike seasoning polemics with strong words, we assert that this Apology only tends to affix with links of steel to the name of Professor Tyndall, the dread imputation against which he struggles." 
Here we have a very fair example of subjective religious vigor. But my quarrel with such exhibitions is that they do not always represent objective fact. No atheistic reasoning can, I hold, disloulge religion from the human heast. Logic cannot deprive us of life, and religion is life to the religions. As an experience of conscionsness it is beyond the assaults of logic. But the religions life is often projected in external forms-I use the word in its widest sense-and this embodiment of the religions sentiment will have to bear more and more, as the world becomes inore enlightener, the stress of scientific tests. We must be careful of projecting into external nature that which belongs to ourselves. If critic commits this mistake: he feels, and takes delight in feeling, that I am struggling, and he obviously experiences the most exquisite pleasures of "the muscular sense" in holding me down. II is feelings are as real as if his imagination of what mine are were equally real. His picture of my "struggles" is, however, a mere delusion. I do not struggle. I do not fear the charge of atheism; nor should I even disavow it, in reference to any definition of the Supreme which he, or his order, would be likely to frame. His "links" and his "steel " and his "dread imputations" are, therefore, even more unsubstantial than my "streaks of morning cloud," and they may be permitted to vanish together.

These minor and more purely personal matters at an end, the weightier allegation remains, that at Belfast I misused my position by quitting the domain of science, and making an unjustifiable raid into the domain of theology. 'This I fail to see. Laying aside abuse, I hope $m y$ accusers will consent to reason with me. Is it not lawful for a scientific man to speculate on the antecedents of the solar gystem? Did Kant, Laplace and William Herschel quit their legitimate spheres, when they prolonged the intellectual vision beyond the boundary of experience, and propounded the nebular theory? Accepting that theory as probable, is it not permitted to a scientific man to follow up, in idea, the series of changes associated with the condensation of the nebulas; to pioture tho succenvise detachument of planets :und moons, and the relation of all of them to the sun? If I look upon our earth, with its orlital revolution sunl wxial rotation, as one small issue of the process which male 
the solar system what it is, will any theologian deny my right to entertain and express this theoretic view? 'Time was when a multitude of theologians would have been found to do so-when that arch enemy of science which now vaunts its tolerance would have made a speedy end of the man who might venture to publish any opinion of the kind. But, that time, unless the world is caught strangely slumbering, is forever past.

As regards inorganic nature, then, we may traverse, without let or hindrance, the whole distance which separates the nebulæ from the worlds of to-day. But only a few years ago this now conceled ground of science was theological ground. I could by no means regard this as the final and safficient concession of theology; and, at Belfast, I thought it not only my right but my duty to state that, as regards the organic world, we must enjoy the freedom which we have already won in regard to the inorganic. I could not discern the shred of a title-deed which gave any man, or any class of men, the right to open the door of one of these worlds to the scientific searcher and to close the other against him. And I considered it frankest, wisest, and in the long run most conducive to permanent peace, to indicate, without evasion or reserve, the ground that belongs to Science, and to which she will assuredly make good her claim.

I have been reminded that an eminent predecessor of mine in the presidential chair expressed a totally different view of the cause of things from that enunciated by me. In doing so he transgressed the bounds of science at least as much as I did; but nobody raised an outcry against him. The freedom he took I claim. And looking at what I must regard as the extravagances of the religious world; at the very inadequate and foolish notions concerning this universe which are entertained by the majority of our authorized religious teachers; at the waste of energy on the part of good men over things unworthy, if I may say it without discourtesy, of the attention of enlightened heathens; the fight about the fripperies of Ritualism, and the verbal quibbles of the Athanasian Creed; the forcing on the public view of Pontigny Pilgrimages; the dating of historic epochs from the definition of the Immaculate Conception; the proclamation of the Divine Glories of the Sacred Heart-standing in the midst of these 
chimeras, which astound all thinking men, it dil not appear to me extraragant to claim the public tolerance for an hour and a half, for the statement of more reason. able views-riews more in accordance with the verities which science has bronght to light, anil which many weary souls would, I thought, welcome with gratification and relief.

But to come to closer quarters. The expression to which the most violent exception has been taken is this: "Abandoning all disguiso, the confession I feel bound to make before yon is, that I prolong the vision backward across the boundary of the experimental evidence, and discern in that Matter which we, in our ignorance, and notwithstamling our professed reverence for its Creator, have hitherto covered with opprobrium, the promise and potency of every form and quality of life." 'To call it a "chorus of dissent," as my Catholic oritic does, is a mild way of describing the storm of opprobrium with which this statement has been assailed. But the first blast of passion being past, I hope I may again ask my opponents to consent to reason. First of all, I am blamed for crossing the boundary of the experimental evidence. This, I reply, is the habitual action of the scientific mind-at least of that portion of it which applies itself to physical investigation. Our theories of light, heat, magnetism, and electricity, all imply the crossing of this boundary. My pajer on the "Scientific Use of the Imagination," and my "lectures on Light," illustrate this point in the amplest manuer; and in the article entitled "Matter and Force" in the present rolume I have sought, incidentally, to make clear, that in physics the experiential incessantly leads to the ultra-experiential; that out of experience there always grows something finer than mere experience, and that in their different powers of ideal extension consists, for the most part, the difference between the great and the merliocre investigator. The kingdom of science, then, cometh not by observation and experiment alone, but is completed by fixing the roots of observation and experiment in a region inacesssible to both, and in dealing with which we are forced to fall back upon the picturing power of the mind.

Passing the boundary of experience, therefore, does not, in the alstract, constitute a sufficient ground for censure. 
'There must have been something in my particular mode of crossing it which provoked this tremendous " chorus of dissent."

Let us calmly reason the point out. I hold the nebular theory as it was held by Kant, Laplace, and Wiliiam Herschel, and as it is held by the best scientific intellects of to-day. According to it, our sun and planets were once diffused through space as an impalpable haze, out of which by condensation, came the solar system. What caused the haze to condense? Loss of heat. What rounded the sun and planets? 'That which rounds a tear-molecular force. For æons, the immensity of which overwhelms man's conceptions, the earth was unfit to maintain what we call life. It is now covered with visible living things. They are not formed of matter different from that of the eurth around them. They are, on the contrary, bone of its bone, and flesh of its flesh. How were they introduced? Was life implicated in the nebula-as part, it may be, of a vaster and wholly Unfathomable Life; or is it the work of a Being standing ouside the nebula, who fashioned it, and vitalized it; but whose own origin and ways are equally past finding out? As far as the eye of science has hitherto ranged through nature, no intrusion of purely creative power into any series of phenomena has ever been observer. The assumption of such a power to account for special phenomena, though often made, has always proved a failure. It is opposed to the very spirit of science; and I therefore assumed the responsibility of holding up, in contrast with it, that method of nature which it has been the vocation and triumph of science to disclose, and in the application of which we can alone hope for further light. Holding, then, that the nebulæ and the solar system, life included, stand to each other in the relation of the germ to the finished organism, I reaffirm here, not arrogantly, or defiantly, but without a shade of indistinctness, the position laid down at Belfast.

Not with the vagueness belonging to the emotions, but with the definiteness belonging to the understanding, the scientific man has to put to himself these questions regarding the introduction of life upon the earth. He will be the last to dogmatize upon the subject, for he knows best that certainty is here for the present unattainable. His refusal of the creative hypothesis is less an assertion of 
knowledge than a protest against the assumption of knowl. ège which inust long, if not forever, lie beyond $\mathrm{ns}$, and the claim to which is the source of perpetual confusion upon earth. With a mind open to conviction he asks his opponents to show him an anthority for the belief they so strenuously and 80 fiercely uphold. They can do no moro than point to the book of Genesis, or some other portion of the Bible. Profoundly interesting, and indeed pathetic, to me are those attempts of the opening mind of man to appease its hunger for a cause. But the book of Genesis has no voice in seientific questions. To the grasp of geology, which it resisted for a time, it at length yielied like potter's clay; its authority as a system of cosmogony being discredited on all hands, by the abandonment of the obvious meaning of its writer. It is 'a poem, not a scientific treatise. In the former aspect it is forever beantiful: in the latter aspect it has been, and it will continue to be, purely obstructive and hurtful. To knouledye its value has been negative, lealing, in rougher ages than ours, to physical, and even in our own "free" age to moral violence.

No incident connected with the proceedings at Belfast is more instructive than the deportment of the Catholic hierarchy of Ireland; a borly usually too wise to confer notoriety upon an alversary by imprudently denouncing him. The Times, to which 1 owe a groat deal on the score of fair play, where so much has been unfair, thinks that the Irish cardinal, archbishops, and bishops, in a recent manifesto, adroitly employed a weapon which I, at an unlucky moment, placed in their hands. The antecedents of their action cause me to regard it in a different light; and a brief reforence to these antecedents will, I think, illuminate not only their proceedings regarding Belfast, but other doings which havo boen recently noised abroad.

Before me lies a document bearing the date of November, 1873, whish, after appearing for a momont, unac. countubly vanished from public view. It is a Memorial addrobsed, by seventy of the students and ex-students of the Catholic University in Ireland, to the Episcopal Board of the University; and it constituteg the plainest and bravest remonstrance ever addressed by Irish laymen to

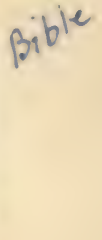


their spiritual pastors and masters. It expresses the profoundest dissatisfaction with the curriculum marked out for the students of the University; setting forth the extraordinary fact that the lecture-list for the faculty of science, published a month before they wrote, did not contain the name of a single professor of the physical or natural sciences.

The memorialists forcibly deprecate this, and dwell upon the necessity of education in science: "The distinguishing mark of this age is its ardor for science. The natural sciences have, within the last fifty years, become the chiefest study in the world; they are in our time pursued with an activity unparalleled in the history of mankind. Searce a year now passes without some discovery being made in these sciences which, as with the touch of the magician's wand, shivers to atoms theories formerly deemed unassailable. It is through the physical and natural sciences that the fiercest assaults are now made on our religion. No more deadly weapon is used against our faith than the facts incontestably proved by modern researches in science."

Such statements must be the reverse of comfortable to a number of gentlemen who, trained in the philosophy of Thomas Aquinas, have been accustomed to the unquestioning submission of all other sciences to their divine science of theology. But this is not all: "One thing seems certain," say the memorialists, viz., "that if chairs for the physical and natural sciences be not soon founded in the Catholic University, very many young men will have their faith exposed to dangers which the creation of a school of science in the University would defend them from. For oul generation of Irish Catholics are writhing under the sense of their inferiority in science, and are deternined that such inferiority shall not long continue; and so, if scientific training be unattainable at our University, they will seek it at Trinity or at the Queen's Colleges, in not one of which is there a Catholic professor of science."

Those who imagined the Catholic University of Kensington to be due to the spontaneous recognition, on the part of the Roman hierarchy, of the intellectual needs of the age, will derive enlightenment from this, and still more from what follows: for the most formidable threat remains. To the picture of Catholic students seceding 
to 'Trinity and the Queen's Colleges, the memorialists ald this darkest stroke of all: "They will, in the solitude of their own homes, unaided by any guiding advice, devour the works of Haeckel, Datrwin, Huxley, 'Tyndall, and Lyell; works innocnous if studied nnder a professor who would point out the difference between established facts and erroneons inferences, but which are calculated to sap the faith of a solitary student, deprived of a discriminating juigment to which he could refer for a solution of his difficulties."

In the light of the knowledge given by this courageous memorial, and of similar knowledge otherwise deriverd, the recent Catholic manifesto did not at all strike me as a ohuckle over the mistake of a maladroit alversary, but rather as an evilence of profonnd uneasiness on the part of the cardinal, the arehbishops, and the bishops who signed it. They acted toward the Student's Memorial, however, with their accustomed practical wistom. As one concession to the spirit which it embodied, the Catholic University at Kensington was brought forth, apparently as the effect of spontaneons inward force, and not of ontward pressure becoming too formidable to be successfully opposed.

The memorialists point with bitterness to the fact, that "the name of no Irish Catholic is known in connection with the phisical and natural sciences." But this, they ought to know, is the complaint of free and cultirated minds. wherever a priesthood exercises dominant power. Preciscly the same complaint has been made with respect to the Catholics of Germany. The great national literature aml the scientific achievements of that country, in molern times, are almost wholly the work of Protestants. A vanishingly small fraction of it only is derised from members of the Roman Ohurch, althongh the number of these in Germany is at least as great as that of the Protestants. "The question arises," says a writer in an able German perioulical, "what is the cause of a phenomenon so humil. bating to the Catholice? It cannot be reforred to want of natural endowment due to climate (for the Protestants of sonthern Germany have contributed powerfully to the creations of the (ferman intellect), but purely to ontwarl circumstances. And these are remolily dimecosered in the pressure exereisud for centuries by the Jesuitical system, 
which has crushed out of Catholics every tendency to free mental productiveness." It is, indeer, in Catholic countries that the weight of Ultramontanism has been most severely felt. It is in such countries that the very finest spirits, who have dared, without quitting their faith, to plead for freedom or reform, have suffered extinction. The extinction, however, was more apparent than real, and Hermes, Hirscher, and Günther, though individually broken and subdued, prepared the way, in Bavaria, for the persecuted but unflinching Frohschammer, for Döllinger, and for the remarkable liberal movement of which Döllinger is the head and guide.

Though molded for centuries to an obedience unparalleled in any other country, except Spain, the Irish intellect is beginning to show signs of independence; demanding a diet more suited to its years than the pabulum of the middle ages. As for the recent manifesto in which pope, cardinal, archbishops, and bishops are united in one grand anathema, its character and fate are shadowed forth by the vision of Nebuchadnezzar recorded in the book of Daniel. It resembles the image, whose form was terrible, but the gold, and silver, and brass, and iron of which rested upon feet of clay. And a stone smote the feet of clay, and the iron, and the brass, and the silver, and the gold, were broken in pieces together, and became like the chaff of the summer threshing-floors, and the wind carried them away.

Monsignor Capel has recently been good enough to proclaim at once the friendliness of his church toward true science, and her right to determine what true science is. Let us dwell for a moment on the proofs of her scientific competence. When Halley's comet appeared in 1456 it was regarded as the harbinger of God's vengeance, the dispenser of war, pestilence, and famine, and by order of the pope the church bells of Europe were rung to scare the monster away. An additional daily prayer was added to the supplications of the faithful. The comet in due time disappeared, and the faithful were comforted by the assurance that, as in previous instances relating to eclipses, droughts, ancl rains, so also as regards this "nefarious" comet, victory had been vouchsafed to the church.

Both Pythagoras and Copernicus had tanght the heliocentric doctrine-that the earth revolves round the 
8un. In the exercise of her right to determine what true science is, the Church, in the pontificato of Paul V., stepped in, and by the mouth of the holy Congregation of the Index, delivered, on March 5, 1616, the following decree:

And whereas it hath also come to the knowledge of the said holy congregation that the false Pythagorean dartrine of the mobility of the earth and the immolitity of the sun, entirely opposed to Holy writ, which is tanght by Nimolas Copernicus, is now published abroad and received by many. In order that this opimion may not furlher sprent, to the damage of Catholic truth, it is ordered that this and all other books tenching the like doctrine be suspended, and by this decree they are all respectively suspended, forbidden, and condemned.

Bat why go back to 1456 and 1616? Far be it from me to charge bygone sins upon Monsignor Capel, were it not for the practices he upholds to-day. The most applanded dogmatist and champion of the Jesuits is, I am informed, Perrone. No less than thirty editions of a work of his have been scattered abroad for the healing of the nations. Il notions of physical astronomy are virtually those of 1456 . He teaches boldly that "God does not rule by unirersal law ... that when God orders a given planet to stand still He does not detract from any law passed by Ilimaelf, but orders that planet to more round the sun for such and such a time, then to stand still, and then again to move, as His pleasure may be." Jesuitism proscribed Frohschammer for questioning its favorite dogma, that every human soul was created by a direct supernatural act of God, and for asserting that man, body and soul, came from his parents. This is the system that now strives for universal power; it is from it, as Monsiguor Capel gracioubly informs us, that we are to learn what is allowable in science, and what is not!

In the fuce of such facts, which might be multiplied at will, it requires extraordinary bravery of mind, or a reliance upon public ignorance almost as extraordinary, to make the claims mate by Monsignor Capel for his Chureh.

Before me is a rery remarkable letfer aldressed in 1855 by the lishop of Montfuellier to the desuss and profesaurs of faculties of Montpellier, in which the writer very clearly lays down the clains of his Chureh. He hat ben 
startled by an incident occurring in a course of lectures on physiology given by a professor, of whose scientific capacity there was no doubt, but who, it was alleged, rightly or wrongly, had made his course the vehicle of materialism. "Je ne me suis point donné," says the bishop, "la mission que je remplis an milieu de vous. "Personne, au tenoignage de saint Paul, ne s'attribue à soi-même un pareil honneur; il y faut être appelé de Dien, comme Aaron.' Et pourquoi en est-il ainsi? C'est parce que, selon le même A pôtre, nous devons être les ambassadeurs de Dieu; et il n'est pas dans les usages, pas plus qu'il n'est dans la raison et le droit, qu'un envoyé s'accrédite lui-même. Mais, si j'ai reçu d'En-Haut une mission; si l'Eglise, au nom de Dieu lui-même, a souscrit mes lettres de créance, me siéraitil de manquer aux instructions qu'elle $m^{\circ} a$ données et d'entendre, en un sens différent du sien, le rôle qu'elle m'a confié?

"Or, Messieurs, la sainte Eglise se croit investie du droit absolu d'enseigner les hommes; elle se croit dépositaire de la vérité, non pas de la vérité fragmentaire, incomplète, mêlée de certitude et d'hésitation, mais de la vérité totale, complete, au point de vue religieux. Bien plus, elle est si sûre de l'infaillibilité que son Fondateur divin lui a communiquée, comme la dot magnifique de leur indissoluble alliance, que, même dans l'ordre naturel, scientifique ou philosophique, moral ou politique, elle n'admet pas qu'un système puisse être soutenu et adopté par des chrétiens, s'il contredit à des dogmes définis. Elle considère que la négation voluntaire et opiniâtre d'un seul point de sa doctrine rend coupable du péché d'hérésie; et elle pense que toute hérésie formelle, si on ne la rejette pas courageusement avant de paraître devant Dieu, entraîne avec soi la perte certaine de la grâce et de l'éternité."

The bishop recalls those whom he addresses from the false philosophy of the present to the philosophy of the past, and foresees the triumph of the latter. "Avant que le dix-neuvième siècle s'achève, la vieille philosophie scolastique aura repris sa place dans la juste admiration du monde. Il lui faudra pourtant bien du temps pour guérir les maux de tout genré, cuusés par son indigne rivale; et pendant de longues années encore, ce nom de philosophie, le plus grand de la langue humaine après celui de religion, sera suspect aux âmes qui se souviendront 
de la science impie et matérialiste de Locke, de Condillac on dHelvetius. IThenre actuello est aux sciences naturelles: cest maintenant l'instrument de combat contre l'Figlise et contre tunte foi religieuse. Nous ne les reiloutons pas." Further on the bishop warns his realers that everything can be abused. Poetry is good, but in excess it may injure practical conduct. ". Les mathématiques sont excellentes: et Bossuet les a lonieg ' comme étant ce qui sert le plus a la justesse du raisonnement; ' mais si on s'accoutume ex. clusivement à leur méthode, rien de co qui appartient a loordre moral ne parait plus pouvoir étre démontré; et Féneton a pu parler de lensurcellement et des attraits diaboliques de la géométrie."

The learned bishop thus finally accentuates the claims of the Cliurch: "Comme le definissait le Pape İéon X., au cinquième concile cecuménique de Latrun," Le vrai ne put pas ètre contraire à lui-méme: par conséquent, toute assertion contraire à une vérité de foi révélée est nécessairement et absolument fausse.' Il suit de là que, sans entrer dans l'examen scientifique de telle ou telle question de physiologre, инаis par la seule certitude de uos dogmes, nous pourons juger du sort de telle on telle hypothise, qui est une machino de guerre anti-chrétienue plutồ qu'une conquête sérieuse sur les secrets et les mystìres de la nature. . . C'est un dogme que l'homme a été formé et façonné des mains de Dieu. Donc il est faux, hérétique, contraire a la dignité du Créateur et offensant pour son chef-d'auvre, de dire que l'homme constitue la srplième espéce des singes. . Hérésie encore de dire que le genre humain n'est pas sorti d'un seul couple, et qu'on $y$ peut compter jusqu'd douze races distinctes!"

The course of life upon earth, as far as science can see, has been one of amelioration-a steady adrance on the whole from the lower to the higher. The continued effort of animated nature is to improve its condition and raise itself to a loftier lovel. In man improvement and amelioration depend largely upon the growth of conscious knowleige, by which the errors of ignorance are continually molded, and truth is orgmuized. It is the alsance of knowlelge that has given a materialistic color to the philosophy of this age. Saterialism is therefore not a thing to 
be mourned over, but to be honestly considered-accepted. if it be wholly true, rejected if it be wholly false, wisely sifted and turned to account if it embrace a mixture of truth and error. Of late years the study of the nervous system, and its relation to thought and feeling, have profoundly occupied inquiring minds. It is our duty not to shirk-it ought rather to be our privilege to accept -the established results of such inquiries, for here assuredly our ultimate weal depends upon our loyalty to the truth. Instructed as to the control which the nervous system exercises over man's moral and intellectual nature, we shall be better prepared, not only to mend their manifold defects, but also to strengthen and purify both. Is mind degraded by this recognition of its dependence? Assuredly not. Matter, on the contrary, is raised to the level it ought to occupy, and from which timid ignorance would remove it.

But the light is dawning, and it will become stronger as time goes on. Even the Brighton "Church Congress" affords evidence of this. From the manifold confusions of that assemblage my memory has rescued two items, which it would fain preserve: the recognition of a relation between health and religion, and the address of the Rev. Harry Jones. Out of the conflict of vanities his words emerge wholesome and strong, because undrugged by dogma, coming directly from the warm brain of one who knows what practical truth means, and who has faith in its vitality anil inherent power of propagation. I wonder whether he is less effectual in his ministry than his more embroidered colleagues? It surely behooves our teachers to come to some definite understanding as to this question of health; to see how, by inattention to it, we are defrumded, negatively and positively: negatively, by the privation of that "sweetness and light" which is the natural concomitant of good health; positively, by the insertion into life of cynicism, ill-temper, and a thousand corroding anxieties which good health would dissipate. We fear and scorn "materialism." But he who knew all about it, and could apply his knowledge, might become the preacher of a new gospel. Not, however, through the ecstatic moments of the individual does such knowledge come, but through the revelations of science, in connection with the history of mankind. 
Why should the Roman Catholic Chureh eall gluttony a mortal sin? Why should fasting occupy a place in the disciplines of religion? What is the meaning of Tather's alvice to the young clergyman who came to him, perplexed with the difhenlties of predestination and election, if it lee not that, in virtue of its action upon the brain, when wisely applied, there is moral and religious virtue even in a hydrocarbon? To use the old language, food and drink are creatures of God, and have therefore a spiritual value. 'Through our neglect of the monitions of a rensonable inaterialism we sin and suffor daily. I might here point to the train of deadly disorders over which science has given molern society such control-disclosing the lair of the material enemy, insuring his destruction, and thus preventing that moral squalor and hopelessness which habitnally tread on the heels of epidemics in the case of the poor.

Rising to higher spheres, the visions of Swedenborg, and the ecstasy of Plotinus and Porphyry, are phases of that psychical condition, obvionsly connected with the nervous system and state of health, on which is based the Vedic dectrine of the absorption of the indivilual into the universal soul. Plotinas taught the derout how to pass into a condition of ecstasy. Porphyry complains of having been only once united to fod in eighty-six years, while his master Plotinus had been so united six times in sixty years." A friend who knew Wordsworth informs me that the poet, in some of his mools, was accustomed to seize hold of an external object to assure himself of his own boulily existence. As states of conscionsuess such phenomena have an undisputed reality, and a substantial identity; but they are connected with the most heterogeneous objective conceptions. 'The subjective experiences are similar, because of the similarity of the underlying organizations.

But for those who wish to look beyond the practical facts, there will always remain ample room for speculation. Take the argument of the Lucretian introduced in the Belfast address. As far as I am aware, not one of my

- I reconumend to the reader's particular attention IDr. Draper's impurtant wotix entitled, " Hintory of the Conflict between Relligion and science." (Messers. H. 8. King and (ंo.) 
assailants has attempted to answer it. Some of them, indeed, rejoice over the ability displayed by Bishop Butler in rolling back the difficulty on his opponent; and they even imagine that it is the bishop's own argument that is there employed. But the raising of a new difficulty does not abolish-does not even lessen-the old one, and the argument of the Lucretian remains untouched by anything the bishop has said or cun say.

And here it may be permitted me to add a word to an important controversy now going on: and which turns on the question: Do states of consciousness enter as links into the chain of antecedence and sequence, which give rise to bodily actions, and to other states of conscionsuess; or are they merely by-products, which are not essential to the physical processes going on in the brain? Speaking for myself, it is certain that I have no power of imagining states of consciousness, interposed between the molecules of the brain, and influencing the transference of motion among the molecules. The thought "eludes all mental presentatiou;" and hence the logic seems of iron strength which claims for the brain an antomatic action, uninfluenced by states of consciousness. But it is, I believe, admitted by those who hold the automaton-theory, that states of consciousness are produced by the marshaling of the molecules of the brain: and this production of consciousness by molecular motion is to me quite as inconceivable on mechanical principles as the production of molecular motion by consciousness. If, therefore, I reject one result, I must reject both. I, howerer, reject neither, and thus stand in the presence of two Incomprehensibles, instead of one Incomprehensible. While accepting fearlessly the facts of materialism dwelt upon in these pages, I bow my head in the dust before that mystery of mind, which has hitherto defied its own penetrative power, and which may ultimately resolve itself into a demonstrable impossibility of self-penetration.

But the secret is an open one-the practical monitions are plain enough, which declare that on our dealings with matter depend our weal and woe, physical and moral. The state of mind which rebels against the recognition of the claims of "materialism" is not unknown to me. I can remember a time when I regarded my body as a weed, 
80 much more highly did I prize the conscions strength and fleasure derived from moral and religious feeling $\rightarrow$ which, I may ald, was mine without the intervention of dogma. 'The error was not an ignoble one, but this did not save it from the penalty attaclsed to error. Saner knowledge taught me that the body is no weed, and that treated as such it would infallibly avenge itself. A m I personally lowered by this change of front? Not so. Give ne their health, and there is no spiritual experience of those earlier years - no resolve of duty, or work of mercy, no work of self-renouncement, no solemnity of thought, no joy in the life and aspects of nature-that would not still be mine; and this without the least reference or regard to any purely personal reward or punishment looming in the future.

And now I have to utter a "farewell" free from bitterness to all $m y$ readers; thanking $m y$ friends for a sympathy more stealfast, I would fain believe, if less noisy, than the antipathy of my foes; and commending to these a passage from Bishop Butler, which they have either not read or failed to lay to heart. "It seems," saith the bishop, "that men would be strangely headstrong and self-willed, and disposed to exert themselves with an impetuosity which would render society insupportable, and the living in it impracticable, were it not for some acquired moderation and self-goverument, some aptitude restraining themselves, and concealing their sense of things."

\section{CHAPTER XXXIII.}

THE REV. JAMES MA RTINEA U AND THE BELPAST ADDRFSS."

Prion to the publication of the fifth edition of these "Fragments" my attention had been directed by several estimable, and indeed eminent, persons, to an essay by the IRer. James Martinean, as demanding serions consideration at my hands. I tried to give the essay the attention claimed for it, and published my views of it as an Introduction to Part II. of the "Fragments." I there referred, and here again refer with pleasure, to the accord subsisting between Mr. Martinean and myself on certain points of

* Fortnightly Review." 
biblical Cosmogony. "In so far," says he, "as Church belief is still committed to a given Cosmogony and natural history of man, it lies open to scientific refutation." And again: "It turns out that with the sun and moon and stars, and in and on the earth, before and after the appearance of our race, quite other things have happened than those which the sacred Cosmogony recites." Unce more: "'The whole history of the genesis of things Religion must surrender to the Sciences." Finally, still more emphatically: "In the investigation of the genetic order of things, 'Theology is an intruder, and must stand aside." 'This expresses, only in words of fuller pith, the views which I ventured to enunciate in Belfast. "The impregnable position of science," I there say, " may be stated in a few words. We claim, and we shall wrest from Theology, the entire domain of Cosmological theory." Thus Theology, so far as it is represented by Mr. Martineau, and Science, so far as I understand it, are in absolute harmony here.

But Mr. Martineau would have just reason to complain of me, if, by partial citation, I left my readers under the impression that the agreement between us is complete. At the opening of the eighty-ninth session of the Manchester New College, London, on October 6, 18\%4, he, its principal, delivered an address bearing the title "Religion as affected by Modern Materialism;" the references and general tone of which make evident the depth of its author's discontent with my previous deliverance at Belfast. I find it difficult to grapple with the exact grounds of this discontent. Indeed, logically considered, the impression left upon iny mind by an essay of great æsthetic merit, con. taining many passages of exceeding beanty, and manysentiments which none but the pure in heart could utter as they are uttered here, is vague and unsatisfactory. The author appears at times so brave and liberal, at times so timid and captious, and at times, if I dare say it, so imperfectly informed, regarding the position he assails.

At the outset of his address $\mathrm{Mr}$. Martineau states with some distinctness his "sources of religious faith." They are two-" the scrutiny of Nature" and "the interpretation of Sacred Books." It would have been a theme worthy of his intelligence to have deduced from these two sources his religion as it stands. But not another word is said about the "Sacred Books." Having swept with the 
besom of Sicience various " books" contemptuously away, lie does not define the Sucred residue; much less give us the reasons why he deems them sacred.* His references to "N Nature," on the other hand, are magnificent tiradeg against Nature, intended, appareritly, to show the wholly abominable character of man's antecelents if the theory of evolution be true. Here also his mood lacks steadiness. While joyfully accepting, at one place, "the willening space, the deepening vistas of time, the detected marrels of physiological structure, and the rapid filling-in of the missing links in the chain of organic life," he falls, at another, into lamentation and mourning over the very theory which renders "organic life" "a chain." He claims the largest liberality for his sect, and avows its contempt for the dangers of possible discovery. But immeliately afterward he damages the claim, and ruins all confidence in the avowal. He professes sympathy with modern Sicience, and almost in the same breath he treats, o: certaiuly will be understood to treat, the Atomic 'Theory, and the doctrine of the Conservation of Energy, as if they were a kind of scientific thimble-riggery.

His ardor, moreover, renders him inaccurate; causing him to see discord between scientific men where nothing but harmony reigns. In his celebrated aldress to the Congress of German Naturforscher, delivered at Leipzig, three years ago, Du Bois-Reymond speaks thus: "What conceirable connection subsists between definite movements of definite atoms in my brain, on the one hand, and on the other hand such primordial, indefinable, undeniable, facts as these: I feel pain or pleasure; I experience a sweet taste, or smell a rose, or hear an organ, or see something red. . . . It is absolutely and forever inconceisablo that an number of carbon, hydrogen, nitrogen, and oxygen atoms should be otherwise than indifferent as to their own position and motion, past, present, or future. It is utterly inconceivable how consciousmess should result from their joint action."

This language, which was spoken in 18\%, Mr. Martineau

* Mr. Martinean's use of the term " sacred " is unintentionally mis lending. In his later essags we are taught that he doen not mean to restriet it to the Bible. He does not, howerer, mention the "lomks" lergond those of the Bible to which he would apply the term. 1879. 
"freely" translates, and quotes against me. The act is due to misapprehension. Evilence is at hand to prove that I employed similar language twenty years ago. It is to be found in the Suturday Review for 1860; but a sufficient illustration of the agreement between my friend Du Bois-Reymond and myself is furnished by the discourse on "Scientific Materialism," delivered in 1868, then widely circulated, and reprinted here. The reader who compares the two discourses will see that the same line of thought is pursued in both, and that perfect agreement reigns between my friend and me. In the very address he criticises, Mr. Martineau might have seen that precisely the same position is maintained. A quotation will prove this: "Thus far," I say, "our way is clear, but now comes my difficulty. Your atoms are individually without sensation, much more are they without intelligence. May I ask you, then, to try your hand upon this problem? 'Take your' dead hydrogen atoms, your dead oxygen atoms, your dead carbon atoms, your dead nitrogen atoms, your dead phosphorus atoms, and all the other atoms, dead as grains of shot, of which the brain is formed. Imagine them separate and sensationless; observe them rumning together and forming all imaginable combinations. This, as a purely mechanical process, is seeable by the mind. But can you see, or dream, or in any way imagine, how out of that mechanical act, and from these individually dead atoms, sensation, thought, and emotion are to rise? Are you likely to extract Homer out of the rattling of dice, or the Differential Calculus out of the clash of billiard balls?... I can follow a particle of musk until it reaches the olfactory nerve; I can follow the waves of sound until their tremor's reach the water of the labyrinth, and set the otoliths and Corti's fibers in motion; I can also visualize the waves of ether as they cross the eye and hit the retina. Nay, more, I am able to pursue to the central organ the motion thus imparted at the periphery, and to see in idea the very molecules of the brain thrown into tremor's. My insight is not baffled by these physical processes. What baffles and bewilders me is the notion that from these physical tremors things so utterly incongruous with them as sensation, thought, and emotion can be derived." It is only a complete misapprehension of our true relationship that could induce Mr. Martineau to represent Du BoisReymond and myself as opposed to each other. 
"The affluence of illustration," writes an able and sympathetic reviewer of this essay, in the New York Tribune, "in which Mr. Martinean delights often impairs the distinctness of his statements by diverting the attention of the reader from the essential points of his discussion to the beanty of his imagery, and thus disminishes their power of conviction." To the beauties here referred to I bear willing testimony; but the reviewer is strictly just in his estimate of their effect npon my critic's logic. The "affluence of illustration," and the heat, and haze, and haste, generated by its reaction upon Mr. Martineau's own mind, often produce vagueness where precision is the one thing needful-poetic fervor where we require judicial calm; and practical unfairness where the strictest justice ought to be, and I willingly believe is meant to be, observeả.

In one of his nobler passages Mr. Martineau tells us how the pupils of his college have been educated hitherto: "They have been trained under the assumptions (1) that the Universe which includes us and folds us round is the life-dwelling of an Eternal Mind; (2) that the world of our abode is the scene of a moral government, incipient but not complete; and (3) that the upper zones of human affection, above the clouds of self and passion, take us into the sphere of a Divine Communion. Into this over-arching scene it is that growing thought and enthusiasm have expanded to catch their light and fire."

Alpine summits seem to kindle above $\mathrm{ns}$ as we read these glowing words; we see their beauty and feel their life. At the close of one of the essays here printed,"* I thus refer to the "Communion" which Mr. Martineau calls “Divine:" “'Two things,' said Immannel Kant, 'fill me with awe-the starry heavens, and the sense of moral responsibility in man.' And in his hours of health and strength and sanity, when the stroke of action has ceased, and the pause of reflection has set in, the scientific investigator finds himself overshadowed by the same awe. Breaking contact with the hampering details of earth, it associates him with a power which gives fullness and tone to his existence, but which he can neither analyze nor 
comprehend." Though "knowledge" is here disavowed, the "feelings" of Mr. Martineau and myself are, I think, very much alike. He, nevertheless, censures me-almost denounces me-for referring religion to the region of emotion. Surely he is inconsistent here. The foregoing words refer to an inward hue or temperature, rather than to an external object of thought. . When I attempt to give the Power which I see manifested in the Universe an objective form, personal or otherwise, it slips away from me, declining all intellectual manipulation. I dare not, save poetically, use the pronoun "He" regarding it; I dare not call it a "Mind;" I refuse to call it even a "Cause." Its mystery overshadows me; but it remains a mystery, while the objective frames which some of my neighbors try to make it fit, seem to me to distort and desecrate it.

It is otherwise with Mr. Martineau, and hence his discontent. He professes to know where I only claim to feel. He could make his contention good against me if, by a process of verification, he would transform his assumptions into "objective knowledge." But he makes no attempt to do so. They remain assumptions from the beginning of his address to its end. And yet he frequently uses the word " unverified," as if it were fatal to the position on which its incidence falls. "The scrutiny of Nature" is one of his sources of "religious faith;" what logical foothold does that scrutiny furnish, on which any one of the foregoing three assumptions could be planted? Nature, according to his picturing, is base and cruel: what is the inference to be drawn regarding its author? If nature be "red in tooth and claw," who is responsible? On a mindless nature Mr. Martineau pours the full torrent of his gorgeous invective; but could the "assumption" of "an Eternal Mind"-even of a Beneficent Eternal Mind-render the world objectively a whit less mean and ugly than it is? Not an iota. It is man's feelings, and not external phenomena, that are influenced by the assumption. It adds not a ray of light nor a strain of music to the objective sum of things. It does not touch the phenomena of physical nature-storm, flood, or fire-nor diminish by a pang the bloody combats of the animal world. But it does add the glow of religious emotion to the human soul, as represented by Mr. Martineau. 
Beyond this I defy him to go; and yet he rashly-it might be said petulantly-kicks away the only philosophic foundation on which it is possible for him to build his religion.

He twits incidentally the modern scientific interpretation of nature because of its want of cheerfulness. "Let the new future," he says, "preach its own gospel, and devise, if it can, the means of making the tidings glad." 'This is a common argument: "If you only knew the comfort of belief!" My reply is that I choose the nobler part of Emerson, when, after various disenchantments, he exclaimed, "I covet truth!" 'The gladness of true heroism visits the heart of him who is really competent to say this. Besides, "gladness" is an emotion, and Mr. Martineau theoretically scorns the emotional. I an not, however, acquainted with a writer who draws more largely upon this source, while mistaking it for something objective. "To reach the Cause," he says, "there is no need to go in to the past, as though being missed here, He could be fousd there. But when once $\mathrm{He}$ has been apprehended by the proper organs of divine apprehension, the whole life of Humanity is recognized as the scene of His agency." That Mr. Martineau should have lived so long, thought so much, and failed to recognize the entirely subjective character of this creed, is highly instructive. IIIs " proper organs of divine apprehension "-given, we must assume, to Mr. Martineau and his pupils, but denied to many of the greatest intellects and noblest men in this and other ages-lie at the very core of his emotions.

In fact, it is when Mr. Martineau is most purely emotional that he scorns the emotions; it is when he is most purely subjective that he rejects subjectivity. He pays a just and liberal tribute to the character of John Stuart Mill. But in the light of Mill's philosophy, benevolence, honor, purity, having "shrunk into mere unaccredited subjective susceptibilities, have lost all support from Omniscient approval, and all presumable accordance with the reality of things." If Mr. Martineau had given them any inkling of the process by which he renders the "subjective susecptibilities" objective, or how he arrives at an objective ground of "Omniscient approval," gratitude from his pupils would hare been his just meed. But, as it is, he leaves them lust in an iridescent cloud of 
words, after exciting a desire which he is incompetent to appease.

"We are," he says, in another place, "forever shaping our representations of invisible things into forms of definite opinion, and throwing them to the front, as if they were the photographic equivalent of our real faith. It is a delusion which affects us all. Yet somehow the essence of our religion never finds its way into these frames of theory: as we put them together it slips away, and, if we turn to pursue it, still retreats behind; ever rearly to work with the will, to unbind and sweeten the affections, and bathe the life with reverence, but refusing to be seen, or to pass from a divine hue of thinking into a human pattern of thought." 'This is very beautiful, and mainly so because the man who utters it obviously brings it all out of the treasury of his own heart. But the "hue " and "pattern" here so finely spoken of, the former refusing to pass into the latter, are neither more nor less than that "emotion," on the one hand, and that "objective knowledge," on the other, which have drawn this suicidal fire from Mr. Martineau's battery.

I riow come to one of the most serious portions of $\mathrm{Mr}$. Martineau's pamphlet-serious far less on account of its "personal errors," than of its intrinsic gravity, though its author has thought fit to give it a witty and sarcastic tone. He analyzes and criticises "the materialist doctrine, which, in our time, is proclaimed with so much pomp, and resisted with so much passion. 'Matter is all I want,' says the physicist; 'give me its atoms alone, and I will explain the universe." It is thought, even by Mr. Martineau's intimate friends, that in this pamphlet he is answering me. I must therefore ask the reader to contrast the foregoing travesty with what I really do say regarding atoms: "I lo not think that he [the materialist] is entitled to say that his molecular groupings and motions explain everything. In reality, they explain nothing. The ntmost he can affirm is the association of two classes of phenomena, of whose real bond of union he is in absolute ignorance." * This is very different from saying, "Give me its atoms alone, and I will explain the universe." Mr. Martineau continues his dialogue with the 
physicist: "'Good,' he says; 'take as many atoms as you please. See that they hitve all that is requisite to body, [a metaphysical 13], being homogeneons extended solids.' 'That is not enongh,' his physicist rephlies; 'it might do for Democritus and the mathematicians, but I must have something more. 'The atoms must not only be in motion, and of various shinpes, but also of as many kinds as there are chemical elements; for how could I ever get water if I liul only hydrogen elements to work with?' "So be it,' Mr. Martineau consents to answer, 'only this is a considerable enlargernent of your sprecitier] datum [where, and by whom specified?]-in fact, a conversion of it into sereral: yet, even at the cost of its monism [put into it by Mr. Martineau], your scheme seems hardly to gain its end; for ly what manipulation of your resourees will you, for example, educe Conscionsmess?"

This reals like pleasantry, but it deals with serious things. For the last seven years the question here proposed by Mr. Martinean, and my answer to it, have been accessible to all. The question, in my words, is briefly this: "A man can say, 'I feel, I think, I love,' but how does conscionsness infuse itself into the problem?" And here is my answer: The passage from the physics of the brain to the corresponding facts of conscionsness is unthinkable. Granted that a definite thought and a definite molecular action in the brain occur simultaneously; we do not possess the intellectual organ, nor apparently any rudiment of the organ, which wonld enalile us to pass, by a process of reasoning, from the one to the other. They appear together, but we do not know why. Were our minds and senses so expanded, strengthened, and illuminaterl, as to enable us to see and feel the very molecules of the brain; were we capable of following all their metions, all their gronpings, all their electric discharges, if such there be; and were we intimately acquainted with the corresponding states of thought and feeling, we shonld be as far as ever from the solution of the problem, "How are these physical processes connected with the facts of conscionsness?" The chasm hetween the two classes of phenomena would still remain intellectually impassable." *

* Bishop Batler's reply to the Lucretian in the "Belfast Address " is all in the same strain. 
Compare this with the answer which Mr. Martineau puts into the mouth of his physicist, and with which I am generally credited by Mr Martineau's readers, both in England and America: " "It [the problem of consciousness] does not daunt me at all. Of course you understand that all along my atoms have been affected by gravitation and polarity; and now I have only to insist with Fechner on a difference amor:g molecules: there are the inorganic, which can change only their place, like the particles in an undulation; and there are the organic, which can change their order, as in a globule that turns itself inside out. With an adequate number of these our problem will be manageable.' 'Likely enough,' we may say [' entirely unlikely,' say I], 'seeing how careful you are to provide for all emergencies; and if any hitch should occur in the next step, where you will have to pass from mere sentiency to thought and will, you can again look in upon your atoms, and fling among them a handful of Leibnitz's monads, to serve as souls in little, and be ready, in a latent form, with that Torstellungs-fähigkeit which our picturesque interpreters of nature so much prize."

"But surely," continues Mr. Martineau, "you must observe that this 'matter' of yours alters its style with every change of serrice: starting as a beggar with scarce a rag of 'property' to cover its bones, it turns up as a prince when large undertakings are wanted. 'We must radically change our notions of matter,' says Professor Tyndall; and then, he ventures to believe, it will answer all demands, carrying 'the promise and potency of all terrestrial life.' If the measure of the required 'change in our notions' had been specified, the proposition wonld have had a real meaning, and been susceptible of a test. It is easy traveling through the stages of such an hypothesis; you deposit at your bank a round sum ere you start, and, drawing on it piecemeal at every pause, complete your grand tour without a debt."

The last paragraph of this argument is forcibly and ably stated. On it I am willing to try conclusions with Mr. Martineau. I may say, in passing, that I share his contempt for the picturesque interpretation of nature, if accuracy of vision be thereby impaired. But the term Vorstellungs-fähigkeit, as used by me, means the power of definite mental presentation, of attaching to words the 
corresponding objects of thought, and of seeing these in their proper relations, withont the interior haze and soft penumbral borders which the theologian loves. 'T'o this mode of " interpreting nature," I shall to the best of my ability now adhere.

Neither of us, I trust, will be afraid or ashamed to begin at the alphabet of this question. Our first effort must be to understand each other, and this mutual understanding can only be ensured by beginning low down. Physically speaking, however, we need not go below the seit-level. Let us then travel in company to the Caribbean Sea, and halt upon the heated water. What is that sea, and what is the sun that heats it? Answering for myself, I say that they are both matter. I fill a glass with the sia-water and expose it on the deck of the vessel; after some time the liquid has all disappeared, and left a solid residue of salt in the glass behind. We have mobility, invisibilityapparent annihilation. In virtue of

The glad and secret aid

The sun unto the ocean paid,

the water has taken to itself wings and flown off as vapor. From the whole surface of the Caribbean Sea such vapor is rising; and now we must follow it-not upon our legs, however, nor in a ship, nor even in a balloon, but by the mind's eye-in other words, by that power of Vorstellung which Mr. Martineau knows so well, and which he so justly scorns when it indulges in loose practices.

Compounding, then, the northward motion of the vapor with the earth's axial rotation, we track our fugitive through the higher atmostpheric regions, obliquely across the Atlantic Ocean to Western Europe, and on to our familiar Alps. Here another wonderful metamorphosis occurs. Floating on the cold calm air, and in presence of the cold firmanent, the vapor condenses, not only to particles of water, but to particles of crystalline water. 'These coalesce to stars of snow, which fall upon the mountains in forms so exquisite that, when first seen, they never fail to excite rapture. As to beanty, indeed, they put the work of the lapillary to shame, while as to accuracy they render concrete the abstractions of the geometer. Are these crystals " matter?" Without presuming to dogmatize, I answer for myself in the affirmative. 
Still, a formative power has obviously here come into plity which did not mauifest itself in either the liquid or the rapor. 'The question now is, was not the power' "potential" in both of them, requiring only the proper conditions of temperature to bring it into action? Again I answer for myself in the affirmative. I am, however, quite willing to discuss with Mr. Martineau the alternative hypothesis, that an imponderable formative soul unites itself with the substance after its escape from the liquid state. If he should espouse this hypothesis, then I should demand of him an immediate exercise of that Vortellungsfähigkeit, with which, in my efforts to think clearly, I can never dispense. I should ask, at what moment did the soul come in? Did it enter at once or by degrees; perfect from the first, or growing and perfecting itself contemporaneously with its own handiwork? I should also ask whether it is localized or diffusel? Does it move about as a lonely builder, putting the bits of solid water in their places as soon as the proper temperature has set in? or is it distributed through the entire mass of the crystal? If the latter, then the soul has the shape of the crystal; but if the former, then I should inquire after its shape. Has it legs or arms? If not, I would ask it to be made clear to me how a thing withont these appliances can act so perfectly the part of a builder? (I insist on definition, and ask unusual questions, if haply I might thereby banish unmeaning words.) What were the condition and residence of the soul before it joined the crystal? What becomes of it when the crystal is dissolred? IVhy should a particular. temperature be needed before it can exercise its vocation? Finally, is the problem before us in any way simplified by the assumption of its existence? I think it probable that, after a full discussion of the question, Mr. Martineau would agree with me in ascribing the building power displayed in the crystal to the bits of water themselves. At all events, I should count upon his sympathy so far as to believe that he would consider any one unmannerly who would denounce me for rejecting this notion of a separate soul, and for holding the snow-crystal to be " matter."

But then what an astonishing addition is here made to the powers of matter! Who would have dreamed, without actually seeing its work, that such a power was locked up 
in a drop of water? All that we needed to make the action of the liquid intelligible was the assumption of $\mathrm{Mr}$. Martineau's "homogeneous extended atomic solids," smoothly gliding over one another. But had we supposed the water to be nothing more than this, we should have ignorantly defranded it of an intrinsic architectural power, which the art of man, even when pushed to its utmost degree of refinement, is incompetent to imitate. I wonld insite Mr. Martineau to consider how inappropiate his figure of a fictitious bank deposit becomes under these circumstances. 'The "account current" of matter receives nothing at my hands which could be honestly kept back from it. If, then, "Democritus and the mathematicians" so defined matter as to exclude the powers here proved to belong to it, they were clearly wrong, and Mr. Martineau, instead of twitting me with my departure from them, ought rather to applaud me for correcting them.*

The reader of my small contributions to the literature which deals with the overlapping nargins of science and theology, will have noticed how frequently I quote Mr. Emerson. I do so mainly because in him we have a poet and a profoundly religious man, who is really and entirely undaunted by the discoveries of science, past, present, or prospectire. In his case Poetry, with the joy of a bacchanal, takes her graver brother Science by the hand, and cheers him with immortal laughter. By Emerson scientific conceptions are continually transmuted into the finer forms and warmer hues of an ideal world. Our present theme is tonched upon in the lines:

The journeying atoms, primordial wholes

Firmily draw, firmly drive by their animate poles.

As regards veracity and insight these few words outweigh, in my estimation, all the formal learning expended by Mr. Martineau in those disqaisitions on Force, where he treats the physicist as a conjuror, and speaks so wittily of atomic

* Definition implies previous examination of the objert defined, and is open to correction or modification as knowledge of the object inereastes. Such incroused knowledge las radically clianged uup conceptions of the luminiferous ether, converting its vilumions from longribulinal into transverse. Such clianges also Mr. Martinean's conceprions of matter are douned to undergo. 
polarity. In fact, without this notion of polarity-this "drawing" and "driving"-this attraction and repulsion, we stand as stupidly dumb before the phenomena of crystallization as a Bushman before the phenomena of the solar system. The genesis and growth of the notion I have endeavored to make clear in my third Lecture on Light, and in the article on "Matter and Force" published in this volume.

Our further course is here foreshadowed. A Sunday or two ago I stood under an oak planted by Sir John Moore, the hero of Corunna. On the ground near the tree little oaklets were successfully fighting for life with the surrounding vegetation. The acorns had dropped into the friendly soil, and this was the result of their interaction. What is the acorn? what the earth? and what the sun, without whose heat and light the tree could not become a tree, however rich the soil, and however healthy the seed? I answer for myself as before-all "matter." And the heat and light which here play so potent a part are acknowledged to be motions of matter. By taking something much lower down in the vegetable kingdom than the oak, we might approach much more nearly to the case of crystallization already discussed; but this is not now necessary.

If, instead of conceding the sufficiency of matter here, Mr. Martineau should fly to the hypothesis of a vegetative soul, all the questions before asked in relation to the snowstar become pertinent. I would invite him to go over them one by one, and consider what replies he will make to them. He may retort by asking me, "Who infused the principle of life into the tree?" I say, in answer, that our present question is not this, but another-not who made the tree, but what is it? Is there anything besides matter in the tree? If so, what, and where? Mr. Martineau may have begun by this time to discern that it is not "picturesqueness," but cold precision, that my Vorstellungs-fähigkeit demands. How, I would ask, is this vegetative soul to be presented to the mind? where did it flourish before the tree grew? and what will become of it when the tree is sawn into planks, or consumed in fire?

Possibly Mr. Martineau may consider the assumption of this soul to be as untenable and as useless as I do. But 
then if the power to build a tree be conceled to pure matter, what an amazing expansion of our notions of the "potency of matter" is implied in the concession! 'Think of the acorn, of the earth, and of the solar light and heat - was ever such necromancy dreamed of as the production of that massive trunk, those swaying boughs and whispering leaves, from the interaction of these three factors? In this interaction, moreover, consists what we call life. It will be seen that I am not in the least insensible to the wonder of the tree; nay, I should not be surprised if, in the presence of this wonder, I feel more perplexed and overwhelmed than Mr. Martiueau himself.

Consider it for a moment. There is an experiment, first male by Wheatstone, where the music of a piano is transferred from its sound-board, through a thin wooden rod, across several silent rooms in succession, and poured out at a distance from the instrument. The strings of the piano vibrate, not singly, but ten at a time. Every string subdivides, yielding not one note, but a dozen. All these vibrations and subvibrations are crowded together into a bit of deal not more than a quarter of a square inch in section. Yet no note is lost. Each vibration asserts its individual rights; and all are, at last, shaken forth into the air by a seeond sound-board, against which the distant end of the rod presses. 'Thought ends in amazement when it seeks to realize the motions of that rod as the music flows through it. I turn to my tree and observe its roots, its trunk, its branches, and its leaves. As the rod conveys the music, and yields it up to the distant air, so does the trunk convey the matter and the motion-the shocks and pulses and other vital actions-which eventually emerge in the umbrageous foliage of the tree. I went some time ago through the greenhouse of a friend. He had ferns from Ceylon, the branches of which were in some cases not much thicker than an ordinary pin-hard, smooth, and cylindrical-often leafless for a foot or more. But at the end of every one of them the unsightly twig unlocked the exuberant beanty hilden within it, and broke forth into a mass of fronds, almost large enough to fill the arms. We stand liere upon a higher level of the wonderful: we are conscious of a music subtler than that of the piano, passing unhearl through these tiny boughs, and issuing in what Mr. Martineau would opulently call the "clustered 
magnificence" of the leaves. Does it lessen my amazement to know that every cluster, and every leaf - their form and texture-lie, like the music in the rod, in the molecular structure of these apparently insignificant stems? Not so. Mr. Martineau weeps for "the beanty of the flower fading into a necessity." I care not whether it comes to me through necessity or through freedom, my delight in it is all the same. I see what he sees with a wonder superadded. 'I'o me, as to him, not even Solomon in all his glory was arrayed like one of these.

I have spoken above as if the assumption of a soul would save Mr. Martineau from the inconsistency of crediting pure matter with the astonishing building power displayed in crystals and trees. This, however, would not be the necessary result; for it would remain to be proved that the soul assumed is not itself matter. When a boy I learned from Dr. Watts that the souls of conscious brutes are mere matter. And the man who would claim for matter the human soul itself, would find himself in very orthodox company. "All that is created," says Fauste, a famous French bishop of the fifth century, "is matter. The soul occupies a place; it is enclosed in a body; it quits the body at death, and returns to it at the resurrection, as in the case of Lazarus; the distinction between hell and heaven, between eternal pleasures and eternal pains, proves that, even after death, souls occupy a place and are corporeal. God only is incorporeal." 'Tertullian, moreover, was quite a physicist in the definiteness of his conceptions regarding the soul. "The materiality of the soul," he says, "is evident from the evangelists. A human soul is there expressly pictured as suffering in hell; it is placed in the middle of a flame, its tongue feels a cruel agony, and it implores a drop of water at the hands of a happier sonl. Wanting materiality," adds Tertullian, "all this would be without meaning." *

* The foregoing extracts, which M. Alglave recently brought to light for the benefit of the bishop of Orleans, are taken from the sixth lecture of the "Cours d'Histoire Moderne" of that most orthodox of statesmen, M. Guizot. " I could multiply," continues M. Guizot, "these citations to infinity, and they prove that in the first centuries of our era the materiality of the soul was an opinion not only permitted, but dominant." Dr. Moriarty, and the synod. which he recently addressed, obviously forget their own antecedents. 
I have glaneed at inorganic nature-at the sea, and the sun, and the vapor, and the snow-flake, and at organic nature as represented by the fern and the oak. 'That same sun which warmed the water and liberated the vapor, exerts a subtler power on the nutriment of the tree. It takes hold of matter wholly unfit for the purpose of nutrition, separates its nutritive from its non-nutritive portions, gires the former to the vegetable, and carries the others away. Planted in the earth, bathed by the air, and tended by the sun, the tree is traversed by its sap, the cells are formed, the woody fiber is spun, and the whole is woven to a texture wonderful even to the naked eye, but a millionfold more so to microscopic vision. Does conscionsmess mix in any way with these processes? No man can tell. Our only gromid for a negatire conclusion is the absence of those outward manifestations from which feeling is usually inferred. But even these are not entirely absent. In the greenhouses of Kew we may see that a leaf can close, in response to a proper stimulus, as promptly as the human fingers themselves; and while there Int. Hooker will tell us of the wondrous fly-catehing and fly-devouring power of the Dionau. No man can say that the feelings of the animal are not represented by a drowsier consciousness in the vegetable world. At all events, no line has ever been drawn between the conscions and the unconscions; for the vegetable shates into the animal by such fine gradations, that is impossible to say where the one ends and the other begins.

In all such inquiries we are necessarily limited by our own powers: we observe what our senses, armed with the aids furnished by science, enable us to observe; nothing more. The evidences as to consciousness in the regetable world depend wholly upon our capacity to observe and weigh them. Alter the eapacity, and the evidence woulil alter too. Would that which to us is a total absence of any manifestation of consciousness be the same to a being with our capacities indefinitely multiplied? To such a being I can imagine not only the vegetable, but the mineral world. responsive to the proper irritauts, the response differing

Their boasted succession from the early Church renclers them the direct offspring of a "uaterialism " uore "brutal " than any ever enunciated by me. 
only in degree from those exaggerated manifestations, which, in virtue of their magnitude, appeal to our weak powers of observation.

Our conclusion, however, must be based, not on powers that we imagine, but upon those that we possess. What do they reveal? As the earth and atmosphere offer themselves as the nutriment of the vegetable world, so does the latter, which contains no constituent not found in inorganic nature, offer itself to the animal world. Mixed with certain inorganic substances-water, for example-the vegetable constitutes, in the long run, the sole sustenance of the animal. Animals may be divided into two classes, the first of which can utilize the vegetable world immediately, having chemical forces strong enough to cope with its most refractory parts; the second class use the vegetable world mediately; that is to say, after its finer portions have been extracted and stored up by the first. But in neither class have we an atom newly created. 'T'he animal world is, so to say, a distillation through the vegetable world from inorganic nature.

From this point of view all three worlds would constitute a unity, in which I picture life as immanent everywhere. Nor am I anxious to shut out the idea that the life here spoken of may be but a subordinate part and function of a Higher Life, as the living moving blood is subordinate to the living man. I resist no such idea as long as it is not dogmatically imposed. Left for the human mind freely to operate upon, the idea has ethical vitality; but, stiffened into a dogma, the inner force disappears, and the outward yoke of a usurping hierarchy takes its place.

The problem before us is, at all events, capable of definite statement. We have on the one hand strong grounds for concluding that the earth was once a molten mass. We now find it not only swathed by an atmosphere, and covered by a sea, but also crowded with living things. The question is, How were they introduced? Certainty may be as unattainable here as Bishop Butler held it to be in matters of religion; but in the contemplation of proba-

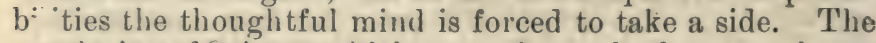
corrlusion of Science which recognizes unbroken causal connection between the past and the present would undonbtedly be that the molten earth contained within it elements of life, which grouped themselves into their present forms as 
the planet cooled. The difficulty and reluctance encountered by this conception arise solely from the fact that the theologic conception obtained a prior footing in the human mind. Did the latter depend upon reasoning alone, it could not hold its ground for an hour against its rival. But it is warmed into life and strength by associated hopes and fears - and not only by these, which are more or less mean, but by that loftiness of thought and feeling which lifts its possessor above the atmosphere of self, and which the theologic idea, in its nobler forms, has engendered in noble minds.

Were not man's origin implicated, we should accept without a murmur the derivation of animal and vegetable life from what we call inorganic nature. The conclusion of pure intellect points this way and no other. But the purity is troubled by our interests in this life, and by our hopes and fears regarding the life to come. Reason is traversed by the emotions, anger rising in the weaker heads to the height of suggesting that the suppression of the inquirer by the arm of the law would be an act agreeable to God, and serviceable to man. But this foolishness is more than neutralized by the sympatliy of the wise; and in England at least, 80 long as the courtesy which befits an earnest theme is ailhered to, such sympathy is ever ready for an honest man. None of us here need shrink from saying all that he has a right to sab. We ought, however, to remember that it is not only a band of Jesuits, weaving their schemes of intellectual slavery, under the innocent guise "of education," that we are opposing. Our foes are to some extent of our own household, including not only the ignorant and the passionate, but a minority of minds of high caliber and culture, lovers of freedom, moreover, who, though its objective hull be riddled by logic, still find the ethic life of their religion unimpaired. But while such considerations ought to inflnence the form of our argument, and prevent it from ever slipping out of the region of courtesy into that of scorn or abuse, its substance, I think, onght to be maintained and presented in unmitigated strength.

In the year 1855 the chair of philosophy in the University of Munich happened to be filled by a Catholic priest of great critical penetration, great learning, and great courage, who had borne the brunt of battle long 
before Döllinger. His Jesuit colleagues, he knew, incnlcated the belief that every human soul is sent into the world from God by a separate and supernatural act of creation. In a work entitled the "Origin of the Human Soul," Professor Frohschammer, the philosopher here alluded to, was hardy enough to question this doctrine, and to affirm that man, body and soul, comes from his parents, the act of creation being, therefore, mediate and secondary only. The Jesuits keep a sharp lookout on all temerities of this kind; and their organ, the "Civilità Cattolica," immediately pounced upon Frohschammer. His book was branded as "pestilent," placed in the Index, and stamped with the condemnation of the Church.* The Jesuit notion does not err on the score of indefiniteness. According to it, the Power whom Goethe does not dare to name, and whom Gassendi and Clerk Maxwell present to us under the guise of a " Manufacturer" of atoms, turns out annually, for England and Wales alone, a quarter of a million of new souls. Taken in connection with the dictum of Mr. Carlyle, that this annual increment to our population are " mostly fools," but little profit to the human heart seems derivable from this mode of regarding the divine operations.

But if the Jesuit notion be rejected, what are we to accept? Physiologists say that every human being comes from an egg not more than the one hundred and twentieth of an inch in diameter. Is this egg matter? I hold it to be so, as much as the seed of a fern or of an oak. Nine monthe go to the making of it into a man. Are the additions made during this period of gestation drawn from matter? I think so undoubtedly. If there be anything besides matter in the egg, or in the infant subsequently slumbering in the womb, what is it? The questions already asked with reference to the stars of snow may be here repeated. Mr. Martineau will complain that I am disenchanting the babe of its wonder; but is this the case?

* King Maximilian II. brought Liebig to Munich, he helped Helmholtz in his researches, and loved to liberate and foster science. But through his liberal concession of power to the Jesuits in the schools, he did far more damage to the intellectual freedom of his country than his superstitious predecessor Iudwig I. Priding himself on being a (terman prince, ludwig would not tolerate the interference of the Roman party with the political affairs of Bavaria. 
I fgure it growing in the womb, woven by a something not itself, withont conscions participation on the part of either father or mother, and appearing in due time a living miracle, with all its organs and all their implications. Consider the work accomplisherl during these nine muntiss in forming the eye alone-with its lens, and its humors, and its miraculous retina behind. Consider the ear with its tympanum, cochlea, and Corti's organ-an instrument of three thousand strings, built abjacent to the brain, and employed by it to sift, separate, and interpret, antecedent to all consciousmess, the sonorous tremors of the external world. All this has been accomplished, not only without man's contrivance, but without his knowledge, the secret of his own organization having been withlield from him since his birth in the immeasurable past, until these latter days. Matter I define as that mysterious thing by which all this is accomplished. How it came to have this power is a question on which I nerer ventured an opinion. If. then, matter starts as "a beggar," it is, in my riew, because the Jacobs of theology have deprived it of its birthright. Mr. Martineau need fear no disenchantment. Theories of evolution go but a short way toward the explanation of this mystery; the Ages, let us hope, will at length give us a poet competent to deal with it aright.

There are men, and they include among them some of the best of the race of man. upon whose minds this mystery falls without producing either warmth or color. 'The "dry light" of the intellect suffices for them, and they live their noble lives untouched by a desire to give the mystery shape or expression. 'There are, on the other hand, men whose minds are warmed and colored by its presence, and who, under its stimulus, attain to moral heights which have never been overtopped. Different spiritual climates are necessary for the healthy existence of these two classes of men; and different climates must be accorded them. The history of humanity, however, proves the experience of the second class to illustrate the most pervading need. 'The world will have religion of some kind, even though it should fly for it to the intellectual whoredom of "spiritnalism." What is really wanted is the lifting power of an ideal element in human life. But the free play of this power must be preceded by its release from the practical materialism of the present, as well as from the torn 
swaddling bands of the past. It is now in danger of being stupefied by the one, or strangled by the other. I look, however, forward to a time when the strength, insight, and elevation which now visit us in mere hints and glimpses, during moments " of clearness and vigor," shall be the stable and permanent possession of purer and mightier minds than ours-purer and mightier, partly because of their deeper knowledge of matter and their more faithful conformity to its laws.

\section{CHAP'TER XXXIV.}

FERMENTATION, AND ITS BEARINGS ON SURGERY AND MEDICINE.*

ONE of the most remarkable characteristics of the age in which we live, is its desire and tendency to connect itself organically with preceding ages-to ascertain how the state of things that now is came to be what it is. And the more earnestly and profoundly this problem is studied, the more clearly comes into view the vast and varied debt which the world of to-day owes to that fore-world, in which man by skill, valor, and well-directed strength first replenished and subdued the earth. Our prehistoric fathers may have been savages, but they were clever and observant ones. They founded agriculture by the discovery and development of seeds whose origin is now unknown. They tamed and harnessed their animal antagonists, and sent them down to us as ministers, instead of rivals in the fight for life. Later on, when the claims of luxury added themselves to those of necessity, we find the same spirit of invention at work. We have no historic account of the first brewer, but we glean from history that his art was practiced, and its produce relished, more than two thousand years ago. Theophrastus, who was born nearly four hundred years before Christ, described beer as the vine of barley. It is extremely difficult to preserve beer in a hot country, still Egypt was the land in which it was first brewed, the desire of man to quench his thirst

* A Discourse delivered before the Glasgow Science Lectures $\Lambda$ ssociation, October 19, 1876. 
with this exhilarating beverage overcoming all the obstacles which a hot climate threw in the way of its manufacture.

Our remote ancestors had also learued by experience that wine maketh glad the heart of man. Noah, we are informed, planted a vineyard, drank of the wine, and experienced the consequences. But, though wine and beer possess 80 old a history, a very fow years ago no man knew the secret of their formation. Indeed, it might be said that until the present year no thorough and scientific account was ever given of the agencies which come into play in the manufucture of beer, of the conditions necessury to its health, and of the maladies and vicissitudes to which it is subject. Hitherto the art and practice of the brewer have resembled those of the physician, both being founded on empirical observation. By this is meant the observation of facts, apart from the principles which explain them, and which give the mind an intelligent mastery over them. The brewer learned from long experience thie conditions, not the reasons, of success. But he had to contend, and has still to contend. against unexplained perplexities. Over and over again his care has been rendered nugatory; his beer has fallen into acidity or rottenness, and disastrous losses have been sustained, of which he has been unable to assign the cause. It is the hidden enemies against which the physician and the brewer have hitherto contended, that recent researches are dragging into the light of day, thus preparing the way for their final extermination.

Let $u$ glance for a moment at the outward and visible signs of fermentation. $\Lambda$ few weeks ago I paid a visit to a private still in a Swiss chalet; and this is what I saw. In the peasant's bedroom was a cask with a very large bunghole carefully closed. 'The cask contained cherries which had lain in it for fourteen days. It was not entirely filled with the fruit, an air-space being left above the cherries whon they were put in. I hal the bung remored, and a small lamp dipped into this space. Its flame was instantly extinguished. The oxygen of the air had entirely disappeared, its place being taken by carbonic acid gas."

* The gas which is exhated from the lungs aftur the oxygen of the air has done its duty in purifying the blood, the same also which effervescus frow suda water and chanpagne. 
I tasted the cherries: they were very sour, though when put into the cask they were sweet. 'The cherries and the liquid associated with them were then placed in a copper boiler, to which a copper head was closely fitted. From the head proceeded a copper tube which passed straight through a vessel of cold water, and issued at the other side. Under the open end of the tube was placed a bottle to receive the spirit distilled. The flame of small woodsplinters being applied to the boiler, after a time vapor rose into the head, passed through the tube, was condensed by the cold of the water, and fell in a liquid fillet into the bottle. On being tasted, it proved to be that fiery and intoxicating spirit known in commerce as Kirsch or Kirschwasser.

The cherries, it should be remembered, were left to themselves, no ferment of any kind being added to them. In this respect what has been said of the cherry applies also to the grape. At the vintage the fruit of the vine is placed in proper vessels, and abandoned to its own action. It ferments, producing carbonic acid; its sweetness disuppears, and at the end of a certain time the unintoxicating grape-juice is converted into intoxicating wine. Here, as in the case of the cherries, the fermentation is spontaneous-in what sense spontaneous will appear more clearly by and by.

It is needless for me to tell a Glasgow andience that the beer-brewer does not set to work in this way. In the first place the brewer deals not with the juice of fruits, but with the juice of barley. The barley having been steeped for a sufficient time in water, it is drained and subjected to a temperature sufficient to cause the moist grain to germinate; after which, it is completely dried upon a kiln. It then receives the name of malt. The malt is crisp to the teeth, and decidedly sweeter to the taste than the original barley. It is ground, mashed up in warm water, then boiled with hops until all the soluble portions have been extracted; the infusion thus produced being called the wort. This is drawn off, and cooled as rapidly as possible; then, instead of abandoning the infusion, as the wine-maker does, to its own action, the brewer mixes yeast with his wort, and places it in vessels each with only one aperture open to the air. Soon after the addition to the yeast, a brownish froth, which is really new yeast, issues 
from the aperture, and falls like a cataract into troughs prepared to receive it. 'This fruthing and foaming of the wort is a proof that the fermentation is actire.

Whence comes the yeast which issues so copiously from the fermenting tub? What is this yeast, and how did the brewer become possessed of it? Examine its quantity before and after fermentation. The brewer introluces, say $10 \mathrm{cwts}$. of yeast; he collects 40 , or it may be $50 \mathrm{cw}$ ts. The yeast has, therefore, augmented from four to fivefold during the fermentation. Shall we conclude that this adlitional yeast has been spontaneously generated by the wort? Are we not rather reminded of that seed which fell into good ground, and brought forth fruit, some thirtyfoli, some sixtyfold, some an hundredfold? On examination, this notion of organic growth turns out to be more than a mere surmise. In the year 1680, when the microscope was still in its infancy, Leeuwenhoek turned the instrument upon this substance, and found it composed of minute globules suspended in a liquid. 'Thus knowledge rested until 1835, when Cagniard de la Tour in France, and Schwann in Germany, independently, but animated by a common thought, turned microscopes of improved definition and heiglitened powers upon yeast, and found it budding and sprouting before their eves. The augmentation of the yeast alluded to above was thus proved to arise from the growth of a minute plant now called Torula (or Saccharomyces) C'erevisice. Spontaneous generation is therefore out of the question. The brewer deliberately sows the yeast-plant, which grows and multiplies in the wort as its proper soil. This discovery marks an epoch in the history of fermentation.

But where did the brewer find his yeast? The reply to this question is similar to that which must be given if it were asked where the brewer found his barley. Ie has received the seels of both of them from preceding generations. Could we comnect without solution of continuity the present with the past, we should probably be able to trace back the yeast employed by my friend Sir Fowell Buxton to-ilay to that employed by some Egyptian brewer two thousand years ago. lint you may urge that there must have been a time when the first yeast-cell was gencrated. Granted-exactly as there was a time when the first barley-corn was generated. Let not the delusion lay 
hold of you that a living thing is easily generated because it is small. Both the yeast-plant and the barley-plant lose themselves in the dim twilight of antiquity, and in this our day there is no more proof of the spontaneous generation of the one, than there is of the spontaneous generation of the other.

I stated a moment ago that the fermentation of grapejuice was spontaneous; but I was careful to add, "in what sense spontaneous will appear more clearly by and by." Now this is the sense meant. The wine-maker does not, like the brewer and distiller, deliberately introduce either yeast, or any equivalent of yeast, into his vats; he does not consciously sow in them any plant, or the germ of any plant; indeed, he has been hitherto in ignorance whether plants or germs of any kind have had anything to do with his operations. Still, when the fermented grape-juice is examined, the living Tor $u$ la concerned in alcoholic fermentation never fails to make its appearance. How is this? If no living germ has been introduced into the wine-vat, whence comes the life so invariably developed there?

You may be disposed to reply, with Turpin and others, that in virtue of its own inherent powers, the grape-juice when brought into contact with the vivifying atmospheric oxygen, runs spontaneously and of its own accord into these low forms of life. I have not the slightest objection to this explanation, provided proper evicence can be adduced in support of it. But the evidence adduced in its favor, as far as I am acquainted with it, snaps asunder under the strain of scientific criticism. It is, as far as I can see, the evidence of men, who however keen and clever as observers, are not rigidly trained experimenters. These alone are aware of the precautions necessary in investigations of this delicate kind. In reference, then, to the life of the wine-vat, what is the decision of experiment when carried out by competent men? Let a quantity of the clear, filtered "must" of the grape be so boiled as to destroy such germs as it may have contracted from the air or otherwise. In contact with germless air the uncontaminated must never ferment. All the materials for spontaneous generation are there, but so long as there is no seed sown, there is no life developed, and no sign of that fermentation which is the concomitant of life. Nor need you resort to a boiled liquid. The grape is sealed by 
its own skin against contamination from without. By an ingenious device l'asteur las extracted from the interior of the grape its pure juice, and proved that in contact with pure air it never acquires the power to ferment itself, nor to produce fermentation in other liquids. ${ }^{*}$ It is not therefore, in the interior of the grape that the origin of the life observed in the rat is to be sought.

What then is its true origin? 'This is Pasteur's answer, which his well-proved accuracy renders worthy of all confidence. At the time of the vintage microscopic particles are observed adherent, both to the outer surface of the grape and of the twigs which support the grape. Brush these particles into a capsule of pure water. It is rendered turbial by the dust. Eximined by a microscope, some of these minute particles are seen to present the appearance of organized cells. Instead of receiving them in water, let them be brushed into the pure inert juice of the grape. Forty-eight hours after this is done, our familiar Torula is observed budding and sproutung, the growth of the plant being accompanied by all the other signs of active fermentation. What is the inference to be drawn from this experiment? Obviously that the particles adherent to the external surface of the grape include the germs of that life which, after they have been sown in the juice, appears in such profusion. Wine is sometimes objected to on the ground that fermentation is " artificial:" but we notice here the responsibility of nature. The ferment of the grape clings like a parasite to the surface of the grape; and the art of the wine-maker from time immemorial has consisted in bringing-and it may be added, ignorantly bringingtwo thiugs thus closely associated by nature into actnal contact with each other. For thousands of years, what has been done conscionsly by the brewer, has been done unconsciously by the wine-grower. The one has sown his leaven just as much as the other.

Nor it is necessary to impregnate the beer-wort with yeast to proroke fermentation. Abandoned to the contact of our common air, it sooner or later ferments; but the

* The liquids of the healthy animal bovly are also soaled from ex sornal contanination. P'ure blomel, for exumple, druwn with due precantions from the beins, will never ferment or putrefy in contmet with pare air. 
shances are that the produce of that fermentation, instead of being agreeable, would be disgusting to the taste. By a rare accident we might get the true alcoholic fermentation, but the odds against obtaining it would be enormous. Pure air acting upon a lifeless liquid will never provoke fermentation; but our ordinary air is the vehicle of numberless germs which act as ferments when they fall into appropriate infusions. Some of them produce acidity, some putrefaction. The germs of our yeastplant are also in the air; but so sparingly distributed that an infusion like beer-wort, exposed to the air, is almost sure to be taken possession of by foreign organisms. In fact, the maladies of beer are wholly due to the admixture of these objectionable ferments, whose forms and modes of nutrition differ materially from those of the true leaven.

Working in an atmosphere charged with the germs of these organisms, you can understand how easy it is to fall into error in studying the action of any one of them. Indeed it is only the most accomplished experimenter, who, moreover, avails himself of every means of checking his conclusions, that can walk without tripping through this land of pitfalls. Such a man the French chemist Pasteur has hitherto proved himself to be. He has taught us how to separate the commingled ferments of our air, and to study their pure individual action. Guided by him, let us fix our attention more particularly upon the growth and action of the true yeast-plant under different conditions. Let it be sown in a fermentable liquir, which is supplied with plenty of pure air. The plant will flourish in the aërated infusion, and produce large quantities of carbonic acid gas-a compound, as you know, of carbon and oxygen. The oxygen thus consumed by the plant is the free oxygen of the air, which we suppose to be abundantly supplied to the liquid. The action is so far similar to the respiration of animals, which inspire oxygen and expire carbonic acid. If we examine the liquid even when the vigor of the plant has reached its maximum, we hardly find in it a trace of alcohol. The yeast has grown and flourished, but it has almost ceased to act as a ferment. And could every individual yeast-cell seize, without any imperliment, free oxygen from the surrounding liquid, it is certain that it would cease to act as a ferment altogether. 
What, then, are the conditions under which the yeastplant must be placed so that it may display its characteristic quality? Reflection on the facts alrealy referred to suggests a reply, and rigid experiment confirms the suggestion. Consider the Alpine cherries in their closed vessel. Consider the beer in its barrel, with a single small aperture open to the air, through which it is observed not to imbibe oxygen, but to pour forth carbonic acid. Whence come the volumes of oxygen necessary to the production of this latter gas? The small quantity of atmospheric air dissolved in the wort and overlying it would be totally incompetent to supply the necessary oxygen. In no other way can the yeast-plant obtain the gas necessury for its respiration than by wrenching it from surrounding substances in which the oxygen exists, not free, but in a slate of combination. It decomposes the sugar of the solution in which it grows, proluces heat, breathes forth carbonic acid gas, and one of the liquid products of the decomposition is our familiar alcohol. The act of fermentation, then, is a result of the effort of the little plant to muintain its respiration by means of combined oxygen, when its supply of free oxygen is cut off. As defined by Pusteur, fermentution is life without air.

But here the knowledge of that thorough: investigator comes to our aid to warn us against errors which have been committed over and over again. It is not all yeast-cells that can thus live without air and provoke fermentation. They must be young cells which have caught their vegetative vigor from contact with free oxygen. But once possessed of this vigor the yeast may be transplanted into a saccharine infusion absolutely purged of air, where it will continue to live at the expense of the oxygen, carbon, and other constituents of the infusion. Under these new conditions its life, as a plant, wi!l bo by 110 means 80 vigorous as when it hat a supply of free oxygen, but its action as a ferment will be indefinitely greater.

Does the yeast-plant stand alone in its power of provoking alcoholic fermentation? It would be singular if amid the multitude of low vegetable forms no other could be found capable of ucting in a similar way. And here again we have occasion to marvel at that sagacity of observation among the ancients to which we owe so vast a debt. 
Not only did they discover the alcoholic ferment of yeast, but they had to exercise a wise selection in picking it out from others, and giving it special prominence. Place an old boot in a moist place, or expose common paste or a pot of jam to the air; it soon becomes coated with a blue-green mold, which is nothing else than the fructification of a little plant called Penicillium glaucum. Do not imagine that the mold has sprung spontaneously from boot, or paste, or jam; its germs, which are abundant in the air, have been sown, and have germinated, in as legal and legitimate a way as thistle-seeds wafted by the wind to a proper soil. Let the minute spores of Penicillium be sown in a fermentable liquid, which has been previously so boiled as to kill all other spores or seeds which it may contain; let pure air have free access to the mixture; the Penicillium will grow rapidly, striking long flaments into the liquid, and fructifying at its surface. Test the infusion at various stages of the plant's growth, you will never find in it a trace of alcohol. But forcibly submerge the little plant, push it down deep into the liquid, where the quantity of free oxygen that can reach it is insufficient for its needs, it immediately begins to act as a ferment, supplying itself with oxygen by the decomposition of the sugar, and producing alcohol as one of the results of the decomposition. Many other low microscopic plants act in a similar manner. In aërated liquids they flourish without any production of alcohol, but cut off from free oxygen they act as ferments, producing alcohol exactly as the real alcoholic leaven produces it, only less copiously. For the right apprehension of all these facts we are indebted to Pasteur.

In the cases hitherto considered, the fermentation is proved to be the invariable correlative of life, being producerl by organisms foreign to the fermentable substance. But the substance itself may also have within it, to some extent, the motive power of fermentation. 'The yeastplant, as we have learned, is an assemblage of living cells; but so at bottom, as shown by Schleiden and Schwann, are all living organisms. Cherries, apples, peaches, pears, plums, and grapes, for example, are composed of cells, each of which is a living unit. And here I have to direct your attention to a point of extreme interest. In 1821, the celebrated French chemist, Bérard, estublished the 
important fact that all ripening fruit, exposed to the free atmosphere, absorbeal the oxygen of the atmosphere ambl liberated an approximately equal volume of carbonic acid. He also found that when ripe fruits were placed in a contined atmosphere, the oxygen of the atmospliere was first absorbed, and an equal volume of carbonic acid given ont. But the process did not end here. After the oxygen ham vanished, carbonic acid, in consilerable quantities, continned to be exhaled by the fruits, which at the same time lost a portion of their sugar, becoming more acid to the taste, though the absolute quantity of acid was not angmented. 'This was an observation of capital importance, and Berard had the sagacity to remark that the process might be regarded as a kind of fermentation.

Thus the living cells of fruits can absorb oxygen and breathe out carbonic acid, exactly like the living cells of the leaven of beer. Supposing the access of oxygen suddenly cut off, will the living fruit.cells as sudienly die, or will chey continue to live as yeast lires, by extracting osygen fron the saccharine juices round them? 'This is a question of suprome theoretic significance. It was tirst answered aftirmatively by the able and conclusive experiments of Lechartier and Bellamy, and the answer wis subsequently confirmed and explisined by the experiments and the reasoning of Pasteur. Bérard only showed the absorption of oxygen and the production of carbonic acid; Lechartier and Bellamy proverl the production of aleohol, thus completing the evidence that it was a case of real fermentation, though the common alcoholie ferment was absent. So full was Pasteur of the idea that the cells of the fruit would continue to live at the expense of the sugar of the fruit, that once in his laboratory, while conversing on these subjects with M. Dumas, he exclaimed, - I will wager that if a grape be plunged into an amosphere of carbonic acid, it will produce alcohol and carbonic acid by the continued life of its own cells - that they will act for a time like the cells of the true alcoholis leaven." He made the experiment, and found the result to be what he had foreseen. He then extenderl the inquiry. Placing under a bell-jur twenty-four plums, he filled the jar with carbonic acid gas; beside it he placed twenty-four similar plums uncorered. At the encl of eight dajs he removed the plums from the jar and comparod them with 
the others. The difference was extraordinary. The uncovered fruits hal become soft, watery, and very sweet; the others were firin and hard, their fleshy portions being not at all watery. 'They had, moreover, lost a considerable quantity of their sugar. They were afterwird bruised, and the juice was distilled. It yielded six and a half grammes of alcohol, or one per cent. of the total weight of the plums. Neither in these plums, nor in the grape first experimented on by Pasteur, could any trace of the ordinary alcoholic leaven be found. As previously proved by Lechartier and Bellamy, the fermentation was the work of the living cells of the fruit itself, after air had been denied to them. When, moreover, the cells were destroyed by bruising, no fermentation ensued. 'The fermentation was the correlative of a vital act, and it ceased when life was extinguished.

Lülersdorf was the first to show by this method that yeast acted, not, as Liebig had assumed, in virtue of its organic, but in virtue of its organized character. He destroyed the cells of yeast by rubbing them on a ground glass plate, and found that with the destruction of the organism, though its chemical constituents remained, the power to act as a ferment totally disappeared.

One word more in reference to Liebig may find a place here. 'I'o the philosophic chemist thoughtfully pondering these phenomena, familiar with the conception of molecular motion, and the changes produced by the interactions of purely chemical forces, nothing could be more natural than to see in the process of fermentation a simple illustration of molecular instability, the ferment propagating to surrounding inolecular groups the overthrow of its own tottering combinations. Broadly considered, indeed, there is a certain amount of truth in this theory; but Liebig, who propounded it, missed the very kernel of the pheomena when he overlooked or contemned the part playerl in fermentation by microscopic life. He looked at the matter too little with the eye of the body, and too much with the spiritual eye. He practically neglected the microscope, and was unmoved by the knowledge which its revelations would have poured in upon his mind. His hypothesis, as I have said, was natural-nay it was a striking illustration of Liebig's power to penetrate and unveil molecular actions; but it was an error, and as such bas 
proved an ignis fatuus instead of a pharos to some of his followers.

I have said that our air is full of the germs of ferments differing from the alcoholic leaven, and sometimes seriously interfering with the latter. They are the weeds of this microscopic garden which often overshadow and choke the flowerg. Let us take an illustrative case. Expose milk to the air. It will, after a time, turn sour, separating like blood into clot and serum. Place a drop of this sour milk under a powerful microscope and watch it closely. You see the minute butter-globules animated by that curious quivering motion called the Brownian motion. But let not this attract your attention too much, for it is another motion that we have now to seek. Here and there you observe a greater disturbance than ordinary anong the globules: keep your eye upon the place of tumult, and you will probably see emerging from it a long eel-like organism, tossing the globules aside and wriggling more or less rapidly across the field of the microseope. Fumiliar with one sample of this organism, which from its motions receives the name of vibrio, you soon detect numbers of them. It is these organisms, and other analogous thongh apparently motionless ones, which by decomposing the milk render it sour and putrid. They are the lactio and putrid ferments, as the yeast-plant is the alcoholic ferment of sugar. Keep them and their germs out of your milk and it will continne sweet. But milk may become putrid without becoming 8our. Examine such putrid milk microscopically, and you find it swarming with shorter organisms, sometimes associated with the vibrios, sometimes alone, and often manifesting a wonderful alacrity of motion. Keep these organisms and their germs out of your milk and it will never putrefy. Expose a mutton-chop to the air and keep it moist; in summer weather it Boon stinks. Place a drop of the juice of the fetid chop under a powerful microscope; it is seen swarming with organisms resembling those in the putrid milk. 'These organisms, which reoeive the common name of buclerin." are the agents of all putrefaction. Keep them and their germs from your meat and

* Doubtless organisms exhibiting grave specifie difforenoes are grouped togrether under this comoun name. 
it will remain forever sweet. Thus we begin to see that within the world of life to which we ourselves belong, there is another living world requiring the microscope for its discerument, but which, nevertheless, has the most important bearing on the welfare of the higher lifeworld.

And now let us reason together as regards the origin of these bacteria. A granular powder is placed in your hands, and you are asked to state what it is. You examine it, and have, or have not, reason to suspect that seeds of some kind are mixed up in it. To determine this point you prepare a bed in your garden, sow in it the powder, and soon after find a mixed crop of docks and thistles sprouting from your bed. Until this powder was sown neither docks nor thistles ever made their appearance in your garden. You repeat the experiment once, twice, ten times, fifty times. From fifty different beds after the sowing of the powder, you obtain the same crop. What will be your response to the question proposed to you? "I am not in a condition," you would say, "to affirm that every grain of the powder is a dock-seed, or a thistle-seed; but I am in a condition to affirm that both dock and thistle-seeds form, at all events, part of the powder." Supposing a succession of such powders to be placed in your hands with grains becoming gradually smaller, until they dwindle to the size of impalpable dust particles; assuming that you treat them all in the same way, and that from every one of them in a few days you obtain a definite crop-it nay be clover, it may be mustard, it may be mignonette, it may be a plant more minute than any of these, the smallness of the particles, or of the plants that spring from them, does not affect the validity of the conclusion. Without a shadow of misgiving you would conclude that the powder must have contained the seeds or germs of the life observed. There is not in the range of physical science an experiment more conclusive nor an inference safer than this one.

Supposing the powder to be light enough to float in the air, and that you are enabled to see it there just as plainly as you saw the heavier powder in the palm of your hand. If the dust sown by the air instear of by the hand produce a definite living crop, with the same logical rigor you would conclude that the germs of this crop must be mixed with the dust. 'T'o take an illustration: 
the spores of the little plant Penirillium glaurum, to which I have alreauly referred, are light enough to float in the air. A cut apple, a prear, a tomato, a slice of regetable marrow, or, as afready mentioned, an old moist boot, a dish of paste, or a pot of jam, constitules a proper soil for the P'enicillum. Now, if it conld be proved that the dust of the air when sown in this soil produces this plant, while, wanting the dust, neither the air, nor the soil, nor both togrether ean produce it, it would be obviously just as certain in this case that the floating dust contains the geraus of Penicillium as that the powilers sown in your garlen concained the germs of the plants which sprung from them.

But how is the floating dust to be rendered risible? In this way. Build a little chamber and provile it with a door, windows, and window-8hutters. let an aperture be made in one of the shutters through which a sunbeam can pass. Close the door and windows so that no light shall enter save through the hole in the shutter. The track of the sunbeam is at first perfectly plain and vivid in the air of the room. If all disturbance of the air of the chamber be aroided, the luminous track will become fainter and fainter, until at last it disappears absolutely, and no trace of the beam is to be seen. What reidered the beam visible at first? 'The floating dust of the air, which, thus illuminated and observec, is as palpable to sense as dust or powder placed on the palm of the hand. In the still air the dust gradually sinks to the floor or stichs to the walls and ceiling, until finally, by this self-cleausing process, the air is entirely freed from mechanically suspendel matter.

Thus far, I think, we have made our footing sure. I Iet us próceed. Chop up a beefsteak and allow it to remain for two or three hours just covered with warm water; you thus extract the juice of the beef in a concentrated form. By properly boiling the liquid and filtering it, you ean obtain from it a perfectly transparent beef-tea. Expose a number of ressels containing this tea to the moteless air of your chamber; and expose a number of vessels containing precisely the same liquid to the dust-laden air. In three days every one of the latter stinks, and examined with the microseope every one of them is found swarming with the bacteria of putrefaction. After three months, or three 
years, the beef-tea within the chamber is found in every case as sweet and clear, and as free from bacteria, as it was at the moment when it was first put in. There is absolutely no difference between the air within and that without save that the one is dustless and the other dust-laden. Clinch the experiment thus: Open the door of your chamber and allow the dust to enter it. In three days afterward you have every vessel within the chamber swarming with bacteria, and in a state of actice putrefaction. Here, also, the inference is quite as certain as in the case of the powder sown in your garden. Multiply your proofs by building fifty chambers instead of one, and by employing every imaginable infusion of wild animals and tame; of flesh, fish, fowl, and viscera; of vegetables of the most various kimls. If in all these cases you find the dust infallibly producing its crop of bacteria, while neither the dustless air nor the nutritive infusion, nor both together, are ever able to produce this crop, your conclusion is simply irresistible that the dust of the air coutains the germs of the crop which has appeared in your infusions. I repeat there is no inference of experimental science more certain than this one. In the presence of such facts, to use the worls of a paper lately published in the " Philosophical Transactions," it would be simply monstrous to affirm that these swarming crops of bacteria are spontaneously generated.

Is there then no experimental proof of spontaneous generation? I answer without hesitation, none! But to doubt the experimental proof of a fact, and to deny its possibility, are two different things, though some writers confuse matters by making them synonymous. In fact, this doctrine of spontaneous generation, in one form or another, falls in with the theoretic beliefs of some of the foremost workers of this age; but it is exactly these men who have the penetration to see, and the honesty to expose, the weakness of the evidence adduced in its support.

And here observe how these discoveries tally with the common practices of life. Heat kills the bacteria, cold numbs them. When my housekeeper has pheasants in charge which she wishes to keep sweet, but which threaten to give way, she partially cooks the birds, kills the infant 
bacteria, and thus postpones the eril day. By boiling her milk she also extends its period of sweetness. Some weeks ago in the Alps I made a few experiments on the influence of cold upon ants. Thongh the sun was strong, patches of snow still maintained themselves on the mountain slopes. The ants were found in the warm grass and on the warm rocks adjacent. Transferred to the snow the rapidity of their paralysis was surprising. In a few seconds a vigorous ant, after a few languid struggles, would wholly lose its power of locomotion and lie practically dead upon the snow. Trunsferred to the warm rock, it would revive, to be again smitten with death-like numbness when retransferred to the snow. What is true of the ant is specially true of our bacteria. 'Their active life is suspended by cold, and with it their power of producing or continuing putrefaction. 'This is the whole philosophy of the preservation of meat by cold. 'The fishmonger, for example, when he surrounds his very assailable wares by lumps of ice, stays the process of putrefaction by reducing to numbness and inaction the organisms which produce it, and in the absence of which his fish would remain sweet and sound. It is the astonishing activity into which these bacteria are pushed by warmth that renders a single summer's day sometimes so disastrous to the great butchers of London and Glaggow. The bodies of guides lost in the crevasses of Alpine glaciers have come to the sut face forty years after their interment, without the flesh showing any sign of putrefaction. But the most astonishing ease of this kind is that of the hairy elephant of Siberia which was found incased in ice. It had been buried for ages, but when laid bare its flesh was sweet, and for some time afforded copious nutriment to the wild beasts which fed upon it.

Beer is assailable by all the organisms here referred to, some of which produce acetic, some lactic, and some butyric acid, while yeast is open to attack from the bacteria of putrefaction. In relation to the partienlar beverage the brewer wishes to produce, these foreign ferments hare been properly called ferments of disease. The cells of the true leaven are globules, nsually somewhat elongated. The other organisms are more or less rod-like or eel-like in shape, some of them being beaied so as to resemble nerklaces. Each of these organisms produces a fermentation 
and a flavor peculiar to itself. Keep them out of your beer and it remains forever maltered. Never without them will your beer contract disease. But their germs are in the air, in the vessels employed in the brewery; even in the yeast used to impregnate the wort. Consciously or unconscionsly, the art of the brewer is directed against them. His aim is to paralyze, if he cannot annihilate them.

For heer, moreover, the question of temperature is one of supreme importance; indeed, the recognized influence of temperature is causing on the continent of Europe a complete revolution in the manufacture of beer. When I was a student in Berlin, in 18j1, there were certain places specially devoted to the sale of Bavarian beer, which was then making its way into public favor. This beer is prepared by what is called the process of low fermentation; the name being given partly because the yeast of the beer, instead of rising to the top and issuing through the bunghole, falls to the bottom of the cask; but partly, also, because it is produced at a low temperature. The other and older process, called high fermentation, is far more handy, expeditious, and cheap. In high fermentation eight days suffice for the production of the beer; in low fermentation, ten, fifteen, even twenty lays are found necessary. Vast quantities of ice, moreover, are consumed in the process of low fermentation. In the single brewery of Dreher, of Vienna, a hundred million pounds of ice are consumed annually in cooling the wort and beer. Notwithstanding these obvious and weighty drawbacks, the low fermentation is rapidly displacing the high upon the Continent. Here are some statistics which show the number of breweries of both kinds existing in Bohemia in 1860,1865 , and $18 \% 0$ :

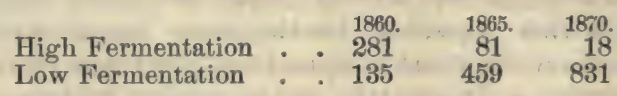

'Thus in ten years the number of high-fermentation breweries fell from 281 to 18 , while the number of lowfermentation breweries rose from 135 to 831 . The sole reason for this vast change-a change which involves a great expenditure of time, labor, and money-is the additional command which it gives the brewer over the 
fortuitous ferments of disense. These ferments, which, it is to be remembered, are living organisms, have their activity suspended by temperatures below 10 degrees C., and as long as they are reduced to torpor the beer remains untainted either by acidity or putrefaction. The beer of low fermentation is brewed in winter, and kept in cool - cellars; the brewer being thus enabled to dispose of it at his leisure, instead of forcing its consumption to avoid the loss involverl in its alteration if kept too long. II ps, it may be remarked, act to some extent as an antiseptic to beer. The essential oil of the hop is bactericidal: hence the strong impregnation with hop juice of all beer intended for exportation.

These low organisms, which one might be disposed to regard as the beginnings of life, were we not warned that the microscope, precious and perfect as it is, has no power to show us the real beginnings of life, are by no means purely useless or purely mischievons in the economy of nature. They are only noxious when out of their proper place. They exercise a useful and ralnable function as the burners and consumers of dead matter, animal and vegetable, reducing such matter, with a rapidity otherwise unattainable, to innocent carbonic acid and water. Furthermore, they are not all alike, and it is only restricted classes of them that are really dangerons to man. One difference in their habits is worthy of special reference here. Air, or rather the oxygen of the air, which is absolutely necessary to the support of the bacteria of putrefaction, is, according to Pasteur, absolntely deadly to the vibrios which provoke the butyric acid fermentation. This has been illustrated by the following beautiful observation.

A drop of the liquid containing those small organisms is placed upon glass, and on the drop is placed a circle of exceedingly thin glass; for, to magnify them sufliciently, it is necessary that the object-glass of the microscope should come very close to the organisms. Round the ealge of the circular plate of glass the liquid is in contact with the air, and incessantly absorbs it, including the oxygen. Here, if the drop be charged with bacteria, we have a zone of very lively ones. But through this living zone, greedy of oxygen and appropriating it, the vivifying gas cannot penetrate to the center of the film. In the middle, there- 
fore, the bacteria die, while their peripheral colleagues continue active. If a bubble of air chance to be enclosed in the film, round it the bacteria will pirouette and wabble until its oxygen has been absorbed, after which all their motions cease. Precisely the reverse of all this occurs with the vibrios of the butyric acid. In their case it is the peripheral organisms that are first killed, the central ones remaining vigorous while ringed by a zone of dead. Pasteur, moreover, filled two vessels with a liquid containing these vibrios; through one ressel he led air, and killed its vibrios in half an hour; through tha other he led carbonic acid, and after three hours found the vibrios fully active. It was while observing these differences of deportment fifteen years ago that the thought of life without air, and its bearing upon the theory of fermentation, flashed upon the mind of this admirable investigator.

We now approach an aspect of this question which concerns us still more closely, and will be best illustrated by an actual fact. A few years ago I was bathing in an Alpine stream, and returning to my clothes from the cascade which had been my shower-bath, I slipped upon a block of granite, the sharp crystals of which stamped themselves into my naked shin. The wound was an awkward one, but being in vigorous health at the time, I hoped for a speedy recovery. Dipping a clean pocket-handkerchief into the stream, I wrapped it round the wound, limped home, and remained for four or five days quietly in bed. There was no pain, and at the end of this time I thought myself quite fit to quit my room. 'The wound, when uncovered, was found perfectly clean, uninflamed, and entirely free from matter. Placing over it a bit of goldbeater's-skin, I walked about all day. Toward evening itching and heat were felt; a large accumulation of matter followed, and I was forced to go to bed again. 'The waterbandage was restored, but it was powerless to check the action now set up; arnica was applied, but it made matters worse. The inflammation increased alarmingly, until finally I had to be carried on men's shoulders down the mountain and transported to Geneva, where, thanks to the kindness of friends, I was immediately placed in the best medical hands. On the morning after my arrival in Geneva, Dr. Gautier discovered an abscess in my instep, at 
a distance of five inches from the wound. The two were conneeted by a channel, or simus, as it is technically called, through which he was able to empty the abscess, without the application of the lance.

By what agency was that channel formed-what was it that thus tore asunder the sound tissue of my instep, and kept me for six weeks a prisoner in bed? In the very room where the water iressing had been removed from my wound and the goldbeater's-skin applied to it, I opened this year a number of tubes, containing perfectly clear and sweet infusions of fish, flesh, and vegetable. These hermetically sealed infusions had been exposed for weeks, both to the sun of the Alps and to the warmith of a kitchen, without showing the slightest turbidity or sign of life. But two days after they were opened the greater number of them swarmed with the bacteria of putrefaction, the germs of which had been contracted from the dust-laden air of the room. And had the matter from my abscess been examined, my memory of its appearance leads me to infer that it would have been found equally swarming with these bacteria-that it was their germs which got into my incautionsly openenl wound, and that they were the subtile workers that burrowed down my shin, dug the abscess in my instep, and produced effects which might easily have proved futal.

This apparent digression brings us face to face with the labors of a man who combines the penetration of the true theorist with the skill and conscientionsmess of the true experimenter, and whose practice is one continued demonstration of the theory that the putrefaction of wounds is to be averted by the destruction of the germs of bacteria. Not only from his own reports of his cases, but from the reports of eminent men who have visited his hospital, and from the opinions expressed to me by continental surgeons, do I gather that one of the greatest steps ever male in the art of surgery was the introduction of the antiseptic system of treatment, introduced by Professor Lister.

The interest of this subject does not slacken ns we proceed. We began with the cherry-cask and beer-pat; we end with the body of man. There aro persons born with the power of interpreting natural facts, ws there 
are others smitten with everlasting incompetence in regard to such interpretation. 'To the former class in an eminent degree belonged the illustrious philosopher Rober Boyle, whose words in relation to this subject have in chem the forecast of prophecy. " And let me add," writes Boyle in his " Essay on the Pathological Part of Physik," "that he that thoroughly understands the nature of ferments and fermentations shall probably be much better able than he that iguores them, to give a fair account of divers phenomena of several diseases (as well fevers as others), which will perhaps be never properly understood without an insight into the doctrine of fermentations."

Two hundred years hare passed since these pregnant words were written, and it is only in this our day that men are beginning to fully realize their truth. In the domain of surgery the justice of Boyle's surmise has bean most strictly demoustrated. But we now pass the bounds of surgery proper, and enter the domain of epidemic disease, including those fevers so sagaciously referred to by Boyle. The most striking analogy between acontagium and a ferment is to be found in the power of indefinite selfmultiplication possessed and exercised by both. You know the exquisitely truthful figures regarding leaven employed in the New Testament. A particle hid in three measures of meal leavens it all. A little leaven leaveneth the whole lump. In a similar manner, a particle of contagium spreads through the human body and may be so multiplied as to strike down whole populations. Consider the effect produced upon the system by a microscopic quantity of the virus of small-pox. That virus is, to all intents and purposes, a seer. It is sown as yeast is sown, it grows and multiplies as yeast grows and multiplies, and it always reproduces itself. To Pasteur we are indebted for a series of masterly researches, wherein he exposes the looseness and general baselessness of prevalent notions regarding the transmutation of one ferment into another. He guards himself against saying it is impossible. The true investigator is sparing in the use of this word, though the use of it is unsparingly ascribed to him; but, as a matter of fact, Pasteur has never been able to effect the alleged transmutation, while he has been always able to point out the open doorways through which the affirmers 
of such transmutations hal allowed error to march in upon them.*

The great source of error here has been alrealy alluded to in this discourse. The observers worked in an atmosphere charged with the germs of different organisms; the mere accident of first possession rendering now one organism, now another, triumphant. In different stages, moreover, of its fermentative or putrefactive changes, the same infusion may so alter as to be successively taken possession of by different organisms. Such cases have been adduced to show that the earlier organisms must have been transformed into the later ones, whereas they are simply cases in which different germs, hecause of changes in the infusion, render themselves valid at different times.

By teaching $\mathrm{us}$ how to cultivate each ferment in its purity-in other words, by teaching us how to rear tho indiridual organism apart from all others-Pasteur has enabled us to avoid all these errors. And where this isolation of a particular organism has been duly effected it grows and multiplies indefinitely, but no change of it into another organism is ever observed. In Pasteur's researches the Bacterium remained a Bacterium, the Vibrio a Vibrio, the Penicillium a Penicillium, and the Torula a Torula. Sow any of these in a state of purity in an appropriate liquid; you get it, and it alone, in the subsequent crop. In like manner, sow small-pox in the human body, your crop is small-pox. Sow there scarlatina, and your crop is scarlatina. Sow typhoid virus, your crop is typhoidcholera, your crop is cholera. The disease bears as constant a relation to its contagium as the microscopic organisms just enumerated do to their germs, or indeed as a thistle does to its seed. No wonder then, with analogies so obvious and 80 striking, that the conviction is sprending and growing daily in strength, that reproductive parasitic life is at the root of epidemic disease-that living ferments finding lodgment in the body increase there and multiply, directly ruining the tissue on which they subsist, or de-

* Those who wish for an illuntration of the care uncessary in thesea reusurches, and of the carelessoess with which they have in some cases been conducted, will do well in comsult the Rev, W. II. Dallin. ger's excellent "Sotes on Heterogenesis" in the Oetuber number of the Popular Beience Revieve. 
stroying life indirectly by the generation of poisonous compounds within the body. This conclusion, which comes to us with a presumption almost amounting to demonstration, is clinched by the fact that virulently infective diseases have been discovered with which living organisms are as closely and as indissolubly associated as the growth of 'T'orula is with the fermentation of beer.

And here, if you will permit me, I would utter a word of warning to well-meaning people. We have now reached a phase of this question when it is of the very last importance that light should once for all be thrown upon the manner in which contagious and infectious diseases take root and spread. To this end the action of various ferments upon the organs and tissues of the living body must be sturlied; the habitat of each special organism concerned in the production of each specific disease must be determined, and the mode by which its germs are spread abroad as sources of further infection. It is only by such rigidly accurate inquiries that we can obtain final and complete mastery over these destroyers. Hence, while abhorring cruelty of all kinds, while shrinking sympathetically from all animal suffering-suffering which my own pursuits never call upon me to inflict--an unbiased surrey of the field of research now opening out before the physiologist causes me to conclude, that no greater calamity could befall the human race than the stoppage of experimental inquiry in this direction. A lady whose philanthropy has rendered her illustrions said to me some time ago, that science was becoming immoral; that the researches of the past, unlike those of the present, were carried on without cruelty. I replied to her that the science of Kepler and Newton, to which she referred, dealt with the laws and phenomena of inorganic nature; but that one great advance made by modern science was in the direction of biology, or the science of life; and that in this new direction scientific inquiry, though at the outset pursued at the cost of some temporary suffering, would in the end prove a thousand times more beneficent than it had ever hitherto been. I said this because I saw that the very researches which the larly deprecated were leading us to such a knowledge of epidemic diseases as will enable us finally to sweep these scourges of the human race from the face of the earth.

This is a point of such capital importance that I should 
like to bring it home to your intelligence by a single trustworthy illustration. In 1850, two distinguished French observers, MM. Davainne and Rayer, noticed in the blood of animals which had died of the virulent disease callecl splenic fever, small microscopic organisms resembling transparent rods, but neither of them at that time attached any significance to the observation. III 186i1. Pasteur published a memoir on the fermentation of butyric acid, wherein he described the organism which provoked it; and after reading this memoir it occurred to Davainne that splenie fever might be a case of fermentation set up within the animal body, by the organisms which had been observed by him and Rayer. This idea has been placed beyond all doubt by subsequent research.

Observations of the highest importance have also been made on splenic fever by Pollender and Brauell. Two years ago, Dr. Burdon Sanderson gave us a very clear account of what was known up to that time of this disorder. With regard to the permanence of the contagium, it hal been proved to hang for years about localities where it had once prevailed; and this seemed to show that the rod-like organisms could not constitute the contagium, becanse their infective power was found to vanish in a few weeks. But other facts established an intimate connection between the organisms and the disease, so that a reriew of all the facts caused Dr. Sanderson to conclude that the contaginm existed in two distinct forms: the one "fugitive" and visible as transparent roils; the other permanent but "latent," and not yet brought within the grasp of the microscope.

At the time that Dr. Sanderson was writing this report. a young German physician, named Koch," ocenpied with the duties of his profession in an obscure country district, was already at work, applying, during his spare time, various original and ingenions devices to the inrestigation of splenic fever. He stadied the habits of the rod-like organisms, and found the squeous humor of an ox's eye to be particularly suitable for their nutrition. With a drop of the aqueous humor he mixed the tiniest speck of a liquid containing the rods, placed the drop under his

* This, I beliove. was the finst reference to the resuearches of Koch made in this country. 1879. 
microscope, warmed it suitably, and observed the subsequent action. During the first two hours hardly any change was noticeable; but at the end of this time the rods began to lengthen, and the action was so rapid that at the end of three or four hours they attained from ten to twenty times their original length. At the end of a few additional hours they had formed filaments in many cases a hundred times the length of the original rods. The same filament, in fact, was frequently observed to stretch through several fields of the microscope. Sometimes they lay in straight lines parallel to each other, in other cases they were bent, twisted, and coiled into the most graceful figures; while sometimes they formed knots of such bewildering complexity that it was impossible for the eye to trace the individual filaments through the confusion.

Had the observation ended here an interesting scientific fact would have been added to our previous store, but the addition would have been of little practical value. Koch, however, continued to watch the filaments, and after a time noticed little dots appearing within them. 'These dots became more and more distinct, until finally the whole length of the organism was studded with minute ovoid bodies, which lay within the outer integument like peas within their shell. By and by the integument fell to pieces, the place of the organisms being taken by a long row of seeds or spores. 'These observations, which were confirmed in all respects by the celebrated naturalist, Cohn of Breslau, are of the highest importance. 'They clear up the existing perplexity regarding the latent and visible contagia of splenic fever; for in the most conclusive manner Koch proved the spores, as distinguished from the rods, to constitute the contagium of the fever in its most deadly and persistent form.

How did he reach this important result? Mark the answer. There was but one way open to him to test the activity of the contagium, and that was the inoculation with it of living animals. He operated upon guinea-pigs and rabbits, but the vast majority of his experiments were made upon mice. Inoculating them with the fresh blood of an animal suffering from splenic fever, they invariably died of the same disease within twenty or thirty hours after inoculation. He then sought to determine how the contagium maintained its vitality. Drying the infectious 
blood containing the rod-like organisms, in which, however, the spores were not developed, he found the contagium to be that which Dr. Sanderson calls "fugitive." It maintained its power of infection for fire weeks at the furthest. Ile then dried blood containing the fully-developed spores, and exposed the substance to a variety of conditions. He permittel the dried blood to assume the form of dust; wetted this dust, allowed it to dry again, permitted it to remain for an indefinite time in the midst of putrefying matter, and subjected it to various other tests. After kecping the spore-charged blood which had been treated in this fashion for four years, he inoculated a number of mice with it, and found its action as fatal as that of blood fresh from the veins of an animal suffering from splenic fever. There was no single escape from deach after inoculation by this deadly contagium. Uncounted millious of these spores are developed in the body of every animal which has died of splenic fever, and every spore of these millions is competent to produce the disease. The name of this formidable parasite is Bacillus anthracis.*

Now the very first step toward the extirpation of theso contagia is the knowledge of their nature; and the knowledge brought to us by Dr. Koch will render as certain the stamping out of splenic fever as the stopyage of the plague of pébine by the researches of Pasteur. $\nmid$ One small item of statistice will show what this implies. In the single district of Novgorod in Russia, between the years 1868 and 1s:0, over fifty-six thousand cases of deach by splenic

* Korls found that to prouluce its characteristic effects the contagium of the sploric fever must enter the blocd: the sirulenty infective splemell of a diseased animal may be eaten with impunity hy mice. (in the other hand, the disesse refuses to be communicateri by inowa lation to digs, partridges, or sparrows. In their blomel Bacillus enthreris ceases to act as a ferment. Pasteur anthouncenl more than six years ago the propagntion of the vibrios of the silk-worm dismse callod fluthirie. Ixith by fission and by spores. He also made some remarkab!e experiments on the permanence of the contagium in the form of spores. Sine " Etudes sur la Maladie des Vers i soje," pp. 168 and sivi.

f Suruiving that the imumaty enjoyed by hirds miglut arise from the lieat of their hlood. which destroyed the tebillun. Pasteur lowered throir temperature artificially, inosulated tlom, and hilled them. He alan raised the eemperata re of guiben pigs after inescalation,

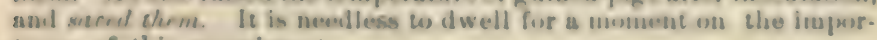
Lance of this experiment. 
fever, among horses, cows, and sheep were recorded. Nor did its ravages confine themselves to the animal world, for during the time and in the district referred to, five hundred and twenty-eight human beings perished in the agonies of the same disease.

A description of the fever will help you to come to a right decision on the point which I wish to submit to your consideration. " An animal," says Dr. Burdon Sanderson, "which perhaps for the previous day has declined food, and shown signs of general disturbance, begins to shudder and to have twitches of the muscles of the back, and soon after becomes weak and listless. In the meantime the respiration becomes frequent and often difficult, and the temperature rises three or four degrees above the normal; but soon convulsions, affecting chiefly the muscles of the back and loins, usher in the final collapse of which the progress is marked by the loss of all power of moving the trunk or extremities, diminution of temperature, mucous and sanguinolent alvine evacuations, and similar discharges from the month and nose." In a single district of Russia, as above remarked, fifty-six thousand horses, cows, and sheep, and five hundred and twenty-eight men and women, perished in this way during a period of two or three yeurs. What the annual fatality is throughout Europe I have no means of knowing. Doubtless it must be very great. 'The question, then, which I wish to submit to your juclgment is this: Is the knowledge which reveals to us the nature, and which assures the extirpation, of a disorder so virulent and so vile, worth the price paid for it? It is exccelingly important that assemblies like the present should see clearly the issues at stake in such questions as this, and that the properly informed sense of the community should temper, if not restrain, the rashness of those who, meaning to be tender, become agents of cruelty by the imposition of short-sighted restrictions upon physiological investigations. It is a modern instance of zeal for Gorl, but not according to knowledge, the excesses of which must be corrected by an instructed public opinion.

And now let us cast a backward glance on the field we have traversed, and try to extract from our labors such further profit as they can yield. For more than two thousand years the attraction of light bodies by amber was 
the sum of human knowlerlge regarding electricity, and for more than two thousand years fermentation was effected without any knowlenge of its cause. In science one discovery grows ont of another, and cannot uppuar without its proper antecedent. Thus, bufore fermentation could be understool, the microscope hat to be invented, and brought to a considerable degree of perfection. Note the growth of knowledge. Leenwenhoek, in $16 \times()$, found yenst to be a mass of floating globules, but he lad no notion that the globules were alive. This was proved in 18.35 by Cagniard de la Tour and Sohwann. Then came the quesstion as to the origin of such microscopic organisms, and in this counection the memoir of Pasteur, publisher in the "Anuales de Chimie" for 1862 , is the inauguration of a new epoch.

On that investigation all Pasteur's subsequent labors were based. Ravages had over and over again occurred among French wines. There was no guarantee that they would not become acid or bitter, particularly when exporterl. The commerce in wines was thus restricted, and disastrous losses were often inflicted on the winegrower. Erery one of these diseases was traced to the lifo of an organism. Pasteur ascertained the temperature which killed these ferments of disense, proving it to be so low as to be perfectly harmless to the wine. By the simple expedient of heating the wine to a temperature of fifty degrees Centigrade, he rendered it inalterable, and thus saved his country the loss of millions. He then went on to vinegar-rinaigre, acid wine-which he proved to be produced by a fermentation set up by a little fungus called Mycolerma aceti. Torula, in fact, converts the grape juice into alcohol, ani Mycoulerma aceti converts the aleohol into vinegar. Here also frequent failures occurred, and severe losses were sustained. Through the operation of unknown causes, the vinegar often became unfit for use, sometimes indeed falling into utter putridity. It had been long known that mere exposure to the air was sufficient to destroy it. Proteur studied all these changes, traced them to their living canses, and showed that the permanent health of the vinegar was chsured by the destruction of this life. He passed from the diseases of rinegur to the stuily of a malady which $n$ dozen years ago had all hut ruined the silk husbandry of France. This plague, which 
received the name of pébrine, was the product of a parasite which first took possession of the intestinal canal of the silk-worm, spreal throughout its body, and filled the sack which ought to contain the viscid matter of the silk. 'I'hus smitten, the worm would go automatically through the process of spinning when it had nothing to spin. Pasteur followed this parasitic destroyer from year to year, and leil by his singular power of combining facts with the logic of facts, discovered eventually the precise phase in the development of the insect when the disease which assailed it could with certainty be stamped out. Pasteur's devotion to this inquiry cost him dear. He restored to France her silk husbandry, rescued thousands of her population from ruin, set the looms of Italy also to work, but emerged from his labors with one of his sides permanently paralyzed. His last investigation is embodied in a work entitled "Studies on Beer," in which he describes a method of renclering beer permanently unchangeable. That method is not so simple as those found effectual with wine and vinegar, but the principles which it involves are sure to receive extensive application at some future day.

There are other reflections connected with this subject which, even were they now passed over without remark, would sooner or later occur to every thoughtful mind in this assembly. I have spoken of the floating dust of the air, of the means of rendering it visible, and of the perfect immunity from putrefaction which accompanies the contact of germless infusions and moteless air. Consider the woes which these wafted particles, during historic and pre-historic ages, have inflicted on mankind; consider the loss of life in hospitals from putrefying wounds; consider the loss in places where there are plenty of wounds, but no hospitals, and in the ages before hospitals were any where founded; consider the slaughter which has hitherto followed that of the battlefield, when those bacterial destroyers are let loose, often producing a mortality far greater than that of the battle itself; add to this the other conception that in times of epidemic disease the selfsame floating matter has frequently, if not always, mingled with it the special germs which produce the epidemic, being thus enabled to sow pestilence and leath over nations and continents-consider all this, and you will come with me to the conclusion that all the havoc of war, ten times multiplied, would be evanescent if compared with the ravages due to atmospheric dust. 
This preventible destruction is going on to-day, and it has been permitted to go on for arres, without a whisper of information regarding its cause being vouchsafed to the suffering sentient world. We have been scourged by invisible throngs, attacked from impenetrable ambuscades, and it is only to-day that the light of science is being let in upon the murderous dominion of our foes. Facts like these excite in me the thought that the rule and govern. ance of this universe are different from what we in our youth supposeal them to be-that the inscrutable Power, at once terrible and beneficent, in whom we live and move and have our being and our end, is to be propitiated by means different to those usually resorted to. 'The first requisite toward such propitiation is knowledge; the second is ation, shaped and illuminated by chat knowledge. Of knowledge we already see the dawn, which will open ont by and by to perfect day; while the action which is to follow has its unfailing source and stimulus in the moral and emotional nature of man-in his desire for personal well-being, in his sense of duty, in his compassionate sympathy with the sufferings of his fellow-men. "How often," says Dr. William Budd in his celebrated work on Typhoid Fever-"How of ten have I seen in past days, in the single narrow chamber of the day-laborer's cottage the father in the coftin, the mother in the sick-bud in muttering delirium, and nothing to relieve the desolation of the children but the devotion of some poor neighbor, who in ton many cases paid the penalty of her kindness in bocoming herself the victim of the same disorder?" From the rantage ground already won I look forward with confident hope to the triumph of medical art over scenes of misery like that here described. The cause of the calamity being once clearly revealed, not only to the physician, but to the public, whose intelligent co-operation is absolutely essential to snccess, the final victory of liumanity is only a question of time. We have already a foretaste of that victory in the triumplis of surgery as practiced at your doors. 


\section{CHAPTER XXXV. \\ SPONTANEOUS GENERATION.*}

WITHIN ten minutes' walk of a little cottage which I have recently built in the Alps, there is a small lake, fed by the melted snows of the upper mountains. During the early weeks of summer no trace of life is to be discerned in this water; but invariably toward the end of July, or the beginning of August, swarms of tailed organisms are seen enjoying the sun's warmth along the shallow margins of the lake, and rushing with audible patter into deeper water at the approach of danger. 'The origin of this periodic crowd of living things is by no means obvious. For years I had never noticed in the lake either an adult frog, or the smallest fragment of frog spawn; so that were I not otherwise informed, I should have found the conclusion of Mathiole a natural one, namely, that tadpoles are generated in lake mud by the vivifying action of the sun.

The checks which experience alone can furnish being absent, the spontaneous generation of creatures quite as high as the frog in the scale of being was assumed for ages to be a fact. Here, as elsewhere, the dominant mind of Aristotle stamped its notions on the world at large. For nearly twenty centuries after him men found no difficulty in believing in cuses of spontaneous generation which would now be rejected as monstrous by the most fanatical sup. porter of the doctrine. Shell fish of all kinds were considered to be without parental origin. Eels were supposed to spring spontaneously from the fat ooze of the Nile. Caterpillars were the spontaneous products of the leaves on which they fed; while winged insects, serpents, rats, and mice were all thought capable of being generated without sexual intervention.

The most copious source of this life without an ancestry was putrefying flesh; and, lacking the checks imposed by fuller investigation, the conclusion that flesh possesses and exerts this generative power is a natural one. I well remember when a child of ten or twelve seeing a joint of imperfectly salted beef cut into, and coils of maggots laid bare within the mass. Without a moment's

* The Nineteenth Century, January, 1878. 
hesitation I jumped to the conclusion that these maggots had been spontaneously generated in the moat. I had no knowledge which could qualify or oppose this conclusion, and for the time it was irresistible. The childhood of the individual typifies that of the race, and the belief here enunciated was that of the world for nearly two thomsand years.

To the exanination of this very point the celebrated Francusco Redi, physician to the Grand Dukes Furdinand 1I. and Cosmo III. of 'Tuscany, and a member of the Academy del Cimento, aldressed himself in 1668. He hal seen the maggots of putrefying flesh, and reflected on their possible origiu. But he was not content with mere reflection, nor with the theoretic guesswork which his predecessors had founded upon their imperfect observations. Watching meat during its passage from freshness to decay, prior to the appearance of maggots he invariably olserved flies buzzing round the meat and frequently alighting on it. The maggots, lie thought, might be the half-developed progeny of these flies.

The inductire guess precedes experiment, by which, however, it must be finally tested. Redi knew this, and acterl accordingly. Placing fresh meat in a jar and cuvering the mouth with paper, he found that, though the meat putrefied in the ordinary way, it never bred maggots, while the same meat placed in open jarssoon swarmed with these organisms. For the paper cover he then substituted fine gauze, throngh which the odor of the meat could rise. Over it the flies buzzed, and on it they laid their eggs, but, the meshes being too small to permit the eggs to fall through, no maggots were generated in the meat. They were, on the contrary, liatched upon the gauze. By u series of such experiments Redi destroyed the belief in the spontaneons generation of maggots in meat, and with it doubtless many rolates beliefs. 'The combat was continued by Vallisneri, Schwanmerdam, and Reanmur, who suceeded in banishing the notion of spontanoous generation from the scientific minds of their day. Indeed, as regards such complex organisms as those which formed the subject of their resenches, the notion was bunished forever.

But the discovery and improvement of the microscope, though giving a deach-blow to much that hat been pre- 
viously written and believed regarding spontaneous generation, brought also into view a world of life formed of individuals so minute-so close as it seemed to the ultimate particles of matter-as to suggest an easy passage from atoms to organisms. Animal and vegetable infusions exposed to the air were found clonded and crowded with creatures far beyond the reach of unaided vision, but perfectly visible to an eye strengthened by the microscope. With reference to their origin these organisms were called "Infusoria." Staguant pools were found full of them, and the obvious difficulty of assigning a germinal origin to existences so minute furnished the precise condition necessary to give new play to the notion of heterogenesis or spontaneous generation.

'The scientific world was soon divided into two hostile camps, the leaders of which only can here be briefly alluded to. On the one side, we have Buffon and Need. ham, the former postulating his "organic molecules," and the latter assuming the existence of a special "vegetative force" which drew the molecules together so as to form living things. On the other side, we have the celebrated Abbé Lazzaro Spallanzani, who in 1777 published results counter to those announced by Needham in 1748, and obtained by methods so precise as to completely overthrow the convictions based upon the labors of his predecessor. Charging his flasks with organic infusions, he sealed their necks with the blowpipe, subjected them in this condition to the heat of boiling water, and subsequently exposed them to temperatures favorable to the development of life. The infusions continued unchanged for months, and when the flasks were subsequently opened no trace of life was found.

Here I may forestall matters so far as to say that the success of Spallauzani's experiments depended wholly on the locality in which he worked. 'The air around him must have been free from the more obdurate infusorial germs, for otherwise the process he followed would, as was long afterward proved by Wyman, have infallibly yielded life. But his refutation of the doctrine of spontaneous generation is not the less valid on this account. Nor is it in any way upset by the fact, that others in repeating his experiments obtained life where he obtained none. Rather is the refutationstrengthened by such differences. Given two experi- 
menters equally skillful and equally careful, operating in different places on the same infusion, in the same way, and assuming the one to obtain life while the other fails to obtain it; then its well-established absence in the one case proves that some ingredient foreign to the infusion must be its cunse in the other.

Spallanzani's sealed flasks containen but small quantities of air, and as oxygen was afterward shown to be generally essential to life, it was thought that the absence of life observed by Spallanzani might have been due to the lack of this vitalizing gas. To dissipate this doubt, Schulze in 1836 half filled a flask with distilled water to which animal and regetable matters were added. First boiling his infusion to destroy whatever life it might contain, Schulze sucked daily into his flask air which has passed through a series of bulbs containing concentrated sulphuric acid, where all germs of life suspended in the air were supposed to be destroyed. From May to August this process was continued without any development of infusorial life.

Here again the success of Schulze was due to his working in comparatively pure air, but even in such air his experiment is a very risky one. Germs will pass unwetted and unscathed through sulphuric acid unless the most special care is taken to detain them. I have repeatedly failed, by repeating Schulze's experiments, to obtain his results. Others have failed likewise. The air passes in bubbles through the bulbs, and to render the method secure, the passage of the air must be so slow as to cause the whole of its floating matter, even to the very core of each bubble, to touch the surrounding liquid. But if this precaution be observed, water will be found quite as effectual as sulphnric acid. By the aid of an air-pump in a highly infective atmosphere I have thus drawn air for weeks without intormission, first throngh bulbs containing water, and afterwarl through vessels containing organic infusions, without any appearance of life. The germs were not killed by the water, but they wero effectually in. tercepted, while the objection that the air had been injured by being brought into contact with strongly corrosive substances was avoided.

The briet paper of Schulze, publisheal in Poggendorf's Annalen for 1836, was followed in 18.37 by another short and pregnant communication by Schwann. Redi, as wo 
have seen, traced the maggots of putrefying flesh to the eggs of flies. But he did not and he could not know the meaning of putrefaction itself. He had not the instrumental means to inform him that it also is a phenomenon attendant on the development of life. This was first proved in the paper now alluded to. Schwann placed flesh in a flask filled to one-third of its capacity with water, sterilized the flask by boiling, and then supplied it for months with calcined air. 'Throughout this time there appeared no mold, 10 infusoria, no putrefaction; the flesh remained unaltered, while the liquid continued as clear as it was immediately after boiling. Schwann then varied his experimental argument, with no alteration in the result. His final conclusion was, that putrefaction is due to decompositions of organic matter attendant on the multiplication therein of minute organisms. These organisms were derived not from the air, but from something contained in the air, which was destroyed by a sufficiently high temperature. There never was a more determined opponent of the doctrine of spontaneous generation than Schwann, though a strange attempt was made a year and a half ago to enlist him and others equally opposed to it on the side of the doctrine.

The physical character of the agent which produces putrefaction was further revealed by Helmholtz in 1843. By means of a membrane he separater a sterilized putrescible liquid from a putrefying one. The sterilized infusion remained perfectly intact. Hence it was not the liquid of the putrefying mass-for that could freely diffuse through the membrane-but something contained in the liquid, and which was stopped by the membrane, that caused the putrefaction. In 1854 Schroeder and Von Dusch struck into this inquiry, which was subsequently followed up by Schroeder alone. These able experimenters employer plugs of cotton-wool to filter the air supplied to their infusions. Fed with such air, in the great majority of cases the putrescible liquids remained perfectly sweet after boiling. Milk formed a conspicuous exception to the general rule. It putrefied after boiling, though supplied with carefully filtered air. The researches of Schroeder bring us up to the year 1859.

In that year a book was published which seemed to overturn some of the best established facts of previous 
inrestigators. Its title was Heterogénie, and its author was F. A. Pouchet, direotor of the Museum of Natural Ilistory at Ronen. Arient, laborions, learned, full not only of scientific but of metaphysical fervor, he threw his whole energy into the inquiry. Never did a subject require the exercise of the cold, critical faculty more than this one-calm study in the unraveling of complex phenomena, care in the preparation of experiments, care in their execution, skillful variation of conditions, and incessint questioning of results until repetition had placed them beyoud doubt or question. 'To a man of Ponchet's temperament the subject was full of danger-danger not lessened by the theoretic bias with which he approached it. This is revealed by the opening words of his preface: "Lorsque, par la múlitation, il fut évident pour, moi que la génération spontanée était encore l'un des moyens quemploie la nature pour la reproduction des êtres, je maspliqnai à déconvrir par quels procédés on pouvait parvenir à en mettre les phénomènes en éridence." It is needless to say that such a prepossession required a strong curb. Pouchet repeated the experiments of Schulze and Schwann with results diametrically opposed to theirs. He heaped experiment upon experiment and argument upon argament, spicing with the sarcasm of the adrocate the logic of the man of science. In view of the multitudes required to prodnce the observed results, he ridiculea the assumption of atmospheric germs. This was one of his strongest points. "Si les Proto-organismes que nous vojons pulluler partout et dans tout, araient leurs germes disséminés dans l’atmosplière, dans la proportion mathématiquement indispensable à cet effet, l'air en serait totalement obscurci, car ils devraient s'y trouver beaucoup plus serrés que les globules d'eau qui forment nos muages epais. II "í a fas là la moindre exagération." Recurring to the subject, he exclaims: "Lair dans lequel nous rivons anrait presque la densité du fer." There is often a virulent contagion in a confident tone, and this hardihood of argumentative assertion was sure to influence minils swared not by knowledge, but by authority. Hal Ponchet knewn that "the blue ethereal sky" is formed of suepended particles, through which the sun freely shines, he would hardly have ventured ngon this line of argument.

Ponchet's pursuit of this inquiry strengthened the con- 
viction with which he began it, and landed him in downright credulity in the end. I do not question his ability as an observer, but the inquiry needed a disciplined experimenter. 'This latter implies not mere ability to look at things as Nature offers them to our inspection, but to force her to show herself under conditions prescribed by the experimenter himself. Here Pouchet lacked the necessary discipline. Yet the vigor of his onset raised clouds of doubt, which for a time obscured the whole field of inquiry. So difficult indeed did the subject seem, and so incapable of definite solution, that when Pasteur made known his intention to take it up, his friends Biot and Dumas expressed their regret, earnestly exhorting him to set a definite and rigid limit to the time he purposed spending in this apparently unprofitable field.*

Schooled by his education as a chemist, and by special researches on the closely related question of fermentation, Pasteur took up this subject under particularly favorable conditions. His work and his culture had given strength and finish to his natural apitudes. In 1862, accordingly, he published a paper "On the Organized Corpuscles existing in the Atmosphere," which must forever remain classical. By the most ingenious devices he collected the floating particles of the air surrounding his laboratory in the Rue d'Ulm, and subjected them to microscopic examination. Many of them he found to be organized particles. Sowing them in sterilized infusions, he obtained abundant crops of microscopic organisms. By more refined methods he repeated and confirmed the experiments of Schwann, which had been contested by Pouchet, Montegazza, Joly, and Musset. He also confirmed the experiments of Schroeder and Von Dusch. He showed that the cause which communicated life to his infusions was not uniformly diffused through the air; that there were aërial interspaces which possessed no power to generate life. Standing on the Mer de Glace, near the Montanvert, he snipped off the ends of a number of hermetically sealed flasks containing organic infusions. One out of twenty of

* "Je ne conseillerais a personne," said Dumas to his already famous pupil, "de rester trop longtemps dans ce sujet."-Annales de Chimie et de Plyysique, 1862, vol. lxiv., p. 22. Since that time the illustrious perpetual secretary of the Acarlemy of Sciences has had good reason to revise this "counsel." 
the flasks thus supplied with glacier air showed signs of life afterward, while eight out of twenty of the same infusions, supplied with the air of the plains, became crowded with life. He took his flasks into the caves under the Otservatory of Paris, and found the still air in these cares devoid of generative power. These and other experiments, carried out with a severity perfectly obvious to the instructed scientific reader, and accompanied by a logic equally severe, restored the conviction that, even in these lower reaches of the scale of being, life does not appear without the operation of antecolent life.

The main position of Pasteur has been strengthened by practical researches of the most momentous kind. He has applied the knowledge won from his inquiries to the preservation of wine and beer, to the manufacture of vinegar, to the staying of the plague which threatened utter destruction of the silk husbandry of France, and to the examination of other formidable discases which assail the higher animals, including man. His relation to the improvements which Professor Lister has introduced into surgery, is shown by a letter quoted in his Ltules sur la Biere. * Professor Lister there expressly thanks Pasteur for having given him the only principle which could have conducted the antiseptic system to a successful issue. The strictures regarding defects of reasoning, to which we have been lately accustomed, throw abundant light upon their author, but no shade upon Pasteur.

Redi, as we have seen, proved the maggots of putrefying flesh to be derived from the eggs of flies; Schwann prosed putrefaction itself to be the concomitant of far lower forms of life than those dealt with by Redi. Our knowledge here, as elsewhere in connection with this subject, has been vastly extended by Professor Cohn, of Breslau. "No putrefaction," he says, "can occur in a nitrogenous substance if its bacteria be destroyed sml now ones prevented from entering it. Putrefaction begins as soon as bacteria, even in the smallest unmhers, are almittol either acridentally or purposely. It progreses in direct proportion to the multiplication of the bacteria, it is retaried when they exlibit low vitality, and is stoppenl hy all influencos which either hinder their development or kill them. All bacte- 
ricidal media are therefore antiseptic and disinfecting." * It was these organisms acting in wound and abscess which so frequently converted our hospitals into charnel-houses, and it is their destruction by the antiseptic system that now renders justifiable operations which no surgeon would have attempted a few yeurs ago. The gain is immenseto the practicing surgeon as well as to the patient practiced upon. Contrast the anxiety of never feeling sure whether the most brilliant operation might not be rendered nugatory by the access of a few particles of unseen hospital dust, with the comfort derived from knowledge that all power of mischief on the part of such dust has been surely and certainly annihilated. But the action of living contagia extends beyond the domain of the surgeon. 'The power of reproduction and indefinite self-multiplication which is characteristic of living things, coupled with the undeviating fact of contagia "breeding true," has given strength and consistency to a belief long entertained by penetrating minds, that epidemic diseases generally are the concomitants of parasitic life. "'There begins to be faintly visible to us a vast and destructive laboratory of nature wherein the diseases which are most fatal to animal life, and the changes to which dead organic matter is passively liable, appear bound together by what must at least be called a very close analogy of causation." $\downarrow$ According to this view, which, as I have said, is daily gaining converts, a contagious disease may be defined as a conflict between the person smitten by it and a specific organism which multiplies at his expense, appropriating his air and moisture, disintegrating his tissues, or poisoning him by the decompositions incident to its growth.

During the ten years extending from 1859 to 1869 , researches on radiant heat in its relations to the gaseous form of matter occupied my continual attention. When air was experimented on, I had to cleanse it effectually of

* In his last excellent memoir Cohn expresses himself thus: “Wer noch heut die Fäulniss von einer spontanen Dissociation der Proteinmolecule, oder von einem unorganisirten Ferment ableitet, oder gar aus 'Stickstoffsplittern' die Balken zur Stütze seiner Fäulnisstheorie zu zimmern versucht, hat zuerst den Satz "keine Fäulniss ohne Bacterium Termo' zu widerlegen."

t Report of the Medical Officer of the Privy Council, 1874, p. 5. 
floating matter, and while doing so I was surprised to notive that, at the ordinary rate of transfer, such matter passed freely through alkalis, acids, aloohols, and ethers. The eye being kept sensitive by darkness, a concentrated beatu of light was fonnd to be a inost searching lest for snspended matter both in water and in air-a test indeed infefinitely more searching and severe than that furnished by the most powerful microscope. With the aid of such a beam I examined air filtered by cotton-wool; air long kejt free from agitation, so as to allow the floating matter to subsile; calcined air, and air filtered by the deopor cells of the human lungs. In all cases the correspondence between any experiments and those of Schroeder, Pasteur, and Lister in regard to spontaneous generation was perfect. The air which they found inoperative was proved by the luminous beam to be optically jure and therefore germless. Maving worked at the subject beth by experiment and reflection, on Frilay evening, January 21, 18\%0, I brought it before the members of the Royal Institution. 'Two or three months subsequently, for sufficient practical reasons, I ventured to direct public attention to the subject in a letter to the Times. Such was my first contact with this important question.

This letter, I believe, gave occasion for the first public utterance of Dr. Bastian in relation to this subject. He did me the honor to inform me, as others hal informed Pasteur, that the subject " pertains to the biologist and physician." He expressed “amazement" at my reasoning, and warned me that before what I had done could be undone "much irroparable mischief might be occusioned." With far less preliminary experience to guide and warn him, the English heterogenist was far bolder than Pouchet in his experiments, and far more adventurous in his conclusions. With organic infusions he obtained the results of his celelorated predecessor, but he did much more-the atoms and molecules of inorganic liquids passing under his manipulation into those more "complex chemieal compounds," which we dignify by calling them "living organisms." * As regards the public who take an interest

- It is further heold that lonecoria or allient organions are prone to

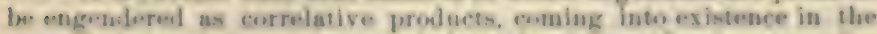

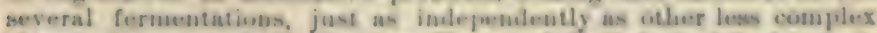

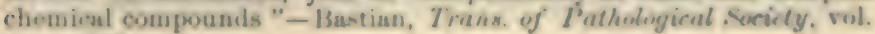
xxvi., 2.5. 
in such things, and apparently also as regards a large portion of the medical profession, our clever countryman succeeded in restoring the subject to a state of uncertainty similar to that which followed the publication of Pouchet's volume in 1859 .

It is desirable that this uncertainty should be removed from all minds, and doubly desirable on practical grounds that it should be removed from the minds of medical men. In the present article, therefore, I propose discussing this question face to face with some eminent and fair-minded member of the medical profession who, as regards spontaneous generation, entertains views adverse to mine. Such a one it would be easy to name; but it is perhaps better to rest in the impersonal. I shall therefore simply call my proposed co-inquirer my friend. With him at my side, I shall endeavor, to the best of my ability, so to conduct this discussion that he who runs may read and that he who reads may understand.

Let us begin at the beginning. I ask my friend to step into the laboratory of the Royal Institution, where I place before him a basin of thin turnip slices barely covered with distilled water kept at a temperature of $1 \geqslant 0$ degrees

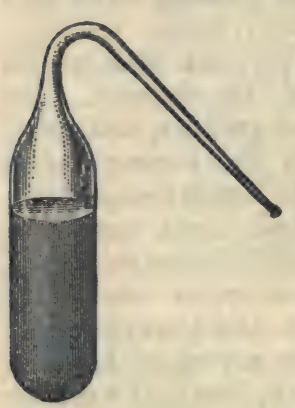
Fahr. After digesting the turnip for four or five hours we pour off the liquid, boil it, filter it, and obtain an infusion as clear as filtered drinking water. We cool the infusion, test its specific gravity, and find it to be $\mathbf{3 0 0 6}$ or higher-water being 1000. A number of small clean empty flasks, of the shape shown on the margin, are before us. One of them is slightly warmed with a spirit-lamp, and its open end is then dipped into turnip the infusion. The warmed glass is afterward ohilled, the air within the flasks cools, contracts, and is followed in its contraction by the infusion. Thus we get a small quantity of liquid into the flask. We now heat this liquid carefully. Steam is produced, which issues from the open neck, carrying the air of the flask along with it. After a few seconds' ebtillition, the open neck is again plunged into the infusion. The steam within the flask condenses, the liquid enters to supply its 
place, and in this way we fill our little flask to about fourlifths of its volume. This description is typical; we may thus fill a thousand flasks with a thousand different infusions.

I now ask my friend to notice a trough made of sheet copper, with two rows of haudy little Bunsen burners underneath it. 'This trough, or bath, is nearly filled with oil: a piece of thin plank constitutes a kimb of lid for the oil-inth. The wood is perforated with circular apertures wide enough to allow our small flask to pass through and plunge itself in the oil, which has been heated, say, to 250 degrees Fahr. Clasped all round by the hot liquil, the infusion in the flask rises to its boiling proint, which is not sensibly over 212 degrees Falır. Steam issues from the open neck of the flask, and the boiling is continued for tive minutes. With a pair of small brass tongg, an assistant now seizes the neck near its junction with the flask, and partially lifts the latter out of the oil. The steam does not cease to issue, but its violence is abated. With a seconil pait of tongs held in one hand, the neck of the flask is seized close to its open end, while with the other hand a Bunsen's flame or an ordinary spirit flame is brought under the midale of the neck. The glass redlens, whitens, softens, and as it is gently drawn out the neek diminishes in diameter, until the canal is complotely blosked up. The tongs with the fragment of severed neek being withil rawn, the flask, with its contents diminished by evaporation, is lifted from the oil-bath perfectly sealed hermetically.

Sixty such flasks filled, boiled, and sealed in the manner described, and cöntaining strong infusions of beef, mutton, turnip, and cucumber, are carefully packed in sawdust, and iransported to the $\Lambda$ lps. Thither, to an elevation of about 7,000 feet above the sea, I invite my co-inquirer to accompany me. It is the month of July, and the weallier is favorable to putrefaction. We open our box at the BulAlp, and count ont fifty-four flasks, with their liquids as clear as filterod drinking wator. In six flasks, however, the infusion is found muildy. We closely examine these, and discover that every one of them has lial its fragile end broken oft in the transit from fondon. Air has entered the flasks, and the observerl muldiues is the result. My collengue knows as wëll as I lo what this means. Fixamined with a pocket-lens, or even with a microsoupo of insuflicient 
power, nothing is seen in the muddy liquid; but regarded with a magnifying power of a thousand diameters or so, what an astonishing appearance does it present! Leeuwenhoek estimated the population of a single drop of stagnant water at 500,000,000: probably the population of a drop of our turbid infusion would be this many times multiplied. The field of the microscope is crowded with organisms, some wabbling slowly, other's shooting rapidly across the microscropic field. They dart hither and thither like a rain of minute projectiles; they piroutete and spin so quickly round, that the retention of the retinal impression transforms the little living rod into a twirling wheel. And yet the most celebrated naturalists tell us they are vegetables. From the rod-like shape which they so frequently assume, these organisms are called "bacteria" - a term, be it here remarked, which covers organisms of very diverse kinds.

Has this multitudinous life been spontaneously generated in these six flasks, or is it the progeny of living germinal matter carried into the flasks by the entering air? If the infusions have a self-generative power, how are the sterility and consequent clearness of the fifty-four uninjured flasks to be accounted for? My colleague may urge-and fairly urge-that the assumption of germinal matter is by no means necessary; that the air itself may be the one thing needed to wake up the dormant infusions. We will examine this point immediately. But meanwhile I would remind him that I am working on the exact lines iaid down by our most conspicuous heterogenist. He distinctly affirms that the withdrawal of the atmospheric pressure above the infusion favors the production of organisms; and he accounts for their absence in tins of preserved meat, fruit, and vegetables, by the hypothesis that fermentation has begun in such tins, that gases have been generated, the pressure of which has stifled the incipient life and stopped its further development.* This is the new theory of preserved meats. Had its author pierced a tin of preserved meat, fruit, or vegetable under water with the view of testing its truth, he would have found it erroneous. In well-preserved tins he would have found, not an outrush of gas, but an inrush of water. I have noticed this recently

* Beginnings of Life, vol. i., p. 418. 
in tins which have lain perfectly good for sixty-three years in the Royal Institution. Moilern tins, subjected to the same test, yielded the sume result. From time to time, moreover, during the last two years, I have placed glass tubes, containing clear infusions of turnip, hay, beef, and mutton, in iron bottles, and subjected then to air-pressures varying from ton to twenty-seven atmospheres-pressures, it is needless to say, far more than suflicient to tear a preserved meat tin to shreds. After ten days these infusions were taken from their bottles rotten with putrefaction and teeming with life. Thus collapses an hypothesis which had no rational foundation, and which conld never have seen the light had the slightest attempt been made to verify it.

Our fifty-four vacuous and pellucid flasks also declare against the heterogenist. We expose them to a warm Alpine sun by day, and at night we suspend them in a warm kitchen. Four of them have been accidentally broken; but at the end of a month we find the fifty remaining ones as clear as at the commencement. There is no sign of putrefaction or of life in any of them. We divide these flasks into two groups of twenty.three and twenty-seren respectively (an accident of counting rendered the division uneven). Tho question now is whether the almission of air can liberate any generative energy in the infusions. Our next experiment will answer this question and something more. Wo carry the flasks to a hayloft, and there, with a pair of steel pliers, snip off the sealed ends of the group of three-and-twenty. Each snipping off is of course followed by an inrush of air. We now carry our twenty-seren flasks, our pliers, and a spirit-lamp, to a ledge overlooking the Aletsoh glacier, about 200 feet ahove the hayloft, from which ledge the mountain falls almost precipitously to the northeast for about a thousancl feet. A gentle wind blows toward us from the northeast -that is, across the crests and snow-fields of the Oberland mountains. We are therefore bathed by air which must have been for a good while out of practical contact with either animal or vegetable life. I stand carefully io leewarl of the flasks, for no dust or particle from my clothes or body must be blown toward them. $\Lambda$ n assistanit innites the spirit-lamp, into the flame of which I plunge the pliers, thereby destroying all attached germs or 
organisms. Then I snip off the sealed end of the flask. Prior to every snipping the same process is gone through, no flask being opened withont the previous cleansing of the pliers by the flame. In this way we charge our seven-and-twenty flasks with clean, vivifying mountain air.

We place the fifty flasks, with their necks open, over a kitchen stove, in a temperature varying from 50 to 90 degrees Fahr., and in three days find twenty-one out of the twenty-three flasks opened on the hayloft invaded by organisms-two only of the group remaining free from them. After three weeks' exposure to precisely the same conditions, not one of the twenty-seven flasks opened in free air had given way. No germ from the kitchen air had ascended the narrow necks, the flasks being shaped to produce this result. They are still in the Alps, as clear, I doubt not, and as free from life as they were when sent off from London.*

What is my colleague's conclusion from the experiment before us? Twenty-seven putrescible infusions, first in vacuo, and afterward supplied with the most invigorating air, have shown no sign of putrefaction or of life. And as to the others, I almost shrink from asking him whether the hayloft has rendered them spontaneously generative. Is not the inference here imperative that it is not the air of the loft-which is connected through a constantly open doer with the general atmosphere-but something contained in the air, that has produced the effects observed? What is this something? A sumbeam entering through a chink in the roof or wall, and traversing the air of the loft, would show it to be laden with suspended dust particles. Indeed the dust is distinctly visible in the diffused daylight. Can it have been the origin of the observed life? If so, are we not bound by all antecedent experience to regard these fruitful particles as the germs of the life observed?

The name of Baron Liebig has been constantly mixed up with these discussions. "We have," it is said, "his authority for assuming that dead decaying matter can produce fermentation." 'True, but with Liebig fermentation was by no means synonymous with life. It. meant, accord-

* An actual experiment made at the Bel Alp is here deseribed. 
ing to him, the shaking asunder by chemical disturbance of unstable molecules. Does the life of our flasks, then, proceed from deud particles? If ung co-inquirer should reply "Yes," then I would ask him, "What warrant does nature offer for such an assumption? Where, anid the multitude of vital phenomena in which her operations have been elearly traced, is the slightest countenance given to the notion that the sowing of dead particles can produce a living crop?" With regard to Baron Liebig, had he studied the revelations of the mieroscope in relation to these questions, a mind so penetrating could never have missed the significance of the facts revealed. He, however, neglerted the microscope, and fell into error-but not into error so gross as that in support of which his anthority has been invoked. Were he now alive, he wonld, I donbt not, repudiate the use often made of his name-Liebig's view of fermentation wats at least a seientific one, founded on profound conceptions of molecular instability. But this view by no means involves the notion that the planting of deal particles -.. Stickstoffsplittern " as Uohn contemptnously calls them -is followed by the sprouting of infusurial life.

Let us now return to London and fix our attention on the dust of its air. Suppose a room in which the honsemaid has just finished her work to be completely closed, with the exception of an aperture in a shutter throngh which a sunbeam enters and crosses the room. 'The floating dust reveals the track of the light. Let a lens be placed in the aperture to condense the beam. Its parallel rays are now conserged to a cone, at the apex of which the dust is raised to almost unbroken whiteness by the intensity of its illumination. Defended from all glare, the eje is peculiarly sensitive to this scattered light. The floating dust of London rooms is organic, and may be burned without leasing visible residue. 'The action of a spirit-lamp flame upon the floating matter has been elsewhere thus deseribed:

In a cylindrical Iwam which strongly flluminated the dust of our laboratury. I placed an ignited spirit-lamp. Mingling w ith the flame. and roumil its rim. were mesn eurious wromelis of darknoss resombling an intersely black stnoke. On placing the flatue at souse distance lelww the lumm, the same dark massess stormed upward. They were blackep tlian the blatkos smoke ever sesen issulag from the funuel of a stesuer; aud their rememblance to smoke was so perfect 
as to prompt the conclusion that the apparently pure flame of the alcohol-lamp required but a beam of sutficient intensity to reveal its clouds of liberated carbon.

But is the blackness smoke? This question presented itself in a moment, and was thus answered: A red-hot poker was placed underneath the beam; from it the black wreaths also ascended. A large hydrogen flame, which emits no smoke, was next employed, and it also produced with augmented copiousness those whirling masses of darkness. Smoke being out of the question, what is the blackness? It is simply that of stellar space; that is to say, blackness resulting from the absence from the track of the beam of all matter competent to scatter its light. When the flame was placed below the beam, the floating matter was destroyed in situ; and the heated air, freed from this matter, rose into the beam, jostled aside the illuminated particles, and substituted for their light the darkness due to its own perfect transparency. Nothing could more forcibly illustrate the invisibility of the agent which renders all things visible. The beam crossed, unseen, the black chasm formed by the transparent air, while, at both sides of the gap, the thick-strewn particles shone out like a luminous solid under the powerful illumination.

Supposing an infusion intrinsically barren, but readily susceptible of putrefaction when exposed to common air, to be brought into contact with this unilluminable air, what would be the result? It would never putrefy. It might, however, be urged that the air is spoiled by its violent calcination. Oxygen passed through a spirit lamp flame is, it may be thought, no longer the oxygen suitable for the development and maintenance of life. We have an easy escape from this difficulty, which is based, however, upon the unproved assumption that the air has been affected by the flame. Let a condensed beam be sent through a large flask or bolthead containing common air. The track of the beam is seen within the flask-the dust revealing the light, and the light revealing the dust. Cork the flask, stuff its neck with cotton-wool, or simply turn it mouth downward and leave it undisturbed for a day or two. Examined afterward with the luminous beam, no track is visible; the light passes throngh the flask as though a vacuum. The floating matter has abolished itself, being now attached to the interior surface of the flask. Were it our object, as it will be subsequently, to effectually detain the dirt, we might coat that surface with some sticky substance. Here, then, without "torturing" the air in any way, we have found a means of ridding it, or rather of enabling it to rid itself, of floating matter. 
We have now to devise a means of testing the action of such spontaneously purified air upon putrescible infusions. Wooden chambers, or cases, are accordingly constructed, having glass fronts, sille-windows, and back-doors. Through the bottoms of the chambers test-tubes pass air-

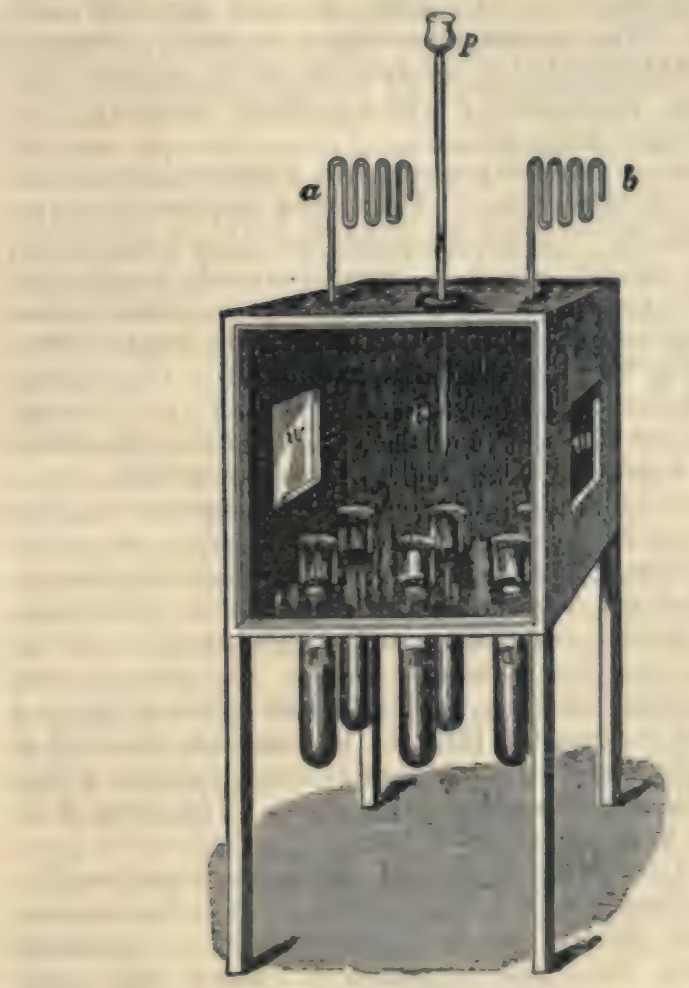

tight; their open ends, for about one-fifth of the length of the tubes, being within the chambers. Provision is mate for a free connection through sinuous channels between the inner and the onter air. Throngh such ehannels, though open, no dast will reach the chamber. 'The top of each chamber is perforated by a circular hole two inches in diameter, closed air-tight by a sheet of india rubber. This 
is pierced in the middle by a pin, and through the pin-bole is pushed the shank of a long pipette, ending above in a small funnel. The shank also passes through a stuffingbox of cotton-wool moistened with glycerine; so that, tightly clasped by the rubber and wool, the pipette is not likely in its motions up and down to carry any dust into the chamber. The annexed woodeut shows a chamber, with six test-tubes, its side-windows $w w$, its pipette $p$, and its sinuous channels $a b$ which connect the air of the chamber with the outer air.

The chamber is carefully closed and permitted to remain quiet for two or three days. Examined at the beginning by a beam sent through its windows, the air is found laden with floating matter, which in three days has wholly disappeared. To prevent its ever rising again, the internal surface of the chamber was at the outset coated with glycerine. The fresh but putrescible liquid is introduced into the six tubes in succession by means of the pipette. Permitted to remain without further precaution, every one of the tubes would putrefy and fill itself with life. The liquid has been in contact with the dust-laden air outside by which it has been infected, and the infection must be destroyed. This is done by plunging the six tubes into a bath of heated oil and boiling the infusion. The time requisite to destroy the infection depends wholly upon its nature. 'Two minutes' boiling suffices to destroy some contagia, whereas two hundred minutes' boiling fails to destroy others. After the infusion has been sterilized, the oil-bath is withdrawn, and the liquid, whose putrescibility has been in no way affected by the boiling, is abandoned to the air of the chamber.

With such chambers I tested, in the autumn and winter of 1875-6, infusions of the most various kinds, embracing natural animal liquids, the flesh and viscera of domestic animals, game, fish and vegetables. Mure than fifty chambers, each with its series of infusions, were tested, many of them repeatedly. There was no shade of uncertainty in any of the results. In every instance we had, within the chamber, perfect limpidity and sweetness, which in some cases lasted for more than a year-without the chamber, with the same infusion, putridity and its characteristic smells. In no instance was the least countenance lent to the notion that an infusion deprived by heat of its 
inherent life, and placed in contact with air cleansed of its visibly suspended matter, has any power to generate life anew.

Remembering then the number and variety of the infusions employed, and the strictness of our alherence to the rules of preparation laid down by the heterogenists themselves; remembering that we have operated upon the very substances recommended by them as capable of furnishing, even in untrained hands, easy and decisive proofs of spoutaneous generation, and that we have added to their substances many others of our own-if this pretended generative power were a reality, surely it must have manifested itself somewhere. Speaking roundly, I should say that in such closed chambers at least five hundred chances have been given to it, but it has nowhere appeared.

The argument is now to be clenched by an experiment which will remore every residne of donbt as to the ability of the infusions here employed to sustain life. We open the back doors of our sealed chambers, and permit the common air with its floating particles to have access to our tubes. For three months they have remained pellucid and 8weet-flesh, fish, and regetable extracts purer than ever cook manufactured. 'Three days' exposure to the dusty air suffices to render them muddy, fetid, and swarming with infusorial life. The liquids are thus proved, one and all, realy for putrefaction when the contaminating agent is applied. I invite my colleague to reflect on these facts. How will he account for the absolute immunity of a liquid exposed for months in a warm room to optically pure air, and its infallible putrefaction in a few days when exposed to dust-laden air? He must, I submit, bow to the conclusion that the dust-particles are the cause of putrefactive life. And nnless he accepts the hypothesis that these parlicles, being dead in the air, are in the liquid miraculously kindled into living things, he must conclude that the life we have observed springs from germs or organisms diffused through the atmosphere.

The experiments with hermetieally sealed flaks have reached the number of 940. A sample group of 130 of them were laid before the Royal Suciety on Jannary 13, 1876. 'They were ntterly free from life, having been completely sterilizen by three minutes briling. Special eare had been taken that the temperatures to which the flasks 
were exposed should include those previously alleged to be efficient. The conditions laid down by the heterogenist were accurately copied, but there was no corroboration of his results. Stress was then laid on the question of warmth, thirty degrees being suddenly added to the temperatures with which both of us had previously worked. Waiving all protest against the caprice thus manifested, I met this new requirement also. 'The sealed tubes, which har proved barren in the Royal Institution, were suspended in perforated boxes, and placed under the supervision of an intelligent assistant in the 'Turkish Bath in Jermyn street. From two to six days had been allowed for the generation of organisms in hermetically sealed tubes. Mine remained in the washing-room of the bath for nine days. 'Thermometers placed in the boxes, and read off twice or three times a day, showed the temperature to vary from a minimum of 101 degrees to a maximum of 112 degrees Fahr. At the end of nine days the infusions were as clear as at the beginning. 'They were then removed to a warmer' position. A temperature of 115 degrees had been mentioned as particularly favorable to spontaneous generation. For fourteen days the temperature of the Turkish Bath hovered about this point, falling once as low as 106 degrees, reaching 116 degrees on three occasions, 118 degrees on one, and 119 degrees on two. 'The result was quite the same as that just recorded. The higher temperatures proved perfectly incompetent to develop life.

Taking the actual experiment we have made as a basis of calculation, if our 940 flasks were opened on the hayloft of the Bel Alp, 858 of them would become filled with organisms. The escape of the remaining 82 strengthens our case, proving as it does conclusively that not in the air, nor in the infusions, nor in anything continuous diffused through the air, but in discrete particles, suspended in the air and nourished by the infusions, we are to seek the cause of life. Our experiment proves these particles to be in some cases so far apart on the hayloft as to permit 10 per cent. of our flasks to take in air without contracting contamination. A quarter of a century ago Pasteur proved the cause of "so-called spontaneous generation" to be discontinuous. I have already referred to his observation that 12 out of 20 flasks opened on the plains escaped 
infection, while 19 ont of ?u flasks opened on the Mer de Glace escaped. Our own experiment at the $\mid 3 \mathrm{el} A \mathrm{lp}$ is a more emplatic instance of the same kimb. 9o per cent. of the flasks opened in the hayloft being smitten, while not one of those opened on the free mountain ledge was attucked.

The power of the air as regards putrefactive infection is incessantly changing through natural causes, and we are able to alter it at will. Of a number of flasks opened in 15i6 in the laboratory of the Royal Institution, 42 per cent. were smitten, while se jer cont. escaped. In 18 s the proportion in the same laboratory was 68 per cent. smitten, to :3: intact. The greater mortality, so to speak, of the infusions in 15\%? was due to the presence of hay which diffused its germinal dust in the laboratory air, catusing it to approximate as regards infectice virulence to the air of the Alpine loft. I would ask my friend to bring his scientific penetration to bear upon all the foregoing facts. They do not prove spontaneous generation to be "impossible." My assertions, however, relate not to "possibilities," but to proofs, and the experiments just hescribed do most distinctly prove the eridence on which the heterogenist relies to be written on waste paper.

My colleague will not, I am persuaded, dispute these resulis; but he may be disposed to urge that other able and honorable men working at the same subject have arrived at conclusions different from mine. Most freely granted; but let me here recur to the remarks alrealy made in speaking of the experiments of Spallanzani, to the effect that the failure of others to confirm his results by no means upsets their evidence. To fix the ideas, let ns suppose that my colleague comes to the laboratory of the Royal Institucion, repeats there my experiments, and obtains confirmatory resnlts; and that he then goes to University or King's College where, operuting with the Rame infusions, he obtains contradiotory results. Will he be disposed to conclube that the eelfsame substance is barren in Albemarle street and fruitfol in Ciower strect or the Sirand? His Alpine experience las alrealy male known to him the literally infinite differenceg existing between different samples of air us regards their cupmeily for putrefactive infection. And, possessing this knowl- 
edge, will he not substitute for the adventurous conclusion that an organic infusion is barren at one place and spontaneously generative at another, the more rational and obvious one that the atmosphere of the two localities which have had access to the infusion are infective in different degrees?

As regards workmanship, moreover, he will not fail to bear in mind, that fruitfulness may be due to errors of manipulation, while barrenness involves the presumption of correct experiment. It is only the careful worker that can secure the latter, while it is open to every novice to obtain the former. Barrenness is the result at which the conscientious experimenter, whatever his theoretic convictions may be, ought to aim, omitting no pains to secure it, and resorting only when there is no escape from it to the conclusion that the life observed comes from no source which correct experiment could nentralize or avoid.

Let us again take a definite case. Supposing my colleague to operate with the same apparent care on 100 infusions-or rather on 100 samples of the same infusion -and that 50 of them prove fruitful and 50 barren. Are we to say that the evidence for and against heterogeny is equally balanced? There are some who would not only say this, but who would treasure up the 50 fruitful flasks as "positive" results, and lower the evidential value of the 50 barren flasks by labeling them " negative" results. This, as shown by Dr. William Roberts, is an exact inversion of the true order of the terms positive and negative.* Not such, I trust, would be the course pursued by my friend. As regards the 50 fruitful flasks he would, I doubt not, repeat the experiment with redoubled care and scrutiny, and not by one repetition only, but by many, assure himself that he had not fallen into error. Such faithful scrutiny fully carried out would infallibly lead him to the conclusion that here, as in all other cases, the evidence in favor of spontaneous generation crumbles in the grasp of the competent inquirer.

The botanist knows that different seeds possess different powers of resistance to heat. $\nmid$ Some are killed by a

* See his truly philosophical remarks on this head in the "British Medical Journal," 18\%6, p. 282.

+ I am indebted to Dr. Thiselton Dyer for various illustrations of such differences. It is, however, surprising that a subject of such 
momentary exposure to the boiling temperature, while others withstand it for several hours. Most of our ordinary seeds are rapidly killed, while l'onchet made known to the Paris Academy of Sciences in 1866, that certain seeds, which had been transported in fleeces of wool from Brazil, germinated after four hours' boiling. 'The germs of the air vary as mach anong themselres as the seeds of the botanist. In somo localities the diffused germs are so tender that boiling for five minutes, or even less, would be sure to destroy them all; in other localities the diffused germs are 80 obstinate, that many hours' bolling would be requisite to deprive them of their power of germination. The absence or presence of a truss of desiccated hay would produce differences as great as those here described. The greatest endurance that I have ever observed-and I believe it is tho greatest on record-was a case of survival after eight hours' boiling.

As regards their power of resisting hcat, the infusorial germs of our atmosphere might be classified under the following and intermediate heads: Killed in five minutes; not killed in tive minutes but killed in fifteen; not killed in fifteen minutes but killed in thirty; not killed in thirty minutes but killed in an hour; not killed in an hour but killed in two hours; not killed in two but killed in three hours; not killed in three but killed in four hours. I have had several cases of survival after four and five hours' boiling, some survivals after six, and one after eight hours' boiling. Thus far has experiment actually reached; but there is no valid warrant for fixing upon even eight hours as the extreme limit of vital resistance. Prubably more extended researches (though mine have been very extensive) would reveal germs more obstinate still. It is also certain that we might begin earlier, and find germs which are destroyed by a temperature far below that of boiling water. In the presence of such facts, to speak of $a$ death-point of bacteria and their germs would be unmeaning-but of this more anon.

"What present warrant," it has been askel, "is there for supposing that a naked, or almost nakerl, speck of

high scientific importance shonld mot hase beren more thoroughly explormi. Here the scioundrels whe denl in killeal sereds mighis be able to add to our knowledge. 
protoplasm can withstand four, six, or eight hours' boiling?" Regarding naked specks of protoplasm I make no assertion. I know nothing about them, save as the creatures of fancy. But I do affirm, not as a "supposition," nor an "assumption," nor a " probable guess," nor as "a wild hypothesis," but as a matter of the most undoubted fact, that the spores of the hay bacillus, when thoroughly desiccated by age, have withstood the ordeal mentioned. And I further affirm that these obdurate germs, under the guidance of the knowledge that they are germs, can be destroyed by five minutes' boiling, or even less. 'This needs explanation. The finished bacterium perishes at a temperature far below that of boiling water, and it is fair to assume that the nearer the germ is to its final sensitive condition the more readily will it succumb to heat. Seeds soften before and during germination. 'This premised, the simple description of the following process will suffice to make its meaning understood.

An infusion infected with the most powerfully resistent germs, but otherwise protected against the floating matters of the air, is gradually raised to its boiling-point. Such germs as have reached the soft and plastic state immediately preceding their development into bacteria are thus destroyed. The infusion is then put aside in a warm room for ten or twelve hours. If for twenty-four, we might have the liquid charged with well-developed bacteria. 'To anticipate this, at the end of ten or twelve hours we raise the infusion a second time to the boiling temperature, which, as before, destroys all germs then approaching their point of final derelopment. The infusion is again put aside for ten or twelve hours, and the process of heating is repeater. We thus kill the germs in the order of their resistance, and finally kill the last of them. No infusion can withstand this process if it be repeated a sufficient number of times. Artichoke, cucumber, and turnip infusions, which had proved specially obstinate when infected with the germs of desiccated hay, were completely broken down by this method of discontinuous heating, three minutes being found sufficient to accomplish what three hundred minutes' continuous boiling failed to accomplish. I applied the method, moreover, to infusions of various kinds of hay, including those most tenacious of life. Not one of them bore the ordeal. These results were clearly foreseen before they 
were realized, so that the germ theory fulfills the test of every true theory, that test being the power of prevision.

When " naked or almost naked specks of protoplasm" are spoken of, the imagination is drawn upon, not the objective truth of Nature. Such words sound like the worls of knowledge where knowledge is really nil. The possibility of a "thin covering" is conceded by those who speak in this way. Such a covering may, however, exereise a powerful protective influence. A thin pellicle of india-rubber, for example, surrounding a pea keeps it hard in boiling water for a time sufficient to rednce an un. cuvered pen to pulp. The pellicle prevents imbibition, diffusion, and the consequent disintegration. A greasy or oily surface, or even the layer of air which clings to certain bodies, would act to some extent in a similar way. "The singular resistance of green regetables to sterilization," says Dr. William Roberts, "appears to be due to some peculiarity of the surface, perhaps their smooth glistening epidermis which prevented complete wetting of their surfaces." I pointed out in 1876 that the process by which an atmospheric germ is wetted would be an interesting subject of investigation. A dry microscope covering glakis may be cansed to float on water for a year. A sewing-needle may be similarly kept floating, though its specifio gravity is nearly eight times that of water. Were it not for some specific relation between the matter of the germ and that of the liquid into which it falls, wetting would be simply impossible. Antecedent to all development there must be an interchange of matter between the gorm and its environment; and this interchange must obviously depend upon the relation of the germ to its encompassing liquid. Anything that hinders this interchange retards the destruction of the germ in boiling water. In my paper published in the "Plilosophical Transactions" for 18\% , I add the following remark:

It is not difficult to see that the surface of a seed or germ may he ans affected Ly desiccation and other causes as practically to prevent contact between it and the surrounding liquid. The body of a germ, unoreover, may the mo indurated loy time and dryuess us to resint jowerfully the insinastion of water twetween its constitnent nomle eules. It would le difleult to cause such a gerun to imbile tlie. moisture necesary to produce the swelling nod softening which frecede itn destruction in a liquid of high temperature. 
However this may be-whatever be the state of the surface of the body, of the spores of Bacillus subtilis, they do as a matter of certainty resist, under some circumstances, exposure for hours to the heat of boiling water. No theoretic skepticism can successfully stand in the wa of this fact, established as it has been by hundreds, if not thousands, of rigidly conducted experiments.

We have now to test one of the principal foundations of the doctrine of spontaneous generation as formulated in this country. With this view, I place before my friend and co-inquirer two liquids which have been kept for six months in one of our sealed chambers, exposed to optically pure air. The one is a mineral solution containing in proper proportions all the substances which enter into the composition of bacteria, the other is an infusion of turnip -it might be any one of a hundred other infusions, animal or vegetable. Both liquids are as clear as distilled water, and there is no trace of life in either of them. They are, in fact, completely sterilized. A mutton-chop, over which a little water has been poured to keep its juices from drying up, has lain for three days upon a plate in our warm room. It smells offensively. Placing a drop of the fetid mutton-juice under a microscope, it is found swarming with the bacteria of putrefaction. With a speck of the swarming liquid I inoculate the clear mineral solution and the clear turnip infusion, as a surgeon might inoculate an infant with vaccine lymph. In four-and-twenty hours the transparent liquids have become turbid thronghout, and instead of being barren as at first they are teeming with life. The experiment may be repeated a thousand times with the same invariable result. To the naked eye the liquids at the beginning were alike, being both equally transparent-to the naked eye they are alike at the end, being both equally muddy. Instead of putrid mutton-juice, we might take as a source of infection any one of a hundred other putrid liquids, animal or vegetable. So long as the liquid contains living bacteria a speck of it communicated either to the clear mineral solution, or to the clear turnip infusion, produces in twenty-four hours the effect here described.

We now vary the experiment thus: Opening the backdoor of another closed chamber which has contained for 
months the pure mineral solution and the pure turnip infusion side by side, I drop into each of them a small pinch of laboratory dust. The effect here is tardier than when the speck of putrid liquid was employed. In three days, however, after its infection with the dust, the turnip infusion is muddy, and swarming as before with bacteria. But what about the mineral solution which, in our first experiment, behaved in a manner undistinguishable from the turnip-juice? At the end of three days there is not a bacterium to be found in it. At the end of three weeks it is equally innocent of bacterial life. We may repeat the experiment with the solution and the infusion a hundred times with the same invariable result. Always in the case of the latter the sowing of the atmospheric dust yields a crop of bacteria - never in the former does the dry germinal matter kindle into active life.* What is the inference which the reflecting mind must draw from this experiment? Is it not as clear as day that while both liquids are able to feed the bacteria alid to enable them to inerease and multiply, after they have been once fully developed, only one of the liquids is able to develop into active bateria the germinal dust of the air?

I invite my friend to reflect upon this conclusion; he will, I think, see that there is no escape from it. He may, if he prefers, hold the opinion, which I consider erroneous, that bacteria exist in the air, not as germs but as desiocated organisms. The inference remains, that while the one liquid is able to force the passuge from the inactive to the active state, the other is not.

But this is not at all the inference which has been drawn from experiments with the mineral solution. Seeing its ability to nourish bucteria when once inoculated with the living active organism, and observing that no bacteria appeared in the solution after long exposure tn the air, the inference was drawn that neither bacteria nor their germs existed in the air. Throughout fermany the ahlest literature of the subject, even that opposed to heterogeny, is infected with this error; while lieterogenists at homo

* This is thes deportunent of the mineral solution as descerilued by others. My own experiments would lead me to say that the develop.

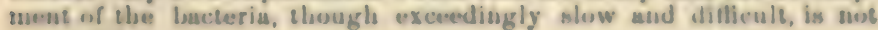
impoasible. 
and abroad have based upon it a triumphant demonstration of their doctrine. It is proved, they say, by the deportment of the mineral solution that neither bacteria nor their germs exist in the air; hence, if, on exposing a thoroughly sterilized turnip infusion to the air, bacteria appear, they must of necessity have been spontaneously generated. In the words of Dr. Bastian: "We can only infer that while the boiled saline solution is quite incapable of engendering bacteria, such organisms are able to arise de novo in the boiled organic infusion." *

I would ask my eminent colleague what he thinks of this reasoning now? The datum is-"A mineral solution exposed to common air does not develop bacteria;" the inference is-"Therefore if a turnip infusion similarly exposed develop bacteria, they must be spontaneously generated." The inference, on the face of it, is an unwarranted one. But while as matter of logic it is inconclusive, as matter of fact it is chimerical. London air is as surely charged with the germs of bacteria as London chimneys are with smoke. The inference just referred to is completely disposed of by the simple question: "Why, when your sterilized organic infusion is exposed to optically pure air, should this generation of life de novo utterly cease? Why should I be able to preserve my turnip-juice side by side with your saline solution for the three hundred and sixty-five days of the year, in free connection with the general atmosphere, on the sole condition that the portion of that atmosphere in contact with the juice shall be visibly free from floating dust, while three days' exposure to that dust fills it with bacteria?" Am I over sanguine in hoping that as regards the argument here set forth he who runs may read, and he who reads may understand?

We now proceed to the calm and thorough consideration of another subject, more important if possible than the foregoing one, but like it somewhat difficult to seize by reason of the very opulence of the phraseology, logical and rhetorical, in which it has been set forth. The subject now to be considered relates to what has been called "the death-point of bacteria." Those who happen to be acquainted with the modern English literature of the question will remember how challenge after challenge has

*“ Proceedings of the Royal Society," vol. xxi., p. 130. 
been issued to panspermatists in general, and to one or two home workers in particular, to come to close quarters on this cardinal point. It is obvionsly the strongholil of the English heterugenist. " Water," he says, " is boiling merrily over a fire when some luckless person upsets the vessel so that the heated fluid exercises its scathing influence upon an uncovered portion of the body-hand, arm, or face. Here, at all events, there is no room for doubt. Boiling water unquestionably exercises a most pernicious and rupially destructive effect upon the living matter of which we are composed." * And lest it should be supposed that it is the high organization which, in this case, renders the body susceptible to heat, he refers to the action of boiling water on the hen's egg to dissipate the notion. "The conclusion," he says, "would seem to force itself upon us that there is something intrinsically deleterious in the action of boiling water upon living matter-whether this matter be of high or of low organization." + Again, at another place: "It has been shown that the briefest exposure to the influence of boiling water is destructive of all living matter." $\ddagger$

The experiments alrealy recorded plainly show that there is a marked difference between the dry bacterial matter of the air, and the wet, soft, and actire bacteria of putrefying organic liquids. The one can be luxuriantly bred in the saline solution, the others refuse to be born there, while both of them are copiously developed in a sterilized turnip infusion. Inferences, as we have already seen, founded on the deportment of the one liguid cannot with the warrant of scientific logic be extended to the other. But this is exactly what the heterogenist has done, thus repeating as regards the denth-point of bacteria the error into which he fell concerning the germs of the air. let us boil our muddy mineral solution with its swarming bacteria for five minutes. In the soft succulent condition in which they exist in the solution not one of them escapes destruction. The same is true of the turnip infusion if it be inoculated with the living bacteria only-the aẹrial dust being carefully excluded. In both cases the

* Bastias, "Evolution," p. 183.

+ Ibid. p. 135.

lbid., p. 46. 
dead organisms sink to the bottom of the liquid, and without re-inoculation no fresh organisms will arise. But the case is entirely different when we inoculate our turnip infusion with the desiccated germinal matter afloat in the air.

The "death-point" of bacteria is the maximum temperature at which they can live, or the minimum temperature at which they cease to live. If, for example they survive a temperature of 140 degrees, and do not survive a temperature of 150 degrees, the death-point lies somewhere between these two temperatures. Vaccine lymph, for example, is proved by Messrs. Braidwood and Vacher to be deprived of its power of infection by brief exposure to a temperature between 140 and 150 degrees Fahr. This may be regarded as the death-point of the lymph, or rather of the particles diffused in the lymph, which constitute the real contagium. If no time, however, be named for the application of the heat, the term "death-point" is a vague one. An infusion, for example, which will resist five hour's' continuous exposure to the boiling temperature, will succumb to five days' exposure to a temperature 50 degrees Fahr. below that of boiling. 'The fully developed soft bacteria of putrefying liquids are not only killed by five minutes' boiling, but by less than a single minute's boiling-indeed, they are slain at about the same temperature as the vaccine. The same is true of the plastic, active bacteria of the turnip infusion.*

But, instead of choosing a putrefying liquid for inoculation, let us prepare and employ our inoculating substance in the following simple way: Let a small wisp of hay, desiccated by age, be washed in a glass of water, and let a perfectly sterilized turnip infusion be inoculated with the washing liquid. After three hours' continuous boiling the infusion thus infected will often develop luxuriant bacterial life. Precisely the same occurs if a turnip infusion be prepared in an atmosphere well charged with desiccated

* In my paper in the "Philosophical Transactions" for 1876, I pointed out and illustrated experimentally the difference, as regards rapidity of development, between water germs and air-germs; the growth from the already softened water-germs proving to be practically as rapid as from developed bacteria. This preparedness of the germ for rapid developinent is associated with its preparedness for rapid destruction. 
hay-germs. The infusion in this ease infects itself without spocial inoculation, and its subsequent resistance to sterilization is often sery great. On the Lst of March last I purposely infected the air of our laboratory with the germinal dust of a sapless kind of hay mown in 1875 . Ten groups of flasks were charged with turnip infusion prepared in the infocted laboratory, and were afterward subjected to the boiling temperature for periods varying from 15 minutes to 240 minutes. Ont of the ten groups only one was sterilized-that, namely, which had been boiled for four hours. Every flask of the nine groups which had been boiled for $15,30,45,60,75,90,105,120$, and 180 minutes respectively, bred organisms afterward. The same is true of other vegetable infusions. On the 28th of February last, for example, I boiled six flasks, containing cucumber infusion prepared in an infected atmosphere, for periods of $15,30,45,60,120$, and 180 minutes. Every flask of the group subsequently developed organisms. On the same day, in the case of three flasks, the boiling was prolonged to 240,300 , and 360 minutes; and these three flasks were completely sterilized. Animal infusions, which under ordinary circumstances are rendered infallibly barren by five minutes' boiling, behare like the regetable infusions in an atmosphere infected with hay. germs. On the 30th of March, for example, five flasks were charged with a clear infusion of beef and boiled for (60) minntes, 120 minutes, 180 minutes, 240 minutes, and 300 minutes, respectively. Every one of them became subsequently crowded with organisms, and the sume happened to a perfectly pellucid mutton infusion prepared at the same time. Tlie cases are to be numbered by hundreds in which similar powers of resistance were manifested by infusions of the most diverse kinds.

In the presence of such facts I would ask my colleagne whether it is necessary to dwell for a single instant on the one-sidedness of the evilence which led to the conclusion that all living matter has its life destroyed by "the bricfest exposure to the influence of boiling water." An infusion proved to be barren by six months' exposure to moteless air maintained at a tomperature of 90 degrees Fahr., when inoculated with foll-grown active bacteria, fills itself in two days with organisms so sensitive as to be killeal by a few minutes' exposure to a temperature much below that 
of boiling water. But the extension of this result to the desiccated germinal matter of the air is without warrant or justification. 'This is obvious without going beyond the argument itself. But we have gone far beyond the argument, and proved by multiplied experiment the alleged destruction of all living matter by the briefest exposure to the influence of boiling water to be a delusion. 'The whole logical edifice raised upon this basis falls therefore to the ground; and the argument that bacteria and their germs, being destroyed at 140 degrees, must, if they appear after exposure to 212 degrees, be spontaneously generated, is, I trust, silenced forever.

Through the precautious, variations, and repetitions observed and executed with the view of rendering its results secure, the separate vessels employed in this inquiry have mounted up in two years to nearly ten thousand.

Besides the philosophic interest attaching to the problem of life's origin, which will be always immense, there are the practical interests involved in the application of the doctrines here discussed to surgery and medicine. The antiseptic system, at which I have already glanced, illustrates the manner in which beneficent results of the gravest moment follow in the wake of clear theoretic insight. Surgery was once a noble art; it is now, as well, a noble science. Prior to the introduction of the antiseptic system, the thoughtful surgeon could not have failed to learn empirically that there was something in the air which often defeated the most consummate operative skill. That something the antiseptic treatment destroys or renders innocuous. At King's College Mr. Lister operates and dresses while a fine shower of mixed carbolic acid and water, produced in the simplest manner, falls upon the wound, the lint and gauze employed in the subsequent dressing being duly saturated with the antiseptic. At St. Bartholomew's Mr. Callender employs the dilute carbolic acid without the spray; but, as regards the real point aimed at-the preventing of the wound from becoming a nidus for the propagation of septic bacteria-the practice in both hospitals is the same. Commending itself as it does to the scientifically trained mind, the antiseptic system has struck deep root in Germany.

Had space allowed, it would have given me pleasure to point out the present position of the "germ theory" in 
reference to the phenomena of infectious discase, distinguishing arguments based on analogy-which, however, are terribly strong-from those based on actual observation. I shonld have liked to follow up the account I have already given* of the truly excellent resesurches of a young and an unknown German physician named Koch, on splenic fever, by an account of what Pasteur has recently done with reference to the same subject. Here we have before us a living contagium of the most deally power, which we can follow from the beginning to the end of its life cycle.t We find it in the blood or spleen of a smitten unimal in the state say of short, motionless rods. When these rods are placed in a nutritive liquid on the warm stage of the microscope, we soon see them lengthening into tilaments which lie, in some cases, side by side, forming in others graceful loops, or becoming coiled into knots of a complexity not to be unrareled. We finally see tl:oso filaments resolving themselves into innumerable spores, each with death potentially housed within it, yet not to be distinguished microscopically from the harmless germs of Bacillus subtilis. The bacterium of splenic fever is called Burillus anthracis. This formidable organism was shown to me by M. Pasteur in Paris last July. His recent investigations regarding the part it plays pathologically certainls rank among the most remarkable labors of that remarkable man. Observer after observer had strayed and fallen in this land of pitfalls, a multitude of opposing conclusions and mutually destructive theories being the result. In association with a younger physiological colleague, M. Joubert, Pasteur struck in amid the chaos, and soon reduced it to harmony. They proved, among other things, that in cases where previous observers in France hal supposed themselves to be dealing solely with splenic fever, another equally virulent factor was simultaneously active. Splenie fever was often overmastered by septicamia, and results due solely to the latter had been frequently male the ground of pathologionl inferences regarding the character and canso of the former.

" Fortnightly Review," Novetuber, 1876, see article "Fermenta. tion

† Dallinger and Dryslale had previously shown what skill and parience can accomplish, by their aduirable observations on the life history of the monads. 
Combining duly the two factors, all the previous irregularities disappeared, every result obtained receiving the fullest explanation. On studying the account of this masterly investigation, the words wherewith Pasteur himself feelingly alludes to the difficulties and dangers of the experimenter's art came home to me with especial force: "J'ai tant de fois éprouvé que dans cet art difficile de l'expérimentation les plus habiles bronchent à chaque pas, et que l'interprétation des faits n'est pas moins périlleuse."*

\section{CHAPTER XXXVI.}

\section{SCIENCE AND MAN. $†$}

A MAGNET attracts iron; but when we analyze the effect we learn that the metal is not only attracted but repelled, the final approach to the magnes being due to the difference of two unequal and opposing forces. Social progress is for the most part typified by this duplex or polar action. As a general rule, every advance is balanced by a partial retreat, every amelioration is associated more or less with deterioration. No great mechanical improvement, for example, is introduced for the benefit of society at large that does not bear hardly upon individuals. Science, like other things, is subject to the operation of this polar law, what is good for it under one aspect being baủ for it under another.

Science demands above all things personal concentration. Its home is the study of the mathematician, the quiet laboratory of the experimenter, and the cabinet of the meditative observer of nature. Different atmospheres are required by the man of science, as such, and the man of action. Thus the facilities of social and international intercourse, the railway, the telegraph, and the post office, which are such undoubted boons to the man of action, react to some extent injuriously on the man of science. Their tendency is to break up that concentrativeness which, as I have said, is an absolute necessity to the scientific investigator.

* "Comptes-Rendus," Ixxxiii., p. 177.

+ Presidential Address, delivered before the Birmingham and Mid. land Institute, October 1, 1877; with additions. 
The men who have uost profoundly influenced the world from the scientific side have habitually sought isolation. Faraday, at a certain period of his eareer, formally renounced dining out. Darwin lives apart from the bustle of the world in his quiet home in Kent. Mayer and Joule dealt in unobtrusive retirement with the weightiest scientific questions. 'There is, however, one motive power in the world which no man, be he a scientific etudent or otherwise, can afford to treat with indifference; and that is, the cultivation of right relations with his follow-men-the performance of his duty, not as an isolated individual, but as a member of society. It is duty in this aspect, overcoming alike the sense of possible danger and the desire for repose, that has placed ine in your presence here to-night.

To look at his picture as a whole, a painter requires distance; and to judge of the total scientific achievement of any age, the standjoint of a succeeding age is desirable. We may, however, transport ourselves in idea into the future, and thus survey with more or less completeness the science of our time. We sometimes hear it decried, and contrasted to its disadvantage with the science of other times. I do not think that this will be the verdict of posterity. I think, on the contrary, that posterity will acknowlerge that in the history of science no higher amples of intellectual conquest are recorded than those which this age has made its own. One of the most salient of these I propose, with your permission, to make the subject of our consideration during the coming hour.

It is now generally admitted that the man of to-day is the child and product of incalculable antecedent time. His physical anil intellectual textures have been woven for him during his pasange through phases of history and forms of existence which lead the mund back to an abysmal past. One of the qualities which he has derired from that past is the yearning to let in the light of prineiples on the otherwise bewildering flux of phenomena. He has been described by the German Lichtenberg as "das rastlose Ursachenthier"-the restless cause seeking animal-in whom facts excito a kind of hunger to know the sources from which they spring. Never, I venture to say, in the listury of the world has this longing been more liberally risponiled to, both among men of science and the general 
public, than during the last thirty or forty year's. I say "the general public," because it is a feature of our time that the man of science no longer limits his labors to the society of his colleagues and his peers, but shares, as far as it is possible to share, with the world at large the fruits of inquiry.

The celebrated Robert Boyle regarded the universe as a machine; Mr. Carlyle prefers regarding it as a tree. He loves the image of the umbrageous Igdrasil better than that of the Strasburg clock. A machine may be defined as an organism with life and direction outside; a tree may be defined as an organism with life and direction within. In the light of these definitions, I close with the conception of Carlyle. The order and energy of the universe I hold to be inherent, and not imposed from without, the expression of fixed law and not of arbitrary will, exercised by what Carlyle would call an Almighty Clockmaker. But the two conceptions are not so much opposed to each other after all. In one fundamental particular they at all events agree. They equally imply the interdependence and harmonious interaction of parts, and the subordination of the individual powers of the universal organism to the working of the whole.

Never were the harmony and interdependence just referred to so clearly recognized as now. O॥r iusight regarding them is not that vague and general insight to which our fathers had attained, and which, in early times, was more frequently affirmed by the synthetic poet than by the scientific man. The interdependence of our day has become quantitative-expressible by numbers-leading, it must be added, directly into that inexorable reigu of law which so many gentle people regard with dread. In the domain now under review men of science had first to work their way from darkness into twilight, and from twilight into day. There is no solution of continuity in science. It is not given to any man, however endowed, to rise spontaneously into intellectual splendor without the parentage of antecedent thought. Great discoveries grow. Here, as in other cases, we have first the seed, then the ear, then the full corn in the ear, the last member of the series implying the first. 'Thus, as regards the discovery of gravitation with which the name of Newton is identified, notions more or less clear concerning it had entered many minds before 
Newton's transcendent mathematieal genius raised it to the level of a demonstration. The whole of his deductions, moreover, rested upon the inductions of Kepler. Newton shot begond his predecessors; but his thoughts were rooted in their thoughts, and a just distribution of merit would assign to them a fair portion of the honor of discovery.

Scientific theories sometimes float like rumors in the air before they recoive complete expression. The doom of a doctrine is often practically sealed, and the truth of one is often practically accepted, long prior to the ciemonstration of either the error or the truth. Perpetual motion was discarted before it was proved to be opposed to natural law; and, as regards the connection and interaction of natural forces, intimations of modern discoveries are strewn through the writings of Leibnitz, Boyle, Hooke, Locke and others.

Confining ourselves to recent times, Dr. Ingleby has pointed ont to me some singularly sagacious remarks bearing upon this question, which were published by an anonymous writer in 1820. Roget's penetration was conspicuous in 1529. Mohr had grasped in 1837 some decplying truth. The writings of Faaday furnish frequent illustrations of his profound belief in the unity of nature. "I have long," he writes in 1845, "held an opinion almost amounting to conviction, in common, I believe, with other lovers of natural knowledge, that the various forms under which the forces of matter are made manifest have one common origin, or, in other words, are so directly related and mutually dependent, that they are couvertible, as it were, one inio another, and possess equivalence of power in their action." His own researches on magneto-electricity, on electro-chemistry, and on the "magnetization of light," led him directly to this belief. At an early date Mr. Justice Grove made his mark upon this question. Colding, thongh starting from a metaphysical basis, grasped eventnally the relation between heat and mechaniend work, and songht to determine it experimentally. And here let me say, that to him who has only the truth at heart, and who in his dealings with scientific history keops his soul unwarped by envy, hatred, or malice, personal or uational, every fresh accession to historio knowlenge mast be welcome. For esery newcomer of proved meril, more especially if that merit should have been previonaly over- 
looked, he makes ready room in his recognition or his reverence. But no retrospect of scientific literature has as yet brought to light a claim which can sensibly affect the position:s accorded to two great Path-hewers, as the Germans call them, whose names in relation to this subject are linked in indissoluble association. 'These names are Julius Robert Mayer and James Prescott Joule.

In his essay on "Circles" Mr. Emerson, if I remember rightly, pictured intellectual progress as rhythmic. At a given moment knowledge is surrounded by a barrier which marks its limit. It gradually gathers clearness and strength until by and by some thinker of exceptional power bursts the barrier and wins a wider circle, within which thought once more entrenches itself. But the internal force again accumulates, the new barrier is in its turn broken, and the mind finds itself surrounded by a still wider horizon. Thus, according to Emerson, knowledge spreads by intermittent victories instead of progressing at a uniform rate.

When Dr. Joule first proved that a weight of one pound, falling through a height of seven hundred and seventy-two feet, generated an amount of heat competent to warm a pound of water one degree Fahrenheit, and that in lifting the weight so much heat exactly disappeared, he broke an Emersonian "circle," releasing by the act an amount of scientific energy which rapidly overran a vast domain, and embodied itself in the great doctrine known as the "Conservation of Energy." This doctrine recognizes in the material universe a constant sum of power made up of items among which the most Protean fluctuations are incessantly going on. It is as if the body of Nature were alive, the thrill and interchange of its energies resembling those of an organism. The parts of the "stupendous whole" shift and change, augment and diminish, appear and disappear, while the total of which they are the parts remains quantitatively immutable. Immutable, because when change occurs it is always polar-plus accompanies minus, gain accompanies loss, no item varying in the slightest degree without an absolutely equal change of some other item in the opposite direction.

The sun warms the tropical ocean, converting a portion of its liquid into vapo!, which rises in the air and is recondensed on mountain heights, returning in rivers to 
the ocean from which it came. Up to the point where condensation begins, an amount of heat exactly equiralent to the molecular work of vaporization and the mechanical work of lifting the vapor wo the mountain-tojs has disappeared from the universe. W bat is the gain corresponding to this loss? It will seem when mentioneal to be expressed in a foreign currency. The loss is a loss of heat; the gain is a gain of distance, both as regarls masses and unolecules Water which was formerly at the sea-lesel has beeu lifted to a position from which it ean fall; molecnles which have been locked together as a liquid are now separate as rapor which can recondense. After condensation gravity comes into effectual play, pulling the showers down upon the hills, and the rivers thus created through their gorges to the sea. Every raindrop which smites the mountain produces its definite amount of heat; every river in its course develops heat by the clash of its cataracts and the friction of its bed. In the act of condensation, moreover, the molecular work of raporization is accurately reversed. Compare, then, the primitive loss of solar warmth with the heat generated by the condensation of the vapor, and by the subsequent fall of the water from cloud to sea. They are mathematically equal to each other. No particle of vapor was formed and lifted withont being paid for in the currency of solar heat; no particle retnrus as water to the sea without the exact quantitatire restitution of that heat. There is nothing gratuitous in physical uature, no expenditure withoụt equivalent gain, no gain without equivalent expenditure. With inexorate constancy the one accompanies the other, leaving no nook or crevice between them for spontaneity to mingle with the pure and necessary play of natural force. If the this uniformity of nature ever been broken? The reply is: "Not to the knowledge of science."

What has boen here stated regarding heat and gravity applies to the whole of inorganie nature. Let us take an illustration from ohemistry. The metal zinc may be burned in oxygen, a perfectly definite amount of heat being prodneed by the combustion of a given weight of the metal. But zine may also be burneal in a liquid which contains at supply of oxygen-in water, for example. It does not in this case proilnee flame or fire, but it does produce heat which is capable of aconrate mensurement. But the hesat 
of zinc burned in water falls shori of that produced in pure oxygen, the reason being that to obtain its oxygen from the water the zinc must first dislodge the hydrogen. It is in the performance of this molecular work that the missing heat is absorbed. Mix the liberated hydrogen with oxygen and cause them to recombine; the heat developed is mathematically equal to the missing heat. 'Thus in pulling the oxygen and hydrogen asunder an amount of heat is consumed which is accurately restored by their reunion.

This leads up to a few remarks upon the voltaic battery. It is not my design to dwell upon the technical features of this wonderful instrument, but simply, by means of it, to show what varying shapes a given amount of energy can assume while maintaining unvarying quantitative stability. When that form of power which we call an electric current passes through Grove's battery, zinc is consumed in acidulated water; and in the battery we are able so to arrange matters that when no current passes no zinc shall be consumed. Now the current, whatever it may be, possesses the power of generating heat outside the battery. We can fuse with it iridium, the most refractory of metals, or we can produce with it the dazzling electric light, and that at any terrestrial distance from the battery itself.

IVe will now, however, content ourselves with causing the current to raise a given length of platinum wire, first to a blood-heat, then to redness, and finally to a white heat. The heat under these circumstances generated in the battery by the combustion of a fixed quantity of zinc is no longer constant, but it varies inversely as the heat generated outside. If the outside heat be nil, the inside heat is a maximum; if the external wire be raised to a blood-heat, the internal heat falls slightly short of the maximum. If the wire be rendered red-hot, the quantity of missing heat within the battery is greater, and if the external wire be rendered white-hot, the defect is greater still. Add together the interual and external heat jroduced by the combustion of a given weight of zinc, and you have an absolutely constant total. The heat generated without is so much lost within, the heat generated within is so much lost without, the polar changes already adverted to :oming here conspicuously into play. Thus in a variety 
of ways we can distribute the items of a never-varying sum, but even the subtle agency of the electric current places no ereative power in our hands.

Insteal of generating external heat, we may cause the current to effect chemical decomposition at a distance from the battery. Let it, for example, decompose water into oxygen and hydrogen. The heat generated in the battery under these circunstances by the combustion of a given weight of rine falls short of what is prodnced when there is no decomposition. How far short? The question admits of a perfectly exact answer. When the oxygen and hydrogen recombine, the heat absorbed in the decomposition is aecurately restored, and it is exactlyequal in amount to that missing in the battery. We may, if we like, bottle up the gases, carry in this form the heat of the battery to the polar regions, and liberate it there. The battery, in fact, is a hearth on which fuel is consumed; but the beat of the combustion, insteal of being confined in the usual manner to the hearth itself, may be first liberated at the other side of the world.

And here we are able to solve an enigma which long perplexed scientific men, and which conld not be solved until the bearing of the mechanical theory of heat upon the phenomena of the voltaic battery was understoot. The puzzle was, that a single cell could not decompose water. The reason is now plain enough. The solution of an equiralent of zinc in a single cell develops not much more than half the amount of heat required to decompose an equivalent of water, and the single cell cannot cede an amount of force which it does not possess. But by forming a battery of two cells instead of one, we develop an amount of heat slightly in excess of that nemled for the decompinsition of the water. The twocelled battery is therefore rich enough to pay for that decompositon, and to maintain the excess referred to within its own cells.

Similar refleotions apply to the thermo-olectric pile, an instrument usually composed of small bars of bismuth and antimony solilered alternately together. The electrio current is here esoked by warming the soldered junctions of one face of the pile. Like the voltaie current, the thermoelectric current can heat wires, produce decomposition, magnetize iron, and deflect a magnelic needle at any dis- 
tance from its origin. You will be disposed, and rightly disposed, to refer those distant manifestations of power to the heat communicated to the face of the pile, but the case is worthy of closer examination. In 1826 'Thomas Seebeck discovered thermo-electricity, and six years subsequently Peltier made an observation which comes with singular felicity to our aid in determining the material used up in the formation of the thermo-electric current. He found that when a weak extraneous current was sent from antimony to bismuth the junction of the two metals was always heated, but that when the direction was from bismuth to antimony the junction was chilled. Now the current in the thermo-pile itself is always from bismuth to antimony, across the heated junction-a direction in which it cannot possibly establish itself without consuming the heat impartel to the junction. This heat is the nutriment of the current. 'Thus the heat generated by the thermocurrent in a distant wire is simply that originally imparted to the pile, which has been first transmuted in to electricity, and then retransmuted into its first form at a distance from its origin. As water in a state of vapor passes from a boiler to a distant condenser, and there assumes its primitive form without gain or loss, so the heat communicated to the thermo-pile distills into the subtler electric current, which is, as it were, recondensed into heat in the distant platinum wire.

In my youth I thought an electro-magnetic engine which was shown to me a veritable perpetual motion-a machine, that is to say, which performed work without the expenditure of power. Let us consider the action of such a machine. Suppose it to be employed to punp water from a lower to a higher level. On examining the battery which works the engine we find that the zinc consumed does not yield its full amount of heat. The quantity of heat thus missing within is the exact thermal equivalent of the mechanical work performed without. Let the water fall again to the lower level; it is warmed by the fall. Add the heat thus produced to that generated by the friction, mechanical and magnetical, of the engine; we thus obtain the precise amount of heat missing in the battery. All the effects obtained from the machine are thus strictly paid for; this " payment for results" being, I would repeat, the inexorable method of nature. 
No engine, however subtly devised, can evade this law of equiralence, or perform on its own account the smallest morlicum of work. The machine distributes, but it eannot create. Is the animal body, then, to be classien among machines? When I lift a weight, or throw a stone, or climb a mountain, or wrestle with $m y$ comrale, am I not conscious of actually creating and expending force? Let us look at the sutecedents of this force. We derive the muscle and fat of our bodies from what we eat. Animal heat you know to be due to the slow combustion of this fuel. My arm is now inactive, and the ordinary slow combustion of $\mathrm{my}$ blood and tissue is going on. For every grain of fuel thus burned a perfectly definite amount of heat has been produced. I now contract my biceps musele withont causing it to perform external work. The combustion is quickened, and the heat is increased; this alditional heat being liberated in the muscle itself. I lay hold of a 56-1b. weight, and by the contraction of my biceps lift it throngh the rertical space of a foot. The blood and tissue consumed during this contraction have not developed in the muscle their due amount of heat. A quantity of heat is at this moment missing in my muscle which wonld raise the temperature of an ounce of water somewhat more than one degroe Fahrenheit. I liberate the weight: it falls to the earth, and by its collision generates the precise amount of beat missing in the muscle. My muscular heat is thus transferred from its local hearth to external space. The fuel is consumed in my body, but the heat of combustion is produced outside my body. The case is substantially the same as that of the voltaic battery when it performs external work, or profucess external heat. All this points to the conclusion that the force we employ in muscular exertion is the force of burning fuel and not of creative will. In the light of these facts the body is seen to be as incapable of generating energy without expentiture, as the solids and liquids of the voltaic battery. The body, in other words, falls into the cutegury of machines.

We can do with the body all that we have alrendy done with the battery - heat platinum wires, deoompose water, magnetize iron, and deflect a magnetic neodle. The combustion of muscle may be made to probluce all there efferts. as the combuation of zinc may be caused to produco them. 
By turning the handle of a magneto-electric machine a coil of wire may be caused to rotate between the poles of a magnet. As long as the two ends of the coil are unconnected we have simply to overcome the ordinary inertia and friction of the machine in turning the handle. But the moment the two ends of the coil are united by a thin platinum wire a sudden addition of labor is thrown upon the turning arm. When the necessary labor is expended, its equivalent immediately appear's. The platinum wire glows. You can readily maintain it at a white heat, or even fuse it. This is a very remarkable result. From the muscles of the arm, with a temperature of 100 degrees, we extract the temperature of molten platinum, which is nearly four thousand degrees. The miracle here is the reverse of that of the burning bush mentioned in Exodus. There the bush burned, but was not consumed: here the body is consumed, but does not burn. The similarity of the action with that of the voltaic battery when it heats iu external wire is too obvious to need pointing out. When the machine is used to decompose water, the heat of the muscle, like that of the battery, is consumed in molecular work, being fully restored when the gases recombine. As before, also, the transmuted heat of the muscles may be bottled up, carried to the polar regions, and there restored to its pristine form.

The matter of the human body is the same as that of the world around us; and here we find the forces of the human body identical with those of inorganic nature. Just as little as the voltaic battery is the animal body a creator of force. It is an apparatus exquisite and effectual beyond all others in transforming and distributing the energy with which it is supplied, but it possesses no creative power. Compared with the notions previously entertained regarding the play of " vital force" this is a great result. The problem of vital dynamics has been described by a competent authority as "the grandest of all." I subscribe to this opinion, and honor correspondingly the man who first successfully grappled with the problem. He was no pope, in the sense of being infallible, but he was a man of genius whose work will be held in honor as long as science endures. I have already named him in connection with our illustrious countryman Dr. Joule. Other eminent 
men took up this subject subsequently and independently. but all that has been done hitherto enhances instead of diminishing the merits of Dr. Mayer.

Consiler the rigor of his reasoning. "Beyond the power of generating internal heat, the animal organism can generate heat external to itself. A blacksmith by hammering can warm a nail, and a savage by friction can lieat wood to its point of ignition. Uniess, then, we abanion the physiological axiom that the animal body cannot create heat out of nothing, we are driven to the conclusion that it is the tolal heal, within and urithont. that ought to be regurdoul as the real anlirifie effect of the oridation within the body." Majer, however, not only states the principle, but illustrates inmerically the transfer of muscular heat to external space. A bowler who imparts a relocity of 30 feet to an 8.16). ball consumes in the act one-tenth of a grain of carbon. The heat of the muscle is here distributed over the track of the ball, being develojed there by mechanical friction. A man weighing 150 the. consumes in lifting his own body to a hoight of 8 feet the heat of a grain of earbon. Jumping from this height the heat is restored. The consumption of $202.4 \mathrm{drs}$. 20 gre. of carbon wonld place the same man on the summit of a mountain 10,000 feet high. In descending the mountain an amount of heat equal to that produced by the combustion of the foregoing amount of carbon is restored. The muscles of a laborer whose weight is $150 \mathrm{lbs}$. weigh 64 Ibs. When dried they are reduced to $15 \mathrm{lbs}$. Were the oxidation corresponding to a day-laborer's ordinary work exorted on the muscles alone, they would be wholly nonsumed in 80 days. Were the oxidation necessary to sustain the heart's action concentrated on the heart itsolf, it would be consumed in 8 dass. And if we confine our attention to the two rentricles, their action would consume the nsoociated muscular tissue in 3 d days. With a fullness and precision of which this is but a sample did Mayer, between 1842 and 1545, deal with the great question of rital dynamics.

In direot opposition, moreover, to the foremost scientifie anthorities of that day, with liebig at their heah, this solitary Heilbronn worker was led by his coloulations to maintain that the muscles, in the main, played the part of machinery, converting the fat, which had been previously 
considered a mere heat-producer, into the motive power of the organism. Mayer's prevision has been justified by events, for the scientific world is now upon his side.

We place, then, food in our stomachs as so much combustible matter. It is first dissolved by purely chemical processes, and the nutritive fluid is poured into the blood. Here it comes into contact with atmospheric oxygen admitted by the lungs. It unites with the oxygen as wood or coal might unite with it in a furnace. The matter-products of the union, if I may use the term, are the same in both cases, viz., carbonic acid and water. The force-products are also the same-heat within the body, or heat and work outside the body. Thus far every action of the organism belongs to the domain either of physics or of chemistry. But you saw me contract the muscle of my arm. What enabled me to do so? Was it or was it not the direct action of $\mathrm{my}$ will? The answer is, the action of the will is mediate, not direct. Over and above the muscles the human organism is provided with long whitish filaments of medullary matter, which issue from the spinal column, being connected by it on the one side with the brain, and on the other side losing themselves in the muscles. Those filaments or cords are the nerves, which you know are divided into two kinds, sensor and motor, or, if you like the terms better, afferent and efferent nerves. The former carry impressions from the external world to the brain; the latter convey the behests of the brain to the muscles. Here, as elsewhere, we find ourselves aided by the sagacity of Mayer, who was the first clearly to formulate the part played by the nerves in the organism. Mayer saw that neither nerves nor brain, nor both together, possessed the energy necessary to animal motion; but he also saw that the nerve could lift a latch and open a door, by which floods of energy are let, loose. "As an engineer," he says with admirable lucidity, "by the motion of his finger in opening a valre or loosening a detent can liberate an amount of mechanical energy almost infinite compared with its exciting cause; so the nerves, acting on the muscles, can unlock an amount of power out of all proportion to the work done by the nerves them. selves." The nerves, according to Mayer, pull the trigger, but the gunpowder which they ignite is stored in the muscles. This is the view now universally entertained. 
The quickness of thought has passed into a proverb, and the notion that any measurable time elapred between the infliction of a wound and the feeling of the injury wonld have been rejected as prefosterous thirty years ago. Nervous impressions, notwithstanding the results of Haller, were thought to be transmitted, if not instantanoonsly, at all events with the rapidity of clectricity. Hence, when Helmholtz, in 1851, athirmod, as the result of experiment, nervous transmission to be a comparatively sluggish process, very few believed him. Ilis experiments may now be mate in the lecture-room. Sotitud in air moves at the rate of 1,100 feet a second; sound in water moves at the rate of 5.000 feet a second; light in ether moves at the rate of 186.000 miles a second, and electricity in freo wires moves probably at the same rate. But the nerves transmit cheir messiges at che rate of only 70 feet a seoumd, a progress which in these quick times might woll be regarded as inordinately slow.

Your townsman, Mr. Gore, has produced by electrolysis a kind of antimony which exhibits an action strikingly analogous to that of nervons propagation. A rod of this antimony is in such a molecular condition that when you serateh or heat one end of the rod, the disturbance propagates itself before your eyes to the other end, the onward march of tho disturbance being announcerl.by the development of heat and fumes along the line of propagation. In some such way the molecules of the nerres are successively overthrown; and if Mr. Gore could only devise some means of winding up his exhatusted antimony, as the nutritive blool winds up exhansted nerves, the comparison would be complete. The subject may be summed up, as Du BoisReymond has summed it up, by reference to the case of a whale struck by a harpoon in the tail. If the animal were 70 feet long, a second would clapse bofore the disturbance could reach the brain. But the impression after its arrival has to diffuse itself and throw the brain into the molecular condition necensary to consciousness. Then, and not till then, the command to the tail to defend itself is shot through the motor norves. Another secumb must elapse before the command can reach the tail, so that moro than two seconds transpire botween the inflivtion of the wound and the muscnlar response of the part wounded. The interval required for the kindling of conscionsuess 
would probably more than suffice for the destruction of the brain by lightning, or even by a rifle-bullet. Before the organ can arrange itself it may, therefore, be destroyed, and in such a case we may safely conclude that death is painless.

The experiences of common life supply us with copious instances of the liberation of vast stores of muscular power by an infinitesimal "priming" of the muscles by the nerves. We all know the effect produced on a "nervous" organization by a slight sound which causes affright. An aërial wave, the energy of which would not reach a minute fraction of that necessary to raise the thousandth of a grain through the thousandth of an inch, can throw the whole human frame into a powerful mechanical spasm, followed by violent respiration and palpitation. The eye, of course, may be appealed to as well as the ear. Of this the lamented Lange gives the following vivid illustration:

A merchant sits complacently in his easy-chair, not knowing whether smoking, sleeping, newspaper reading, or the digestion of food occupies the largest portion of his personality. A servant enter's the room with the telegram bearing the words, "Antwerp, etc. . . Jonas and Co. have failed." "Tell James to harness the horses!" The servant flies. Up starts the merchant, wide awake; makes a dozen paces through the room, descends to the counting-house, dictates letters, and forwards despatches. $\mathrm{He}$ jumps into his carriage, the horses snort, and their driver is immediately at the bank, on the Bourse, and among his commercial friends. Before an hour has elapsed he is again at home, where he throws himself once more into his easy-chair with a deep-drawn sigh, "Thank God I am protected against the worst, and now for further reflection."

This complex mass of action, emotional, intellectual, and mechanical, is evoked by the impart upon the retiuin of the infinitesimal wares of light coming from a few pencil marks on a bit of paper. We have, as Lange says, terror, hope, sensation, calculation, possible ruin, and victory compressed into a moment. What caused the merchant to spring out of his chair? The contrac. tion of his muscles. What made his muscles contract? An impulse of the nerves, which lifted the proper latch, 
and liberated the muscular power. Whence this impulse? From the center of the nervous system. But how did it originate there? This is the critical question, to which some will reply that it had its origin in the human soul.

The aim and effort of seience is to explain the unk nown in terms of the known. Fxplanation, therefore, is conditioned by knowledge. You lave probably heard the story of the German peasant, who, in early railway days, was taken to see tho performanee of a locomotive. He had never known carriages to be moved except by animal jower. Every explanation outside of this conception lay begond his experience, and could not be invoked. After long reflection therefore, and seeing no possible cscape from the conclusion, he exclaimed confidently to his companion, "Fs mulssen doch Pferde darin sein"-There must be horses inside. Amusing as this locomotive theory may seem, it illustrates a deop-lying truth.

With reference to our present question, some may be disposed to press upon me such considerations as these: Your motor nerves are so many speaking-tubes, tlwough which messages are sent from the man to the world; and your sensor nerves are so many conduits through which the whispers of the world are sent back to the man. But you have not told us where is the man. Who or what is it that sends and receives those messiges throngh the bodily organism? Do not the phenomena point to the existence of a self within the self, which aots through the bouly as through a skillfully constructed instrument? You picture the muscles as hearkening to the commands sent throngh the motor nerres, and you pieture the sensor nerves as the vehicles of incoming intelligence; are you not bound to supplement this mechanism by the assumption of an entity which uses it? In other worils, are you not foreenl by your ow a exposition into the hypothesis of a free human soul?

This is fair reasoning now, and at a certain stage of the world's knowledge it might well have been deomed conclusire. Adequate reflection, however, shows that inatead of introducing light into our minds, this hypothesis considered scientifically increases our darkness. You do not in this easo explain the muknewn in terms of the known, which, as stated abose, is the method of science, but gou 
explain the unknown in terms of the more unknown. Try to mentally visualize this soul as an entity distinct from the body, and the difficulty immediately appears. From the side of science all that we are warranted in stating is that the terror, hope, sensation, and calculation of Lange's merchant, are psychical phenomena produced by, or associated with, the molecular processes set up by waves of light in a previousiy prepared brain.

When facts present themselves let us dare to face them, but let the man of science equally dare to confess ignorance where it prevails. What then is the causal connection, if any, between the objective and subjective-between molecular motions and states of consciousness? My answer is: I do not see the connection, nor have I as yet met anybody who does. It is no explanation to say that the objective and subjective effects are two sides of one and the same phenomenon. Why should the phenomenon have two sides? This is the very core of the difficulty. There are plenty of molecular motions which do not exhibit this two-sidedness. Does water think or feel when it runs into frosîferns upon a window-pane? If not, why should the molecular motion of the brain be yoked to this mysterious companion-consciousness? We can form a coherent picture of the physical processes-the stirring of the brain, the thrilling of the nerves, the discharging of the muscles, and all the subsequent mechanical motions of the organism. But we can present to our minds no picture of the process whereby consciousness emerges, either as a necessary link or as an accidental by-product of this series of actions. Yet it certainly does emerge-the prick of a pin suffices to prove that molecular motion can produce consciousness. The reverse process of the production of motion by consciousness is equally unpresentable to the mind. We are here, in fact, upon the boundary line of the intellect, where the ordinary canons of science fail to extricate us from our difficulties. If we are true to these canons, we must deny to subjective phenomena all influence on physical processes. Observation proves that they interact, but in passing from one to the uther we meet a blank which mechanical deduction is unable to fill. Frankly stated, we have here to deal with facts almost as difficult to seize mentally as the idea of a soul. And if you are content to make your "soul " a peetic render- 
ing of a phenomenon which refuses the yoke of ordinary physical laws, I, for one, would not object to this exercise of ideality. Amid all our speculative nncertainty, however, there is one practical point as clear as the day; namely, that the brightriess and the usefulness of life, as well as its darknews and disaster, depend to a great extent upon our own use or abuse of this miraculous organ.

Accustomed as I am to harsh language, I am quite prepared to hear my "poetic rendering" branded as a "falsehood" and a "fib." The vituperation is unmerited, for poetry, or ideality, and untruth are assuredly very different things. The one may vivify, while the other kills. When St. John extends the notion of a soul to "souls washed in the blood of Christ" does he "fib?" Indeed, if the appeal to ideality is censurable, Christ himself ought not to have escaped censure. Nor did he escape it. "How can this man gire us his flesh to eat?" expressed the skeptical flouting of mmpoetic natures. Such are still among us. Cardinal Manning would doubtless tell any Protestant who rejects the doctrine of transubstantiation that he "fibs" away the plain words of his Saviour when he reduces "the Body of the Lord" in the sacrament to a mere figure of speech.

Though misuse may render it grotesque or insincere, the idealization of ancient conceptions, when done consciously and above board, has, in my opinion, an important future. We are not ralically different from our historic ancestors, and any feeling which affected them profoundly requires only appropriate clothing to affect 18 . 'The world will not lightly relinquish its heritage of poetio freling, and motaphysic will be welcomed when it abandons its pretensions to scientific discovery and consents to be ranked as a kind of poetry. "A good symbol," savs Emerson, "is a missionary to persuade thonsands. The Vedas, the Edda, the Koran, are each remembered by its happiest figure. There is no more welcome gift to men than a new symbol. They assimilate themselves to it, deal with it in all ways, and it will last a hnndren years. Then comesa new genius and brings another." Our ideas of God and the soul are obviously subject to this symbolio mntation. They are not now what they were a cenilury ago. They will not be a century hence what they are now. Such ideas constitute a 
kind of central energy in the human mind, capable, like the energy of the physical universe, of assuming various shapes and undergoing various transformations. They baffle and elude the theological mechanic who would carve them to dogmatic forms. They offer themselves freely to the poet who understands his vocation, and whose function is, or ought to be, to find "local habitation" for thoughts woven into our subjective life, but which refuse to be mechanically defined.

We now stand face to face with the final problem. It is this: Are the brain, and the moral and intellectual processes known to be associated with the brain-and, as far as our experience goes, indissolubly associated-subject to the laws which we find paramount in physical nature? Is the will of man, in others words, free, or are it and nature equally "bound fast in fate?" From this latter conclusion, after he had established it to the entire satisfaction of his understanding, the great German thinker Fichte recoiled. You will find the record of this struggle between head and heart in his book, entitled "Die Bestimmung des Menschen"-The Vocation of Man.* Fichte was determined at all hazards to maintain his freedom, but the price he paid for it indicates the difficulty of the task. To escape from the iron necessity seen everywhere reigning in physical nature, he turned defiantly round upon nature and law, and affirmed both of them to be the products of his own mind. He was not going to be the slave of a thing which he had himself created. There is a good deal to be said in favor of this view, but few of us probably would be able to bring. into play the solvent transcendentalism whereby Fichte melted his chains.

Why do some regard this notion of necessity with terror, while others do not fear it at all? Has not Carlyle somewhere said that a belief in destiny is the bias of all earnest minds? "It is not Nature," says Fichte, "it is Freedom itself, by which the greatest and most terrible disorders incident to our race are produced. Man is the cruelest enemy of man." But the question of moral responsibility here emerges, and it is the possible loosening of this responsibility that so many of us dread. The notion of

* Trauslated by Dr. William Smitl of Edinburgh; Trübner, 18 \%3. 
necessity certainly failed to frighten Bishop Butler. He thought it untrue - even absurd-bat he did not fear its practical consequences. He showed, on the contrary, in the "Analogy," that as far as human conduct is concerned, the two theories of free-will and necessity would come to the same in the end.

What is meant by free-will? Does it imply the power of probucing events withont anterentents-of starting, as it were, upon a creative tour of occurrences withont any impulse from within or from without? Let us consider the point. If there be absolutely or relatively no reason why a tree should fall, it will not fall; and if there be absolutely or relatively no reason why a man should act, he will not act. It is true that the united roice of this assembly could not persuale me that I have not, at this moment, the power to lift my arm if I wished to do 80 . Within this range the conscions freedom of my will cannot be questioned. But what about the origin of the "wish?" Are we, or are we not, complete masters of the circumstances which create our wishes, motives and tendencies to action? Adequate reflection will, I think, prove that we are not. What, for example, hare I had to do with the generation and development of that which some will consider my total being, and others a most potent factor of my totat being-the living, speaking organism which now addresser you? As stated at the beginning of this discourse, my phissical and intellectual textures were woren for me, not by me. Processes in the conduct or regulation of which I had no share have made me what I am. Here, surely, if anywhere, we are as clay in the hands of the pother. It is the greatest of delusions to suppose that we come into this world as sheets of white paper on which the age can write anything it likes, making us good or bad, noble or mean, ws the age plenses. The age can stunt, promote, or pervert pre-existent capacities, but it cannot ereate them. The worthy Robert Owen, who saw in external circumstances the great molders of human ehar. acter, was obliged to supplement his duetrine by making the man himself one of the circumstances. It is as fatial as it is cowardly to blink facls because they are not to our taste. How many disorders, ghosily and bodily, are transmitted to us by inheritance? In vir courts of law, when. ever it is a question whether a crime has been committed 
under the influence of insanity, the best guidance the judge and jury can have is derived from the parental antecedents of the accused. If among these insanity be exhibited in any marked degree, the presumption in the prisoner's favor is enormously enhanced, because the experience of life has taught both judge and jury that insanity is frequently transmitted from parent to child.

I met, some years ago, in a railway carriage the governor of one of our largest prisons. He was evidently an observant and reflective man, possessed of wide experience gathered in various parts of the world, and a thorough student of the duties of his vocation. He told me that the prisoners in his charge might be divided into three distinct classes. 'The first class consisted of persons who ought never to have been in prison. Exterual accident, and not internal taint, had brought them within the grasp of the law, aid what had happened to them might happen to most of us. They were essentially men of sound moral stamina, though wearing the prison garb. Then came the largest class, formed of individuals possessing no strong bias, moral or immoral, plastic to the touch of circumstances, which could mold them into either good or evil members of society. Tliirdly came a class-happily not a large one-whom no kindness could conciliate and no discipline tame. They were sent into this world labeled "incorrigible," wickedness being stamped, as it were, upon their organizations. It was an unpleasant truth, but as a truth it ought to be faced. For such criminals the prison over which he ruled was certainly not the proper place. If confined at all, their prison should be on a desert island where the deadly contagium of their example could not taint the moral air. But the sea itself he was disposed to regard as a cheap and appropriate substitute for the island. It seemed to him evident that the state would benefit if prisoners of the first class were liberated; prisoners of the second class educated; and prisoners of the third class put compendiously under water.

It is not, however, from the observation of individuals that the argument against "free-will," as commonly understood, derives its principal force. It is, as already hinted, indefinitely strengthened when extended to the race. Most of you have been forced to listen to the out- 
cries and denunciations which rang discordant through the land for some years after the publication of Mr. Darwin's "Origin of Species." Well, the world-even the clerical world - has for the most part settled down in the belief that MIr. Darwin's book simply reflects the truth of nature: that we who are now "foremost in the files of time" have come to the front through almost endless stages of promotion from lower to higher forms of life.

If to any one of us were given the privilege of looking back through the aons acruss which life has crept toward its present outcome, his vision, according to Darwin, would ultimately reach a point when the progenitors of this assembly could not be called human. From that humble society, through the interaction of its members and the storing up of their best qualities, a bettor one emerged; from this again a better still; until at length, by the integration of infinitesimals through ages of amelioration, we came to be what we are to-day. We of this generation had no conscious share in the production of this grand and beneficent result. Any and every generation which preceled 18 had just as little share. The farored organisms whose gamered excellence constitutes our present store owed their advantages, first, to what we in our ignorance are obliged to call "accidental variation;" and, secondly, to a law of heredity in the passing of which our suffrages were not collected. With characteristic felicity and precision Mr. Matthew Arnold lifts this question into the free air of poetry, but not out of the atmos. phere of truth, when he ascribes the process of amelioration to "a power not ourselves which makes for righteonsness." If, then, our organisms, with all their tendencies and capacities, are given to 18 without our being consulted; and if, while capable of acting within certain limits in accordance with our wishes, we are not imasters of the circumstances in which motives and wishes originate; if, finally our motives and wishes determine our aotions-in what sense can these actions be said to be the result of free-will?

Here, again, we are confronted with the question of moral responsibility, which, at it has been mueh talked of lately, it is desirable to meet. IVith the siew of remoring the fear of our falling back into the condition of " the ape and tiger," so sedulously excited by certain writers, I 
propose to grapple with this question in its rudest form, and in the most uncompromising way. "If," says the robber, the ravisher, or the murderer, "I act because I must act, what right have you to hold me responsible for my deeds?" The reply is, "S'The right of society to protect itself against aggressive and injurious forces, whether they be bound or free, forces of nature or forces of man." " 'Then," retorts the criminal, "you punish me for what I camnot help." "Let it be granted," says society, "but had you known that the treadmill or the gallows was certainly in store for you, you might have 'helped.' Let us reason the matter fully and frankly out. We may entertain no malice or hatred against you; it is enough that with a view to our own safety and purification we are determined that you and such as you shall not enjoy liberty of evil action in our midst. You, who have behaved as a wild beast, we claim the right to cage or kill as we should a wild beast. 'The public safety is a matter of more importance than the very limited chance of your moral renovation, while the knowledge that you have been hanged by the neck may furnish to others about to do as you have done the precise motive which will hold them back. If your act be such as to invoke a minor penalty, then not only others, but yourself, may profit by the punishment which we inflict. On the homely principle that ' a burnt child dreads the fire,' it will make you think twice before venturing on a repetition of your crime. Observe, finally, the consistency of our conduct. You offend, you say, because you cannot help offending, to the public detriment. We punish, is our reply, because we cannot help punishing, for the public good. Practically, then, as Bishop Butler predicted, we act as the world acted when it supposed the evil deeds of its criminals to be the products of free-will." *

" What," I have heard it argued, "is the use of preaching about duty, if a man's predetermined position in the moral world renders him incapable of profiting by advice?" Who knows that he is incapable? The preacher's last word is a factor in the man's conduct, and it may be a

\footnotetext{
* An eminent church dignitary describes all this, not unkindly, as "truculent logic." I think it worthy of his grace's graver consideration.
} 
most important factor, unlocking moral energies which might otherwise remain imprisoned and unused. If the preacher thoroughly feels that worls of enlightenment, conrage, and admonition enter into the list of forces employed by Nature herself for man's amelioration, since she gifted man with speech, he will suffer no paralysis to fall upon his tongue. Dung the fig-tree hopefully, and not until its barrenness has been demonstrated beyond a doubt let the sentence go forth, "Cut it down, why cumbereth it the ground?"

I remember when a youth in the town of Halifax, some two-and-thirty years ago, attending a lecture given by a young man to a small but select audience. The aspect of the lecturer was earnest and practical, and his voice soon rireted attention. He spoke of duty, defining it as a debt owed, and there was a kindling vigor in his words which must hare strengthened the sense of duty in the minds of those who heard him. No speculations regarding the freedom of the will could alter the fact that the words of that young man did me good. His name was George Dawson. He also spoke, if you will allow me to alude to it, of a social subject much discussed at the time-the Chartist subject of "leveling." Suppose, he says, two men to be equal at night, and that one rises at six, while the other sleeps till nine next morning, what becomes of your leveling? And in so speaking he made himself the mouthpiece of Nature, which, as we have seen, secures advance, not by the reduction of all to a common level, but by the encouragement and conservation of what is best.

It may be urged that, in dealing as above with my hypothetical criminal, I am assuming a state of things brought about by the influence of religions which include the dogmas of theology and the belief in free-will-a state, namely, in which a moral majority control and keep in awe an immoral minority. The heart of man is deceitful above all things, and desperately wicked. Withdraw, then, our theologio sanctions, including the belief in free-will, and the condition of the race will be typified by the samples of individual wickedness which have been above addnced. We shall all, that is, beeome robbers, and rarishers, and murderers. From much that has been written of late it would seem that this astounding inference finds house- 
room in many minds. Possibly, the people who hold such views might be able to illustrate them by individual instances.

The fear of hell's a hangman's whip,

To keep the wretch in order.

Remove the fear, and the wretch, following his natural instinct, may become disorderly; but I refuse to accept him as a sample of humanity. "Let us eat and drink, for to-morrow we die" is by no means the ethical consequence of a rejection of dogma. To many of you the name of George Jacob Holyoake is doubtless familiar, and you are probably aware that at no man in England has the term " atheist" been more frequently pelted. There are, moreover, really few who have more completely liberated themselves from theologic notions. Among working-class politicians Mr. Holyoake is a leader. Does he exhort his followers to "Eat and drink, for to-morrow we die?" Not so. In the August number of the Nineteenth Century you will find these words from his pen: "'The gospel of dirt is bad enough, but the gospel of mere material comfort is much worse." He contemptuously calls the Comtist championship of the workingman, "the championship of the trencher." He would place "the leanest liberty which brought with it the dignity and power of self-help" higher than "any prospect of a full plate without it." Such is the moral doctrine taught by this "atheistic" leader; and no Christian, I apprehend, need be ashamed of it.

Most heartily do I recognize and admire the spiritual radiance, if I may use the term, shed by religion on the minds and lives of many personally known to me. At the same time I cannot but observe how signally, as regards the production of anything beantiful, religion fails in other cases. Its professor and defender is sometimes at bottom a brawler and a clown. These differences depend upon primary distinctions of character which religion does not remove. It may comfort some to know that there are among us many whom the gladiators of the pulpit would call "atheists" and " materialists," whose lives, nevertheless, as tested by any accessible standard of morality, would contrast more than favorably with the lives of those who seek to stamp them with this offensive brand. When I say " offensive," I refer simply to the intention of those who use such terms, and not because atheism or material- 
ism, when compared with many of the notions ventilated in the columns of religious newspapers, has any particular offensireness for me. If I wished to find men who are scrupulous in their adherence to engagements, whose words are their bond, and to whom moral shiftiness of any kind is subjectively unknown; if I wanted a loving facher, a faithful husband, an honomble neighbor, anil a just citizen-I should seek him, and find him among the baud of "atheists" to which I refer. I have known some of the most pronounced anong them not only in life but in death-seen them approaching with open eyes the inexorable goal, with no dread of a " hangman's whip," with no hope of a hearenly crown, and still as mindful of their duties, and as faithful in the discharge of them, as if their etermal future dependel upon their lateet deeds.

In lettersaldressed to myself, and in utterances addressed to the public, Faraday is often referred to as a sample of the association of religious faith with moral elevation. I was locally intimate with him for fourteen or fifteen years of my life, and had thus occusion to observe liow nearly his character approached what might, withont extruv. agance, be caller perfection. He was strong but gentle, impetuons but self-restrained; a sweet and lofty courtesy marked his dealings with men and women; and thongh he sprang from the body of the people, a nature so fine might well have been distilled from the flower of antecedent chivalry. Not only in its broader sense was the Christian religion necessary to Faraday's spiritual peace, but in what many would call the narrow sense held by those deseribed by Faralay himself as " a very small and despised sect of Christians, known, if known at all, us Sandemanians," it constituter the light and comfort of his days.

Were our experience confined to such cases, it would furnish an irrestatible argument in faror of the association of dogmatic religion with moral purity and grace. But, as already intimated, our experience is not thus confined. In further illustration of this point, we may compare with Faraday a philosopher of equal magnitude, whose character, including gentlenese and strength, candor und simplicity. intellectnal power and moral elevation, singularly resembles that of the great Sundemanian, but who has neithur shared the theologic views nor the religious emotions which 
formed so dominant a factor in Faraday's life. I allude to Mr. Charles Darwin, the Abraham of scientific men-a searcher as obedient to the command of truth as was the patriarch to the command of God. I cannot therefore, as so many desire, look upon Faraday's religious belief as the exclusive source of qualities shared so conspicnously by one uninfluenced by that belief. To a deeper virtue belonging to human nature in its purer forms I am disposed to refer the excellence of both.

Superstition may be defined as constructive religion which has growu incongruous with intelligence. We may admit, with Fichte, "that superstition has unquestionably constrained its subjects to abandon many pernicious practices and to adopt many useful ones;" the real loss accompanying its decay at the present day has been thus clearly stated by the same philosopher: "In so far as these lamentations do not proceed from the priests themselveswhose grief at the loss of their dominion over the human mind we can well understand-but from the politicians, the whole matter resolves itself into this, that government has thereby become more difficult and expensive. The judge was spared the exercise of his own sagacity and penetration when, by threats of relentless dammation, he could compel the accused to make confession. The evil spirit formerly performed without reward services for which in later times judges and policemen have to be paid."

No man ever felt the need of a high and ennobling religion more thoroughly than this powerful and fervid teacher, who, by the way, did not escape the brand of " atheist." But Fichte asserted emphatically the power and sufficiency of morality in its own sphere. "Let us consider," he says, " the highest which man can possess in the absence of religion-I mean pure morality. The moral man obeys the law of duty in his breast absolutely, because it is a law unto him; ani he does whatever reveals itself to him as his duty simply because it is duty. Let not the impudent assertion be repeated that such an obedience, without regard for consequences, and without desire for consequences, is in itself impossible and opposed to human nature." So much for Fichte. Faraday was equally distinct. "I have no intention," he says, "of substituting anything for religion, but I wish to take that part of human nature which is independent of it. Moral- 
ity, philosophy, commerce, the various institutions and habits of society, are independent of religion and may exist without it." "These were the worls of his youth, but they expressed his latest convictions. I would add, that the muse of Tumyson never reacherl a higher strain than when it embodied the sentiment of daty in Anone:

And, because right is right, io follow right

Were wisloum in the scorn of consequence.

Not in the way assumed by our dogmatic teachers has the morality of limman nature been built "p. The power which has molded us thus far has worked with stern tools upon a very rigid stuff. What it has done cannot be so readily undone; and it has endowed us with moral constitutions which take pleasure in the noble, the beantiful, and the true, just as surely as it has endowed us with sentient organisms, which find aloes bitter and sugar sweet. That power did not work with delusions, nor will it stay ils hand when such are removed. Facts, rather than dogmas, have been its ministers - hunger and thirst, heat and cold, pleasure and pain, ferror, sympathy, aspiration, shame, pride, love, hate, terror, awe-snch were the forces whose interaction and adjustment throughout an immensurable past wove the triplex web of man's pliysical, intellectual and moral nature, and such are the forces that will be effectual to the end.

You may retort that even on my own showing "the power which makes for righteousness" has dealt in delusions: for it cannot be dened that the beliefs of religion, ineluding the dogmas of theology and the freedoun of the will, have had some effect in molding the moral world. Granted; but I do not think that this goes to the root of the matter. Are you quite aure that those beliefs and dogmas are primary, and not derived?- that they are not the proulnets. instead of being the creators, of man's moral nature? I think it is in one of the Latter- Day Pamphlets that Carlyle corrects a reasoner, who deduced the notility of man from a belief in leaven, by telling him that he puts the eart before the horse, the real truth being that the belief in heaven is derived from the nobility of man. The hird's instinet to weare its nest is referred to by Emerson as ivpical of the force which built cathedrals, temples, and pyrauids: 
Knowest thou what wove yon woodbird's nest

Of leaves and feathers from her breast,

Or how the fish outbuilt its shell,

Painting with morn each annual cell?

Such and so grew these holy piles

While love and terror laid the tiles;

Earth proudly wears the Parthenon

As the best gem upon her zone;

And Morning opes with haste her lids

To gaze upon the Pyramids;

O'er England's abbeys bends the sky

As on its friends with kindred eye;

For out of 'Thought's interior sphere

These wonders rose to upper air,

And Nature gladly gave them place,

Adopted them into her race,

And granted them an equal date

With Andes and with Ararat.

Surely, many utterances which have been accepted as descriptions ought to be interpreted as aspirations, or as having their roots in aspiration instead of in objective knowledge. Does the song of the herald angels, "Glory to God in the highest, and on earth peace, goodwill toward men," express the exaltation and the yearning of a human soul? or does it describe an optical and acoustical fact-a visible host and an andible song? If the former, the exaltation and the yearning are man's imperishable possession-a ferment loug confined to individuals, but which may by and by become the leaven of the race. If the latter, then belief in the entire transaction is wrecked by non-fulfillment. Look to the East at the present moment as a comment on the promise of peace on earth and goodwill toward men. That promise is a dream ruined by the experience of eighteen centuries, and in that ruin is involved the claim of the "heavenly host" to prophetic vision. But though the mechanical theory proves untenable, the immortal song and the feelings it expresses are still ours, to be incorporated, let us hope, in purer and less shadowy forms in the poetry, philosophy, and practice of the future.

Thus, following the lead of physical science, we are brought without solution of continuity into the presence of problems which, as usually classified, lie entirely outside the domain of physics. To these problems thoughtful and penetrative minds are now applying those methods of research which in physical science have proved their truth 
by their fruits. There is on all hands a growing repug. nance to invoke the supernatural in accounting for the phenomena of human life; and the thoughtful minds just referred to, finding no trace of evidence in favor of any other origin, are driven to seck in the interaction of social forces the genesis and development of man's moral nature. If they succeed in their search-and I think they are sure to succeed-8ocial duty will be raised to a higher level of significance and the deepening sense of social duty will, it is to be hoped, lessen, if not obliterate, the strifes and heartburnings which now beset and disfigure our social life. 'Toward this great end it behoves us one and all to work; and devoutly wishing its consummation, I have the honor, ladies and gentlemen, to bid you a friendly farewell.

\section{CHAP'TER XXXVII.}

\section{PROFESSOE VIRCHOW AND FVOLUTION.}

THIs WORLD of ours has, on the whole, been an inclement region for the growth of natural truth; but it may be that the plant is all the hardier for the bendings and buffetings it has undergone. The torturing of a shrub, withn certain limits, strengthens it. 'Throngh the struggles and passions of the brute, man reaches his estate; through saragery and barburism his cirilization; and throngh illusion and persecution his knowledge of nature, inclading that of his own frame. 'The bias toward natural truth must have been strong to have withstood and overcome the opposing forces. Feeling appeared in the world before Knowledge; and thoughts, conceptions, and creeds, founded on emotion, had, before the dawn of science. taken root in man. Such thoughts, conceptions, and creeds must have met a deep and general want; otherwise their growth conld not have been so laxuriant, nor their sbiding power so strong. This generul need-this hunger for the ideal and wonderful-led erentually to the differentiation of a caste, whose vocation it was to enltivate the myatery of life and its surroundings, and to gise shape, name, and habitation to the emotions which thiat mystery aronsed. Fivell the sasage lised, not by breal alone, bit in a mental world peoplod with forms answering to his 
capacities and needs. As time adranced-in other words, as the savage opened out into civilized man-these forms were purified and ennobled until they finally emerged in the mythology and art of Greece:

\section{Where still the magic robe of Poesy \\ Wound itself lovingly around the Truth.*}

As poets, the priesthood would have been justified, their deities, celestial and otherwise, with all their retinue and appliances, being more or less legitimate symbols and personifications of the aspects of nature and the phases of the human soul. 'The priests, however, or those among them who were mechanics, and not poets, claimed objective validity for their conceptions, and tried to base upon external evidence that which sprang from the innermost need and nature of man. It is against this objective rendering of the emotions-this thrusting into the region of iact and positive knowledge of conceptions essentially ideal and poetic-that science, consciously or unconsciously, wages war. Religious feeling is as much a verity as any other part of human consciousness; and against it, on its subjective side, the waves of science beat in vain. But when, manipulated by the constructive imagination, mixed with imperfect or inaccurate historic data, and molded by misapplied logic, this feeling makes claims which traverse our knowledge of nature, science, as in duty bound, stands as a hostile power in its path. It is against the mythologic scenery, if I may use the term, rather than against the life and substance of religion, that Science enters her protest. Sooner or later among thinking people, that scenery will be taken for what it is worth-as an effort on the part of man to bring the mystery of life and nature within the range of his capacities; as a temporary and essentially fluxional rendering in terms of knowlerlge of that which transcends all knowledge, and admits only of ideal approach.

'The signs of the times, I think, point in this direction. It is, for example, the obvious aim of Mr. Matthew Arnold to protect, amid the wreck of dogma, the poetic basis of religion. And it is to be remembered that under the circumstances poetry may be the purest accessible truth. In

* " Da der Dichtung zauberische Hülle

Sich noch lieblich una die Wahrheit wand."-Schiller. 
other influential quarters a similar spirit is at work. In a remarkable article published by Professor Knight of Sit. Andrews in the september number of the Sineleenth Century. amid other free utherances, we have this one: - If mitter is not eternal, its first emergence into being is a miracle beside which all others dwindle into absolute insignificance. But, as has often been pointed out, the process is unthinkable; the sudden apocaly pse of a material world ont of blank nonentity cannot be imagined; * its emergence into orler out of chaos when "without form and roid" of life, is merely "poetic rendering of the doctrine of its slow evolution.' These are all bold words to be spoken before the moral philosophy elass of a Sontch university, while those I have underlined show a remarkable freedom of dealing with the sacred text. They repeat in terser language what 1 ventured to utter four years ago regarding the Book of Genesis. "Profoundly interesting anil indeed pathetic to me are those attempts of the opening mind of man to appease its hunger for a Cause. But the Book of Cenesis has no voice in scientifie questions. It is a poem, nol a screntific treatise. In the former aspect it is forever beautiful; in the latter it has beon, and it will continue to be, purely obstructive and hurtful." My agreement with Professor Knight extemis still further. "Does the vital," he ask8, "proceed by a still remoter levelopment from the non-vital? Or was it created by a fiat of volition? Or." - and here he emphasizes his question"has it always existed in some form or olher as an eternal constituent of the universe? I do not see," he replies, " how we can escape from the last alternative." With the whole force of my conviction I say, Nor do I, though our modes of regarding the "eternal constituent" may not be the same.

When matler was defined by Descartes, he deliberately excluded the idea of force or motion from its attributos and from his definition. Erlension only was tuken into acconnt. And, inasmuch as the impotence of matter to generate motion was assumed, its observed motions were referred to an external canse. God, resident outside of matter, gare the impulse. In this connection the

- I'rofensor finighe will have to reckun with the English uarriage services one of whose estlecets legeins thus! "O (iod, whos by thy auiglity juwer lasat made all things of mothing." 
argument in Young's "Night Thoughts" will occur to most readers:

Who Motion foreign to the smallest grain

Shot through vast masses of enormous weight?

Who bid brute Matter's restive lump assume

Such various forms, and gave it wings to fly?

Against this notion of Descartes the great deist John T'oland, whose ashes lie unmarked in Putney churchyard, strenuously contended. He affirmed motion to be an inherent attribute of matter-that no portion of matter was at rest, and that even the most quiescent solids were animated by a motion of their ultimate particles. The success of his contention, according to the learned and laborious Dr. Berthold,* entitles Toland to be regarded as the founder of that monistic doctrine which is now so rapidly spreading.

It seems to me that the idea of vitality entertained in our day by Professor Knight, closely resembles the idea of motion entertained by his opponents in Toland's day. Motion was then virtually asserted to be a thing suigeneris, distinct from matter, and incapable of being generated ont of matter. Hence the obvious inference when matter was observed to move. It was the vehicle of an energy not its own-the repository of forces impressed on it from without -the purely passice recipient of the shock of the Divine. The logical form continues, but the subjectmatter is changed. "The evolution of nature," says Professor Knight, " may be a fact; a daily and hourly apocalypse. But we have no evidence of the non-vital passing into the vital. Spontaneous generation is, as yet, an imaginative guess, unverified by scientific tests. And matter is not itself alive. Vitality, whether seen in a single cell of protoplasm or in the human brain, is a thing sui generis, distinct from matter, and incapable of being generated out of matter." It may be, however, that in process of time, vitality will follow the example of motion, and, after the necessary antecedent wrangling, take its place among the attributes of that "universal mother" who has been so often misdefined.

* "John Toland und der Monismus der Gegenwart," Heidelberg, Carl Winter. 
That " matter is not itself alive" Professor Knight seems to regard as an axiomatic truth. Let us place in contrast with this the notion entertained by the philosopher Ueberweg, one of the subtlest hends that Germany has produced. "What oocurs in the brain," says Ueberweg, " would, in my opinion, not be possible, if the process which here appears in its greatest concentration did not obtain generally, only in a vastly diminished degree. Take a pair of mice and a cask of flour. By copious nourishment the animals insrease and multiply, and in the same proportion zansations and feelings augment. 'The quantity of these latter possessed by the first pair, is not simply diffused among their descendants, for in that case the last must feel more feebly than the first. - The sensations and feelings must necessarily be referred back to the flour, where they exist, weak and pale it is true, and not concentrated as they are in the brain." " We may not be able to taste or smell alcohol in a tub of fermented cherries, but by distillation we obtain from them concentrated Kirsch. wasser. Hence Ueberweg's comparison of the brain to a still, which concentrates the sensation and feeling, preexisting, but diluted in the food.

"Definitions," says Mr. Holyoake, f "grow as the horizon of experience expands. They are not inventions, but descriptions of the state of a question. No man sees all through a discovery at once." Thus Descartes' notion of matter, and his explanation of motion, would be put aside as trivial by a physiologist or a crystallogrupher of the present day. They are not descriptions of the state of the question. And yet a desire sometimes shows itself in distinguished quarters to bind us down to conceptions which passed mnster in the infancy of knowledge, but which are wholly incompatible with our present enlightenment. Mr. Martinean, I think, errs when he seeks to hold me to views enunciated by "Democritus and the mathematicians." That definitions should chango as knowledge adrances is in accordance both with sound sense and scientitic practice. When, for example, the undulatory theory was started, it was not imagined that the vibrations of light could be

- Letter uo Lange: "Ueschichte des Materialisuus," zwoite Aufl. vol. I1., p. 521.

† Ninetconth Century, September, 1878. 
transverse to the direction of propagation. The example of sound was at hand, which was a case of longitudinal vibration. Now the substitution of transverse for longitudinal vibrations in the case of light involved a radical change of conception as to the mechanical properties of the luminiferous medium. But though this change went so far as to fill space with a substance possessing the properties of a solid, rather than those of a gas, the change was accepted, because the newly discovered facts imperatively demanded it. Following Mr. Martineau's example, the opponent of the undulatory theory might effectually twit the holder of it on his change of front. "T'This ether of. yours," he might say, "alters its style with every change of service. Starting as a beggar, with scarce a rag of 'property' to cover its bones, it turns up as a prince when large undertakings are wanted. You had some show of reason when, with the case of sound before you, you assumed your ether to be a gas in the last extremity of attenuation. But now that new service is rendered necessary by new facts, you drop the beggar's rags, and accomplish an undertaking, great and princely enough in all conscience; for it implies that not only planets of enormous weight, but comets with hardly any weight at all, fly through your hypothetical solid without perceptible loss of motion." This wonld sound very cogent, but it would be very vain. Equally vain, in my opinion, is $\mathrm{Mr}$. Martineau's contention that we are not justified in modifying, in accordance with advancing knowledge, our notions of matter.

Before parting from Professor Knight, let me commend his courage as well as his insight. We have heard much of late of the peril to morality involved in the decay of religious belief. What Mr. Knight says under this head is worthy of all respect and attention. " I admit," he writes, "that were it proved that the moral faculty was derived as well as developed, its present decisions would not be invalidated. 'The child of experience has a father whose teachings are grave, peremptory, and angust; and an earthborn rule may be as stringent as any derived from a celestial source. It does not even follow that a belief in the material origin of spiritual existence, accompanied by a corresponding decay of belief in immortality, must necessarily lead to a relaxation of the moral fiber of the 
race. It is certain that it has often done so." But it is equally certain that there have been individuals, and great historical communities, in which the absence of the latter belief has neither weakened moral earnestness, nor prerented devotional ferror." I have elsewhere stated that some of the best men of my acquaintance-men lofty in thought and beneficent in act-belong to a class who assiduously let the belief referred to alone. They derive from it neither stimulus nor inspiration, while-I say it with regret-were I in quest of persons who, in regard to the finer endowments of human character, are to be ranked with the unendowed, I should find some characteristic samples among the noisier defenders of the orthodox belief. These, however, are but "hand-specimens" on both siles; the wider data referred to by Professor Kright constitute, therefore, a welcome corroboration of my experience. Again, my excellent critic, Professor Blackie, describes Buddha as heing "a great deal more than a prophet; a rare, exceptional, and altogether transcendental incarnation of moral perfection." $\dagger$ And yet, "what Buddha preached was a gospel of pure human ethics, divorced not only from Brahma and the Brahminic Trinity, but eren from the existence of God." $\$$ These civilized and gallant voices from the North contrast pleasantly with the barbarous whoops which sometimes come to us along the same meridian.

Looking backward from my present standpoint over the earnest past, a boybood fond of play and physical action, but arerse to schoolwork, lies befure me. The arersion did not arise from intellectual apathy or want of appetite for knowledge, but simply from the fact that my earliest teachers lacked the power of imparting vitality to what they tanght. Athwart all play and amueement, however, a threal of serionsness ran throngh my character; and many a sleepless night of my childhood has been pussed. fretted by the question "Who made God?" I was well

* Is this really certain? Inmtead of standing iu the relation of causen snel .ffect, may not the "docery" and " relaxation" be me.rely

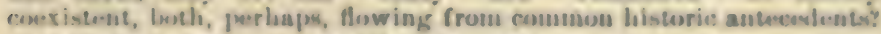

$+\cdots$. Nutural ITintory of Atheison," p. 136.

\$ Ibld., p. 125. 
versed in Scripture; for I loved the Bible, and was prompted by that iove to commit large portions of it to memory. Later on I became adroit in turning my Scriptural knowledge against the Church of Rome, but the characteristic doctrines of that Uhurch marked only for a time the limits of inquiry. 'The eternal Sonship of Christ, for example, as enunciated in the Athanasian Creed, perplexed me. The resurrection of the body was also a thorn in my mind, and here I remember that a passage in Blair's "Grave" gave me momentary rest.

\section{Sure the same power}

That rear'd the piece at first and took it down

Can reassemble the loose, scatter'd parts

And put them as they were.

The conclusion seemed for the moment entirely fair, but with further thought, my difficulties came back to me. I had seen cows and sheep browsing upon churchyard grass, which sprang from the decaying mold of dead men. The flesh of these animals was undoubtedly a modification of human flesh, and the persons who fed upon them were as undoubtedly, in part a more remote modification of the same substance. I figured the selfsame molecules as belonging first to one body and afterward to a different one, and I asked myself how two bodies so related could possibly arrange their claims at the day of resurrection. The scattered parts of each were to be reassembled and set as they were. But if handed over to the one, how could they possibly enter into the composition of the other? Omnipotence itself, I concluded, could not reconcile the contradiction. 'Thus the plank which Blair's mechanical theory of the resurrection brought momentarily into sight disappeared, and I was again cast abroad on the waste ocean of speculation.

At the same time I could by no means get rid of the idea that the aspects of nature and the consciousness of man implied the operation of a power altogether beyond $\mathrm{my}$ grasp-an energy the thought of which raised the temperature of the mind, though it refused to accept shape, personal or otherwise, from the intellect. Perhaps the able critics of the Saturday Review are justified in speaking as they sometimes do of Mr. Carlyle. They owe him nothing, and have a right to announce the fact in 
their own way. I, however, owe him a great deal, and an also in honor bound to acknowleslge the delit. Few, perlaps, who are privileged to come into contact with that illustrious man have ghown him a sturier frout than I have, or in discussing modern science have more frequently withstood him. But I could see that his contention at bottom always was that the human sonl has claime and yearnings which physical science cannot satisfy. Englami to come will assuredly thank him for his aftirmation of the ethical and ideal side of human nature. Be this as it may, at the period now reached in my story the feeling referred to was indefinitely strengthened, my whole life being at the same time rendered inore earnest, resolute, and lahorious by the writings of Carlyle. Others also ministered to this result. Emerson kindled me, while Fichte powerfully stirred my moral pulse." In this relation I cared little for political theories or philosophic systems, but a great deal for the propagated life and strength of pure and powerful minds. In my later schooldays, under a clever teacher, some knowledge of mathematies and physics had been picked up: my stock of both was, howerer, scanty, and I resolved to augment it. But it was really with the view of learning whether mathematics and pliysics could help ne in other spheres, rather than with the desire of acquiring distinction in either science, that I ventured, in 1848 , to break the continuity of my life, und derote the meager funds then at my disposal to the study of science in Germany.

But science soon fascinated me on its own account. 'To carry it duly and honestly ont, moral qualities were incessantly invoked. 'There was no room allowed for insin. cerity-no room even for carelessness. The mlifice of science had been raised by men who had unswervingly followerl the truth as it is in nature; and in doing so lial often sacrificed interests which are nsnally potent in this world. Among thene rationalistic men of Ciermany I found conscientionsness in work as much insisted on us it could be smong theologians. And why, since they had not the rewards or penalties of the theologian to offer $w$

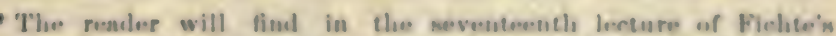

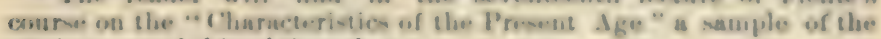
vital power of this philomopher. 
their disciples? Because they assumed, and were justified in assuming, that those whom they addressed had that within them which would respond to their appeal. If Germany should ever change for something less noble the simple earnestness and fidelity to duty, which in those days characterized her teachers, and through them her sons generally, it will not be because of rationalism. Such a decadent Germany might coexist with the most rampant rationalism without their standing to each other in the relation of cause and effect.

My first really laborious investigation, conducted jointly with my friend Professor Knoblauch, landed me in a region which harmonized with my speculative tastes. It was essentially an inquiry in molecular physics, having reference to the curious, and then perplexing, phenomena exhibited by crystals when freely suspended in the magnetic field. I here lived amid the most complex operations of magnetism in its twofold aspect of an attractive and a repellent force. Iron was attracted by a magnet, bismuth was repelled, and the crystals operated on ranged themselves under these two heads. Faraday and Plücker had worked assiduously at the subject, and had invoked the aid of new forces to account for the phenomena. It was soon, however, found that the displacement in a crystal of an atom of the iron class by an atom of the bismuth class, involving no change of crystalline form, produced a complete reversal of the phenomena. The lines through the crystal which were in the one case drawn toward the poles of the magnet, were driven, in the other case, from these poles. By such instances and the reasoning which they suggested, magne-crystallic action was proved to be due, not to the operation of new forces, but to the modification of the old ones by molecular arrangement. Whether diamagnetism, like magnetism, was a polar force, was in those days a subject of the most lively contention. It was finally proved to be so; and the most complicated cases of magne-crystallic action were immediately shown to be simple meshanical consequences of the principle of diamagnetic polarity. 'These early researches, which occupied in all five years of my life, and throughout which the molecular architecture of crystals was an incessant subject of mental contemplation, gave a tinge and bias to my subsequent scientific thought, and their influence 
is ensily traced in my subsequent impuiries. For example, during nine years of labor on the subject of radiation, heat and light were handled throughont by me, not as ends, but as instruments by the aid of which the mind might perchance lay hold upon the ultimato particles of matter.

Scientific progress depends mainly upon two factors which incessantly interact-the strengthening of the mind by exercise, and the illumination of phenomena by knowl. edge. There seoms no limit to the insight regarling physical processes which this interaction carries in its train. Through such insight we are enabled to enter and explore that subsensible world into which all natural phenomena strike their roots, and from which theyderive nutrition. $\mathrm{By}$ it we are enabled to place before the mind's eye atoms and atomic motions which lie far beyond the range of the senses, and to apply to them reasoning as stringent as that applied by the mechanician to the motions and collisions of sensible masses. But once committed to such conceptions, there is a risk of being irresistibly led buyoud the bounds of inorganic nature. Fven in those early stages of scientific growth, I found myself more and more complelled to regard not only crystals, but organic structures, the body of man inclusive, as cases of moleoular architecture, infinitely more complex, it is true, than those of inorganic nature, but reducible, in the long run, to the same mechanical laws. In aucient jouruals I find recorded ponderings and speculations relating to these subjects, and attempts male, by reference to magnetic and orystalline phenomena, to present some satisfactory image to the mind of the way in which plants and animals are built up. Perhaps I may be exeused for noting a sample of these early speculations, alruady possibly kuown to a fow of my readers, but which here finils a more suitable place than that which it formerly occupied.

Sitting, in the summer of 1855, with my friend Dr. Debus under the shadow of a uassive elm on the hank of a river in Normandy, the current of our thonghts and conversation was substantially this: We regardent the tree above us. In opposition to gravity its molecules had ascended, direrged into branches, and budded into innumerable lesses. What camsed them to do so-a power 
external to themselves, or an inherent force? Science rejects the outside builder; let us, therefore, consider from the other point of view the experience of the present year. A low temperature had kept back for weeks the life of the vegetable world. But at length the sun gained power-or, rather, the cloud-screen which our atmosphere had drawn between him and us was removed-and life immediately kindled under his warmth. But what is life, and how can solar light and heat thus affect it? Near our elm was a silver birch, with its leaves rapidly quivering in the morning air. We had here motion, but not the motion of life. Each leaf moved as a mass under the influence of an outside force, while the motion of life was inherent and molecular. How are we to figure this molecular motionthe forces which it implies, and the results which flow from them? Suppose the leares to be shaken from the tree and enabled to attract and repel each other. To fix the ideas, suppose the point of each leaf to repel all the uther points and to attract the roots, and the root of each leaf to repel all other roots but to attract the points. The leaves would then resemble an assemblage of little magnets abandoned freely to the interaction of their own forces. In obedience to these they would arrange themselves, and finally assume positions of rest, forming a coherent mass. Let us suppose the breeze, which now causes them to quiver, to disturb the assumed equilibrium. As often as disturbed there would be a constant effort on the part of the leaves to re-establish it; and in making this effort the mass of leaves would pass through different shapes and forms. If other leaves, moreover, were at hand endowed with similar forces, the attraction would extend to them-a growth of the mass of leaves being the consequence.

We have strong reason for assuming that the ultimate particles of matter-the atoms and molecules of which it is made up-are endowed with forces coarsely typified by those here ascribed to the leaves. The phenomena of crystallization lead, of necessity, to this conception of molecular polarity. Under the operation of such forces the molecules of a seed, like our fallen leaves in the first instance, take up positions from which they would never move if undisturbed by an external impulse. But solar light and heat, which come to us as waves through space, 
are the great agents of molecular disturbance. On the inert molecules of seed and soil these waves impinge, disturbing the atomic equilibrium, which there is an immediate effort to restore. The effort, incessantly defentedfor the waves continue to pour in-is incessantly renewed: in the molecular struggle matter is gathered from the soil and from the atmosphere, and buili, in obedience to the forces which guide the molecules, into the special form of the tree. In a general way, therefore, the life of the tree might be defined as an unceasing effort to restore a disturbed equilibrium. In the building of crystals Nature makes her first structural effort; we have here the esiliest groping of the 8o-called "vital force," and the manifertations of this force in plants and animals, theugh, as already stated, indefinitely more complex, are to be regarded of the same mechanical quality as those concerned in the building of the crystal.

Consider the cycle of operations by which the seed produces the plant, the plant the flower, the flower again the seed, the cansal line, returning with the fidelity of a planetary orbit to its original point of departure. Who or what plauned this molecular rhythm? We do not knowscience fails even to inform us whether it was ever "planned" at all. Yonder butterfly has a spot of orange on its wing; and if we look at a drawing made a century ago, of one of the ancestors of that butterfly, we probably finit the selfsame spot upon the wing. For a century the molecules have described their cycles. Butterflies have been begotten, have been born, and have died; still we find the molecular arohitecture unchanged. Who or what determined this persistency of recurrence? We do not know; but we stand within our intellectual range when we say that there is probably nothing in that wing which may not yet find its Newton to prove that the principles involsed in its con. struction are qualitatively the same as those brought into play in the formation of the solar system. We may eren take a step furthor, and affirm that the brain of man-the organ of his renson-without which he cum neither think nor feel, is also an assemblage of molecules, acting and reacting according to law. Ifere, however, the methenls prursued in mechanical science come to an end; and if askerl to deduce from the plysical interaction of the brain molecules the least of the phenomena of sonsation or 
thought, I acknowledge my helplessuess. The association of both with the matter of the brain may be as certain as the association of light with the rising of the sun. But whereas in the latter case we have unbroken mechanical connection between the sun and our organs, in the former cuse logical continuity disappears. Between molecular mechanics and consciousness is interposed a fissure over which the ladder of physical reasoning is incompetent to carry us. We must, therefore, accept the observed associ. ation as an empirical fact, without being able to bring it under the yoke of à priori deduction.

Such were the ponderings which ran habitually through my mind in the days of my scientific youth. They illustrate two things-a determination to push physical considerations to their utmost legitimate limit; and an acknowledgment that physical considerations do not lead to the final explanation of all that we feel and know. 'This acknowledgment, be it said in passing, was by no means made with the view of providing room for the play of considerations other than physical. The same intellectual duality, if I may use the phrase, manifests itself in the following extract from an article entitled "Physics and Metaphysics," published in the Saturday Review for August 4, 1860:

6'The philosophy of the future will assuredly take more account than that of the past of the dependence of thought and feeling on physical processes; and it may be that the qualities of the mind will be studied through organic combinations as we now study the character of a force through the affections of ordinary matter. We believe that every thought and every feeling has its definite mechanical correlative - that it is accompanied by a certain breaking up and remarshaling of the atoms of the brain. This latter process is purely physical; and were the faculties we now possess sufficiently expanded, without the creation of any new faculty, it would doubtless be within the range of our augmented powers to infer from the molecular state of the brain the character of the thought acting on it, and, conversely, to infer from the thought the exact molecular condition of the brain. We do not say-and this, as will be seen, is all-important-that the inference here reforred to would be an à priori one. But by observing, with the 
faculties we assume, the state of the brain and the associated mental affections, both might be so tabulated side by side that, if one were given, a mere reference to the table would declare the other. Our present powers, it is true, shrivel into nothingness when brought to bear on such a problem, but it is because of its complexity and our limits that this is the case. 'The quality of the problem and of our powers are, we believe, so relited, that a mere expansion of the latter would enable them to cope with the former. Why, then, in scientific speculation should we turn our eyes exclusively to the past? May it not be that a time is coming-ages no doubt distant, but still advancing-when the dwellers upon this fair earth, starting from the gross human brain of to-day as a rudiment, may be able to apply to these mighty questions faculties of commensurate extent? Given the requisite expansibility to the present senses and intelligence of man-given also the time necessary for their expansion-and this high goal may be attained. Development is all that is required, and not a change of quality. There need be no absolute breach of continuity between us and our loftier brothers yet to come.

"We have guarded ourselves against saying that the inferring of thought from material combinations and arrangements would be an inference à priori. 'The inference meant would be the same in kind as that which the observation of the effects of food and drink upon the mind would enable us to make, differing only from the latter in the degree of analytical insight which we suppose attained. Given the masses and distances of the planets, we can infer the perturbations consequent on their mutual attractions. Given the nature of a disturbance in water, air, or etherknowing the physical qualities of the medium we can infer how its particles will be affected. In all this we deal with physical laws. The mind runs with certainty along the line of thought which connects the phenomena, and from beginning to end there is no break in the chain. But when we endeavor to pass by a similar process from the phenomena of physics to those of thought, we meet a problem which transcends any conceivable expansion of the powers which we now possess. We may think over the subject again and again, but it eludes all intellectual presentation. We staud at length face to face with the Incomprehensible. 
The territory of physics is wide, but it has its limits from which we look with vacant gaze into the region beyond. Let us follow matter to its utmost bounds, let us claim it in all its forms-even in the muscles, blood, and brain of man himself-as ours to experiment with and to speculate upon. Casting the term "vital force" from our vocabulary, let us reduce, if we can, the visible phenomena of life to mechanical attractions and repulsions. Having thus exhausted physics, and reached its very rim, a mighty Mystery still looms beyond us. We have, in fact, made no step toward its solution. And thus it will ever loom, compelling the philosophies of successive ages to confess that

$$
\text { “. We are such stuff }
$$

As dreams are made of, and our little life Is rounded by a sleep.'

In my work on " Heat," published in 1863 and republished many times since, I employ the precise language thus extracted from the Saturday Review.

'The distinction is here clearly brought out which I had resolved at all hazards to draw - that, namely, between what men knew or might know, and what they could never hope to know. Impart simple magnifying power to our present vision, and the atomic motions of the brain itself might be brought into view. Compare these motions with the corresponding states of consciousness, and an empirical nexus might be established; but "we try to soar in a vacuum when we endeavor to pass by logical deduction from the one to the other." Among these brain-effects a new product appears which defies mechanical treatment. We cannot deduce motion from consciousness or consciousness from motion as we deduce one motion from another. Nevertheless observation is open to us, and by it relations may be established which are at least as valid as those of the deductive reason. The difficulty may really lie in the attempt to convert a datum into an inference-an ultimate fact into a product of logic. My desire for the moment, however, is not to theorize, but to let facts speak in reply to accusation.

The most " materialistic" speculation for which I was responsible, prior to the "Belfast Address," is embodied in the following extract from a brief article written as far back as 1865: "Supposing the molecules of the human 
body, instead of replacing others, and thus renewing a pre-existing form, to be gathered first-hand from nature, and placed in the exact relative positions which they occupy in the body. Supposing them to have the same forces and distribution of forces, the same motions and distribution of motions-would this organized concourse of molecules stand before us as a sentient, thinking being? There seems no valid reason to assume that it would not. $\mathrm{Or}^{\circ}$ supposing a planet carved from the sun, set spinning round an axis, and sent revolving round the sun at a distance equal to that of our earth, would one consequence of the refrigeration of the mass be the development of organic forms? I lean to the affirmative." This is plaiu speaking, but it is without " dogmatism." An opinion is expressed, a belief, a leaning-not an established "doctrine."

The burden of my writings in this connection is as much a recognition of the weakness of science as an assertion of its strength. In $186 \%$, I told the workingmen of Dundee that while making the largest demand for freedom of investigation; while considering science to be alike powerful as an instrument of intellectual culture, and as a ministrant to the material wants of men; if asked whether science has solved, or is likely in our day to solve, "the problem of the universe," I must shake my bead in doubt. I compare the mind of man to a musical instrument with a certain range of notes, beyond which in both directions exists infinite silence. The phenomena of matter and force come within our intellectual range; but behind, and above, and around us the real mystery of the universe lies unsolved, and, as far as we are concerned, is incapable of solution.

While refreshing my mind on these old themes I appear to myself as a person possessing one idea, which so overmasters him that he is never weary of repeating it. That idea is the polar conception of the grandeur and the littleness of man-the vastness of his range in some respects and directions, and his powerlessuess to take a singie step in others. In 1868, before the Mathematical and Physical Section of the British Association, then assembled at Norwich, I repeat the same well-worn note:

"In thus affirming the growth of the human body to be mechanical, and thought as exercised by us to have its 
correlative in the physics of the brain, the position of the 'Materialist,' as far as that position is tenable, is stated. I think the materialist will be able finally to maintain this position against all attacks, but I do not think he can pass beyond it. The problem of the connection of body and soul is as insoluble in its modern form as it was in the prescientific ages. Phosphorus is a constituent of the human brain, and a trenchant German writer has exclaimed, 'Ohne Phosphor kein gedanke!' 'That may or may not be the case; but, even if we knew it to be the case, the knowledge would not lighten our darkness. On both sides of the zone here assigned to the materialist he is equally helpless. If you ask him whence is this 'matter' of which we have been discoursing-who or what divided it into molecules, and impressed upon them this necessity of running into organic forms-he has no answer. Science is also mute in regard to such questions. But if the materialist is confounded and science is rendered dumb, who else is prepared with an answer? Let us lower our heals and acknowledge our ignorauce, priest and philosopher, one and all."

The roll of echoes which succeeded the lecture delivered by Professor Virchow at Munich on September 2\%, 187\%, was long and loud. 'The Times published a nearly full translation of the lecture, and it was eagerly commented on in other journals. Glances from it to an address delivered by me before the Midland Institute in the autumn of $18 \% \%$, and published in this volume, were very frequent. Professor Virchow was held up to me in some quarter's as a model of philosophic caution, who by his reasonableness reproved my rashness, and by his depth reproved my shallowness. With true theologic courtesy I was sedulously emptied, not only of the "principles of scientific thought," but of " common modesty" and "common sense." And though I am indebted to Professor Clifford for recalling in the Nineteenth Century for April the public mind in this connection from heated fancy to sober fact, I do not think a brief additional examination of Virchow's views, and of my relation to them, will be out of place here.

The keynote of his position is struck in the preface to the excellent English translation of his lecture-a preface 
written expressly by himself. "Nothing," he says, "was farther from his intention than any wish to disparage the great services rendered by Mr. Darwin to the advancement of biological science, of which no one has expressed more admiration than himself. On the other hand, it seemed high time to him to enter an energetic protest against the attempts that are made to proclaim the problems of research as actual facts, and the opinions of scientists as established science." On the ground, among others, that it promotes the pernicious delusions of the Socialist, Virchow considers the theory of evolution dangerous; but his fidelity to truth is so great that he would brave the danger and teach the theory, if it were only proved. "However dangerous the state of things might be, let the confederates be as mischievous as they might, still I do not hesitate to say that from the moment when we had become convinced that the evolution theory was a perfectly established doctrine-so certain that we could pledge our oath to it, so sure that we could say, 'Thus it is'-from that moment we could not dare to feel any scruple about introducing it into our actual life, so as not only to communicate it to every educated man, but to impart it to every child, to make it the foundation of our whole ideas of the world, of society, and the state, and to base upon it our whole system of education. 'This I hold to be a necessity."

It would be interesting to know the persons designated by the pronoun "we" in the first sentence of the foregoing quotation. No doubt Professor Haeckel would accept this canon in all its fullness, and found on it his justification. He would say without hesitation: "I am convinced that the theory of evolution is a perfectly established doctrine, and hence on your own showing I am justified in urging its introduction into our schools." It is plain, however, that Professor Virchow would not accept this retort as valid. His "we" must cover something more than Professor Haeckel. It would probably cover more even than the andience he addressed; for he would hardly affirm, even if every one of his hearers accepted the theory of evolution, that that would be a sufficient warrant for forcing it upon the public at large. His "we," I submit, needs definition. If he means that the theory of evolution ought to be introduced into our schools, not when experts are agreed as to its truth, but when the community is pre- 
pared for its introduction, then, I think, he is right, and that, as a matter of social policy, Dr. Haeckel would be wrong in seeking to antedate the period of its introduction. In dealing with the community great changes must have timeliness as well as truth upon their side. But if the mouths of thinkers be stopped, the necessary social preparation will be impossible; an unwholesome divorce will be established between the expert and the public, and the slow and natural process of learening the social lump by discovery and discussion will be displaced by something far less safe and salutary.

The burden, however, of this celebrated lecture is a warning that a marked distinction ought to be made between that which is experimentally proved, and that which is still in the region of speculation. As to the latter, Virchow by no means imposes silence. He is far too sagacious a man to commit himself, at the present time of day, to any such absurdity. But he insists that it ought not to be put on the same evidential level as the former. "It ought," as he poetically expresses it, " to be written in small letter's under the text." The audience ought to be warned that speculative matter is only possible, not actual truth-that it belongs to the region of "belief," and not to that of demonstration. As long as a problem continues in this speculative stage it would be mischievous, he considers, to teach it in our schools. "We ought not," he urges, " to represent our conjecture as a certainty, nor our hypothesis as a doctrine: this is inadmissible." With regard to the connection between physical processes and mental phenomena he says: "I will, indeed, willingly grant that we can find certain gradations, certain definite points at which we trace a passage from mental processes purely physical, or of a physical character. Throughout this discourse I am not asserting that it will never be possible to bring psychical processes into an immediate connection with those that are physical. All I say is that we have at present no right to set up this possible connection as a doctrine of science." In the next paragraph he reiterates his position with reference to the introduction of such topics into school teaching. "We must draw," he says, " a strict distinction between what we wish to teach, and what we wish to search for. The objects of our research are expressed as problems (or hypotheses). We 
need not keep them to ourselves; we are ready to communicute them to all the world, and say 'There is the problem; that is what we strive for.'. . . The investigation of such problems, in which the whole nation may be interested, cannot be restricted to any one. This is Freedom of Inquiry. But the problem (or hypothesis) is not, without further debate, to be made a doctrine." He will not concede to Dr. Haeckel " that it is a question for the schoolmasters to decide, whether the Darwinian theory of man's descent should be at once laid down as the basis of instruction, and the protoplastic soul be assumed as the foundation of all ideas concerning spiritual being." The professor concludes his lecture thus: " With perfect truth did Bacon say of old 'Scientia est potentia.' But he also defined that knowledge; and the knowledge he meant was not speculative knowledge, not the knowledge of hypotheses, but it was objective and actual knowledge. Gentlemen, I think we should be abusing our power, we should be imperiling onr power, unless in our teaching we restrict ourselves to this perfectly safe and unassailable domain. From this domain we may make incursions into the field of problems, and I ain sure that every venture of that kind will then find all needful security and support." I have emphasized by italics two sentences in the foregoing series of quotations; the other italics are the author's own.

Virchow's position could not be made clearer by any comments of mine than he has made it himself. That position is one of the highest practical importance. "'Throughout our whole German Fatherland," he says, "men are busied in renovating, extending, and developing the system of education, and in inventing fixed forms in which to mold it. On the threshold of coming events stands the Prussian law of education. In all the German states larger schools are being built, new educational establishments are set up, the universities are extended, ' higher' and 'middle' schools are founded. Finally comes the question, What is to be the chief substance of the teaching?" What Virchow thinks it ought and ought not to be, is disclosed by the foregoing quotations. There ought to be a clear distinction made between science in the state of hypothesis, and science in the state of fact. In school teaching the former ought to be excluded. And, as he assumes it to be still in its hypothetical stage, the 
ban of exclusion ought, he thinks, to fall upon the theory of evolution.

I now freely offer myself for judgment before the tribunal whose law is here laid down. First and foremost, then, I have never advocated the introduction of the theory of evolution into our schools. I should even be disposed to resist its introduction before its meaning had been better understood and its utility more fully recognized than it is now by the great body of the community. The theory ought, I think, to bide its time until the free conflict of discovery, argument, and opinion has won for it this recognition. A necessary condition here, however, is that free discussion should not be prevented, either by the ferocity of reviewers or the arm of the law; otherwise, as I said before, the work of social preparation cannot go on. On this count, then, I claim acquittal, being for the moment on the side of Virchow.

Besides the duties of the chair, which I have been privileged to occupy in London for more than a quarter of a century, and which never involved a word on my part, pro or con, in reference to the theory of evolution, I have had the honor of addressing audiences in Liverpool, Belfast, and Birmingham; and in these addresses the theory of evolution, and the connected doctrine of spontaneous generation, have been more or less touched upon. Let us now examine whether in my references I have departed from the views of Virchow or not.

In the Liverpool discourse, after speaking of the theory of evolution when applied to the primitive condition of matter, as belonging to " the dim twilight of conjecture," and affirming that " the certainty of experimental inquiry is here shut out," I sketch the nebular theory as enunciated by Kant and Laplace, and afterward proceed thus: "Accepting some such view of the construction of our system as probable, a desire immediately arises to connect the present life of our planet with the past. We wish to know something of our remotest ancestry. On its first detachment from the sun, life, as we understand it, could not have been present on the earth. How, then, did it come there? 'The thing to be encouraged here is a reverent freedom-a freedom preceded by the hard discipline which 
checks licentiousness in speculation-while the thing to be repressed, both in science and out of it, is dogmatism. And here I am in the hands of the meeting, willing to end but ready to go on. I have no right to intrude upon you unasked the unformed notions which are floating like clouds, or gathering to more solid consistency in the modern speculative mind."

I then notice more especially the basis of the theory. "Those who hold the doctrine of evolution are by no means ignorant of the uncertainty of their data, and they only yield to it a provisional assent. They regard the nebular hypothesis as probable; and, in the utter absence of any proof of the illegality of the act, they prolong the method of nature from the present into the past. Here the observed uniformity of nature is their only guide. Having determined the elements of their curve in a world of observation and experiment, they prolong that curve in to an antecedent world, and accept as probable the unbroken sequence of development from the nebula to the present time." 'Thus it appears that, long antecedent to the publication of his advice, I did exactly what Professor Virchow recommends, showing myself as careful as he could be not to claim for a scientific doctrine a certainty which did not belong to it.

I now pass on to the Belfast Address, and will cite at once from it the passage which has given rise to the most violent animadversion. "Believing as I do in the continuity of nature, I cannot stop abruptly where our microscopes cease to be of use. At this point the vision of the mind authoritatively supplements that of the eye. By an intellectual necessity I cross the boundary of the experimental evidence, and discern in that ' matter' which we, in our ignorance of its latent powers, and notwithstanding our professed reverence for its Creator, have hitherto covered with opprobrium, the promise and potency of all terrestrial life." Without halting for a moment I go on to do the precise thing which Professor Virchow declares to be necessary. "If you ask me," I say, "whether there exists the least evidence to prove that any form of life can be developed ont of matter independently of antecedent life, my reply is that evidence considered perfectly con-. clusive by many has been adduced, and that were we to follow a common example, and accept testimony because 
it falls in with our belief, we should eagerly close with the evidence referred to. But there is in the true man of science a desire stronger than the wish to have his beliefs upheld; namely, the desire to have them true. And those to whom I refer as having studied this question, believing the evidence offered in favor of 'spontaneous generation' to be vitiated by error, cannot accept it. 'T'hey know full well that the chemist now prepares from inorganic matter a vast array of substances, which were some time ago regarded as the products solely of vitality. They are intimately acquainted with the structural power of matter, as evidenced in the phenomena of crystallization. They can justify scientifically their belief in its potency, under the proper conditions, to produce organisms. But, in reply to your question, they will frankly admit their inability to point to any satisfactory experimental proof that life can be developed, save from demonstrable antecedent life." *

Comparing the theory of evolution with other theories, I thus express myself : "The basis of the doctrine of evolution consists, not in an experimental demonstrationfor the subject is hardly accessible to this mode of proofbut in its general harmony with scientific thought. From contrast, moreover, it derives enormous relative strength. On the one side we have a theory, which converts the Power whose garment is seen in the visible universe into an Artificer, fashioned after the human model, and acting by broken efforts, as man is seen to act. On the other side we have the conception that all we see around us and feel within us - the phenomena of physical nature as well as those of the human mind-have their unsearchable roots in a cosmical life, if I dare apply the term, an infinitesimal span of which is offered to the investigation of man." Among thinking people, in my opinion, this last conception has a higher ethical value than that of a personal artificer. Be that as it may, I make here no claim for the theory of evolution which can reasonably be refused.

"'Ten years have elapsed," said Dr. Hooker at Norwich in 1868, $\nmid$ "since the publication of "The Origin of Species by Natural Selection,' and it is therefore not too

*Quoted by Clifford, Nineteenth Century, 3, p. 726.

† President's Address to the British Association. 
early now to ask what progress that bold theory has made in scientific estimation. Since the 'Origin' appeared it has passed through four English editions, ${ }^{*}$ two American, two German, two French, several Russian, a Dutch, and an Italian edition. So far from Natural Selection being a thing of the past [the 'Athenæum' had stated it to be so] it is an accepted doctrine with almost every philosophical naturalist, including, it will always be understood, a considerable proportion who are not prepared to admit that it accounts for all Mr. Darwin assigns to it." In the following year, at Innsbruck, Helmholtz took up the same ground. $\dagger$ Another decade has now passed, and he is simply blind who cannot see the enormous progress made by the theory during that time. Some of the outward and visible signs of this advance are readily indicated. The hostility and fear which so long prevented the recognition of Mr. Darwin by his own university have vanished, and this year Cambridge, amid universal acclamation, conferred on him her Doctor's degree. The Academy of Sciences in Paris, which had so long persistently closed its doors against Mr. Darwin, has also yielded at last; while sermons, lectures, and published articles plainly show that even the clergy have, to a great extent, become acclimatized to the Darwinian air. My brief reference to Mr. Darwin in the Birmingham Address was based upon the knowledge that such changes had been accomplished, and were still going on.

That the lecture of Professor Virchow can, to any practical extent, disturb this progress of public faith in the theory of evolution, I do not believe. That the special lessons of caution which he inculcates were exemplified by me, years before his voice was heard upon this subject, has

* Published by Mr. John Murray, the English publisher of Virchow's lecture. Bane and antidote are thus impartially distributed by the same hand.

+ " Noch besteht lebhafter Streit um die Wahrheit oder Wahrscheinlichkeit von Darwin's Theorie; er dreht sich aber doch eigentlich nur um die Grenzen, welche wir für die Veränderlichkeit der Arten annehmen dürfen. Dass innerhalb derselben Species erbliche Racenverschiedenheiten auf die von Darwin beschriebene Weise zu kommen können, ja dass viele der bisher als verschiedene Species derselben Gattung betrachteten Formen von derselben Urform abstammen, werden auch seine Gegner kaum leugnen."-(Populäre Vorträge.) 
been proved in the foregoing pages. In point of fuct, if he had preceded me instead of following me, and if my desire had been to incorporate his wishes in my words, I could not have accomplished this more completely. It is possible, moreover, to draw the coincident lines still further, for most of what he has said about spontaneous generation might have been uttered by me. I share his opinion that the theory of evolution in its complete form involves the passage from matter which we now hold to be inorganic into organized matter; in other words, involves the assumption that at some period or other of the earth's history there occurred what would be now called "spontaneous generation." I agree with him that "the proofs of it are still wanting." "Whoever," he says, "recalls to mind the lamentable failure of all the attempts made very recently to discover a decided support for the generatio cequivoca in the lower forms of transition from the inorganic to the organic world will feel it doubly serious to demand that this theory, so utterly discredited, should be in any way accepted as the basis of all our views of life." I hold with Virchow that the failures have been lamentable, that the doctrine is utterly discredited. But my position here is so well known that I need not dwell upon it further.

With one special utterance of Professor Virchow his translator connects me by name. "I have no objection," observes the professor, "to your saying that atoms of carbon also possess mind, or that in their connection with the Plistidule company they acquire mind; only $I$ do not know how I am to perceive this." This is substantially what I had said seventeen years previously in the Suturduy Review. The professor continues: "If I explain attraction and repulsion as exhibitions of mind, as psychical phenomena, I simply throw the Psyche out of the window, and the Psyche ceases to be a Psyche." I may say, in passing, that the Psyche that could be cast out of the window is not worth house-room. At this point the translator, who is evidently a man of culture, strikes in with a foot-note. "As an illustration of Professor Virchow's meaning, we may quote the conclusion at which Doctor Tyndall arrives respecting the hypothesis of a human soul, offered as an explanation or a simplification of a series of obscure phenomena-psychical phenomena, as he calls them. 'If you 
are content to make your soul a poetic rendering of a phenomenon which refuses the yoke of rdinary physical laws, I, for one, would not object to this exercise of ideality." * Professor Virchow's meaning, I admit, required illustration; but I do not clearly see how the quotation from me subserves this purpose. I do not even know whether I am cited as meriting praise or deserving opprobrium. In a far coarser fashion this utterance of mine has been dealt with in other places: it may therefore be worth while to spend a few words upon it.

The sting of a wasp at the finger-end announces itself to the brain as pain. The implession made by the sting travels, in the first place, with comparative slowness along the nerves affected; and only when it reaches the brain have we the fact of consciousness. Those who think most pofoundly on this subject hold that a chemical change, which, strictly interpreted, is atomic motion, is in such a case, propagated along the nerve, and communicated to the brain. Again, on feeling the sting I flap the insect violently away. What has caused this motion of my hand? The command from the brain to remove the insect travels along the motor nerves to the proper muscles, and, their force being unlocked, they perform the work demanded of them. But what moved the nerve molecules which unlocked the muscle? The sense of pain, it may be replied. But how can a sense of pain, or any other state of consciousness, make matter move? Not all the sense of pain or pleasure in the world could lift a stone or move a billiardball; why should it stir a molecule? Try to express the motion numerically in terms of the sensation, and the difficulty immediately appears. Hence the idea long ago entertained by philosophers, but lately brought into special prominence, that the physical processes are complete in themselves, and would go on just as they do if consciousness were not at all implicated. Consciousness, on this view, is a kind of by-product inexpressible in terms of force and motion, and unessential to the molecular changes going on in the brain.

Four years ago, I wrote thus: "Do states of consciousness enter as links into the chain of antecedence and

* Presidential address delivered before the Birmingham and Midland Institute, October 1, 1877. Fortnightly Review, Nov. 1, 1877 , p. 607 . 
sequence, which gives rise to bodily actions? Speaking for myself, it is certain that I have no power of imagining such states interposed between the molecules of the brain, and influencing the transference of motion among the molecules. The thing 'eludes all mental presentation.' Hence an iron strength seems to belong to the logic which claims for the brain an automatic action uninfluenced by consciousness. But it is, I believe, admitted by those who hold the antomaton theory, that states of consciousness are produced by the motion of the molecules of the brain; and this production of consciousness by molecular motion is to me quite as unpresentable to the mental vision as the production of molecular motion by consciousness. If I reject one result I must reject both. I, however, reject neither, and thus stand in the presence of two Incomprehensibles, instead of one Incomprehensible." Here I secede from the automaton theory, though maintained by friends who have all my esteem, and fall back upon the avowal which occurs with such wearisome iteration throughout the foregoing pages; namely, my own utter incapacity to grasp the problem.

This avowal is repeated with emphasis in the passage to which Professor Virchow's translator draws attention. What, I there ask, is the causal connection between the objective and the subjective-between molecular motions and states of conscionsness? My answer is: I do not see the connection, nor am I acquainted with anybody who does. It is no explanation to say that the objective and subjective are two sides of one and the same phenomenon. Why should the phenomenon have two sides? This is the very core of the difficulty. There are plenty of molecular motions which do not exhibit this two-sidedness. Does water think or feel when it runs into frost-ferns upon a window pane? If not, why should the molecular motion of the brain be yoked to this mysterious companionconsciousness? We can form a coherent picture of all the purely physical processes-the stirring of the brain, the thrilling of the nerves, the discharging of the muscles, and all the subsequent motions of the organism. We are here dealing with mechanical problems which are mentally presentable. But we can form no picture of the process whereby consciousness emerges, either as a necessary link, or as an accidental by-product, of this series of actions. 
The reverse process of the production of motion by consciousness is equally unpresentable to the mind. We are here in fact on the boundary line of the intellect, where the ordinary canons of science fail to extricate us. If we are true to these canons, we must deny to subjective phenomena all influence on physical processes. 'T'he mechanical philosopher, as such, will never place a state of consciousness and a group of molecules in the relation of mover and moved. Observation proves them to interact; but, in passing from the one to the other, we meet a blank which the logic of deduction is unable to fill. This, the reader will remember, is the conclusion at which I harl arrived more than twenty years ago. I lay bare unsparingly the central difficulty of the materialist, and tell him that the facts of observation which he considers so simple are "almost as difficult to be seized mentally as the idea of a soul." I go further, and say, in effect, to those who wish to retain this idea, "If you abandon the interpretations of grosser minds, who image the soul as a Psyche which could be thrown out of the window-an entity which is usually occupied, we know not how, among the molecules of the brain, but which on due occasion, such as the intrusion of a bullet or the blow of a club, can fly away into other regions of space-if, abandoning this heathen notion, you consent to approach the subject in the only way in which approach is possible-if you consent to make your soul a poetic rendering of a phenomenon which, as I have taken more pains than anybody else to show you, refuses the yoke of ordinary physical laws-then I, for one, would not object to this exercise of ideality." I say it strongly, but with good temper, that the theologian, or the defender of theology, who hacks and scourges me for putting the question in this light is guilty of black ingratitude.

Notwithstanding the agreement thus far pointed out, there are certain points in Professor Virchow's lecture to which I should feel inclined to take exception. I think it was hardly necessary to associate the theory of evolution with socialism; it may be even questioned whether it was correct to do so. As Lange remarks, the aim of socialism, or of its extreme leaders, is to overthrow the existing systems of government, and anything that helps them to 
this end is welcomed, whether it be atheism or papal in. fallibility. For long years the socialists saw church and state united against them, and both were therefore regarded with a common hatred. But no sooner does a serious difference arise between church and state, than a portion of the socialists begin immediately to dally with the former.* The experience of the last German elections illustrates Lange's position. Far nobler and truer to my mind than this fear of promoting socialism by a scientific theory which the best and soberest hearls in the world have substantially accepted, is the position assumed by Helmholtz, who in his " Popular Lectures" describes Darwin's theory as embracing "an essentially new creative thought" (einen wesentlich neuen schöpferischen Gedanken), and who illustrates the greatness of this thought by copious references to the solutions, previuusly undreamed of, which it offers of the enigmas of life and organization. He points to the clouds of error and confusion which it has already dispersed, and shows how the progress of discovery since its first enunciation is simply a record of the approach of the theory toward complete demonstration. One point in this "popular" exposition deserves especial mention here. Helmholtz refers to the dominant position acquired by Germany in physiology and medicine, while other nations have kept abreast of her in the investigation of inorganic nature. He claims for German men the credit of pursuing with unfiagging and self-denying industry, with purely ideal aims, and without any immediate prospect of practical utility, the cultivation of pure science. But that which has determined German superiority in the fields referred to was, in his opinion, something different from this. Inquiries into the nature of life are intimately connected with psychological and ethical questions; and he claims for his countrymen a greater fearlessness of the consequences which a full knowledge of the truth may here carry along with it, than reigns among the inquirers of other nations. And why is this the case? "England and France," he says, "possess distinguished investigatorsmen competent to follow up and illustrate with vigorous energy the methods of natural science; but they have hitherto been compelled to bend before social and theolog-

* "Geschichte des Materialismus," 2e Auflage, vol. ii., p. 538. 
ical prejudices, and could only utter their convictions under the penalty of injuring their social influence and usefulness. Germany has gone forward more courageously. She has cherished the trust, which has never been deceived, that complete truth carries with it the antidote against the bane and danger which follow in the train of half knowledge. A cheerfully laborious and temperate people -a people morally strong-can well afford to look truth full in the face. Nor are they to be ruined by the enunciation of one-sided theories, even when these may appear to threaten the bases of society." 'These words of Helmholtz are, in my opinion, wiser and more applicable to the condition of Germany at the present moment than those which express the fear's of Professor Virchow. It will be remembered that at the time of his lecture his chief anxieties were directed toward France; but France has since that time given ample evidence of her ability to crush, not only socialists, but anti-socialists, who would impose on her a yoke which she refuses to bear.

In close connection with these utterances of Helmholtz, I place another utterance not less noble, which I trust was understood and appreciated by those to whom it was addressed. " "If," said the president of the British Association in his opening address in Dublin, "we could lay down beforehand the precise limits of possible knowledge, the problem of physical science would be already half solved. But the question to which the scientific explorer has often to address himself is, not merely whether he is able to solve this or that problem; but whether he can so far unravel the tangled threads of the matter with which he has to deal, as to weave them into a definite problem at all. . . If his eye seem dim, he must look steadfastly and with hope into the misty vision, until the very clouds wreathe themselves into definite forms. If his ear seem dull, he must listen patiently and with sympathetic trust to the intricate whisperings of Nature-the goddess, as she has been called, of a hundred voices - until here and there he can pick out a few simple notes to which his own powers can resound. If, then, at a moment when he finds himself placed on a pinnacle from which he is called upon to take a perspective survey of the range of science, and to tell us what he can see from his vantage ground; if at such a moment, after straining his gaze to the very verge of the horizon, and 
after describing the most distant of well-defined objects, he should give utterance also to some of the subjective impressions which he is conscious of receiving from regions beyond; if he should depict possibilities which seem opening to his view; if he should explain why he thinks this a mere blind alley and that an open path; then the fault and the loss would be alike ours if we refused to listen calmly, and temperately to form our own judgment on what we hear; then assuredly it is we who would be committing the error of confounding matters of fact with matters of opinion, if we failed to discriminate between the various elements contained in such a discourse, and assumed that they had been all put on the same footing."

While largely agreeing with him, I cannot quite accept the setting in which Professor Virchow places the confessedly abortive attempts to secure an experimental basis for the doctrine of spontaneous generation. It is not a doctrine "so discredited" that some of the scientific thinkers of England accept " as the basis of all their views of life." Their induction is by no means thus limited. They have on their side more than the "reasonable probability" deemed sufficient by Bishop Butler for practical guidance in the gravest affairs that the members of the solar system which are now discrete once formed a continuous mass; that in the course of untold ages, during which the work of condensation, through the waste of heat in space, went on, the planets were detached; and that our present sun is the residual nucleus of the flocculent or gaseous ball from which the planets were successively separated. Life, as we define it, was not possible for xons subsequent to this separation. When and how did it appear? I have already pressed this question, but have received no answer.* If, with Professor Knight, we regard the Bible account of the introduction of life upon the earth as a poem, not as a statement of fact, where are we to seek for guidance as to the fact? 'There does not exist a barrier possessing the strength of a cobweb to oppose to the hypothesis which ascribes the appearance of life to that " potency of matter"

*In the "Apology for the Belfast Address," the question is reasoned out. 
which finds expression in natural evolution.* This hypothesis is not without its difficulties, but they vanish when compared with those which encumber its rivals. There are various facts in science obviously connected, and whose connections we are unable to trace; but we do not think of filling the gap between them by the intrusion of a separable spiritual agent. In like manner though we are unable to trace the course of things from the nebula, when there was no life in our sense, to the present earth where life abounds, the spirit and practice of science pronounce against the intrusion of an anthropomorphic creator. Theologians must liberate and refine their conceptions or be prepared for the rejection of them by thoughtful minds. It is they, not we, who lay claim to knowledge never given to man. Our refusal of the creative hypothesis is less an assertion of knowledge than a protest against the assumption of knowledge which must long, if not always, lie beyond us, and the claim to which is a source of perpetual confusion. At the same time, when I look with strenuous gaze into the whole problem as far as my capacities allow, overwhelming wonder is the predominant feeling. This wonder has come to me from the ages just as much as my understanding, and it has an equal right to satisfaction. Hence I say, if, abandoning your illegitimate claim to knowledge, you place, with Job, your forehead in the dust and acknowledge the authorship of this universe to be past finding out-if, having made this confession, and relinquished the views of the mechanical theologian, you desire for the satisfaction of feelings which I admit to be, in great part, those of humanity at large, to give ideal form to the Power that moves all things - it is not by me that you will find objections raised to this exercise of ideality, if it be only consciously and worthily carried out.

Again, I think Professor Virchow's position, in regard to the question of contagium animatum, is not altogether that of true philosophy. He points to the antiquity of the doctrine. "It is lost," he says, " in the darkness of the

* "We feel it an undeniable necessity," says Professor Virchow, " not to sever the organic world from the whole, as if it were something disjoined from the whole." This grave statement cannot be weakened by the subsequent pleasantry regarding " C'arbon \& Co." 
mildle ages. We have received this name from our forefathers, and it already appears distinctly in the sixteenth century. We possess several works of that time which put forward contagium animatum as a scientific doctrine, with the same confidence, with the same sort of proof, with which the 'Plastidulic soul' is now set forth."

These speculations of our "forefathers" will appeal differently to different minds. By some they will be dismissed with a sneer; to others they will appeal as proofs of genius on the part of those who enunciated them. 'There are men, and by no means the minority, who, however wealthy in regard to facts, can never rise into the region of principles; and they are sometimes intolerant of those who can. They are formed to plod meritoriously on the lower levels of thought, unpossessed of the pinions necessary to reach the heights. They cannot realize the mental act-the act of inspiration it might well be called -by. which a man of genius, after long pondering and proving, reaches a theoretic conception which unravels and illuminates the tangle of centuries of observation and experiment. 'There are minds, it may be said in passing, who at the present moment stand in this relation to $\mathrm{Mr}$. Darwin. For my part, I should be inclined to ascribe to penetration rather than to presumption the notion of a contagium animatum. He who invented the term ought, I think, to be held in esteem; for he had before him tho quantity of fact, and the measure of analogy, that would justify a man of genius in taking a step so bold. "Nevertheless," says Professor Virchow, "no one was able throughout a long time to discover these living germs of disease. The sixteenth century did not find them, nor did the seventeenth, nor the eighteenth." But it may be urged, in reply to this, that the theoretic conjecture often legitimately comes first. It is the forecast of genius which anticipates the fact and constitutes a spur toward its discovery. If, instead of being a spur, the theoretic guess rendered men content with imperfect knowledge, it would be a thing to be deprecated. But in modern investigation this is distinctly not the case; Darwin's theory, for example, like the undulatory theory, has been a motive power and not an anodyne. "At last," continues Professor Virchow, "in the nineteenth century we have begun little by little really to find contayia animala." So much 
the more honor, I infer, is due to those who, three celituries in advance, so put together the facts and analogies of contagious disease as to divine its root and character. Professor Virchow seems to deprecate the "obstinacy" with which this notion of a contagium vivum emerged. Here I should not be inclined to follow him; because I do not know, nor does he tell me, how much the discovery of facts in the nineteenth century is indebted to the stimulus derived from the theoretic discussions of preceding centuries. The genesis of scientific ideas is a subject of profound interest and importance. He would be but a poor philosopher who would sever modern chemistry from the efforts of the alchemists, who would detach modern atomic doctrines from the speculations of Lucretius and his predecessors, or who would claim for our present knowledge of contagia an origin altogether independent of the efforts of our "forefathers" to penetrate this enigma.

Finally, I do not know that I should agree with Professor Virchow as to what a theory is or ought to be. I call a theory a principle or conception of the mind which accounts for observed facts, and which helps us to look for and predict facts not yet observed. Every new discovery which fits into a theory strengthens it. The theory is not a thing complete from the first, but a thing which grows, as it were asymptotically, toward certainty. Darwin's theory, as pointed out nine and ten years ago by Helmholtz and Hooker, was then exactly in this condition of growth; and had they to speak of the subject to-day they would be able to announce an enormous strengthening of the theoretic fiber. Fissures in continuity which then existed, and which left little hope of being ever spanned, have been since filled in, so that the further the theory is tested the more fully does it harmonize with progressive experience and discovery. We shall probably never fill all the gaps; but this will not prevent a profound belief in the truth of the theory from taking root in the general mind. Much less will it justify a total denial of the theory. The man of science who assumes in such a case the position of a denier is sure to be stranded and isolated. The proper attitude, in my opinion, is to give to the theory during the phases of its growth as nearly as 
possible a proportionate assent; and, if it be a theory which influences practice, our wisdom is to follow its probable suggestions where more than probability is for the moment unattainable. I write thus with the theory of contagium vivum more especially in my mind, and must regret the attitude of denial assumed by Professor Virchow toward that theory. "I must beg my friend Klebs to pardon me," he says, "if, notwithstanding the late advances made by the doctrine of infectious fungi, I still persist in my reserve so far as to admit only the fungus which is really proved, while I deny all other fungi so long as they are not actually brought before me." Professor Virchow, that is to say, will continue to deny the germ theory, however great the probabilities on its side, however Humerous be the cases of which it renders a just account, until it has ceased to be a theory at all, and has become a congeries of sensible facts. Had he said, "As long as a single fungus of disease remains to be discorered, it is your bounden duty to search for it," I should cordially agree with him. But by his unreserved denial he quenches the light of probability which ought to guide the practice of the medical man. Both here and in relation to the theory of evolution excess upon one side has begotten excess upon the other.

\section{CHAPTER XXXVIII.}

\section{THE ELECTRIC LIGHT.*}

THE SUBJECT of this evening's discourse was proposed by our late honorary secretary. $\dagger$ That word "late" has for me its own connotations. It implies, among other things, the loss of a comrade by whose side I have worked for thirteen years. On the other hand, regret is not without its opposite in the feeling with which I have seen him rise by sheer intrinsic merit, moral and intellectual, to the highest official position which it is in the power of English science to bestow. Well, he, whose constant desire and

* A discourse delivered at the Royal Institution of Great Britain on Friday, January 17, 1879, and introduced here as the latest Fragment.

+ Mr. William Spottis woode, late president of the Royal Society. 
practice were to promote the interests and extend the usefulness of this institution, thought that at a time when the electric light occupied so much of public attention, a few sound notions regarding it, on the more purely scientific side, might, to use his own pithy expression, be " planted" in the public mind. I am here to-night with the view of trying, to the best of my ability, to realize the idea of our friend.

In the year 1800, Volta announced his immortal discovery of the pile. Whetted to eagerness by the previous conflict between him and Galvani, the scientific men of the age flung themselves with ardor upon the new discovery, repeating Volta's experiments, and extending them in many ways. The light and heat of the voltaic circuit attracted marked attention, and in the innumerable tests and trials to. which this question was subjected, the utility of platinum and charcoal as means of exalting the light was on all hands recognized. Mr. Children, with a battery surpassing in strength all its predecessors, fused platinum wires eighteen inches long, while "points of charcoal produced a light so vivid that the sunshine, compared with it, appeared feeble." * Such effects reached their culmination when, in 1808 , through the liberality of a few members of the Royal Institution, Davy was enabled to construct a battery of two thousand pairs of plates, with which he afterward obtained calorific and luminous effects far transcending anything previously observed. The arc of flame between the carbon terminals was four inches long, and by its heat quartz, sapphire, magnesia, and lime, were melted like wax in a candle flame; while fragments of diamond and plumbago rapidly disappeareu as if reduced to vapor. $t$

The first condition to be fulfilled in the development of leat and light by the electric current is that it shall en-

* Davy, "Chemical Philosophy," p. 110.

+ In the concluding lecture at the Royal Institution in June, 1810 , Davy showed the action of this battery. He then fused iridium, the alloy of iridium and osmium, and other refractory substances. Philosophical Magazine, vol. xxxv., p. 463. Quetelet assigns the first production of the spark between coal-points to Curtet in 1802. Davy certainly in that year showed the carbon light with a battery of 150 pairs of plates in the theater of the Royal Institution ("Jour. Roy. Inst.," vol. i., p. 166). 
counter and overcome resistance. Flowing through a perfect conductor, no matter what the strength of the current might be, neither beat nor light could be developed. A rod of unresisting copper carries away uninjured and unwarmed an atmospheric discharge competent to shiver to splinter's a resisting oak. I send the selfsame current through a wire composed of alternate lengths of silver and platinum. The silver offers little resistance, the platinum offers much. The consequence is that the platinum is raised to a white heat, while the silver is not visibly warmed. The same holds good with regard to the carbon terminals employed for the production of the electric light. 'The interval between them offers a powerful resistance to the passage of the current, and it is by the gathering up of the force necessary to burst across this interval that the voltaic current is able to throw the carbon into that state of violent intestine commotion which we call heat, and to which its effulgence is due. The smallest interval of air usually suffices to stop the current. But when the carbon points are first brought together and then separated, there occurs between them a discharge of incandescent matter which carries, or may carry, the current over a considerable space. The light comes almost wholly from the incandescent carbons. 'The space between them is filled with a blue flame which, being usually bent by the earth's magnetism, receives the name of the Voltaic Arc.*

For seventy years, then, we have been in possession of this transcendent light without applying it to the illumination of our streets and houses. Such applications suggested themselves at the outset, but there were grave

* The part played by resistance is strikingly illustrated by the deportment of silver and thallium when mixed together and volatilized ir the arc. The current first selects as its carrier the most volatile metal, which in this case is thallium. While it continues abundant, the passage of the current is so free-the resistance to it is so small-that the heat generated is incompetent to volatilize the silver. As the thallium disappears the current is forced to concentrate its power; it presses the silver into its service, and finaily fills the space between the carbons with a vapor which, as long as the necessary resistance is absent, it is incompetent to produce. I have on a former nccasion drawn attention to a danger which besets the spectroscopist when operating upon a mixture of constituents volatile in different degrees. When, in 1872, I first observed the effect here described had I not known that silver was present, I should have inferred its absence. 
difficulties in their way. The first difficulty arose from the waste of the carbons, which are dissipated in part by ordinary combustion, and in part by the electric transfer of matter from the one carbon to the other. To keep the carbons at the proper distance asunder regulators were devised, the earliest, I believe, by Staite, and the most successful by Duboseq, Foucault, and Serrin, who have been succeeded by Holmes, Siemens, Browning, Carré, Gramme, Lontin and others. By such arrangements the first difficulty was practically overcome; but the second, a graver one, is probably inseparable from the construction of the voltaic battery. It arises from the operation of that inexorable law which throughout the material universe demands an eye for an eye, and a tooth for a tooth, refusing to yield the faintest glow of heat or glimmer of light without the expenditure of an absolutely equal quantity of some other power. Hence, in practice, the desirability of any transformation must depend upon the value of the product in relation to that of the power expended. The metal zinc can be burned like paper; it might be ignited in a flame, but it is possible to avoid the introduction of all foreign heat and to burn the zine in air of the temperature of this room. This is done by placing zinc foil at the focus of a concave mirror, which concentrates to a point the divergent electric beam, but which does not warm the air. The zinc burns at the focus with a violet flame, and we could readily determine the amount of heat generated by its combustion. But zinc can be burned not only in air but in liquids. It is thus burned when acidulated water is poured over it; it is also thus burned in the voltaic battery. Here, however, to obtain the oxygen necessary for its combustion, the zinc has to dislodge the hydrogen with which the oxygen is combined. The consequence is that the heat due to the combustion of the metal in the liquid falls short of that developed by its combustion in air, by the exact quantity necessary to separate the oxygen from the hydrogen. Fully four-fifths of the total heat are used up in this molecular work, only one-fifth remaining to warm the battery. It is upon this residue that we must now fix our attention, for it is solely ont of it that we manufacture our electric light

Before you are two small voltaic batteries of ten cells each. The two ends of one of them are united by a thick 
copper wire, while into the circuit of the other a thin platinum wire is introduced. The platinum glows with a white heat, while the copper wire is not sensibly warmed.

Now an ounce of zinc, like an ounce of coal, produces by its complete combustion in air a constant quantity of heat. The total heat developed by an ounce of zinc through its union with oxygen in the battery is also absolutely invariable. Let our two batteries, then, continue in action until an ounce of zine in each of them is consumed. In the one case the heat generated is purely domestic, being liberated on the hearth where the fuel is burned, that is to say in the cells of the battery itself. In the other case, the heat is in part domestic and in part foreign-in part within the battery and in part outside. One of the fundamental truths to be borne in mind is that the sum of the foreign and domestic-of the external and internal-heats is fixed and invariable. Hence, to have heat outside, you must draw upon the heat within. These remarks apply to the electric light. By the intermediation of the electric current the moderate warmth of the battery is not only carried away, but concentrated so as to produce, at any distance from its origin, a heat next in order to that of the sun. The current might therefore be defined as the swift carrier of heat. Loading itself here with invisible power, by a process of transmutation which outstrips the dreams of the alchemist, it can discharge its load, in the fraction of a second, as light and heat, at the opposite side of the world.

Thus, the light and heat produced outside the battery are derived from the metallic fuel burned within the battery; and, as zinc happens to be an expensive fuel, though we have possessed the electric light for more than seventy years, it has been too costly to come into general use. But within these walls, in the autumn of 1831, Faraday discovered a new source of electricity, which we have now to investigate. On the table before me lies a coil of covered copper wire, with its ends disunited. I lift one side of the coil from the table, and in doing so exert the muscular effort necessary to overcome the simple weight of the coil. I unite its two ends and repeat the experiment. The effort now required, if accurately measured, would be found greater than before. In lifting the coil I cut the lines of the earth's magnetic force, such cutting, as proved 
by Faraday, being always accompanied, in a closed conductor, by the production of an "induced" electric current which, as long as the ends of the coil remained separate, had no circuit through which it could pass. The current here evoked subsides immediately as heat; this heat being the exact equivalent of the excess of effort just referred to as over and above that necessary to overcome the simple weight of the coil. When the coil is liberated it falls back to the table, and when its ends are united it encounters a resistance over and above that of the air. It generates an electric current opposed in direction to the first, and reaches the table with a diminished shock. The amount of the diminution is accurately represented by the warmth which the momentary current develops in the coil. Various devices were employed to exalt these induced currents, among which the instruments of Pixii, Clarke, and Saxton were long conspicuous. Faraday, indeed, foresaw that such attempts were sure to be made; but he chose to leave them in the hands of the mechanician, while he himself pursued the deeper study of facts and principles. "I have rather," he writes in 1831, "been desirous of discovering new facts and new relations dependent on magneto-electric induction, than of exalting the force of those alrealy obtained; being assured that the latter would find their full development hereafter."

For more than twenty years magneto-electricity had subserved its first and noblest purpose of augmenting our knowledge of the powers of nature. It had been discovered and applied to intellectual ends, its application to practical ends being still unrealized. The Drummond light had raised thoughts and hopes of vast improvements in public illumination. Many inventors tried to obtain it cheaply; and in 1853 an attempt was made to organize a company in Paris for the purpose of procuring, through the decomposition of water by a powerful magneto-electric machine constructed by M. Nollet, the oxygen and hydrogen necessary for the lime light. The experiment failed, but the apparatus by which it was attempted suggested to $\mathrm{Mr}$. Holmes other and more hopeful applications. Abandoning the attempt to produce the lime light, with persevering skill IIolmes continued to improve the apparatus and to angment its power, until it wasfinally able to yield a magneto-electric light comparable to that of 
the voltaic battery. Judged by later knowledge, this first machine would be considered cumbrous and defective in the extreme; but judged by the light of antecedent events, it marked a great step forward.

Faraday was profoundly interested in the growth of his own discovery. The Elder Brethren of the Trinity House had had the wisdom to make him their "Scientific Adviser;" and it is interesting to notice in his reports regarding the light, the mixture of enthusiasm and caution which characterized him. Enthusiasm was with him a motive power, guided and controlled by a disciplined judgment. He rode it as a charger, holding it in by a strong rein. While dealing with Holmes, he states the case of the light pro and con. He checks the ardor of the in ventor, and, as regards cost, rejecting sanguine estimates, he insists over and over again on the necessity of continued experiment for the solution of this important question. His matured opinion was, however, strongly in favor of the light. With reference to an experiment made at the South Foreland on the 20th of April, 1859, he thus expresses himself: "The beauty of the light was wonderful. At a mile off, the apparent streams of light issuing from the lantern were twice as long as those from the lower lighthouse, and apparently three or four times as bright. The horizontal plane in which they chiefly took their way made all above or below it black. The tops of the hills, the churches, and the houses illuminated by it were striking in their effect upon the eye." Further on in his report he expresses himself thus:- "In fulfillment of this part of my duty, I beg to state that, in my opinion, Professor Holmes has practically established the fitness and sufficiency of the magneto-electric light for lighthouse purposes, so far as its nature and management are concerned. The light produced is powerful beyond any other that I have yet seen so applied, and in principle may be accumulated to any degree; its regularity in the lantern is great; its management easy, and its care there may be confided to attentive keepers of the ordinary degree of intellect and knowledge." Finally, as regards the conduct of Professor Holmes during these memorable experiments, it is only fair to add the following remark with which Faralay closes the report submitted to the Elder Brethren of the 'Trinity IIouse on the 29th of April, 1859: "I 
must bear my testimony," he says, "to the perfect openness, candor, and honor of Professor Holmes. He has answered every question, concealed no weak point, explained every applied principle, given every reason for a change either in this or that direction, during several periods of close questioning, in a manner that was very agreeable to rne, whose duty it was to search for real faults or possible objections, in respect both of the present time and the future."*

Soon afterward the Elder Brethren of the Trinity Ilouse had the intelligent courage to establish the machines of Itolmes permanently at Dungeness, where the magneto-electric light continued to shine for many years.

The magneto-electric machine of the Alliance Company soon succeeded to that of Holmes, being in various ways a very marked improvement on the latter. Its currents were stronger and its light was brighter than those of its predecessor. In it, moreover, the commutator, the flashing and destruction of which were sources of irregularity and deterioration in the machine of Holmes, was, at the suggestion of M. Masson, $\nmid$ entirely abandoned; alternating currents instead of the direct current being employed. M. Serrin modified his excellent lamp with the express view of enabling it to cope with alternating currents. During the International Exhibition of 1862, where the machine was shown, M. Berlioz offered to dispose of the invention to the Elder Brethren of the Trinity House. 'They referred the matter to Faraday, and he replied as follows: "I am not aware that the Trinity House authorities have advanced so far as to be able to decide whether they will require more magneto-electric machines, or whether, if they should require them, they see reason to suppose the means of their supply in this country, from the source already open to them, would not be sufficient. Therefore I do not see that at present they want to purchase a machine." Faraday was obviously swayed by the desire to protect the interests of Holmes, who had borne the burden and heat which fall upon the pioneer. The Alliance

* Holmes' first offer of his machine to the 'Trinity House bears date February 2, 1857.

† Du Moncel, "l'Electricité," August, 1878, p. 150. 
machines were introduced with success at Cape la Hève, near Havre; and the Elder Brethren of the 'Trinity House, determined to have the best available apparatus, lecided, in 1868, on the introduction of machines on the Alliance principle into the lighthouses at Souter Point and the South Foreland. 'These machines were constructed by Professor Holmes, and they still continue in operation. With regard, then, to the application of electricity to lighthouse purposes, the course of events was this: The Dungeness light was introduced on January 31, 1862; the light at La Iìve on December 26, 1863, or nearly two years later. But Faraday's experimental trial at the South Foreland preceded the lighting of Dungeness by more than two years. 'The electric light was afterward established at Cape Grisnez. The light was started at Souter Point on January 11, 18\%1; and at the South Foreland on January 1, 1872. At the Lizard, which enjoys the newest and most powerful development of the electric light, it began to shine on January 1, 1878.

I have now to revert to a point of apparently small moment, but which really constitutes an important step in the development of this subject. I refer to the form given in $185 \%$ to the rotating armature by Dr. Werner Siemens, of Berlin. Instead of employing coils wound transversely round cores of iron, as in the machine of Saxton, Siemens, after giving a bar of iron the proper shape, wound his wire longitudinally round it, and obtained thereby greatly angmented effects between suitably placed magnetic poles. Such an armature is employed in the small magneto-electric machine which I now introduce to your notice, and for which the institution is indebted to Mr. Henry Wilde, of Manchester. 'There are here sixteen permanent horseshoe magnets placed parallel to each other, and between their poles a Siemens armature. The two ends of the wire which surrounds the armature are now disconnected. In turning the handle and causing the armature to rotate, I simply overcome ordinary mechanical friction. But the two ends of the armature coil can be united in a moment, and when this is done I immediately experience a greatly increased resistance to rotation. Something over and above the ordinary friction of the machine is now to be overcome, and by the expenditure of 
an additional amount of muscular force $I$ am able to overcome it. The excess of labor thus thrown upon my arm has its exact equivalent in the electric currents generated, and the heat produced by their subsidence in the coil of the armature. A portion of this heat may be rendered visible by connecting the two ends of the coil with a thin platinum wire. When the bandle of the machine is rapidly turned the wire glows, first with a red heat, then with a white heat, and finally with the heat of fusion. The moment the wire melts, the circuit round the armature is broken, an instant relief from the labor thrown upon the arm being the consequence. Clearly realize the equivalent of the heat here developed. During the period of turning the machine a certain amount of combustible substance was oxidized or burned in the muscles of my arm. Had it done no external work, the matter consumed would have produced a definite amount of heat. Now, the muscular heat actually developed during the rotation of the machine fell short of this definite amount, the missing heat being reproduced to the last fraction in the glowing platinum wire and the other parts of the machine. Here, then, the electric current intervenes between my muscles and the generated heat, exactly as it did a moment ago between the voltaic battery and its generated heat. The electric current is to all intents and purposes a vehicle which transports the heat both of muscle and battery to any distance from the hearth where the fuel is consumed. Not only is the current a messenger, but it is also an intensifier of magical power. The temperature of my arm is, in round numbers, 100 degrees Fahr., and it is by the intensification of this heat that one of the most refractory of metals, which requires a heat of 3,600 degrees Fahr. to fuse it, has been reduced to the molten condition.

Zinc, as I have said, is a fuel far too expensive to permit of the electric light produced by its combustion being ased for the common purposes of life, and you-will readily perceive that the human muscles, or even the muscles of a horse, would be more expensive still. Here, however, we can employ the force of burning coal to turn our machine, and it is this employment of our cheapest fuel, rendered possible by Faraday's discovery, which opens out to us the prospect of being able to apply the electric light to public use. 
In 1866 a great step in the intensification of induced currents, and the consequent augmentation of the magnetoelectric light, was taken by Mr. Henry Wilde. It fell to my lot to report upon them to the Royal Society, but before doing so I took the trouble of going to Manchester to witness Mr. Wilde's experiments. He operated in this way: starting from a small machine like that worked in your presence a moment ago, he employed its current to excite an electro-magnet of a peculiar shape, between whose poles rotated a Siemens armature; * from this armature currents were obtained vastly stronger than those generated by the small magneto-electric machine. These currents might have been immediately employed to produce the electric light; but instead of this they were conducted round a second electro-magnet of vast size, between whose poles rotated a Siemens armature of corresponding dimensions. Three armatures therefore were involved in this series of operations: first, the armature of the small magneto-electric machine; secondly, the armature of the first electro-magnet, which was of considerable size; and, thirdly, the armature of the second electro-magnet, which was of vast dimensions. With the currents drawn from this third armature, Mr. Wilde obtained effects, both as regards heat and light, enormously transcending those previously known. $†$

But the discovery which, above all others, brought the practical question to the front is now to be considered. On the 4 th of February, $186 \%$, a paper was received by the Royal Society from Dr. William Siemens bearing the title, "On the Conversion of Dynanic into Electrical Force without the use of Permanent Magnetism." $\ddagger$ On the 14 th

* Page and Moigno had previously shown that the magneto-electric current could produce powerful electro-magnets.

† Mr. Wilde's paper is published in the "Philosophical 'Transactions" for $186 \%$, p. 89 . My opinion regarding Wilde's machine was briefly expressed in a report to the Elder Brethren of the 'Trinity House on May 17, 1866: "It gives me pleasure to state that the machine is exceedingly effective, and that it far transcends in power all other apparatus of the kind."

‡ A paper on the same subject, by Dr. Werner Siemens, was read on January 17, 1867 , before the Academy of Ściences in Berlin. In a letter to Engineering, No. 622, p. 45, Mr. Robert Sabine states that Professor Wheatstone's machines were constructed by Mr. Stroh in the months of July and August, 1866. I do not doubt Mr. 
of February a paper from Sir Charles Wheatstone was received, bearing the title, "On the Augmentation of the Power of a Magnet by the reaction thereon of Currents induced by the Magnet itself." Both papers, which dealt with the same discovery, and which were illustrated by experiments, were read upon the same night, viz., the 14th of February. It would be difficult to find in the whole field of science a more beautiful example of the interaction of natural forces than that set forth in these two papers. You can hardly find a bit of iron-you can hardly pick up an old horseshoe, for example-that does not possess a trace of permanent magnetism; and from such a small beginning Siemens and Wheatstone have taught us to rise by a series of interactions between magnet and armature to a magnetic intensity previously unapproached. Conceive the Siemens armature placed between the poles of a suitable electro-magnet. Suppose this latter to possess at. starting the faintest trace of magnetism; when the armature rotates, currents of infinitesimal strength are generated in its coil. Let the ends of that coil be connected with the wire surrounding the electro-magnet. 'The infinitesimal current generated in the armature will then circulate round the magnet, augmenting its intensity by an infinitesimal amount. The strengthened magnet instantly reacts upon the coil which feeds it, producing a current of greater strength. 'This current again passes round the magnet, which immediately brings its enhanced power to bear upon the coil. By this play of mutual give and take between magnet and armature, the strength of the former is raised in a very brief interval from almost nothing to complete magnetic saturation. Such a magnet and armature are able to produce currents of extraordinary power, and if an

Sabine's statement; still it would be dangerous in the highest degree to depart from the canon, in asserting which Faraday was specially strenuous, that the date of a discovery is the date of its publication. 'Toward the end of December, 1866, Mr. Alfred Varley also lodged a provisional specification (which, I beliere, is a sealed document) embodying the principles of the dynamo-electric machine, but some years elapsed before he male anything public. His brother, Mr. ('romwell Varley, when writing on this sulject in 1867, does not mention him (Proc. Roy. Soc., March 14, 186\%). It probably marks a national trait, that sealed communications, though allowed in France, have never been recognized by the scientific societies of England. 
electric lamp be introduced into the common circuit of magnet and armature, we can rearlily obtain a most powerful light.* By this discovery, then, we are enabled to avoid the trouble and expense involved in the employment of permanent magnets; we are also enabled to drop the exciting magneto-electric machine, and the duplication of the electro-magnets. By it, in short, the electric generator is so far simplified, and reduced in cost, as to enable electricity to enter the lists as the rival of our present means of illumination.

Soon after the announcement of their discovery by Siemens and Wheatstone, Mr. Holmes, at the instance of the Elder Brethren of the 'Trinity House, endeavored to turn this discovery to accomt for lighthouse purposes. Already, in the spring of 1869, he had constructed a machine which, though hampered with defects, exhibited extraordinary power. The light was developed in the focus of a dioptric apparatus placed on the Trinity Wharf at Blackwall, and witnessed by the Elder Brethren, Mr. Douglass, and myself, from an observatory at Charlton, on the opposite side of the Thames. Falling upon the suspended haze, the light illuminated the atmosphere for miles all round. Anything so sunlike in splendor had not, I imagine, been previously witnessed. The apparatus of Holmes, however, was rapidly distanced by the safer and more powerful machines of Siemens and Gramme.

As regards lighthouse illumination, the next step forward was taken by the Elder Brethren of the Trinity House in 18\%6-7\%. Having previously decided on the establishment of the electric light at the Lizard in Cornwall, they instituted, at the time referred to, an elaborate series of comparative experiments wherein the machines of Holmes, of the Alliance Company, of Siemens, and of Gramme, were pitted against each other. The Siemens and the Gramme machines delivered direct currents, while those of Holmes and the Alliance Company delivered alternating currents. The light of the latter was of the same intensity in all azimuths; that of the former was different in different azimuths, the discharge being so regulated as to yield a gush of light of special intensity in one direction.

* In 1867 Mr. I add introluced the modification of dividing the armature into two separate coils, one of which fed the electro-magnets, while the other yielded the induced currents. 
The following table gives in standard candles the performance of the respective machines: *

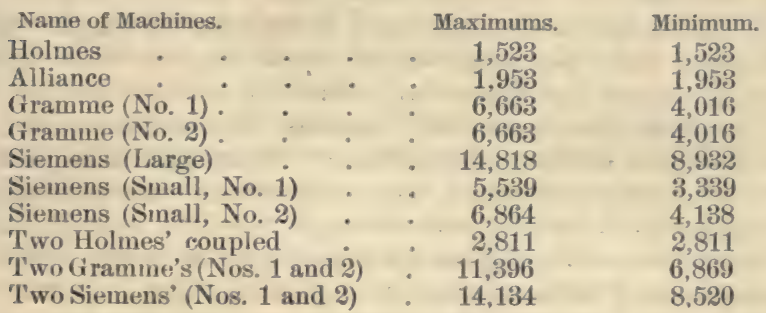

These deterninations were made with extreme care and accuracy by $\mathrm{Mr}$. Douglass, the engineer-in-chief, and $\mathrm{Mr}$. Ayres, the assistant engineer of the Trinity House. It is practically impossible to compare photometrically and directly the flame of the candle with these suulike lights. A light of intermediate intensity-that of the six-wick I'rinity oil lamp-was therefore in the first instance compared with the electric light. The candle power of the oil lamp being afterward determined, the intensity of the electric light became known. The numbers given in the table prove the superiority of the Alliance machine over that of Holmes. They prove the great superiority both of the Gramme machine and of the small Siemens machine over the Alliance. The large Siemens machine is shown to yield a light far exceeding all the others, while the coupling of two Grammes, or of two Siemens together, here effected for the first time, was followed by a very great augmentation of the light, rising in the one case from 6,663 candles to 11,396 , and in the other case from 6,864 candles to 14,134 . Where the arc is single and the external resistance small, great advantages attach to the Siemens light. After this contest, which was conducted

* Observations from the sea on the night of November 21, 1876, made the Gramme and small Siemens practically equal to the Alliance. But the photometric observations, in which the external resistance was abolished, and previous to which the light-keepers had become more skilled in the management of the direct current showed the differences recorded in the table. A close inspection of these powerful lights at the South Foreland caused my face to peel, as if it had been irritated by an Alpine sun. 
throughout in the most amicable manner, Siemens machines of type No. ¿2 were chosen for the Lizard.*

We have machines capable of sustaining a single light, and also machines capable of sustaining several lights. The Gramme machine, for example, which ignites the Jablochkoff candles on the 'Thames Embankment and at the Holborn Viaduct, delivers four currents, each passing through its own circuit. In each circuit are five lamps through which the current belonging to the circuit passes in succession. The lights correspond to so many resisting spaces, over which, as already explained, the current has to leap; the force which accomplishes the leap being that which produces the light. Whether the current is to be competent to pass through five lamps in succession, or to sustain only a single lamp, depends entirely upon the will and skill of the maker of the machine. He has, to guide him, definite laws laid down by Ohm half a century ago, by which he must abide.

Ohm has taught us how to arrange the elements of a voltaic battery so as to augment indefinitely its electro-motive force-that force, namely, which urges the current forward and enables it to surmount external obstacles. We have only to link the cells together so that the current generated by each cell shall pass through all the others, and add its electro-motive force to that of all the others. We increase, it is true, at the same time, the resistance of the battery, diminishing thereby the quantity of the current from each cell, but we augment the power of the integrated current to overcome external hindrances. The resistance of the battery itself may, indeed, be rendered so great that the external resistance shall vanish in comparison. What is here said regarding the voltaic battery is equally true of magneto-electric machines. If we wish our current to leap over five intervals, and produce five lights in succession, we must invoke a sufficient electro-motive force. This is done through multiplying, by the use of thin wires, the convolutions of the rotating armature as, a moment ago, we augmented the cells of our voltaic battery. Each alditional convolution, like each additional cell, adds

* As the result of a recent trial by Mr. Schwendler, they have been also chosen for India. 
its electro-motive force to that of all the others; and though it also adds its resistance, thereby diminishing the quantity of current contributed by each convolution, the integrated current becomes endowed with the power of leaping across the successive spaces necessary for the production of a series of lights in its course. The current is, as it were, rendered at once thinner and more piercing by the simultaneous addition of internal resistance and elertromotive power. 'The machines, on the other hand, which produce only a single light have a small internal resistance associated with a small electro-motive force. In such machines the wire of the rotating armature is comparatively short and thick, copper riband instead of wire being commonly employed. Such machines deliver a large quantity of electricity of low tension-in other words, of low leaping power. Hence, though competent when their power is converged upon a single interval, to produce one splendid light, their currents are unable to force a passage when the number of intervals is increased. Thus, by augmenting the convolutions of our machines we sacrifice quantity and gain electro-motive force; while by lessening the number of the convolutions we sacrifice electromotive force and gain quantity. Whether we onght to choose the one form of machine or the other depends entirely upon the external work the machine has to perform. If the object be to obtain a single light of great splendor, machines of low resistance and large quantity must be employed. If we want to obtain in the same circuit several lights of moderate intensity, machines of high internal resistance and of correspondingly high electromotive power must be invoked.

When a coil of covered wire surrounds a bar of iron, the two ends of the coil being connected together, every alteration of the magnetism of the bar is accompanied by the development of an induced current in the coil. 'The current is only excited during the period of magnetic change. No matter how strong or how weak the magnetism of the bar may be, as long as its condition remains permanent no current is developed. Conceive, then, the pole of a magnet placed near one end of the bar to be noved along it toward the other end. During the time of the pole's motion there will be an incessant change in the magnetism of the bar, and accompanying this change we 
shall have an induced current in the surrounding coil. If, instead of moving the magnet, we move the bar and its surrounding coil past the magnetic pole, a similar alteration of the magnetism of the bar will occur, and a similar current will be induced in the coil. You have here the fundamental conception which led M. Gramme to the construction of his beautiful machine.* He aimed at giving continuous motion to such a bar as we have here described; and for this purpose he bent it into a continuous ring, which, by a suitable mechanism, he caused to rotate rapidly close to the poles of a horseshoe magnet. 'The direction of the current varied with the motion and with the character of the influencing pole. The result was that the currents in the two semicircles of the coil surrounding the ring flowed in opposite directions. But it was easy, by the mechanical arrangement called a commutator, to gather up the currents and cause them to flow in the same direction. The first machines of Gramme, therefore, furnished direct currents, similar to those yielded by the voltaic pile. M. Gramme subsequently so modified his machine as to produce alternating currents. Such alternating machines are employed to produce the lights now exhibited on the Holborn Viaduct and Thames Embankment.

Another machine of great alleged merit is that of $M$. Lontin. It resembles in shape a toothed iron wheel, the teeth being used as cores, round which are wound coils of copper wire. 'The wheel is caused to rotate between the opposite poles of powerful electro-magnets. On passing each pole the core or tooth is strongly magnetized, and instantly evokes in its surrounding coil an induced current of corresponding strength. The currents excited in approaching to and retreating from a pole, and in passing different poles, move in opposite directions, but by means of a commutator these conflicting electric streams are gathered up and caused to flow in a common bed. The bobbins, in which the currents are induced, can be so increased in number as to augment indefinitely the power of the machine. 'To excite his electro-magnets, M. Lontin applies the principle of Mr. Wilde. A small machine

* “Comptes Rendus," 1871, p. 176. See also Gaugain on the Gramme machine, "Ann. de Chem. et de Phys.," vol. xxviii., p. 324. 
furnishes a direct current, which is carried round the electro-magnets of a second and larger machine. Wilde's principle, it may be added, is also applied on the 'Thames Embankment and the Holborn Viaduct; a small Gramme machine being used in each case to excite the electromagnets of the large one.

The Farmer-Wallace machine is also an apparatus of great power. It consists of a combination of bobbins for inducerl curients, and of inducing electro-magnets, the latter being excited by the method discovered by Siemens and Wheatstone. In the machines intended for the production of the electric light, the electro-motive force is so great as to permit of the introduction of several lights in the same circuit. A peculiarly novel feature of the Farmer-IVallace system is the shape of the carbons. Instead of rods, two large plates of carbons with beveled edges are employed, one above the other. 'The electric discharge passes from edge to edge, and shifts its position according as the carbon is dissipated. The duration of the light in this case far exceeds that obtainable with rods. I have myself seen four of these lights in the same circuit in Mr. Ladd's workshop in the City, and they are now, I beiieve, employed at the Liverpool Street Station of the Metropolitan Railway. The Farmer-Wallace "quantity machine" pours forth a flood of electricity of low tension. It is unable to cross the interval necessary for the production of the electric light, but it can fuse thick copper wires. When sent through a short bar of iridium, this refractory metal emits a light of extraordinary splendor.*

The machine of M. de Méritens, which he has generously brought over from Paris for our instruction, is the newest of all. In its construction he falls back upon the principle of the magneto-electric machine, employing permanent magnets as the exciters of the induced currents. Using the magnets of the Alliance Company, by a skillful disposition of his bobbins, M. de Méritens produces with eight magnets a light equal to that produced by forty magnets in the Alliance machines. While the space occupied is only one-fifth, the cost is little more than one-fourth

* The iridium light was shown by Mr. Ladd. It brilliantly illuminated the theater of the Royal Institution. 
of the latter. In the de Méritens machine the commu. tator is abolished. The internal heat is hardly sensible, and the absorption of power, in relation to the effects produced, is small. With his larger machines M. de Méritens maintains a considerable number of lights in the same circuit. *

In relation to this subject, inventors fall into two classes, the contrivers of regulators and the constructors of machines. M. Rapieff has hitherto belonged to inventors of the first class, but I have reason to know that he is engaged on a machine which, when complete, will place him in the other class also. Instead of two single carbon rods, M. Rapieff employs two pair's of rods, each pair forming a $\mathrm{V}$. The light is produced at the common junction of the four carbons. 'The device for regulating the light is of the simplest character. At the bottom of the stand which supports the carbons are two small electromagnets. One of them, when the current passes, draws the carbons together, and in so doing throws itself out of circuit,leaving the control of the light to the other. The carbons are caused to approach each other by a descending weight, which acts in conjunction with the electro-magnet. 'I'hrough the liberality of the proprietors of the Times, every facility has been given to M. Rapieff to develop and simplify his invention at Printing House Square. 'The illumination of the press-room, which I had the pleasure of witnessing, under the guidance of M. Rapieff himself, is extremely effectual and agreeable to the eye. There are, I believe, five lamps in the same circuit, and the regulators are so devised that the extinction of any lamp does not compromise the action of the others. M. Rapieff has lately improved his regulator.

Many other inventors might here be named, and fresh ones are daily crowding in. Mr. Werdermann has been long known in comnection with this subject. Employing as negative carbon a disc, and as positive carbon a rod, he has, I am assurel, obtained very satisfactory results. 'The small resistances brought into play by his minute ares enable $\mathrm{Mr}$. Werdermann to introduce a number of lamps

* The small machine transforms one-and-a-quarter horse-power into heat and light, yielding abont 1,900 candles; the large machine transforms five-horse power, yielding about 9,000 candles. 
into a circuit traversed by a current of only molerate electro-motive power. M. Reynier is also the inventor of a very beantiful little lamp, in which the point of a thin carbon rod, properly adjusted, is cansed to touch the circumference of a carbon wheel which rotates underneath the point. 'The light is developed at the place of contact of rod and wheel. One of the last steps, though I am informed not quite the last, in the improvement of regulators is this: 'The positive carbon wastes more profusely than the negative, and this is alleged to be due to the greater heat of the former. It occurred to Mr. William Siemens to chill the negative artificially, with the view of diminishing or wholly preventing its waste. 'This he accomplishes by making the negative pole a hollow cone of copper, and by ingeniously discharging a small jet of cold water against the interior of the cone. His negative copper is thus caused to remain fixed in space, for it is not dissipated, the positive carbon only needing control. I have seen this lamp in action, and can bear witness to its success.

I might go on to other inventions, achieved or projected. Indeed, there is something bewildering in the recent rush of constructive talent into this domain of applied electricity. 'The question and its prospects are modified from day to day, a steady advance being made toward the improvement both of machines and regulators. With regard to our public lighting, I strongly lean to the opinion that the electric light will at no distant day triumph over gas. I am not so sure that it will do so in our private houses. As, however, I am anxions to avoid dropping a word here that could influence the share market in the slightest degree, I limit myself to this general statement of opinion.

To one inventor in particnlar belongs the honor of the idea, and the realization of the idea, of causing the carbon rods to burn away like a candle. It is needless to say that I here refer to the young Russian officer, M. Jablochkoff. He sets two carbon rods upright at a small listance apart, and fills the space between them with an insulating substance like plaster of Paris. I'lie carbon rods are fixed in metallic holders. A momentary contact is established between the two carbons by a little cross-piece of the sime substance placed horizontally from top to top. This cross- 
piece is immediately dissipated or removed by the current, the passage of which once established is afterward maintained. 'The carbons gradually waste, while the substance between them melts like the wax of a candle. 'The comparison, however, only holds good for the act of melting; for, as regards the current, the insulating plaster is practically inert. Indeed, as proved by M. Rapieff and Mr. Wilie, the plaster may be dispensed with altogether, the current passing from point to point between the naked carbons. M. de Méritens has recently brought out a new candle, in which the plaster is abandoned, while between the two principal carbons is placel a third insulated rod of the same material. With the small de Méritens machine two of these candles can be lighted before you; they produce a very brilliant light. * In the Jablochkoff candle it is necessary that the carbons should be consumed at the sime rate. Hence the necessity for alternating currents by which this equal consumption is secured. It will be seen that M. Jablochkoff has abolished regulators altogether, introdncing the candle principle in their stead. In my julgment, the performance of the Jablochkoff candle on the 'Thames Embankment and the Holborn Viaduct is highly creditable, notwithstanding a considerable waste of light toward the sky. The Jabloch koff lamps, it may be added, would be more effective in a street, where their light, would be scattered abroad by the adjacent houses, than in the positions which they now occupy in London.

It was my custom some years ago, whenever I needed a new and complicated instrument, to sit down beside its proposed constructor, and to talk the matter over with Jim. The study of the inventor's mind which this habit opened out was always of the highest interest to me. I particularly well remember the impression made upon me on such occasions by the late Mr. Darker, a philosophical instrument maker in Lambeth. 'This man's life was a struggle, and the reason of it was not far to seek. No matter how commercially lucrative the work upon which

* The machine of M. de Méritens and the Farmer-Wallace machine were worked by an excellent gras-engine, leut for the occasion by the Messrs. Crossley, of Manchester. The Siemens machine was worked by steam. 
he was engaged might be, he would instantly turn aside from it to scize and realize the ideas of a scientific man. He had an inventor's power, and an inventor's delight in its exercise. 'The late Mr. Becker possessed the same power in a very considerable degree. On the Continent, Froment, Breguet, Sauerwald, and others might be mentioned as eminent instances of ability of this kind. Such minds resemble a liquid on the point of crystallization. Stirred by a hint, crystals of coustructive thought immediately shoot through them. 'T'hat Mr. Edison possesses this intuitive power in no common measure, is proved by what he has already accomplished. He has the penetration to seize the relationship of facts and principles, and the art to reduce them to novel and concrete combinations. Hence, though he has thus far accomplisherl nothing that we can recognize as new in relation to the electric light, an adverse opinion as to his ability to solve the complicated problem on which he is engaged would be unwarranted.

I will endeavor to illustrate in a simple manner $\mathrm{Mr}$. Edison's alleged mode of electric illumination, taking advantage of what $\mathrm{Ohm}$ has taught us regarding the laws of the current, and what Joule has tanght us regarding the relation of resistance to the development of light and heat. From one end of a voltaic battery runs a wire, dividing at a certain point into two branches, which reunite in a single wire connected with the other end of the battery. From the positive end of the battery the current passes first through the single wire to the point of junction, where it divides itself between the branches according to a well-known law. If the branches be equally resistant, the current divides itself equally between them. If one branch be less resistant than the other, more than half the current will choose the freer path. The strict law is that the quantity of current is inversely proportional to the resistance. A clear image of the process is derived from the deportment of water. When a river meets an island it divides, passing right and left of the obstacle, and afterward reuniting. If the two branch beils be equal in depth, width, and inclination, the water will divide itself equally between them. If they be unequal, the larger quantity of water will flow through the more open course. And, as in the case of the water we 
mily have an indefinite number of islands, producing an indefinite subdivision of the trunk strean, so in the case of elestricity we may have instead of two branches, any number of branches, the current dividing itself among them, in accordance with the law which fixes the relation of How to resistance.

Let us apply this knowledge. Suppose an insulated colper rod, which we may call an "electric main," to be laid down along one of our streets, say along the Strand. Let this rod be connected with one end of a powerful voltaic battery, a good metallic connection being established between the other end of the battery and the water-pipes under the street. As long as the electric main continues unconnected with the water-pipes, the circuit is incomplete and no current will flow; but if any part of the main, however distant from the battery, be comnected with the adjacent water-pipes, the circuit will be completed and the current will flow. Supposing our battery to be at Charing Cross, and our rod of copper to be tapped opposite Somerset House, a wire can be carried from the rod into the building, and the current passing through the wire may be subdivided into any number of subordinate branches, which reunite afterward and return through the water-pipes to the battery. The branch currents may be employed to raise to vivid incandescence a refractory metal like iridium or one of its alloys. Instead of being tapped at one point, our main may be tapped at one hundred points. The current will divide in strict accordance with law, its power to produce light being solely limited by its strength. The process of division closely resembles the circulation of the blood; the electric main carrying the outgoing current representing a great artery, the water-pipes carrying the return current representing a great vein, while the intermediate branches represent the various vessels by which the blood is distributed through the system. This, if I understand aright, is $\mathrm{Mr}$. Edison's proposed mode of illumination. The electric force is at hand. Metals sufficiently refractory to bear being raised to vivid incandescence are also at hand. The principles which regulate the division of the current and the development of its light and heat are perfectly well known. There is no room for a "discovery," in the scientific sense of the term, but there is ample room for the exercise of that mechanical ingenuity 
which has given us the sewing machine and so many other useful inventions. Knowing something of the intricacy of the practical problem, I should certainly prefer seeing it in Mr. Edison's hands to having it in mine.*

It is sometimes stated as a recommendation to the electric light, that it is light without heat; but to disprove this, it is only necessary to point to the experiments of Davy, which show that the heat of the voltaic arc transcends that of any other terrestrial source. The emission from the carbon points is capable of accurate analysis. To simplify the subject, we will take the case of a platinum wire at first slightly warmed by the current, and then gradually raised to a white heat. When first warmed, the wire sends forth rays which have no power on the optic nerve. They are what we call invisible rays; and not until the temperature of the wire has reached nearly 1,000 degrees Fahr., does it begin to glow with a faint, red light. 'The rays which it emits prior to redness are all invisible rays which can warm the hand but cannot excite vision. When the temperature of the wire is raised to whiteness, these dark rays not only persist, but they are enormously angmented in intensity. They constitute about 95 per' cent. of the total radiation from the white-hot platinum wire. They make up nearly 90 per cent. of the emission from a brilliant electric light. You can by no means have the light of the carbons without this invisible emission as an accompaniment. 'The visible radiation is as it were, built upon the invisible as its necessary foundation.

It is easy to illustrate the growth in intensity of these invisible rays as the visible ones enter the radiation and angment in power. The transparency of the elementary gases and metalloids-of oxygen, hydrogen, nitrogen, chlorine, iodine, bromine, sulphur, phosphorus, and even of carbon, for the invisible heat rays is extraordinary. Dissolved in a proper vehicle, iodine cuts the visible radiation sharply off, but allows the invisible free transmission. By dissolving iodine in sulphur, Professor Dewar has recently added to the number of our effectual rayfilters. The mixture may be made as black as pitch for

* More than thirty years ago the radiation from incandescent platinum was admirably investigated by Dr. Draper of New York. 
the visible, while remaining transparent for the invisible rays. By such filters it is possible to detach the invisible rays from the total radiation, and to watch their augmentation as the light increases. Expressing the radiation from a platinum wire when it first feels warm to the touchwhen, therefore, all its rays are invisible-by the number 1 , the invisible radiation from the same wire raised to a white heat may be 500 or more. * It is not, then, by the diminution or transformation of the non-luminous emission that we obtain the luminous; the heat rays maintain their ground as the necessary antecedents and companions of the light rays. When detached and concentrated, these powerful heat rays can produce all the effects ascribed to the mirrors of Archimedes at the siege of Syracuse. While incompetent to produce the faintest glimner of light, or to affect the most delicate air-thermometer, they will inflame paper, burn up wood, and even ignite combustible metals. When they impinge upon a metal refractory enough to bear their shock without fusion, they can raise it to a heat so white and luminous as to yield, when analyzed, all the colors of the spectrum. In this way the dark rays emitted by the incandescent earbons are converted into light rays of all colors. Still, so powerless are these invisible rays to excite vision, that the eye has been placed at a focus competent to raise platinum foil to bright redness without experiencing any visual impression. Light for light, no doubt, the amount of heat imparted by the incandescent carbons to the air is far less than that imparted by gas flames. It is less, because of the smaller size of the carbons, and of the comparative smallness of the quantity of fuel consumed in a given time. It is also less because the air cannot penetrate the carbons as it penetrates a flame. The temperature of the flame is lowered by the admixture of a gas which constitutes four-fifths of our atmosphere, and which, while it appropriates and diffuses the heat, does not aid in the combustion; and this lowering of the temperature by the inert atmospheric nitrogen renders necessary the cumbustion of a greater amount of gas to produce the necessary light. In fact, though the statement may appear paradoxical, it is entirely because of its enormous actual temperature that the 
electric light seems so cool. It is this temperature that renders the proportion of luminous to non-luminous heat greater in the electric light than in our brightest flames. The electric light, moreover, requires no air to sustain it. It glows in the most perfect air vacuum. Its light and heat are therefore not purchased at the expense of the vitalizing constituent of the atmosphere.

'I'wo orders of minds have been implicated in the development of this subject; first, the investigator and discoverer, whose object is purely scientific, and who cares little for practical ends; secondly, the practical mechanician, whose object is mainly industrial. It would be easy, and probably in many cases true to say that the one wants to gain knowledge, while the other wishes to make money; but I am persuaded that the mechanician not unfrequently merges the hope of profit in the love of his work. Members of each of these classes are sometimes scornful toward those of the other. 'There is, for example, something superb in the disdain with which Cuvier hands over the discoveries of pure science to those who apply them: "Your grand practical achievements are only the easy application of truths not sought with a practical intenttruths which their discoverers pursued for their own sake, impelled solely by an ardor for knowledge. 'I'hose who turned them into practice could not have discovered them, while those who discovered them had neither the time nor the inclination to pursue them to a practical result. Your rising workshops, your peopled colonies, your vessels which furrow the seas; this abundance, this lixury, this tumult," - "this commotion," he would have added, were he now alive, "regarding the electric light"_-"all come from discoveries in science, though all remain strange to them. The day that a discovery enters the market they abandon it; it concerns them no more."

In writing thus, Cuvier probably did not sufficiently take into account the reaction of the applications of science upou science itself. The improvement of an old instrument or the invention of a new one is often tantamount to an enlargement and refinement of the senses of the scientific investigator. Beyond this, the amelioration of the community is also an object worthy of the best efforts of the human brain. Still, assuredly it is well and wise for a nation to bear in mind that those practical 
applications which strike the public eye, and excite pubic almiration, are the outgrowth of long antecedent labors begun, continued, and ended, under the operation of a purely intellectual stimulus. "Few," says Pasteur, "seem to comprehend the real origin of the marvels of industry and the wealth of nations. I need no other proof of this than the frequent employment in lectures, speeches, and official language of the erroneous expression, 'applied science.' A statesman of the greatest talent stated some time ago that in our day the reign of theoretic science had rightly yielded place to that of applied science. Nothing, I venture to say, could be more dangerous, even to practical life, than the consequences which might flow from these words. They show the imperious necessity of a reform in our higher education. 'There exists no category of sciences to which the name of 'applied science' could be given. We have science and the applications of science which are united as tree and fruit."

A final reflection is here suggested. We have among us a small cohort of social regenerators-men of high thoughts and aspirations-who would place the operations of the scientific mind under the control of a hierarchy which should dictate to the man of science the course that he ought to pursue. How this hierarchy is to get its wisdom they do not explain. They decry and denounce scientific theories; they scorn all reference to ether, and atoms, and molecules, as subjects lying far apart from the world's needs; and yet such ultra-sensible conceptions are often the spur to the greatest discoveries. 'The source, in fict, from which the true natural philosopher derives inspiration and unifying power is essentially ideal. Faraday lived in this ideal world. Nearly half a century ago, when he first obtained a spark from the magnet, an Oxford don expressed regret that such a discovery should have been made, as it placed a new and facile implement in the hands of the incendiary. To regret, a Comtist hierarchy would have probably added repression, sending Faraday back to his bookbinder's bench as a more dignified and practical sphere of action than peddling with a magnet. And yet it is Faraday's spark which now shines upon our coasts, and promises to illuminate our streets, halls, quays, squares, warehouses, and, perhaps at no listant day, our homes.

THE END. 


\title{
BURT'S LIBRARY
}

\author{
OF THE
}

\section{WORLD'S BEST BOOKS}

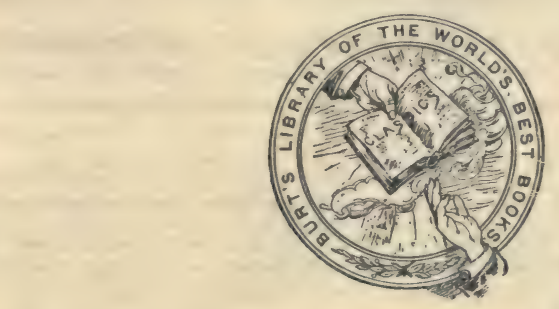

A series which will include the standard works of the voorld's literature. The books will be neatly and durubly bound, printed on good paper, in large, clear type, uniform, in all respects with this volume. The text will be in every case thoroughly reliable and unabridyed, hence mecting equally the needs of the student and general reader. Indexes, biographical sketches and explanatory notes will be givein where these are likely to prove of value and interest. All who have at heart the best interests of literature, and would further the spread of a taste for the world's classics, will velcoms the appearance of this series, which will pluce a complete, rich and uniform library of the highest character within the reach of all. 


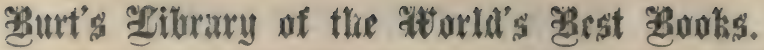

The Essays or Counsels Civil and Moral of Francis Bacon. First published in 1597 , and as he left them newly written and published in 1625. Including also his A pophthegms, Elegant Sentenees, and Wisdom of the Ancients. With an introduction by IIENRY Mortey, Ll.D., Professor of English Literature at University Col. lege, London. Portrait. 12mo, cloth, gilt top, $\$ 1.00$.

They (Bacon's Essays) contain the condensed wisdom gathered during the whole long life-time of one of the mightiest minds of modern times. - Frank Pursons.

Vanity Fair. A Novel Without a Hero. By Wmimam Make. PEACE 'T'HACKeray. Portrait, $12 \mathrm{mo}$, eloth, gilt top, $\$ 1.00$.

As a whole the book is full of quiet sarcasm and severe rebuke. It is replete with humor and morality, and rivets attention to the end by the vivid reality of all the persuns and scenes. - From "A Manual of English Literature" by T, B. Shaw.

Other Worlds Than Ours. The plurality of worlds studied under the light of recent scientific researches. By RICIAARD A. Proctor. With an introductory note by Frank Parsons. Portrait. Cloth, gilt top, $\$ 1.00$.

Like Huxley and Tyndall, Mr. Proetor sees the poetry of his subject and knows how to bring the largest trutlis within the comprehension of a clild, and make the deepest researches as interesting to the general reader as a novel.-Frank Parsons.

The Fifteen Decisive Battles of the World, from Marathon to Waterloo. By E. S. Creasy, M.A., Professor of Ancient and Mod. ern History in University College, London; late Fellow of King's College, Cambridge. With an introductory note by FranK PARsons. Portrait. $12 \mathrm{mo}$, cloth, gilt top, $\$ 1.00$.

So vivid are his descriptions that one feels as though he were present at the seene himself, listering to the counsels of the generals, hearing the tread of marching columns, watching the gleaming spears and bayonets, armies of infantry, charying cavalry, breach, rally and retreat, deafened with the roar of batteries, saddented by the death of friends, and flushed with triumph; and at last the reader lays the bouk away exhausied with the rush of feeling through his heart.-Frank Parsons.

The Essays of Elia. By Cirarles Lamb. With an introduc. tion and notes by Alfred Ainger, and a Biographical Sketch of Charles Lamb, by Henry Morley. Portrait. 12mo, cloth, gilt top, $\$ 1.00$.

The Essays of Elia have been characterized as the "finest things for humor taste, penetration and vivacity which have appeared since the days of Montaigne." In his bits of criticism charles Lamb shows a most delicate and acute eritical faculty; in his few poems, much graces and sweetness, but first and foremost, he is an essayist of rare power. The refined wit, genuine pleas. antry, deep and tender pathos, and subtle diserimination of his essays, are unexcelled by any compositions in the language.-Robert Thorme.

Essays. By Raiph Waldo Emerson. First and second series. Portrait. 12mo, cloth, gilt top, $\$ 1.00$.

He exercised a great power over men; he brought them wide comfort, and to him more than to any man of his time belongs the glory of having taught them that life was worth the living.-From the "Optimism of Emerson." by $W$. E. Dana.

For sale by ail binoksellers, or will be sent post-paid on receipt of price, by the pub. iisher, A. L. BUIT, 66 Reade Street, New York. 


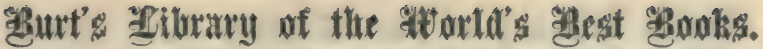

Faust. By johann Wolfgang von Goethe. Complete in two parts. Translated by Anna Swanwick. Portrait. Cloth, gilt top, $\$ 1.00$.

Deeper meanings are disoovered with every reading, and familiarity does not cause it to grow trite, but ever the more strongly to lay hold on the soul with the irresistible fascination of an eternal problem and the charm of an endless variety.-Robert Thorne.

The Sketch-Book of Geoffrey Crayon, Gent. By Washington inving. With an introductory note by Frank Parsons. Portrait. Cloth, gilt top, $\$ 1.00$.

The book is refined, pnetical and picturesque, full of quaint humor, exquis ite feeling, and a thorough knowledge of human nature.-Fr'ank Pursons.

Lorna Doone. A Romance of Exmoor. By R. D. BLAckmone. $12 \mathrm{mo}$, cloth, gilt top, $\$ 1.00$.

These wonderfully reproduced scenes, and the men and women with whom they are peopled, and finally the beautiful language in which the narrative is set forth, unite to make a delightful, and, what is more, a wholesome, invigorating, inspiring book. - E. S. Howes.

Hypatia, or New Foes with an Old Face. By Charles Kings. LEY, F.S.A., F.L.S. Portrait. $12 \mathrm{mo}$, cloth, gilt top, $\$ 1.00$.

The plot is well developed, the characters are vigorously drawn, and the scenes and incidents show great dramatie power, while the language and word-painting are exquisite. The book holds throughout, with a firm grasp, our syrapathy and interest, Kingsley being one of the very few who have sue. ceeded in throwing a strong human interest into a historical novel.-Robert Thorne.

Romola. By George Eliot. Portrait. 12mo, cloth, gilt top, $\$ 1.00$.

George Ellot is admitted by thoughtful persons to have been endowed with one of the greatest minds of this century. . . . Romola, which is one of her earlier works, is also one of the most popular. The morement is so rapid, and the situations are so dramatic, that the interest never flags; . . . the book has nowhere the air of tiresome preaching, but it stands the test of a great novel-it may be read again and again with pleasure, $-E$. S. Hawes.

The Data of Ethics. By Herbert Spencer. Portrait. 12mo, eloth, gilt top, $\$ 1.00$.

Herbert Spencer is the foremost name in the philosophic literature of the world. He is the Shakespeare of science. He has a grander grasp of knowledge and more perfect conscious correspondence with the external universe than any other human being who ever looked wonderingly out into the starry depths: and his few errors flow from an orer-anxiety to exert his splendid power of making beautiful generalizations. Plato and spencer are brothers. Plato would have done what Spencer has had be lived in the nineteenth cen tury.-From "The World's Best Books," by Frank Parsons.

The Origin of Species, by Means of Natural Selection, or the Preservation of a Favored Race in the Struggle for Life. By Charles DARWIN, M.A., LL.D., F.R.S. Portrait. 12mo, cloth, gilt top, $\$ 1.00$.

This hook is the grandest achievement of modern scientific thought and research. It has passed through many editions in Furlish, has been translated into almost all the languages of Europe, and has neen the subject of more reviews, pamphlets and separate books than any other volume of the age.Robert Thorne.

For sale by all Booksellers, or will be sent post-paid on receipt of price, by the publisher, A. L. BURT, G6 Reade Street, New York. 


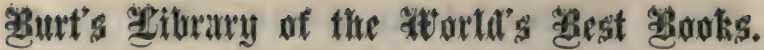

Past and Present. By Thomas Carlyie, with an introductory note by Robert 'T'horne, M.A. Portrait. $12 \mathrm{mo}$, cloth, gilt top, $\$ 1.00$.

His (Carlyle's) bidding is to do the allotted work of life silently and bravely. and there is probably no person who has not gained strength by the reading of his strong and earnest writings,-Robert Thorne.

The History of Civilization in Europe. By Francois Pierre Guillaume Guizot. Translated by William Hazlitt, with a biographical sketch of the author. Portrait. 12mo, cloth, gilt top, $\$ 1.00$.

These lectures made a profound impression at the time they were delivered and published, and indeed marked an epoch in the history of edueation, rais. ing the reputation of their author at onee to the highest point of fame, and placing him among the best writers of France and of Europe.-Robert Thorne.

Ivanhoe. A Romance. By Sir Walter Scott, Bart. Reprinted from the author's edition, unaltered and unabridged. Portrait. $12 \mathrm{mo}$, cloth, gilt top, $\$ 1.00$.

Ivanhoe is one of the most famous and brilliant of all the master romances of Sir Walter Scott, who is placed by many at the head of modern novelists. . T The breadth and power of Scott's style and his charm as a story-teller are too well known to need cemment, and in this volume we have him at his best.-Robert Thorne.

The Vicar of Wakefield, The Traveller, and The Deserted Village. By OLIVER Goldsmith. With a Life of Goldsmith by William Black. Portrait. 12mo, eloth, gilt top, $\$ 1.00$.

The style is easy and delightful. The humor is delicate and all good humor; there is hardly a trace of satire or ill-nature in the whole book, which is a true expression of the spirit of Goldsmith himself, one of the most lovable personalities in the world of letters.-Robert Thorne.

The Discourses of Epictetus, with the Encheiridion and Fragments. 'Translated with notes, a life of Epictetus, a view of his philosophy, and index. By George Long, M.A. Portrait. 12mo, cloth, gilt top, $\$ 1.00$.

Great purity, sustained reflection, wealth of illustration and allusion, vivid revelations of eharacter and brilliant bursts of eloquence, mark the utterances of this great teacher and insure their immortality.-Frank Parsons.

The Crown of Wild Olive and Sesame and Lilies. By JoHN Ruskin, LL.D. Portrait. $12 \mathrm{mo}$, cloth, gilt top, $\$ 1.00$.

As a great and fearless leader of thought and antagonistic to many features of our social order, he is naturally the object of nuch violent criticism, but is warmly admired and loved by a great part of the reading world, and coming ages will aecord him his due. He has told the world new truth and the world will grow up to his majestic stature.-Robert Thome.

The Meditations of the Emperor Marcus Aurelius Antoninus. Translated by GEORGE LONA, M.A., with a biographical sketch and a view of the philosophy of Antoninus by the translator. Including also an essay on Marcus Aurelius by Canon Farrar. Portrait. 12mo, cloth, gils top, \$1.00.

"The noblest book of antiquity" is Canon Farrar's estimate of the "Meditations of Mareus Aurelius;" and his regard is shared by thousands who bave been made better and trucr men by the enmobling influence of the great soul and lofty character of this pagan emperor.-Roberl Thorne.

Fin sale by all Booksellers, or will be sent post-paid on receipt of price, by the pub. lisher, A. L. BURT, 66 Reade Street, New York. 
John Halifax, Gentleman. A Norel. By Mrss Mulock. Portrait. $12 \mathrm{mo}$, cloth, gilt top, $\$ 1.00$.

The book is from the pen of one who combines a careful study of life with a rare genius in depicting its real experiences, and who renders charming even a simple story of actual life, by the glory of a warm and loving heart with which she transfuses it.-Frederick Mynon Cooper.

Undine and Other Tales. By De LA Motre Fouque. Trans lated from the German by F. E. BUnNeTt. Portrait. 12mo, cloth, gilt top, $\$ 1.00$.

Undine has become a household book for old and young in Germany, and has been translated into almost every European language. There is in it a simplieity of style unsurpassed, and plenty of sweet pathos which wets the eye but never wrings the heart.-Henry Prentice.

Uarda, A Romance of Ancient Egypt. By George EBers. Translated from the German by CLARA BELL. 12mo, cloth, gilt top, $\$ 1.00$.

Amid all the tempest of passion and expectation incidental to such a tale the novelist evolves a charming story of love and constancy rising superior to class prejudices, and of the sweet amenities of social ties and family affection. - Frederick Myron Cooper.

Confessions of an English Opium-Eater and Selected Essays. By Thomas De Quincey. Edited with notes by David Masson, Professor of English Literature in the University of Edinburgh. Portrait. 12mo, cloth, gilt top, $\$ 1.00$.

De Quincey's skill in narration, his rare pathos, his wide sympathies, the pomp of his dream-deseriptions, his abounding though subtle humor, commend him to a large class of readers. - Encyclopedia Britannica.

On the Heights. By Berthold Auerbach. Translated from the German by F. E. BunnetT. Portrait. 12mo, cloth, gilt top, $\$ 1.00$.

Auerbach has been called the Charles Dickens of Germany. He is not only a brilliant writer of fiction, but is at the same time a profound thinker and elevated moralist. In "On the Heiglits," his most powerful work, education, labor, wealth, poverty, and the relations of rich and poor; aristocracy, religfon and philosophy, the rights of the individual, and their various applications to our daily life, are illumed and illustrated by its progress and development. It is a beautiful story, sad in its ending, but free from any tinge of coarseness or sensationalism; pure, sweet, warm with human love and tenderness. Erederick Mynon Cooper.

The Last Days of Pompeii. By Sir Edward Bulwer-LytTon, Bart. Pertrait. $12 \mathrm{mo}$, eloth, gilt top, $\$ 1.00$.

The fate of the rich Campanian eity, the most awful catastrophe whieh history records, supplies a superb climax to the story. This is dramatic and powerful throughout, and of absorbing interest. The characters arise naturally from the scene of the story, and they move and speak in perfect accord with their surroundings; with a human sympathy which easily bridges the eighteen centuries which have rolled over the buried city, we follow with eager interest this tale of the men and women of ancient Pompeil-Robert Thorne.

For sale by all Booksellers, or will te sent post-paid on receipt of price, by the pub kisher, A. L. BURT, 66 Reade street, New York. 
Westward Ho! or, The Voyages and Adventures of Sir Amyas Leigh, Knight. By C'HAklé Kingsiey. Portrait. 12mo, cloth, gilt top, $\$ 1.00$.

"Westward Ho!" is one of the nost vigorous, powerful, and fascinating of novels. It is strong and graphice in its pertraiture, intense and drantatic in its diversitiel coloring. 'The nervous and effective style, the skilful blending of the matsifuld protraits inte one comprehensive picture, are anomg the merits which have male this Kingsley's greatest work. - rrederic Mynon Conper.

The Pilgrim's Progress. By Joun Bunyan, with a life of Bun yan by James Anthony Froude. Portrait. 12mo, cloth, gilt top, $\$ 1.00$.

No other book execut the Bible has gone through so many eolitions and at tained to so wile a popularity in all lansuages as "The: I'ilgrim's l'rogrens." . It narrates the strugses, the experiences, and the trials of a cliristian in his pasiage from a life of sin to everlasting felicity: and it aboumls with those little inimitable tourhes of natural feeling and description which have placed its author among the most picturesque of writers. . . . J3unyan may truly be called the prince of allegrorists, and he is also the most perfect revresentative of the piain, vigorous, idimatic, and sometimes picturesque and poetical language of the common people.-Taken from "A Ilanual of Einglish Literature," by T. B. Shaw.

Self-Help, with Illustrations of Character, Conduct, and Perseverance. By SAMUEL SMILES. Portrait. 12mo, cloth, gilt top, $\$ 1.00$.

"Self-Help" is a burk which helps and stimulates men to elevate and improre themselves. It teaches them that the humblest person who sets before lis fellows an example of industry, subriety, and upright honesty of purpose in life, has a present as well as a fiture influence upou the well-being of his eountry. - Humblreds of its terse and happy phrases have become the common property of mankind, and it has been already translated into four or five of the European languages.-Frederic Mynon Cooper.

Jane Eyre. By C'harlotte Bronté. Portrait. 12mo, cloth, gilt top, $\$ 1.00$.

Few novels have gained such immediate popularity as was accorded to "Jane Evre." This was doubtless due in part to the freshness and vigor of mind it evincerl ; but it was obtained not so muceli by these quatities as hy the frecuent dealings in moral paradox, and by the hardilood of its assauls ujom the joejudices of proper people. Throughout the tale the author exlithits a percention of character and the power of delineating it, which is, considering her youth, remarkable.-Frederic Mynon Cooper.

The Moonstone. A Novel. By Wilkie Collins. Portrait. $12 \mathrm{mo}$, cloth, gilt top, $\$ 1.00$.

Iike the generality of his romances, the interest of "The Monnstone" depends chiefly upon the development of a plot whose systematic intricucies pigue the curiosity until the last moment, and upon the conceglment of a mystery which haffles and defies solution until it shall bave contributed to no end of cross purposes and caused a prodigious amount of incertitude and wretchedness.Frederic Mynon Cooper.

For sale by all Bonksellers, or will be sent post-paid on receipt of price. by the pub. isher, A. I. BURT, 66 Reade Street. New York. 


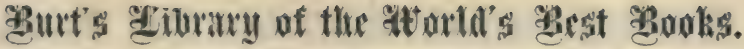

A Thousand Miles Up The Nile. By Ameli B. Edwands. Portrait. 12mo, illustrated, cloth, gilt top, $\$ 1.00$.

The Mill on the Floss. By George Eliot. Portrait. 12mo, cloth, gilt top, $\$ 1.00$.

This is a charmine story of middle-class English life, for which George Eliot is justly celebratert. . . " "The Mill on the Floss" commends itself strongly to the reader by its tine analyses of motives, its vivid force in description and its quality as a work of literary art.

The Adventures of Oliver Twist. By Charles Dickens. Portrait. $12 \mathrm{mo}$, cloth, gilt top, $\$ 1.00$.

It is in the Enclish parochial work-house that we first meet oliver, and his sufferings while under the charge of that benign creature, Mr. Bumble, are alone sufficient to secure for him our warmest sympathy. . . . There is passion and feeling in every page of the book, and it can be read; not alone once, but again and again, with renewed delight.

The Holy Roman Empire. By James Bryce, D. C. L. Portrait. $12 \mathrm{mo}$, cloth, gilt top, $\$ 1.00$.

"The Holy Roman Empire" is a work of great learning, and is universally conceded to show a high degree of historical power, thoush written at au early age it immediately established the reputation of the distinguished author as one of the most profound thinkers of the century, and has steadily grown into the highest favor with scholars.

Daniel Deronda. By George Eliot. Portrait, 12mo, cloth, gilt top, $\$ 1.00$.

"Daniel Deronda " is a love story, but at the same time a treasure-house of information regarding the manners, customs, and traditions of the Hebrew race. It belongs to the enduring literature of the age, durable, not for the fashionableness of its pattern, but for the texture of its stuff.

Corinne; or, Italy. By MAdAme De Stael. Portrait. 12mo, cloth, gilt top, $\$ 1.00$.

"Corimue," the success of which was instant, and won for the author a really European reputation, is a love story which emphasizes strength and nolility of character and purity of life. The seene of the tale is laid principally in Italy and interspersed throughout the narrative are vivid glimpses of Italian scenery. life, manners, and its historical and literary remains.

The Divine Comedy; or, Vision of Hell, Purgatory and Paradise. By Dante Alighieri. Translated by the Rev. Henry Francis Cary, M. A. Portrait. 12mo, cloth, gilt top. $\$ 1.00$.

The Divina Comedia is one of the grandest monuments of human genius, with the epics of Homer and Milton it forms a supreme trinity of poens, which have summer up the spirit of great eras of civilization and formed the educa tion of succeeding centuries.

Consuelo. By George Sand. Portrait. 12mo, cloth, gilt top, $\$ 1.00$.

In the character of Consuelo Marame Sand has pictured for us a woman as chaste, as noble and as lovable as any in all fiction. . . " Consuelo" is an ideal romance of remarkable power and fascination and it will long live a monument to its author's genius.

For sale by all Bonksellers, or will be sent jost-paid on receipt of price, by the put lisher, A. L. BURT, 66 Reade $8 t .9$ New York. 


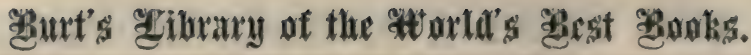

The Descent of Man. By Charles Darwin. Portrait. 12mo, illustrated, cloth, gilt top, $\$ 1.00$.

The Life and Adventures of Nicholas Nickleby. By Charles Dickens. Portrait. $12 \mathrm{mo}$, cloth, gilt top, $\$ 1.00$.

Nicholas, the hero of the tale is a young man of impetuous temper, not always blameless in his actions, indeed, not always agreoable, yet upon the: whole, so manly, so honest and so lovable, that we overionk his faults, and sympathize with him in his misfortunes, and rejoice with him in his successes.

Lucile. By Owen Merediti (Edward Robert Bulwer-Lytton) Portrait. 12mo, cloth, gilt top, $\$ 1.00$.

In the character of Lucile we have the author's highest and purest embodiment of intellect and virtue. First subduing her own nature, she is content to spend all the treasures of her life and genius in offices of weil-iloing, and from the heart of a woman thoroughly true and good, and ever roariy for self-sactfice, she finally diffuses bealth and strength into the hearts of all aruund her.

The Posthumous Papers of the Pickwick Club. By Charles Drckens. Portrait. $12 \mathrm{mo}$, cloth, gilt top $\$ 1.00$.

The Pickwick Papers chronicle the travels and adventures of the immortal Mr. Pickwick and his fellow members of the Pickwick (clul, and the varied pictures of life through which we follow the kind oid bachelor, his three friends and his attached servant, the inimitable Sam Weller, are of absorbing interest.

First Principles. By Herbert Spencer. Portrait. 12mo, cloth, gilt top, $\$ 1.00$.

The Personal History of David Copperfield. By CHARles Dickens. Portrait. $12 \mathrm{mo}$, cloth, gilt top, $\$ 1.00$.

David Copperfield is a novel full of tenderness and purity of feeling. and in it Dickens presents to the full that comprehensiveness of sympathy which springs from a sense of brotherhood with all mankind.

The Old Curiosity Shop. By Charles Dickens. Portrait. $12 \mathrm{mo}$, cloth, gilt top, $\$ 1.00$.

"The Old Curiosity Shop " abounds with vivid deseriptions of human life and character, and the reader's attention is held until the very end . . . Of all of Jickens' works there is none which appeals more strongly to our heart than this story of childish abnegation and devotion.

Middlemarch: A Study of Provincial Life. By George ELIOT. Portrait. 12mo, cloth, gilt top, $\$ 1.00$.

"Middlemarch" is a study of Provincial life, and is unquestionably one of the strongest of English novels. . . I It is a picture, vast, swarminin, deen? colored, crowded with episodes, with vivid images, with lurking master strokes. with brilliant passages of expression, and as such we may freely accept and enjoy it.

The Life of Christ. By Frederic W. Farrar, D.D., F. R. S. Portrait. $12 \mathrm{mo}$, cloth, gilt top, $\$ 1.00$.

Great ability, ripe literary skill, graphic description and a fine spiritual insight are conspicuous in every chapter and taken altogether it is the most marked of all the many attempts in our own days to present to us the human life of the Savior of mankind.

For sale by all Booksellers, or will be sent post-paid on receipt of price, by the pub Gher, A. L. BURT, 66 Reade st., New York. 


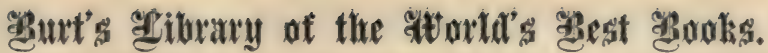

An Egyptian Princess. By George Ebers. Portrait. 12mo, cloth, gilt top, $\$ 1.00$.

Kenilworth. By Sir Walter Scott. Portrait. 12mo, cloth, gilt top, $\$ 1.00$.

The History of Henry Esmond, Esq. By WiLliam MAKEPEACE Thackeray. Portrait. 12mo, cloth, gilt top, $\$ 1.00$.

Mosses from an Old Manse. By Nathaniel Hawthorne. Portrait. 12mo, eloth, gilt top, $\$ 1.00$.

The Light of Asia, or The Great Renunciation. By EDwn Aknold, M.A. Portrait. $12 m o$, cloth, gilt top, $\$ 1.00$.

Les Miserables. A Novel. By Victor Hugo. Illustrated. Two vols., $12 \mathrm{mo}$, cloth, gilt top, each $\$ 1.00$.

The Count of Monte Cristo. By Alexandre Dumas. Illustrated. Two vols., $12 \mathrm{mo}$, cloth, gilt top, each $\$ 1.00$.

Heroes, Hero-Worship and the Heroic in History. By Thомas CARLYLE. Portrait. 12mo, cloth, gilt top, $\$ 1.00$.

Around the World in the Yacht Sunbeam. By Mrs. Brasser. Portrait. $12 \mathrm{mo}$, cloth, gilt top, $\$ 1.00$.

Picciola, or the Prison Flower. By X. B. Sarntine. Portrait. 12mo, cloth, gilt top, $\$ 1.00$.

The Scarlet Letter. By Natraniel Hawthorne. Portrait. $12 \mathrm{mo}$, cloth, gilt top, $\$ 1.00$.

East Lynne. Ry Mrs. Henry Wood. Portrait. 12mo, cloth, gilt top, $\$ 1.00$.

The Woman in White. By WILkIE Colisins. Portrait. 12mo, cloth, gilt top, $\$ 1.00$.

For sale by all Bonksellers, or will be sent post-paid on receipt of price, by the pub. lisher, A. L. BURT, 66 Reade st., New York. 







\section{BINDING SECT. JUN 131983}

\section{PLEASE DO NOT REMOVE CARDS OR SLIPS FROM THIS POCKET}

\section{UNIVERSITY OF TORONTO LIBRARY}

Physical \&

Applied Sci. 


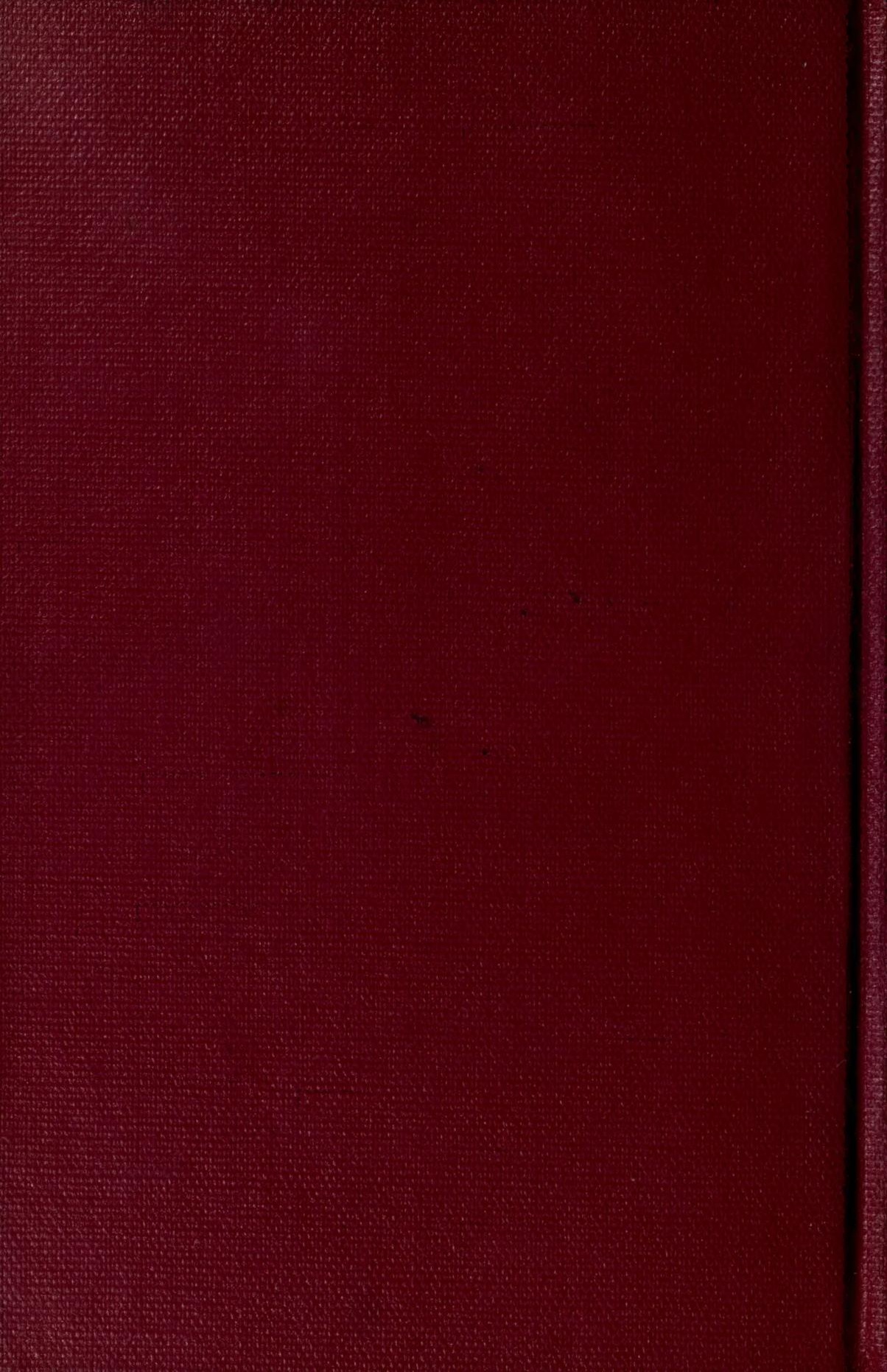

